\title{
Hydrologic Investigations Concerning Lead Mining Issues in Southeastern Missouri
}

Scientific Investigations Report 2008-5140

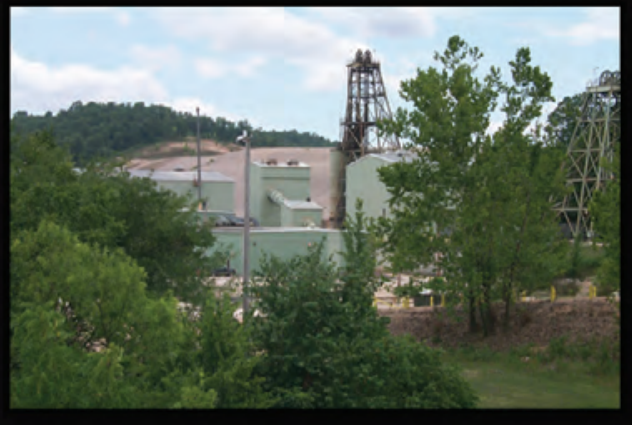


Cover photographs. Current River (photograph courtesy of David J. Weary, U.S. Geological Survey). Insets: Top, Blue Spring near Owls Bend (photograph courtesy of Jeffrey L. Imes, U.S. Geological Survey). Middle, Woodland crayfish (photograph courtesy of John M.

Besser, U.S. Geological Survey). Bottom, West Fork Mine (photograph courtesy of Michael J. Kleeschulte, U.S. Geological Survey). 


\title{
Hydrologic Investigations Concerning Lead Mining Issues in Southeastern Missouri
}

\author{
Edited by Michael J. Kleeschulte
}

Contributions by Cheryl M. Seeger, Michael J. Kleeschulte, Lopaka Lee, Gary W. Krizanich, Suzanne R. Femmer, and John G. Schumacher

Volume comprises Chapters 1, 2, 3, 4, 5, 6, and 7

Scientific Investigations Report 2008-5140 


\title{
U.S. Department of the Interior DIRK KEMPTHORNE, Secretary
}

\author{
U.S. Geological Survey \\ Mark D. Myers, Director
}

U.S. Geological Survey, Reston, Virginia: 2008

This and other USGS information products are available at http://store.usgs.gov/
U.S. Geological Survey
Box 25286, Denver Federal Center
Denver, CO 80225
To learn more about the USGS and its information products visit http://www.usgs.gov/
1-888-ASK-USGS

Any use of trade, product, or firm names is for descriptive purposes only and does not imply endorsement by the U.S. Government.

Although this report is in the public domain, permission must be secured from the individual copyright owners to reproduce any copyrighted materials contained within this report.

Suggested citation:

Kleeschulte, M.J., ed., 2008, Hydrologic investigations concerning lead mining issues in southeastern Missouri: U.S. Geological Survey Scientific Investigations Report 2008-5140, 238 p. 


\section{Forward}

Good stewardship of our Nation's natural resources demands that the extraction of exploitable, minable ore deposits be conducted in harmony with the protection of the environment, a dilemma faced by many land and water management agencies in the Nation's mining areas. As ore is mined, milled, and sent to the smelter, it leaves footprints where it has been in the form of residual trace metals. Often these footprints become remnants that can be detrimental to other natural resources. This emphasizes the importance of understanding the earth's complex physical and biological processes and their interactions at increasingly smaller scales because subtle changes in one component can substantially affect others. Understanding these changes and resulting effects requires an integrated, multidisciplinary scientific approach.

As ore reserves are depleted in one area, additional exploitable deposits are required to replace them, and at times these new deposits are discovered in previously unmined areas. Informed decisions concerning resource management in these new, proposed mining areas require an understanding of the potential consequences of the planned mining actions. This understanding is usually based on knowledge that has been accumulated from studying previously mined areas with similar geohydrologic and biologic conditions. If the two areas experience similar mining practices, the information should be transferable.

Lead and zinc mining along the Viburnum Trend Subdistrict of southeastern Missouri has occurred for more than 40 years. Additional potentially exploitable deposits have been discovered 30 miles to the south, within the Mark Twain National Forest. It is anticipated that the observation of current (2008) geohydrologic conditions in the Viburnum Trend can provide insight to land managers that will help reasonably anticipate the potential mining effects should additional mining occur in the exploration area.

The purpose of this report is to present a compilation of previously unpublished information that was collected as part of a larger multidisciplinary study of lead mining issues in southeastern Missouri. The report resulted from the application of a multidisciplinary approach to investigate current hydrologic and biologic conditions in streams of the Viburnum Trend and the exploration area in the Mark Twain National Forest.

Michael E. Slifer

Director, Missouri Water Science Center 



\section{Volume Contents}

1. History of Mining in the Southeast Missouri Lead District and Description of Mine Processes, Regulatory Controls, Environmental Effects, and Mine Facilities in the Viburnum Trend Subdistrict. 1

by Cheryl M. Seeger

2. Seepage Runs on Streams Draining the Viburnum Trend Subdistrict, Southeastern Missouri, August 2003-0ctober 2006

by Michael J. Kleeschulte

3. Distribution of Mining-Related Trace Elements and Sulfide-Mineral Occurrence in Streambed Sediment of the Viburnum Trend Subdistrict and Non-Mining Areas, Southeastern Missouri, 1992-2002

by Lopaka Lee

4. Spatial and Temporal Patterns of Trace-Element Deposition in Bed Sediment from Clearwater Lake, Southeastern Missouri, 2002.

by Gary W. Krizanich

5. National Water-Quality Assessment (NAWQA) Program Black River Synoptic Study, Southeastern Missouri, 1993 and 1995 by Suzanne R. Femmer

6. Water Quality of the Viburnum Trend Subdistrict, Exploration Area, and Strother Creek, Southeastern Missouri, 1964-2006.

by Michael J. Kleeschulte

7. Water-Quality Trends and Effects of Lead and Zinc Mining on Upper Logan Creek and Blue Spring, Southeastern Missouri, 1925-2006 .193

by John G. Schumacher 


\section{Conversion Factors, and Datums}

Inch/Pound to SI

\begin{tabular}{|c|c|c|}
\hline Multiply & By & To obtain \\
\hline \multicolumn{3}{|c|}{ Length } \\
\hline inch (in.) & 2.54 & centimeter $(\mathrm{cm})$ \\
\hline inch (in.) & 25.4 & millimeter $(\mathrm{mm})$ \\
\hline foot $(\mathrm{ft})$ & 0.3048 & meter $(\mathrm{m})$ \\
\hline mile (mi) & 1.609 & kilometer $(\mathrm{km})$ \\
\hline \multicolumn{3}{|c|}{ Area } \\
\hline acre & 4,047 & square meter $\left(\mathrm{m}^{2}\right)$ \\
\hline square mile $\left(\mathrm{mi}^{2}\right)$ & 2.590 & square kilometer $\left(\mathrm{km}^{2}\right)$ \\
\hline \multicolumn{3}{|c|}{ Volume } \\
\hline gallon (gal) & 3.785 & liter (L) \\
\hline million gallons (Mgal) & 3,785 & cubic meter $\left(\mathrm{m}^{3}\right)$ \\
\hline \multicolumn{3}{|c|}{ Flow rate } \\
\hline foot per second (ft/s) & 0.3048 & meter per second $(\mathrm{m} / \mathrm{s})$ \\
\hline cubic foot per second $\left(\mathrm{ft}^{3} / \mathrm{s}\right)$ & 0.02832 & cubic meter per second $\left(\mathrm{m}^{3} / \mathrm{s}\right)$ \\
\hline \multicolumn{3}{|c|}{ Mass } \\
\hline ounce, avoirdupois (oz) & 28.35 & $\operatorname{gram}(\mathrm{g})$ \\
\hline pound, avoirdupois (lb) & 0.4536 & kilogram (kg) \\
\hline ton, short $(2,000 \mathrm{lb})$ & 0.9072 & megagram (Mg) \\
\hline ton per day (ton/d) & 0.9072 & metric ton per day \\
\hline
\end{tabular}

Temperature in degrees Celsius $\left({ }^{\circ} \mathrm{C}\right)$ may be converted to degrees Fahrenheit $\left({ }^{\circ} \mathrm{F}\right)$ as follows:

$$
{ }^{\circ} \mathrm{F}=\left(1.8 x^{\circ} \mathrm{C}\right)+32
$$

Temperature in degrees Fahrenheit $\left({ }^{\circ} \mathrm{F}\right)$ may be converted to degrees Celsius $\left({ }^{\circ} \mathrm{C}\right)$ as follows:

$$
{ }^{\circ} \mathrm{C}=\left({ }^{\circ} \mathrm{F}-32\right) / 1.8
$$

Vertical coordinate information is referenced to the North American Vertical Datum of 1988 (NAVD 88).

Horizontal coordinate information is referenced to the North American Datum of 1983 (NAD 83).

Altitude, as used in this report, refers to distance above the vertical datum.

Specific conductance is given in microsiemens per centimeter at 25 degrees Celsius $(\mu \mathrm{S} / \mathrm{cm}$ at $\left.25^{\circ} \mathrm{C}\right)$.

Concentrations of chemical constituents in water are given either in milligrams per liter (mg/L) or micrograms per liter ( $\mu \mathrm{g} / \mathrm{L})$. 


\section{Acronyms}

\begin{tabular}{|c|c|}
\hline ANC & Acid Neutralizing Capacity \\
\hline BM-60 & Streambed Sediment Sampler \\
\hline C3 & Non-magnetic Heavy Minerals Fraction in Streambed-Sediment Samples \\
\hline DGLS & Division of Geology and Land Survey \\
\hline DH-81 & Hand-held, Depth Integrating Water-Quality Sampler \\
\hline EPT & Ephemeroptera, Plecoptera, and Tricoptera \\
\hline EWI & Equal-Width Increment \\
\hline HWP & Hazardous Waste Program \\
\hline ICP-AES & Inductively Coupled Plasma-Atomic Emission Spectrometry \\
\hline ICP-MS & Inductively Coupled Plasma Mass Spectrometry \\
\hline IOR & Interquartile Range \\
\hline LDS & Latter Day Saints \\
\hline LRP & Land Reclamation Program \\
\hline MAG-1 & Marine Mud from Gulf of Mexico \\
\hline MDC & Missouri Department of Conservation \\
\hline MRL & Method Reporting Level \\
\hline MVT & Mississippi Valley Type \\
\hline $\mathbf{N}$ & Normal \\
\hline NAWQA & National Water-Quality Assessment \\
\hline NPDES & National Pollution Discharge Elimination System \\
\hline NWOL & National Water Quality Laboratory \\
\hline ONSR & Ozark National Scenic Riverways \\
\hline PEC & Probable Effects Concentration \\
\hline PVC & Polyvinyl Chloride \\
\hline RCRA & Resource Conservation and Recovery Act \\
\hline RSD & Relative Standard Deviation \\
\hline SCUBA & Self-Contained Underwater Breathing Apparatus \\
\hline TDS & Total Dissolved Solids \\
\hline TEC & Threshold Effects Concentration \\
\hline USGS & U.S. Geological Survey \\
\hline WMA & Waste Management Area \\
\hline
\end{tabular}





\section{History of Mining in the Southeast Missouri Lead District and Description of Mine Processes, Regulatory Controls, Environmental Effects, and Mine Facilities in the Viburnum Trend Subdistrict}

By Cheryl M. Seeger ${ }^{1}$

Chapter 1 of

Hydrologic Investigations Concerning Lead Mining Issues in Southeastern Missouri

Edited by Michael J. Kleeschulte

${ }^{1}$ Missouri Department of Natural Resources, Division of Geology and Land Survey

Scientific Investigations Report 2008-5140 


\section{Contents}

Abstract

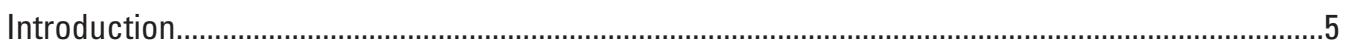

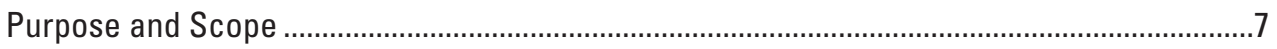

Previous and Current Studies ....................................................................................................

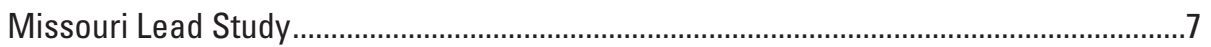

U.S. Geological Survey Studies .............................................................................

Other Viburnum Trend Studies........................................................................................

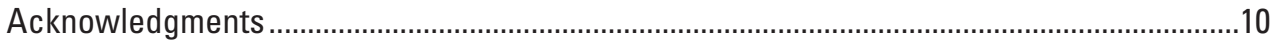

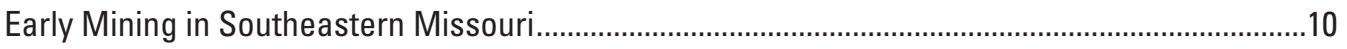

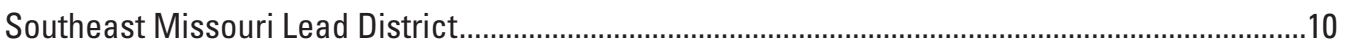

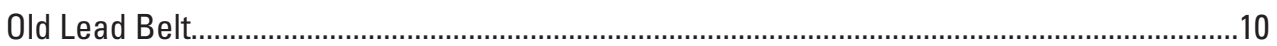

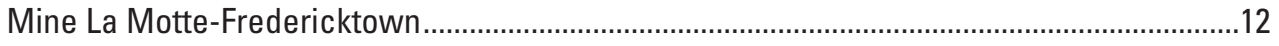

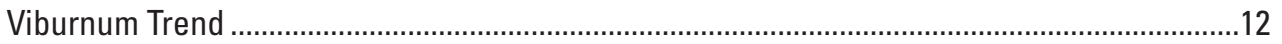

Other Lead-Zinc Subdistricts of the Southeast Missouri Lead District..................................13

General Mining Processes, Regulatory Controls, and Environmental Effects in the

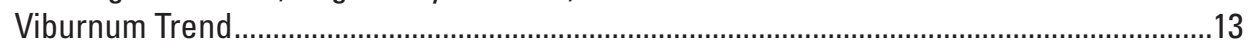

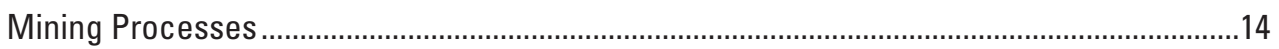

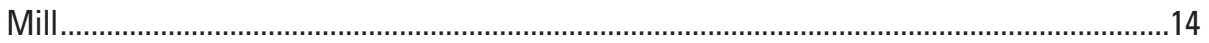

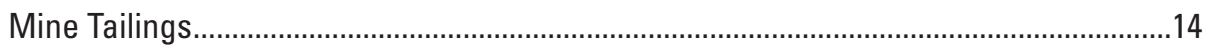

Transportation of Ore and Concentrate ………………..........................................15

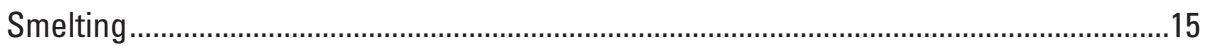

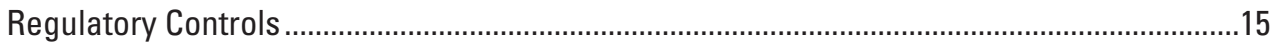

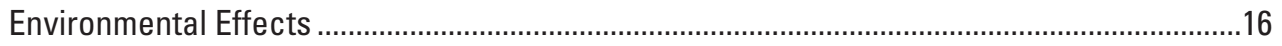

Mine Facility Descriptions in the Viburnum Trend Subdistrict.....................................................17

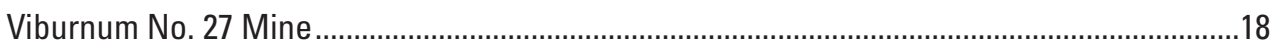

Viburnum No. 28 Mine and Central Mill ................................................................................

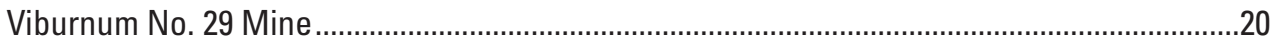

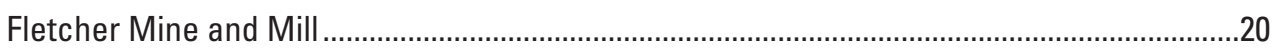

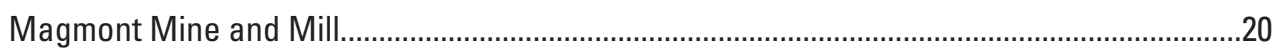

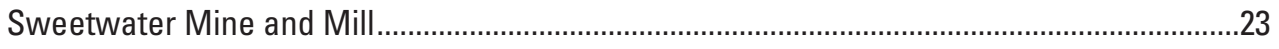

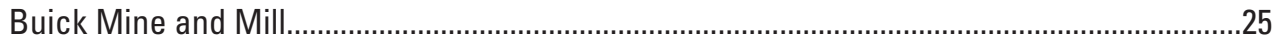

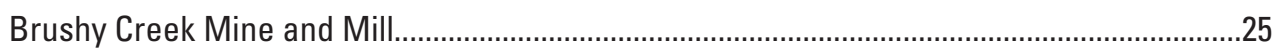

Casteel Mine (Viburnum No. 35 Mine)..............................................................................26

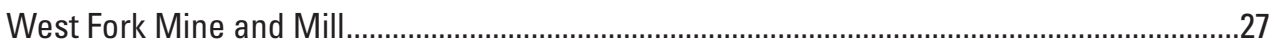

Buick Resource Recovery Facility (Buick Smelter) ...............................................................30

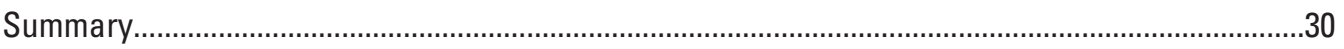

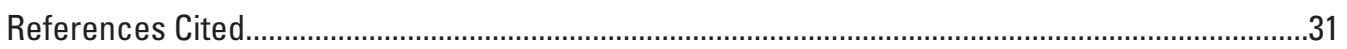




\section{Figures}

1-2. Maps showing-

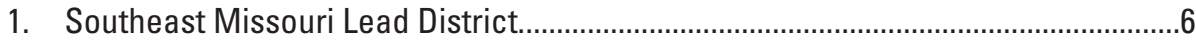

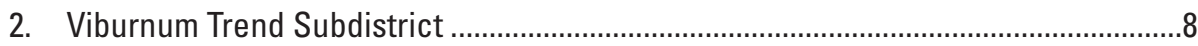

3. Stratigraphic column for an exploration hole in Reynolds County, Missouri, and a general lithologic description of formations in the Viburnum Trend...............................11

4-14. Aerial photographs showing-

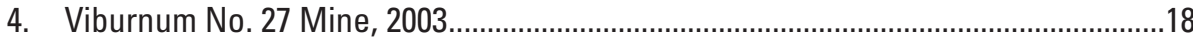

5. Viburnum No. 28 Mine and Central Mill, 2003 …………........................................19

6. Viburnum No. 29 Mine, 2003..........................................................................21

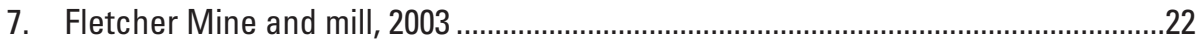

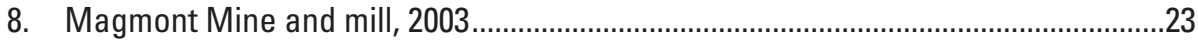

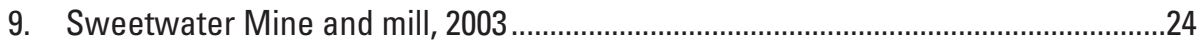

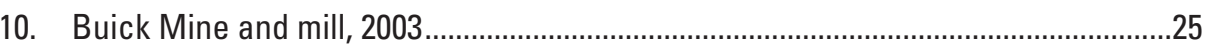

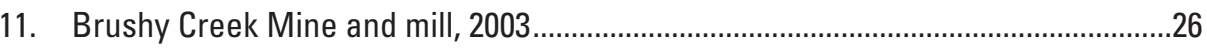

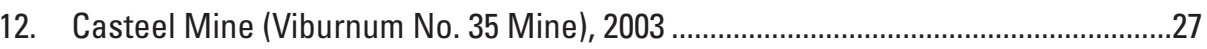

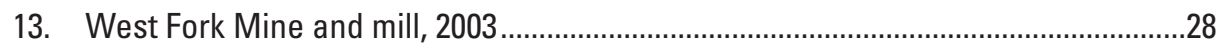

14. Buick Resource Recovery Facility (Buick Smelter), 2003 .....................................29

\section{Table}

1. Summary of features and facilities at the Viburnum Trend mine complexes. 



\title{
History of Mining in the Southeast Missouri Lead District and Description of Mine Processes, Regulatory Controls, Environmental Effects, and Mine Facilities in the Viburnum Trend Subdistrict
}

\author{
By Cheryl M. Seeger ${ }^{1}$
}

\section{Abstract}

Missouri has three world-class lead/zinc subdistricts (Old Lead Belt, Mine La Motte-Fredericktown, and Viburnum Trend) and several minor subdistricts that are in a region referred to as the Southeast Missouri Lead District. Arsenic, cadmium, cobalt, copper, lead, nickel, and zinc are the primary trace elements associated with the sulfide minerals of the Mississippi Valley Type ore deposits present in the district. As more ore bodies were discovered, a relation between Precambrian structural highs and mineralization that developed in complex carbonate facies in the surrounding Bonneterre Formation was observed. This observation aided in the discovery of the Viburnum Trend Subdistrict.

Production began in the Viburnum Trend in 1960; 10 mines eventually were opened in the subdistrict. Galena $(\mathrm{PbS})$ is the primary ore mineral, and sphalerite $(\mathrm{ZnS})$ is the second most common ore mineral. Total ore production from individual mines range from 20 to more than 50 million tons, and contain as much as 8 percent lead and about 3 percent zinc.

Mining and milling processes have remained essentially the same throughout the history of the Viburnum Trend, although the mills have undergone changes and improvements for recovery enhancement or for recovery of additional metals. Mining is done using a room and pillar method that follows the ore trend. Of the 10 mines in the Viburnum Trend, 7 have or had mills onsite to concentrate the ore. Milling processes follow four major stages; crushing and grinding, flotation, filtering and dewatering, and tailings disposal. All mills in the Viburnum Trend have lead and zinc circuits; most mills had or have copper circuits. Mine tailings (waste rock) from the milling process are contained in a surface disposal area (tailings pond) and consists of gangue minerals (primarily dolomite) and minor amounts of ore minerals; flotation reagents also may be present.

\footnotetext{
${ }^{1}$ Missouri Department of Natural Resources, Division of Geology and Land Survey.
}

The Missouri Department of Natural Resources, Hazardous Waste Program inspects facilities for violations of hazardous waste handling. The primary environmental issue for The Missouri Department of Natural Resources, Land Reclamation Program is wind-blown transport of tailings material outside the waste management area. Inspections of other Viburnum Trend mines and the Buick Smelter have indicated no violations since the mid-1990s. The Hazardous Waste Program has one Superfund action in the Viburnum Trend area-public highways used as transportation routes by trucks hauling ore mineral concentrates to smelters or shipment points.

Waste products from mining, milling, and smelting have affected the environment immediately surrounding the operation. The environmental effects of concern in the Viburnum Trend include wind-blown dust or water-transported trace elements from ore mineral concentrates and tailings, deposition from gases related to smelting, and unusual widespread growth of benthic bacterial/algal mats downstream from operations.

\section{Introduction}

Missouri has a long and diverse mining heritage, beginning with Native Americans, followed by French-Canadian explorers, and later by European settlers. Lead and fur were the most important exports from Missouri during its early years as a Spanish, French, and then United States territory (Burford, 1978). Southeastern Missouri, with the largest known concentration of galena (lead sulfide) in the world, was the site of the first prolonged mining in the state and has produced lead almost continuously since 1721 . It has three world-class lead/zinc subdistricts surrounding the St. Francois Mountains (fig. 1): the Old Lead Belt; the Mine La MotteFredericktown, which also produced nickel, copper, silver, and cobalt; and the Viburnum Trend, currently (2008) producing lead, zinc, copper, and silver. The Old Lead Belt is north of the St. Francois Mountains, Mine La Motte-Fredericktown is 


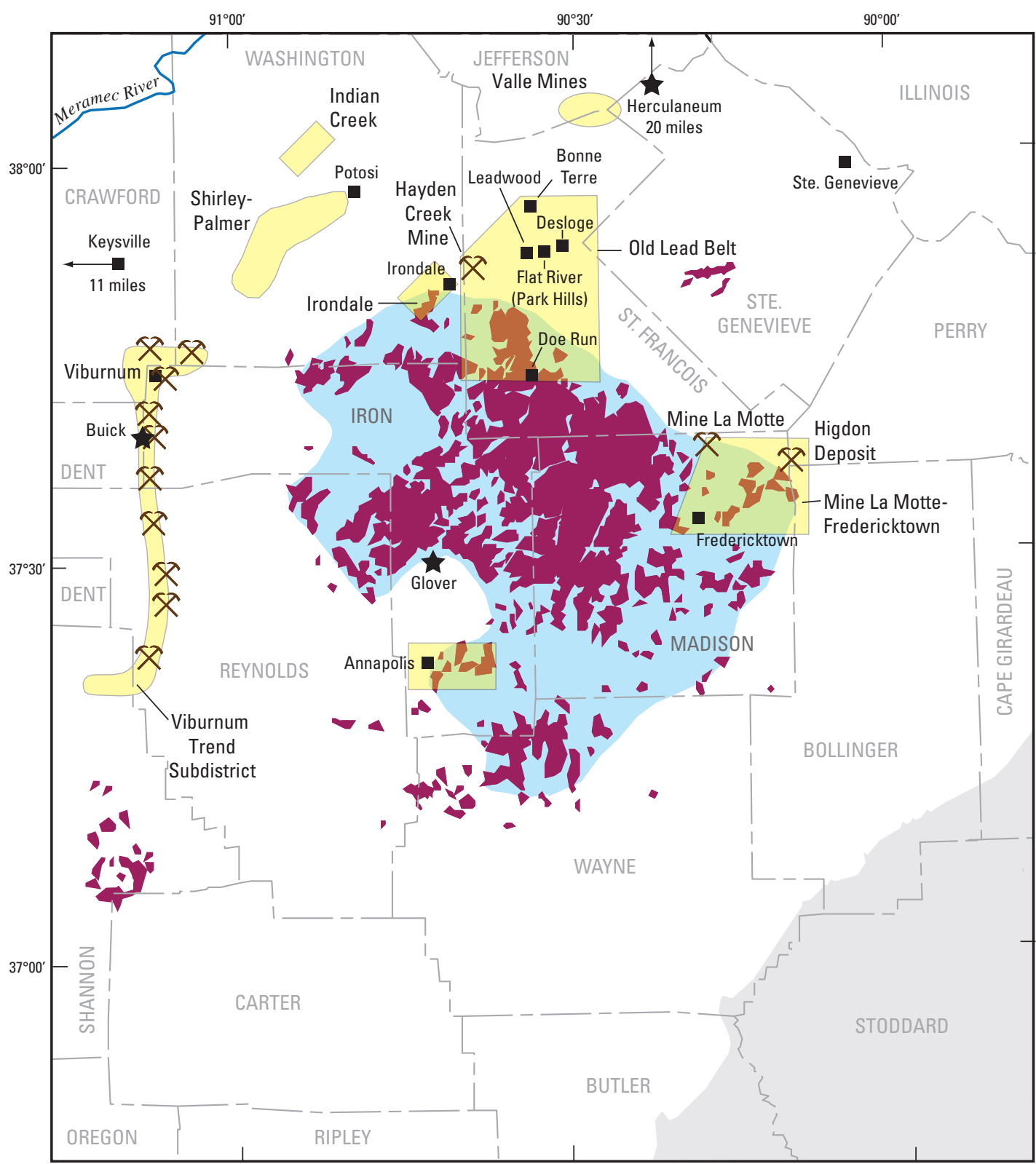

Base from U.S. Geological Survey digital data, 1:100,000, 1994 Universal Transverse Mercator projection,

Subdistrict boundaries from Snyder and Gerdemann, 1968 Zone 15

\section{EXPLANATION}

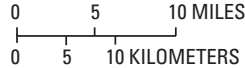

Physiographic areas
Mississippi Lowlands
Salem Plateau
St. Francois Mountains
Precambrian outcrop

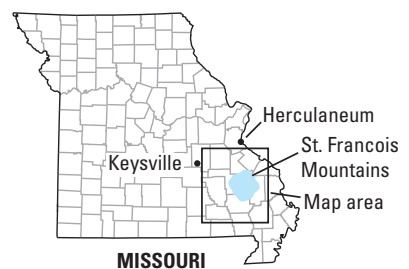

Figure 1. Southeast Missouri Lead District. 
to the east, and the Viburnum Trend is to the west. The entire region is referred to as the Southeast Missouri Lead District.

The deposits of the Southeast Missouri Lead District are a class known as Mississippi Valley Type (MVT) deposits. Although MVT deposits are named for the region in the United States where they were first described and studied, they occur on every continent of the world (Leach and Sangster, 1993). The MVT deposits of the Southeast Missouri Lead District are metal-sulfide deposits that are hosted in Paleozoic dolostone, limestone, and to a lesser extent sandstone. Arsenic, cadmium, cobalt, copper, lead, nickel, and zinc are the primary trace elements associated with the sulfide minerals of MVT. The primary metal-sulfide minerals of these deposits are pyrite-marcasite $\left(\mathrm{FeS}_{2}\right)$, galena $(\mathrm{PbS})$, and sphalerite $(\mathrm{ZnS})$. In localized areas, mixed-metal sulfide minerals also occur in minor amounts. These include copper sulfide minerals such as bornite $\left(\mathrm{Cu}_{5} \mathrm{FeS}_{4}\right)$, chalcopyrite $\left(\mathrm{CuFeS}_{2}\right)$, and enargite $\left(\mathrm{Cu}_{3} \mathrm{AsS}_{4}\right)$; nickel sulfide minerals such as millerite $(\mathrm{NiS})$ and vaesite $\left(\mathrm{NiS}_{2}\right)$; and iron sulfide minerals such as arsenopyrite (FeAsS) (Rakovan, 2007).

Missouri has been and continues to be the largest lead producer in the United States, and generally ranks in the top five states in the nation for zinc production (U.S. Geological Survey, 2008). The Old Lead Belt and the Viburnum Trend also account for most of historical and current copper production from the State. Missouri copper production often ranks in the top 10 states in the country based on production from Viburnum Trend mines (U.S. Geological Survey, 2008). Silver recovered during smelting of the Viburnum Trend deposits causes Missouri to be ranked in the top 10 silver producing states in the nation. Cobalt and nickel are present in the Viburnum Trend ore deposits, but are not recovered (Denis Murphy, The Doe Run Company, written commun., 2007).

\section{Purpose and Scope}

This chapter briefly describes the history of lead and zinc mining in the Southeast Missouri Lead District, including location of mining activity, the extent of mineralization, and the period during which each subdistrict was mined. Emphasis is placed on the Viburnum Trend, which is an active subdistrict. A general description of the mine processes, regulatory controls, environmental effects, and mine facilities in the Viburnum Trend is provided to explain possible contaminant sources and transport routes of mining-related materials to the surrounding environment.

\section{Previous and Current Studies}

Numerous environmental studies have been done in the Old Lead Belt, Mine La Motte-Fredericktown, and Viburnum Trend in southeastern Missouri. Most have been in the Old Lead Belt. The Viburnum Trend has been the focus of two primary investigations, the Missouri Lead Study (Wixson, 1977) and studies by the U.S. Geological Survey (USGS). Stud- ies completed as part of the Missouri Lead Study that have been referenced in this chapter are Wixson and others (1972), Escalera (1973), Wixson and Anderson (1973), Bolter and others (1974a), Gale (1974), Jennett (1974), Butherus (1975), and Arseneau (1976). Several studies unrelated to or built upon the Missouri Lead Study also are discussed in this section. Studies completed by the USGS that are referenced in this chapter include Kleeschulte (2001), Kleeschulte and Seeger (2003), Femmer (2004), and Kleeschulte (2006).

\section{Missouri Lead Study}

The most comprehensive study previously completed for the Viburnum Trend is the Missouri Lead Study. The study was done between 1972 and 1977 by an interdisciplinary team from the University of Missouri-Rolla (now the Missouri University of Science and Technology), the University of Missouri-Columbia, and mining companies operating in the Viburnum Trend. The study covered the major aspects of air and water quality, and soil, geochemical, and vegetation studies; remote sensing data also were examined. Air-quality studies examined air-quality characteristics, source concentrations and characterization, transport of trace-element particulates, and distribution of trace element particulates around the Buick Smelter near the Magmont Mine (fig. 2) (Wixson, 1977).

Water-quality studies examined chemical and biological aspects of the streams. Chemical analyses of samples determined the effects of mining on surface water and streambed sediment, trace organic material from mining and milling, toxicity of milling reagents, and sorption and desorption by soils in contact with water and industrial wastes. Biologic aspects included the concentration of trace elements in vegetation, toxicity of reagents to aquatic life, and the potential for Clearwater Lake (fig. 2) to be a sink for trace elements. Studies were done in Strother Creek, Bee Fork of the Black River, and Crooked Creek Basins (Wixson, 1977).

Soil and geochemical studies examined the distribution patterns of trace elements near possible contamination sources, the chemical and mineralogical properties of baghouse and flue dusts from the Buick Smelter, and identification of contamination sources. Studies also examined the accumulation of lead and other trace elements in vegetation in the vicinity of the mine operations, lead contamination along roadways, and lead accumulation in specific vegetable crops (Wixson, 1977).

\section{U.S. Geological Survey Studies}

The USGS performed several studies concerning the hydrology in the Viburnum Trend. For most of the approximately 40 years the mines have been in operation along the Viburnum Trend, 27 million gallons (Mgal) of water per day have been pumped from the St. Francois aquifer for mine dewatering (Kleeschulte, 2001). To determine if mine dewatering was affecting water levels in the shallower Ozark aquifer, the USGS compared a pre-mining (before 1960) potentiomet- 


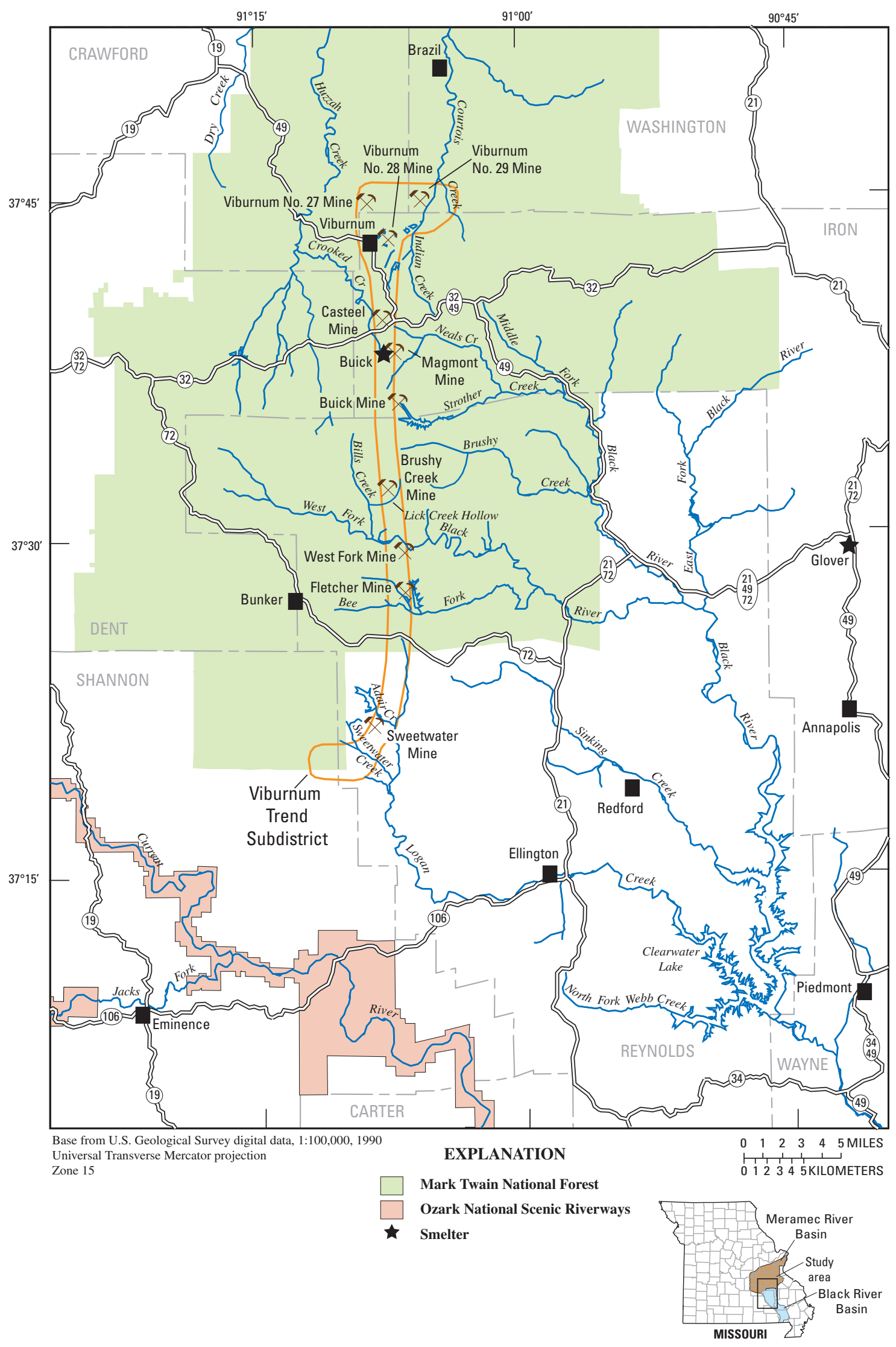

Figure 2. Viburnum Trend Subdistrict. 
ric surface to a 1999 potentiometric surface for the Viburnum Trend. The report concluded no large cones of depression are apparent in the potentiometric surface of the Ozark aquifer in the Viburnum Trend as a consequence of mining activity. Leakage of water from the Ozark aquifer into the St. Francois aquifer probably is occurring at shafts, ventholes, and inadequately plugged exploration drill holes; therefore, there may be localized areas of small drawdowns.

A deficiency of the previous ground-water level report was that there were few water-level measurements directly over the active mine works (Kleeschulte, 2006). During 2001, eight monitoring wells were installed along the Viburnum Trend directly over or in proximity to active mine works. Results of this study reinforced the conclusions of Kleeschulte (2001) in that in most wells no long-term, continuous waterlevel declines occurred from 2002 to 2005. One well did have a substantial water-level decline [about 30 feet $(\mathrm{ft})$ ] that developed during the study. This indicated that small areas of drawdown in the Ozark aquifer may be present along the Viburnum Trend as a result of mine dewatering.

The St. Francois confining unit separates the shallow ground water in the Ozark aquifer from that in the deeper St. Francois aquifer. The vertical hydraulic conductivity of the confining unit in the Viburnum Trend was compared to a potential lead and zinc exploration area about 30 miles (mi) south that has similar geohydrologic characteristics as the Viburnum Trend (Kleeschulte and Seeger, 2003). The report concluded that based on the calculated vertical hydraulic conductivity ranges, the St. Francois confining unit is considered 'tight', meaning the unit does not readily transmit water, at both locations. The effective vertical hydraulic conductivity range for the St. Francois confining unit in the Viburnum Trend is $2 \times 10^{-13}$ to $3 \times 10^{-12}$ foot per second (ft/s).

Femmer (2004) studied baseline conditions of streams in non-mining basins of the Viburnum Trend, stream reaches upstream from mining activity, and a lead and zinc exploration area in Shannon and Oregon Counties about $30 \mathrm{mi}$ south of the Viburnum Trend. Biologic, water-quality, and streambedsediment data were collected for three sites in the Viburnum Trend in 1995; similar data were collected for four sites in the exploration area in 2001. Water samples were analyzed for specific conductance, $\mathrm{pH}$, temperature, dissolved oxygen, concentrations of major ions, trace elements, nutrients, and bacteria densities. Streambed-sediment samples also were collected and analyzed for major and trace elements.

Fish-tissue samples were collected at one site in the Viburnum Trend and one site in the exploration area, and both were assessed for bioaccumulation of trace elements. Benthic invertebrate community data were collected and habitat characteristics noted. Results indicated that, with the exception of potassium, dissolved major ion concentrations generally were larger in samples from the exploration area than in samples from the Viburnum Trend sites. Trace-element concentrations were small in all samples, but did not differ greatly between sample areas; in general, concentrations were slightly larger in samples from the exploration area. Most samples were less than minimum reporting levels for nutrients. Habitat characteristics varied from site to site in both areas, with differences in substrate, level of disturbance, canopy, and woody plant densities. The report concluded that "...background conditions at sites in the exploration study area are not substantially different from non-mining sites in the Viburnum Trend study area in relation to nutrients, trace elements, streambed sediment, and fish tissue. Data for physical parameters, major ions, bacteria, and habitat characteristics indicated slight differences between the two study areas. Invertebrate communities were diverse and demonstrated differences between study areas" (Femmer, 2004).

\section{Other Viburnum Trend Studies}

Tibbs (1969) determined baseline concentrations for copper, lead, and zinc in streams in the Viburnum Trend area. This work was incorporated as part of a larger project in the Missouri Lead Study by Bolter and others (1974b). Constituent concentrations were not stated to be either dissolved or total; however, the concentrations determined for lead, zinc, and copper ranged from 1 to 20 milligrams per liter (mg/L), with most concentrations from 4 to $6 \mathrm{mg} / \mathrm{L}$. Other reported concentrations were calcium ( 1.5 to $27 \mathrm{mg} / \mathrm{L}$ ), potassium ( 0.5 to 0.9 $\mathrm{mg} / \mathrm{L}$ ), sodium ( 0.8 to $5 \mathrm{mg} / \mathrm{L}$ ), and manganese [as much as 30 $\mu \mathrm{g} / \mathrm{L}$ (micrograms per liter)]. The $\mathrm{pH}$ values ranged from 7.0 to 8.8 (Bolter and others, 1974b).

Fernandes (1987) conducted geochemical and bioassay tests on mine and process waters to study the effects of lead, zinc, and cadmium on water quality and stream biota. The study reported that mine and process waters increased the hardness derived from calcium of receiving stream waters from 55 to 70 percent of the total hardness.

Erten (1988) discussed the adequacy of the Buick Mine meander system that modified Strother Creek downstream from the tailings dam. The constructed oscillating channel lowers stream velocities, thus increasing the detention time of water discharged from the tailings pond into the stream. The study noted that the trace elements mainly were transported in a particulate state. Major removal of these particles occurs in the meander system within $1.5 \mathrm{mi}$ downstream from the tailings dam. Particle removal was induced by carbonate complexation, precipitation, entrapment and detention, and adsorption by aquatic vegetation. Removal rates of the trace elements and other waste products were improved by decreasing velocities within the meander system. Downstream distance and meander widths were predominant factors in the determination of velocity and detention time in the system. Erten (1988) concluded, as did Wixson (1977), that the meander system provided adequate treatment for mine and mill wastewater according to the trace-element (heavy-metal) ratios defined by Missouri. The heavy-metal ratio is the ratio of the concentration of a particular metal to the concentration of that metal that is allowed in effluent (Wixson, 1977).

Bornstein (1989) compared lead concentrations in leaf litter and soil samples collected in 1975 and 1988 to exam- 
ine long-term effects of the Buick Smelter. The leaf and soil samples had increased lead concentrations within $2 \mathrm{mi}$ of the smelter, near background concentrations within the 2- to 5-mi radius, and background concentrations outside a 5 -mi radius from the smelter. Lead concentrations in the 1988 leaf litter and soils samples were essentially the same as in the 1975 samples, indicating no appreciable increase in the lead concentrations during this period (Bornstein, 1989).

A study at the University of Missouri-Rolla (Faeth and others, 2004) examined the transport of trace elements in the Black River as they relate to mining in the Viburnum Trend. They reported lead and zinc concentrations in Clearwater Lake sediment downstream from the Viburnum Trend.

\section{Acknowledgments}

Denis Murphy of The Doe Run Company provided information and assistance on changes and enhancements to the facilities in the Viburnum Trend. Larry Hopkins of The Missouri Department of Natural Resources, Land Reclamation Program (LRP) provided assistance obtaining information regarding Metallic Mine Waste permits, National Pollution Discharge Elimination System outfalls, and compliance issues. The staff of The Missouri Department of Natural Resources, Hazardous Waste Program provided information on compliance and enforcement issues.

\section{Early Mining in Southeastern Missouri}

The first written record of a lead mine in Missouri was made by Father James Gravier, who, in the story of his voyage to Missouri in 1700, noted the potential for lead mining on the Meramec River in what is now Washington County (Burford, 1978). The first major lead mining in Missouri began in 1721, when French explorer Phillipe Francois Renault brought slave labor into the area north of the present town of Potosi (fig. 1) to mine the surface and near-surface lead ores. Numerous small mines rapidly opened and produced as much as 1,500 pounds (lbs) of ore per day that was shipped through the town of Ste. Genevieve, down the Mississippi River, and ultimately to France. Mining also occurred at Mine La Motte, near the present-day city of Fredericktown (fig. 1) (Burford, 1978).

Mining activity tapered off by the 1750s, with only intermittent metal production until 1799, when Moses Austin settled in Potosi (Burford, 1978). Austin brought improved mining and beneficiation methods to the local lead mining industry. Ore in the region previously had been recovered from shallow pits less than $10 \mathrm{ft}$ deep; Austin sank his first shaft to a depth of $80 \mathrm{ft}$. He erected the first reverberatory furnace and, by 1802 , was smelting ore for the entire Potosi region. The new process tripled the per pound yield. Austin also developed the town of Herculaneum (fig. 1), the present (2008) site of The Doe Run Company smelter operations, to establish a shipping point closer to the mines than the port at Ste. Gen- evieve (Burford, 1978). Additional lead ore discoveries during this time led to the development of about 45 larger mines and numerous smaller ones in what are now Washington, Jefferson, Madison, and St. Francois Counties (Burford, 1978).

Production declined in many of the mines by 1820 , although intermittent production continued until the mid1860s (Burford, 1978). In 1864, a group of New York investors formed the St. Joseph Lead Company, predecessor of The Doe Run Company. The company purchased lands in the vicinity of Bonne Terre (fig. 1), which contained rich deposits of galena at or near the surface (Burford, 1978).

\section{Southeast Missouri Lead District}

The Southeast Missouri Lead District is made of several subdistricts. Because mineralization is so widespread, both geographically and stratigraphically, the Southeast Missouri Lead District is defined as the area in which MVT stratiform deposits of lead-zinc-copper primarily occur around the exposed Precambrian rocks of the St. Francois Mountains (fig. 1) (Snyder and Gerdemann, 1968). The ore deposits primarily occur in the Bonneterre Formation, but can extend into the underlying Lamotte Sandstone and the overlying Davis Formation (fig. 3). Ore has been mined in rocks as young as the Potosi Dolomite in some of the subdistricts. Three of the most productive subdistricts include the Old Lead Belt, Mine La Motte-Fredericktown Subdistrict, and the Viburnum Trend. Minor subdistricts include Annapolis, Indian Creek, Irondale, and other small mines (fig. 1). Currrently (2008), the only active mines are in the Viburnum Trend.

\section{Old Lead Belt}

The lead-producing subdistrict commonly known as the Old Lead Belt (fig. 1; primarily located in St. Francois County, with minor production in Washington and Ste. Genevieve Counties) encompassed mines in the vicinity of Bonne Terre, Desloge, Park Hills, Doe Run, and Leadwood. The district produced more than 90 percent of the lead and zinc mined in southeastern Missouri before the advent of mining in the Viburnum Trend.

Between 1864 and 1972, the St. Joseph Lead Company (referred to as St. Joe in this chapter) operated numerous mines and mills in the Old Lead Belt. Lead and zinc were the primary commodities produced. During the first 70 years, as many as 14 other companies operated in the Old Lead Belt, including the American Smelting and Refining Company, St. Louis Smelting and Refining (later National Lead Company), and the Flat River Lead Company. By the mid-1930s, St. Joe had absorbed all of the competing operations; National Lead Company was the last company to be purchased (Kiilsgaard and others, 1967).

Nearly all mining in the Old Lead Belt before 1869 was from scattered and shallow workings above the water table. 


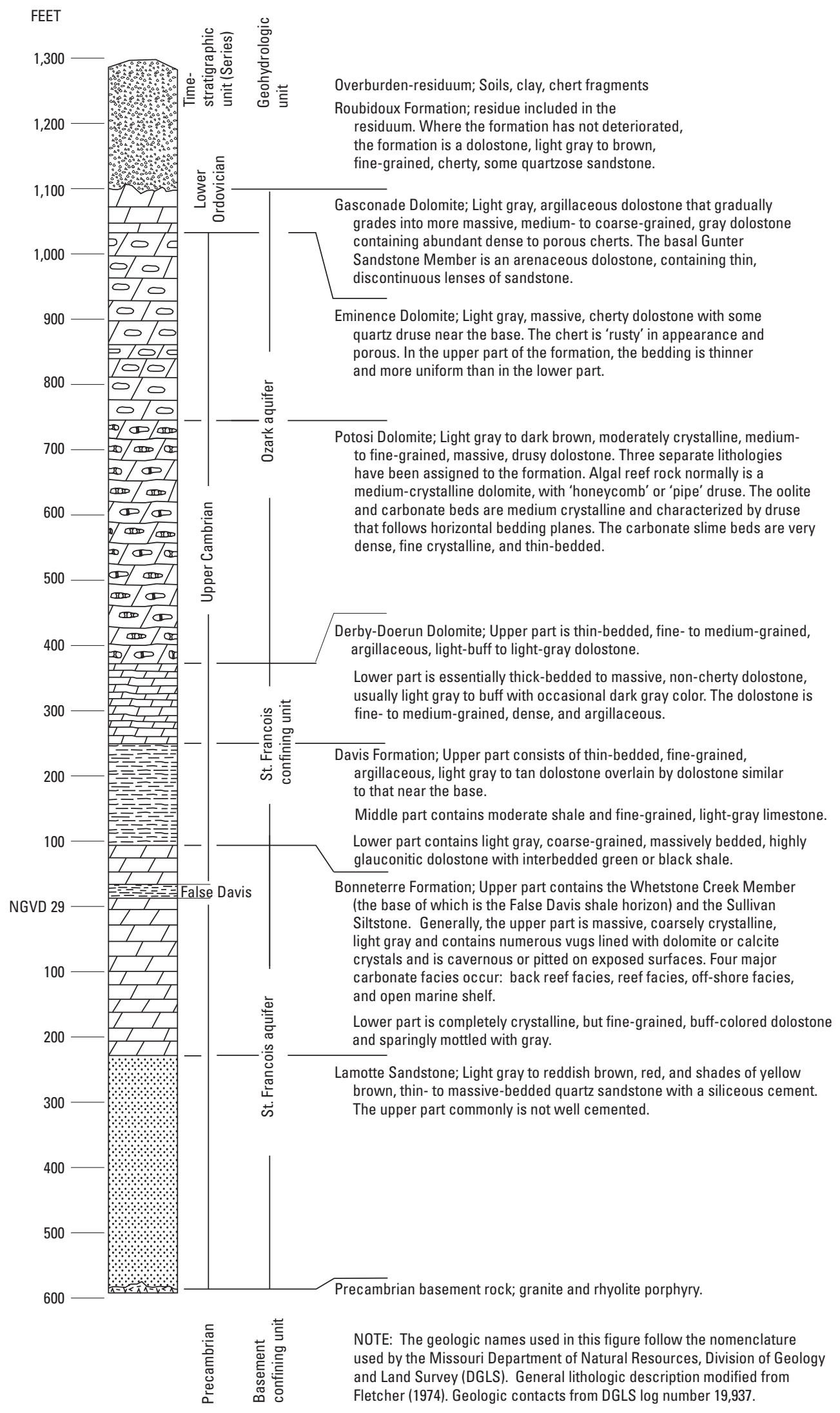

Figure 3. Stratigraphic column for an exploration hole in Reynolds County, Missouri, and a general lithologic description of formations in the Viburnum Trend. 
Exploratory drilling led to the discovery of deeper ores, and underground mining soon commenced, resulting in a complex of underground mines 200 to $300 \mathrm{ft}$ deep that were connected by more than $250 \mathrm{mi}$ of underground railroad. Mining was active in Bonne Terre from 1864 to 1961, Doe Run from 1887 to 1914 , Desloge from 1929 to 1958 , and Leadwood from 1915 to 1962 . The first St. Joe mine at Bonne Terre closed in 1961 after 97 years of continuous mining. The last mine to close was at Flat River and production ceased in 1972. St. Joe opened a lead smelter at Herculaneum (fig. 1) in 1891; its smelting operations have been continuously based there since (Kiilsgaard and others, 1967).

Galena was the principal ore mineral, although in the early days of mining, oxidized lead ores comprised of cerussite $\left(\mathrm{PbCO}_{3}\right)$ also were mined. Much of this ore was contained in residual material at the ground surface. Minor zinc (from sphalerite) and copper (from chalcopyrite) were recovered at the Federal Mill in Flat River. Total production figures for the Old Lead Belt are not readily available; however, more than 8.5 million tons of lead were estimated to have been produced (Wharton, 1975).

Mineralization in the Old Lead Belt occurred throughout the Bonneterre Formation and extended $100 \mathrm{ft}$ into the underlying Lamotte Sandstone. Ore generally followed depositional features and post-lithification faulting. Much of the mineralization was in reef and related carbonate facies; some also occurred in bar-reef complexes (Snyder and Gerdemann, 1968) and where the Bonneterre Formation onlapped or pinched out against Precambrian knobs. Lead mineralization primarily is galena replacing the dolomite; however, ore also was present along bedding-plane contacts, in fracture zones, and occasionally as breccia cement. The orebodies spread laterally for hundreds of feet and vertically up to $200 \mathrm{ft}$.

\section{Mine La Motte-Fredericktown}

Lead was discovered north of what is now Fredericktown (Madison County) in 1720 at the site of Mine La Motte (fig. 1). The mine was active intermittently until 1959; when it closed, it had produced more than 325,000 tons of lead (Kiilsgaard and others, 1967). A number of mines were worked around Fredericktown from the 1860s until 1961. Early mining was from surface and near surface areas similar to mining in the Old Lead Belt. The St. Louis Smelting and Refining Division of the National Lead Company operated the most productive mines, located southeast of Fredericktown, as well as a mill (Kiilsgaard and others, 1967).

The ores generally were shallow, usually 250 to $400 \mathrm{ft}$ below the surface. Orebodies were nearly flat lying, sinuous lenses as much as $40 \mathrm{ft}$ thick, 50 to $250 \mathrm{ft}$ wide, and several hundred feet long; ore was restricted to the lower $50 \mathrm{ft}$ of the Bonneterre Formation and, in places, the upper $15 \mathrm{ft}$ of the Lamotte Sandstone. Mineralization was strongly controlled by Lamotte Sandstone pinchouts against Precambrian knobs, although algal facies also controlled some ore deposition (Snyder and Gerdemann, 1968).

Mine La Motte-Fredericktown ores contained lead, copper, silver, nickel, and cobalt. Average ore grades for the subdistrict were 3 percent lead, 0.9 percent copper, 0.25 percent nickel, and 0.2 percent cobalt. Copper primarily was in chalcopyrite; cobalt and nickel were in siegenite $\left[(\mathrm{Ni}, \mathrm{Co})_{3} \mathrm{~S}_{4}\right]$ and bravoite $\left[(\mathrm{Ni}, \mathrm{Fe}) \mathrm{S}_{2}\right]$. Cobalt and nickel ores were distributed erratically within individual deposits, making mine planning for their recovery difficult. Difficulty also was encountered in separating the cobalt and nickel ores from the other sulfide minerals; consequently cobalt and nickel ores may be concentrated in mine tailings and mine dumps (disposal areas containing waste rock not processed through mill). Some of the tailings and dumps were re-worked for recovery of metals during World Wars I and II (Burford, 1978).

No continuous cobalt and nickel production was from the subdistrict. Attempts were made to recover the ores from 1903 to 1910,1917 to 1920 , and 1944 to 1961 . Mine La Motte was the primary domestic source of nickel at various periods in the 1800s. The National Lead Company, assisted by the Defense Minerals Exploration Administration, explored southeast and east of Fredericktown during the 1950s and discovered the Higdon deposit (fig. 1). This deposit contains an unspecified tonnage of lead-copper-cobalt-nickel ore. Two shafts were installed and construction was begun on a surface plant and concentrator; work was suspended in 1967 (Kiilsgaard and others, 1967) and currently (2008) the mine is inactive. The Anschutz Company evaluated the property in the early 1980s; in recent years, The Doe Run Company (hereinafter, referred to as Doe Run in this chapter) purchased the property and submitted an application to reactivate the mine, which is being reviewed by LRP and other programs within the Missouri Department of Natural Resources (Missouri Department of Natural Resources, Division of Environmental Quality, 2008).

\section{Viburnum Trend}

Declining reserves in the Old Lead Belt led to exploration for additional orebodies in the late 1940s. Exploration was conducted on the northern and western margins of the St. Francois Mountains. St. Joe discovered an isolated orebody, Indian Creek (fig. 1), north of the St. Francois Mountains in 1948; the mine began production in 1953 and closed in 1982.

Exploration extended southwestward from Indian Creek toward Viburnum (fig. 2), where St. Joe drilled the discovery hole for the Viburnum Trend in 1955. Further drilling defined the orebody for what was to become the Viburnum No. 27 Mine (fig. 2). Continued exploration by St. Joe and other companies, including Amax Inc., Cominco, Kennecott Copper, and Asarco, Inc., led to the eventual opening of 10 mines along the 60-mi long ore trend in Crawford, Washington, Iron, Dent, Reynolds, and Shannon Counties (fig. 2). The Viburnum Trend produces lead, zinc, copper, and silver, and the ore deposits also contain substantial cobalt and nickel mineralization. Con- 
struction began on smelters near the Buick Mine (fig. 2) and at Glover (fig. 1) in 1966, and both smelters were opened in 1967 (Missouri Department of Labor and Industrial Relations, 1967).

Exploration in the Viburnum Trend is a "story of geology successfully applied" (Vineyard, 1977) and was in part based on discoveries made in the Old Lead Belt and Indian Creek Subdistricts. In these subdistricts, a relation between Precambrian structural highs and mineralization that developed in complex carbonate facies in the surrounding Bonneterre Formation was observed. This relation also applies to the Viburnum Trend. Lead-zinc mineralization is associated with and seaward of stromatolite reefs that grew around Precambrian knobs that were islands or shallow submerged highs during Bonneterre Formation deposition. Mineralization also is related to limestone/dolostone interfaces, solution-collapse breccias, and fault-related breccias. Exploration was completed by development of detailed stratigraphic and structural studies. Gravity and magnetic surveys were used to determine the locations of Precambrian structural highs. Because Davis Formation shales were a barrier (confining unit) to mineralizing fluids, exploration centered on areas where Davis Formation shales are present (Vineyard, 1977).

Galena is the primary ore mineral in the Viburnum Trend, and sphalerite is the second most common ore mineral. Copper is recovered from chalcopyrite and bornite. Minor potential ore minerals include cobalt- and nickel-bearing siegenite and bravoite. In addition, sulfur is recovered from pyrite, and silver is recovered during the smelting process. Common gangue minerals (waste rock) include dolomite, calcite, marcasite, and quartz.

The deposits are sinuous tabular stratabound bodies that generally trend north-south and exhibit varying structural control. The average depth to ore is $1,200 \mathrm{ft}$. Deposits average 30 to $85 \mathrm{ft}$ thick and have a width of 200 to $2,000 \mathrm{ft}$. Total ore production from individual mines range from 20 to more than 50 million tons, and ore contains as much as 8 percent lead and about 3 percent zinc.

According to Hagni and Trancynger (1977), the ore is open-space fill and replacement (disseminations and along bedding planes) that exhibits a general paragenesis of pyrite and marcasite $\rightarrow$ sphalerite $\rightarrow$ cubic and octahedral galena $\rightarrow$ marcasite blades $\rightarrow$ cubic galena $\rightarrow$ sphalerite $\rightarrow$ chalcopyrite, pyrite, quartz, siegenite, bornite, and millerite. Early disseminated mineralization was followed by sulfides with colloform (typically irregularly banded) texture. Colloform sulfides were then followed by open-space fill. Ore fluids were brines with suggested fluid temperatures of 85 to 150 degrees Celsius $\left({ }^{\circ} \mathrm{C}\right)$. Sulfur isotopes indicate that there were different fluid sources for different sulfide generations; this implies fluid mixing and multiple mineralizing events. The positioning of different minerals indicate that the fluids had varying stages of deposition, nondeposition, dissolution, and redeposition (Hagni and Trancynger, 1977).

\section{Other Lead-Zinc Subdistricts of the Southeast Missouri Lead District}

Several small subdistricts in southeastern Missouri also have produced lead and zinc. The Indian Creek Subdistrict (fig. 1) was the first major discovery outside the Old Lead Belt and Mine La Motte-Fredericktown. The deposits are unusual in that a substantial part of the ore was in the Lamotte Sandstone; major mineralization was in the Bonneterre Formation.

The Valle Mines Subdistrict (fig. 1), located primarily in southern Jefferson County and extending into Ste. Genevieve and St. Francois Counties, began production in 1824 and was most active from the late-1800s to 1917 . With the exception of dump material shipped during World War II, Valle Mines has been inactive since 1917. Ore was mined from shallow workings generally less than $200 \mathrm{ft}$ deep; smithsonite $\left(\mathrm{ZnCO}_{3}\right)$ was the most common ore mineral. Residual barite $\left(\mathrm{BaSO}_{4}\right)$ was common in the ore; other lead-bearing minerals were present, but never recovered (Kiilsgaard and others, 1967).

The Irondale (Washington County) and Annapolis (Iron County) Subdistricts (fig. 1) were small orebodies concentrated around igneous Precambrian knobs. Mineralization at Irondale was similar to that in the Old Lead Belt; ore at Annapolis occurred in vuggy dolomite near the Bonneterre Formation-Precambrian contact. The Hayden Creek Mine (fig. 1) in St. Francois County, near Irondale, produced ore from granite conglomerate beds located where the Bonneterre Formation and Lamotte Sandstone pinch out against a Precambrian knob (Snyder and Gerdemann, 1968).

Galena also was produced from the Shirley-Palmer Subdistrict (Washington County). Most production was from residual deposits and from solution channels in the Potosi Dolomite, with minor production from the Eminence Dolomite. This later became the Washington County Barite District (Kiilsgaard and others, 1967).

\section{General Mining Processes, Regulatory Controls, and Environmental Effects in the Viburnum Trend}

Most Viburnum Trend mines operate using the same general steps and procedures during the mining and milling processes; and these processes have remained essentially the same throughout the history of the Viburnum Trend, although, the mills have undergone changes and improvements for recovery enhancement or for recovery of additional metals. An overview of these processes helps in understanding the complexity of turning ore into concentrate and potential sources of environmental effects. The section on "Regulatory Controls" in this chapter details regulations, permit application, and inspection procedures. 


\section{Mining Processes}

Mining is done using a room and pillar method that follows the ore trend. First-pass mine passages are about $30 \mathrm{ft}$ wide and about $20 \mathrm{ft}$ high and trend in a generally orthogonal grid pattern. Spacing between the centers of adjoining passages varies, but usually is $60 \mathrm{ft}$. Subsequent back (ore above the first pass) mining and bench (ore beneath the first pass) mining can result in mine openings as large as $100 \mathrm{ft}$ or more. The remaining rocks are support pillars. Pillars may be recovered near the end of mining in specific areas of the mine. Ore is moved using diesel equipment, and facilities for equipment maintenance are located underground. Primary crushing is completed underground, then the ore is hoisted to the surface for beneficiation. Hoisted ore is put directly into ore feed bins for the mill.

Mine water from dewatering is pumped to the surface and detained in either a reservoir in a tailings pond or in a mine water detention pond, where any remaining fine sediments (fines) in the water are allowed to settle before the mine water is discharged into surrounding surface streams. These waters are discharged under National Pollution Discharge Elimination System (NPDES) permits from the Missouri Department of Natural Resources with specific limits on trace elements, oil and grease, $\mathrm{pH}$, and total suspended solids (Boggess and Wixson, 1977). The water can contain increased trace element concentrations, other inorganic and organic constituents, fines from mining, unused blasting agents, and fluid from minor oil or hydraulic fluid spills. Mine water also has a slightly basic $\mathrm{pH}$ and large carbonate concentration (Boggess and Wixson, 1977), which gives the water a high buffering capacity, and can be used in the milling process.

\section{Mill}

The beneficiation process consists of four major stages: crushing and grinding, flotation, filtering and dewatering, and tailings disposal. Primary crushing occurs underground and decreases the ore to less than 6 inches (in.). After the ore is hoisted to the surface, it is put in ore feed bins and is carried by conveyer for secondary crushing. Secondary crushing uses a combination of rod and ball mills. Ore from the secondary crushers is separated into coarse material that is sent back into a rod or ball mill and fine material that is slurried and sent to flotation cells. The slurry is comprised of 50 percent ore and 50 percent water. The process water is mine water collected during dewatering, water recovered from the tailings ponds, a mixture of both, or water recycled from the mill.

At the flotation cells, a small quantity of frothing reagent is added to the slurry. Impellers within each cell agitate the slurry, keeping the particles in suspension and generating air bubbles. The reagent causes the lead and copper mineral grains (if copper is recovered) to attach to the air bubbles, which are then collected from the cell. The lead- and copperbearing mineral concentrate is then sent through a circuit that separates the lead minerals from the copper minerals and remaining gangue minerals. Further concentration separates the copper minerals from the gangue minerals at mills that have copper circuits. The remaining slurry from the first separation, which contains the zinc ore minerals, is sent to another flotation circuit for a similar recovery process. The collected concentrate is passed through additional flotation cells for further concentration. Water is added at all stages of this process. All mills in the Viburnum Trend have lead and zinc circuits; mills at the Viburnum No. 28 Mine (Central Mill), Fletcher Mine, Magmont Mine, Buick Mine, and Brushy Creek Mine also have or had copper circuits.

The concentrated ore mineral slurries are sent to thickeners where the solids are allowed to settle. The mineral concentrates are then dewatered, often on a drum or vacuum filter, and are stored briefly before transport to the smelter or buyer. Gangue minerals are sent to the tailings pond. Process water may be retained in either the tailings pond or in a separate impoundment, or recycled for use in the mill. The process water may contain trace elements and gangue mineral fines, other inorganic constituents, as well as excess flotation reagents.

Doe Run upgraded the stream x-ray analyzers at the Buick, Brushy Creek, and Fletcher Mills. These analyzers provide "real-time" data that allow better control of the addition or removal of mill reagents as needed, based on analysis of the ore feed to the mill (Denis Murphy, The Doe Run Company, written commun., 2005). In addition to improving ore recovery, the analyzers helped minimize the quantity of unused reagents disposed of in the tailings pond.

The Buick and Brushy Creek Mills have the highest zinc ore grade. Because of this, Doe Run installed auxiliary zinc and copper collectors at each mill for improved recovery of these minerals. At the Fletcher Mill, column cells installed for zinc circuit flotation have improved zinc recovery. Column cell flotation is a relatively new process that provides the ability to remove more impurities from the concentrates with additional reagents (Denis Murphy, The Doe Run Company, written commun., 2005).

\section{Mine Tailings}

Mine tailings are composed of gangue minerals (primarily dolomite) and minor amounts of ore minerals; flotation reagents also may be present in the tailings ponds. Tailings generally are transported to the disposal area through pipes as slurry consisting of about 35 percent solid grains and 65 percent water. Coarse tailings are placed on the downstream side of the tailings dam and are used to build and maintain the dam structure. Fine tailings are placed on the upstream side of the dam. Mill water pumped out with the tailings may be reused in the mill and usually is collected by a barge pump. A water pool is maintained in the tailings pond to provide a water source and to allow mill reagents to biodegrade. Upon property closure, tailings ponds have been drained, capped, 
and revegetated (Missouri Department of Natural Resources, Division of Environmental Quality, 2008).

Tailings pond discharges are monitored for trace elements that may leach from the tailings. Dolomite in the tailings is a buffer, decreasing production of acidic surface water. Surface water contamination by non-degraded mill reagents is a concern. Mine waters pumped into the impoundment also may contain chemical residue from explosive agents used during mining. To prevent contamination of local streams, most impoundments have catchment ponds downstream from the tailings dam to contain normal discharge and unplanned discharges such as stormwater runoff. Water in these catchment ponds commonly is pumped back into the impoundments. Several mines employ meander systems in the stream channel to increase detention time and allow additional biodegradation and particulate settling (Asarco, Inc., 1991a; McLaren/Hart Environmental Engineering Company, 1991b).

\section{Transportation of Ore and Concentrate}

For mines that do not have mill operations onsite, the ore must be transported to a mill. Transportation generally is by truck on haul roads on property owned or leased by the company; however, part of this transportation occurs on public highways. After ore mineral concentrates have been prepared for shipment, they are transported by truck directly to a smelter, or in the case of copper and zinc, by truck to a rail system for shipment to a smelter. Potential exists for ore or ore mineral concentrates to be released into the environment if not handled properly during transportation.

\section{Smelting}

Smelting is the process of separating the metal from impurities (primarily sulfur) by heating the concentrate, separating the sulfur from the metal-sulfide concentrates, and causing the metal to melt. Smelter dust (fine-particulate fraction) and gas releases probably are the greatest potential source of contamination in the production process. Air from the smelting process is first passed through a cooling chamber to condense as much gas as possible. The air then passes through a baghouse, where it is forced through Teflon bags that capture particulate material. The air can then pass through a sulfuric acid production plant. Air from the blast furnace usually is routed through a cooling chamber and baghouse filtration system before release to the atmosphere; air emissions are monitored for sulfur dioxide. Hazardous materials can be spilled and carried into the offsite environment by stormwater runoff. Material can be released to the environment by spillage while loading onto truck or rail transport or may be windblown from ore concentrate piles (Boggess and Wixson, 1977).

\section{Regulatory Controls}

The Metallic Minerals Waste Management Act is implemented by the LRP. Missouri legislation requires that a permit application be made for the operation of a waste management area (WMA) that is associated with metallic mineral mining. The WMA includes areas designated and used for the disposal of metallic mining waste. The waste includes material from mining, beneficiation, and processing, as well as mine water and topsoil and vegetation over mine waste. The WMA does not include mine shafts, shop areas, or smelters. Issues covered under the permit include wind or water erosion, sedimentation of materials beyond permit boundaries, affected ground not included in the permit, and ground water. Inspections are made annually, at a minimum, and the permit application is reviewed every 5 years (Missouri Department of Natural Resources, Division of Environmental Quality, 2008).

The WMA permit application requires information pertaining to the physical aspects of the tailings pond dams and to outfall locations regulated under the federally enforceable NPDES permits. The NPDES permit program was authorized as part of the Clean Water Act and these federally enforceable permits control water pollution by regulating point sources that discharge pollutants into waters of the United States (U.S. Environmental Protection Agency, 2008). The NPDES discharge points are monitored monthly; non-scheduled monitoring is done for intermittent discharge. Mine and mill sites also are monitored for air emissions.

Companies conducting metallic minerals mining must provide financial assurance information and must file an inspection-maintenance plan and a permanent closure plan that includes permanent stabilization of tailings disposal and storage areas. The plans must establish and explain the steps planned to complete and maintain closure after mining has ceased and metallic mine waste disposal is completed. Issues addressed in the plan include design and construction of waste control structures and tailings dams; characterization of waste products; methods for control and protection of surface water; methods for protection of ground water and aquifers; geology and seismicity of the area; potential of subsidence; reuse and offsite removal of wastes; and surface reclamation of waste management areas (Missouri Department of Natural Resources, 2003).

Tailings pond monitoring includes measurements of precipitation, pumping rates, tailings levels, reservoir water volumes, and seepage volumes. These data are recorded monthly. Tailings dams are inspected and re-permitted yearly by Missouri Department of Natural Resources, Dam and Reservoir Safety Program.

Because permitting and legislation affecting the mines, mills, and smelters only have been in effect for relatively few years, compliance/enforcement information is limited to more recent years. The primary environmental issue for the LRP is wind-blown transport of tailings material outside of the WMA (Larry Hopkins, Missouri Department of Natural Resources, Land Reclamation Program, written commun., 2005). The 
LRP has, in the past, taken enforcement action against the Fletcher Mine for wind-blown tailings leaving the WMA and crossing State Route TT. Abatement requirements, determined in conjunction with The Missouri Department of Natural Resources, Dam and Reservoir Safety Program, included covering part of the tailings dam with rock, installing a sprinkler system to keep the tailings wet and minimize wind transport, and planting trees and grasses between the tailings pond and the highway. These have all been implemented. Other mines and the Buick Smelter have not had documented violations (Larry Hopkins, Missouri Department of Natural Resources, Land Reclamation Program, written commun., 2005).

The Missouri Department of Natural Resources, Hazardous Waste Program (HWP) inspects facilities for violations of hazardous waste handling. During 1997, the Sweetwater Mine was issued a notice of violation as an unauthorized hazardous waste treatment/disposal facility. Violations included failure to label and mark containers, and the presence of an open container. Compliance was met in 2 to 3 weeks. Inspections of other Viburnum Trend mines and the Buick Smelter have indicated no violations since the mid-1990s (Robert Hinkson, Missouri Department of Natural Resources, Hazardous Waste Program, oral commun., 2005).

The HWP has one Superfund site in the Viburnum Trend area-public highways used as transportation routes by trucks hauling ore mineral concentrates to smelters or shipment points. Highways within the Superfund site are located in Iron, Reynolds, and Dent Counties. Reports of mining-related sediment being transported downstream from the Sweetwater Mine have led to sampling and investigation, but have not triggered a formal Superfund action at this time (Robert Hinkson, Missouri Department of Natural Resources, Hazardous Waste Program, oral commun., 2005).

\section{Environmental Effects}

The potential exists for waste products from mining, milling, and smelting to affect the environment immediately surrounding the operation. Studies completed in and around the Viburnum Trend, including the Missouri Lead Study, document some of the effects. The environmental effects of concern in the Viburnum Trend include wind-blown dust or water-transported trace elements from ore mineral concentrates and tailings, deposition from gases related to smelting, and unusual widespread growth of benthic bacterial/algal mats downstream from operations (Wixson and others, 1972; Wixson, 1977). A decline in the number and type of benthic organisms downstream from operations was noted by Jennett (1974). Responses by the industry to these effects have resulted in improved air quality from smelter stack releases and further remediation of mine and process waters (Wixon, 1977).

The Buick Smelter had the most immediate noted effect on the surrounding environment. By the summer of 1970, smelter emissions were affecting the foliage of trees in the surrounding woodlands (Wixson and Anderson, 1973). Geochemical sampling indicated increased sulfur and lead concentrations in the soil and vegetation immediately surrounding the smelter. By 1973, a mist eliminator and extra cooling capacity had been added to the smelter to decrease sulfur dioxide emissions. In addition, two dams were built to catch and allow particulate matter to settle from stormwater runoff from the plant area, and baghouse maintenance and operation were improved. The baghouse in the plant producing sulfuric acid was replaced by an electrostatic dust precipitator. A new dust handling system was implemented, and the onsite roads and yard were paved. The in-plant ventilation system also was improved at the smelter (Wixson and Anderson, 1973).

The distribution of trace elements near the Buick Smelter as part of the Missouri Lead Study was examined by Bolter and others (1974a). They noted an extended retention of trace elements in the leaf litter, with minimal contamination of soils during the first 5 years of smelter operations. This indicated that the metals may be transportable with intense rains and movement of the leaf litter. Butherus (1975) noted that where leaf litter is absent, metal contamination moved quickly down the soil column. The report also described a maximum contamination radius for lead of 20 to $25 \mathrm{mi}$ around the smelter; the cadmium, copper, and zinc contamination radii were not as extensive.

Also, as part of the Missouri Lead Study, Arseneau (1976) studied flue and baghouse dust and noted large enrichments of cadmium in the dust that could easily escape the system. Lead and cadmium concentrations in dust surrounding the smelter, however, indicated that "fugitive sources" or nonregulated sources also were responsible for the contamination. The report also stated that older, more decayed leaf litter had higher lead to cadmium ratios than the more recent leaf litter, indicating a faster removal rate for cadmium than for lead.

In response to data from the Missouri Lead Study (Wixson, 1977), the Buick Smelter also installed four wet scrubbers that, along with the electrostatic dust precipitator, returned dust to the smelting system. The paved parts of the site were washed by sprinkler trucks several times a day, and the dust was collected and recycled into the smelter (Wixson, 1977).

During the first few years of production from the Viburnum Trend mines, releases of process and mine water stimulated downstream algal and bacterial growth (Gale, 1974). Natural nutrients were being supplemented by nitrogen and ammonia from unused and degraded blasting agents, by manganese in mine water, and by phosphate, sulfate, and nitrate from milling agents that had not biodegraded.

Jennett (1974) noted that most metals were transported in water in fine materials (less than 63-micrometer fraction). Large concentrations of lead and zinc were related to excessive surface runoff and turbulent streamflow. The report also noted incomplete biodegradation of constituents in process water that were diluted with mine water in tailings ponds, detention ponds that were too deep for effective light penetration, and inadequate detention time. 
In response to concerns about the water quality of tailings pond discharges, the Buick Mine implemented a meander system for water treatment (Wixson and Anderson, 1973). The meander system allowed growth of algal/bacterial mats within property boundaries, consuming nutrients before the water left the property. Trace-element and gangue fines were trapped by the mats (Wixson and Anderson, 1973; Jennett, 1974) and these fines settled within the meander system (Escalera, 1973). An additional sediment pond was constructed to retain any remaining fines, as well as material and algal/bacterial mats transported by stormwater runoff (Wixson and Anderson, 1973; Jennett, 1974). A decrease in the quantity of trace elements trapped in the algal mats occurred after tailings pond size increased and the meander system was implemented (Jennett, 1974). The primary tailings pond and meander system at Buick Mine provided adequate treatment of mine/mill wastes (Wixson, 1977).

Water recycling and treatment of process waters were implemented at mine sites to help alleviate water-quality issues. The Fletcher Mine constructed a process-water recycling system. Brushy Creek Mine constructed a complete recycling system for water discharged from milling and flotation (Wixson, 1977). By 1972, efforts were made at the existing mines to limit the direct release of mine and process waters to the environment. Additionally, cycloned water from coarse tailings deposited at the dams was pumped into the tailings pond, rather than released directly into streams (Wixson and others, 1972).

Increased concentrations of trace elements on roads and railways used for ore, concentrate, and smelter product shipment were noted by Butherus (1975) and Wixson (1977). Covers on vehicles and rail cars transporting the ore and concentrate were recommended and companies implemented this recommendation (Wixson, 1977).

\section{Mine Facility Descriptions in the Viburnum Trend Subdistrict}

St. Joe, which became Doe Run in 1986, opened the first mine in the Viburnum Trend in 1960; it was followed by five other St. Joe mines and mines operated by Cominco American Inc., Amax Inc., Kennecott Copper, and Asarco, Inc. Most of the mines opened during the 1960s, one opened in the 1970s, and two during the 1980s (table 1).

Table 1. Summary of features and facilities at the Viburnum Trend mine complexes.

[NPDES, National Pollution Discharge Elimination System; NA, not applicable; L, lead; Z, zinc; C, copper]

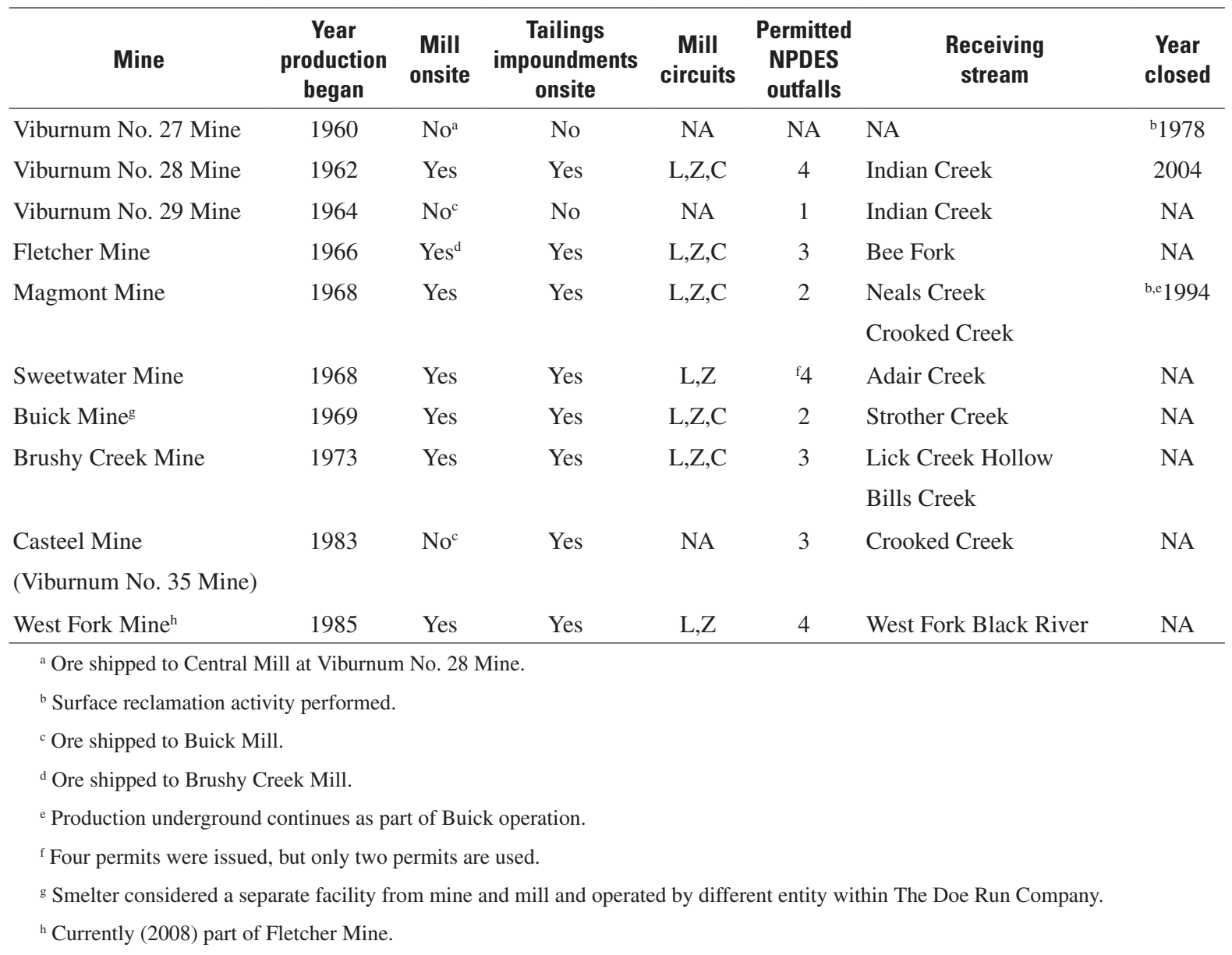


All mine sites in the Viburnum Trend have certain features in common - at a minimum, they have a production shaft, detention pond(s) for mine water and surface runoff, and buildings for offices, supplies, change room, and hoist room. At sites that do not have a mill, loading facilities are present for transportation of ore to a mill. Of the 10 mines in the Viburnum Trend, 7 have or had mills onsite, as well as facilities for transportation of ore concentrates. Provisions for detention of process waters used in the milling operations are present. The mine sites in the Viburnum Trend are discussed below in chronological order of their development, and the Buick Smelter is discussed last.

\section{Viburnum No. 27 Mine}

The Viburnum No. 27 Mine (figs. 2, 4) in Crawford County was opened by St. Joe and was the first mine in the Viburnum Trend. The discovery hole was drilled in 1955, and it intersected $31 \mathrm{ft}$ of 29 percent lead ore. Ore reserves were

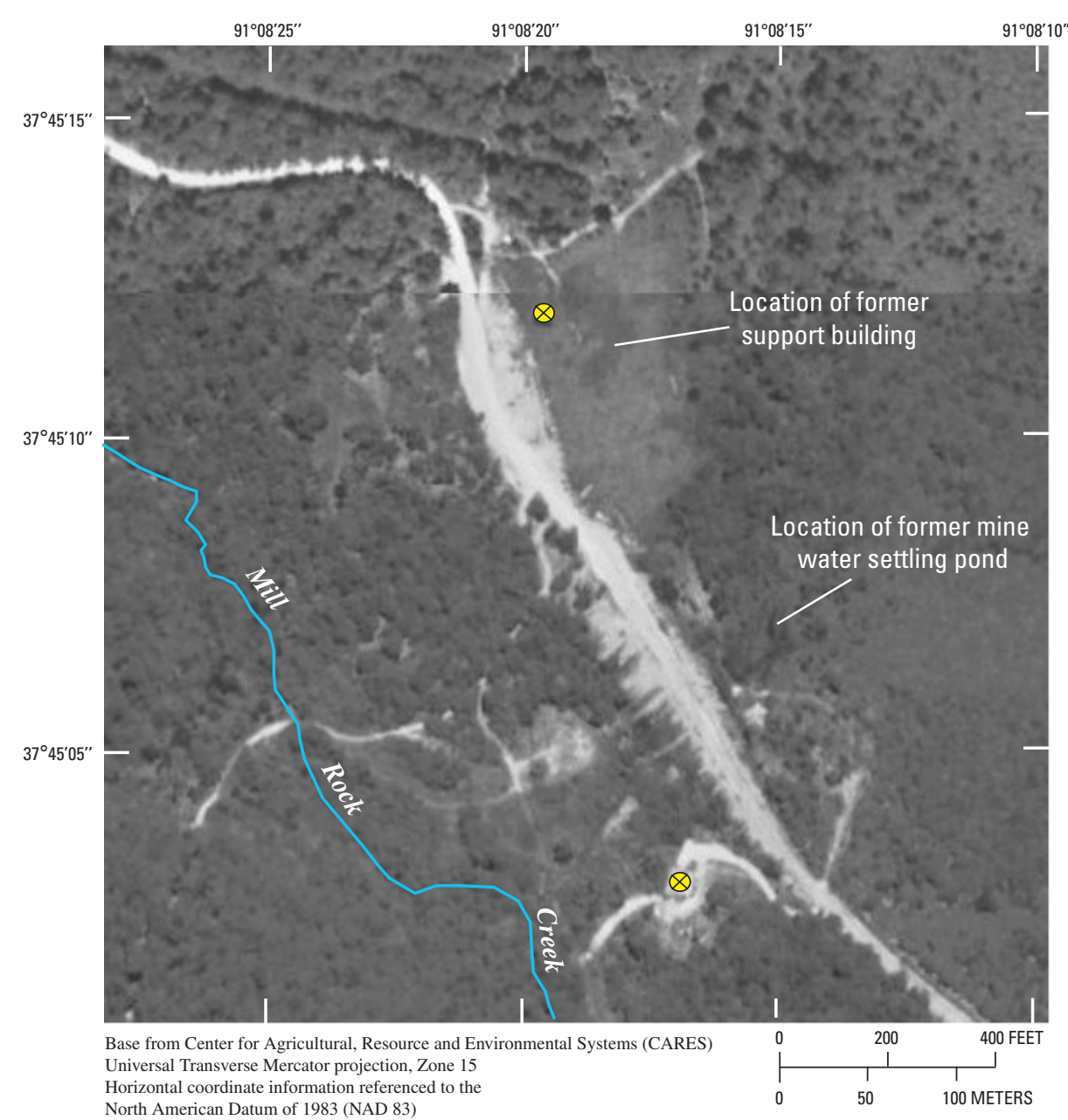

EXPLANATION

$\otimes$ Former mine shaft location proven by 1958 and production began in 1960 . The mine, located 2 mi north of the town of Viburnum, ceased operations in 1978.

The Viburnum No. 27 Mine had a minimal surface effect because no mill or tailings pond was constructed at the site; all ore was shipped to the Central Mill located at Viburnum No. 28 Mine. Onsite structures included the headframe and a building that contained offices, a change room, and the hoist room. After closure in 1978, the structures were removed for use at the Casteel Mine (Viburnum No. 35 Mine) (McLaren/ Hart Environmental Engineering Company, 1991d). The mine currently (2008) is used as the drinking-water supply for the city of Viburnum and consistently meets the water-quality standards required for a public drinking-water supply (Missouri Department of Natural Resources, 2007, 2008) .

During production, mine water was pumped to a settling pond that discharged to Mill Rock Creek. After closure, the pond was allowed to drain and native vegetation was established (McLaren/Hart Environmental Engineering Company, 1991d). Because mine closure and drainage of the pond predated legislation, no NPDES outfall permits were issued for the mine.

\section{Viburnum No. 28 Mine and Central Mill}

The Viburnum No. 28 Mine (figs. 2, 5), a St. Joe and later Doe Run facility, is located in Iron County on the east edge of Viburnum. The mine began full production in 1962 ; mining ceased and the pumps were removed from the mine in August 2004, allowing the mine to flood (Denis Murphy, The Doe Run Company, written commun., 2004). The Viburnum Central Mill, located at Viburnum No. 28 Mine, began operation in 1961 and closed in 2000. While active, the mill processed ore from the Viburnum No. 27, No. 28, and No. 29 Mines, and Casteel Mine (Viburnum No. 35; fig. 2) (Grundmann, 1977). Since construction, mill capacity doubled from 6,000 to 12,000 tons per day (ton/d) when it closed.

Tailings from the Central Mill (fig. 5) were pumped to the old and new Viburnum tailings ponds that are located within the Indian Creek Basin. The old Viburnum tailings pond received tailings and process water from 1960 to 1975 . From 1975

Figure 4. Viburnum No. 27 Mine, 2003. 


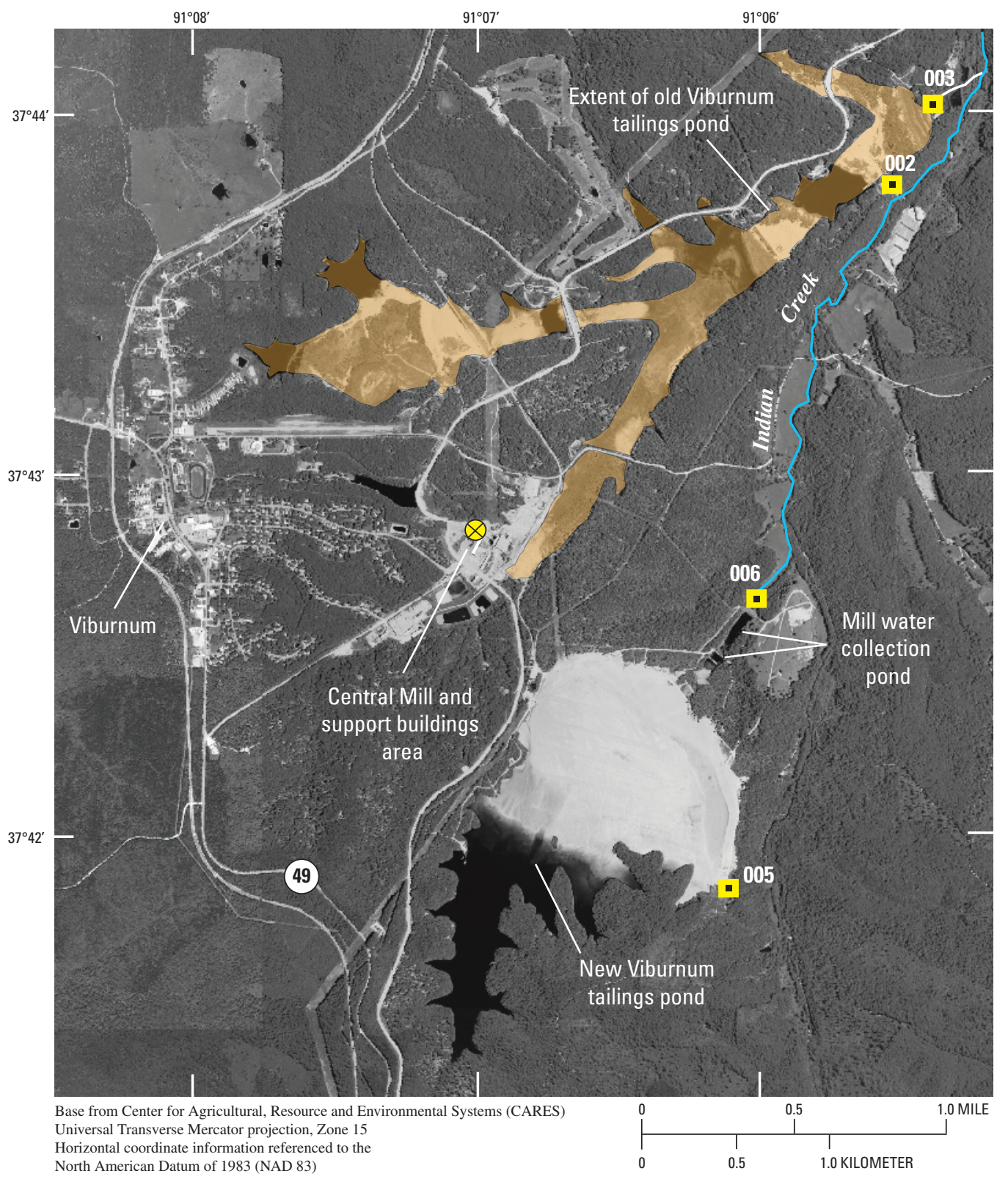

EXPLANATION

002 - National Pollution Discharge Elimination System outfall and number

Q Shaft

Figure 5. Viburnum No. 28 Mine and Central Mill, 2003.

until closure, it received mine water and stormwater runoff, with some possible input from nearby springs. The mine waters were treated by settling. The tailings pond originally had a primary dam and six small dams that formed separate reservoir areas. Subsequent tailings emplacement buried the small dams. A decant system allowed overflow in the tailings pond to discharge beyond the toe of the dam. The water flowed into a series of three settling ponds before its release into Indian Creek at outfall 002. The discharge from tailings dewatering was released at a pipe located at the toe of the primary dam [outfall 003 (McLaren/Hart Environmental Engineering Company, 1991d)].

The new Viburnum tailings pond received tailings and process water from 1975 until closure. A reservoir pool, located near the headwaters of the tailings pond, provided water for the mill. A seepage collection pond system located downstream from the toe of the dam embankment (outfall 006) was composed of two mill water collection ponds and a pump-back system. The pump-back system recycled water back to the tailings pond. Outfall 005 discharged floodwater 
over a spillway into Indian Creek; precipitation must equal or surpass 27 in. in 24 hours for discharge to occur. Outfalls 005 and 006 had no discharge during normal operation (McLaren/ Hart Environmental Engineering Company, 1991d).

\section{Viburnum No. 29 Mine}

The Viburnum No. 29 Mine in Washington County (figs. $2,6)$ began production in 1964. Similar to the Viburnum No. 27 Mine, the Viburnum No. 29 Mine has no mill or tailings pond onsite, and ore was trucked to the Central Mill for processing until 2000 and currently (2008) is trucked to the Buick Mill (Denis Murphy, The Doe Run Company, written commun., 2008). Mine water is pumped to five surface ponds that were constructed in 1974 and are used as settling basins for mine water and stormwater runoff. The ponds were built as a "step-down," using mine waste rock and clay-rich soil. The water eventually drains into a tributary of Indian Creek at outfall 004. Eight shallow bermed impoundments immediately to the east of the ponds were used for the same purpose from 1964 to 1974 . These bermed impoundments were allowed to dry and naturally revegetate (McLaren/Hart Environmental Engineering Company, 1991d). Because these bermed impoundments pre-dated legislation, no NPDES outfall permits were issued for them.

\section{Fletcher Mine and Mill}

The first ore discovery for the Fletcher Mine (figs. 2, 7), in Reynolds County, was drilled by St. Joe in 1958. The production shaft, which extends to a depth of $1,334 \mathrm{ft}$ (Paarlberg and Evans, 1977), was completed in 1965; ore was hoisted in 1966 and mill production began in 1967. The mine briefly was placed on standby in 1986; before 1989, all ore from the Fletcher Mine was processed at the mill onsite. Beginning in 1989, approximately 300 ton/d of high grade copper ore were trucked to the Brushy Creek Mill for processing (Denis Murphy, The Doe Run Company, written commun., 2005).

The Fletcher Mine has three NPDES outfall permits that were issued in 1989. The mine water pond was constructed in 1976; before this, all mine water was stored in the tailings pond. Mine water is treated by allowing the particulate matter to settle. The pond is designed to contain mine water and runoff from a 48-hour storm. In the event of a larger storm, floodwaters are routed downstream from the dam. Discharge is from outfall 001 into a tributary of Bee Fork of the Black River (fig. 7) (McLaren/Hart Environmental Engineering Company, 1991c).

The tailings pond originally had two dams that retained small reservoirs. These have since been buried by tailings infill after construction of a single larger dam downstream. A return water basin is located at the toe of the dam from which water is pumped back into the tailings pond. Discharge from the tailings pond is through outfall 002. Outfall 003 is a spillway discharge for the tailings pond. Both outfalls are designed to be zero-discharge during normal operation (McLaren/Hart Environmental Engineering Company, 1991c). A process water recycling system also has been constructed so that process water can be reused in the mill (Wixson, 1977).

\section{Magmont Mine and Mill}

The Magmont Mine (figs. 2, 8) is primarily in Iron County; however, subsurface workings extend into Dent County. The deposit was first discovered in 1962 at a depth of approximately 1,200 ft. Exploration was performed jointly by Cominco American Inc. (operating as Montana Phosphate Products Company) and Dresser Industries (operating as Magnet Cove Barium). The name "Magmont" was created from the operating names of the companies. The first ore was produced from the mine-mill complex in 1968 (Sweeney and others, 1977), and the mill continued to operate at or above capacity from 1971 until closure in 1994 (Hydro-Search, Inc., 1991; Milton Bradley, Cominco American, oral commun., 2005), when Cominco American Inc. and Dresser Industries ceased operations. Surface buildings and the mill were removed and the site was reclaimed. Doe Run purchased the underground operations soon after closure and currently (2008) produces ore from the mine as part of its Buick operation.

Surface waters at the Magmont Mine site drained into two separate basins, resulting in two NPDES outfall permits issued in 1989. Most of the surface water drained into the tailings pond (fig. 8), which also contained process and mine waters. A return water basin for overflow was located immediately downstream from the tailings dam in the Left Fork of Neals Creek. A second dam was located further downstream and formed a settling pond. Water from this pond was monitored at outfall 001 (Hydro-Search Inc., 1991).

The remaining surface water drained west into a small runoff collection pond with an emergency outfall (002) that discharged into the headwaters of Crooked Creek. Water that collected in the runoff collection pond was pumped into the tailings pond, which was designed as zero-discharge (HydroSearch, Inc., 1991).

Upon closure, the surface structures at the site were removed. Two temporary solid waste landfills that contained scrap steel, tires, drums, and other materials were remediated as part of the closure and reclamation process and the tailings pond was reclaimed. The tailings were allowed to drain; dry areas received a clay cap and were covered by local soil, then seeded with native grasses and other flora (Hydro-Search, Inc., 1991; Swenty, 1996). The tailings pond was modified to improve its drainage characteristics and to provide a single outlet for all storm water. The decant pipe in the tailings pond was plugged, and a concrete channel was constructed as a principal spillway (Swenty, 1996). All closure and reclamation work was completed by 1998 . 


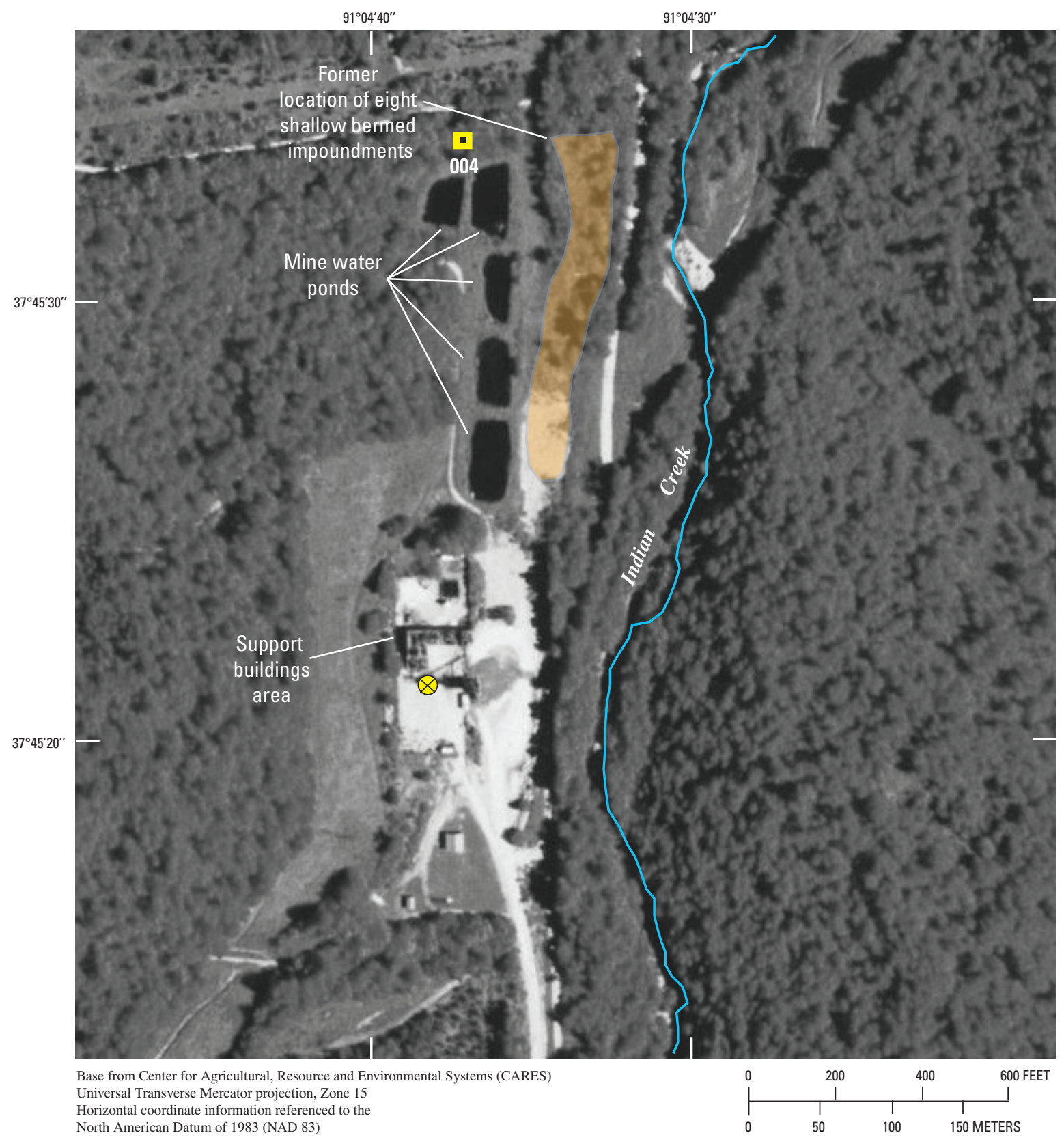

EXPLANATION

004 - National Pollution Discharge Elimination System outfall and number

$\otimes$ Shaft

Figure 6. Viburnum No. 29 Mine, 2003. 


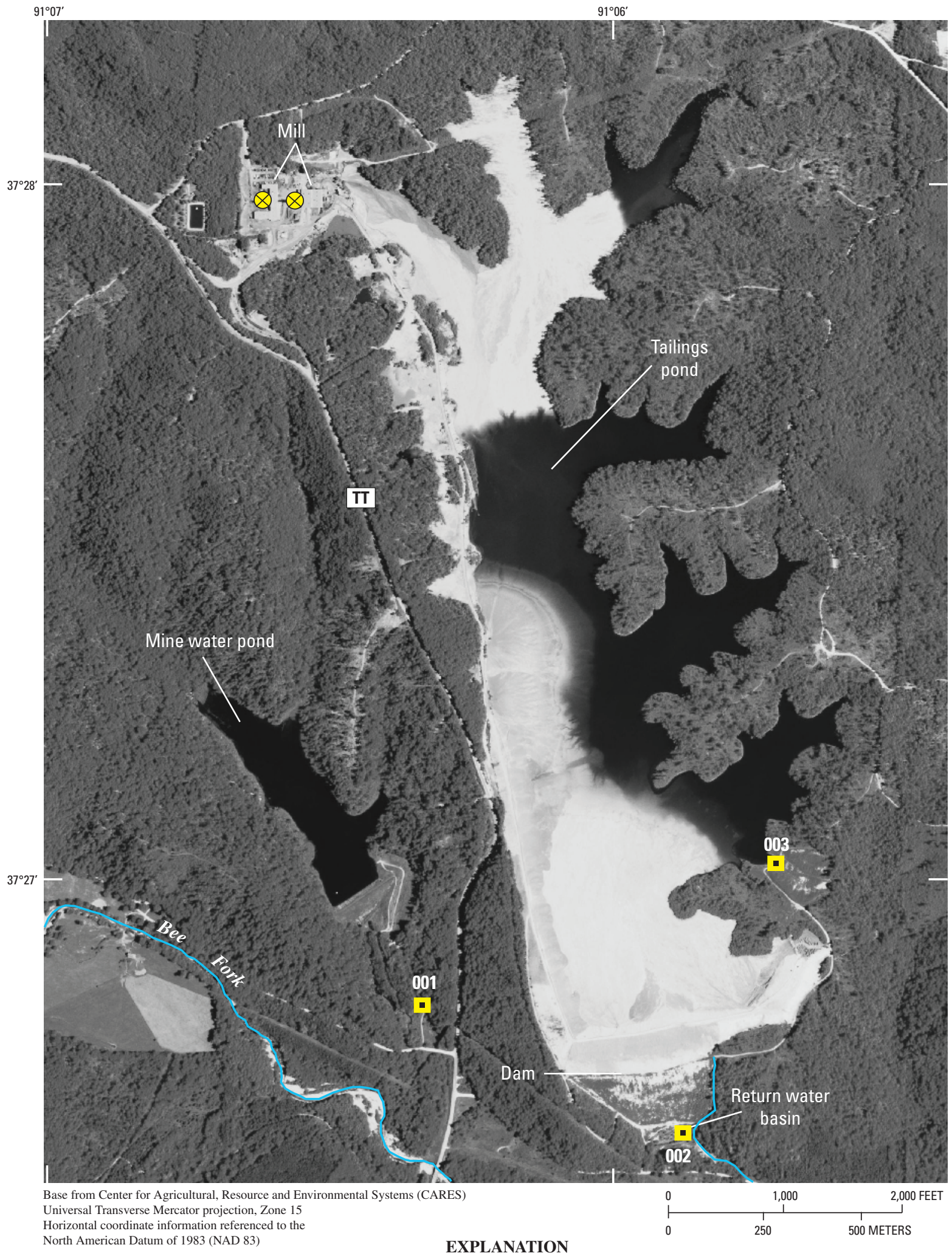

002 - National Pollution Discharge Elimination System outfall and number

$\otimes$ Shaft

Figure 7. Fletcher Mine and mill, 2003. 


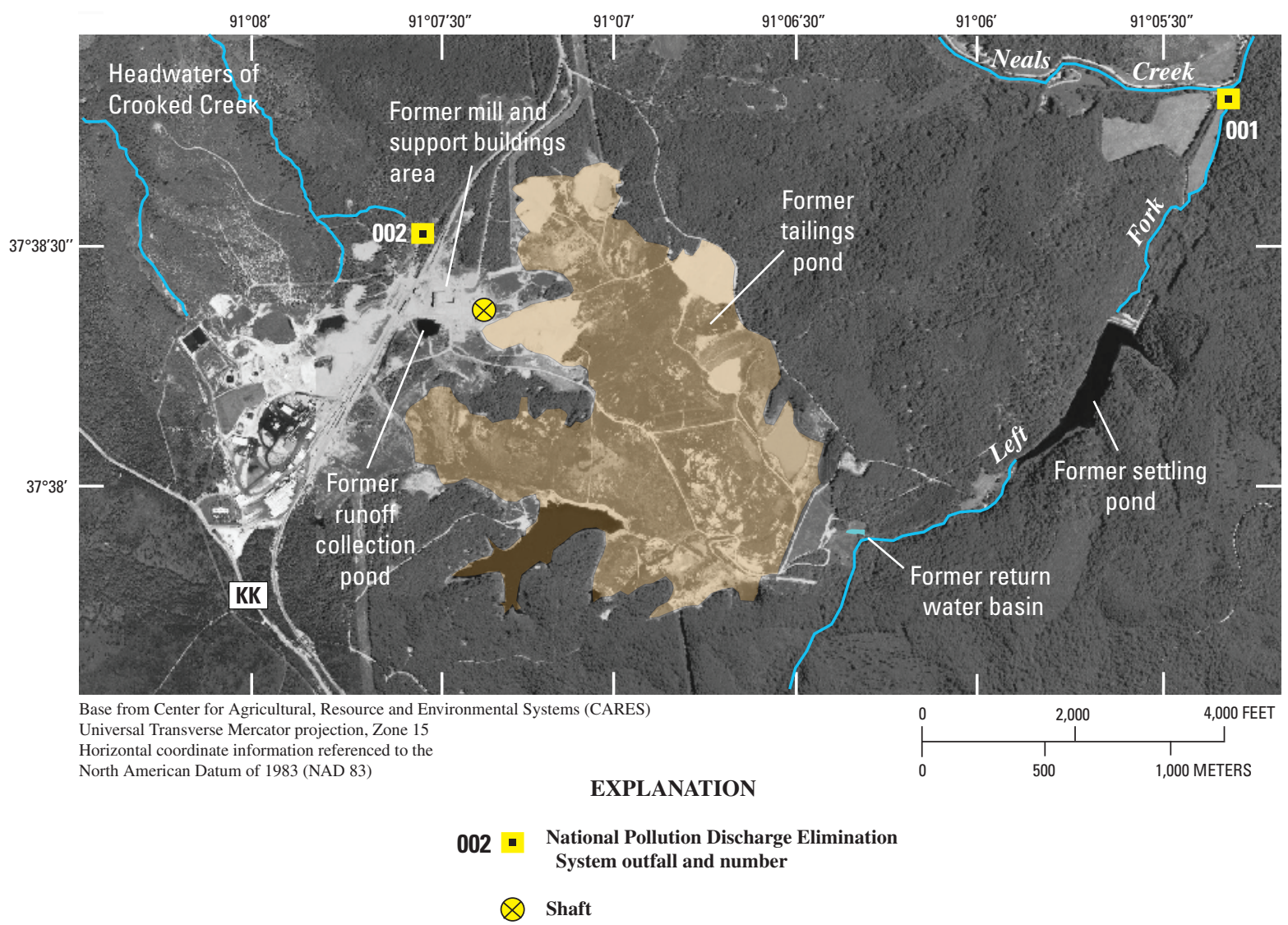

Figure 8. Magmont Mine and mill, 2003.

\section{Sweetwater Mine and Mill}

The Sweetwater Mine (figs. 2, 9), in Reynolds County, was originally known as the Ozark Lead Company Mine or the Frank R. Milliken Mine. It is the southernmost mine in the Viburnum Trend. The orebody, located at 1,400 ft deep, was discovered by Bear Creek Mining Company, an exploration subsidiary of Kennecott Copper (Mouat and Clendenin, 1977). The discovery hole was drilled in 1962 and production began in 1968. Lead concentrates were sold to the Asarco, Inc. Smelter at Glover (fig. 1). Because of depressed lead prices, the Ozark Lead Company placed the mine on standby in 1983 and ceased operations. The mine was purchased by Asarco, Inc. in 1986 and renamed the Sweetwater Mine; production was restarted in 1987. The mine was purchased by Doe Run in 1997. The Sweetwater Mill recovers lead and zinc concentrates. Lead concentrates are now (2008) shipped to the Herculaneum Smelter.

The original NPDES permits for the Sweetwater Mine were issued to Kennecott Copper in 1986. The permits were modified in 1987 because of the change in ownership from Kennecott Copper to Asarco, Inc. and the accompanying name change to Sweetwater Mine. The current (2008) outfall 001 (fig. 9) discharges non-mining related wastewater into a tributary of Adair Creek after the water has undergone settling and aerobic digestion (Asarco, Inc., 1991a). Process water from the mill is decanted and reused from two return water basins at the base of the tailings pond dam. Excess mill water is routed through a 6,800-ft meander system ending at outfall 002 in Adair Creek. Runoff to the tailings pond from excessive rainfall is channeled through an emergency spillway at the north end of the tailings pond dam and routed to Adair Creek. The tailings pond is designed to discharge only with storm waters that equal or exceed a 25 -year storm. Surface water from the mill and support buildings area is channeled into a drainage east of the mill, where it flows through settling ponds and discharges into the meander system upstream from outfall 002 (Asarco, Inc., 1991a).

The original permits included two additional outfalls to Sweetwater Creek (formerly designated outfalls 001 and 002). One was for domestic wastewater near the production shaft, and the other was for stormwater runoff from a proposed mine impoundment. Neither site was ever developed. With the permit modifications, these outfalls were renamed outfalls 003 and 004 (Asarco, Inc., 1991a), for a total of four permitted outfalls. There are no plans to build the wastewater treatment site or impoundment (Asarco, Inc., 1991a). The outfalls are not included in figure 9 . 


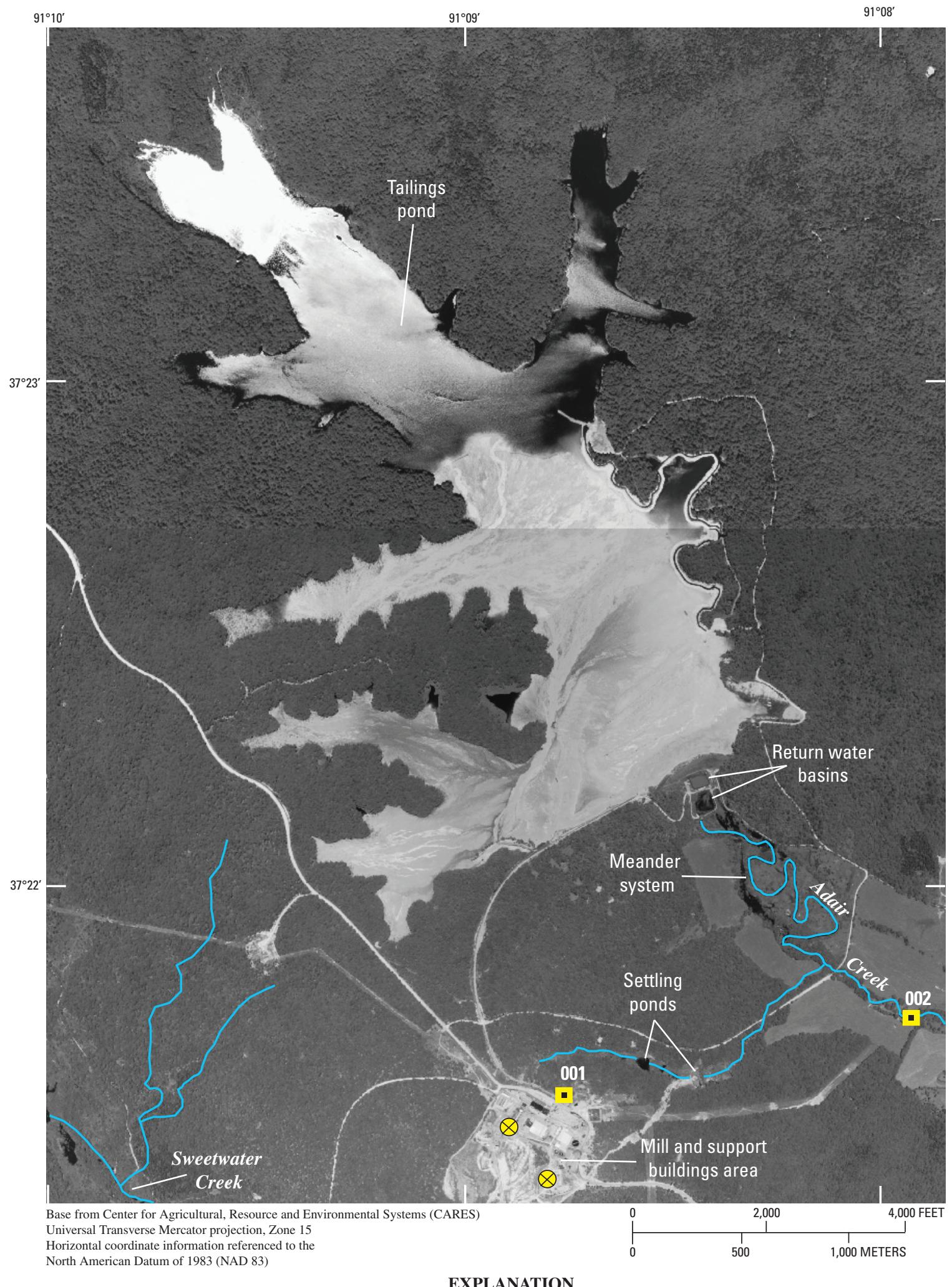

002 - National Pollution Discharge Elimination System outfall and number

$\otimes$ Shaft

Figure 9. Sweetwater Mine and mill, 2003. 


\section{Buick Mine and Mill}

The Buick Mine (figs. 2, 10) is located in Iron and Reynolds Counties. The deposit was discovered in 1960 and production began in 1969. The tailings dam was constructed in 1966. Mineralization has been located at depths averaging $1,100 \mathrm{ft}$. The mine was opened as a joint venture of Amax Inc. and Homestake Mining Company; the site was operated by AMAX Lead Company of Missouri under the name Missouri Lead Operating Company. A mill and smelter also were constructed (Rogers and Davis, 1977). In 1967, 32 mi of railroad track were completed from Keysville (fig. 1) to the Buick Mine to transport the products from the mill and smelter. In 1986, Homestake Mining Company assumed ownership and suspended mine/mill/smelter operations. Later in 1986, the operation was purchased by Doe Run and reactivated. During 1989, Doe Run installed a copper recovery circuit at the mill. Before installation of this circuit, all copper minerals transported to the surface with the lead and zinc ore were sent to the tailings. A particle-size indicator also was installed, allowing better control of grinding, density of particles, and the flotation separation process. In addition, in 2004 a copper pre-float circuit was added to the mill. The pre-float circuit improved the recovery of copper minerals during flotation, resulting in fewer copper minerals being disposed of in the tailings pond (Denis Murphy, The Doe Run Company, written commun., 2005).

The Buick Mine received NPDES outfall permits in 1990. Outfall 001 is the designated discharge point for water from a sewage lagoon system upstream from outfall 001 (McLaren/ Hart Environmental Engineering Company, 1991b). Effluent from the sewage lagoon originally was disposed in the tailings pond (Escalera, 1973).

The tailings pond contains mine water, process water, and stormwater runoff, and discharges to Strother Creek. Excess water in the pond is decanted to a 3-mi long meander system that begins at the pond decant discharge pipe. The meander system enters a 25-acre final settling pond for the Buick Mine water discharge. Overflow from this pond travels through additional meanders before discharging at outfall 002 . The system is designed as a biologic remediation system for the decanted water. An emergency spillway discharges water from major storms.

\section{Brushy Creek Mine and Mill}

Drilling for the Brushy Creek Mine (figs. 2, 11) shaft began in 1968, and production began in 1973. St. Joe constructed a mine-mill complex at the site, located in Reynolds County (Evans, 1977). The mine briefly was placed on standby

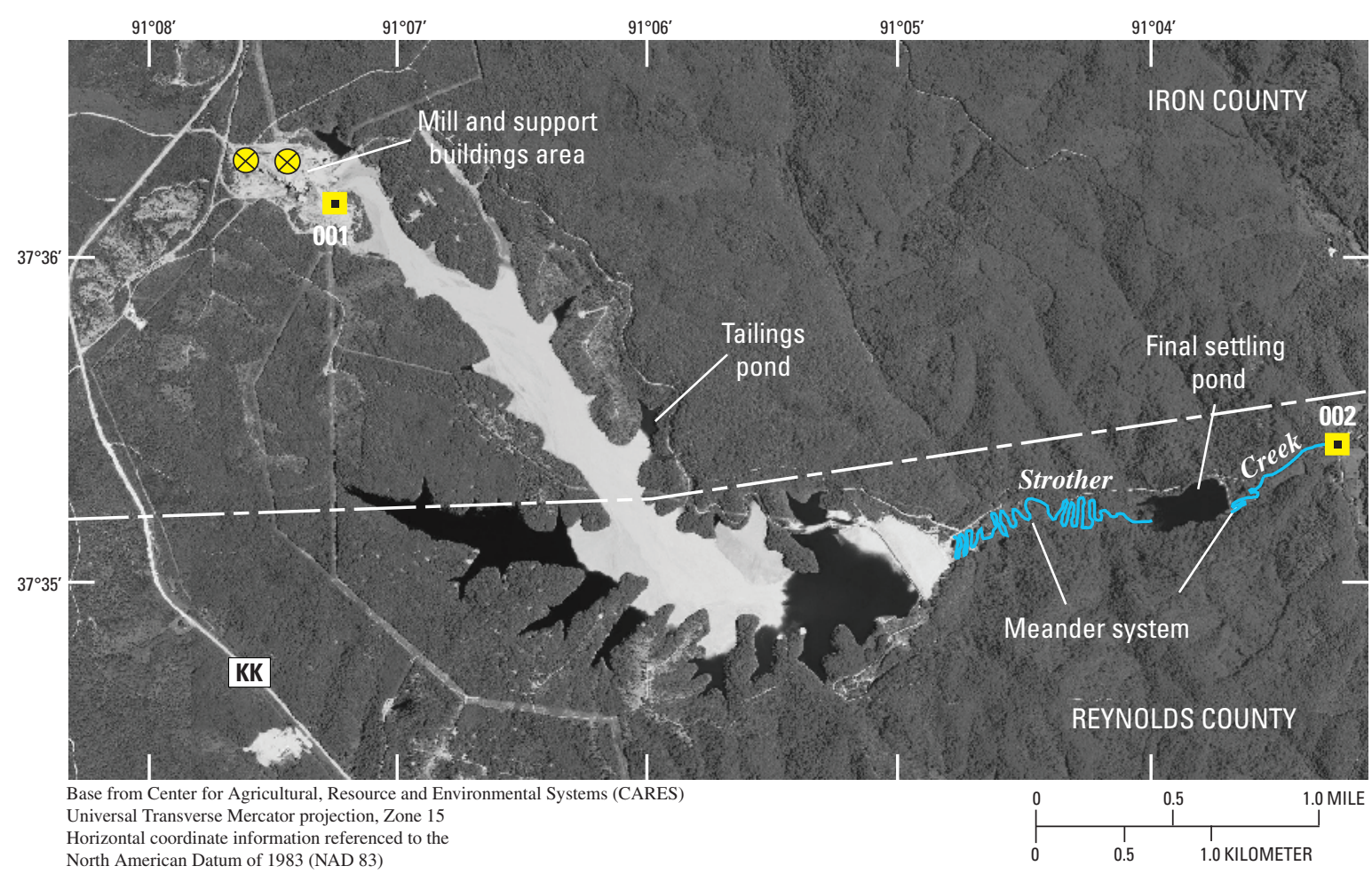

EXPLANATION

Figure 10. Buick Mine and mill, 2003. 


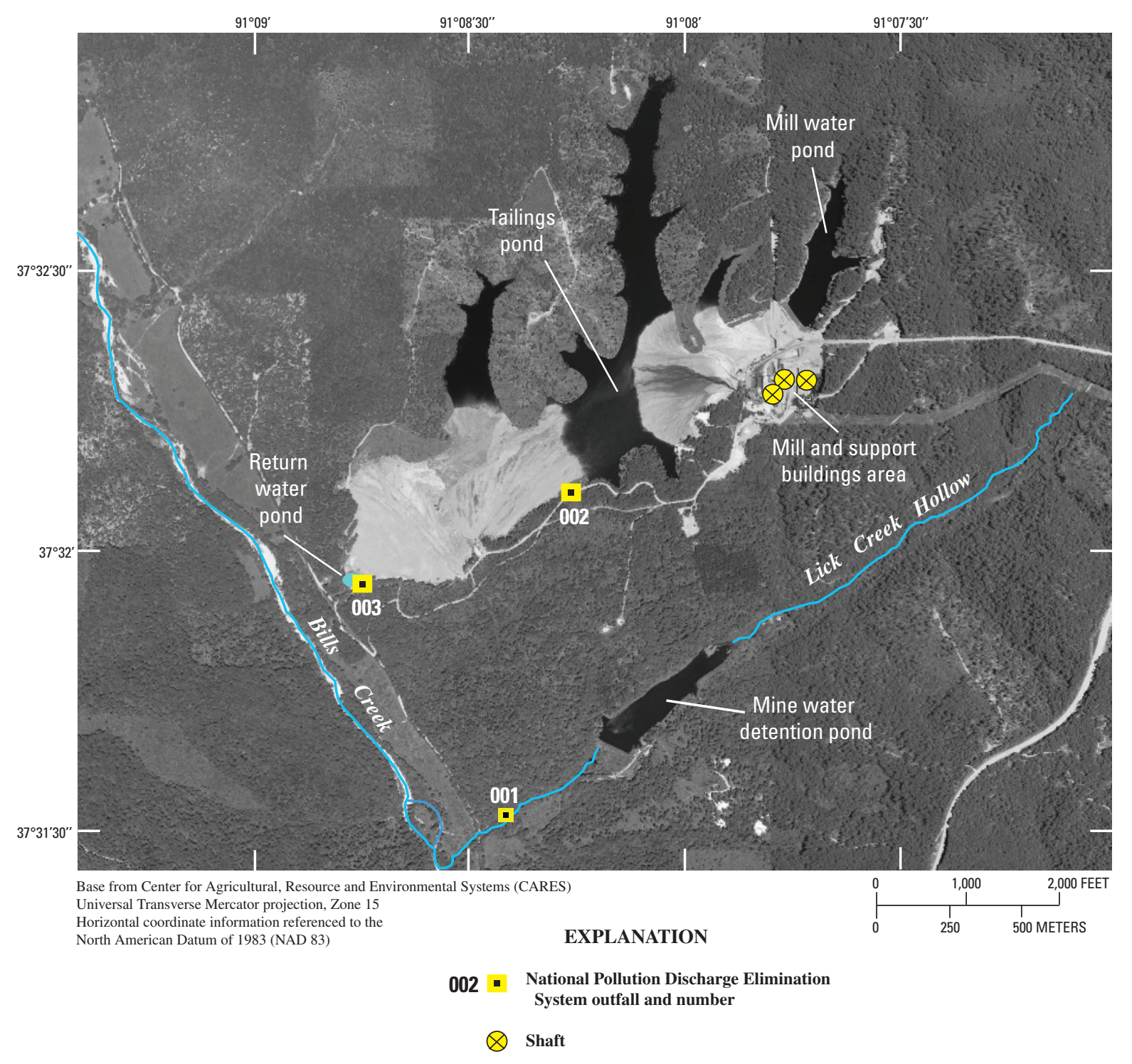

Figure 11. Brushy Creek Mine and mill, 2003.

in 1986. The mill processes all ore from the Brushy Creek Mine and about 20 percent of the ore from the Casteel Mine (Viburnum No. 35 Mine). In addition, starting in 1989, about 300 ton/d of high grade copper ore from the Fletcher Mine has been trucked to the Brushy Creek Mill for processing (Denis Murphy, The Doe Run Company, oral commun., 2005).

The Brushy Creek Mine has three NPDES outfall permits, issued in 1989. Mine water is pumped to a detention pond in Lick Creek Hollow; the pond also retains stormwater runoff. Treatment is by settling. Excess water is released at outfall 001 (McLaren/Hart Environmental Engineering Company, 1991a).

The tailings pond retains process water and stormwater runoff and is designed as zero discharge. Water collected in a return water (seepage collection) pond at the toe of the dam is pumped back into the tailings pond. The seepage collection pond is zero discharge during normal operation; any discharge would be at outfall 003. An emergency spillway on the south- east side of the tailings pond (outfall 002) discharges into a tributary of Bills Creek. The spillway is zero discharge during normal rainfall (McLaren/Hart Environmental Engineering Company, 1991a).

Brushy Creek Mine constructed a complete recycling system for water discharged from milling and flotation (Wixson, 1977). A mill water pond, constructed in 1969, contains water pumped back from the tailings pond. Excess water is piped to the tailings pond. No discharge point exists for this reservoir (McLaren/Hart Environmental Engineering Company, 1991a). The reservoir is part of a complete recycling system for water used in milling and flotation (Wixson, 1977).

\section{Casteel Mine (Viburnum No. 35 Mine)}

The Casteel Mine (figs. 2, 12), also called the Viburnum No. 35 Mine, is in Iron County and was opened by St. 


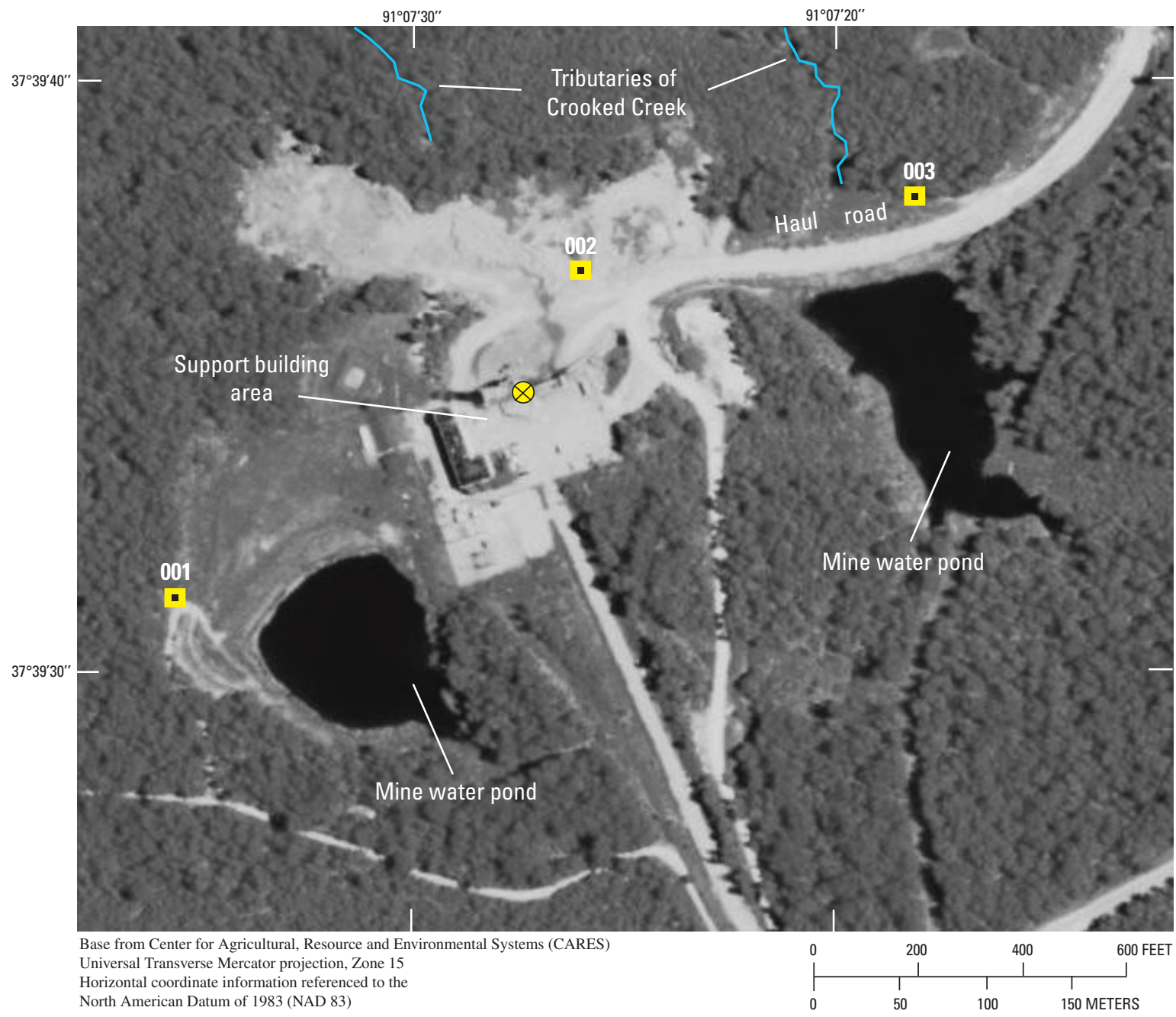

EXPLANATION

002 - National Pollution Discharge Elimination System outfall and number

$\otimes$ Shaft

Figure 12. Casteel Mine (Viburnum No. 35 Mine), 2003.

Joe in 1983. Similar to Viburnum No. 27 and No. 29 Mines, the Casteel Mine has no mill or tailings pond. Until 2000, about 80 percent of the ore from the mine was trucked to the Central Mill and about 20 percent was trucked to the Brushy Creek Mill. Currently (2008) ore is trucked to the Buick Mill (Denis Murphy, The Doe Run Company, written commun., 2008). Mine water is pumped to two mine water ponds, then discharged into tributaries of Crooked Creek. The dam for the east pond also is a bridge structure for the haul road to the Central Mill. Outfalls for the ponds are 001 (west pond) and 003 (east pond). Sewage is passed through a treatment system, followed by a single cell lagoon; this water is released at outfall 002 (McLaren/Hart Environmental Engineering Company, 1991d).

\section{West Fork Mine and Mill}

The West Fork ore body was discovered in Reynolds County in the early 1960s by Asarco, Inc. Various factors, including depressed lead prices, delayed development of the deposit until the early 1980s. The mine (figs. 2, 13) began limited production in 1985 and reached full production in 1988. The mine was purchased by Doe Run in 1997. The West Fork Mill produces lead and zinc concentrates. Lead concentrate was shipped to the Glover Smelter until operations at the smelter were suspended in 2003 because of a decreased demand for lead; the concentrate is now shipped to the Herculaneum Smelter. West Fork Mine is currently (2008) considered to be part of the Fletcher Mine. 


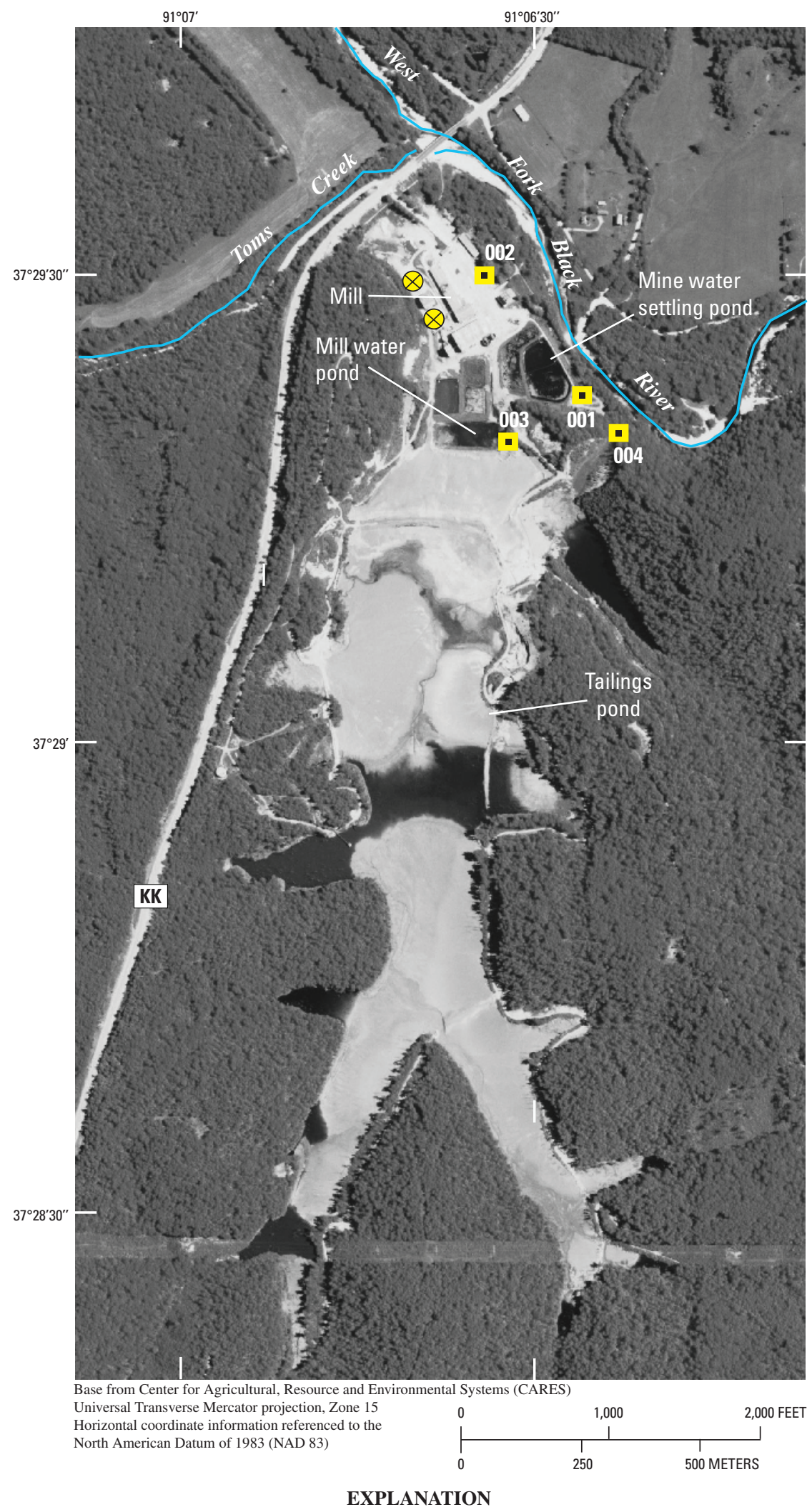

002 - National Pollution Discharge Elimination System outfall and number

$\otimes$ Shaft

Figure 13. West Fork Mine and mill, 2003. 
The West Fork Mine has four NPDES outfall permits originally issued in 1983. Outfall 001 discharges excess mine water after treatment. The water is pumped from an underground settling complex to a surface settling pond and the receiving tributary is the West Fork Black River (Asarco, Inc., 1991b).

Outfall 002 discharges sanitary wastewater, after treatment in an extended aeration treatment plant. Discharge is into a tributary of West Fork Black River (Asarco, Inc., 1991b). The sanitary wastewater originally was potable well water. The solid waste is transported to public or commercial landfills.

Outfall 003 discharges water from a mill water pond that collects stormwater runoff, water from the coarse and fine tailings, and mill seepage. Solids are decanted and reclaimed; the water is pumped to the tailings pond that is upstream from outfall 004. Floating pumps return water from the tailings pond to the mill via a storage tank. Outfalls 003 and 004 are designed to discharge only during storms that exceed a 25 -year storm. The mill water circuit is designed as a total recycle circuit with no discharge (Asarco, Inc., 1991b)

The West Fork mine and mill complex has a "de minimis" air permit that pertains to the ore bin (no controls and product is wet), enclosed ore conveyor tubes and storage bunkers, sample preparation area and storage bins (both with baghouse control), and underground storage tanks. The primary crusher underground uses a wet scrubber to control dust emissions. The secondary crusher is enclosed in a tower and has a wet scrubber (Asarco, Inc., 1991b).

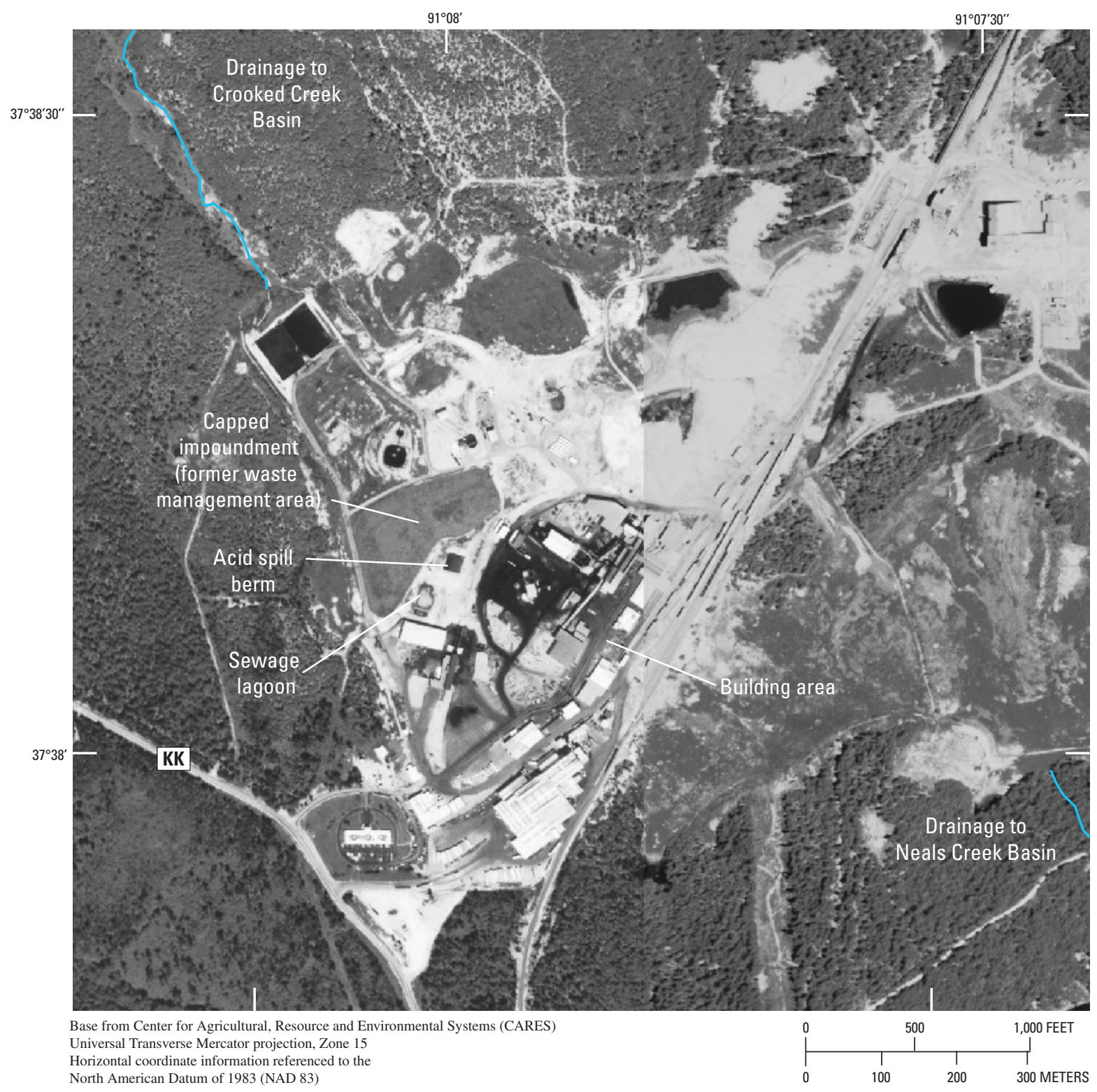

Figure 14. Buick Resource Recovery Facility (Buick Smelter), 2003. 


\section{Buick Resource Recovery Facility (Buick Smelter)}

The Buick Smelter (figs. 2, 14) is located about $2 \mathrm{mi}$ north of the Buick Mine and mill and immediately west of the Magmont Mine on the drainage divide between Crooked Creek and Neals Creek. The Buick Smelter and Buick Mine and mill are separate facilities and are operated by different entities within Doe Run. The smelter operated intermittently for several years. In 1991, the Buick Smelter was converted from primary recovery of lead from ore concentrates to a secondary smelter recovering lead as a recycled product. At this time, the facility was renamed the Buick Resource Recovery Facility (The Doe Run Company, 2007). Because of its location near the drainage divide, smelter emissions potentially can affect Neals Creek Basin as well as Crooked Creek Basin. Water impoundments onsite are for stormwater runoff control (Barr Engineering, 2005).

The Buick Smelter has a WMA primarily consisting of a slag disposal area. Closure of the former WMA was initiated with closure of a surface impoundment and with the grading and covering (capped) of the WMA. The WMA currently (2008) is being evaluated as a potential future secondary slag storage area under a Resource Conservation and Recovery Act (RCRA) permit modification. This modification may affect some closure and post-closure aspects of the WMA (Barr Engineering, 2005).

\section{Summary}

Missouri has three world-class lead/zinc subdistricts (Old Lead Belt, Mine La Motte-Fredericktown, and Viburnum Trend) and several minor subdistricts that are in a region referred to as the Southeast Missouri Lead District. Arsenic, cadmium, cobalt, copper, lead, nickel, and zinc are the primary trace elements associated with the sulfide minerals of the Mississippi Valley Type ore deposits present in the district. Although the history of lead and zinc mining in the Southeast Missouri Lead District is discussed in this report, emphasis is placed on the Viburnum Trend, which is an active subdistrict. A general description of the mine processes, regulatory controls, environmental effects, and mine facilities in the Viburnum Trend are provided to explain possible contaminant sources and transport routes of mining-related materials to the surrounding environment.

Earliest mining occurred in pits less than 10-foot deep, but with time companies had to mine deeper (depths more than 1,000 feet) to find ore. As more ore bodies were discovered, a relation between Precambrian structural highs and mineralization that developed in complex carbonate facies in the surrounding Bonneterre Formation was observed. This observation aided in the discovery of the Viburnum Trend Subdistrict.

Production began in the Viburnum Trend in 1960; 10 mines eventually were opened in the subdistrict. Galena is the primary ore mineral; sphalerite is the second most common ore mineral. Total ore production from individual mines range from 20 to more than 50 million tons, and ore contains as much as 8 percent lead and several percent zinc.

Most Viburnum Trend mines operate using the same general steps and procedures. Mining is done using a room and pillar method that follows the ore trend with mine passages that are approximately 30 feet wide and at least 20 feet high. Mine water from dewatering is pumped to the surface and retained in either a tailings pond or mine water detention pond, then discharged to surrounding surface streams.

Of the 10 mines in the Viburnum Trend, 7 have or had mills onsite to concentrate the ore, as well as facilities for transportation of ore mineral concentrates. Milling processes have remained essentially the same throughout the history of the Viburnum Trend and follows four primary stages; crushing and grinding, flotation, filtering and dewatering, and tailings disposal. The mills have undergone changes and improvements for recovery enhancement or for recovery of additional metals. All mills in the Viburnum Trend have lead and zinc circuits; most mills had or have copper circuits. Mine tailings (waste rock) from the milling process generally are transported through pipes as slurry and are contained in a surface disposal area (tailings pond). Materials in the tailings ponds consist of gangue minerals (primarily dolomite) and minor amounts of ore minerals; flotation reagents also may be present. Mines that do not have mill operations onsite transport the ore to either the Buick Mill or Brushy Creek Mill generally by truck on haul roads or on property owned or leased by the company; some transportation occurs on public highways.

Smelter dust (fine-particulate fraction) and gas releases probably are the greatest potential source of contamination in the production process. Air emissions at the smelters are monitored for sulfur dioxide. Material can be released to the environment by spillage while being loaded onto truck or rail transport or may be windblown from ore concentrate piles at the smelter. These materials can be carried into the offsite environment by stormwater runoff.

Permitting and legislation affecting mines and smelters only have been in effect for relatively few years. The Missouri Department of Natural Resources, Hazardous Waste Program inspects facilities for violations of hazardous waste handling. The major environmental issue for The Missouri Department of Natural Resources, Land Reclamation Program is wind-blown transport of tailings material outside the waste management area. During 1997, the Sweetwater Mine was issued a notice of violation as an unauthorized hazardous waste treatment/disposal facility. Violations included failure to label and mark containers and the presence of an open container. Compliance was met in 2 to 3 weeks. Inspections of other Viburnum Trend mines and the Buick Smelter have indicated no violations since the mid 1990s. The Missouri Departmemt of Natural Resources, Hazardous Waste Program has one Superfund site in the Viburnum Trend area-public highways used as transportation routes by trucks hauling ore mineral concentrates to smelters or shipment points. Highways 
within the Superfund site are located in Iron, Reynolds, and Dent Counties.

Waste products from mining, milling, and smelting have affected the environment immediately surrounding the operation. Studies completed in and around the Viburnum Trend document some of the effects. The environmental effects of concern in the Viburnum Trend include wind-blown dust or water-transported trace elements from ore mineral concentrates and tailings, deposition from gases related to smelting, and unusual widespread growth of benthic bacterial/algal mats downstream from operations. One study noted a decline in the number and type of benthic organisms downstream from operations. Another study observed the Buick Smelter emissions affecting the foliage of trees in the surrounding woodlands. Responses by the industry to these effects have resulted in improved air quality from smelter stack releases and further remediation of mine and process waters.

All mine sites in the Viburnum Trend have certain features in common - at a minimum, they have a production shaft, detention pond(s) for mine water and surface runoff, and buildings for offices, supplies, change room, and hoist room. At sites that do not have a mill, loading facilities are present for transportation of ore to a mill. There also are provisions for detention of process waters used in the milling operations.

\section{References Cited}

Arseneau, J.G., 1976, Chemical and mineralogical characteristics of particulates in stack emission from two lead smelters in southeast Missouri: University of Missouri-Rolla, unpublished M.S. thesis, $63 \mathrm{p}$.

Asarco, Inc., 1991a, Application for metallic minerals waste management permit (Asarco, Inc. Missouri Lead Division Sweetwater Unit): Tucson, Ariz., 147 p., 5 pl.

Asarco, Inc., 1991b, Application for metallic minerals waste management permit (Asarco, Inc. Missouri Lead Division West Fork Unit): Tucson, Ariz., 173 p., 5 pl.

Barr Engineering, 2005, 2004 annual groundwater monitoring report, The Doe Run Company Buick Resource Recovery Facility: Minneapolis, Minn., 212 p.

Boggess, W.R., and Wixson, B.G., 1977, Lead in the environment: A report and analysis of research at Colorado State University, University of Illinois at Urbana-Champaign, and University of Missouri-Rolla: Washington, D.C., National Science Foundation, 272 p.

Bolter, Ernst, Wixson, B.G., Butherus, D.L., Jennett, J.C., 1974a, Distribution of trace elements in soils near an active lead smelter: University of Missouri-Rolla, 17 p.
Bolter, Ernst, Jennett, J.C., and Wixson, B.G., 1974b, Geochemical impact of lead-mining wastewaters on streams in southeastern Missouri: University of Missouri-Rolla, 9 p.

Bornstein, R.E., 1989, Long term geochemical effects of lead smelting within the New Lead Belt, southeast Missouri: University of Missouri-Rolla, unpublished M.S. thesis, $84 \mathrm{p}$.

Burford, J., 1978, Underground treasures: The story of mining in Missouri, in Johnson, K.M., ed., Official Manual State of Missouri 1977-1978: Jefferson City, Mo., p. 1-33.

Butherus, D.L., 1975, Trace element contamination in soil around a lead smelter in southeast Missouri: University of Missouri-Rolla, unpublished M.S. thesis, 131 p.

Erten, Z.M., 1988, Toxicity evaluation and biological treatment of lead and zinc mine-mill effluents in southeast Missouri: University of Missouri-Rolla, unpublished Ph.D. dissertation, $292 \mathrm{p}$.

Escalera, W.A., 1973, Pollution abatement of mining effluents in the New Lead Belt of Missouri: University of MissouriRolla, unpublished M.S. thesis, 87 p.

Evans, L.L., 1977, Geology of the Brushy Creek Mine, Viburnum Trend, southeast Missouri: Economic Geology, v. 72, no. 3, p. 381-390.

Faeth, A.M., Wronkiewicz, D.J., Adams, C.D., Mendoza, C., McBurnett, J., Krizanich, G.W., Struttman, S.R., Hemman, R., and Wolf, S.F., 2004, Trace element transport processes in the Black River of Missouri's New Lead Belt: Geological Society of America Abstracts with Program 38th Annual Meeting 2004, v. 36, no. 3, p. 4.

Femmer, S.R., 2004, Background and comparison of waterquality, streambed-sediment, and biological characteristics of streams in the Viburnum Trend and the exploration study areas, southern Missouri, 1995 and 2001: U.S. Geological Survey Water-Resources Investigations Report 03-4285, $18 \mathrm{p}$.

Fenneman, N.M., 1938, Physiography of eastern United States: New York, McGraw-Hill, 714 p.

Fernandes, D.L., 1987, A study on chemical extraction and bioassays for lead, zinc and cadmium and effluent characterization in the New Lead Belt of Missouri: University of Missouri-Rolla, unpublished M.S. thesis, 98 p.

Fletcher, C.S., 1974, The geology and hydrogeology of the New Lead Belt, Missouri: University of Missouri-Rolla, unpublished M.S. thesis, $91 \mathrm{p}$.

Gale, N.L., 1974, The impact of lead mine and mill effluent on aquatic life: University of Missouri-Rolla, 22 p. 
Grundmann, W.H. Jr., 1977, Geology of the Viburnum No. 27 Mine, Viburnum Trend, southeast Missouri: Economic Geology, v. 72, no. 3, p. 349-364.

Hagni, R.D., and Trancynger, T.C., 1977, Sequence of deposition of the ore minerals at the Magmont Mine, Viburnum Trend, southeast Missouri: Economic Geology, v. 72, no. 3, p. 451-464.

Hydro-Search, Inc., 1991, Revised application for metallic minerals waste management permit, Magmont Mine tailings pond, Cominco American Incorporated, Bixby, Missouri: Golden, Colo., 146 p., 3 pls.

Jennett, J.C., 1974, Transport mechanisms of lead industry wastes: University of Missouri-Rolla, 19 p.

Kiilsgaard, T.H., Hayes, W.C., and Heyl, A.V., 1967, Lead and zinc, in Mineral and Water Resources of Missouri: Rolla, Missouri Department of Natural Resources, Division of Geology and Land Survey, v. 43, p. 41-63.

Kleeschulte, M.J., 2001, Effects of lead-zinc mining on ground-water levels in the Ozark Aquifer in the Viburnum Trend, Southeastern Missouri: U.S. Geological Survey Water-Resources Investigations Report 00-4293, 28 p.

Kleeschulte, M.J., 2006, Ground-water levels in the Ozark Aquifer along the Viburnum Trend, Southeastern Missouri, 2001-05: U.S. Geological Survey Scientific Investigations Report 2006-5220, 21 p.

Kleeschulte, M.J., and Seeger, C.M., 2003, Stratigraphy and vertical hydraulic conductivity of the St. Francois confining unit in the Viburnum Trend and evaluation of the unit in the Viburnum Trend and exploration areas, Southeastern Missouri: U.S. Geological Survey Water-Resources Investigations Report 03-4329, 57 p.

Leach, D.L., and Sangster, D., 1993, Mississippi ValleyType lead-zinc deposits, in Kirkham, R.V., Sinclair, W.D., Thorpe, R.I., and Duke, J.M., eds., Mineral deposit modeling: Geological Association of Canada, Special Paper, v. 40, p. 289-314.

McLaren/Hart Environmental Engineering Company, 1991a, Application for metallic minerals waste management area permit, The Doe Run Company Brushy Creek operations, Missouri: Lester, Pa., 120 p.

McLaren/Hart Environmental Engineering Company, 1991b, Application for metallic minerals waste management area permit, The Doe Run Company Buick operations, Missouri: Lester, Pa., 144 p.

McLaren/Hart Environmental Engineering Company, 1991c, Application for metallic minerals waste management area permit, The Doe Run Company Fletcher facility, Missouri: Lester, Pa., 122 p.
McLaren/Hart Environmental Engineering Company, 1991d, Application for metallic minerals waste management area permit, The Doe Run Company Viburnum operations, Missouri: Lester, Pa., 402 p.

Missouri Department of Labor and Industrial Relations, 1967, Eightieth annual report of the Division of Mine Inspection: Jefferson City, Mo., 38 p.

Missouri Department of Natural Resources, 2007, 2006 Annual Compliance Report of Missouri Public Drinking Water Systems: Jefferson City, Public Drinking Branch, Water Protection Program, accessed January 14, 2008, at URL: http://dnr.missouri.gov/pubs/pub2228.pdf

Missouri Department of Natural Resources, 2008, Census of Missouri public water systems: accessed January 15, 2008, at URL, http://dnr.missouri.gov/env/wpp/census.htm

Missouri Department of Natural Resources, Division of Environmental Quality, 2008, Metallic minerals permitting: accessed January 14, 2008, at URL http://www.dnr.mo.gov/ env/lrp/homemm.htm

Missouri Department of Natural Resources, Land Reclamation Program, 2003, 2001 and 2002 Biennial Report: Jefferson City, Mo., 30 p.

Mouat, M.M., and Clendenin, C.W., 1977, Geology of the Ozark Lead Company Mine, Viburnum Trend, southeast Missouri: Economic Geology, v. 72, no. 3, p. 398-407.

Paarlberg, N.L., and Evans, L.L., 1977, Geology of the Fletcher Mine, Viburnum Trend, southeast Missouri: Economic Geology, v. 72, no. 3, p. 391-397.

Rakovan, John, 2007, Mississippi Valley Type deposits: Oxford, Ohio, Miami University, accessed October 5, 2007 , at URL http://www.users.muohio.edu/rakovajf/WTTW\%20 MVT.pdf

Rogers, R.K., and Davis, J.H., 1977, Geology of the Buick Mine, Viburnum Trend, southeast Missouri: Economic Geology, v. 72, no. 3, p. 372-380.

Snyder, F.G., and Gerdemann, P.E., 1968, Geology of the Southeast Missouri Lead District, in Ridge, J.D., ed., Ore deposits of the United States, 1933-1967: New York, The American Institute of Mining Metallurgical and Petroleum Engineers, Inc., p. 327-358.

Sweeney, P.H., Harrison, E.D., and Bradley, M., 1977, Geology of the Magmont Mine, Viburnum Trend, southeast Missouri: Economic Geology, v. 72, no. 3, p. 365-371.

Swenty, B.J., 1996, Construction permit application for the modification of the Magmont Mine tailings dam, Iron County, Missouri: Jefferson City, Mo., 104 p. 
The Doe Run Company, 2007, Company information, accessed November 6, 2007, at URL http://www.doerun. com/about/company.aspx\#timeline

Tibbs, N.H., 1969, The background concentrations of copper, lead, and zinc in streams of the "New Lead Belt": University of Missouri-Rolla, unpublished M.S. thesis, 86 p.

U.S. Environmental Protection Agency, 2008, National Pollutant Discharge Elimination System (NPDES), accessed January 15, 2008, at URL http://cfpub.epa.gov/npdes/

U.S. Geological Survey, 2008, Mineral commodity summary, accessed January 18, 2008, at URL http://minerals.usgs. gov/minerals/pubs/commodity/statistical_summary/

Vinyard, J.D., 1977, Preface: Economic Geology, v. 72, no. 3, p. 337-338.

Wharton, H.M., 1975, Introduction to the Southeast Missouri Lead District, in Guidebook to the geology and ore deposits of selected mines in the Viburnum Trend, Missouri: Rolla, Missouri Department of Natural Resources, Division of Geology and Land Survey, Report of Investigations Number 58 , p. $2-14$.

Wixson, B.G., 1977, The Missouri lead study: An interdisciplinary investigation of environmental pollution by lead and other trace elements from industrial development in the New Lead Belt of southeastern Missouri: University of Missouri-Rolla and University of Missouri-Columbia, $1,108 \mathrm{p}$.

Wixson, B.G., and Anderson, M.N., 1973, Missouri Lead Belt project: University of Missouri-Rolla, $11 \mathrm{p}$.

Wixson, B.G., Bolter, Ernst, Gale, N.G., Jennett, J.C., Purushothaman, K., 1972, The lead industry as a source of trace elements in the environment: University of MissouriRolla, 22 p. 



\section{Seepage Runs on Streams Draining the Viburnum Trend Subdistrict, Southeastern Missouri, August 2003-0ctober 2006}

By Michael J. Kleeschulte

Chapter 2 of

Hydrologic Investigations Concerning Lead Mining Issues in Southeastern Missouri

Edited by Michael J. Kleeschulte

Scientific Investigations Report 2008-5140 


\section{Contents}

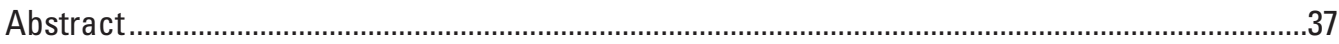

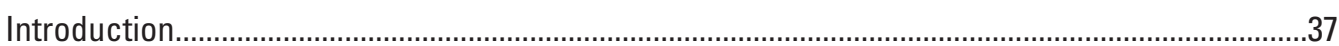

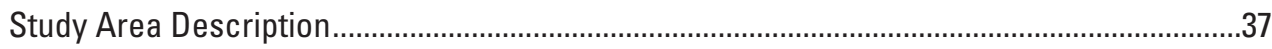

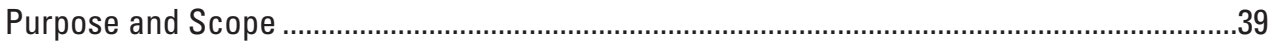

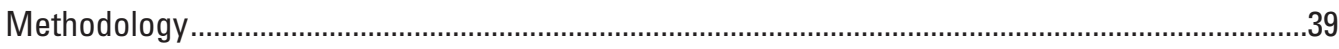

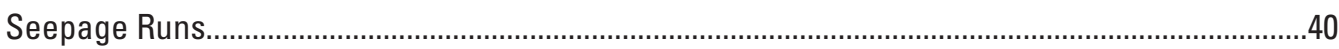

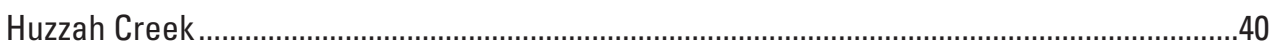

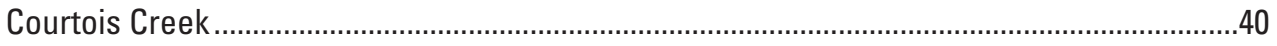

West Fork Black River ....................................................................................................... 40

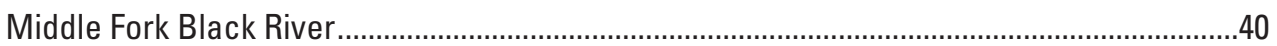

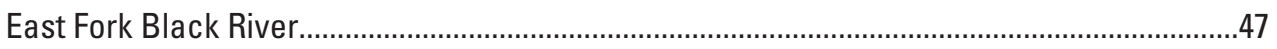

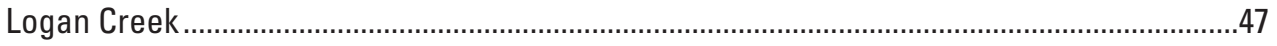

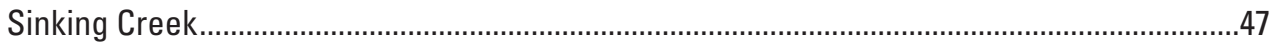

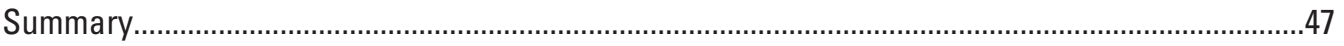

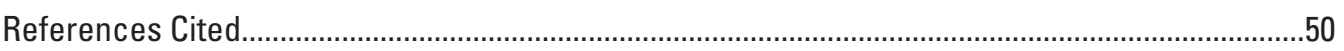

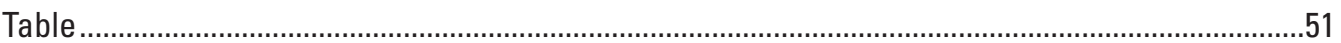

\section{Figures}

1-2. Maps showing-

1. Study area and seepage run sites on streams draining the Viburnum Trend Subdistrict...

2. Seepage run sites on streams in the Meramec River Basin draining the Viburnum Trend Subdistrict.

3-4. Graphs showing -

3. Seepage run data for West and East Forks Huzzah Creek and Crooked Creek, August 2003 through August 2006.

4. Seepage run data for Courtois Creek and Indian Creek, August 2003 through October 2006

5. Map showing seepage run sites on streams in the Black River Basin draining the central part of the Viburnum Trend Subdistrict.

6. Graphs showing weepage run data for the upper Black River Basin, August 2003 through August 2006...

7. Map showing seepage run sites on streams in the Black River Basin draining the southern part of the Viburnum Trend Subdistrict

8. Graphs showing seepage run data for the Black River Basin, August 2004 through September 2004 ...

\section{Table}

1. Seepage run data for streams draining the Viburnum Trend Subdistrict. 


\title{
Seepage Runs on Streams Draining the Viburnum Trend Subdistrict, Southeastern Missouri, August 2003- October 2006
}

\author{
By Michael J. Kleeschulte
}

\section{Abstract}

A study was conducted to identify streams or reaches of streams that lose discharge to the ground-water system in and around active lead and zinc mining areas along the Viburnum Trend Subdistrict that have potential to release mining-related trace elements or chemicals into receiving streams. The data collection began in August 2003 and was not completed until October 2006.

All the streams in the study area have sand and gravelfilled channels (streambed sediment) that include frequent, large bars composed of loose sand, gravel, and cobbles. During low flow, a large percentage of water can be traveling through the streambed sediment instead of flowing on the surface. In many cases, the water flowing through the streambed sediment resurfaces farther downstream and is not being lost to the underlying bedrock aquifer.

Stream reaches in East Fork Huzzah Creek; Strother Creek, Neals Creek, and Brushy Creek in the Middle Fork Black River Basin; and East Fork Black River had less discharge measured in a downstream mainstem site than was expected based on the upstream discharge measurements. None of these reaches were classified as losing. This classification is based on the quantity of gravel in the stream channel, the hydrologic assessment of the personnel making the measurements, and the rated discharge measurement accuracy for the sites.

Logan Creek had about a 13-mile reach with no flow that began losing discharge downstream from Sweetwater Creek. Discharge in Logan Creek was observed again near Ellington and continued to increase downstream to Clearwater Lake.

\section{Introduction}

The lead and zinc ore deposits of the Viburnum Trend Subdistrict in southeastern Missouri are part of the largest known lead reserve within the United States (U.S. Geological Survey, 2008). The initial discovery of lead and zinc deposits near Viburnum, Missouri, was made in 1955 and initial ore production began in 1960. Continued exploration led to the eventual opening of 10 operating underground mines along a 60-mile (mi) north-south trending band (C.M. Seeger, Missouri Department of Natural Resources, Division of Geology and Land Survey, written commun., 2008) that ranges from less than 500 feet (ft) wide to, in rare cases, about $1 \mathrm{mi}$ wide (Wharton, 1975).

Ore mined underground is brought to the surface and milled at several of the mines. Tailings (waste rock) and other wastes from the milling process are pumped as slurry to large surface impoundments that fill headwater stream valleys. One environmental effect of concern is water-transported trace elements from tailings piles into local streams.

The Viburnum Trend lies within a large region of welldeveloped karst terrain that is characterized by the presence of caves, springs, sinkholes, and gaining and losing streams. Karst terrain is used to describe a particular type of topography that forms when carbonate or other soluble rocks are dissolved by water percolating underground and enlarging subsurface openings. With time, some of these enlarged openings can cause the movement of water in the aquifer to change from diffuse flow through small, scattered openings in the rock to discrete flow that is concentrated within well-developed conduits. As these openings continue to enlarge, caves are formed and the ground-water level can decline below the level of surface streams. As the water table declines below the stream elevation, the stream loses water and any substances dissolved in water into the underlying bedrock (U.S. Geological Survey, 1985). Streams with this characteristic are known as losing streams.

\section{Study Area Description}

Much of the Viburnum Trend and study area is within the boundaries of the Mark Twain National Forest, which is managed by the U.S. Department of Agriculture, Forest Service (fig. 1). Much of the area inside the National Forest boundaries is uninhabited, hickory-oak forest. The area includes scattered parcels of private property that contain either isolated homesteads or small communities. Most of the private property is concentrated along major roads or along stream valleys. 


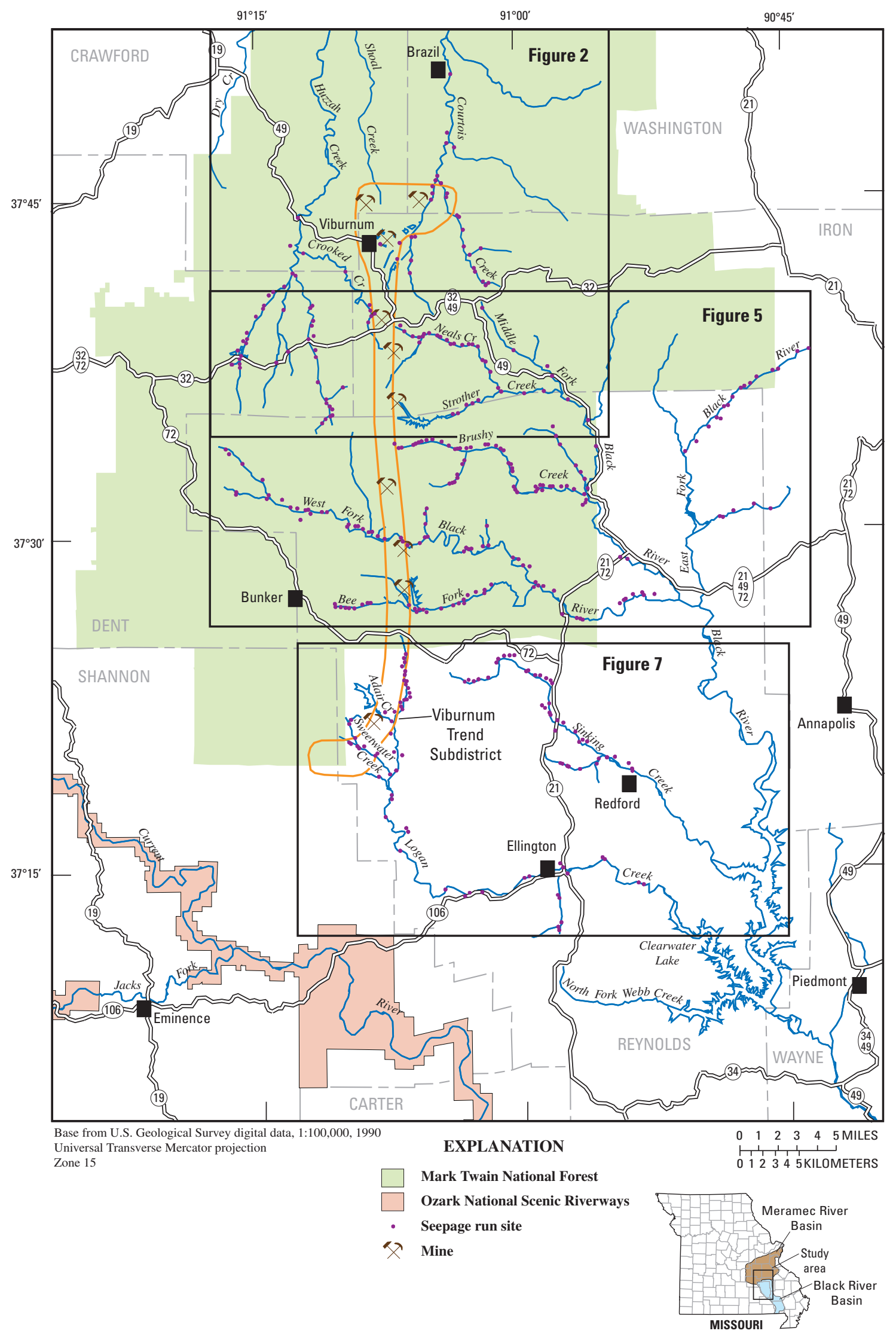

Figure 1. Study area and seepage run sites on streams draining the Viburnum Trend Subdistrict. 
This area is characterized by deep, narrow valleys, and narrow, steep-sided ridges. A regional surface-water divide trends northeast across the northern part of the study area (U.S. Geological Survey, 1977). The northward flowing streams are part of the Meramec River Basin and the southward flowing streams are part of the Black River Basin. Most of the springs in the study area are small, discharging 0.1 to 1.0 cubic foot per second $\left(\mathrm{ft}^{3} / \mathrm{s}\right)$ (Vineyard and Feder, 1974)

\section{Purpose and Scope}

The purpose of this chapter is to describe seepage runs on streams draining the Viburnum Trend Subdistrict (an active lead and zinc mining area in southeast Missouri; fig. 1) to identify streams or reaches of streams that lost discharge to the ground-water system between August 2003 and October 2006. Emphasis was placed on streams in and around active mines in the subdistrict that have potential to release mining-related trace elements or chemicals into receiving streams. Streams included Huzzah and Courtois Creeks in the Meramec River Basin and tributaries of the Black River upstream from Clearwater Lake (fig. 1).

\section{Methodology}

A seepage run is a series of discharge measurements made along a stream within a short time to identify gaining and losing reaches. Typically, measurements were made consecutively downstream at stream sections where channel morphology and velocity were favorable to make accurate discharge measurements. Seepage runs are designed to be made during periods of minimum streamflow and minimum daily fluctuations (base flow) when discharge is sustained by diffuse ground-water and spring inflow, not by surface runoff.

Discharge measurements were made using acoustic Doppler velocity and current meters and followed the methods described by Rantz and others (1982). Discharge measurements were rated according to stream channel conditions and uniformity of flow. The rated measurement accuracies are defined as follows: "good" indicates that the measured discharge is estimated to be within 5 percent of the true discharge; "fair" between 5 and 8 percent; and "poor" greater than 8 percent. The criteria for rating a discharge measurement are based on guidelines using flow and stream channel characteristics observed at the measuring section (Sauer and Meyer, 1992; Nolan and Shields, 2000). However, the measurement rating is subjective based on the assessment of the personnel making the measurement. Discharge was estimated at sites where there was minimal discharge and conditions were not adequate for making discharge measurements, either because of insufficient water depths, obstructions in the stream channel, or minimal flow velocities. Discharge was estimated by measuring the stream width, estimating an average stream depth, and estimating an average velocity for the measuring section. The product of these three components is the estimated discharge. All estimated discharges are rated poor.

The measurement accuracy and associated error defined by the measurement rating became an important determining factor in classifying several stream reaches as non-losing, even though less discharge was measured at a downstream site than was measured upstream. Along these reaches, the measurement error was considered at the adjacent mainstem measuring sites along with the measured discharge from tributary inflow between the mainstem sites. To calculate the range of possible discharge for a given measurement (error bars), a "good" measurement rating has error bars limited to plus or minus 5 percent of the actual measurement; "fair" is limited to plus or minus 8 percent; and poor is limited to plus or minus 10 percent. The resulting error bars then were compared. If successive error bars overlapped, indicating that the observed decrease in discharge could be the result of measurement error, the reach was not classified as losing.

The stream length used to identify the seepage run sites in the figures and table in this chapter was calculated from the beginning of the stream channel as defined by the hydrologic coverage from the U.S. Geological Survey 1:100,000 digital data. Stream length shown in all figures and tables increases downstream.

Specific conductance and water temperature generally were measured at the seepage-run sites at the time of the discharge measurement. These measurements were made according to procedures described by Wilde and Radtke (1998). Specific conductance was measured with a temperature compensating meter calibrated to read in microsiemens per centimeter at 25 degrees Celsius $\left({ }^{\circ} \mathrm{C}\right)$. Water temperature was measured with a thermistor to the nearest $0.1{ }^{\circ} \mathrm{C}$.

The seepage runs described in this chapter were conducted on the northward flowing Huzzah Creek and Courtois Creek of the Meramec River Basin and tributaries to the Black River upstream from Clearwater Lake (fig.1). The tributaries of the Black River included three forks (West Fork, Middle Fork, and East Fork), Logan Creek, and Sinking Creek and their tributaries. The study was cursory in that the stream discharge was monitored at road crossings or at other convenient locations rather than traversing the entire length of these streams. When rainfall caused runoff to occur and base-flow conditions to cease, the seepage run was stopped and not restarted until base-flow conditions existed again. These postponements occurred several times. Discharge measurements along several reaches were repeated at later dates because of either inconsistencies or a sparse distribution in the original data, and additional streams were added. Because of these postponements, restarts, reexaminations, and expansion of the study area, the data collection for this study that began in August 2003 was not completed until October 2006. This extensive timeframe is contrary to the desired method of completing seepage runs within a short period.

All streams in the study area have sand and gravel-filled stream channels (streambed sediment) that include frequent, large bars composed of loose sand, gravel, and cobbles. Dur- 
ing low flow, a large percentage of the water can be traveling through the streambed sediment instead of flowing on the surface. In many cases, the water flowing through the streambed sediment resurfaces farther downstream and is not being lost to the underlying bedrock aquifer. Discharge measurements were avoided when possible in areas where large gravel bars were present, but on occasion alternative sites were not a viable option. These features were more frequently encountered in the vicinity of a tributary mouth where the stream channels widen and gravel deposits increase in thickness. Discharge measurements at sites where flow through streambed sediment occurred hampered the analysis of the seepage run data because the quantity of water lost through the streambed sediment could not be determined, and the assigned discharge measurement rating was based solely on observed flow and stream-channel conditions. Based on the hydrologic assessment of the personnel making the discharge measurement, occasionally these reaches were not classified as losing even though considerably more discharge was measured upstream. These assessments typically were affected by hydrologic observations of smaller tributaries in the immediate area of the site of concern. If water was flowing in these smaller tributaries or if the quantity of water lost at a particular site resurfaced at the next downstream mainstem measuring site, the site or reach with decreased discharge was not classified as losing.

\section{Seepage Runs}

\section{Huzzah Creek}

No losing stream reaches were identified in Huzzah Creek or its major tributaries (figs. 2, 3; table 1, at the back of this chapter); however, the East Fork Huzzah Creek (fig. 3B) had less measured discharge at the mouth (site hce 18; 2.59 $\mathrm{ft}^{3} / \mathrm{s}$ ) than at the previous upstream mainstem measuring site (site hce 16; $2.99 \mathrm{ft}^{3} / \mathrm{s}$ ). The quantity of lost discharge between the two sites exceeded that which could be attributed to the error defined by the measurement rating at the sites; therefore, the reach could be classified as losing. However, because of a large sand and gravel bar at the mouth of East Fork Huzzah Creek, a considerable quantity of water likely was flowing through the streambed sediment and could not be measured. This conclusion is supported by the discharge measured at the downstream Huzzah Creek site hc03 (figs. 2, 3A; table 1), which was larger than the combined discharge from West Fork Huzzah Creek (site hcw17), East Fork Huzzah Creek (site hce18), Crooked Creek (site hc01), and Indian Creek (site hc02).

\section{Courtois Creek}

No losing stream reaches were identified in Courtois Creek or its major tributary, Indian Creek, during this study (figs. 2, 4A, 4B; table 1). However, the Indian Creek seep- age run had somewhat inconsistent results. Indian Creek and its tributaries, specifically West Prong, drain an area that has been affected by considerable anthropogenic land disturbances associated with Viburnum No. 28 Mine and contains two mine tailings ponds with outfalls (Chapter 1 of this report). In the tailings ponds areas, there were physical changes to the stream channel, a lack of adequate discharge measuring sites, and a disruption of the steady, base-flow conditions that would otherwise be present in the stream. These factors made the analyses of the Indian Creek and West Prong data difficult. A large increase in discharge between two successive mainstem measuring sites on Indian Creek-site cc $14 \mathrm{~d}\left(0.43 \mathrm{ft}^{3} / \mathrm{s}\right.$; figs. 2, 4B) and site cc $14 \mathrm{~g}\left(9.82 \mathrm{ft}^{3} / \mathrm{s}\right)$ could not be attributed to measured inflows. The most probable explanation for the larger than expected discharge at site cc14g is additional unmeasured inflow to West Prong between the downstreammost measured site in West Prong (site cc14f; $2.77 \mathrm{ft}^{3} / \mathrm{s}$ ) and the mouth of West Prong.

\section{West Fork Black River}

The seepage run on West Fork Black River, which included Bee Fork, did not identify any losing stream reaches. The headwaters of West Fork Black River (Parker Branch and Crossville Branch) (fig. 5; table 1) were first investigated in August 2003, but because of rainfall the seepage run was suspended before the mainstem of the West Fork Black River was investigated. The seepage run resumed in September 2004, and discharges at the mouths of both Parker Branch (site wfb05; fig. 6A) and Crossville Branch (site wfb08, fig. 6A) were larger than when the initial seepage run ended in 2003. Discharge continued to increase in the mainstem of the West Fork Black River to the junction with Middle Fork Black River (fig. 5; table 1), with the largest discharge contributions from Bee Fork (site wfb43) and Reeds Spring (site wfb48).

Flow began in the headwaters of Bee Fork and in Kitchel Branch upstream from its junction with Bee Fork (fig. 5). Discharge increased in Bee Fork at each successive mainstem measurement downstream to the mouth (fig. 6B). The largest contributor of inflow to Bee Fork was an unnamed tributary that discharged water from the Fletcher Mine clarifying pond (site wfb43k, fig. 5; table 1).

\section{Middle Fork Black River}

The seepage run on Middle Fork Black River and its tributaries (fig. 5) did not conclusively identify any losing stream reaches; however, three tributaries (Strother Creek, Neals Creek, and Brushy Creek) had less discharge at downstream sites than was anticipated based on previous upstream measurements. The middle reach of the Middle Fork Black River initially was investigated in August 2003 and then reexamined at a later date because of inconsistencies in the original data. This resulted in the seepage run data for this stream being 

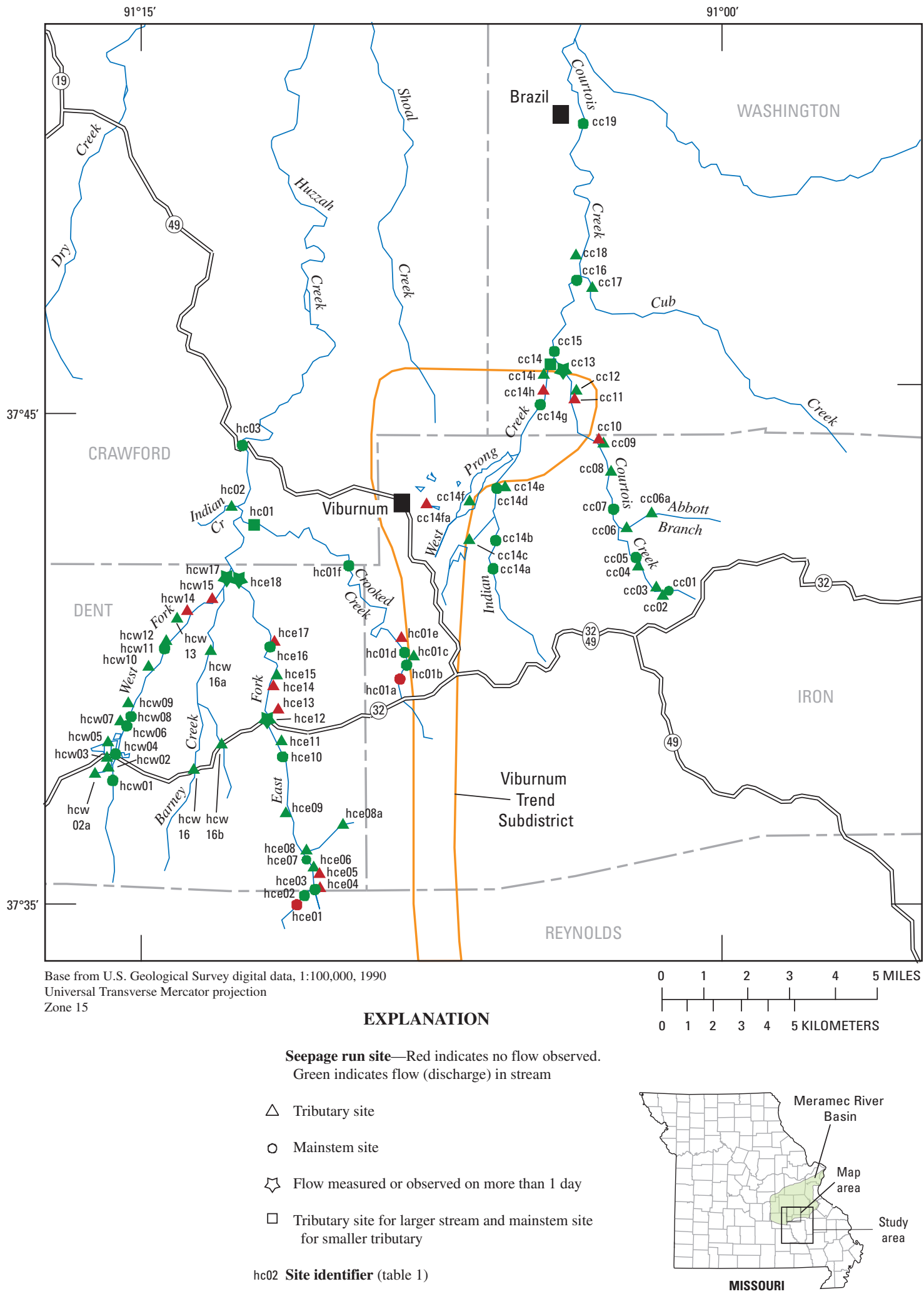

Figure 2. Seepage run sites on streams in the Meramec River Basin draining the Viburnum Trend Subdistrict. 


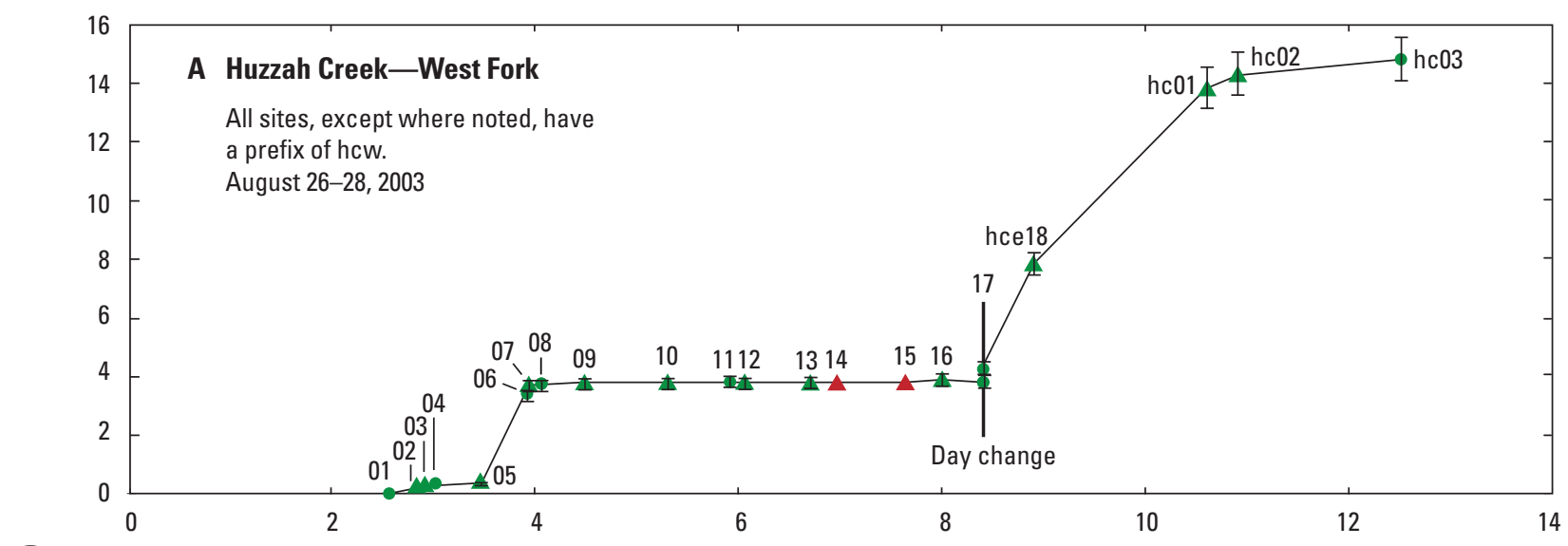

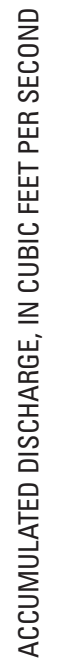
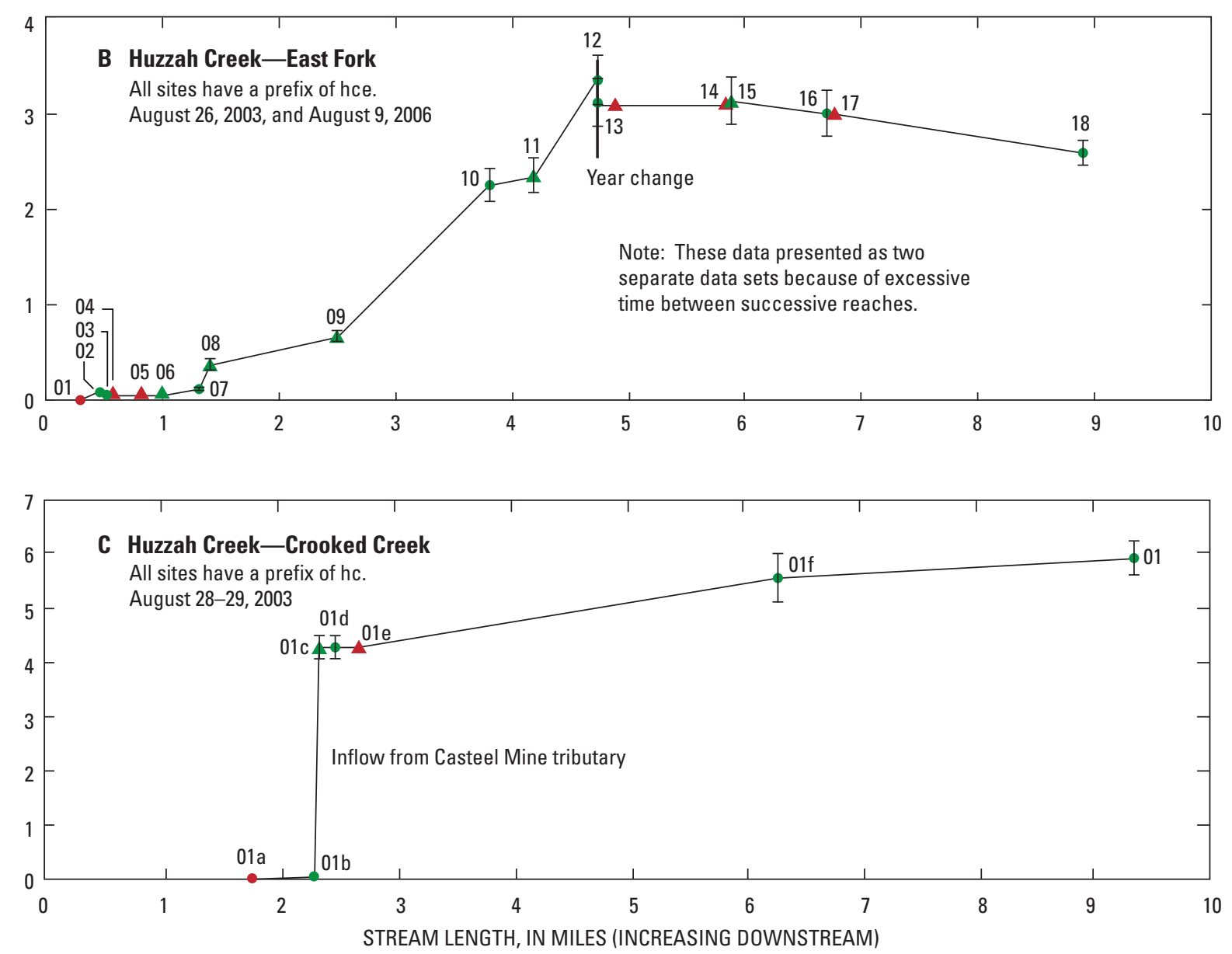

EXPLANATION

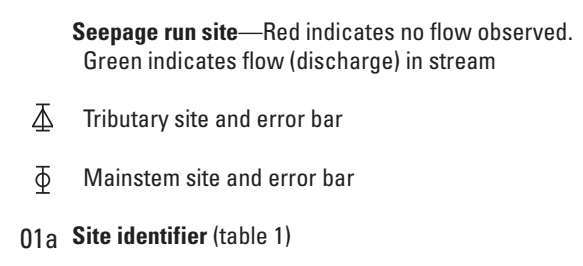

Figure 3. Seepage run data for West and East Forks Huzzah Creek and Crooked Creek, August 2003 through August 2006. 

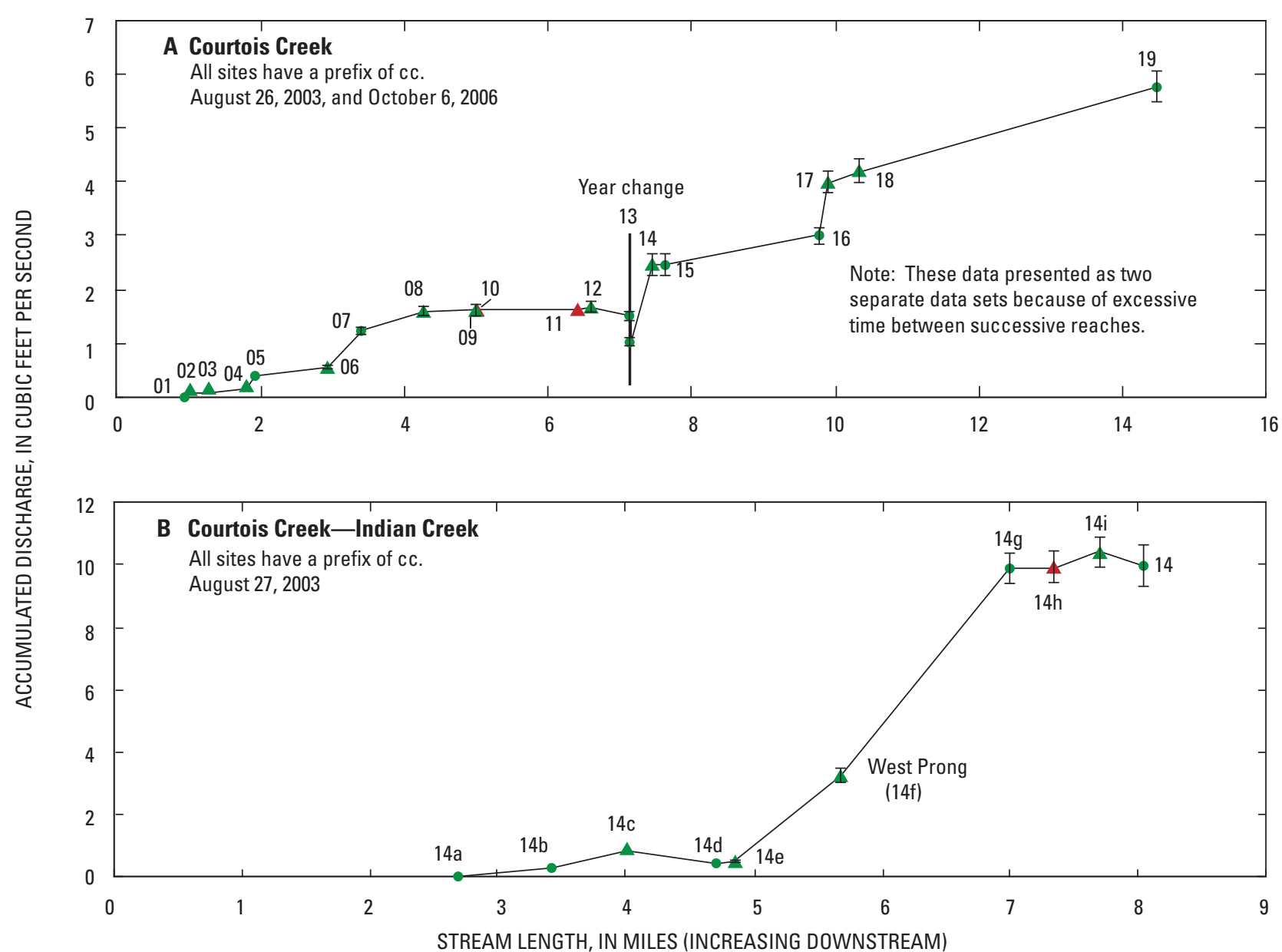

EXPLANATION

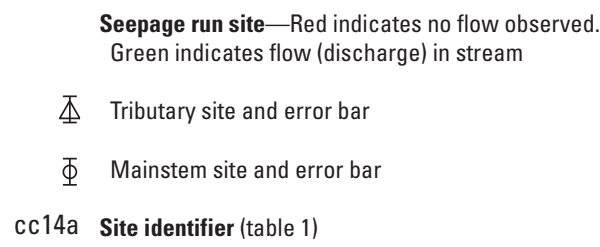

Figure 4. Seepage run data for Courtois Creek and Indian Creek, August 2003 through October 2006.

presented in three segments (fig. 6C; table 1). None of these segments indicated losing reaches.

The three aforementioned tributaries of Middle Fork Black River also were investigated (fig. 6D-F). In each of these tributaries, the sum of the measured discharge at a mainstem site and the contributing inflows from downstream tributaries was greater than the discharge at the next downstream mainstem site. For example, discharge decreased from site mf05b to site mf05d (figs. 5, 6D; table 1) along Strother Creek and from site nc18 to site nc21 along Neals Creek (figs. $5,6 \mathrm{E}$; table 1). However, if the measurement accuracy is considered for all the discharge measurements made within these two reaches, the sum of the upstream mainstem discharge plus all the downstream tributary inflows is within the error associated with the measurement rating accuracy at the downstream mainstem site (table 1). Therefore, these two reaches were not classified as losing. The reported discharge at the upstream mainstem site of each reach is thought to be greater than the actual discharge in the stream in both cases.

The 3-mi reach upstream from the mouth of Brushy Creek was another occurrence of discharge decreasing downstream (figs. 5, 6F; table 1). Because of limited access along this reach, the mainstem measurements were widely spaced. At the upstream mainstem site mf11zf, a discharge of $5.44 \mathrm{ft}^{3} / \mathrm{s}$ was measured in September 2004. Between this site and the mouth of Brushy Creek (site mf11), $1.42 \mathrm{ft}^{3} / \mathrm{s}$ was added from tributary inflow and springs; however, the measured discharge at the mouth of Brushy Creek (site $\mathrm{mf} 11$ ) was $0.69 \mathrm{ft}^{3} / \mathrm{s}$, about $6.2 \mathrm{ft}^{3} / \mathrm{s}$ less discharge than expected. 


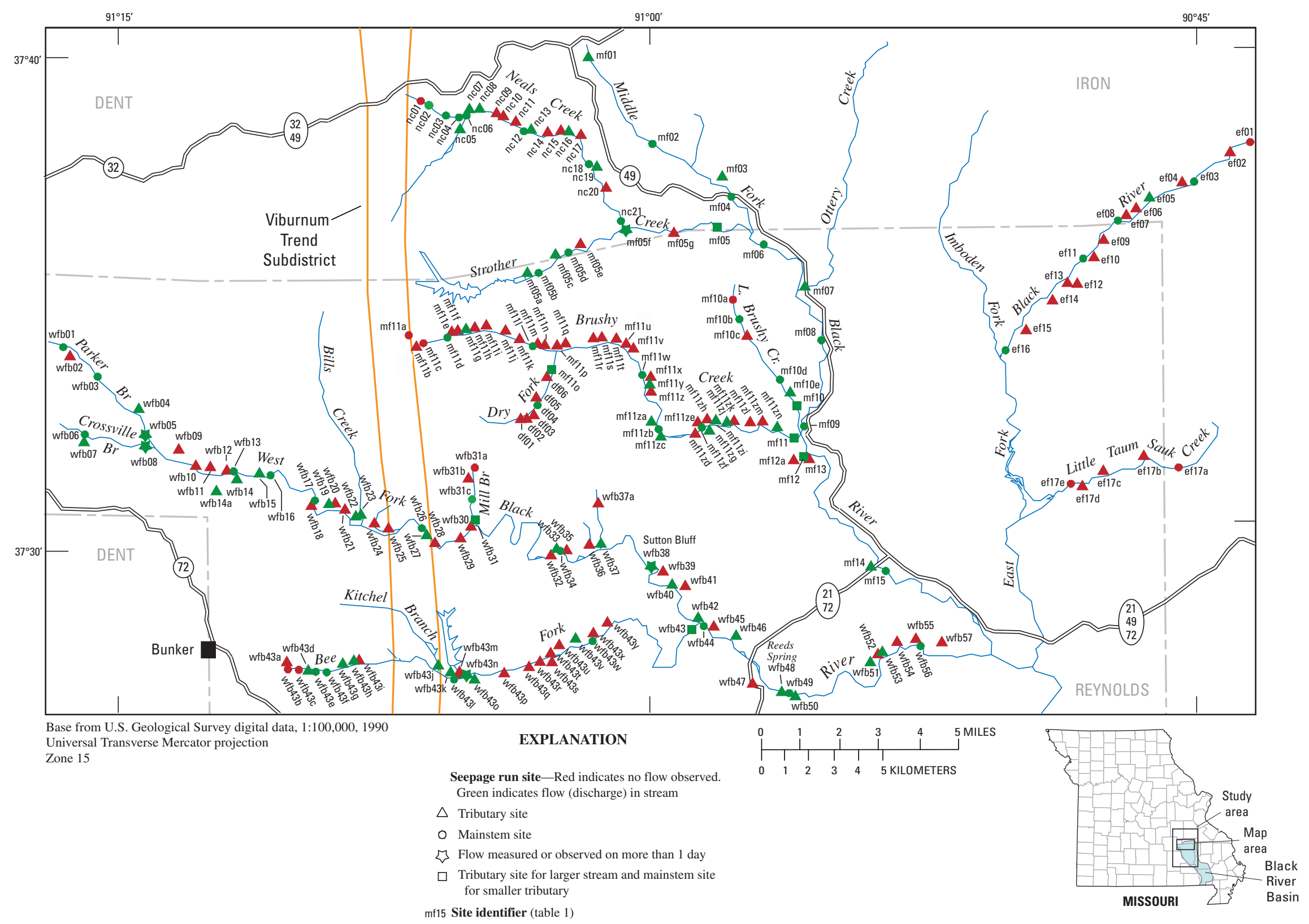

Figure 5. Seepage run sites on streams in the Black River Basin draining the central part of the Viburnum Trend Subdistrict. 

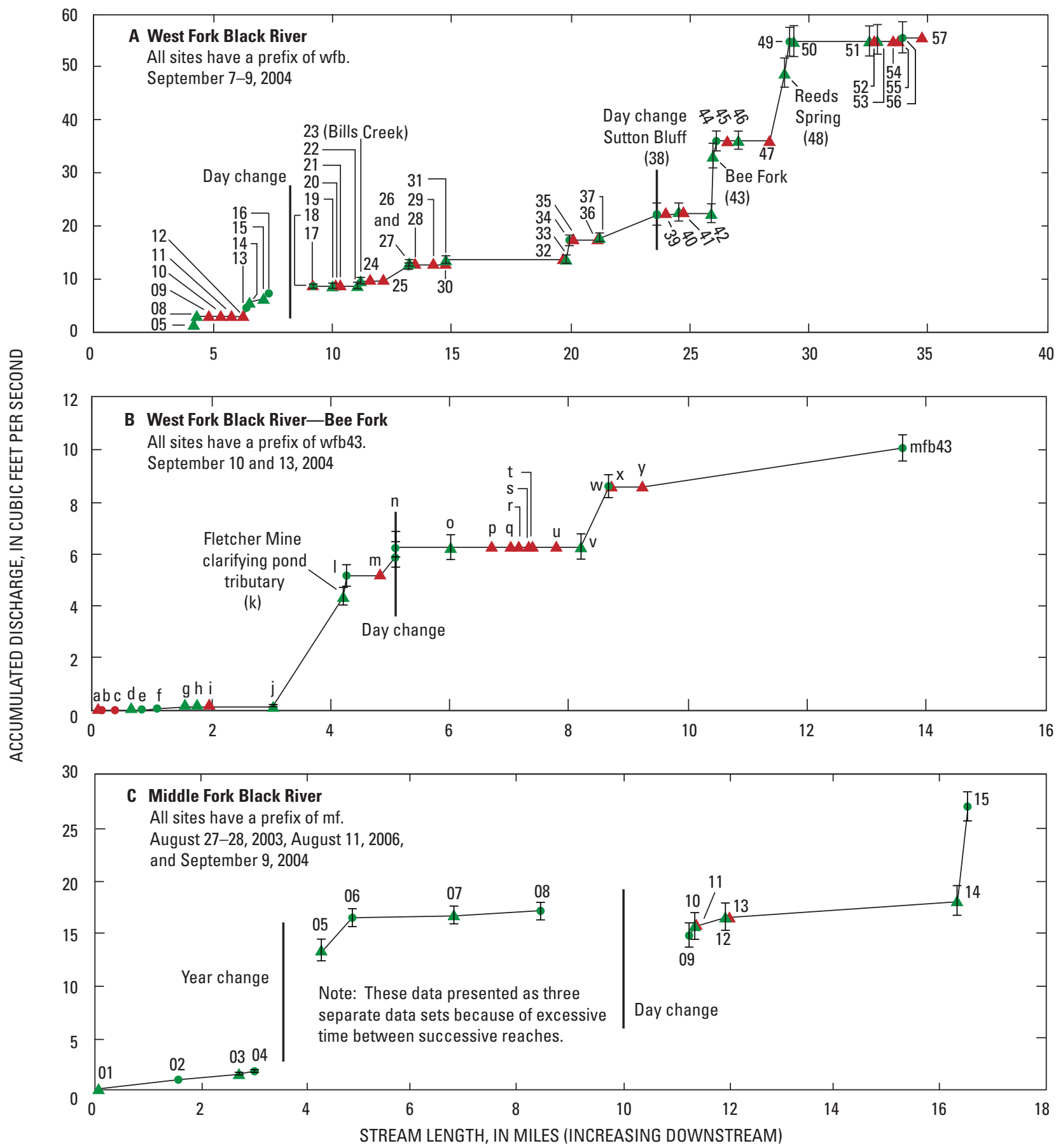

EXPLANATION

\footnotetext{
Seepage run site- Red indicates no flow observed. Green indicates flow (discharge) in stream

本 Tributary site and error bar

$\Phi \quad$ Mainstem site and error bar

05 Site identifier (table 1)
}

Figure 6. Seepage run data for the upper Black River Basin, August 2003 through August 2006. 


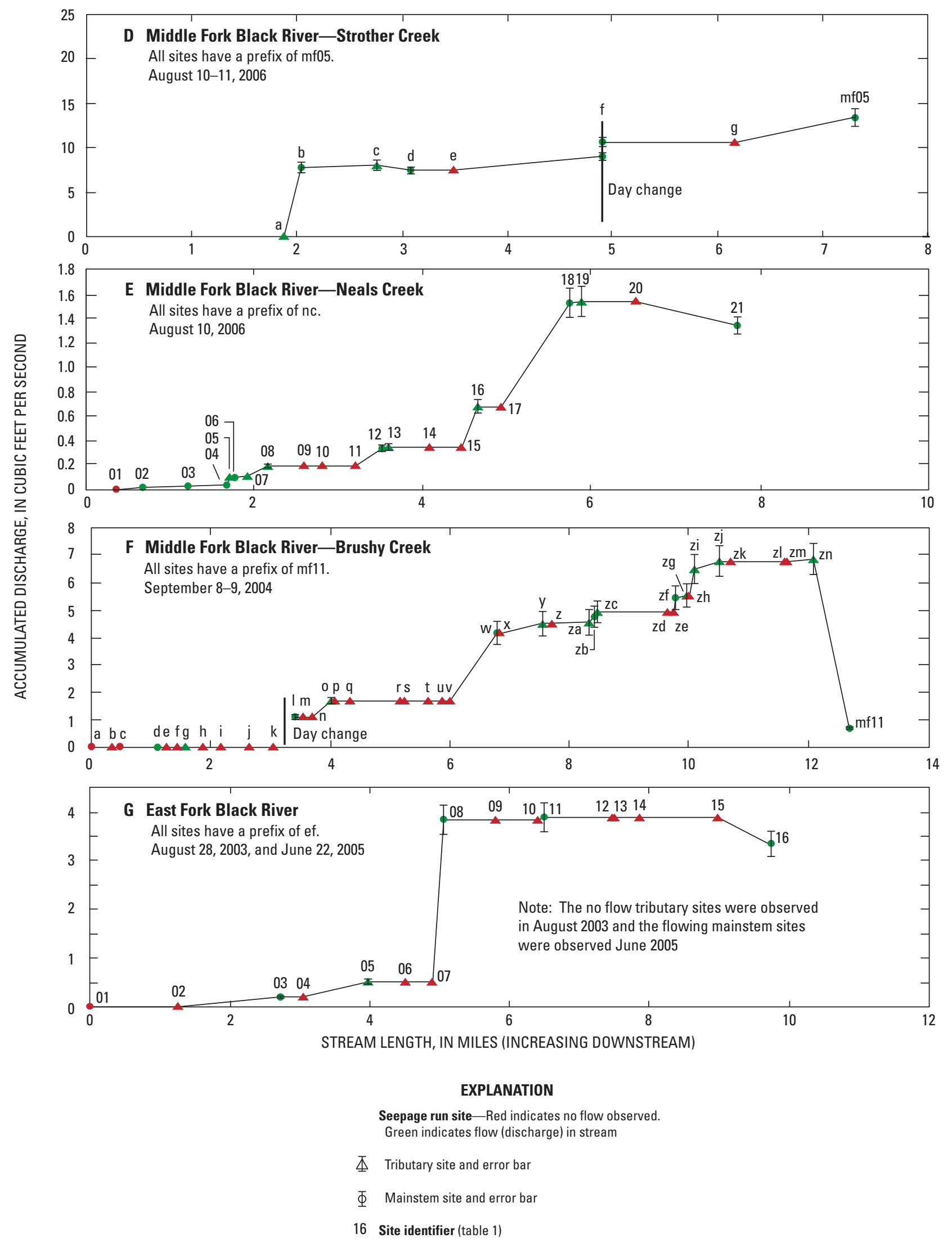

Figure 6. Seepage run data for the upper Black River Basin, August 2003 through August 2006. - Continued 
No conclusive explanation is available as how this discharge was lost; however, circumstantial evidence indicates that this water is not lost to the bedrock aquifer, but instead continued to flow through the gravel-filled channel at the mouth of Brushy Creek. A year earlier, on August 28, 2003, the reach of Middle Fork Black River containing the mouth of Brushy Creek was being investigated, and no visible discharge was observed at the mouth (site mf11). The discharge of the Middle Fork Black River upstream from Brushy Creek (site mf09; figs. 5, 6C; table 1) was $14.7 \mathrm{ft}^{3} / \mathrm{s}$ and the discharge downstream from Brushy Creek was $26.9 \mathrm{ft}^{3} / \mathrm{s}$ (site mf15). Between these two mainstem sites, an additional $3.31 \mathrm{ft}^{3} / \mathrm{s}$ was contributed to the Middle Fork Black River from the tributaries Little Brushy Creek (site mf10), Carty Branch (site mf12), and Paynes Branch (site mf14). Consequently, the additional $8.9 \mathrm{ft}^{3} / \mathrm{s}$ measured at site $\mathrm{mf} 15$ could not be attributed to tributary inflow. Because Brushy Creek had no visible discharge at the mouth, no further inspection of Brushy Creek was performed at that time. Much of the additional discharge $\left(8.9 \mathrm{ft}^{3} / \mathrm{s}\right)$ at site $\mathrm{mf} 15$ probably resurfaced in the mainstem of Middle Fork Black River from subsurface flow in the sand and gravel-filled channel at the mouth of Brushy Creek; therefore, the lost discharge in Brushy Creek was not lost to the bedrock aquifer.

\section{East Fork Black River}

The East Fork Black River was studied from its headwaters to the junction with Imboden Fork (fig. 5). No flow was observed in tributary sites in August 2003 and only the mainsteam sites were measured in June 2005 (table 1). No reaches were classified as losing in the East Fork Black River. Discharge increased in each successive mainstem measurement between the headwaters of East Fork Black River and Imboden Fork, except at the last discharge measurement site near the junction. This measurement (site ef16; figs. 5, 6G; table 1) indicated a decrease in discharge of $0.55 \mathrm{ft}^{3} / \mathrm{s}$ from the previous upstream measurement (site ef11). The stream channel at site ef16 is wide and filled with sand and gravel. Substantial flow likely is through the subsurface into the streambed sediment at this site. The flow is contained in the channel and is not considered to be lost to the bedrock aquifer. Little Taum Sauk Creek also was investigated in August 2004 and had no observed discharge.

\section{Logan Creek}

Logan Creek was studied from its headwaters to Clearwater Lake (fig. 7) and contained about a 13-mi losing stream reach (from sites lc28 to lc36; figs. 7, 8A; table 1). Discharge increased in the headwaters of Logan Creek (sites lc05 to lc25) from various sources, such as tributary streams, flowing wells, and springs. The discharge in Adair Creek (site lc19) was greater than the discharge in the mainstem of Logan Creek upstream from the junction of these two streams at site lc17 (figs. 7, 8A). The accumulated discharge in Logan Creek continued to increase downstream to site lc25 (table 1). Between sites 1c26 and lc28, Logan Creek lost all its discharge and went dry. No discharge was observed in Logan Creek or its tributaries downstream to mainstem site lc36. The next observed discharge was in Dry Valley (fig. 7; site lc $38,0.49 \mathrm{ft}^{3} / \mathrm{s}$ ) which entered Logan Creek near Ellington (fig. 7). Downstream from Ellington, discharge continued to increase in Logan Creek from tributary and spring inflow. This increase continued to Clearwater Lake.

Sweetwater Creek also was investigated and no reaches were classified as losing, in spite of the less than expected discharge measured at site lc24h (figs. 7, 8B; table 1). The reach of Sweetwater Creek from where tributary discharge enters the creek at site $1 \mathrm{c} 24 \mathrm{~g}$ (accumulated discharge of $0.87 \mathrm{ft}^{3} / \mathrm{s}$ ) to site $1 \mathrm{c} 24 \mathrm{~h}\left(0.55 \mathrm{ft}^{3} / \mathrm{s}\right)$ was not classified as losing because of the measurement accuracy ratings (poor; measurement error greater than 8 percent) at both these sites. Also, the discharge further downstream at the mouth of Sweetwater Creek (site $1 \mathrm{c} 24 ; 0.95 \mathrm{ft}^{3} / \mathrm{s}$ ) was consistent with the accumulated discharge calculated at site lc24g (fig. 8B; table 1). The discharge at site lc24h possibly was under-measured because water may have been flowing through the streambed sediment.

\section{Sinking Creek}

No losing stream reaches were identified in Sinking Creek. The upper 8-mi reach of Sinking Creek had no discharge until Pyrtle Spring (site sk21; figs. 7, 8C; table 1), where the first visible discharge $\left(0.34 \mathrm{ft}^{3} / \mathrm{s}\right)$ was measured. Between Pyrtle Spring and the next Sinking Creek mainstem measurement (site sk26), discharge had increased in the stream to $5.76 \mathrm{ft}^{3} / \mathrm{s}$ (fig. 8C). Discharge continued to increase in Sinking Creek to the downstream most measuring site at Redford (site sk34, fig. 7), where the study ended.

\section{Summary}

A study was conducted to identify streams or reaches of streams that lose discharge to the ground-water system in and around active lead and zinc mining areas along the Viburnum Trend Subdistrict that have potential to release mining-related trace elements or chemicals into receiving streams. The study was cursory in that the discharge was monitored at road crossings or at other convenient locations rather than traversing the entire length of these streams. Rainfall caused the seepage run to be stopped and restarted several times, several completed reaches were reexamined, and additional streams were added; therefore, the data collection that began in August 2003 was not completed until October 2006.

All the streams in the study area have sand and gravelfilled channels (streambed sediment) that include frequent, large bars composed of loose sand, gravel, and cobbles. During low flow, a large percentage of water can be travel- 


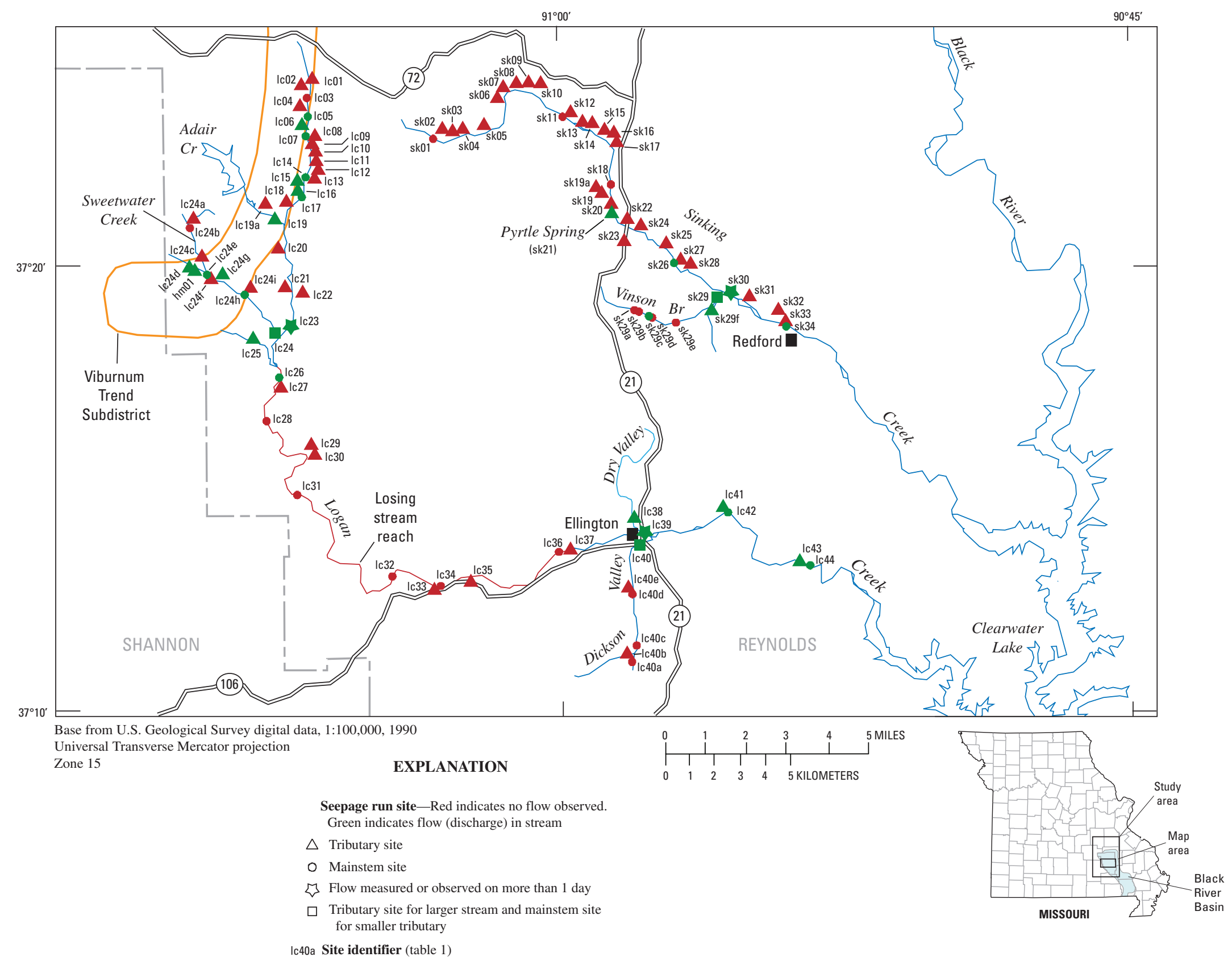

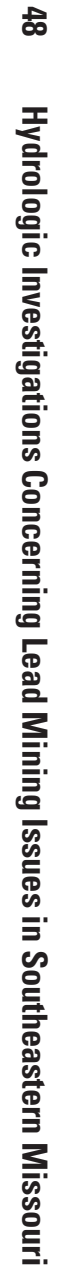

Figure 7. Seepage run sites on streams in the Black River Basin draining the southern part of the Viburnum Trend Subdistrict. 

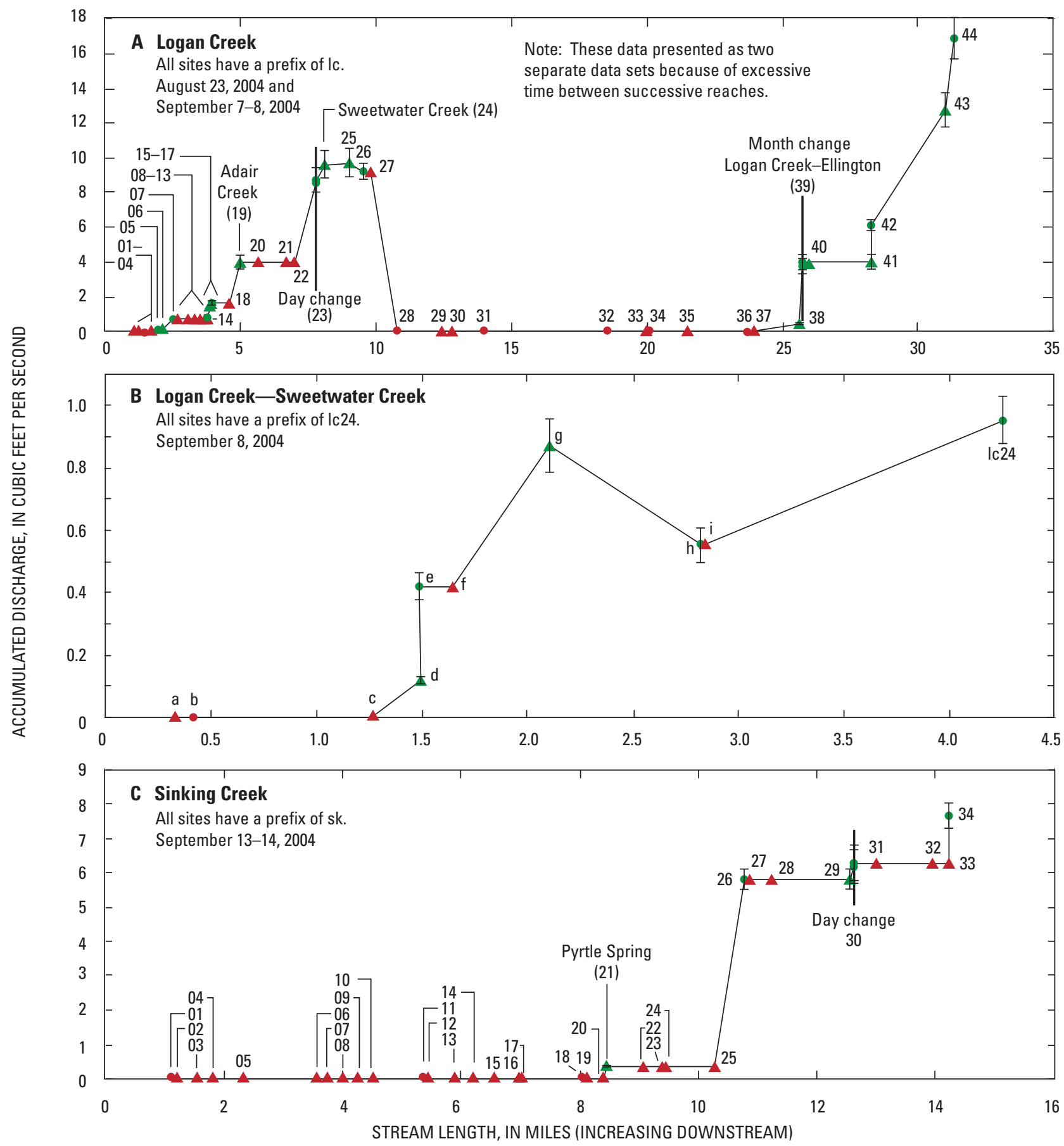

EXPLANATION

\footnotetext{
Seepage run site-Red indicates no flow observed.

Green indicates flow (discharge) in stream

$\triangle$ Tributary site and error bar

$\Phi \quad$ Mainstem site and error bar

22 Site identifier (table 1)
}

Figure 8. Seepage run data for the Black River Basin, August 2004 through September 2004. 
ing through the streambed sediment instead of flowing on the surface. In many cases, the water flowing through the streambed sediment resurfaces farther downstream and is not being lost to the underlying bedrock aquifer. Based on the hydrologic assessment of the personnel making the discharge measurement, occasionally these reaches were not classified as losing even though considerably more discharge was measured upstream. These assessments typically were influenced by hydrologic observations of smaller tributaries in the immediate area of the site of concern. If water was flowing in these smaller tributaries, or if the quantity of water lost at a particular site resurfaced at the next downstream mainstem measuring site, the site or reach with decreased discharge was not classified as losing.

The measurement accuracy and associated error defined by the measurement rating also became an important determining factor in classifying several stream reaches as non losing even though less discharge was measured at a downstream site than was measured upstream. Whenever a decrease in discharge was observed downstream, the measurement error (defined by measurement rating) of the relevant upstream discharge measurements (mainstem and tributary measurements) was considered and the range of discharges as defined by the associated error bars was compared. If successive error bars overlapped, the reach was not classified as losing.

Stream reaches in East Fork Huzzah Creek; Strother Creek, Neals Creek, and Brushy Creek in the Middle Fork Black River Basin; and East Fork Black River had less discharge measured in a downstream mainstem site than was expected based on the upstream discharge measurements; however, none of these reaches were classified as losing. This classification is based on the quantity of gravel in the stream channel, the hydrologic assessment of the personnel making the measurements, and the rated discharge measurement accuracy for the sites.

Logan Creek had about a 13-mile reach with no flow that began losing discharge downstream from Sweetwater Creek. Discharge in Logan Creek was observed again near Ellington and continued to increase downstream to Clearwater Lake.

\section{References Cited}

Nolan, K.M., and Shields, R.R., 2000, Measurement of stream discharge by wading: U.S. Geological Survey Water-Resources Investigations Report 00-4036, on CD-ROM.

Rantz, S.E., and others, 1982, Measurement and computation of streamflow-Volume 1. Measurement of stage and discharge: U.S. Geological Survey Water-Supply Paper 2175, $284 \mathrm{p}$.

Sauer, S.P., and Meyer, R.W., 1992, Determination of error in individual discharge measurements: U.S. Geological Survey Open-File Report 92-144, 21 p.
U.S. Geological Survey, 1977, Hydrologic unit map—1974, State of Missouri: scale 1:500,000, 1 sheet.

U.S. Geological Survey, 1985, Water fact sheet-Hydrologic hazards in karst terrain: U.S. Geological Survey Open-File Report 85-677, 2 p.

U.S. Geological Survey, 2008, Mineral commodity summary, accessed January 18, 2008, at URL http://minerals.usgs. gov/minerals/pubs/commodity/statistical_summary/

Vineyard, J.D. and Feder, G.L., 1974, Springs of Missouri: Rolla, Missouri Department of Natural Resources, Division of Geology and Land Survey, Water Resources Report Number 26, $266 \mathrm{p}$.

Wharton, H.M., 1975, Introduction to the southeast Missouri lead district, in Vineyard, J.D. ed., The geology and ore deposits of selected mines, Viburnum Trend, Missouri: Rolla, Missouri Department of Natural Resources, Division of Geology and Land Survey Report of Investigations 58, p. $3-14$.

Wilde, F.D., and Radtke, D.B., eds., 1998, National field manual for the collection of water-quality data-Field measurements: U.S. Geological Survey Techniques of WaterResources Investigations, book 9, chap. A6, 238 p. 
Table 
Table 1. Seepage run data for streams draining the Viburnum Trend Subdistrict.

[DDMMSS, degrees, minutes, seconds; MM/DD/YYY, month/day/year; mi, mile; $\mathrm{ft}^{3} / \mathrm{s}$, cubic feet per second; $\mu \mathrm{S} / \mathrm{cm}$, microsiemens per centimeter at 25 degrees Celsius; ${ }^{\circ} \mathrm{C}$, degrees Celsius; --, no data; WFHC, West Fork Huzzah Creek; Est, estimated; trib, tributary; Calc, calculated; Obs, observation; shaded boxes are identical sites measured on different days; EFHC, East Fork Huzzah Creek; WFBR, West Fork Black River; MFBR, Middle Fork Black River; EFBR, East Fork Black River]

\begin{tabular}{|c|c|c|c|c|c|c|c|c|c|c|c|}
\hline \multirow[b]{2}{*}{ Stream site } & \multirow[b]{2}{*}{$\begin{array}{c}\text { Site } \\
\text { identifier }\end{array}$} & \multirow[b]{2}{*}{$\begin{array}{c}\text { Latitude } \\
\text { (DDMMSS) }\end{array}$} & \multirow[b]{2}{*}{$\begin{array}{c}\text { Longitude } \\
\text { (DDDMMSS) }\end{array}$} & \multirow[b]{2}{*}{$\begin{array}{c}\text { Date } \\
\text { (MM/DD/YYYY) }\end{array}$} & \multirow{2}{*}{$\begin{array}{l}\text { Stream } \\
\text { length } \\
\text { (mi) }\end{array}$} & \multicolumn{2}{|c|}{ Measurement location } & \multirow{2}{*}{$\begin{array}{c}\text { Accumulated } \\
\text { discharge } \\
\text { (figs. } 3,4,6,8) \\
\left(\mathrm{ft}^{3} / \mathrm{s}\right)\end{array}$} & \multirow{2}{*}{$\begin{array}{l}\text { Measure- } \\
\text { ment } \\
\text { rating }\end{array}$} & \multirow{2}{*}{$\begin{array}{c}\text { Specific } \\
\text { conductance } \\
(\mu \mathrm{S} / \mathrm{cm} \text { at } \\
\left.25^{\circ} \mathrm{C}\right)\end{array}$} & \multirow[b]{2}{*}{$\begin{array}{c}\text { Temperature } \\
\left({ }^{\circ} \mathrm{C}\right)\end{array}$} \\
\hline & & & & & & $\begin{array}{c}\text { Tributary } \\
\left(\mathrm{ft}^{3} / \mathrm{s}\right)\end{array}$ & $\begin{array}{l}\text { Mainstem } \\
\left(\mathrm{ft}^{3} / \mathrm{s}\right)\end{array}$ & & & & \\
\hline \multicolumn{12}{|c|}{ Huzzah Creek - West Fork (figs. 2, 3A) } \\
\hline WFHC & hcw01 & 373734 & 0911541 & $08 / 26 / 2003$ & 2.6 & -- & 0.02 & 0.02 & Est & 383 & 26.9 \\
\hline WFHC - Rowe Branch & hcw02 & 373747 & 0911542 & $08 / 26 / 2003$ & 2.8 & 0.15 & -- & .17 & Good & 445 & 21.2 \\
\hline WFHC - trib & hcw03 & 373752 & 0911539 & $08 / 26 / 2003$ & 2.9 & .04 & -- & .21 & Fair & 321 & 24.0 \\
\hline WFHC & hcw04 & 373756 & 0911534 & $08 / 26 / 2003$ & 3.0 & -- & .30 & .30 & Good & 415 & 23.0 \\
\hline WFHC - trib & hcw05 & 373816 & 0911545 & $08 / 26 / 2003$ & 3.5 & .01 & -- & .31 & Est & 477 & 20.2 \\
\hline WFHC & hcw06 & 373840 & 0911516 & $08 / 26 / 2003$ & 3.9 & -- & 3.48 & 3.48 & Good & 358 & 21.7 \\
\hline WFHC - Martin Spring Branch & hcw07 & 373841 & 0911526 & $08 / 26 / 2003$ & 3.9 & .20 & -- & 3.68 & $\mathrm{Calc}^{\mathrm{a}}$ & 447 & 22.2 \\
\hline WFHC & hcw08 & 373844 & 0911514 & $08 / 26 / 2003$ & 4.0 & -- & 3.68 & 3.68 & Good & 366 & 22.5 \\
\hline WFHC - trib & hcw09 & 373903 & 0911513 & $08 / 26 / 2003$ & 4.5 & .04 & -- & 3.72 & Est & 391 & 27.7 \\
\hline WFHC - Rooney Hollow & hcw 10 & 373942 & 0911441 & $08 / 26 / 2003$ & 5.3 & .05 & -- & 3.77 & Est & 391 & 27.2 \\
\hline WFHC & hcw11 & 374010 & 0911417 & $08 / 26 / 2003$ & 5.9 & -- & 3.72 & 3.72 & Good & 366 & 26.2 \\
\hline WFHC - Bates Hollow & hcw12 & 374016 & 0911413 & $08 / 26 / 2003$ & 6.1 & .03 & -- & 3.75 & Est & 400 & 30.1 \\
\hline WFHC - trib & hcw13 & 374043 & 0911356 & $08 / 26 / 2003$ & 6.7 & .02 & -- & 3.77 & Est & 418 & 23.6 \\
\hline WFHC - trib & hcw14 & 374052 & 0911341 & $08 / 26 / 2003$ & 7.0 & 0 & -- & 3.77 & Obs & -- & -- \\
\hline WFHC - trib & hcw15 & 374106 & 0911303 & $08 / 26 / 2003$ & 7.6 & 0 & -- & 3.77 & Obs & -- & -- \\
\hline WFHC - Barney Creek & hcw16 & 373741 & 0911335 & $08 / 26 / 2003$ & 8.0 & .05 & -- & 3.82 & Good & 440 & 22.2 \\
\hline WFHC mouth & hcw17 & 374141 & 0911234 & $08 / 26 / 2003$ & 8.4 & -- & 3.77 & 3.77 & Good & 334 & 25.7 \\
\hline WFHC mouth & hcw17 & 374141 & 0911234 & $08 / 28 / 2003$ & 8.4 & -- & 4.23 & 4.23 & Good & 381 & 25.2 \\
\hline EFHC mouth & hce18 & 374140 & 0911230 & $08 / 28 / 2003$ & 8.9 & 3.61 & -- & 7.84 & Good & 393 & 24.4 \\
\hline Huzzah Creek - Crooked Creek & hc01 & 374238 & 0911153 & $08 / 28 / 2003$ & 10.6 & 5.93 & -- & 13.8 & Good & 604 & 25.6 \\
\hline Huzzah Creek - Indian Creek & hc02 & 374256 & 0911240 & $08 / 28 / 2003$ & 10.9 & .40 & -- & 14.2 & Fair & 440 & 23.5 \\
\hline Huzzah Creek & hc03 & 374411 & 0911212 & $08 / 28 / 2003$ & 12.5 & -- & 14.7 & 14.7 & Good & 490 & 28.3 \\
\hline \multicolumn{12}{|c|}{ Miscellaneous tributary and headwater measurement or observation sites (not shown on fig. 3A) } \\
\hline WFHC - Rowe Branch & hcw02a & 373738 & 0911607 & $08 / 26 / 2003$ & -- & .01 & -- & -- & Obs & -- & -- \\
\hline WFHC - Barney Creek & hcw16a & 374003 & 0911307 & $08 / 28 / 2003$ & -- & 0.66 & -- & -- & Good & 415 & 23.1 \\
\hline WFHC - Barney Creek trib & hcw16b & 373810 & 0911254 & $08 / 26 / 2003$ & -- & .01 & -- & -- & Est & 301 & 21.1 \\
\hline
\end{tabular}

hcw $16 \mathrm{~b}$

373810

0911254

$08 / 26 / 2003$

Est

21.1 
Table 1. Seepage run data for streams draining the Viburnum Trend Subdistrict.—Continued

[DDMMSS, degrees, minutes, seconds; MM/DD/YYY, month/day/year; mi, mile; ft $3 / \mathrm{s}$, cubic feet per second; $\mu \mathrm{S} / \mathrm{cm}$, microsiemens per centimeter at 25 degrees Celsius; ${ }^{\circ} \mathrm{C}$, degrees Celsius; --, no data; $\mathrm{WFHC}$, West Fork Huzzah Creek; Est, estimated; trib, tributary; Calc, calculated; Obs, observation; shaded boxes are identical sites measured on different days; EFHC, East Fork Huzzah Creek; WFBR, West Fork Black River; MFBR, Middle Fork Black River; EFBR, East Fork Black River]

\begin{tabular}{|c|c|c|c|c|c|c|c|c|c|c|c|}
\hline \multirow[b]{2}{*}{ Stream site } & \multirow[b]{2}{*}{$\begin{array}{c}\text { Site } \\
\text { identifier }\end{array}$} & \multirow[b]{2}{*}{$\begin{array}{c}\text { Latitude } \\
\text { (DDMMSS) }\end{array}$} & \multirow[b]{2}{*}{$\begin{array}{c}\text { Longitude } \\
\text { (DDDMMSS) }\end{array}$} & \multirow[b]{2}{*}{$\begin{array}{c}\text { Date } \\
\text { (MM/DD/YYYY) }\end{array}$} & \multirow{2}{*}{$\begin{array}{l}\text { Stream } \\
\text { length } \\
\text { (mi) }\end{array}$} & \multicolumn{2}{|c|}{ Measurement location } & \multirow{2}{*}{$\begin{array}{c}\text { Accumulated } \\
\text { discharge } \\
\text { (figs. } 3,4,6,8) \\
\left(\mathrm{ft}^{3} / \mathrm{s}\right) \\
\end{array}$} & \multirow{2}{*}{$\begin{array}{l}\text { Measure- } \\
\text { ment } \\
\text { rating }\end{array}$} & \multirow{2}{*}{$\begin{array}{c}\text { Specific } \\
\text { conductance } \\
(\mu \mathrm{S} / \mathrm{cm} \text { at } \\
\left.25^{\circ} \mathrm{C}\right)\end{array}$} & \multirow[b]{2}{*}{$\begin{array}{c}\text { Temperature } \\
\left({ }^{\circ} \mathrm{C}\right)\end{array}$} \\
\hline & & & & & & $\begin{array}{c}\text { Tributary } \\
\left(\mathrm{ft}^{3} / \mathrm{s}\right)\end{array}$ & $\begin{array}{c}\text { Mainstem } \\
\left(\mathrm{ft}^{3} / \mathbf{s}\right)\end{array}$ & & & & \\
\hline \multicolumn{12}{|c|}{ Huzzah Creek - East Fork (figs. 2, 3b) } \\
\hline EFHC & hce01 & 373455 & 0911103 & $08 / 26 / 2003$ & 0.3 & -- & 0 & 0 & Obs & -- & -- \\
\hline EFHC & hce02 & 373513 & 0911035 & $08 / 26 / 2003$ & .4 & -- & .10 & .10 & Est & 74 & 20.1 \\
\hline EFHC & hce 03 & 373510 & 0911049 & $08 / 26 / 2003$ & .5 & -- & .05 & .05 & Est & -- & -- \\
\hline EFHC - trib & hce04 & 373516 & 0911028 & $08 / 26 / 2003$ & .5 & 0 & -- & .05 & Obs & -- & -- \\
\hline EFHC - trib & hce05 & 373532 & 0911028 & $08 / 26 / 2003$ & .8 & 0 & -- & .05 & Obs & -- & -- \\
\hline EFHC - trib & hce06 & 373542 & 0911033 & $08 / 26 / 2003$ & 1.0 & .01 & -- & .06 & Obs & -- & -- \\
\hline EFHC & hce 07 & 373554 & 0911052 & $08 / 26 / 2003$ & 1.3 & -- & .11 & .11 & Poor & -- & 25.1 \\
\hline EFHC - Asher Creek & hce 08 & 373557 & 0911048 & $08 / 26 / 2003$ & 1.4 & .25 & -- & .36 & Poor & -- & 23.5 \\
\hline EFHC - Bucksnort Fork & hce09 & 373646 & 0911115 & $08 / 26 / 2003$ & 2.5 & .29 & -- & .65 & Poor & 418 & 24.1 \\
\hline EFHC & hce10 & 373756 & 0911119 & $08 / 26 / 2003$ & 3.8 & -- & 2.26 & 2.26 & Fair & -- & 26.5 \\
\hline EFHC - Big Branch & hce11 & 373815 & 0911118 & $08 / 26 / 2003$ & 4.2 & .08 & -- & 2.34 & Poor & -- & 22.8 \\
\hline EFHC & hce12 & 373841 & 0911141 & $08 / 26 / 2003$ & 4.7 & -- & 3.34 & 3.34 & Fair & 384 & 24.7 \\
\hline EFHC & hce12 & 373841 & 0911141 & 08/09/2006 & 4.7 & -- & 3.10 & 3.10 & Fair & 423 & 24.9 \\
\hline EFHC - trib & hce13 & 373841 & 0911125 & 08/09/2006 & 4.8 & 0 & -- & 3.10 & Obs & -- & -- \\
\hline EFHC - trib & hce14 & 373919 & 0911132 & 08/09/2006 & 5.8 & 0 & -- & 3.10 & Obs & -- & -- \\
\hline EFHC - Tinker Branch & hce15 & 373932 & 0911127 & 08/09/2006 & 5.9 & .02 & -- & 3.12 & Est & 520 & 23.3 \\
\hline EFHC & hce16 & 374007 & 0911136 & 08/09/2006 & 6.7 & -- & 2.99 & 2.99 & Fair & 414 & 26.6 \\
\hline EFHC - trib & hce17 & 374008 & 0911135 & 08/09/2006 & 6.8 & 0 & -- & 2.99 & Obs & -- & -- \\
\hline EFHC mouth & hce18 & 374140 & 0911230 & 08/09/2006 & 8.9 & -- & 2.59 & 2.59 & Good & 420 & 26.2 \\
\hline \multicolumn{12}{|c|}{ Miscellaneous tributary and headwater measurement or observation sites (not shown on fig. 3B) } \\
\hline EFHC - Asher Creek & hce08a & 373633 & 0910947 & $08 / 26 / 2003$ & -- & 0.05 & -- & -- & Est & -- & -- \\
\hline \multicolumn{12}{|c|}{ Huzzah Creek - Crooked Creek (figs 2, 3C) } \\
\hline Crooked Creek & hc01a & 373925 & 0910820 & $08 / 29 / 2003$ & 1.8 & -- & 0 & 0 & Obs & -- & -- \\
\hline Crooked Creek & hc01b & 373950 & 0910809 & $08 / 28 / 2003$ & 2.3 & -- & 0.05 & .05 & Est & 2,305 & 22.3 \\
\hline Crooked Creek trib - Casteel Mine & hc01c & 373949 & 0910802 & $08 / 28 / 2003$ & 2.4 & 4.22 & -- & 4.27 & $\mathrm{Calc}^{\mathrm{a}}$ & 825 & 23.8 \\
\hline Crooked Creek & hc01d & 373957 & 0910812 & $08 / 28 / 2003$ & 2.5 & -- & 4.27 & 4.27 & Good & 840 & 24.0 \\
\hline Crooked Creek trib & hc01e & 374008 & 0910811 & $08 / 28 / 2003$ & 2.7 & 0 & -- & 4.27 & Obs & -- & -- \\
\hline Crooked Creek & hc01f & 374144 & 0910938 & $08 / 28 / 2003$ & 6.3 & -- & 5.57 & 5.57 & Fair & 808 & 25.5 \\
\hline Crooked Creek - near mouth & hc01 & 374238 & 0911153 & $08 / 28 / 2003$ & 9.4 & -- & 5.93 & 5.93 & Good & 604 & 25.6 \\
\hline
\end{tabular}


Table 1. Seepage run data for streams draining the Viburnum Trend Subdistrict.-Continued

[DDMMSS, degrees, minutes, seconds; MM/DD/YYY, month/day/year; mi, mile; $\mathrm{ft}^{3} / \mathrm{s}$, cubic feet per second; $\mathrm{uS} / \mathrm{cm}$, microsiemens per centimeter at 25 degrees Celsius; ${ }^{\circ} \mathrm{C}$, degrees Celsius; --, no data; $\mathrm{WFHC}$, West Fork Huzzah Creek; Est, estimated; trib, tributary; Calc, calculated; Obs, observation; shaded boxes are identical sites measured on different days; EFHC, East Fork Huzzah Creek; WFBR, West Fork Black River; MFBR, Middle Fork Black River; EFBR, East Fork Black River]

\begin{tabular}{|c|c|c|c|c|c|c|c|c|c|c|c|}
\hline \multirow[b]{2}{*}{ Stream site } & \multirow[b]{2}{*}{$\begin{array}{c}\text { Site } \\
\text { identifier }\end{array}$} & \multirow[b]{2}{*}{$\begin{array}{c}\text { Latitude } \\
\text { (DDMMSS) }\end{array}$} & \multirow[b]{2}{*}{$\begin{array}{c}\text { Longitude } \\
\text { (DDDMMSS) }\end{array}$} & \multirow[b]{2}{*}{$\begin{array}{c}\text { Date } \\
\text { (MM/DD/YYYY) }\end{array}$} & \multirow[b]{2}{*}{$\begin{array}{l}\text { Stream } \\
\text { length } \\
\text { (mi) }\end{array}$} & \multicolumn{2}{|c|}{ Measurement location } & \multirow{2}{*}{$\begin{array}{c}\text { Accumulated } \\
\text { discharge } \\
\text { (figs. } 3,4,6,8) \\
\left(\mathrm{ft}^{3} / \mathrm{s}\right)\end{array}$} & \multirow{2}{*}{$\begin{array}{c}\text { Measure- } \\
\text { ment } \\
\text { rating }\end{array}$} & \multirow{2}{*}{$\begin{array}{c}\text { Specific } \\
\text { conductance } \\
\text { ( } \mu \mathrm{S} / \mathrm{cm} \text { at } \\
\left.25^{\circ} \mathrm{C}\right)\end{array}$} & \multirow[b]{2}{*}{$\begin{array}{c}\text { Temperature } \\
\left({ }^{\circ} \mathrm{C}\right)\end{array}$} \\
\hline & & & & & & $\begin{array}{l}\text { Tributary } \\
\left(\mathrm{ft}^{3} / \mathrm{s}\right)\end{array}$ & $\begin{array}{l}\text { Mainstem } \\
\left(\mathrm{ft}^{3} / \mathrm{s}\right)\end{array}$ & & & & \\
\hline \multicolumn{12}{|c|}{ Courtois Creek (figs 2, 4A) } \\
\hline Courtois Creek & $\operatorname{cc} 01$ & 374050 & 0910136 & $08 / 26 / 2003$ & 0.9 & -- & 0.01 & 0.01 & Est & -- & -- \\
\hline Courtois Creek - spring & $\operatorname{cc} 02$ & 374054 & 0910138 & $08 / 26 / 2003$ & 1.0 & 0.05 & -- & .06 & Est & -- & -- \\
\hline Courtois Creek - spring & $\operatorname{cc} 03$ & 374109 & 0910147 & $08 / 26 / 2003$ & 1.2 & .05 & -- & .11 & Est & -- & -- \\
\hline Courtois Creek - Hoover Hollow & $\operatorname{cc} 04$ & 374136 & 0910213 & $08 / 26 / 2003$ & 1.8 & .05 & -- & .16 & Est & -- & -- \\
\hline Courtois Creek & $\operatorname{cc} 05$ & 374146 & 0910217 & $08 / 26 / 2003$ & 1.9 & -- & .40 & .40 & Good & 346 & 21.0 \\
\hline Courtois Creek - Abbott Branch & $\operatorname{cc} 06$ & 374222 & 0910230 & $08 / 26 / 2003$ & 2.9 & .14 & -- & .54 & Good & 294 & 23.6 \\
\hline Courtois Creek & $\operatorname{cc} 07$ & 374245 & 0910250 & $08 / 26 / 2003$ & 3.4 & -- & 1.22 & 1.22 & Good & 143 & 26.7 \\
\hline Courtois Creek - Warren Hollow & $\operatorname{cc} 08$ & 374330 & 0910252 & $08 / 26 / 2003$ & 4.3 & .36 & -- & 1.58 & Good & 434 & 22.0 \\
\hline Courtois Creek - spring & $\operatorname{cc} 09$ & 374405 & 0910304 & $08 / 26 / 2003$ & 5.0 & .05 & -- & 1.63 & Est & -- & -- \\
\hline Courtois Creek - Cave Hollow & $\operatorname{cc} 10$ & 374413 & 0910305 & $08 / 26 / 2003$ & 5.0 & 0 & -- & 1.63 & Obs & -- & -- \\
\hline Courtois Creek - trib & $\mathrm{cc} 11$ & 374505 & 0910346 & $08 / 26 / 2003$ & 6.4 & 0 & -- & 1.63 & Obs & -- & -- \\
\hline Courtois Creek - Andys Branch & $\mathrm{cc} 12$ & 374509 & 0910343 & $08 / 26 / 2003$ & 6.6 & .05 & -- & 1.68 & Est & -- & -- \\
\hline Courtois Creek & $\operatorname{cc} 13$ & 374537 & 0910403 & $08 / 26 / 2003$ & 7.2 & -- & 1.52 & 1.52 & Good & 358 & 25.2 \\
\hline Courtois Creek & $\operatorname{cc} 13$ & 374537 & 0910403 & $10 / 06 / 2006$ & 7.2 & -- & 1.03 & 1.03 & Fair & 382 & 18.0 \\
\hline Courtois Creek - Indian Creek & cc14 & 374545 & 0910421 & $10 / 06 / 2006$ & 7.4 & 1.41 & -- & 2.44 & $\mathrm{Calc}^{\mathrm{a}}$ & -- & -- \\
\hline Courtois Creek & $\operatorname{cc} 15$ & 374557 & 0910415 & $10 / 06 / 2006$ & 7.6 & -- & 2.44 & 2.44 & Fair & 465 & 17.4 \\
\hline Courtois Creek & $\operatorname{cc} 16$ & 374725 & 0910342 & $10 / 06 / 2006$ & 9.8 & -- & 3.01 & 3.01 & Good & 473 & 18.7 \\
\hline Courtois Creek - Cub Creek & $\operatorname{cc} 17$ & 374713 & 0910316 & $10 / 06 / 2006$ & 9.9 & .96 & -- & 3.97 & Good & 435 & 17.5 \\
\hline Courtois Creek - Bailey Branch & $\operatorname{cc} 18$ & 374753 & 0910339 & $10 / 06 / 2006$ & 10.3 & .20 & -- & 4.17 & Est & 460 & 14.0 \\
\hline Courtois Creek & $\operatorname{cc} 19$ & 375031 & 0910324 & $10 / 06 / 2006$ & 14.5 & -- & 5.74 & 5.74 & Good & 448 & 19.0 \\
\hline \multicolumn{12}{|c|}{ Miscellaneous tributary and headwater measurement or observation sites (not shown on fig. 4A) } \\
\hline Courtois Creek - Abbott Branch & $\operatorname{cc} 06 \mathrm{a}$ & 374239 & 0910152 & $08 / 26 / 2003$ & -- & 0.01 & -- & -- & Est & -- & -- \\
\hline \multicolumn{12}{|c|}{ Courtois Creek - Indian Creek (figs. 2, 4B) } \\
\hline 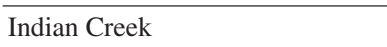 & cc14a & 374136 & 0910555 & $8 / 27 / 2003$ & 2.7 & -- & 0.01 & 0.01 & Est & 420 & 28.6 \\
\hline Indian Creek & $\mathrm{cc} 14 \mathrm{~b}$ & 374210 & 0910550 & $8 / 27 / 2003$ & 3.4 & -- & .28 & .28 & Good & 904 & 25.1 \\
\hline Indian Creek - tailings discharge & $\mathrm{cc} 14 \mathrm{c}$ & 374215 & 0910650 & $8 / 27 / 2003$ & 4.0 & 0.56 & -- & .84 & Good & 3,818 & 28.3 \\
\hline Indian Creek & $\mathrm{cc} 14 \mathrm{~d}$ & 374313 & 0910547 & $8 / 27 / 2003$ & 4.7 & -- & .43 & .43 & Fair & 688 & 22.4 \\
\hline Indian Creek - East Prong & $\mathrm{cc} 14 \mathrm{e}$ & 374314 & 0910541 & $8 / 27 / 2003$ & 4.8 & .03 & -- & .46 & Est & 415 & 22.7 \\
\hline Indian Creek - West Prong & $\operatorname{cc} 14 \mathrm{f}$ & 374258 & 0910628 & $8 / 27 / 2003$ & 5.7 & 2.77 & -- & 3.23 & Fair & 706 & 19.9 \\
\hline Indian Creek & $\mathrm{cc} 14 \mathrm{~g}$ & 374453 & 0910438 & $8 / 27 / 2003$ & 7.0 & -- & 9.82 & 9.82 & Good & 614 & 28.6 \\
\hline Indian Creek - Mason Hollow & $\mathrm{cc} 14 \mathrm{~h}$ & 374510 & 0910433 & $8 / 27 / 2003$ & 7.4 & 0 & -- & 9.82 & Obs & -- & -- \\
\hline Indian Creek - trib & cc14i & 374529 & 0910432 & $8 / 27 / 2003$ & 7.7 & .55 & -- & 10.4 & Fair & 504 & 23.3 \\
\hline Indian Creek & $\mathrm{cc} 14$ & 374545 & 0910421 & $8 / 27 / 2003$ & 8.1 & -- & 9.94 & 9.94 & Fair & 599 & 27.3 \\
\hline
\end{tabular}


Table 1. Seepage run data for streams draining the Viburnum Trend Subdistrict.—Continued

[DDMMSS, degrees, minutes, seconds; MM/DD/YYY, month/day/year; mi, mile; $\mathrm{ft}^{3} / \mathrm{s}$, cubic feet per second; $\mu \mathrm{S} / \mathrm{cm}$, microsiemens per centimeter at 25 degrees Celsius; ${ }^{\circ} \mathrm{C}$, degrees Celsius; --, no data; $\mathrm{WFHC}$, West Fork Huzzah Creek; Est, estimated; trib, tributary; Calc, calculated; Obs, observation; shaded boxes are identical sites measured on different days; EFHC, East Fork Huzzah Creek; WFBR, West Fork Black River; MFBR, Middle Fork Black River; EFBR, East Fork Black River]

\begin{tabular}{|c|c|c|c|c|c|c|c|c|c|c|c|}
\hline \multirow[b]{2}{*}{ Stream site } & \multirow[b]{2}{*}{$\begin{array}{c}\text { Site } \\
\text { identifier }\end{array}$} & \multirow[b]{2}{*}{$\begin{array}{c}\text { Latitude } \\
\text { (DDMMSS) }\end{array}$} & \multirow[b]{2}{*}{$\begin{array}{c}\text { Longitude } \\
\text { (DDDMMSS) }\end{array}$} & \multirow[b]{2}{*}{$\begin{array}{c}\text { Date } \\
\text { (MM/DD/YYYY) }\end{array}$} & \multirow{2}{*}{$\begin{array}{l}\text { Stream } \\
\text { length } \\
\text { (mi) }\end{array}$} & \multicolumn{2}{|c|}{ Measurement location } & \multirow{2}{*}{$\begin{array}{c}\text { Accumulated } \\
\text { discharge } \\
\text { (figs. } 3,4,6,8) \\
\left(\mathrm{ft}^{3} / \mathrm{s}\right)\end{array}$} & \multirow{2}{*}{$\begin{array}{l}\text { Measure- } \\
\text { ment } \\
\text { rating }\end{array}$} & \multirow{2}{*}{$\begin{array}{c}\text { Specific } \\
\text { conductance } \\
(\mu \mathrm{S} / \mathrm{cm} \text { at } \\
\left.25^{\circ} \mathrm{C}\right)\end{array}$} & \multirow[b]{2}{*}{$\begin{array}{c}\text { Temperature } \\
\left({ }^{\circ} \mathrm{C}\right)\end{array}$} \\
\hline & & & & & & $\begin{array}{c}\text { Tributary } \\
\left(\mathrm{ft}^{3} / \mathbf{s}\right)\end{array}$ & $\begin{array}{c}\text { Mainstem } \\
\left(\mathrm{ft}^{3} / \mathbf{s}\right)\end{array}$ & & & & \\
\hline \multicolumn{12}{|c|}{ Miscellaneous tributary and headwater measurement or observation sites (not shown on fig. 4b) } \\
\hline Indian Creek - West Prong trib & cc14fa & 374256 & 0910734 & $8 / 27 / 2003$ & -- & 0 & -- & -- & Obs & -- & -- \\
\hline \multicolumn{12}{|c|}{ West Fork Black River (figs. 5, 6A) } \\
\hline WFBR - Parker Branch mouth & wfb05 & 373133 & 0911402 & 09/07/2004 & 4.2 & 1.25 & -- & 1.25 & Good & 267 & 21.0 \\
\hline WFBR - Crossville Branch mouth & wfb08 & 373133 & 0911403 & 09/07/2004 & 4.3 & 1.63 & -- & 2.88 & Good & 317 & 22.5 \\
\hline WFBR - Moore Branch & wfb09 & 373130 & 0911324 & 09/07/2004 & 4.8 & 0 & -- & 2.88 & Obs & -- & -- \\
\hline WFBR - trib & wfb10 & 373109 & 0911256 & 09/07/2004 & 5.3 & 0 & -- & 2.88 & Obs & -- & -- \\
\hline WFBR - trib & wfb11 & 373106 & 0911233 & 09/07/2004 & 5.8 & 0 & -- & 2.88 & Obs & -- & -- \\
\hline WFBR - trib & wfb12 & 373101 & 0911205 & 09/07/2004 & 6.3 & 0 & -- & 2.88 & Obs & -- & -- \\
\hline WFBR & wfb13 & 373057 & 0911202 & 09/07/2004 & 6.4 & -- & 4.53 & 4.53 & Good & 321 & 23.0 \\
\hline WFBR - Henpeck Creek & wfb14 & 373046 & 0911155 & 09/07/2004 & 6.5 & .84 & -- & 5.37 & Good & 312 & 18.7 \\
\hline WFBR - McMurtrey Creek & wfb15 & 373055 & 0911110 & 09/07/2004 & 7.1 & .80 & -- & 6.17 & Good & 420 & 19.1 \\
\hline WFBR & wfb16 & 373052 & 0911059 & 09/07/2004 & 7.3 & -- & 7.14 & 7.14 & Good & 343 & 20.9 \\
\hline WFBR & wfb17 & 373020 & 0910940 & 09/08/2004 & 9.2 & -- & 8.44 & 8.44 & Good & 341 & 18.5 \\
\hline WFBR - Smalls Creek & wfb18 & 373019 & 0910945 & 09/08/2004 & 9.2 & 0 & -- & 8.44 & Obs & -- & -- \\
\hline WFBR - trib & wfb19 & 373015 & 0910911 & 09/08/2004 & 10.0 & .03 & -- & 8.47 & Est & 395 & 18.0 \\
\hline WFBR - trib & wfb20 & 373015 & 0910908 & 09/08/2004 & 10.1 & 0 & -- & 8.47 & Obs & -- & -- \\
\hline WFBR - trib & wfb21 & 373007 & 0910852 & 09/08/2004 & 10.3 & 0 & -- & 8.47 & Obs & -- & -- \\
\hline WFBR - Bills Creek & wfb22 & 372958 & 0910826 & 09/08/2004 & 11.0 & .18 & -- & 8.65 & Good & 517 & 20.0 \\
\hline WFBR - Bills Creek & wfb23 & 372959 & 0910821 & 09/08/2004 & 11.1 & .79 & -- & 9.44 & Fair & 495 & 21.2 \\
\hline WFBR - trib & wfb24 & 372947 & 0910802 & 09/08/2004 & 11.5 & 0 & -- & 9.44 & Obs & -- & -- \\
\hline WFBR - trib & wfb25 & 372939 & 0910739 & 09/08/2004 & 12.1 & 0 & -- & 9.44 & Obs & -- & -- \\
\hline WFBR & wfb26 & 372931 & 0910637 & 09/08/2004 & 13.2 & -- & 12.2 & 12.2 & Good & 359 & 21.8 \\
\hline WFBR - Toms Creek & wfb27 & 372931 & 0910638 & 09/08/2004 & 13.2 & .27 & -- & 12.5 & Good & 288 & 19.9 \\
\hline WFBR - Brown Branch & wfb28 & 372921 & 0910622 & 09/08/2004 & 13.4 & 0 & -- & 12.5 & Obs & -- & -- \\
\hline WFBR - trib pools & wfb29 & 372926 & 0910540 & 09/08/2004 & 14.3 & 0 & -- & 12.5 & Obs & -- & -- \\
\hline WFBR - trib pools & wfb30 & 372941 & 0910516 & 09/08/2004 & 14.7 & 0 & -- & 12.5 & Obs & -- & -- \\
\hline WFBR - Mill Branch mouth & wfb31 & 372943 & 0910513 & 09/08/2004 & 14.8 & 1.03 & -- & 13.5 & Fair & 300 & 19.0 \\
\hline WFBR - trib & wfb32 & 372901 & 0910311 & 09/08/2004 & 19.7 & 0 & -- & 13.5 & Obs & -- & -- \\
\hline WFBR - Radford Hollow & wfb33 & 372911 & 0910303 & 09/08/2004 & 19.8 & .23 & -- & 13.7 & Fair & 374 & 17.5 \\
\hline WFBR & wfb34 & 372907 & 0910255 & 09/08/2004 & 20.0 & -- & 17.1 & 17.1 & Good & 377 & 23.2 \\
\hline WFBR - trib & wfb35 & 372908 & 0910245 & 09/08/2004 & 20.1 & 0 & - & 17.1 & Obs & -- & -- \\
\hline WFBR - trib & wfb36 & 372914 & 0910206 & 09/08/2004 & 21.2 & 0 & -- & 17.1 & Obs & -- & -- \\
\hline WFBR - Cooks Spring & wfb37 & 372916 & 0910147 & 09/08/2004 & 21.2 & .57 & -- & 17.7 & Fair & 296 & 16.0 \\
\hline WFBR - Sutton Bluff & wfb38 & 372835 & 0910026 & 09/08/2004 & 23.6 & -- & 21.9 & 21.9 & Poor & 358 & 21.8 \\
\hline
\end{tabular}


Table 1. Seepage run data for streams draining the Viburnum Trend Subdistrict.-Continued

[DDMMSS, degrees, minutes, seconds; MM/DD/YYY, month/day/year; mi, mile; $\mathrm{ft}^{3} / \mathrm{s}$, cubic feet per second; $\mathrm{uS} / \mathrm{cm}$, microsiemens per centimeter at 25 degrees Celsius; ${ }^{\circ} \mathrm{C}$, degrees Celsius; --, no data; $\mathrm{WFHC}$, West Fork Huzzah Creek; Est, estimated; trib, tributary; Calc, calculated; Obs, observation; shaded boxes are identical sites measured on different days; EFHC, East Fork Huzzah Creek; WFBR, West Fork Black River; MFBR, Middle Fork Black River; EFBR, East Fork Black River]

\begin{tabular}{|c|c|c|c|c|c|c|c|c|c|c|c|}
\hline \multirow[b]{2}{*}{ Stream site } & \multirow[b]{2}{*}{$\begin{array}{c}\text { Site } \\
\text { identifier }\end{array}$} & \multirow[b]{2}{*}{$\begin{array}{c}\text { Latitude } \\
\text { (DDMMSS) }\end{array}$} & \multirow[b]{2}{*}{$\begin{array}{c}\text { Longitude } \\
\text { (DDDMMSS) }\end{array}$} & \multirow[b]{2}{*}{$\begin{array}{c}\text { Date } \\
\text { (MM/DD/YYYY) }\end{array}$} & \multirow{2}{*}{$\begin{array}{l}\text { Stream } \\
\text { length } \\
\text { (mi) }\end{array}$} & \multicolumn{2}{|c|}{ Measurement location } & \multirow{2}{*}{$\begin{array}{c}\text { Accumulated } \\
\text { discharge } \\
\text { (figs. } 3,4,6,8) \\
\left(\mathrm{ft}^{3} / \mathrm{s}\right)\end{array}$} & \multirow{2}{*}{$\begin{array}{l}\text { Measure- } \\
\text { ment } \\
\text { rating }\end{array}$} & \multirow{2}{*}{$\begin{array}{c}\text { Specific } \\
\text { conductance } \\
(\mu \mathrm{S} / \mathrm{cm} \text { at } \\
\left.25^{\circ} \mathrm{C}\right)\end{array}$} & \multirow[b]{2}{*}{$\begin{array}{c}\text { Temperature } \\
\left({ }^{\circ} \mathrm{C}\right)\end{array}$} \\
\hline & & & & & & $\begin{array}{c}\text { Tributary } \\
\left(\mathrm{ft}^{3} / \mathrm{s}\right)\end{array}$ & $\begin{array}{l}\text { Mainstem } \\
\left(\mathrm{ft}^{3} / \mathrm{s}\right)\end{array}$ & & & & \\
\hline \multicolumn{12}{|c|}{ West Fork Black River (figs. 5, 6A) —Continued } \\
\hline WFBR - Sutton Bluff & wfb38 & 372835 & 0910026 & 09/09/2004 & 23.6 & -- & 22.0 & 22.0 & Fair & 359 & 20.6 \\
\hline WFBR - trib & wfb39 & 372837 & 0910006 & 09/09/2004 & 24.0 & 0 & -- & 22.0 & Obs & -- & -- \\
\hline WFBR - Stillwell Hollow & wfb40 & 372820 & 0905951 & 09/09/2004 & 24.6 & .29 & -- & 22.3 & Good & 268 & 18.4 \\
\hline WFBR - trib & wfb41 & 372817 & 0905929 & 09/09/2004 & 24.8 & 0 & -- & 22.3 & Obs & -- & -- \\
\hline WFBR - trib & wfb42 & 372734 & 0905910 & 09/09/2004 & 25.9 & .03 & -- & 22.3 & Est & 410 & 15.6 \\
\hline WFBR - Bee Fork mouth & wfb43 & 372731 & 0905916 & 09/09/2004 & 26.0 & 10.8 & -- & 33.1 & Good & 372 & 19.6 \\
\hline WFBR & wfb44 & 372725 & 0905901 & 09/09/2004 & 26.1 & -- & 36.1 & 36.1 & Good & 358 & 21.2 \\
\hline WFBR - trib & wfb45 & 372723 & 0905844 & 09/09/2004 & 26.6 & 0 & -- & 36.1 & Obs & -- & -- \\
\hline WFBR - McLean Hollow & wfb46 & 372710 & 0905808 & 09/09/2004 & 27.0 & .02 & -- & 36.1 & Est & 290 & 20.5 \\
\hline WFBR - Hunter Hollow & wfb47 & 372606 & 0905742 & 09/09/2004 & 28.3 & 0 & -- & 36.1 & Obs & -- & -- \\
\hline WFBR - Reeds Spring & wfb48 & 372555 & 0905653 & 09/09/2004 & 29.0 & 11.9 & -- & 48.0 & Fair & 229 & 13.2 \\
\hline WFBR & wfb49 & 372553 & 0905641 & 09/09/2004 & 29.2 & -- & 55.0 & 55.0 & Good & 313 & 20.8 \\
\hline WFBR - Russell Hollow & wfb50 & 372550 & 0905633 & 09/09/2004 & 29.3 & .04 & -- & 55.0 & Est & 187 & 18.9 \\
\hline WFBR - Barn Hollow & wfb51 & 372632 & 0905426 & 09/09/2004 & 32.6 & .02 & -- & 55.1 & Est & 350 & 23.0 \\
\hline WFBR - trib & wfb52 & 372641 & 0905412 & 09/09/2004 & 32.8 & 0 & -- & 55.1 & Obs & -- & -- \\
\hline WFBR - Middle Hollow & wfb53 & 372644 & 0905406 & 09/09/2004 & 32.8 & .01 & -- & 55.1 & Est & 339 & 20.7 \\
\hline WFBR - Russell Hollow & wfb54 & 372656 & 0905341 & 09/09/2004 & 33.7 & 0 & -- & 55.1 & Obs & -- & -- \\
\hline WFBR - trib & wfb55 & 372700 & 0905303 & 09/09/2004 & 33.9 & 0 & -- & 55.1 & Obs & -- & -- \\
\hline WFBR & wfb56 & 372653 & 0905304 & 09/09/2004 & 34.0 & -- & 55.6 & 55.6 & Good & 311 & 23.0 \\
\hline WFBR - Hawk Hollow & wfb57 & 372655 & 0905228 & 09/09/2004 & 34.8 & 0 & -- & 55.6 & Obs & -- & -- \\
\hline \multicolumn{12}{|c|}{ Miscellaneous tributary and headwater measurement or observation sites (not shown on fig. 6A) } \\
\hline WFBR - Henpeck Creek & wfb14a & 373035 & 0911223 & 09/07/2004 & -- & 0.09 & -- & -- & Good & 112 & 19.5 \\
\hline WFBR - Cooks Creek & wfb37a & 373009 & 0910157 & $09 / 08 / 2004$ & -- & 0 & -- & -- & Obs & -- & -- \\
\hline \multicolumn{12}{|c|}{ West Fork Black River - Parker Branch (not shown on fig. 6A) } \\
\hline Parker Branch & wfb01 & 373349 & 0911632 & $08 / 29 / 2003$ & 0.4 & -- & 0.05 & 0.05 & Est & 192 & 23.1 \\
\hline Parker Branch trib & wfb02 & 373336 & 0911621 & $08 / 29 / 2003$ & .7 & 0 & -- & .05 & Obs & -- & -- \\
\hline Parker Branch & wfb03 & 373309 & 0911536 & $08 / 29 / 2003$ & 1.7 & -- & .17 & .17 & Good & 240 & 21.9 \\
\hline Parker Branch - Radford Branch & wfb04 & 373225 & 0911429 & $08 / 29 / 2003$ & 3.2 & .02 & -- & .19 & Est & 391 & 23.6 \\
\hline Parker Branch mouth & wfb05 & 373133 & 0911402 & $08 / 29 / 2003$ & 4.2 & -- & .54 & .54 & Fair & 333 & 23.8 \\
\hline \multicolumn{12}{|c|}{ West Fork Black River - Crossville Branch (not shown on fig. 6A) } \\
\hline Crossville Branch & wfb06 & 373153 & 0911559 & $08 / 29 / 2003$ & -- & -- & 0.05 & 0.05 & Est & 313 & 27.3 \\
\hline Crossville Branch - Hayes Branch & wfb07 & 373151 & 0911600 & $08 / 29 / 2003$ & -- & 0.02 & -- & .07 & Est & -- & -- \\
\hline Crossville Branch mouth & wfb08 & 373133 & 0911403 & $08 / 29 / 2003$ & -- & -- & .39 & .39 & Fair & 335 & 22.5 \\
\hline
\end{tabular}


Table 1. Seepage run data for streams draining the Viburnum Trend Subdistrict.—Continued

[DDMMSS, degrees, minutes, seconds; MM/DD/YYY, month/day/year; mi, mile; $\mathrm{ft}^{3} / \mathrm{s}$, cubic feet per second; $\mu \mathrm{S} / \mathrm{cm}$, microsiemens per centimeter at 25 degrees Celsius; ${ }^{\circ} \mathrm{C}$, degrees Celsius; --, no data; $\mathrm{WFHC}$, West Fork Huzzah Creek; Est, estimated; trib, tributary; Calc, calculated; Obs, observation; shaded boxes are identical sites measured on different days; EFHC, East Fork Huzzah Creek; WFBR, West Fork Black River; MFBR, Middle Fork Black River; EFBR, East Fork Black River]

\begin{tabular}{|c|c|c|c|c|c|c|c|c|c|c|c|}
\hline \multirow[b]{2}{*}{ Stream site } & \multirow[b]{2}{*}{$\begin{array}{c}\text { Site } \\
\text { identifier }\end{array}$} & \multirow[b]{2}{*}{$\begin{array}{c}\text { Latitude } \\
\text { (DDMMSS) }\end{array}$} & \multirow[b]{2}{*}{$\begin{array}{c}\text { Longitude } \\
\text { (DDDMMSS) }\end{array}$} & \multirow[b]{2}{*}{$\begin{array}{c}\text { Date } \\
\text { (MM/DD/YYYY) }\end{array}$} & \multirow{2}{*}{$\begin{array}{l}\text { Stream } \\
\text { length } \\
\text { (mi) }\end{array}$} & \multicolumn{2}{|c|}{ Measurement location } & \multirow{2}{*}{$\begin{array}{c}\text { Accumulated } \\
\text { discharge } \\
\text { (figs. } 3,4,6,8) \\
\left(\mathrm{ft}^{3} / \mathrm{s}\right) \\
\end{array}$} & \multirow{2}{*}{$\begin{array}{l}\text { Measure- } \\
\text { ment } \\
\text { rating }\end{array}$} & \multirow{2}{*}{$\begin{array}{c}\text { Specific } \\
\text { conductance } \\
(\mu \mathrm{S} / \mathrm{cm} \text { at } \\
\left.25^{\circ} \mathrm{C}\right)\end{array}$} & \multirow[b]{2}{*}{$\begin{array}{c}\text { Temperature } \\
\left({ }^{\circ} \mathrm{C}\right)\end{array}$} \\
\hline & & & & & & $\begin{array}{c}\text { Tributary } \\
\left(\mathrm{ft}^{3} / \mathrm{s}\right)\end{array}$ & $\begin{array}{c}\text { Mainstem } \\
\left(\mathrm{ft}^{3} / \mathbf{s}\right)\end{array}$ & & & & \\
\hline \multicolumn{12}{|c|}{ West Fork Black River - Mill Branch (not shown on fig. 6A) } \\
\hline Mill Branch & wfb31a & 373103 & 0910519 & $09 / 08 / 2004$ & -- & -- & 0 & 0 & Obs & -- & -- \\
\hline Mill Branch trib & wfb31b & 373052 & 0910521 & $09 / 08 / 2004$ & -- & 0 & -- & 0 & Obs & -- & -- \\
\hline Mill Branch & wfb31c & 373023 & 0910517 & $09 / 08 / 2004$ & -- & -- & 1.00 & 1.00 & Est & 293 & 17.4 \\
\hline Mill Branch mouth & wfb31 & 372943 & 0910513 & $09 / 08 / 2004$ & -- & -- & 1.03 & 1.03 & Fair & 300 & 19.0 \\
\hline \multicolumn{12}{|c|}{ West Fork Black River - Bee Fork (figs. 5, 6B) } \\
\hline Bee Fork trib & wfb43a & 372641 & 0911032 & $09 / 10 / 2004$ & 0.1 & 0 & -- & 0 & Obs & -- & -- \\
\hline Bee Fork & wfb43b & 372638 & 0911030 & 09/10/2004 & .1 & -- & 0 & 0 & Obs & 64 & 16.3 \\
\hline Bee Fork spring pool & wfb43c & 372637 & 0911012 & $09 / 10 / 2004$ & .4 & -- & 0 & 0 & Obs & -- & -- \\
\hline Bee Fork trib & wfb43d & 372635 & 0910956 & 09/10/2004 & .6 & .01 & -- & .01 & Est & 146 & 15.3 \\
\hline Bee Fork & wfb43e & 372634 & 0910944 & 09/10/2004 & .8 & -- & .02 & .02 & Est & 213 & 15.4 \\
\hline Bee Fork & $w f b 43 f$ & 372633 & 0910926 & 09/10/2004 & 1.1 & -- & .06 & .06 & Fair & 126 & 14.3 \\
\hline Bee Fork spring & wfb43g & 372643 & 0910859 & 09/10/2004 & 1.6 & .02 & -- & .08 & Est & 290 & 14.3 \\
\hline Bee Fork spring & wfb43h & 372647 & 0910841 & 09/10/2004 & 1.8 & .01 & -- & .09 & Est & 391 & 16.9 \\
\hline Bee Fork trib & wfb43i & 372647 & 0910837 & $09 / 10 / 2004$ & 1.9 & 0 & -- & .09 & Obs & -- & -- \\
\hline Bee Fork - Kitchel Branch & $w f b 43 j$ & 372702 & 0910743 & 09/10/2004 & 3.0 & .05 & -- & .14 & Est & 164 & 16.9 \\
\hline Bee Fork - Fletcher Mine CP trib & wfb43k & 372639 & 0910621 & $09 / 10 / 2004$ & 4.2 & 4.17 & -- & 4.31 & Fair & 633 & 21.5 \\
\hline Bee Fork & wfb431 & 372629 & 0910620 & 09/10/2004 & 4.3 & -- & 5.13 & 5.13 & Fair & 453 & 20.8 \\
\hline Bee Fork - West Fork Hollow & $w f b 43 m$ & 372627 & 0910546 & 09/10/2004 & 4.8 & 0 & -- & 5.13 & Obs & -- & -- \\
\hline Bee Fork & $w f b 43 n$ & 372626 & 0910533 & $09 / 10 / 2004$ & 5.1 & -- & 5.82 & 5.82 & Fair & 507 & 22.4 \\
\hline Bee Fork & wfb43n & 372626 & 0910533 & 09/13/2004 & 5.1 & -- & 5.86 & 5.86 & Fair & 515 & 22.5 \\
\hline Bee Fork - Grasshopper Hollow & wfb43o & 372625 & 0910530 & $09 / 13 / 2004$ & 5.1 & .38 & -- & 6.24 & Fair & 228 & 19.9 \\
\hline Bee Fork trib & wfb43p & 372628 & 0910432 & 09/13/2004 & 6.0 & 0 & -- & 6.24 & Obs & -- & -- \\
\hline Bee Fork trib & wfb43q & 372636 & 0910351 & 09/13/2004 & 6.7 & 0 & -- & 6.24 & Obs & -- & -- \\
\hline Bee Fork trib & $w f b 43 r$ & 372641 & 0910332 & $09 / 13 / 2004$ & 7.0 & 0 & -- & 6.24 & Obs & -- & -- \\
\hline Bee Fork trib & $\mathrm{wfb} 43 \mathrm{~s}$ & 372645 & 0910322 & 09/13/2004 & 7.2 & 0 & -- & 6.24 & Obs & -- & -- \\
\hline Bee Fork trib & wfb43t & 372651 & 0910315 & 09/13/2004 & 7.3 & 0 & -- & 6.24 & Obs & -- & -- \\
\hline Bee Fork trib & wfb43u & 372703 & 0910301 & 09/13/2004 & 7.8 & 0 & -- & 6.24 & Obs & -- & -- \\
\hline Bee Fork trib & wfb $43 \mathrm{v}$ & 372711 & 0910233 & 09/13/2004 & 8.2 & .03 & -- & 6.27 & Est & 291 & 19.5 \\
\hline Bee Fork & $w f b 43 w$ & 372712 & 0910158 & 09/13/2004 & 8.7 & -- & 8.53 & 8.53 & Good & 416 & 22.1 \\
\hline Bee Fork trib & wfb43x & 372716 & 0910203 & 09/13/2004 & 8.7 & 0 & -- & 8.53 & Obs & -- & -- \\
\hline Bee Fork trib & wfb43y & 372730 & 0910140 & 09/13/2004 & 9.2 & 0 & -- & 8.53 & Obs & -- & -- \\
\hline Bee Fork mouth & wfb43 & 372731 & 0905916 & 09/13/2004 & 13.6 & -- & 10.1 & 10.1 & Good & 372 & 21.3 \\
\hline
\end{tabular}


Table 1. Seepage run data for streams draining the Viburnum Trend Subdistrict.-Continued

[DDMMSS, degrees, minutes, seconds; MM/DD/YYY, month/day/year; mi, mile; $\mathrm{ft}^{3} / \mathrm{s}$, cubic feet per second; $\mathrm{uS} / \mathrm{cm}$, microsiemens per centimeter at 25 degrees Celsius; ${ }^{\circ} \mathrm{C}$, degrees Celsius; --, no data; $\mathrm{WFHC}$, West Fork Huzzah Creek; Est, estimated; trib, tributary; Calc, calculated; Obs, observation; shaded boxes are identical sites measured on different days; EFHC, East Fork Huzzah Creek; WFBR, West Fork Black River; MFBR, Middle Fork Black River; EFBR, East Fork Black River]

\begin{tabular}{|c|c|c|c|c|c|c|c|c|c|c|c|}
\hline \multirow[b]{2}{*}{ Stream site } & \multirow[b]{2}{*}{$\begin{array}{c}\text { Site } \\
\text { identifier }\end{array}$} & \multirow[b]{2}{*}{$\begin{array}{l}\text { Latitude } \\
\text { (DDMMSS) }\end{array}$} & \multirow[b]{2}{*}{$\begin{array}{c}\text { Longitude } \\
\text { (DDDMMSS) }\end{array}$} & \multirow[b]{2}{*}{$\begin{array}{c}\text { Date } \\
\text { (MM/DD/YYYY) }\end{array}$} & \multirow{2}{*}{$\begin{array}{l}\text { Stream } \\
\text { length } \\
\text { (mi) }\end{array}$} & \multicolumn{2}{|c|}{ Measurement location } & \multirow{2}{*}{$\begin{array}{c}\text { Accumulated } \\
\text { discharge } \\
\text { (figs. } 3,4,6,8) \\
\left(\mathrm{ft}^{3} / \mathrm{s}\right)\end{array}$} & \multirow{2}{*}{$\begin{array}{c}\text { Measure- } \\
\text { ment } \\
\text { rating }\end{array}$} & \multirow{2}{*}{$\begin{array}{c}\text { Specific } \\
\text { conductance } \\
(\mu \mathrm{S} / \mathrm{cm} \text { at } \\
\left.25^{\circ} \mathrm{C}\right)\end{array}$} & \multirow[b]{2}{*}{$\begin{array}{c}\text { Temperature } \\
\left({ }^{\circ} \mathrm{C}\right)\end{array}$} \\
\hline & & & & & & $\begin{array}{c}\text { Tributary } \\
\left(\mathrm{ftt}^{3} / \mathrm{s}\right)\end{array}$ & $\begin{array}{l}\text { Mainstem } \\
\left(\mathrm{ft}^{3} / \mathrm{s}\right)\end{array}$ & & & & \\
\hline \multicolumn{12}{|c|}{ Middle Fork Black River (figs. 5, 6C) } \\
\hline MFBR - Brooks Creek & $\mathrm{mf01}$ & 373958 & 0910151 & $08 / 27 / 2003$ & 0 & 0.15 & -- & 0.15 & Est & -- & -- \\
\hline MFBR & $\mathrm{mf02}$ & 373802 & 0910008 & $08 / 27 / 2003$ & 1.6 & -- & 0.94 & .94 & Good & 319 & 25.0 \\
\hline MFBR - Clayton Creek & $\mathrm{mf03}$ & 373717 & 0905814 & $08 / 27 / 2003$ & 2.7 & .63 & -- & 1.57 & Good & 332 & 23.6 \\
\hline MFBR & mf04 & 373650 & 0905759 & $08 / 27 / 2003$ & 3.0 & -- & 1.81 & 1.81 & Good & 318 & 25.0 \\
\hline MFBR - Strother Creek near mouth & $\mathrm{mf05}$ & 373610 & 0905828 & 08/11/2006 & 4.3 & 13.3 & -- & 15.1 & Fair & 708 & 23.8 \\
\hline MFBR & mf06 & 373546 & 0905707 & $08 / 11 / 2006$ & 4.9 & -- & 16.4 & 16.4 & Good & 646 & 23.5 \\
\hline MFBR - Ottery Creek & $\mathrm{mf07}$ & 373449 & 0905600 & $08 / 11 / 2006$ & 6.8 & .25 & -- & 16.7 & Good & 252 & 23.5 \\
\hline MFBR & mf08 & 373338 & 0905535 & $08 / 11 / 2006$ & 8.4 & -- & 17.0 & 17.0 & Good & 545 & 25.2 \\
\hline MFBR & mf09 & 373107 & 0905559 & $08 / 28 / 2003$ & 11.2 & -- & 14.7 & 14.7 & Fair & 469 & 27.1 \\
\hline $\begin{array}{l}\text { MFBR - Little Brushy Creek near } \\
\text { mouth }\end{array}$ & $\mathrm{mf} 10$ & 373210 & 0905616 & $08 / 28 / 2003$ & 11.4 & .85 & -- & 15.6 & Est & -- & -- \\
\hline MFBR - Brushy Creek mouth & $\operatorname{mf} 11$ & 373129 & 0905618 & $08 / 28 / 2003$ & 11.4 & 0 & -- & 15.6 & Obs & -- & -- \\
\hline MFBR - Carty Branch mouth & $\mathrm{mf} 12$ & 373106 & 0905609 & $08 / 28 / 2003$ & 11.9 & .85 & -- & 16.4 & Good & 262 & 23.4 \\
\hline MFBR - trib & $\mathrm{mf} 13$ & 373112 & 0905607 & 09/09/2004 & 12.0 & 0 & -- & 16.4 & Obs & -- & -- \\
\hline MFBR - Paynes Branch & $\mathrm{mf} 14$ & 372837 & 0905415 & $08 / 28 / 2003$ & 16.3 & 1.61 & -- & 18.0 & Good & 190 & 24.1 \\
\hline MFBR & $\mathrm{mf} 15$ & 372834 & 0905403 & $08 / 28 / 2003$ & 16.5 & -- & 26.9 & 26.9 & Good & 369 & 25.8 \\
\hline \multicolumn{12}{|c|}{ Middle Fork Black River - Little Brushy Creek (not shown on fig. 6C) } \\
\hline Little Brushy Creek & mf10a & 373434 & 0905760 & $09 / 09 / 2004$ & 1.7 & -- & 0 & 0 & Obs & -- & -- \\
\hline Little Brushy Creek & $\mathrm{mf} 10 \mathrm{~b}$ & 373408 & 0905750 & 09/09/2004 & 2.0 & -- & .01 & .01 & Est & 298 & 19.6 \\
\hline Little Brushy Creek trib & $\mathrm{mf} 10 \mathrm{c}$ & 373345 & 0905737 & $09 / 09 / 2004$ & 2.5 & 0 & -- & .01 & Obs & -- & - \\
\hline Little Brushy Creek & $\operatorname{mf} 10 \mathrm{~d}$ & 373247 & 0905645 & 09/09/2004 & 3.9 & -- & .05 & .05 & Est & 440 & 16.8 \\
\hline Little Brushy Creek - spring & $\mathrm{mf10e}$ & 373229 & 0905629 & 09/09/2004 & 4.8 & .25 & -- & .30 & Est & 388 & 13.3 \\
\hline Little Brushy Creek near mouth & $\mathrm{mf} 10$ & 373210 & 0905616 & 09/09/2004 & 5.1 & -- & 1.14 & 1.14 & Fair & 270 & 19.8 \\
\hline \multicolumn{12}{|c|}{ Middle Fork Black River sites (not shown on fig. 6C) } \\
\hline MFBR - Carty Branch trib & $\mathrm{mf} 12 \mathrm{a}$ & 373060 & 0905618 & $09 / 09 / 2004$ & -- & 0 & -- & -- & Obs & -- & -- \\
\hline MFBR - Carty Branch mouth & $\mathrm{mf} 12$ & 373106 & 0905609 & 09/09/2004 & -- & 1.61 & -- & -- & Fair & 251 & 19.2 \\
\hline
\end{tabular}


Table 1. Seepage run data for streams draining the Viburnum Trend Subdistrict.—Continued

[DDMMSS, degrees, minutes, seconds; MM/DD/YYY, month/day/year; mi, mile; $\mathrm{ft}^{3} / \mathrm{s}$, cubic feet per second; $\mu \mathrm{S} / \mathrm{cm}$, microsiemens per centimeter at 25 degrees Celsius; ${ }^{\circ} \mathrm{C}$, degrees Celsius; --, no data; $\mathrm{WFHC}$, West Fork Huzzah Creek; Est, estimated; trib, tributary; Calc, calculated; Obs, observation; shaded boxes are identical sites measured on different days; EFHC, East Fork Huzzah Creek; WFBR, West Fork Black River; MFBR, Middle Fork Black River; EFBR, East Fork Black River]

\begin{tabular}{|c|c|c|c|c|c|c|c|c|c|c|c|}
\hline \multirow[b]{2}{*}{ Stream site } & \multirow[b]{2}{*}{$\begin{array}{c}\text { Site } \\
\text { identifier }\end{array}$} & \multirow[b]{2}{*}{$\begin{array}{l}\text { Latitude } \\
\text { (DDMMSS) }\end{array}$} & \multirow[b]{2}{*}{$\begin{array}{l}\text { Longitude } \\
\text { (DDDMMSS) }\end{array}$} & \multirow[b]{2}{*}{$\begin{array}{c}\text { Date } \\
\text { (MM/DD/YYYY) }\end{array}$} & \multirow{2}{*}{$\begin{array}{l}\text { Stream } \\
\text { length } \\
\text { (mi) }\end{array}$} & \multicolumn{2}{|c|}{ Measurement location } & \multirow{2}{*}{$\begin{array}{c}\text { Accumulated } \\
\text { discharge } \\
\text { (figs. } 3,4,6,8) \\
\left(\mathrm{ft}^{3} / \mathrm{s}\right)\end{array}$} & \multirow{2}{*}{$\begin{array}{l}\text { Measure- } \\
\text { ment } \\
\text { rating }\end{array}$} & \multirow{2}{*}{$\begin{array}{c}\text { Specific } \\
\text { conductance } \\
(\mu \mathrm{S} / \mathrm{cm} \text { at } \\
\left.25^{\circ} \mathrm{C}\right)\end{array}$} & \multirow[b]{2}{*}{$\begin{array}{c}\text { Temperature } \\
\left({ }^{\circ} \mathrm{C}\right)\end{array}$} \\
\hline & & & & & & $\begin{array}{c}\text { Tributary } \\
\left(\mathrm{ft}^{3} / \mathbf{s}\right)\end{array}$ & $\begin{array}{c}\text { Mainstem } \\
\left(\mathrm{ft}^{3} / \mathrm{s}\right)\end{array}$ & & & & \\
\hline \multicolumn{12}{|c|}{ Middle Fork Black River - Strother Creek (figs. 5, 6D) } \\
\hline Strother Creek trib & mf05a & 373513 & 0910339 & 08/10/2006 & 1.9 & 0.02 & -- & 0.02 & Est & -- & -- \\
\hline Strother Creek & mf05b & 373515 & 0910328 & 08/10/2006 & 2.0 & -- & 7.63 & 7.63 & Fair & 1,155 & 29.9 \\
\hline Strother Creek - Little Creek & $\mathrm{mf} 05 \mathrm{c}$ & 373537 & 0910252 & 08/10/2006 & 2.8 & .37 & -- & 8.00 & Fair & 409 & 25.7 \\
\hline Strother Creek & mf05d & 373541 & 0910231 & 08/10/2006 & 3.1 & -- & 7.37 & 7.37 & Good & 1,093 & 28.6 \\
\hline Strother Creek trib & mf05e & 373549 & 0910211 & 08/10/2006 & 3.5 & 0 & -- & 7.37 & Obs & -- & -- \\
\hline Strother Creek & mf05f & 373611 & 0910053 & 08/10/2006 & 4.9 & -- & 8.90 & 8.90 & Good & 774 & 25.3 \\
\hline Strother Creek & mf05f & 373611 & 0910053 & 08/11/2006 & 4.9 & -- & 10.5 & 10.5 & Good & 829 & 24.7 \\
\hline Strother Creek trib & $\mathrm{mf} 05 \mathrm{~g}$ & 373605 & 0905935 & 08/11/2006 & 6.2 & 0 & -- & 10.5 & Obs & -- & -- \\
\hline Strother Creek near mouth & mf05 & 373610 & 0905828 & 08/11/2006 & 7.3 & -- & 13.3 & 13.3 & Fair & 708 & 23.8 \\
\hline \multicolumn{12}{|c|}{ Middle Fork Black River - Neals Creek (figs. 5, 6E) } \\
\hline Neals Creek & nc01 & 373915 & 0910631 & $08 / 10 / 2006$ & 0.4 & -- & 0 & 0 & Obs & -- & -- \\
\hline Neals Creek & nc02 & 373859 & 0910618 & 08/10/2006 & .7 & -- & .01 & .01 & Est & -- & -- \\
\hline Neals Creek & nc03 & 373845 & 0910550 & 08/10/2006 & 1.2 & -- & .03 & .03 & Est & 410 & 24.5 \\
\hline Neals Creek & nc04 & 373842 & 0910522 & 08/10/2006 & 1.7 & -- & .04 & .04 & Est & 445 & 23.3 \\
\hline Neals Creek - Left Fork & nc05 & 373838 & 0910521 & 08/10/2006 & 1.7 & 0.05 & -- & .09 & Est & 379 & 23.6 \\
\hline Neals Creek & nc06 & 373844 & 0910516 & 08/10/2006 & 1.8 & -- & .09 & .09 & Fair & 408 & 23.5 \\
\hline Neals Creek trib & nc07 & 373851 & 0910511 & 08/10/2006 & 1.9 & .02 & -- & .11 & Est & 486 & 23.3 \\
\hline Neals Creek trib & nc08 & 373853 & 0910454 & 08/10/2006 & 2.2 & .08 & -- & .19 & Fair & 449 & 22.6 \\
\hline Neals Creek trib & nc09 & 373846 & 0910426 & 08/10/2006 & 2.6 & 0 & -- & .19 & Obs & -- & -- \\
\hline Neals Creek trib & $\mathrm{nc} 10$ & 373842 & 0910416 & 08/10/2006 & 2.8 & 0 & -- & .19 & Obs & -- & -- \\
\hline Neals Creek trib & nc11 & 373833 & 0910354 & 08/10/2006 & 3.2 & 0 & -- & .19 & Obs & -- & -- \\
\hline Neals Creek & $\mathrm{nc} 12$ & 373822 & 0910335 & 08/10/2006 & 3.5 & -- & .33 & .33 & Fair & 434 & 23.5 \\
\hline Neals Creek trib & nc13 & 373823 & 0910330 & 08/10/2006 & 3.6 & .01 & -- & .34 & Est & -- & -- \\
\hline Neals Creek trib & nc14 & 373819 & 0910302 & 08/10/2006 & 4.1 & 0 & -- & .34 & Obs & -- & -- \\
\hline Neals Creek trib & nc15 & 373821 & 0910240 & 08/10/2006 & 4.5 & 0 & -- & .34 & Obs & -- & -- \\
\hline Neals Creek - Henderson Creek & nc16 & 373820 & 0910227 & 08/10/2006 & 4.7 & .34 & -- & .68 & Fair & 372 & 23.5 \\
\hline Neals Creek trib & nc17 & 373814 & 0910209 & 08/10/2006 & 4.9 & 0 & -- & .68 & Obs & -- & -- \\
\hline Neals Creek & nc18 & 373737 & 0910154 & 08/10/2006 & 5.7 & -- & 1.52 & 1.52 & Fair & 393 & 24.0 \\
\hline Neals Creek - Gunstock Hollow & nc19 & 373733 & 0910150 & 08/10/2006 & 5.9 & .02 & -- & 1.54 & Est & 440 & 24.6 \\
\hline Neals Creek trib & nc20 & 373705 & 0910128 & 08/10/2006 & 6.5 & 0 & -- & 1.54 & Obs & -- & -- \\
\hline Neals Creek & nc21 & 373616 & 0910103 & 08/10/2006 & 7.7 & -- & 1.35 & 1.35 & Good & 512 & 24.5 \\
\hline
\end{tabular}


Table 1. Seepage run data for streams draining the Viburnum Trend Subdistrict.-Continued

[DDMMSS, degrees, minutes, seconds; MM/DD/YYY, month/day/year; mi, mile; $\mathrm{ft}^{3} / \mathrm{s}$, cubic feet per second; $\mathrm{uS} / \mathrm{cm}$, microsiemens per centimeter at 25 degrees Celsius; ${ }^{\circ} \mathrm{C}$, degrees Celsius; --, no data; $\mathrm{WFHC}$, West Fork Huzzah Creek; Est, estimated; trib, tributary; Calc, calculated; Obs, observation; shaded boxes are identical sites measured on different days; EFHC, East Fork Huzzah Creek; WFBR, West Fork Black River; MFBR, Middle Fork Black River; EFBR, East Fork Black River]

\begin{tabular}{|c|c|c|c|c|c|c|c|c|c|c|c|}
\hline \multirow[b]{2}{*}{ Stream site } & \multirow[b]{2}{*}{$\begin{array}{c}\text { Site } \\
\text { identifier }\end{array}$} & \multirow[b]{2}{*}{$\begin{array}{c}\text { Latitude } \\
\text { (DDMMSS) }\end{array}$} & \multirow[b]{2}{*}{$\begin{array}{l}\text { Longitude } \\
\text { (DDDMMSS) }\end{array}$} & \multirow[b]{2}{*}{$\begin{array}{c}\text { Date } \\
\text { (MM/DD/YYYY) }\end{array}$} & \multirow{2}{*}{$\begin{array}{l}\text { Stream } \\
\text { length } \\
\text { (mi) }\end{array}$} & \multicolumn{2}{|c|}{ Measurement location } & \multirow{2}{*}{$\begin{array}{c}\text { Accumulated } \\
\text { discharge } \\
\text { (figs. } 3,4,6,8) \\
\left(\mathrm{ft}^{3} / \mathrm{s}\right) \\
\end{array}$} & \multirow{2}{*}{$\begin{array}{c}\text { Measure- } \\
\text { ment } \\
\text { rating }\end{array}$} & \multirow{2}{*}{$\begin{array}{c}\text { Specific } \\
\text { conductance } \\
(\mu \mathrm{S} / \mathrm{cm} \text { at } \\
\left.25^{\circ} \mathrm{C}\right)\end{array}$} & \multirow[b]{2}{*}{$\begin{array}{c}\text { Temperature } \\
\left({ }^{\circ} \mathrm{C}\right)\end{array}$} \\
\hline & & & & & & $\begin{array}{c}\text { Tributary } \\
\left(\mathrm{ft}^{3} / \mathbf{s}\right)\end{array}$ & $\begin{array}{l}\text { Mainstem } \\
\quad\left(\mathrm{ft}^{3} / \mathbf{s}\right)\end{array}$ & & & & \\
\hline \multicolumn{12}{|c|}{ Middle Fork Black River - Brushy Creek (figs. 5, 6F) } \\
\hline Brushy Creek & mf11a & 373356 & 0910659 & 09/08/2004 & 0 & -- & -- & 0 & Obs & -- & -- \\
\hline Brushy Creek - Bowen Branch & $\operatorname{mf} 11 \mathrm{~b}$ & 373344 & 0910645 & 09/08/2004 & .4 & 0 & -- & 0 & Obs & -- & -- \\
\hline Brushy Creek & $\operatorname{mf} 11 \mathrm{c}$ & 373345 & 0910635 & 09/08/2004 & .5 & -- & 0 & 0 & Obs & 333 & 19.1 \\
\hline Brushy Creek & $\operatorname{mf} 11 \mathrm{~d}$ & 373352 & 0910555 & 09/08/2004 & 1.1 & -- & .01 & .01 & Est & 317 & 20.5 \\
\hline Brushy Creek trib & $\mathrm{mf} 11 \mathrm{e}$ & 373355 & 0910547 & 09/08/2004 & 1.2 & 0 & -- & .01 & Obs & -- & -- \\
\hline Brushy Creek trib & mf11f & 373358 & 0910537 & 09/08/2004 & 1.4 & 0 & -- & .01 & Obs & -- & -- \\
\hline Brushy Creek trib & $\mathrm{mf} 11 \mathrm{~g}$ & 373401 & 0910527 & 09/08/2004 & 1.6 & .01 & -- & .02 & Est & 255 & 17.7 \\
\hline Brushy Creek trib & $\mathrm{mf} 11 \mathrm{~h}$ & 373404 & 0910510 & 09/08/2004 & 1.9 & 0 & -- & .02 & Obs & -- & -- \\
\hline Brushy Creek trib & $\operatorname{mf11i}$ & 373406 & 0910450 & 09/08/2004 & 2.2 & 0 & -- & .02 & Obs & -- & -- \\
\hline Brushy Creek trib & $\operatorname{mf} 11 \mathrm{j}$ & 373359 & 0910419 & 09/08/2004 & 2.6 & 0 & -- & .02 & Obs & 210 & 20.7 \\
\hline Brushy Creek trib & $\mathrm{mf} 11 \mathrm{k}$ & 373348 & 0910355 & 09/08/2004 & 3.0 & 0 & -- & .02 & Obs & -- & -- \\
\hline Brushy Creek & mf111 & 373338 & 0910334 & 09/09/2004 & 3.4 & -- & 1.09 & 1.09 & Fair & 313 & 17.1 \\
\hline Brushy Creek trib & $\mathrm{mf} 11 \mathrm{~m}$ & 373339 & 0910326 & 09/09/2004 & 3.5 & 0 & -- & 1.09 & Obs & -- & -- \\
\hline Brushy Creek trib & $\operatorname{mf} 11 \mathrm{n}$ & 373340 & 0910317 & 09/09/2004 & 3.7 & 0 & -- & 1.09 & Obs & -- & -- \\
\hline Brushy Creek - Dry Fork near mouth & $\operatorname{mf} 110$ & 373307 & 0910303 & 09/09/2004 & 4.0 & .63 & -- & 1.72 & Good & 194 & 17.1 \\
\hline Brushy Creek trib & $\mathrm{mf} 11 \mathrm{p}$ & 373340 & 0910255 & 09/09/2004 & 4.0 & 0 & -- & 1.72 & Obs & -- & -- \\
\hline Brushy Creek trib & $\mathrm{mf} 11 \mathrm{q}$ & 373342 & 0910240 & 09/09/2004 & 4.3 & 0 & -- & 1.72 & Obs & -- & -- \\
\hline Brushy Creek trib & $\operatorname{mf11r}$ & 373349 & 0910144 & 09/09/2004 & 5.1 & 0 & -- & 1.72 & Obs & -- & -- \\
\hline Brushy Creek trib & $\mathrm{mf} 11 \mathrm{~s}$ & 373349 & 0910141 & 09/09/2004 & 5.2 & 0 & -- & 1.72 & Obs & -- & -- \\
\hline Brushy Creek - Henry Branch & $\operatorname{mf} 11 \mathrm{t}$ & 373345 & 0910114 & 09/09/2004 & 5.6 & 0 & -- & 1.72 & Obs & -- & -- \\
\hline Brushy Creek - Tucker Hollow & $\operatorname{mf} 11 \mathrm{u}$ & 373337 & 0910059 & 09/09/2004 & 5.9 & 0 & -- & 1.72 & Obs & -- & -- \\
\hline Brushy Creek trib & $\operatorname{mf} 11 \mathrm{v}$ & 373332 & 0910049 & 09/09/2004 & 6.0 & 0 & -- & 1.72 & Obs & -- & -- \\
\hline Brushy Creek & $\operatorname{mf} 11 w$ & 373252 & 0910033 & 09/09/2004 & 6.8 & -- & 4.15 & 4.15 & Poor & 246 & 19.7 \\
\hline Brushy Creek - Black Hollow & $\operatorname{mf} 11 x$ & 373254 & 0910025 & 09/09/2004 & 6.8 & 0 & -- & 4.15 & Obs & -- & -- \\
\hline Brushy Creek - spring & $\operatorname{mf11y}$ & 373243 & 0910021 & 09/09/2004 & 7.6 & .34 & -- & 4.49 & Poor & 224 & 13.2 \\
\hline Brushy Creek trib & $\operatorname{mf} 11 \mathrm{z}$ & 373240 & 0910020 & 09/09/2004 & 7.7 & 0 & -- & 4.49 & Obs & -- & -- \\
\hline Brushy Creek trib & mf11za & 373148 & 0910017 & 09/09/2004 & 8.3 & .05 & -- & 4.54 & Est & 290 & 22.6 \\
\hline Brushy Creek & $\mathrm{mf} 11 \mathrm{zb}$ & 373145 & 0910012 & 09/09/2004 & 8.4 & -- & 4.77 & 4.77 & Fair & 248 & 21.0 \\
\hline Brushy Creek - Gunnis Creek & $\operatorname{mf} 11 z c$ & 373143 & 0910012 & 09/09/2004 & 8.5 & .16 & -- & 4.93 & Poor & 294 & 17.9 \\
\hline Brushy Creek trib & $\operatorname{mf} 11 z d$ & 373139 & 0905902 & 09/09/2004 & 9.6 & 0 & -- & 4.93 & Obs & -- & -- \\
\hline Brushy Creek trib & mf11ze & 373152 & 0905903 & 09/09/2004 & 9.8 & 0 & -- & 4.93 & Obs & -- & -- \\
\hline Brushy Creek & $\operatorname{mf} 11 z f$ & 373146 & 0905856 & 09/09/2004 & 9.8 & -- & 5.44 & 5.44 & Fair & 255 & 22.3 \\
\hline Brushy Creek - Cash Hollow & $\mathrm{mf} 11 \mathrm{zg}$ & 373142 & 0905847 & 09/09/2004 & 10.0 & .05 & -- & 5.49 & Est & 236 & 18.7 \\
\hline
\end{tabular}


Table 1. Seepage run data for streams draining the Viburnum Trend Subdistrict.—Continued

[DDMMSS, degrees, minutes, seconds; MM/DD/YYY, month/day/year; mi, mile; ft $3 / \mathrm{s}$, cubic feet per second; $\mu \mathrm{S} / \mathrm{cm}$, microsiemens per centimeter at 25 degrees Celsius; ${ }^{\circ} \mathrm{C}$, degrees Celsius; --, no data; $\mathrm{WFHC}$, West Fork Huzzah Creek; Est, estimated; trib, tributary; Calc, calculated; Obs, observation; shaded boxes are identical sites measured on different days; EFHC, East Fork Huzzah Creek; WFBR, West Fork Black River; MFBR, Middle Fork Black River; EFBR, East Fork Black River]

\begin{tabular}{|c|c|c|c|c|c|c|c|c|c|c|c|}
\hline \multirow[b]{2}{*}{ Stream site } & \multirow[b]{2}{*}{$\begin{array}{c}\text { Site } \\
\text { identifier }\end{array}$} & \multirow[b]{2}{*}{$\begin{array}{c}\text { Latitude } \\
\text { (DDMMSS) }\end{array}$} & \multirow[b]{2}{*}{$\begin{array}{c}\text { Longitude } \\
\text { (DDDMMSS) }\end{array}$} & \multirow[b]{2}{*}{$\begin{array}{c}\text { Date } \\
\text { (MM/DD/YYYY) }\end{array}$} & \multirow{2}{*}{$\begin{array}{l}\text { Stream } \\
\text { length } \\
\text { (mi) }\end{array}$} & \multicolumn{2}{|c|}{ Measurement location } & \multirow{2}{*}{$\begin{array}{c}\text { Accumulated } \\
\text { discharge } \\
(\mathrm{figs.} 3,4,6,8) \\
\left(\mathrm{ft}^{3} / \mathrm{s}\right)\end{array}$} & \multirow{2}{*}{$\begin{array}{l}\text { Measure- } \\
\text { ment } \\
\text { rating }\end{array}$} & \multirow{2}{*}{$\begin{array}{c}\text { Specific } \\
\text { conductance } \\
(\mu \mathrm{S} / \mathrm{cm} \text { at } \\
\left.25^{\circ} \mathrm{C}\right)\end{array}$} & \multirow[b]{2}{*}{$\begin{array}{c}\text { Temperature } \\
\left({ }^{\circ} \mathrm{C}\right)\end{array}$} \\
\hline & & & & & & $\begin{array}{c}\text { Tributary } \\
\left(\mathbf{f t}^{3} / \mathbf{s}\right)\end{array}$ & $\begin{array}{l}\text { Mainstem } \\
\quad\left(\mathrm{ft}^{3} / \mathrm{s}\right)\end{array}$ & & & & \\
\hline \multicolumn{12}{|c|}{ Middle Fork Black River - Brushy Creek (figs. 5, 6F)—Continued } \\
\hline Brushy Creek trib & mf11zh & 373156 & 0905847 & 09/09/2004 & 10.0 & 0 & -- & 5.49 & Obs & -- & -- \\
\hline Brushy Creek trib & mf11zi & 373155 & 0905838 & $09 / 09 / 2004$ & 10.1 & 1.00 & -- & 6.49 & Est & 268 & 17.0 \\
\hline Brushy Creek - spring & $\operatorname{mf11zj}$ & 373152 & 0905814 & $09 / 09 / 2004$ & 10.5 & .25 & -- & 6.74 & Est & 412 & 14.4 \\
\hline Brushy Creek trib & $\operatorname{mf} 11 \mathrm{zk}$ & 373152 & 0905805 & 09/09/2004 & 10.7 & 0 & -- & 6.74 & Obs & -- & -- \\
\hline Brushy Creek trib & $\operatorname{mf11zl}$ & 373151 & 0905736 & $09 / 09 / 2004$ & 11.6 & 0 & -- & 6.74 & Obs & -- & -- \\
\hline Brushy Creek trib & $\mathrm{mf} 11 \mathrm{zm}$ & 373152 & 0905717 & $09 / 09 / 2004$ & 11.6 & 0 & -- & 6.74 & Obs & -- & -- \\
\hline Brushy Creek trib & mf11zn & 373143 & 0905653 & 09/09/2004 & 12.1 & .12 & -- & 6.86 & Est & 348 & 22.6 \\
\hline Brushy Creek mouth & $\operatorname{mf} 11$ & 373129 & 0905618 & $09 / 09 / 2004$ & 12.7 & -- & 0.69 & .69 & Fair & 259 & 22.3 \\
\hline \multicolumn{12}{|c|}{ Middle Fork Black River - Brushy Creek - Dry Fork (not shown on fig. 6F) } \\
\hline Brushy Creek - Dry Fork trib & df01 & 373205 & 0910355 & 09/09/2004 & 1.2 & 0 & -- & 0 & Obs & -- & -- \\
\hline Brushy Creek - Dry Fork trib & df02 & 373207 & 0910345 & $09 / 09 / 2004$ & 1.3 & 0 & -- & 0 & Obs & -- & -- \\
\hline Brushy Creek - Dry Fork trib & df03 & 373209 & 0910338 & $09 / 09 / 2004$ & 1.4 & 0 & -- & 0 & Obs & -- & -- \\
\hline Brushy Creek - Dry Fork & df04 & 373220 & 0910328 & $09 / 09 / 2004$ & 1.7 & -- & 0.05 & .05 & Est & 214 & 14.2 \\
\hline Brushy Creek - Dry Fork trib & df05 & 373229 & 0910328 & $09 / 09 / 2004$ & 1.9 & 0 & -- & .05 & Obs & -- & -- \\
\hline Brushy Creek - Dry Fork trib & df06 & 373257 & 0910310 & $09 / 09 / 2004$ & 2.5 & 0 & -- & .05 & Obs & -- & -- \\
\hline Brushy Creek - Dry Fork near mouth & $\operatorname{mf110}$ & 373307 & 0910303 & $09 / 09 / 2004$ & 2.7 & -- & .63 & .63 & Good & 194 & 17.1 \\
\hline \multicolumn{12}{|c|}{ East Fork Black River (figs. 5, 6G) } \\
\hline EFBR & ef03 & 373656 & 0904511 & $06 / 22 / 2005$ & 2.7 & -- & 0.20 & 0.20 & Fair & 349 & 20.4 \\
\hline EFBR - Womble Hollow & ef05 & 373636 & 0904625 & $06 / 22 / 2005$ & 4.0 & 0.32 & -- & .52 & Fair & 330 & 22.7 \\
\hline EFBR & ef08 & 373607 & 0904719 & $06 / 22 / 2005$ & 5.1 & -- & 3.82 & 3.82 & Fair & 161 & 23.1 \\
\hline EFBR & ef11 & 373518 & 0904818 & $06 / 22 / 2005$ & 6.5 & -- & 3.86 & 3.86 & Fair & 170 & 22.4 \\
\hline EFBR & ef16 & 373319 & 0905033 & $06 / 22 / 2005$ & 9.8 & -- & 3.31 & 3.31 & Fair & 181 & 27.0 \\
\hline EFBR & ef01 & 373759 & 0904254 & $08 / 28 / 2003$ & 0 & -- & 0 & 0 & Obs & -- & -- \\
\hline EFBR trib & ef02 & 373742 & 0904404 & $08 / 28 / 2003$ & 1.2 & 0 & -- & 0 & Obs & -- & -- \\
\hline EFBR trib & ef04 & 373653 & 0904530 & $08 / 28 / 2003$ & 3.0 & 0 & -- & .20 & Obs & -- & -- \\
\hline EFBR trib & ef06 & 373619 & 0904647 & $08 / 28 / 2003$ & 4.5 & 0 & -- & .52 & Obs & -- & -- \\
\hline EFBR trib & ef07 & 373612 & 0904707 & $08 / 28 / 2003$ & 4.9 & 0 & -- & .52 & Obs & -- & -- \\
\hline EFBR trib & ef09 & 373543 & 0904748 & $08 / 28 / 2003$ & 5.8 & 0 & -- & 3.82 & Obs & -- & -- \\
\hline EFBR trib & ef10 & 373521 & 0904804 & 08/28/2003 & 6.4 & 0 & -- & 3.82 & Obs & -- & -- \\
\hline EFBR trib & ef12 & 373445 & 0904830 & $08 / 28 / 2003$ & 7.5 & 0 & -- & 3.86 & Obs & -- & -- \\
\hline EFBR trib & ef13 & 373443 & 0904837 & $08 / 28 / 2003$ & 7.5 & 0 & -- & 3.86 & Obs & -- & -- \\
\hline EFBR trib & ef14 & 373424 & 0904911 & $08 / 28 / 2003$ & 7.9 & 0 & -- & 3.86 & Obs & -- & -- \\
\hline EFBR trib & ef15 & 373345 & 0904954 & $08 / 28 / 2003$ & 9.0 & 0 & -- & 3.86 & Obs & -- & -- \\
\hline
\end{tabular}


Table 1. Seepage run data for streams draining the Viburnum Trend Subdistrict.-Continued

[DDMMSS, degrees, minutes, seconds; MM/DD/YYY, month/day/year; mi, mile; $\mathrm{ft}^{3} / \mathrm{s}$, cubic feet per second; $\mathrm{uS} / \mathrm{cm}$, microsiemens per centimeter at 25 degrees Celsius; ${ }^{\circ} \mathrm{C}$, degrees Celsius; --, no data; $\mathrm{WFHC}$, West Fork Huzzah Creek; Est, estimated; trib, tributary; Calc, calculated; Obs, observation; shaded boxes are identical sites measured on different days; EFHC, East Fork Huzzah Creek; WFBR, West Fork Black River; MFBR, Middle Fork Black River; EFBR, East Fork Black River]

\begin{tabular}{|c|c|c|c|c|c|c|c|c|c|c|c|}
\hline \multirow[b]{2}{*}{ Stream site } & \multirow[b]{2}{*}{$\begin{array}{c}\text { Site } \\
\text { identifier }\end{array}$} & \multirow[b]{2}{*}{$\begin{array}{c}\text { Latitude } \\
\text { (DDMMSS) }\end{array}$} & \multirow[b]{2}{*}{$\begin{array}{c}\text { Longitude } \\
\text { (DDDMMSS) }\end{array}$} & \multirow[b]{2}{*}{$\begin{array}{c}\text { Date } \\
\text { (MM/DD/YYYY) }\end{array}$} & \multirow{2}{*}{$\begin{array}{c}\text { Stream } \\
\text { length } \\
\text { (mi) }\end{array}$} & \multicolumn{2}{|c|}{ Measurement location } & \multirow{2}{*}{$\begin{array}{c}\text { Accumulated } \\
\text { discharge } \\
\text { (figs. } 3,4,6,8) \\
\left(\mathrm{ft}^{3} / \mathrm{s}\right)\end{array}$} & \multirow{2}{*}{$\begin{array}{c}\text { Measure- } \\
\text { ment } \\
\text { rating }\end{array}$} & \multirow{2}{*}{$\begin{array}{c}\text { Specific } \\
\text { conductance } \\
(\mu \mathrm{S} / \mathrm{cm} \text { at } \\
\left.25^{\circ} \mathrm{C}\right)\end{array}$} & \multirow[b]{2}{*}{$\begin{array}{c}\text { Temperature } \\
\left({ }^{\circ} \mathrm{C}\right)\end{array}$} \\
\hline & & & & & & $\begin{array}{c}\text { Tributary } \\
\left(\mathrm{ft}^{3} / \mathrm{s}\right)\end{array}$ & $\begin{array}{c}\text { Mainstem } \\
\left(\mathrm{ft}^{3} / \mathbf{s}\right)\end{array}$ & & & & \\
\hline \multicolumn{12}{|c|}{ East Fork Black River - Little Taum Sauk (not shown on fig. 6G) } \\
\hline Little Taum Sauk & ef $17 \mathrm{a}$ & 373039 & 0904548 & $8 / 23 / 2004$ & 1.8 & -- & 0 & 0 & Obs & -- & -- \\
\hline Little Taum Sauk trib & ef17b & 373053 & 0904644 & $8 / 23 / 2004$ & 2.7 & 0 & -- & 0 & Obs & -- & -- \\
\hline Little Taum Sauk trib & ef $17 \mathrm{c}$ & 373036 & 0904754 & $8 / 23 / 2004$ & 4.0 & 0 & -- & 0 & Obs & -- & -- \\
\hline Little Taum Sauk trib & ef $17 d$ & 373019 & 0904830 & $8 / 23 / 2004$ & 4.6 & 0 & -- & 0 & Obs & -- & -- \\
\hline Little Taum Sauk & ef $17 \mathrm{e}$ & 373021 & 0904834 & $8 / 23 / 2004$ & 15.7 & -- & 0 & 0 & Obs & -- & -- \\
\hline \multicolumn{12}{|c|}{ Logan Creek (figs. 7, 8A) } \\
\hline Logan Creek trib & lc01 & 372422 & 0910635 & $09 / 07 / 2004$ & 1.1 & 0 & -- & 0 & Obs & -- & -- \\
\hline Logan Creek trib & $1 \mathrm{c} 02$ & 372416 & 0910635 & $09 / 07 / 2004$ & 1.3 & 0 & -- & 0 & Obs & -- & -- \\
\hline Logan Creek & $\mathrm{lc} 03$ & 372405 & 0910639 & $09 / 07 / 2004$ & 1.5 & -- & 0 & 0 & Obs & -- & -- \\
\hline Logan Creek trib & lc04 & 372354 & 0910642 & 09/07/2004 & 1.7 & 0 & -- & 0 & Obs & -- & -- \\
\hline Logan Creek & lc05 & 372341 & 0910638 & $09 / 07 / 2004$ & 1.9 & -- & .05 & .05 & Est & 342 & 11.8 \\
\hline Logan Creek well & lc06 & 372331 & 0910640 & $09 / 07 / 2004$ & 2.1 & .09 & -- & .14 & Poor & 325 & 14.9 \\
\hline Logan Creek & lc07 & 372316 & 0910642 & 09/07/2004 & 2.5 & -- & .65 & .65 & Poor & 67 & 20.1 \\
\hline Logan Creek trib & lc08 & 372314 & 0910640 & 09/07/2004 & 2.5 & 0 & -- & .65 & Obs & -- & -- \\
\hline Logan Creek trib & lc09 & 372305 & 0910637 & $09 / 07 / 2004$ & 2.7 & 0 & -- & .65 & Obs & -- & -- \\
\hline Logan Creek - Willis Branch & $\operatorname{lc} 10$ & 372255 & 0910629 & 09/07/2004 & 2.9 & 0 & -- & .65 & Obs & -- & -- \\
\hline Logan Creek - Corridon Hollow & $\operatorname{lc} 11$ & 372242 & 0910625 & 09/07/2004 & 3.1 & 0 & -- & .65 & Obs & -- & -- \\
\hline Logan Creek trib & lc12 & 372229 & 0910629 & $09 / 07 / 2004$ & 3.6 & 0 & -- & .65 & Obs & -- & -- \\
\hline Logan Creek trib & $\operatorname{lc} 13$ & 372215 & 0910636 & 09/07/2004 & 3.7 & 0 & -- & .65 & Obs & -- & -- \\
\hline Logan Creek & $\operatorname{lc} 14$ & 372215 & 0910648 & 09/07/2004 & 3.8 & -- & .75 & .75 & Poor & 97 & 20.3 \\
\hline Logan Creek trib & lc15 & 372215 & 0910647 & $09 / 07 / 2004$ & 3.8 & .64 & -- & 1.39 & Poor & 62 & 21.8 \\
\hline Logan Creek spring & lc16 & 372210 & 0910649 & 09/07/2004 & 3.9 & .09 & -- & 1.48 & Poor & 334 & 13.9 \\
\hline Logan Creek & lc17 & 372208 & 0910650 & $09 / 07 / 2004$ & 3.9 & -- & 1.60 & 1.60 & Fair & 117 & 21.5 \\
\hline Logan Creek trib & $\operatorname{lc} 18$ & 372152 & 0910723 & $09 / 07 / 2004$ & 4.6 & 0 & -- & 1.60 & Obs & -- & - \\
\hline Logan Creek - Adair Creek & lc19 & 372124 & 0910723 & $09 / 07 / 2004$ & 5.0 & 2.39 & -- & 3.99 & Poor & 492 & 21.2 \\
\hline Logan Creek trib & $\operatorname{lc} 20$ & 372053 & 0910731 & 09/07/2004 & 5.7 & 0 & -- & 0 & Obs & -- & -- \\
\hline Logan Creek trib & $\operatorname{lc} 21$ & 372003 & 0910715 & 09/07/2004 & 6.7 & 0 & -- & 0 & Obs & -- & -- \\
\hline Logan Creek - Dixon Hollow & $\operatorname{lc} 22$ & 371954 & 0910654 & $09 / 08 / 2004$ & 7.0 & 0 & -- & 0 & Obs & -- & -- \\
\hline Logan Creek & $\operatorname{lc} 23$ & 371913 & 0910715 & 09/07/2004 & 7.7 & -- & 8.58 & 8.58 & Fair & 344 & 21.0 \\
\hline Logan Creek & lc23 & 371913 & 0910715 & 09/08/2004 & 7.7 & -- & 8.68 & 8.68 & Fair & 344 & 18.4 \\
\hline Logan Creek - Sweetwater Creek & lc24 & 371903 & 0910736 & 09/08/2004 & 8.1 & .95 & -- & 9.63 & Fair & 146 & 20.8 \\
\hline Logan Creek - Suses Branch & lc25 & 371857 & 0910814 & 09/08/2004 & 9.0 & .08 & -- & 9.71 & Poor & 156 & 19.2 \\
\hline Logan Creek & $\operatorname{lc} 26$ & 371801 & 0910738 & $09 / 08 / 2004$ & 9.5 & -- & 9.22 & 9.22 & Good & 322 & 22.6 \\
\hline Logan Creek - Crabtree Hollow & $\operatorname{lc} 27$ & 371756 & 0910732 & 09/08/2004 & 9.8 & 0 & -- & 9.22 & Obs & -- & -- \\
\hline
\end{tabular}


Table 1. Seepage run data for streams draining the Viburnum Trend Subdistrict.—Continued

[DDMMSS, degrees, minutes, seconds; MM/DD/YYY, month/day/year; mi, mile; ft $3 / \mathrm{s}$, cubic feet per second; $\mu \mathrm{S} / \mathrm{cm}$, microsiemens per centimeter at 25 degrees Celsius; ${ }^{\circ} \mathrm{C}$, degrees Celsius; --, no data; $\mathrm{WFHC}$, West Fork Huzzah Creek; Est, estimated; trib, tributary; Calc, calculated; Obs, observation; shaded boxes are identical sites measured on different days; EFHC, East Fork Huzzah Creek; WFBR, West Fork Black River; MFBR, Middle Fork Black River; EFBR, East Fork Black River]

\begin{tabular}{|c|c|c|c|c|c|c|c|c|c|c|c|}
\hline \multirow[b]{2}{*}{ Stream site } & \multirow[b]{2}{*}{$\begin{array}{c}\text { Site } \\
\text { identifier }\end{array}$} & \multirow[b]{2}{*}{$\begin{array}{c}\text { Latitude } \\
\text { (DDMMSS) }\end{array}$} & \multirow[b]{2}{*}{$\begin{array}{c}\text { Longitude } \\
\text { (DDDMMSS) }\end{array}$} & \multirow[b]{2}{*}{$\begin{array}{c}\text { Date } \\
\text { (MM/DD/YYYY) }\end{array}$} & \multirow{2}{*}{$\begin{array}{l}\text { Stream } \\
\text { length } \\
\text { (mi) }\end{array}$} & \multicolumn{2}{|c|}{ Measurement location } & \multirow{2}{*}{$\begin{array}{c}\text { Accumulated } \\
\text { discharge } \\
\text { (figs. } 3,4,6,8) \\
\left(\mathrm{ft}^{3} / \mathrm{s}\right)\end{array}$} & \multirow[b]{2}{*}{$\begin{array}{c}\text { Measure- } \\
\text { ment } \\
\text { rating }\end{array}$} & \multirow{2}{*}{$\begin{array}{c}\text { Specific } \\
\text { conductance } \\
(\mu \mathrm{S} / \mathrm{cm} \text { at } \\
\left.25^{\circ} \mathrm{C}\right)\end{array}$} & \multirow[b]{2}{*}{$\begin{array}{c}\text { Temperature } \\
\left({ }^{\circ} \mathrm{C}\right)\end{array}$} \\
\hline & & & & & & $\begin{array}{c}\text { Tributary } \\
\left(\mathrm{ft}^{3} / \mathrm{s}\right)\end{array}$ & $\begin{array}{l}\text { Mainstem } \\
\left(\mathrm{ft}^{3} / \mathrm{s}\right)\end{array}$ & & & & \\
\hline \multicolumn{12}{|c|}{ Logan Creek (figs. 7, 8A)-Continued } \\
\hline Logan Creek & lc28 & 371708 & 0910754 & $09 / 08 / 2004$ & 10.7 & -- & 0 & 0 & Obs & -- & -- \\
\hline Logan Creek trib & $\operatorname{lc} 29$ & 371637 & 0910645 & $09 / 08 / 2004$ & 12.5 & 0 & -- & 0 & Obs & -- & -- \\
\hline Logan Creek trib & $\operatorname{lc} 30$ & 371627 & 0910639 & $09 / 08 / 2004$ & 12.8 & 0 & -- & 0 & Obs & -- & -- \\
\hline Logan Creek & $\operatorname{lc} 31$ & 371536 & 0910707 & $09 / 08 / 2004$ & 13.9 & -- & 0 & 0 & Obs & -- & -- \\
\hline Logan Creek & $\operatorname{lc} 32$ & 371349 & 0910437 & $09 / 08 / 2004$ & 18.6 & -- & 0 & 0 & Obs & -- & -- \\
\hline Logan Creek - Pumpkin Hollow & $1 \mathrm{c} 33$ & 371330 & 0910331 & $09 / 08 / 2004$ & 20.0 & 0 & -- & 0 & Obs & -- & -- \\
\hline Logan Creek & $\operatorname{lc} 34$ & 371334 & 0910315 & $09 / 08 / 2004$ & 20.0 & -- & 0 & 0 & Obs & -- & -- \\
\hline Logan Creek - Brawley Hollow & $\operatorname{lc} 35$ & 371340 & 0910232 & $09 / 08 / 2004$ & 21.4 & 0 & -- & 0 & Obs & -- & -- \\
\hline Logan Creek & $1 \mathrm{c} 36$ & 371420 & 0905948 & $09 / 08 / 2004$ & 23.8 & -- & 0 & 0 & Obs & 249 & 22.9 \\
\hline Logan Creek trib & $\operatorname{lc} 37$ & 371423 & 0910003 & $09 / 08 / 2004$ & 23.9 & 0 & -- & 0 & Obs & -- & -- \\
\hline Logan Creek - Dry Valley & $\operatorname{lc} 38$ & 371443 & 0905801 & $09 / 08 / 2004$ & 25.6 & .49 & -- & .49 & Poor & 257 & 20.7 \\
\hline Logan Creek - Ellington & lc39 & 371442 & 0905757 & $09 / 08 / 2004$ & 25.7 & -- & 3.62 & 3.62 & Fair & 272 & 23.1 \\
\hline Logan Creek - Ellington & lc39 & 371442 & 0905757 & $08 / 23 / 2004$ & 25.7 & -- & 4.03 & 4.03 & Poor & 300 & 22.0 \\
\hline Logan Creek - Dickson Valley mouth & lc 40 & 371426 & 0905808 & $08 / 23 / 2004$ & 26.0 & .03 & -- & 4.06 & Poor & 269 & 23.5 \\
\hline Logan Creek - Spring Valley & lc41 & 371507 & 0905537 & $08 / 23 / 2004$ & 28.2 & .01 & -- & 4.07 & Est & -- & -- \\
\hline Logan Creek & $\operatorname{lc} 42$ & 371502 & 0905536 & $08 / 23 / 2004$ & 28.3 & -- & 6.13 & 6.13 & Good & 276 & 23.1 \\
\hline Logan Creek spring & lc43 & 371357 & 0905343 & $08 / 23 / 2004$ & 31.0 & 6.59 & -- & 12.7 & Poor & 247 & 15.5 \\
\hline Logan Creek & lc44 & 371352 & 0905326 & $08 / 23 / 2004$ & 31.3 & -- & 17.0 & 17.0 & Fair & 261 & 20.6 \\
\hline \multicolumn{12}{|c|}{ Miscellaneous tributary and headwater measurement or observation sites (not shown on fig. 8A) } \\
\hline Logan Creek - Adair Creek trib & lc19a & 372151 & 0910749 & $09 / 07 / 2004$ & & 0 & -- & -- & Obs & -- & -- \\
\hline \multicolumn{12}{|c|}{ Logan Creek - Dickson Valley (not shown on fig. 8A) } \\
\hline Dickson Valley & lc40a & 371153 & 0905815 & $08 / 23 / 2004$ & 0.2 & -- & 0 & 0 & Obs & -- & -- \\
\hline Dickson Valley - Darr Valley & lc $40 \mathrm{~b}$ & 371202 & 0905818 & $08 / 23 / 2004$ & .4 & 0 & -- & 0 & Obs & -- & -- \\
\hline Dickson Valley & lc $40 \mathrm{c}$ & 371214 & 0905807 & $08 / 23 / 2004$ & .7 & -- & 0 & 0 & Obs & -- & -- \\
\hline Dickson Valley & lc $40 \mathrm{~d}$ & 371323 & 0905811 & $08 / 23 / 2004$ & 2.0 & -- & 0 & 0 & Obs & -- & -- \\
\hline Dickson Valley trib & lc $40 \mathrm{e}$ & 371325 & 0905812 & $08 / 23 / 2004$ & 2.1 & 0 & -- & 0 & Obs & -- & -- \\
\hline Dickson Valley mouth & lc40 & 371426 & 0905808 & $08 / 23 / 2004$ & 3.5 & -- & .03 & .03 & Poor & 269 & 23.5 \\
\hline
\end{tabular}


Table 1. Seepage run data for streams draining the Viburnum Trend Subdistrict.-Continued

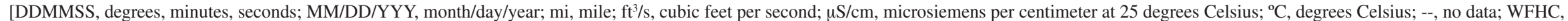

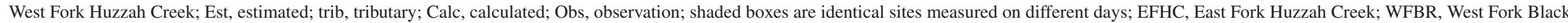
River; MFBR, Middle Fork Black River; EFBR, East Fork Black River]

\begin{tabular}{|c|c|c|c|c|c|c|c|c|c|c|c|}
\hline \multirow[b]{2}{*}{ Stream site } & \multirow[b]{2}{*}{$\begin{array}{c}\text { Site } \\
\text { identifier }\end{array}$} & \multirow[b]{2}{*}{$\begin{array}{c}\text { Latitude } \\
\text { (DDMMSS) }\end{array}$} & \multirow[b]{2}{*}{$\begin{array}{c}\text { Longitude } \\
\text { (DDDMMSS) }\end{array}$} & \multirow[b]{2}{*}{$\begin{array}{c}\text { Date } \\
\text { (MM/DD/YYYY) }\end{array}$} & \multirow[b]{2}{*}{$\begin{array}{l}\text { Stream } \\
\text { length } \\
\text { (mi) }\end{array}$} & \multicolumn{2}{|c|}{ Measurement location } & \multirow{2}{*}{$\begin{array}{c}\text { Accumulated } \\
\text { discharge } \\
\text { (figs. } 3,4,6,8) \\
\left(\mathrm{ft}^{3} / \mathrm{s}\right)\end{array}$} & \multirow[b]{2}{*}{$\begin{array}{l}\text { Measure- } \\
\text { ment } \\
\text { rating }\end{array}$} & \multirow{2}{*}{$\begin{array}{c}\text { Specific } \\
\text { conductance } \\
\text { ( } \mu \mathrm{S} / \mathrm{cm} \text { at } \\
\left.25^{\circ} \mathrm{C}\right)\end{array}$} & \multirow[b]{2}{*}{$\begin{array}{c}\text { Temperature } \\
\left({ }^{\circ} \mathrm{C}\right)\end{array}$} \\
\hline & & & & & & $\begin{array}{c}\text { Tributary } \\
\left(\mathbf{f t}^{3} / \mathbf{s}\right)\end{array}$ & $\begin{array}{l}\text { Mainstem } \\
\quad\left(\mathrm{ft}^{3} / \mathbf{s}\right)\end{array}$ & & & & \\
\hline \multicolumn{12}{|c|}{ Logan Creek - Sweetwater Creek (figs. 7, 8B) } \\
\hline Sweetwater Creek trib & lc24a & 372127 & 0910951 & $09 / 08 / 2004$ & 0.3 & 0 & -- & 0 & Obs & -- & -- \\
\hline Sweetwater Creek & $\operatorname{lc} 24 b$ & 372121 & 0910951 & 09/08/2004 & .4 & -- & 0 & 0 & Obs & -- & -- \\
\hline Sweetwater Creek trib & $\operatorname{lc} 24 \mathrm{c}$ & 372043 & 0910932 & 09/08/2004 & 1.3 & 0 & -- & 0 & Obs & -- & -- \\
\hline Sweetwater Creek - Hominy Creek & $\operatorname{lc} 24 d$ & 372032 & 0910940 & 09/08/2004 & 1.5 & .12 & -- & .12 & Poor & 86 & 18.6 \\
\hline Sweetwater Creek & $1 \mathrm{c} 24 \mathrm{e}$ & 372031 & 0910931 & 09/08/2004 & 1.5 & -- & .42 & .42 & Poor & 95 & 17.2 \\
\hline Sweetwater Creek trib & lc24f & 372025 & 0910925 & 09/08/2004 & 1.6 & 0 & -- & .42 & Obs & -- & -- \\
\hline Sweetwater Creek tailings trib & lc24g & 372020 & 0910902 & 09/08/2004 & 2.1 & .45 & -- & .87 & Poor & 374 & 19.0 \\
\hline Sweetwater Creek & lc24h & 371954 & 0910825 & 09/08/2004 & 2.8 & -- & .55 & .55 & Poor & 136 & 21.4 \\
\hline Sweetwater Creek trib & $1 \mathrm{c} 24 \mathrm{i}$ & 371955 & 0910824 & 09/08/2004 & 2.8 & 0 & -- & .55 & Obs & -- & -- \\
\hline Sweetwater Creek & lc24 & 371903 & 0910736 & 09/08/2004 & 4.3 & -- & .95 & .95 & Fair & 146 & 20.8 \\
\hline \multicolumn{12}{|c|}{ Miscellaneous tributary and headwater measurement or observation sites (not shown on fig. 8B) } \\
\hline Hominy Creek spring & $\mathrm{hm} 01$ & 372031 & 0910940 & 09/08/2004 & -- & 0.02 & -- & -- & Est & 136 & 17.7 \\
\hline \multicolumn{12}{|c|}{ Sinking Creek (figs. 7, 8C) } \\
\hline Sinking Creek & sk01 & 372259 & 0910311 & $09 / 13 / 2004$ & 1.1 & - & 0 & 0 & Obs & -- & -- \\
\hline Sinking Creek trib & sk02 & 372304 & 0910304 & 09/13/2004 & 1.2 & 0 & -- & 0 & Obs & -- & -- \\
\hline Sinking Creek trib & sk03 & 372310 & 0910246 & 09/13/2004 & 1.6 & 0 & -- & 0 & Obs & -- & -- \\
\hline Sinking Creek trib & sk04 & 372315 & 0910230 & 09/13/2004 & 1.8 & 0 & -- & 0 & Obs & -- & -- \\
\hline Sinking Creek trib & sk05 & 372319 & 0910157 & 09/13/2004 & 2.3 & 0 & -- & 0 & Obs & -- & -- \\
\hline Sinking Creek trib & sk06 & 372359 & 0910128 & 09/13/2004 & 3.6 & 0 & -- & 0 & Obs & -- & -- \\
\hline Sinking Creek trib & sk07 & 372409 & 0910122 & 09/13/2004 & 3.8 & 0 & -- & 0 & Obs & -- & -- \\
\hline Sinking Creek trib & sk08 & 372417 & 0910102 & 09/13/2004 & 4.0 & 0 & -- & 0 & Obs & -- & -- \\
\hline Sinking Creek trib & sk09 & 372418 & 0910043 & 09/13/2004 & 4.3 & 0 & -- & 0 & Obs & -- & -- \\
\hline Sinking Creek trib & sk10 & 372418 & 0910027 & 09/13/2004 & 4.5 & 0 & -- & 0 & Obs & -- & -- \\
\hline Sinking Creek & sk11 & 372334 & 0905948 & 09/13/2004 & 5.4 & -- & 0 & 0 & Obs & -- & -- \\
\hline Sinking Creek trib & sk12 & 372336 & 0905941 & 09/13/2004 & 5.5 & 0 & -- & 0 & Obs & -- & -- \\
\hline Sinking Creek trib & sk13 & 372323 & 0905918 & 09/13/2004 & 5.9 & 0 & -- & 0 & Obs & -- & -- \\
\hline Sinking Creek trib & sk14 & 372322 & 0905901 & 09/13/2004 & 6.2 & 0 & -- & 0 & Obs & -- & -- \\
\hline Sinking Creek trib & sk15 & 372314 & 0905841 & 09/13/2004 & 6.6 & 0 & -- & 0 & Obs & -- & -- \\
\hline Sinking Creek trib & sk16 & 372308 & 0905826 & 09/13/2004 & 7.0 & 0 & -- & 0 & Obs & -- & -- \\
\hline Sinking Creek trib & sk17 & 372305 & 0905821 & 09/13/2004 & 7.0 & 0 & -- & 0 & Obs & -- & -- \\
\hline Sinking Creek & sk18 & 372155 & 0905836 & 09/13/2004 & 8.0 & -- & 0 & 0 & Obs & -- & -- \\
\hline Sinking Creek - Amsden Hollow & sk19 & 372201 & 0905857 & 09/13/2004 & 8.1 & 0 & -- & 0 & Obs & -- & -- \\
\hline Sinking Creek trib & sk20 & 372141 & 0905832 & 09/13/2004 & 8.4 & 0 & -- & 0 & Obs & -- & -- \\
\hline Sinking Creek - Pyrtle Spring & sk21 & 372135 & 0905829 & 09/13/2004 & 8.5 & .34 & -- & .34 & Good & 169 & 19.2 \\
\hline
\end{tabular}


Table 1. Seepage run data for streams draining the Viburnum Trend Subdistrict.-Continued

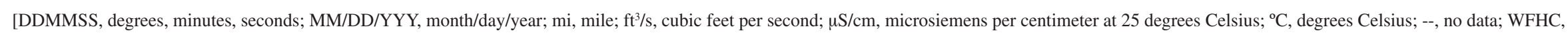

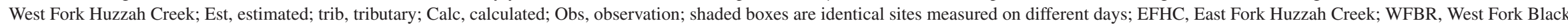
River; MFBR, Middle Fork Black River; EFBR, East Fork Black River]

\begin{tabular}{|c|c|c|c|c|c|c|c|c|c|c|c|}
\hline \multirow[b]{2}{*}{ Stream site } & \multirow[b]{2}{*}{$\begin{array}{c}\text { Site } \\
\text { identifier }\end{array}$} & \multirow[b]{2}{*}{$\begin{array}{c}\text { Latitude } \\
\text { (DDMMSS) }\end{array}$} & \multirow[b]{2}{*}{$\begin{array}{c}\text { Longitude } \\
\text { (DDDMMSS) }\end{array}$} & \multirow[b]{2}{*}{$\begin{array}{c}\text { Date } \\
\text { (MM/DD/YYYY) }\end{array}$} & \multirow{2}{*}{$\begin{array}{l}\text { Stream } \\
\text { length } \\
\text { (mi) }\end{array}$} & \multicolumn{2}{|c|}{ Measurement location } & \multirow{2}{*}{$\begin{array}{c}\text { Accumulated } \\
\text { discharge } \\
\text { (figs. } 3,4,6,8) \\
\left(\mathrm{ft}^{3} / \mathrm{s}\right) \\
\end{array}$} & \multirow{2}{*}{$\begin{array}{l}\text { Measure- } \\
\text { ment } \\
\text { rating }\end{array}$} & \multirow{2}{*}{$\begin{array}{c}\text { Specific } \\
\text { conductance } \\
\text { ( } \mu \mathrm{S} / \mathrm{cm} \text { at } \\
\left.25^{\circ} \mathrm{C}\right)\end{array}$} & \multirow[b]{2}{*}{$\begin{array}{c}\text { Temperature } \\
\left({ }^{\circ} \mathrm{C}\right)\end{array}$} \\
\hline & & & & & & $\begin{array}{c}\text { Tributary } \\
\left(\mathrm{ft}^{3} / \mathrm{s}\right)\end{array}$ & $\begin{array}{l}\text { Mainstem } \\
\quad\left(\mathrm{ft}^{3} / \mathbf{s}\right)\end{array}$ & & & & \\
\hline \multicolumn{12}{|c|}{ Sinking Creek (figs. 7, 8C)—Continued } \\
\hline Sinking Creek trib & sk22 & 372120 & 0905806 & $09 / 14 / 2004$ & 9.1 & 0 & -- & 0.34 & Obs & -- & -- \\
\hline Sinking Creek - Brawley Hollow & sk23 & 372052 & 0905814 & $09 / 13 / 2004$ & 9.4 & 0 & -- & .34 & Obs & -- & -- \\
\hline Sinking Creek trib & sk24 & 372113 & 0905746 & 09/14/2004 & 9.5 & 0 & -- & .34 & Obs & -- & -- \\
\hline Sinking Creek trib & sk25 & 372048 & 0905706 & 09/14/2004 & 10.3 & 0 & -- & .34 & Obs & -- & -- \\
\hline Sinking Creek & sk26 & 372020 & 0905654 & $09 / 14 / 2004$ & 10.8 & -- & 5.76 & 5.76 & Good & 246 & 18.8 \\
\hline Sinking Creek - Pogue Hollow & sk27 & 372026 & 0905642 & 09/14/2004 & 10.9 & 0 & -- & 5.76 & Obs & -- & -- \\
\hline Sinking Creek trib & sk28 & 372021 & 0905627 & 09/14/2004 & 11.3 & 0 & -- & 5.76 & Obs & -- & -- \\
\hline $\begin{array}{l}\text { Sinking Creek - Vinson Branch } \\
\text { mouth }\end{array}$ & sk29 & 371934 & 0905549 & 09/13/2004 & 12.6 & .04 & -- & 5.80 & Est & 160 & 19.3 \\
\hline Sinking Creek & sk30 & 371937 & 0905521 & $09 / 13 / 2004$ & 12.6 & -- & 6.16 & 6.16 & Fair & 225 & 20.6 \\
\hline Sinking Creek & sk30 & 371937 & 0905521 & $09 / 14 / 2004$ & 12.6 & -- & 6.31 & 6.31 & Fair & 225 & 19.9 \\
\hline Sinking Creek trib & sk31 & 371938 & 0905454 & $09 / 14 / 2004$ & 13.0 & 0 & -- & 6.31 & Obs & -- & -- \\
\hline Sinking Creek - Harrison Valley & sk32 & 371920 & 0905408 & 09/14/2004 & 14.0 & 0 & -- & 6.31 & Obs & -- & -- \\
\hline Sinking Creek - Harrison Valley & sk33 & 371905 & 0905357 & $09 / 14 / 2004$ & 14.2 & 0 & -- & 6.31 & Obs & -- & -- \\
\hline Sinking Creek at Redford & sk34 & 371859 & 0905356 & $09 / 14 / 2004$ & 14.2 & -- & 7.65 & 7.65 & Good & 218 & 19.6 \\
\hline \multicolumn{12}{|c|}{ Miscellaneous tributary and headwater measurement or observation sites (not shown on fig. 8C) } \\
\hline Sinking Creek - Amsden Hollow & sk19a & 372159 & 0905847 & $09 / 13 / 2004$ & -- & 0 & -- & -- & Obs & -- & -- \\
\hline \multicolumn{12}{|c|}{ Sinking Creek - Vinson Branch (not shown on fig. 8C) } \\
\hline Vinson Branch & sk29a & 371924 & 0905753 & $09 / 13 / 2004$ & 1.2 & -- & 0 & 0.00 & Obs & -- & -- \\
\hline Vinson Branch & sk29b & 371922 & 0905752 & 09/13/2004 & 1.3 & -- & 0 & 0.00 & Obs & -- & -- \\
\hline Vinson Branch & sk29c & 371916 & 0905736 & 09/13/2004 & 1.6 & -- & .01 & 0.01 & Est & 430 & 21.2 \\
\hline Vinson Branch & sk29d & 371914 & 0905731 & 09/13/2004 & 1.7 & -- & 0 & 0.00 & Obs & -- & -- \\
\hline Vinson Branch & sk29e & 371907 & 0905653 & 09/13/2004 & 2.3 & -- & 0 & 0.00 & Obs & -- & -- \\
\hline Vinson Branch trib & sk29f & 371933 & 0905548 & $09 / 13 / 2004$ & 3.6 & 0.02 & -- & 0.02 & Est & 166 & 21.1 \\
\hline Vinson Branch mouth & sk29 & 371934 & 0905549 & 09/13/2004 & 3.9 & -- & .04 & 0.04 & Est & 160 & 19.3 \\
\hline
\end{tabular}

${ }^{\text {a }}$ Site discharge calculated by subtracting discharge measured immediately upstream from site from discharge measured immediately downstream from site. 



\section{Distribution of Mining-Related Trace Elements and Sulfide-Mineral Occurrence in Streambed Sediment of the Viburnum Trend Subdistrict and Non-Mining Areas, Southeastern Missouri, 1992-2002}

By Lopaka Lee

Chapter 3 of

Hydrologic Investigations Concerning Lead Mining Issues in Southeastern Missouri

Edited by Michael J. Kleeschulte

Scientific Investigations Report 2008-5140 


\section{Contents}

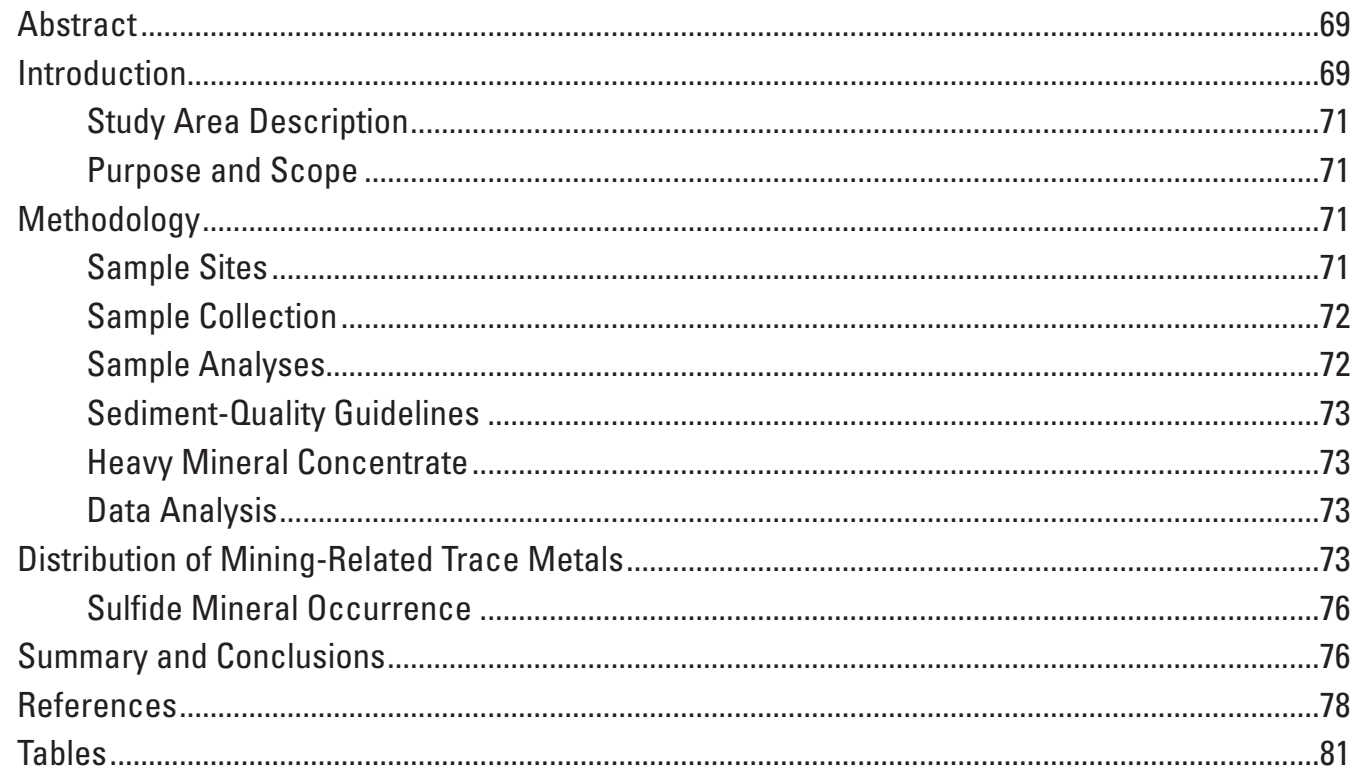

\section{Figures}

1. Map showing study area and streambed-sediment sampling sites

2-3. Box plots showing-

2. Observed abundance of Mississippi Valley Type-related metals in streambed-sediment samples, 1992-2002

3. Concentrations of Mississippi Valley Type-related metals and chromium in streambed sediment samples, threshold effects concentrations (TEC), and probable effects concentrations (PEC)

4. Graphs showing exceedance of probable effects concentrations (PEC) of copper, lead, nickel, and zinc in streambed-sediment samples

\section{Tables}

1. Quantitative elemental analysis of the less than 63-micrometer fraction and bulk sample grain size distribution of streambed-sediment samples, threshold effects concentrations (TEC), and probable effects concentrations (PEC) for selected trace elements, 1992-2002

2. Optical mineralogy of non-magnetic fraction (C3) of heavy mineral concentrate for streambed-sediment samples, 1995-2002

3. Summary statistics for Mississippi Valley Type-related metals and chromium in streambed-sediment samples, 1992-2002. 


\title{
Distribution of Mining-Related Trace Elements and Sulfide-Mineral Occurrence in Streambed Sediment of the Viburnum Trend Subdistrict and Non-Mining Areas, Southeastern Missouri, 1992-2002
}

\author{
By Lopaka Lee
}

\section{Abstract}

The Mississippi Valley Type deposits of the Viburnum Trend Subdistrict in southeastern Missouri are mined for lead and zinc sulfides containing the primary trace elements arsenic, cadmium, cobalt, copper, lead, nickel, and zinc (hereafter referred to as metals). The waste material produced during this mining is stored in headwater valleys of the area and thus can be transported downstream into larger streams and rivers. Streambed-sediment sampling at sites in non-mining basins, sites upstream, and sites at varying distances downstream from mining activities indicates streambed sediments are significantly enriched in Mississippi Valley Type-related metals. The degree of this enrichment depends on the constituent considered and the distance downstream from mining activities.

Statistical analysis indicated that the distribution of Mississippi Valley Type-related metals in the less than 63 micrometer fraction of streambed-sediment samples upstream from mining areas and in non-mining stream basins were similar. This indicated if Mississippi Valley Type-related metal enrichment was occurring in the Viburnum Trend, it was not caused by geographical location. Near-mining sites (mining activity within 7.5 miles upstream from the site) were enriched significantly in cobalt, lead, nickel, and zinc. Statistical analysis indicated the distribution of nickel and zinc in non-mining and distal-mining sites were similar, but these metals were significantly enriched in near-mining sites. This indicates concentrations of these metals in streambed sediment at sites within 7.5 miles of mining activity were elevated, but returned to baseline-like conditions at distal-mining sites (distances of 7.5 miles or more downstream from mining activity). However, cobalt and lead concentrations do not decrease to the lower non-mining concentrations at the distal-mining sites, but stay slightly elevated.

Median lead concentrations at near-mining sites were about 10 times greater than median lead concentrations observed at non-mining sites. Similarly, median zinc concentrations were elevated about seven times, cobalt about five times, and nickel about four times. Arsenic, cadmium, copper, and chromium median concentrations at near-mining sites were similar to non-mining sites.

At near-mining sites, every lead and nickel concentration, and all but one zinc concentration, exceeded their respective probable effects concentration thresholds, even though these same metals only exceeded these thresholds occasionally in the samples from non-mining sites. No distal-mining sites exceeded the probable effects concentration thresholds for copper or zinc, but three sites exceeded the lead probable effects concentration threshold, and two sites exceeded the nickel probable effects concentration threshold.

Pyrite and galena were the only sulfide minerals observed in the non-magnetic, heavy mineral fraction samples. Pyrite was the most commonly observed sulfide mineral, occurring in 55 percent (18 of 33) of all samples. Galena was observed far less frequently, occurring in 12 percent ( 4 of the 33 ) of all samples. The proportion of samples in which pyrite occurs increased from 33 percent at non-mining sites to 86 percent at near-mining sites, and then decreased to 75 percent at distalmining sites. Pyrite is common in all Paleozoic rocks in this region and occurs in trace- and ore-grade mineralized areas. This may explain the increased occurrence rate of pyrite in areas upstream from mining and overall lower occurrence of galena.

\section{Introduction}

The lead and zinc ore deposits of the Viburnum Trend Subdistrict (hereafter referred to as Viburnum Trend in this chapter) in southeastern Missouri are a part of the largest known lead reserves within the United States (U.S. Geological Survey, 2008) (fig. 1). The genesis of these deposits is associated with regional mineralization (Leach, 1994) and the deposits are a class known as Mississippi Valley Type (MVT). Although MVT deposits are named for the region in the United States where they were first described and studied, they occur on every continent of the world (Leach and Sangster, 1993). 


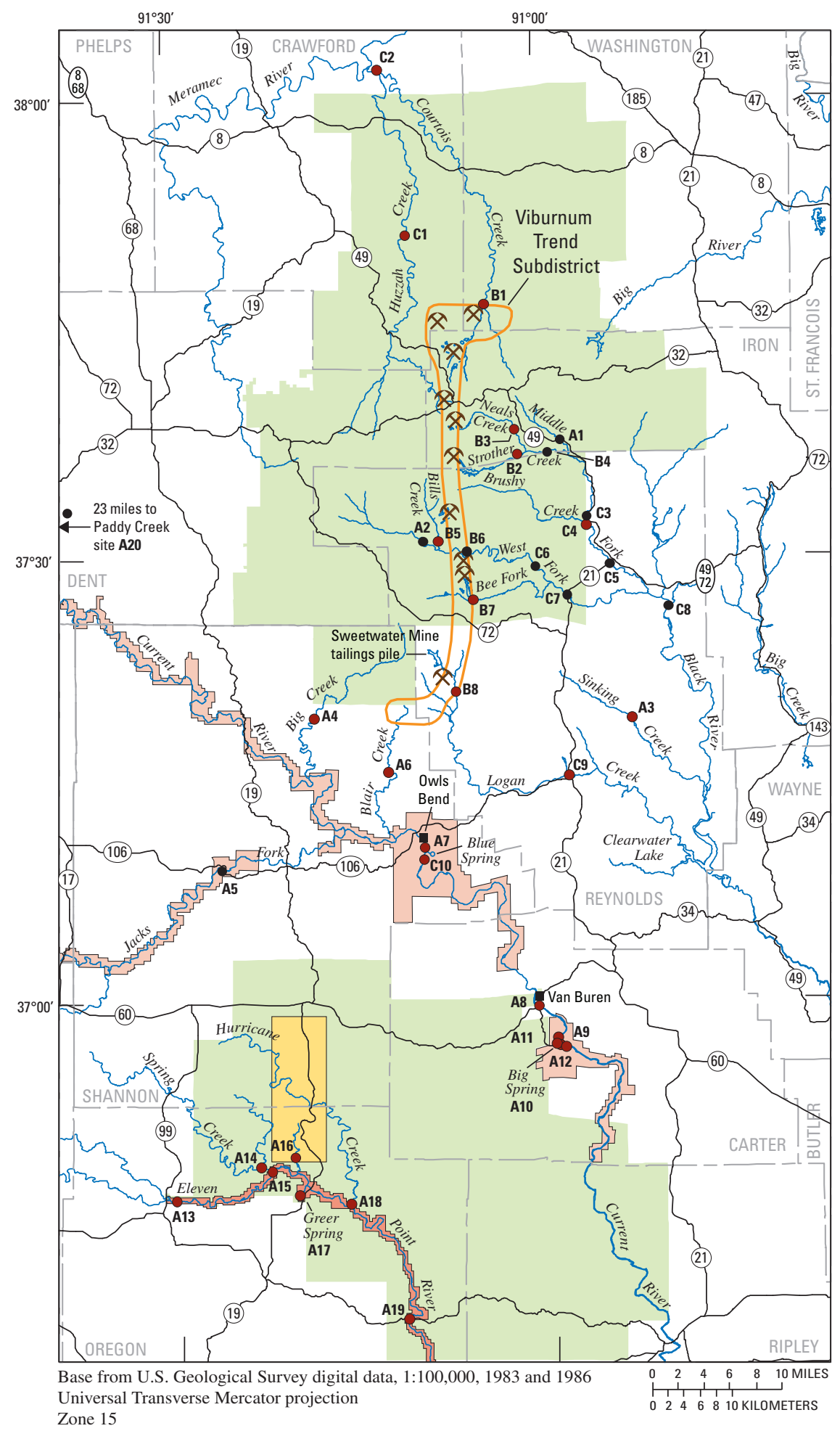

Zone 15

EXPLANATION

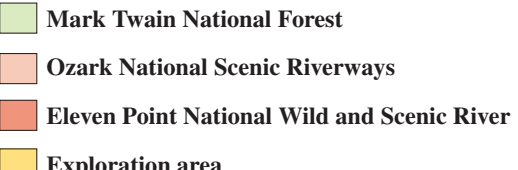

Exploration area
A9 Streambed-sediment sampling site and identifier-Black circle indicates site sampled as part of the National WaterQuality Assessment (NAWQA) Program

x Mine

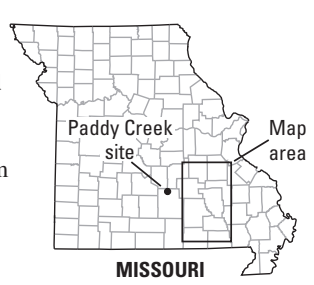

Figure 1. Study area and streambed-sediment sampling sites. 
The MVT deposits of the Viburnum Trend are metal-sulfide deposits that are hosted in Paleozoic dolostone, limestone, and to a lesser extent sandstone. The primary metal-sulfide minerals of these deposits are pyrite-marcasite $\left(\mathrm{FeS}_{2}\right)$, galena $(\mathrm{PbS})$, and sphalerite $(\mathrm{ZnS})$. In localized areas, mixed-metal sulfides also occur in minor amounts. These include copper sulfides, such as bornite $\left(\mathrm{Cu}_{5} \mathrm{FeS}_{4}\right)$, chalcopyrite $\left(\mathrm{CuFeS}_{2}\right)$, and enargite $\left(\mathrm{Cu}_{3} \mathrm{AsS}_{4}\right)$; nickel sulfides, such as millerite $(\mathrm{NiS})$ and vaesite $\left(\mathrm{NiS}_{2}\right)$; and iron sulfides, such as arsenopyrite (FeAsS) (Rakovan, 2007). Arsenic, cadmium, cobalt, copper, lead, nickel, and zinc are the primary trace elements associated with the sulfide minerals of MVT ores and, therefore, are referred to as MVT-related metals in this chapter.

Mining in the northern part of the Viburnum Trend began in 1960, and mining began in the Black River Basin in 1966 and has continued uninterrupted since. As many as 10 mines have operated along the Viburnum Trend (fig. 1); presently (2008), 6 mines are in operation. These mines are large-scale underground workings that implement a room and pillar method of mining that has resulted in an extensive, interconnected system of underground workings. Ore material mined underground is brought to the surface and milled near the mine shafts. Wastes from the milling process are pumped as a slurry to large surface impoundments that fill headwater stream valleys.

\section{Study Area Description}

The study area includes streams in the Huzzah Creek and Black River Basins that drain the Viburnum Trend and streams draining non-mining areas to the south and west in the Current River, Eleven Point, and Paddy Creek Basins (fig. 1). The area lies within a large region of well-developed karst terrain that is characterized by the presence of caves, springs, sinkholes, and gaining and losing streams. The topography is characterized by deep, narrow valleys and narrow, steep-sided ridges that resulted from deep dissection of the carbonate bedrock by surface and karst drainage. More than 300 feet (ft) of relief is common between the ridge top and the adjacent valley.

Two federally designated scenic rivers, the Eleven Point River and the Current River, are immediately south of the Viburnum Trend. The Eleven Point River flows through the Mark Twain National Forest and contains the Eleven Point National Wild and Scenic River, which is managed by the U.S. Department of Agriculture, Forest Service. The adjacent Current River Basin contains the Ozark National Scenic Riverways, which is managed by the National Park Service. Additionally, these basins contain the two largest springs in Missouri, Big Spring and Greer Spring (Vineyard and Feder, 1982). As ore reserves are being depleted in the Viburnum Trend, exploration for new deposits is expanding south into the Mark Twain National Forest (fig. 1).

\section{Purpose and Scope}

The U.S. Geological Survey (USGS) collected 44 streambed-sediment samples between 1992 and 2002 from streams draining the Viburnum Trend and nearby non-mining basins to determine the distribution of mining-related trace metals and sulfide-mineral occurrence. The purpose of this chapter is to quantify the degree of trace-metal enrichment in the less than 63 micrometer $(\mu \mathrm{m})$ fraction of streambed sediment as a result of mining in the Viburnum Trend and to determine if this enrichment is caused by geographical location or proximityto-mining activity. Trace-metal enrichment is characterized with respect to baseline conditions as defined by sampling sites upstream from active mining within the Viburnum Trend and in non-mining river basins, which include streams in the exploration area. No time trends were investigated. Chromium is a metal not associated with MVT ores, but it is included in the analysis of trace metals as an indicator for alternative trace-metal sources in streambed sediment other than lead and zinc mining.

\section{Methodology}

The streambed-sediment data described in this chapter are a compilation of data that were collected as part of four different studies that investigated the effects of lead and zinc mining on southeastern Missouri streams. Each study had a different purpose; consequently, different reporting limits were used for some of the analyzed elemental concentrations.

All streambed-sediment data used in this analysis came from samples collected in a similar manner by USGS personnel, and all samples were analyzed by the same laboratory using the same analytical methods. For these reasons, all the data are considered comparable.

\section{Sample Sites}

Most of the data (26 samples) were collected from April through June 2002 at stream and spring sites in the Huzzah Creek and Black River Basins of the Viburnum Trend (fig. 1) and river basins to the south near the exploration area. The analyses of these data were performed to characterize streambed sediments along flow paths from lower-order, or smaller streams in headwater areas, to higher-order streams. In the Black River and Huzzah Creek Basins of the Viburnum Trend, the lower-order streams are used as containment areas for mine tailings.

The data collected in 2002 were supplemented with National Water-Quality Assessment (NAWQA) Program streambed-sediment data from 11 sites collected from 1992 through 1995. These data were collected as part of a reconnaissance investigation to evaluate, by using multiple lines of evidence, the effects of lead and zinc mining on area streams in the Viburnum Trend. Nine of the 11 sites sampled for the 
NAWQA program were in the Black River Basin and 2 additional non-mining (baseline) sites were Paddy Creek (A20) and Jacks Fork (A5; fig. 1).

Also added to the data set were three streambed-sediment samples collected in June 1995 from two sites on the Current River near Owls Bend. The Current River sample collected upstream from Blue Spring (A7) is considered a baseline site, but the two samples collected downstream from Blue Spring (C10, east side and west side of the Current River) are considered to be from a distal-mining site. During 1977 and 1978, part of the Sweetwater Mine tailings pile upstream from site B8 (fig. 1) was breached on three occasions causing mine tailings to be released into Logan Creek (Duchrow and others, 1980). Increased turbidity caused by the tailings releases extended more than 40 miles (mi) downstream in Logan Creek, and Blue Spring also reportedly became turbid. A dyetrace investigation conducted by Feder and Barks (1972) verified the connection between Logan Creek downstream from the tailings pile and Blue Spring.

Four streambed-sediment samples collected in October 1997 from Big Spring and the Current River near Van Buren completes the data set (sites A9-A12). These data were collected to document baseline conditions in a non-mining area that has potential to be affected if future mining occurs in the exploration area.

\section{Sample Collection}

All streambed-sediment samples used for this study consisted of alluvium that was collected and composited from several dozen localities across each stream and spring sampling site to obtain a representative sample of available sediment. Samples from springs were collected at or near the spring orifice. About 20 individual subsamples at wadable sections were obtained from a hand-held sampler that used a 2-inch (in.) cup made of polyvinyl chloride (PVC) or Teflon. The sampling cup was scraped across the top 2 in. of the streambed and the collected sediment was deposited into a polyethylene container. In stream reaches that were too deep to wade, an epoxy-coated BMH-60 sampler was used (Radtke, 1997). This sampler contains an impact-activated scoop (stainless steel bucket) that penetrates into the streambed about 2 in.

The faster flowing sections of the stream were the most difficult to sample using the hand-held sampler because, in swift current, a part of the sample typically was flushed from the sampler as it was brought to the surface. Consequently, the resulting composite samples were more representative of the finer sediment from the slower current areas typically near the sides of the stream. However, because the silt and clay fraction (less than $63 \mu \mathrm{m}$ ) is the standard fraction for which to perform chemical analyses, these results are expected to be comparable to other streambed-sediment studies.

\section{Sample Analyses}

All samples used in this study were sent to the USGS mineralogic laboratory in Lakewood, Colorado, for further processing and were analyzed using the same analytical methods. The composited samples from each site were sieved to obtain the less than 2-millimeter ( $\mathrm{mm}$ ) fraction and oven dried at 105 degrees Fahrenheit $\left({ }^{\circ} \mathrm{F}\right)$ before subsequent preparation or analyses. After drying, each sample was divided into two subsamples; one was analyzed for total elemental abundance and the other for selective concentration of heavy minerals.

Grain-size analyses of the streambed-sediment samples indicate the mass of coarse material ( $2 \mathrm{~mm}$ to $63 \mu \mathrm{m}$ ) ranged from 91.6 to 99.5 percent, the silt fraction (less than 63 to 2 $\mu \mathrm{m}$ ) ranged from 0.1 to 7.0 percent, and the clay fraction (less than $2 \mu \mathrm{m}$ ) ranged from less than 0.1 to 1.4 percent (table 1 , at the back of this chapter). However, only the silt and clay fraction (less than $63 \mu \mathrm{m}$ ) of samples was used to determine the total elemental abundances. This fraction was used to remove some of the large variability in sediment trace-element concentrations that is caused by analysis on bulk samples with differing grain size distribution (Horowitz, 1985). The samples were digested using a mixture of hydrochloric, nitric, perchloric, and hydrofluoric acids at low temperature (Crock and others, 1983). The digested sample was then aspirated into the inductively coupled plasma-atomic emission spectrometry (ICP-AES) discharge where the elemental emission signals were measured simultaneously for 40 elements. Additional details about this method are documented in Briggs (2003). Chemical constituents detected at concentrations less than limits deemed reliable for reporting as numerical values were considered less than the reporting limit. This situation is indicated by a less than $(<)$ symbol in front of the reporting limit value in table 1.

No stringent quality assurance or quality control measures were incorporated in any of the four studies for which these samples were collected; therefore, there were no duplicate or split samples for comparison or quality control. Whereas mining activity may have increased or diminished during this 10 -year period, mining and milling practices probably changed little. Five sites (A2, A10, B6, C7, and C8; fig. 1) were sampled twice between 1992 and 2002; and these data provide an indication of temporal variability of the sediment released from mining facilities into area streams during the period.

Using the criterion of a greater than 50 percent difference in elemental concentration between the two samples, Big Spring orifice (A10) and West Fork Black River at West Fork (B6) were the two sites that exceeded the criterion most often (table 1). Compared to the other sites, A10 had more exceedances for the major elements (concentrations given in percent weight) and site B6 had more exceedances for the MVT-related metals. Site B6 was the closest of the five sites to active mining operations. 


\section{Sediment-Quality Guidelines}

Consensus-based sediment-quality guidelines have been developed to measure the potential effects of sediment trace-element concentrations on freshwater ecosystems. Two guidelines are used to place the detected concentrations of arsenic, cadmium, copper, lead, nickel, zinc, and chromium (table 1) within the context of element toxicity. The threshold effects concentration (TEC) is the concentration below which adverse biological effects to sediment-dwelling organisms are not expected to occur or rarely occur. The probable effects concentration (PEC) is the concentration above which adverse biological effects to sediment-dwelling organisms are expected to usually or frequently occur (MacDonald and others, 2000).

\section{Heavy Mineral Concentrate}

The selective concentration of heavy minerals was performed on all of the streambed-sediment samples, except for the 11 NAWQA samples, to isolate sulfide ore minerals that may be present in streambed sediment. These minerals commonly are a small part of the total minerals in bulk material. The heavy-mineral fraction of streambed-sediment samples was used to determine if the occurrence of sulfide minerals changes downstream from mining operations.

To isolate the heavy-mineral fraction, the less than 2-mm fraction of sediments was wet panned until most of the quartz, feldspar, organic material, and clay-size sediment were removed. The samples were then sieved with a $0.5-\mathrm{mm}$ (35 mesh) screen and floated through bromoform (specific gravity 2.85) to remove the remaining quartz, feldspar, and other light minerals. The remaining sediment was collected, air dried, and separated into three magnetic fractions using a modified Frantz Isodynamic Separator. The most magnetic fraction (primarily magnetite) and the moderately to weakly magnetic fraction (largely ferromagnesian silicates and oxides) were isolated and saved, but not analyzed for this study. The fraction that includes nonmagnetic sulfide minerals, such as galena, pyrite, and sphalerite, was retained and characterized. This fraction is referred to as the $\mathrm{C} 3$ fraction.

Sulfide minerals in the $\mathrm{C} 3$ fraction were identified using the standard methods of optical mineralogy that are based on morphology and optical properties of grains (table 2, at the back of this chapter). The absence or presence of sulfide minerals in samples was documented, and the relative proportions of detection or non-detection of sulfide minerals were used for characterizing spatial trends in sulfide occurrence.

\section{Data Analysis}

The computer software SYSTAT (SYSTAT Software Inc., 2002) was used for statistical hypothesis tests, summary statistics, and the preparation of boxplots. A level of significance ( $\alpha$-value) less than 0.05 caused rejection of the null hypothesis that states the streambed-sediment concentrations for compared areas were similar. The "attained significance level" ( $\mathrm{p}$-value) is a probability value determined from the data (Helsel and Hirsch, 1992) and measures the "believability" of the null hypothesis. The larger the p-value, the more likely is the observed test statistic when the null hypothesis is true and the weaker the evidence to reject the null hypothesis. Both logarithmic and linear scales were used to graphically present the concentration data. When a constituent concentration was less than the reporting limit, the reporting limit value was substituted for the "less than" value during the statistical calculations. A "less than" symbol was inserted before the appropriate calculated value listed in the summary statistics (table 3, at the back of this chapter).

\section{Distribution of Mining-Related Trace Metals}

The results of the entire 40-element ICP-AES are reported in table 1; however, only the total elemental abundances of the MVT-related metals and chromium are discussed here. A statistical summary of the MVT-related metals and chromium total elemental abundances are listed in table 3. The observed abundance of MVT-related metals in all samples ordered from largest to smallest median concentration is: zinc greater than $(>)$ copper $>$ lead $>$ nickel $>$ cobalt $>$ arsenic $>$ cadmium (fig. 2; table 3). The median concentrations of zinc, copper, lead, nickel, and cobalt equaled or exceeded 25 milligrams per kilogram $(\mathrm{mg} / \mathrm{kg})$. Median concentrations of arsenic $(<10 \mathrm{mg} / \mathrm{kg})$ and cadmium $(<2 \mathrm{mg} / \mathrm{kg})$ were equal to the reporting limit. The four largest maximum concentrations of MVT-related metals detected were zinc $>$ lead $>$ cobalt $>$ nickel (fig.2; table 3). Using the interquartile range (IQR) as a robust measure of variability, lead concentrations are the most variable, followed by zinc, copper, nickel, cobalt, arsenic, and cadmium (table 3 ).

Data from the 20 non-mining sites ( 22 samples) were analyzed to determine if trace-metal enrichment is caused by geographical location. These baseline data were subgrouped based on drainage basin and included sites in the Huzzah Creek and Black River Basins upstream from active Viburnum Trend mining and sites in non-mining river basins to the south and west of the Viburnum Trend. The concentrations of MVTrelated metals and chromium in the less than $63 \mu \mathrm{m}$ fraction of streambed sediment in these two subgroups were analyzed using the non-parametric two-sample Kolmogorov-Smirnov test (SYSTAT Software Inc., 2002) to determine if concentrations in both areas were similar.

The results of this statistical test indicated that the MVT-related metals in the two areas have a similar distribution (p-values ranged from 0.272 to 0.992 ). This indicated if MVT-related metal enrichment was occurring in the Viburnum Trend, it was not caused by geographical location; therefore, data from both areas were combined to form a larger data set and improve the power of the statistical tests. 


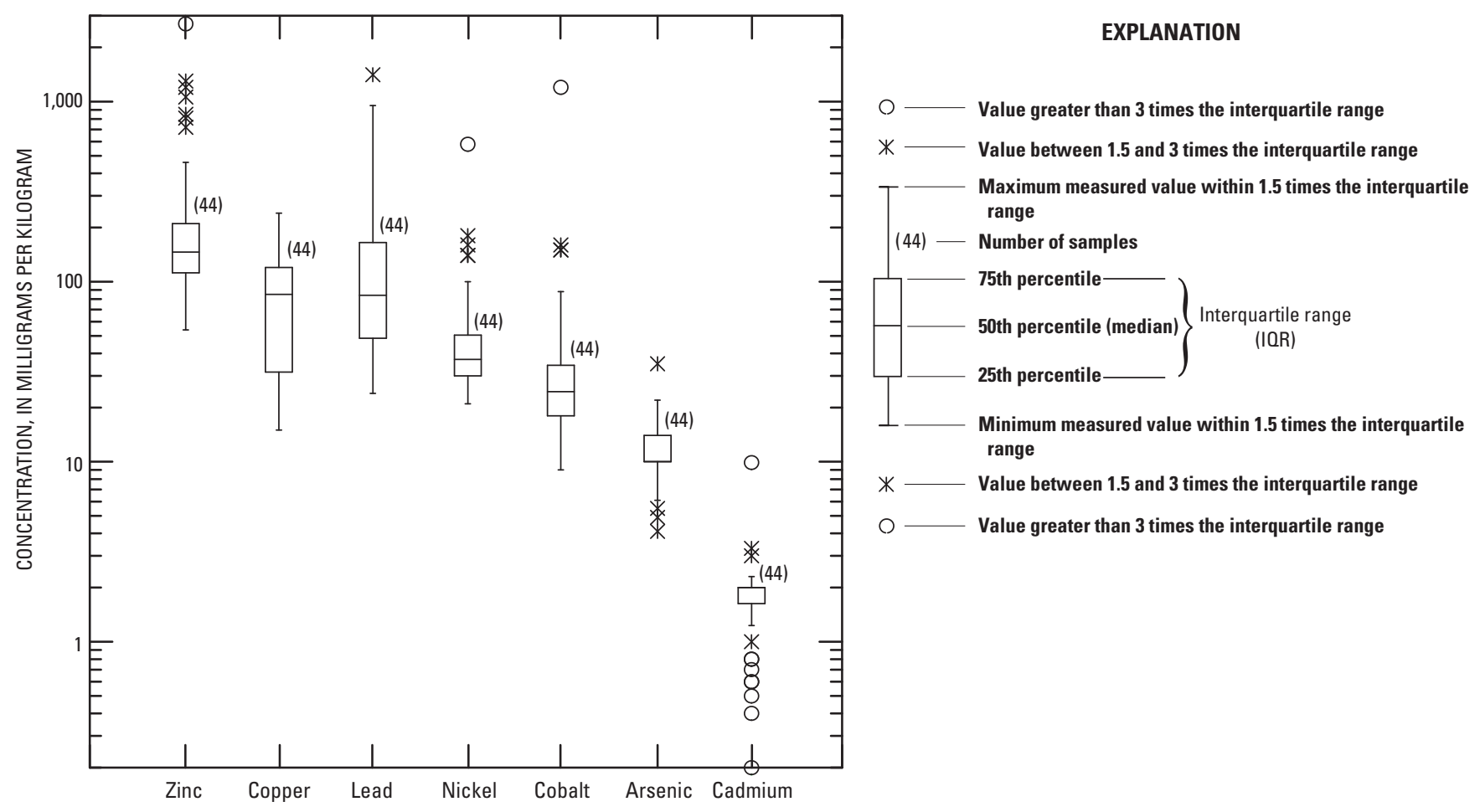

Figure 2. Observed abundance of Mississippi Valley Type-related metals in streambed-sediment samples, 1992-2002.

The distribution of the chromium concentrations, a trace metal not related to MVT deposits but included as an indicator for alternative sources of trace elements other than lead and zinc mining in the basins, was statistically different (p-value = 0.003 ) for the two subgroups. Chromium concentrations were significantly larger at sites in non-mining basins than at sites upstream from active mining in the Viburnum Trend. This indicates geographical location affects chromium concentrations.

Because the streambed-sediment concentrations for MVT-related metals from both non-mining areas were similar statistically, any statistical changes to these metal concentrations downstream from mines would be a result of activity at the mines. Therefore, all the sampling sites were classified into three groups based on the location and proximity of the sampling site to mining activity. The grouping was performed to determine if distance downstream from active mines or tailings piles affects the total elemental concentrations of the MVT-related metals. The three groups include: (A) non-mining sites; sampling sites upstream from mining activity or tailings ponds, or sites where no mining activity is present in the basin, (B) near-mining sites; mining activity or tailings ponds within 7.5 mi upstream from sampling site, and (C) distal-mining sites; mining activity greater than $7.5 \mathrm{mi}$ upstream from the sampling site. The arbitrary 7.5-mi distance that was used as the criterion to separate near-mining and distal-mining sites was based on the distribution of the sampling sites.

The non-parametric Kruskal-Wallis statistical test (Helsel and Hirsch, 1992; SYSTAT Software Inc., 2002) was performed on the grouped data to determine if there was similar distribution between the three groups. Statistical analyses for cadmium were difficult because many cadmium concentrations were less than the reporting limit (28 of 44 values). Various reporting limits were used in the cadmium analysis $(0.1,2$, and $3 \mathrm{mg} / \mathrm{kg}$ ) and the substitution method was used to resolve the numerous less than reporting limit situation. The median values for the cadmium concentrations (fig. 3) indicate the distribution in the three proximity-to-mining groups to be similar; however, the statistical analysis indicated the three groups were different. This discrepancy was caused by the substitution of different reporting limits; therefore, the cadmium concentrations are considered to be similar in the three groups.

Arsenic, copper, and chromium concentrations had p-values greater than 0.05 , which indicates these concentrations are similar statistically in all three proximity-to-mining groups. Although the boxplot of copper concentrations (fig. 3 ) appears to show a significant difference, the p-value for the copper analysis indicated marginal similarity ( $\mathrm{p}$-value $=0.053$ ) between the three groups. Cobalt, lead, nickel, and zinc concentrations were different statistically for the three groups.

The Tukey method for multiple comparisons (SYSTAT Software Inc., 2002) was applied to the ranked data of cobalt, lead, nickel, and zinc to identify which groups were different statistically. The distribution of nickel and zinc concentrations in non-mining sites and distal-mining sites (groups A and C) were similar ( $\mathrm{p}$-value $>0.05$ ), but concentrations of these trace metals in near-mining sites (group B) were significantly larger (p-value < 0.05) than the other two groups (fig. 3). Cobalt and lead concentrations were different statistically in all three groups, with the largest concentrations at near-mining sites 

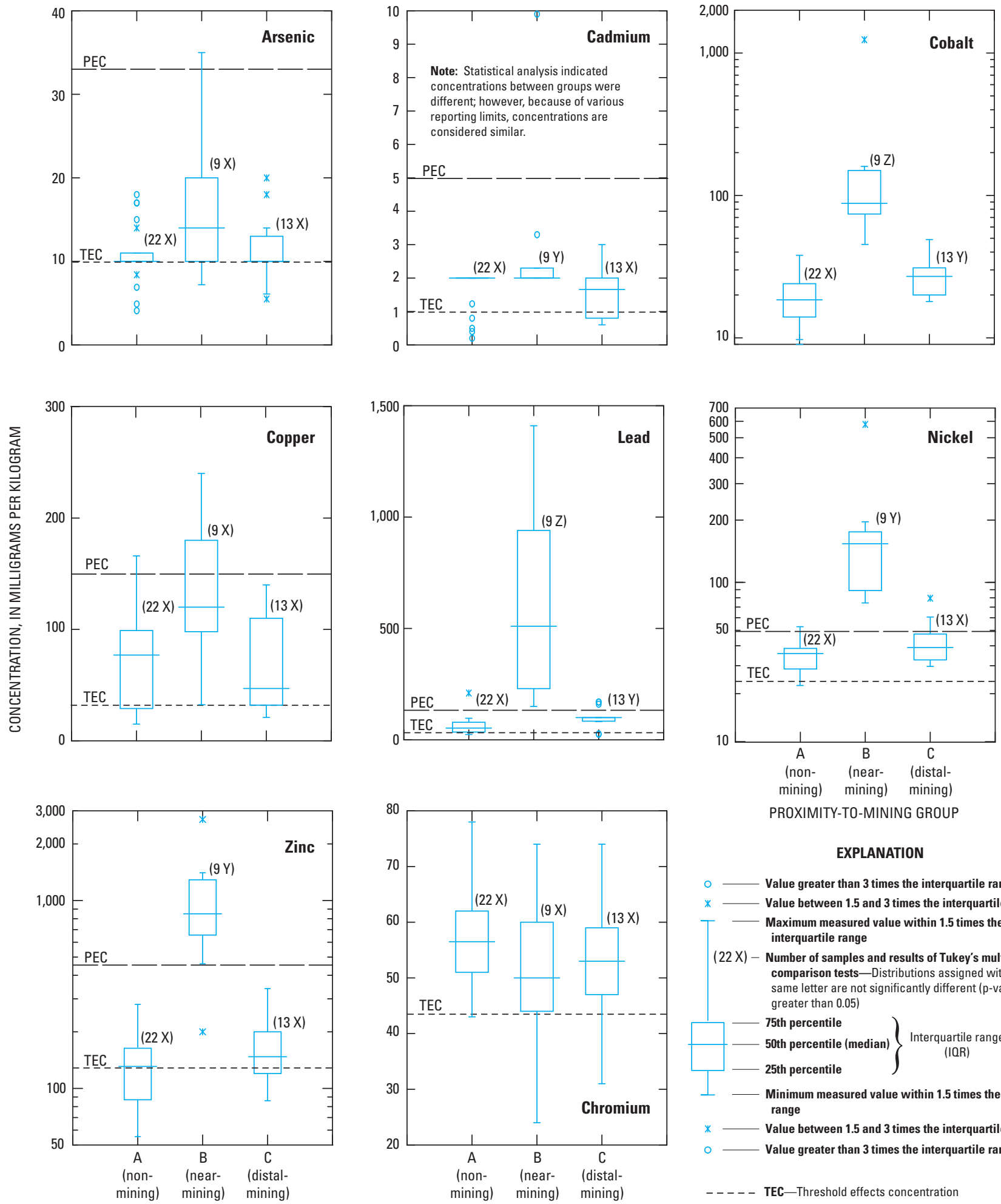

PROXIMITY-TO-MINING GROUP

\section{EXPLANATION}

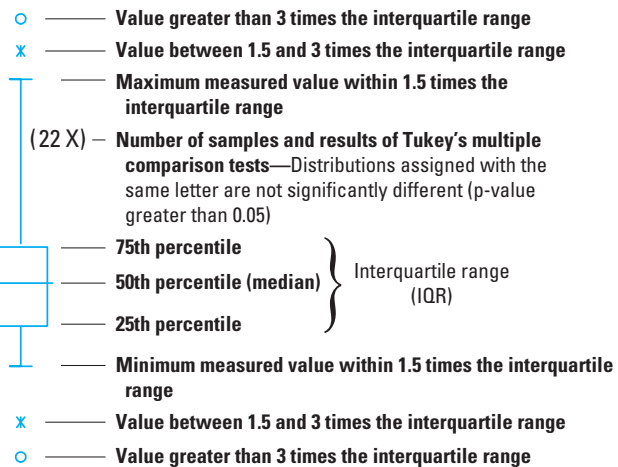

PROXIMITY-TO-MINING GROUP

- - - - - TEC-Threshold effects concentration

— $—$ PEC—Probable effects concentration

Figure 3. Concentrations of Mississippi Valley Type-related metals and chromium in streambed sediment samples, threshold effects concentrations (TEC), and probable effects concentrations (PEC). 
and the smallest concentration at the non-mining sites. These concentrations at the distal-mining sites did not decrease to the smaller non-mining concentrations, but stayed slightly elevated.

Thus, for MVT-related metals in streambed-sediment samples, near-mining sites had significantly elevated cobalt, lead, nickel, and zinc concentrations when compared to baseline-like conditions in non-mining areas. Distal-mining sites (7.5 mi or more downstream from mining activity) had nickel and zinc concentrations that were not significantly different from sites with no mining activity; however, cobalt and lead concentrations were more persistent in the streambed-sediment samples, even at sites greater than 7.5 mi downstream from mining activities.

With the exception of one elevated arsenic concentration $(35 \mathrm{mg} / \mathrm{kg}$ ) at Strother Creek near Goodland (B2; fig. 1) and one elevated cadmium concentration $(9.9 \mathrm{mg} / \mathrm{kg})$ at Logan Creek near Corridon (B8; fig. 1), which were both near-mining sites, concentrations of these two metals were small in all samples (table 1). Although arsenic and cadmium concentrations increased in group B samples, these are minor constituents of Viburnum Trend sulfides when compared to cobalt, lead, nickel, and zinc (Hagni, 1983). This is perhaps the primary reason why these metals were detected in lower concentrations in streambed-sediment samples downstream from mining operations.

In contrast to the MVT-related metals discussed above, other metals were not expected to indicate a relation with the degree of mining activity upstream. For example, chromium is not associated with MVT ore minerals. Consistent with this, the distribution of chromium concentrations in streambed-sediment samples was similar between all three groups ( $\mathrm{p}$-value $=$ 0.391 ) and not significantly elevated at sites downstream from mining activity (fig. 3 ).

Comparing median concentrations of MVT-related metals in streambed-sediment samples at near-mining sites (group B) to non-mining sites (group A) indicated the most enriched metals were lead and zinc followed by cobalt and nickel. Median lead concentrations at near-mining sites were about 10 times greater than the median lead concentrations detected at non-mining sites. Similarly, median zinc concentrations were elevated about seven times, cobalt about five times, and nickel about four times in samples from near-mining sites. In contrast, arsenic, cadmium, copper, and chromium median concentrations at near-mining sites were similar to median concentrations observed at non-mining sites (table 3; fig. 3).

Few samples in each of the three proximity-to-mining groups met the TEC for the MVT-related metals and chromium (table 1). No samples from any of the proximity-tomining groups exceeded the PEC threshold for chromium. One sample (Strother Creek at Goodland, B2; fig. 1) exceeded the PEC for arsenic and one sample (Logan Creek at Corridon, B8; fig. 1) exceeded the PEC for cadmium (table 1). Both samples were from near-mining sites (group B). The percentage of near-mining samples that exceeded the PEC for copper, lead, nickel, and zinc was substantially greater as compared to the percentage of samples from non-mining sites (table 1; fig. 4). Four copper concentrations, all lead and nickel concentrations, and all but one zinc concentration exceeded their respective PEC threshold in near-mining sites. This occurred even though these same metals only occasionally exceeded these thresholds in samples from the non-mining sites. A trace of anthropogenic lead contamination (processed lead, such as lead shot or fishing sinkers, not galena) was contained in the sample from the non-mining site (A17, West Fork Black River near Greeley; table 2), which exceeded the lead PEC threshold. No distal-mining sites exceeded the PEC thresholds for copper or zinc, but three sites exceeded the lead PEC threshold and two sites exceeded the nickel PEC threshold.

\section{Sulfide Mineral Occurrence}

The selective concentration of heavy minerals indicated pyrite and galena were the only sulfide minerals observed in the $\mathrm{C} 3$ fraction samples (table 2). Pyrite was the most commonly observed sulfide mineral, occurring in 55 percent (18 of 33) of all samples (table 2). Galena was observed far less frequently, occurring in 12 percent ( 4 of the 33) of all samples. Pyrite occurrence increased from non-mining sites (group A) to near-mining sites (group B). The proportion of samples where pyrite occurs increased from 33 percent at non-mining sites to 86 percent at near-mining sites, and then decreased to 75 percent at distal-mining sites (group C). Because of the small number of sites where galena was detected, no trends will be inferred.

Pyrite is common in all Paleozoic rocks in this region and occurs in trace- and ore-grade mineralized areas (Erickson and others, 1978; Lee, 2001; Lee and Goldhaber, 2002). The occurrence of galena, however, is confined to ore-grade mineralization, which may explain the higher occurrence rate of pyrite in areas upstream from mining and overall lower occurrence of galena.

\section{Summary and Conclusions}

The Viburnum Trend Subdistrict (Viburnum Trend) in southeastern Missouri is part of the largest known lead reserve within the United States and is mined for lead and zinc sulfides containing the primary trace elements arsenic, cadmium, cobalt, copper, lead, nickel, and zinc (hereafter referred to as metals). The waste material produced during this mining is stored in headwater valleys of the area and thus may be transported downstream into larger streams and rivers.

Streambed-sediment samples were collected from sites along the Viburnum Trend and from non-mining areas to the south and west of the subdistrict. The samples were divided into three groups that included: (A) non-mining sites; sites upstream from mining activity or mine tailings ponds, or sites where no mining activity is present in the basin, (B) nearmining sites; mining activity or mine tailings ponds within 


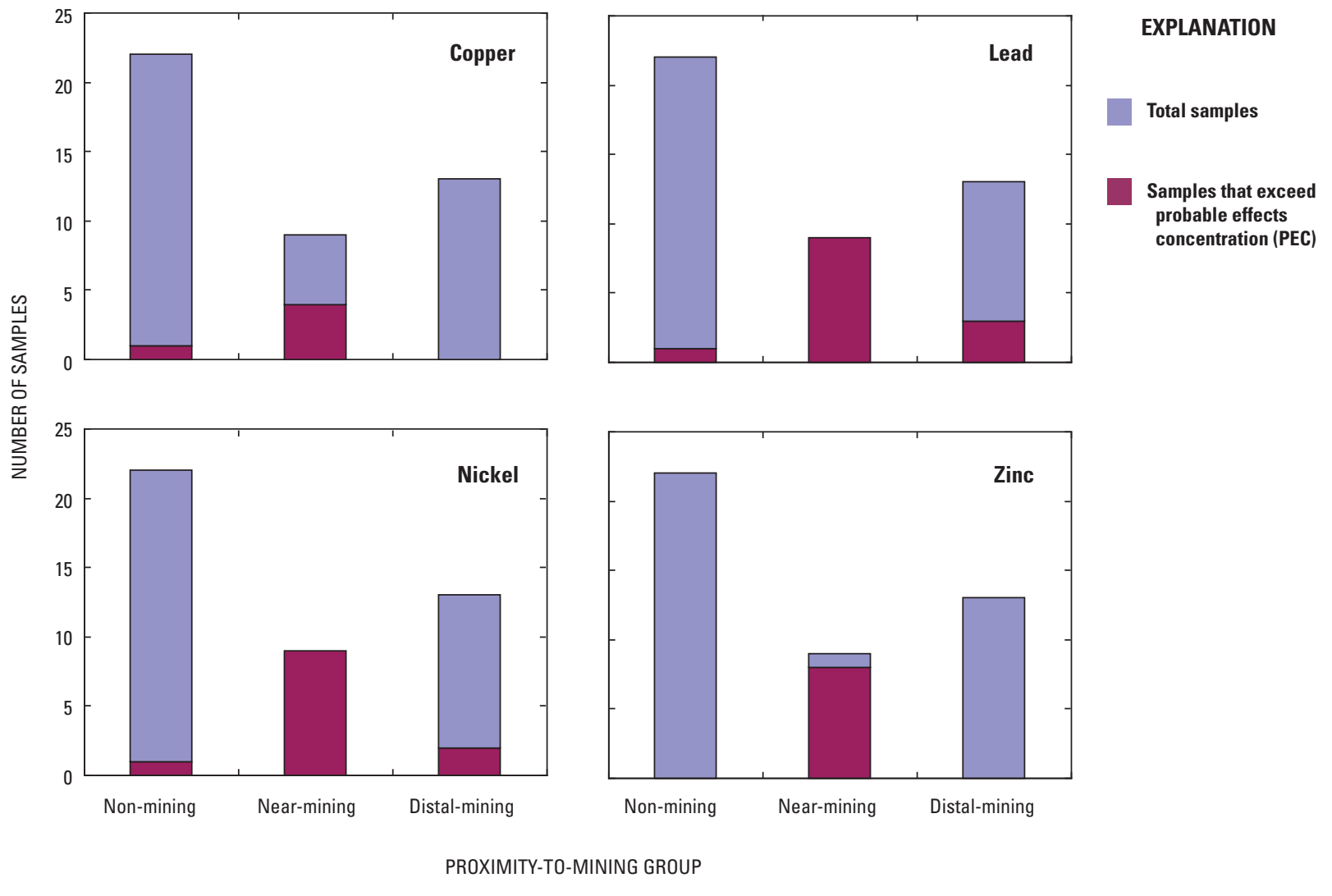

Figure 4. Exceedance of probable effects concentrations (PEC) of copper, lead, nickel, and zinc in streambed-sediment samples.

7.5 miles upstream from the site, and (C) distal-mining sites; mining activity distally upstream at distances greater than 7.5 miles. The total elemental concentrations in the less than 63 micrometer fraction of streambed sediment from the three groups were compared to quantify the degree of heavy metal enrichment that occurred as a result of mining. Chromium (a non-Mississippi Valley Type-related metal) concentrations also were analyzed as an indicator for alternative trace metal sources in streambed material other than lead and zinc mining in the basins.

Data from the 20 non-mining sites (22 samples) were analyzed to determine if trace-metal enrichment was caused by geographical location. These data were divided into two subgroups and compared. One group consisted of sites in the mining area, but upstream from any mining activity; the other group consisted of sites in stream basins outside of the Viburnum Trend where no mining has occurred. Statistical analysis indicated that the MVT-related metals in these two areas were distributed similarly in the streambed sediment. This indicated that if MVT-related metal enrichment was occurring in the Viburnum Trend, it was not caused by geographical location; therefore, data from both areas were combined to form a larger data set.

A comparison of concentrations of MVT-related metals in streambed sediment indicates a significant relation between the proximity-to-mining groups and the degree of metal enrichment. Near-mining sites were enriched significantly in the primary MVT-related metals - cobalt, lead, nickel, and zinc. Statistical analyses indicate that the distribution of nickel and zinc in non-mining and distal-mining sites were similar, but the concentrations of these metals in near-mining sites were significantly larger. This indicated the elevated nickel and zinc concentrations in streambed sediment at near-mining sites returned to near baseline conditions at distal-mining sites (7.5 miles or more downstream from mining activity). However, cobalt and lead concentrations did not decrease to the lower non-mining concentrations at the distal-mining sites but stayed slightly elevated.

When comparing median concentrations of MVT-related metals in streambed sediment at near-mining (group B) sites to non-mining (group A) sites, lead concentrations were about 10 times greater. Similarly, zinc concentrations were elevated about seven times, cobalt about five times, and nickel about four times. Arsenic, cadmium, and copper concentrations at near-mining sites were similar to non-mining sites. Chromium (a non-MVT related metal) concentrations also were similar at the two groups.

Consensus based sediment-quality guidelines were used as a means of measuring the potential effects of the sediment trace-metal concentrations of arsenic, cadmium, copper, lead, nickel, zinc, and chromium on freshwater ecosystems. The threshold effects concentration (TEC) is the level below which 
adverse biological effects to sediment dwelling organisms are not expected to occur or rarely occur; the probable effects concentration (PEC) is the level above which adverse biologic effects to sediment dwelling organisms are expected to usually or frequently occur. Few samples in each of the three proximity-to-mining groups met the TEC for the MVT-related metals and chromium. At near-mining sites, every lead and nickel concentration and all but one zinc concentration, exceeded their respective PEC threshold, even though these same metals only occasionally exceeded these thresholds in samples from the non-mining sites. No distal-mining sites exceeded the PEC thresholds for copper or zinc, but three sites exceeded the lead PEC threshold, and two sites exceeded the nickel PEC threshold.

Pyrite and galena were the only sulfide minerals observed in the non-magnetic, heavy mineral fraction (C3) samples. Pyrite was the most commonly observed sulfide mineral, occurring in 55 percent (18 of 33) of all samples. Galena was observed far less frequently, occurring in 12 percent ( 4 of the 33) of all samples. The proportion of samples that pyrite occurs increased from 33 percent at non-mining sites to 86 percent at near-mining sites, and then decreased to 75 percent at distal-mining sites. Pyrite is common in all Paleozoic rocks in this region and occurs in trace- and ore-grade mineralized areas. The occurrence of galena, however, is confined to oregrade mineralization. This may explain the higher occurrence rate of pyrite in areas upstream from mining and overall lower occurrence of galena.

\section{References}

Briggs, P.H., 2003, The determination of 40 elements in geological and botanical samples by inductively coupled plasma-atomic emission spectrometry: U.S. Geological Survey Open-File Report 02-223, chap. G, 18 p.

Crock, J.G., Lichte, F.E., and Briggs, P.H., 1983, Determination of elements in National Bureau of Standards Geological reference materials SRM 278 Obsidian and SRM 688 Basalt by inductively coupled argon plasma-atomic emission spectrometry: Geostandards Newsletter, v. 7, p. 335-340.

Duchrow, R.M., Robinson-Wilson, E., and Trial, Linden, 1980, The effects of lead mine tailings on the water quality of Logan Creek, Reynolds County, Missouri: Missouri Department of Conservation, $29 \mathrm{p}$.

Erickson, R., Mosier, E., and Viets, J., 1978, Generalized geologic and summary geochemical maps of the Rolla 1 x 2 degree quadrangle, Missouri: U.S. Geological Survey Miscellaneous Field Studies Map MF-1004-A, 1 sheet.
Feder, G.L., and Barks, J.H., 1972, A losing drainage basin in the Missouri Ozarks identified by side-looking radar imagery, in Geological Survey Research 1972: U.S. Geological Survey Professional Paper 800-C, p. C249-C252.

Hagni, R.D., 1983, Minor elements in Mississippi Valley-Type ore deposits, in Shanks, W.C., ed., Cameron symposium on unconventional mineral deposits, p. 71-88.

Helsel, D.R., and Hirsch, R.M., 1995, Statistical methods in water resources, in Studies in Environmental Science 49: Amsterdam, The Netherlands, Elsevier Science, 529 p.

Horowitz, A.J., 1985, A primer on trace metal-sediment chemistry: U.S. Geological Survey Water-Supply Paper 2277, $67 \mathrm{p}$.

Leach, D.L., 1994, Genesis of the Ozark Mississippi ValleyType metallogenic province, Missouri, Arkansas, Kansas, and Oklahoma, USA, in Fontbote, L., and Boni, M. eds., Sediment-hosted zinc-lead ores: Springer Verlag, Special Publication, no. 10, p. 104-138.

Leach, D.L., and Sangster, D., 1993, Mississippi ValleyType lead-zinc deposits, in Kirkham, R.V., Sinclair, W.D., Thorpe, R.I., and Duke, J.M., eds., Mineral deposit modeling: Geological Association of Canada, Special Paper, v. 40, p. 289-314.

Lee, Lopaka, 2001, The distribution of MVT-related metals in acid-insoluble residues of Paleozoic rocks in Ozark Plateaus region of the United States: U.S. Geological Survey OpenFile Report 01-0042, 32 p.

Lee, Lopaka, and Goldhaber, M.B., 2002, Geologic cross sections showing the concentrations of arsenic, cadmium, cobalt, copper, chromium, iron, molybdenum, nickel, lead, and zinc, in Acid-Insoluble residues of paleozoic rocks within the Doniphan/Eleven Point Ranger District of the Mark Twain National Forest, Missouri, USA: U.S. Geological Survey Open-File Report 02-0055, 24 p.

MacDonald, D.D., Ingersoll, C.G., and Berger, T.A., 2000, Development and evaluation of consensus-based sediment quality guidelines for freshwater ecosystems: Environmental Contamination and Toxicology, v. 39, p. 20-31.

Radtke, D.B., 1997, National field manual for the collection of water-quality data: Bottom-material samples: U.S. Geological Survey Techniques of Water-Resources Investigations, book 9, chap. A8, 48 p. with appendices.

Rakovan, John, 2007, Mississippi Valley-Type deposits: Oxford, Ohio, Miami University, accessed October 5, 2007, at URL http://www.users.muohio.edu/rakovajf/WTTW\%20 MVT.pdf

SYSTAT Software Inc., 2002, SYSTAT user's guide-Statistics I and II: Richmond, Calif., version 10.2, 1,376 p. 
U.S. Environmental Protection Agency, 1998, The incidence and severity of sediment contamination in surface waters of the United States, v. 1-National sediment survey: U.S. Environmental Protection Agency Report EPA823R97006.

U.S. Geological Survey, 2008, Mineral commodity summary, accessed January 18, 2008, at URL http://minerals.usgs. gov/minerals/pubs/commodity/statistical_summary/

Vineyard, J.D., and Feder, G.L., 1982, Springs of Missouri: Rolla, Missouri Department of Natural Resources, Division of Geology and Land Survey Water Resources Report 29, 212 p. 

Tables 
Table 1. Quantitative elemental analysis of the less than 63-micrometer fraction and bulk sample grain size distribution of streambed-sediment samples, threshold effects concentrations (TEC), and probable effects concentrations (PEC) for selected trace elements, 1992-2002.

[mm/dd/yyyy, month/day/year; \% wt, percent by weight; NA, not applicable; es, east side; ws, west side; mg/kg, milligrams per kilogram; <, less than; shaded cells indicate PEC exceedance; --, no data]

\begin{tabular}{|c|c|c|c|c|c|c|c|c|}
\hline $\begin{array}{c}\text { Site } \\
\text { identifier } \\
\text { (fig. 1) }\end{array}$ & $\begin{array}{c}\text { Date } \\
\text { (mm/dd/yyyy) }\end{array}$ & $\begin{array}{c}\text { Distance } \\
\text { downstream } \\
\text { from mining } \\
\text { activity } \\
\text { (miles) }\end{array}$ & Site name & $\begin{array}{c}\text { Alumimun } \\
(\% \mathrm{wt})\end{array}$ & $\begin{array}{c}\text { Calcium } \\
\text { (\% wt) }\end{array}$ & $\begin{array}{l}\text { Iron } \\
\text { (\% wt) }\end{array}$ & $\begin{array}{c}\text { Magnesium } \\
\text { (\% wt })\end{array}$ & $\begin{array}{c}\text { Phosphorous } \\
\text { (\% wt) }\end{array}$ \\
\hline \multicolumn{9}{|c|}{ Group A (Non-mining sites) } \\
\hline A1 & $09 / 14 / 1995$ & NA & Middle Fork Black River at Redmondville ${ }^{a}$ & 4.7 & 4.9 & 2.1 & 0.83 & 0.07 \\
\hline \multirow[t]{2}{*}{ A2 } & 09/12/1995 & NA & West Fork Black River near Greeley ${ }^{\mathrm{a}}$ & 3.8 & 1.4 & 1.6 & .40 & .06 \\
\hline & 05/06/2002 & & & 3.7 & .91 & 1.9 & .59 & .05 \\
\hline A3 & 05/07/2002 & NA & Sinking Creek near Redford & 3.6 & .52 & 2.8 & .37 & .07 \\
\hline A4 & $05 / 29 / 2002$ & NA & Big Creek at Mauser Mill & 5.1 & .68 & 2.6 & .49 & .07 \\
\hline A5 & $10 / 21 / 1993$ & NA & Jacks Fork at Alley Spring ${ }^{a}$ & 4.5 & .50 & 1.9 & .40 & .06 \\
\hline A6 & $05 / 29 / 2002$ & NA & Blair Creek & 4.9 & 1.10 & 3.1 & .73 & .07 \\
\hline A7 & 06/20/1995 & NA & Current River upstream from Blue Spring & 5.7 & .79 & 2.7 & .59 & .08 \\
\hline A8 & $06 / 11 / 2002$ & NA & Current River at Van Buren & 4.5 & .52 & 2.4 & .37 & .06 \\
\hline A9 & $10 / 16 / 1997$ & NA & Current River upstream from Big Spring & 4.7 & .89 & 2.5 & .47 & .07 \\
\hline \multirow[t]{2}{*}{ A10 } & $10 / 16 / 1997$ & NA & Big Spring orifice & 6.0 & 2.60 & 3.3 & 1.80 & .13 \\
\hline & $06 / 11 / 2002$ & & & 4.7 & .77 & 2.3 & .53 & .08 \\
\hline A11 & $10 / 16 / 1997$ & NA & Big Spring branch & 4.2 & 1.80 & 2.2 & 1.20 & .10 \\
\hline A12 & $10 / 16 / 1997$ & NA & Current River downstream from Big Spring & 4.4 & 1.10 & 2.4 & .59 & .09 \\
\hline A13 & $06 / 10 / 2002$ & NA & Eleven Point River at Thomasville & 3.5 & .35 & 1.8 & .27 & .06 \\
\hline A14 & $06 / 12 / 2002$ & NA & Spring Creek tributary & 5.2 & .62 & 3.2 & .44 & .09 \\
\hline A15 & $06 / 25 / 2002$ & NA & Spring Creek & 4.6 & .55 & 2.4 & .36 & .09 \\
\hline A16 & $06 / 12 / 2002$ & NA & McCormack Spring & 5.4 & .70 & 2.4 & .46 & .08 \\
\hline A17 & $06 / 10 / 2002$ & NA & Greer Spring & 4.6 & 1.40 & 2.6 & .80 & .09 \\
\hline A18 & $06 / 12 / 2002$ & NA & Hurricane Creek & 3.9 & .48 & 1.8 & .30 & .07 \\
\hline A19 & $06 / 10 / 2002$ & NA & Eleven Point River at Bardley & 3.2 & .66 & 1.6 & .30 & .05 \\
\hline A20 & $10 / 28 / 1992$ & NA & Paddy Creek near Slabtown Spring ${ }^{\mathrm{a}}$ & 6.2 & .47 & 1.7 & .53 & .04 \\
\hline
\end{tabular}


Table 1. Quantitative elemental analysis of the less than 63-micrometer fraction and bulk sample grain size distribution of streambed-sediment samples, threshold effects concentrations (TEC), and probable effects concentrations (PEC) for selected trace elements, 1992-2002.—Continued

[mm/dd/yyyy, month/day/year; \% wt, percent by weight; NA, not applicable; es, east side; ws, west side; mg/kg, milligrams per kilogram; <, less than; shaded cells indicate PEC exceedance; --, no data]

\begin{tabular}{|c|c|c|c|c|c|c|c|c|}
\hline $\begin{array}{c}\text { Site } \\
\text { identifier } \\
\text { (fig. 1) }\end{array}$ & $\begin{array}{c}\text { Date } \\
\text { (mm/dd/yyyy) }\end{array}$ & $\begin{array}{c}\text { Distance } \\
\text { downstream } \\
\text { from mining } \\
\text { activity } \\
\text { (miles) }\end{array}$ & Site name & $\begin{array}{c}\text { Alumimun } \\
\text { (\% wt })\end{array}$ & $\begin{array}{l}\text { Calcium } \\
\text { (\% wt) }\end{array}$ & $\begin{array}{l}\text { Iron } \\
\text { (\% wt) }\end{array}$ & $\begin{array}{c}\text { Magnesium } \\
\text { (\% wt) }\end{array}$ & $\begin{array}{c}\text { Phosphorous } \\
\text { (\% wt) }\end{array}$ \\
\hline \multicolumn{9}{|c|}{ Group B (Near-mining sites; within 7.5 miles downstream from mining activity) } \\
\hline B1 & $04 / 29 / 2002$ & 2.5 & Courtois Creek near Courtois & 5.4 & 2.10 & 3.8 & 1.20 & 0.07 \\
\hline B2 & $04 / 30 / 2002$ & 4.0 & Strother Creek near Goodland & 4.2 & 3.00 & 2.5 & 1.80 & .07 \\
\hline B3 & $04 / 30 / 2002$ & 4.7 & Neals Creek near Goodland & 3.8 & 5.10 & 2.2 & .77 & .07 \\
\hline B4 & 09/18/1995 & 6.3 & Strother Creek near Redmondville ${ }^{a}$ & 2.5 & 3.60 & 1.2 & .39 & .06 \\
\hline B5 & 05/06/2002 & 2.3 & Bills Creek near Greeley & 4.2 & 1.20 & 2.5 & .54 & .06 \\
\hline \multirow[t]{2}{*}{ B6 } & 09/11/1995 & 6 & West Fork Black River at West Fork ${ }^{\mathrm{a}}$ & 4.2 & .89 & 1.9 & .52 & .04 \\
\hline & 05/06/2002 & & & 4.3 & .87 & 2.5 & .54 & .07 \\
\hline B7 & 05/06/2002 & .4 & Bee Fork Black River near Reynolds & 4.0 & .96 & 2.9 & .62 & .08 \\
\hline B8 & $05 / 29 / 2002$ & 2.7 & Logan Creek near Corridon & 3.4 & 2.00 & 3.0 & 1.20 & .05 \\
\hline \multicolumn{9}{|c|}{ Group C (Distal-mining sites; more than 7.5 miles downstream from active mining) } \\
\hline $\mathrm{C} 1$ & $04 / 29 / 2002$ & 20.9 & Huzzah Creek near Davisville & 4.7 & 1.3 & 2.6 & 0.56 & 0.07 \\
\hline $\mathrm{C} 2$ & $04 / 29 / 2002$ & 32.2 & Huzzah Creek near Scotia & 4.0 & .96 & 2.6 & .64 & .07 \\
\hline $\mathrm{C} 3$ & 09/14/1995 & 15.7 & Middle Fork Black River at Black ${ }^{a}$ & 5.6 & .49 & 2.9 & .48 & .07 \\
\hline $\mathrm{C} 4$ & $04 / 30 / 2002$ & 15.7 & Middle Fork Black River near Black & 4.9 & .76 & 3.2 & .57 & .08 \\
\hline $\mathrm{C} 5$ & $10 / 27 / 1993$ & 20.3 & Middle Fork Black River near Lesterville ${ }^{a}$ & 4.6 & .40 & 2.3 & .35 & .05 \\
\hline C6 & 09/13/1995 & 9.9 & West Fork Black River near Centerville ${ }^{a}$ & 3.6 & 3.40 & 1.5 & .44 & .05 \\
\hline \multirow[t]{2}{*}{$\mathrm{C} 7$} & 09/13/1995 & 10.9 & West Fork Black River at Centerville ${ }^{a}$ & 5.2 & 60 & 2.2 & .43 & .04 \\
\hline & 05/07/2002 & & & 3.7 & .57 & 2.2 & .38 & .05 \\
\hline \multirow[t]{2}{*}{$\mathrm{C} 8$} & $10 / 26 / 1993$ & 21.4 & Black River near Lesterville ${ }^{a}$ & 3.7 & .36 & 1.9 & .29 & .04 \\
\hline & 05/07/2002 & & & 4.8 & .55 & 3.1 & .47 & .06 \\
\hline C9 & $05 / 29 / 2002$ & 21.8 & Logan Creek at Ellington & 3.8 & .52 & 2.7 & .39 & .07 \\
\hline \multirow[t]{2}{*}{$\mathrm{C} 10$} & 06/20/1995 & 16.5 & Current River downstream Blue Spring-es & 5.3 & 1.28 & 2.3 & .95 & .09 \\
\hline & 06/20/1995 & 16.5 & Current River downstream Blue Spring_ws & 4.9 & 1.44 & 2.6 & .82 & .10 \\
\hline $\mathrm{TEC}^{\mathrm{c}}$ & & & & NA & NA & NA & NA & NA \\
\hline $\mathrm{PEC}^{\mathrm{c}}$ & & & & NA & NA & NA & NA & NA \\
\hline
\end{tabular}


Table 1. Quantitative elemental analysis of the less than 63-micrometer fraction and bulk sample grain size distribution of streambed-sediment samples, threshold effects concentrations (TEC), and probable effects concentrations (PEC) for selected trace elements, 1992-2002.—Continued

[mm/dd/yyyy, month/day/year; \% wt, percent by weight; NA, not applicable; es, east side; ws, west side; mg/kg, milligrams per kilogram; <, less than; shaded cells indicate PEC exceedance; --, no data]

\begin{tabular}{|c|c|c|c|c|c|c|c|c|c|c|}
\hline $\begin{array}{c}\text { Site } \\
\text { identifier }\end{array}$ & $\begin{array}{l}\text { Potassium } \\
\text { (\% wt) }\end{array}$ & $\begin{array}{c}\text { Sodium } \\
\text { (\% wt) }\end{array}$ & $\begin{array}{c}\text { Titanium } \\
\text { (\% wt) }\end{array}$ & $\begin{array}{l}\text { Arsenic } \\
(\mathrm{mg} / \mathrm{kg})\end{array}$ & $\begin{array}{l}\text { Barium } \\
\text { (mg/kg) }\end{array}$ & $\begin{array}{l}\text { Beryllium } \\
\text { (mg/kg) }\end{array}$ & $\begin{array}{l}\text { Bismuth } \\
\text { (mg/kg) }\end{array}$ & $\begin{array}{l}\text { Cadmium } \\
\text { (mg/kg) }\end{array}$ & $\begin{array}{l}\text { Cerium } \\
\text { (mg/kg) }\end{array}$ & $\underset{\text { (mg/kg) }}{\text { Chromium }}$ \\
\hline \multicolumn{11}{|c|}{ Group A (Non-mining sites) } \\
\hline A1 & 2.1 & 0.19 & 0.23 & 6.9 & 350 & 2.0 & $<10$ & 0.8 & 61 & 43 \\
\hline \multirow[t]{2}{*}{$\mathrm{A} 2$} & .88 & .26 & .25 & 4.9 & 340 & 1.0 & $<10$ & .5 & 61 & 32 \\
\hline & .85 & .21 & .24 & $<10$ & 370 & 1.4 & $<10$ & $<2$ & 74 & 45 \\
\hline A3 & .68 & .14 & .24 & $<10$ & 420 & 1.5 & $<10$ & $<2$ & 88 & 51 \\
\hline A4 & .82 & .14 & .29 & 14 & 390 & 2.2 & $<10$ & $<2$ & 91 & 63 \\
\hline A5 & 1.20 & .20 & .28 & 8.4 & 380 & 2.0 & $<10$ & .4 & 69 & 52 \\
\hline A6 & .73 & .10 & .24 & $<10$ & 315 & 2.7 & $<10$ & $<2$ & 74 & 73 \\
\hline A7 & 1.09 & .19 & .33 & $<10$ & 386 & 2.3 & $<10$ & 1.23 & 78 & 62 \\
\hline A8 & .93 & .17 & .30 & $<10$ & 406 & 2.0 & $<10$ & $<2$ & 78 & 58 \\
\hline A9 & 1.00 & .19 & .32 & $<10$ & 370 & 2.0 & $<10$ & $<2$ & 84 & 60 \\
\hline \multirow[t]{2}{*}{ A10 } & 1.10 & .09 & .28 & 17 & 230 & 3.0 & $<10$ & $<2$ & 95 & 61 \\
\hline & 1.00 & .20 & .31 & 11 & 402 & 2.2 & $<10$ & $<2$ & 85 & 54 \\
\hline A11 & .86 & .13 & .23 & 11 & 220 & 2.0 & $<10$ & $<2$ & 77 & 54 \\
\hline A12 & .89 & .16 & .29 & 10 & 310 & 2.0 & $<10$ & $<2$ & 78 & 60 \\
\hline A13 & .84 & .16 & .28 & 10 & 360 & 1.4 & $<10$ & $<2$ & 74 & 47 \\
\hline A14 & .86 & .12 & .30 & 18 & 362 & 2.2 & $<10$ & $<2$ & 90 & 67 \\
\hline A15 & .85 & .13 & .31 & $<10$ & 367 & 2.1 & $<10$ & $<2$ & 79 & 59 \\
\hline A16 & .87 & .14 & .32 & 17 & 321 & 2.4 & $<10$ & $<2$ & 90 & 55 \\
\hline A17 & 1.00 & .14 & .33 & 15 & 438 & 2.2 & $<10$ & $<2$ & 97 & 66 \\
\hline A18 & .78 & .14 & .31 & 11 & 355 & 1.8 & $<10$ & $<2$ & 84 & 54 \\
\hline A19 & .83 & .15 & .25 & $<10$ & 328 & 1.5 & $<10$ & $<2$ & 63 & 44 \\
\hline A 20 & 1.20 & .24 & .36 & 4.1 & 410 & 2.0 & $<10$ & .2 & 84 & 78 \\
\hline
\end{tabular}


Table 1. Quantitative elemental analysis of the less than 63-micrometer fraction and bulk sample grain size distribution of streambed-sediment samples, threshold effects concentrations (TEC), and probable effects concentrations (PEC) for selected trace elements, 1992-2002.—Continued

[mm/dd/yyyy, month/day/year; \% wt, percent by weight; NA, not applicable; es, east side; ws, west side; mg/kg, milligrams per kilogram; <, less than; shaded cells indicate PEC exceedance; --, no data]

\begin{tabular}{|c|c|c|c|c|c|c|c|c|c|c|}
\hline $\begin{array}{c}\text { Site } \\
\text { identifier }\end{array}$ & $\begin{array}{c}\text { Potassium } \\
\text { (\% wt) }\end{array}$ & $\begin{array}{l}\text { Sodium } \\
\text { (\% wt) }\end{array}$ & $\begin{array}{c}\text { Titanium } \\
\text { (\% wt) }\end{array}$ & $\begin{array}{l}\text { Arsenic } \\
(\mathrm{mg} / \mathrm{kg})\end{array}$ & $\begin{array}{l}\text { Barium } \\
(\mathrm{mg} / \mathrm{kg})\end{array}$ & $\begin{array}{l}\text { Beryllium } \\
\text { (mg/kg) }\end{array}$ & $\begin{array}{l}\text { Bismuth } \\
\text { (mg/kg) }\end{array}$ & $\begin{array}{l}\text { Cadmium } \\
\text { (mg/kg) }\end{array}$ & $\begin{array}{c}\text { Cerium } \\
(\mathrm{mg} / \mathrm{kg})\end{array}$ & $\begin{array}{c}\text { Chromium } \\
\text { (mg/kg) }\end{array}$ \\
\hline \multicolumn{11}{|c|}{ Group B (Near-mining sites; within 7.5 miles downstream from mining activity) } \\
\hline B1 & 0.95 & 0.18 & 0.28 & 22 & 580 & 1.8 & $<10$ & 2.3 & 78 & 74 \\
\hline $\mathrm{B} 2$ & .73 & .14 & .20 & 35 & 230 & 2.2 & $<10$ & $<2$ & 62 & 53 \\
\hline B3 & .70 & .15 & .20 & 10 & 300 & 1.4 & $<10$ & $<2$ & 57 & 44 \\
\hline B4 & .63 & .23 & .14 & 7.2 & 240 & 1.0 & $<10$ & 2.2 & 38 & 24 \\
\hline B5 & .79 & .16 & .24 & 17 & 300 & 1.7 & $<10$ & $<2$ & 78 & 60 \\
\hline \multirow[t]{2}{*}{ B6 } & .93 & .26 & .29 & 10 & 310 & 2.0 & $<10$ & 3.3 & 65 & 38 \\
\hline & .83 & .17 & .26 & 14 & 390 & 1.7 & $<10$ & $<2$ & 71 & 50 \\
\hline B7 & .74 & .16 & .26 & $<10$ & 470 & 1.9 & $<10$ & $<2$ & 83 & 50 \\
\hline B8 & .60 & .11 & .20 & $<20$ & 375 & 2.0 & $<20$ & 9.9 & 76 & 60 \\
\hline \multicolumn{11}{|c|}{ Group C (Distal-mining sites; more than 7.5 miles downstream from active mining) } \\
\hline $\mathrm{C} 1$ & 0.94 & 0.24 & 0.27 & $<10$ & 400 & 1.5 & $<10$ & $<2$ & 78 & 51 \\
\hline $\mathrm{C} 2$ & .91 & .23 & .27 & 10 & 450 & 1.3 & $<10$ & $<2$ & 72 & 72 \\
\hline $\mathrm{C} 3$ & 1.60 & .20 & .32 & 14 & 390 & 3.0 & $<10$ & .8 & 98 & 53 \\
\hline $\mathrm{C} 4$ & 1.30 & .16 & .26 & 18 & 370 & 2.1 & $<10$ & $<2$ & 89 & 61 \\
\hline $\mathrm{C} 5$ & 1.40 & .25 & .26 & 11 & 410 & 2.0 & $<10$ & .7 & 78 & 47 \\
\hline C6 & .77 & .22 & .25 & 5.5 & 310 & 2.0 & $<10$ & 1 & 59 & 31 \\
\hline \multirow[t]{2}{*}{$\mathrm{C} 7$} & .84 & .21 & .29 & 6.1 & 290 & 2.0 & $<10$ & .6 & 79 & 45 \\
\hline & .71 & .16 & .24 & $<10$ & 310 & 1.6 & $<10$ & $<2$ & 68 & 55 \\
\hline \multirow[t]{2}{*}{$\mathrm{C} 8$} & .99 & .26 & .27 & 7.6 & 370 & 2.0 & $<10$ & .6 & 76 & 42 \\
\hline & 1.10 & .19 & .30 & 13 & 400 & 2.0 & $<10$ & $<2$ & 87 & 52 \\
\hline C9 & .68 & .11 & .22 & $<20$ & 383 & $<2$ & $<20$ & $<3$ & 74 & 59 \\
\hline \multirow[t]{2}{*}{$\mathrm{C} 10$} & .99 & .15 & .25 & $<10$ & 278 & 2.3 & $<10$ & 1.60 & 64 & 58 \\
\hline & 1.02 & .19 & .24 & $<10$ & 369 & 2.1 & $<10$ & 1.66 & 70 & 74 \\
\hline $\mathrm{TEC}^{\mathrm{c}}$ & NA & NA & NA & 9.79 & NA & NA & NA & 0.99 & NA & 43.4 \\
\hline $\mathrm{PEC}^{\mathrm{c}}$ & NA & NA & NA & 33.0 & NA & NA & NA & 4.98 & NA & 111 \\
\hline
\end{tabular}


Table 1. Quantitative elemental analysis of the less than 63-micrometer fraction and bulk sample grain size distribution of streambed-sediment samples, threshold effects concentrations (TEC), and probable effects concentrations (PEC) for selected trace elements, 1992-2002.-Continued

[mm/dd/yyyy, month/day/year; \% wt, percent by weight; NA, not applicable; es, east side; ws, west side; mg/kg, milligrams per kilogram; <, less than; shaded cells indicate PEC exceedance; --, no data]

\begin{tabular}{|c|c|c|c|c|c|c|c|c|c|c|}
\hline $\begin{array}{c}\text { Site } \\
\text { identifier }\end{array}$ & $\begin{array}{l}\text { Cobalt } \\
\text { (mg/kg) }\end{array}$ & $\begin{array}{c}\text { Copper } \\
\text { (mg/kg) }\end{array}$ & $\begin{array}{c}\text { Europium } \\
\text { (mg/kg) }\end{array}$ & $\begin{array}{l}\text { Gallium } \\
\text { (mg/kg) }\end{array}$ & $\begin{array}{c}\text { Gold } \\
(\mathrm{mg} / \mathrm{kg})\end{array}$ & $\begin{array}{c}\text { Holmium } \\
\text { (mg/kg) }\end{array}$ & $\begin{array}{l}\text { Lanthanum } \\
\text { (mg/kg) }\end{array}$ & $\begin{array}{c}\text { Lead } \\
(\mathrm{mg} / \mathrm{kg})\end{array}$ & $\begin{array}{l}\text { Lithium } \\
\text { (mg/kg) }\end{array}$ & $\begin{array}{c}\text { Manganese } \\
(\mathrm{mg} / \mathrm{kg})\end{array}$ \\
\hline \multicolumn{11}{|c|}{ Group A (Non-mining sites) } \\
\hline A1 & 15 & 26 & $<2$ & 9 & $<8$ & $<4$ & 32 & 80 & 20 & 510 \\
\hline \multirow[t]{2}{*}{ A2 } & 16 & 17 & $<2$ & 7 & $<8$ & $<4$ & 30 & 33 & 20 & 610 \\
\hline & 20 & 130 & $<2$ & 8.7 & $<8$ & $<4$ & 33 & b210 & 21 & 660 \\
\hline A3 & 38 & 85 & $<2$ & 10 & $<8$ & $<4$ & 32 & 53 & 22 & 2,000 \\
\hline A4 & 24 & 142 & $<2$ & 11 & $<8$ & $<4$ & 42 & 97 & 30 & 1,740 \\
\hline A5 & 16 & 19 & $<2$ & 10 & $<8$ & $<4$ & 37 & 24 & 31 & 760 \\
\hline A6 & 29 & 97 & $<2$ & 11 & $<8$ & $<4$ & 36 & 61 & 29 & 1,760 \\
\hline A7 & 22 & 27 & $<2$ & 14 & $<8$ & $<4$ & 45 & 24 & 37 & 843 \\
\hline A8 & 18 & 138 & $<2$ & 10 & $<8$ & $<4$ & 40 & 85 & 26 & 1,480 \\
\hline A9 & 26 & 29 & $<2$ & 12 & $<8$ & $<4$ & 42 & 33 & 31 & 1,600 \\
\hline \multirow[t]{2}{*}{ A10 } & 28 & 40 & 2 & 24 & $<8$ & $<4$ & 57 & 53 & 43 & 1,300 \\
\hline & 16 & 61 & $<2$ & 10 & $<8$ & $<4$ & 43 & 49 & 26 & 1,190 \\
\hline A11 & 19 & 39 & $<2$ & 14 & $<8$ & $<4$ & 42 & 45 & 28 & 750 \\
\hline A12 & 31 & 31 & $<2$ & 13 & $<8$ & $<4$ & 42 & 35 & 29 & 1,500 \\
\hline A13 & 12 & 166 & $<2$ & 7.9 & $<8$ & $<4$ & 36 & 72 & 20 & 892 \\
\hline A14 & 20 & 85 & $<2$ & 12 & $<8$ & $<4$ & 42 & 79 & 31 & 1,740 \\
\hline A15 & 14 & 108 & $<2$ & 9.9 & $<8$ & $<4$ & 42 & 72 & 26 & 1,230 \\
\hline A16 & 10 & 89 & $<2$ & 12 & $<8$ & $<4$ & 50 & 72 & 32 & 823 \\
\hline A17 & 20 & 99 & $<2$ & 9.9 & $<8$ & $<4$ & 46 & 84 & 27 & 2,210 \\
\hline A18 & 12 & 74 & $<2$ & 9 & $<8$ & $<4$ & 42 & 48 & 22 & 1,280 \\
\hline A19 & 10 & 80 & $<2$ & 7.4 & $<8$ & $<4$ & 33 & 44 & 19 & 739 \\
\hline A20 & 9 & 15 & $<2$ & 14 & $<8$ & $<4$ & 55 & 27 & 38 & 170 \\
\hline
\end{tabular}


Table 1. Quantitative elemental analysis of the less than 63-micrometer fraction and bulk sample grain size distribution of streambed-sediment samples, threshold effects concentrations (TEC), and probable effects concentrations (PEC) for selected trace elements, 1992-2002.-Continued

[mm/dd/yyyy, month/day/year; \% wt, percent by weight; NA, not applicable; es, east side; ws, west side; mg/kg, milligrams per kilogram; <, less than; shaded cells indicate PEC exceedance; --, no data]

\begin{tabular}{|c|c|c|c|c|c|c|c|c|c|c|}
\hline $\begin{array}{c}\text { Site } \\
\text { identifier }\end{array}$ & $\begin{array}{c}\text { Cobalt } \\
\text { (mg/kg) }\end{array}$ & $\begin{array}{l}\text { Copper } \\
\text { (mg/kg) }\end{array}$ & $\begin{array}{l}\text { Europium } \\
(\mathrm{mg} / \mathrm{kg})\end{array}$ & $\begin{array}{l}\text { Gallium } \\
\text { (mg/kg) }\end{array}$ & $\begin{array}{c}\text { Gold } \\
(\mathrm{mg} / \mathrm{kg})\end{array}$ & $\begin{array}{l}\text { Holmium } \\
\text { (mg/kg) }\end{array}$ & $\begin{array}{c}\text { Lanthanum } \\
\text { (mg/kg) }\end{array}$ & $\begin{array}{c}\text { Lead } \\
(\mathrm{mg} / \mathrm{kg})\end{array}$ & $\begin{array}{l}\text { Lithium } \\
\text { (mg/kg) }\end{array}$ & $\begin{array}{c}\text { Manganese } \\
(\mathrm{mg} / \mathrm{kg})\end{array}$ \\
\hline \multicolumn{11}{|c|}{ Group B (Near-mining sites; within 7.5 miles downstream from mining activity) } \\
\hline B1 & 88 & 240 & $<2$ & 13 & $<8$ & $<4$ & 37 & 510 & 32 & 1,500 \\
\hline B2 & 1,200 & 180 & $<2$ & 6.6 & $<8$ & $<4$ & 30 & 860 & 28 & 4,200 \\
\hline B3 & 76 & 120 & $<2$ & 8.2 & $<8$ & $<4$ & 28 & 150 & 22 & 1,400 \\
\hline B4 & 150 & 31 & $<2$ & 5 & $<8$ & $<4$ & 21 & 200 & 20 & 910 \\
\hline B5 & 160 & 110 & $<2$ & 10 & $<8$ & $<4$ & 34 & 940 & 29 & 1,500 \\
\hline \multirow[t]{2}{*}{ B6 } & 43 & 36 & $<2$ & 8 & $<8$ & $<4$ & 34 & 950 & 30 & 490 \\
\hline & 150 & 98 & $<2$ & 12 & $<8$ & $<4$ & 33 & 420 & 27 & 4,700 \\
\hline B7 & 67 & 150 & $<2$ & 11 & $<8$ & $<4$ & 36 & 230 & 26 & 4,300 \\
\hline B8 & 74 & 180 & $<4$ & 8.8 & $<10$ & $<7$ & 32 & 1,410 & 24 & 6,470 \\
\hline \multicolumn{11}{|c|}{ Group C (Distal-mining sites; more than 7.5 miles downstream from active mining) } \\
\hline $\mathrm{C} 1$ & 18 & 110 & $<2$ & 12 & $<8$ & $<4$ & 37 & 100 & 27 & 950 \\
\hline $\mathrm{C} 2$ & 19 & 110 & $<2$ & 10 & $<8$ & $<4$ & 34 & b88 & 24 & 1,000 \\
\hline $\mathrm{C} 3$ & 31 & 37 & $<2$ & 11 & $<8$ & $<4$ & 46 & 100 & 30 & 760 \\
\hline $\mathrm{C} 4$ & 49 & 120 & $<2$ & 12 & $<8$ & $<4$ & 38 & 160 & 26 & 1,400 \\
\hline C5 & 29 & 32 & $<2$ & 11 & $<8$ & $<4$ & 38 & 100 & 26 & 1,500 \\
\hline C6 & 18 & 22 & $<2$ & 7 & $<8$ & $<4$ & 31 & 170 & 20 & 450 \\
\hline \multirow[t]{2}{*}{ C7 } & 20 & 29 & $<2$ & 10 & $<8$ & $<4$ & 40 & 100 & 30 & 280 \\
\hline & 30 & 120 & $<2$ & 9.4 & $<8$ & $<4$ & 30 & 170 & 25 & 1,100 \\
\hline \multirow[t]{2}{*}{$\mathrm{C} 8$} & 25 & 21 & $<2$ & 8 & $<8$ & $<4$ & 37 & 84 & 22 & 1,500 \\
\hline & 31 & 87 & $<2$ & 13 & $<8$ & $<4$ & 39 & b82 & 26 & 1,700 \\
\hline C9 & 31 & 140 & $<3$ & 9.1 & $<10$ & $<6$ & 29 & 93 & 23 & 2,330 \\
\hline \multirow[t]{2}{*}{$\mathrm{C} 10$} & 20 & 35 & $<2$ & 13 & $<8$ & $<4$ & 40 & 27 & 40 & 388 \\
\hline & 27 & 47 & $<2$ & 11 & $<8$ & $<4$ & 38 & 25 & 32 & 1,580 \\
\hline $\mathrm{TEC}^{\mathrm{c}}$ & NA & 31.6 & NA & NA & NA & NA & NA & 35.8 & NA & NA \\
\hline $\mathrm{PEC}^{\mathrm{c}}$ & NA & 149 & NA & NA & NA & NA & NA & 128 & NA & NA \\
\hline
\end{tabular}


Table 1. Quantitative elemental analysis of the less than 63-micrometer fraction and bulk sample grain size distribution of streambed-sediment samples, threshold effects concentrations (TEC), and probable effects concentrations (PEC) for selected trace elements, 1992-2002.-Continued

[mm/dd/yyyy, month/day/year; \% wt, percent by weight; NA, not applicable; es, east side; ws, west side; mg/kg, milligrams per kilogram; <, less than; shaded cells indicate PEC exceedance; --, no data]

\begin{tabular}{|c|c|c|c|c|c|c|c|c|c|c|}
\hline $\begin{array}{c}\text { Site } \\
\text { identifier }\end{array}$ & $\begin{array}{l}\text { Molybdenum } \\
\text { (mg/kg) }\end{array}$ & $\begin{array}{l}\text { Neodybium } \\
\text { (mg/kg) }\end{array}$ & $\begin{array}{l}\text { Nickel } \\
\text { (mg/kg) }\end{array}$ & $\begin{array}{l}\text { Niobium } \\
(\mathrm{mg} / \mathrm{kg})\end{array}$ & $\begin{array}{l}\text { Scandium } \\
\text { (mg/kg) }\end{array}$ & $\begin{array}{c}\text { Silver } \\
(\mathrm{mg} / \mathrm{kg})\end{array}$ & $\begin{array}{l}\text { Strontium } \\
(\mathrm{mg} / \mathrm{kg})\end{array}$ & $\begin{array}{l}\text { Tantalum } \\
\text { (mg/kg) }\end{array}$ & $\begin{array}{l}\text { Thorium } \\
\text { (mg/kg) }\end{array}$ & $\underset{(\mathrm{mg} / \mathrm{kg})}{\mathrm{Tin}}$ \\
\hline \multicolumn{11}{|c|}{ Group A (Non-mining sites) } \\
\hline A1 & $<2$ & 28 & 28 & 2 & 7 & 0.2 & 65 & $<40$ & 7.0 & 5 \\
\hline \multirow[t]{2}{*}{ A2 } & $<2$ & 28 & 21 & 12 & 5 & .1 & 51 & $<40$ & 10.0 & 5 \\
\hline & $<2$ & 31 & 27 & 14 & 5.9 & $<2$ & 41 & $<40$ & 9.0 & 11 \\
\hline A3 & 2.1 & 32 & 37 & 6.3 & 6.6 & $<2$ & 34 & $<40$ & 7.7 & 11 \\
\hline A4 & 2 & 41 & 40 & 8.4 & 9.2 & $<2$ & 34 & $<40$ & 7.3 & 17 \\
\hline A5 & $<2$ & 34 & 30 & 9 & 8 & .2 & 46 & $<40$ & 9.0 & $<5$ \\
\hline A6 & 2.6 & 36 & 45 & 8.3 & 9.5 & $<2$ & 30 & $<40$ & 8.9 & 16 \\
\hline A7 & $<2$ & 41 & 36 & 16 & 9.7 & $<2$ & 48 & $<40$ & 11.9 & $<5$ \\
\hline A8 & $<2$ & 34 & 33 & 8.8 & 7.5 & $<2$ & 36 & $<40$ & 7.1 & 23 \\
\hline A9 & $<2$ & 41 & 29 & 17 & 8 & $<2$ & 43 & $<40$ & 10.0 & $<5$ \\
\hline \multirow[t]{2}{*}{ A10 } & 2 & 67 & 51 & 12 & 12 & $<2$ & 40 & $<40$ & 11.0 & $<5$ \\
\hline & $<2$ & 40 & 35 & 11 & 8.5 & $<2$ & 39 & $<40$ & 6.5 & 7.3 \\
\hline A11 & $<2$ & 49 & 35 & 10 & 8 & $<2$ & 38 & $<40$ & 6.0 & $<5$ \\
\hline A12 & $<2$ & 41 & 34 & 16 & 8 & $<2$ & 40 & $<40$ & 9.0 & $<5$ \\
\hline A13 & 2.3 & 28 & 24 & 8.1 & 5.7 & $<2$ & 36 & $<40$ & 6.4 & 16 \\
\hline A14 & 3.1 & 36 & 41 & 9.4 & 8.8 & $<2$ & 29 & $<40$ & 6.8 & 12 \\
\hline A15 & 2 & 35 & 34 & 11 & 7.5 & $<2$ & 34 & $<40$ & 6.8 & 8.9 \\
\hline A16 & $<2$ & 47 & 35 & 13 & 10 & $<2$ & 33 & $<40$ & 7.9 & 7.9 \\
\hline A17 & 2.9 & 38 & 42 & 5.7 & 7.6 & $<2$ & 37 & $<40$ & 7.3 & 18 \\
\hline A18 & $<2$ & 35 & 27 & 7.1 & 6.8 & $<2$ & 31 & $<40$ & 6.7 & 12 \\
\hline A19 & $<2$ & 28 & 24 & 7.5 & 5.4 & $<2$ & 34 & $<40$ & 4.9 & 8.6 \\
\hline A20 & $<2$ & 46 & 23 & 11 & 10 & .1 & 52 & $<40$ & 15.0 & $<5$ \\
\hline
\end{tabular}


Table 1. Quantitative elemental analysis of the less than 63-micrometer fraction and bulk sample grain size distribution of streambed-sediment samples, threshold effects concentrations (TEC), and probable effects concentrations (PEC) for selected trace elements, 1992-2002.-Continued

[mm/dd/yyyy, month/day/year; \% wt, percent by weight; NA, not applicable; es, east side; ws, west side; mg/kg, milligrams per kilogram; <, less than; shaded cells indicate PEC exceedance; --, no data]

\begin{tabular}{|c|c|c|c|c|c|c|c|c|c|c|}
\hline $\begin{array}{c}\text { Site } \\
\text { identifier }\end{array}$ & $\begin{array}{c}\text { Molybdenum } \\
(\mathrm{mg} / \mathrm{kg})\end{array}$ & $\begin{array}{c}\text { Neodybium } \\
\text { (mg/kg) }\end{array}$ & $\begin{array}{c}\text { Nickel } \\
\text { (mg/kg) }\end{array}$ & $\begin{array}{l}\text { Niobium } \\
\text { (mg/kg) }\end{array}$ & $\begin{array}{c}\text { Scandium } \\
\text { (mg/kg) }\end{array}$ & $\begin{array}{c}\text { Silver } \\
(\mathrm{mg} / \mathrm{kg})\end{array}$ & $\begin{array}{c}\text { Strontium } \\
\text { (mg/kg) }\end{array}$ & $\begin{array}{c}\text { Tantalum } \\
\text { (mg/kg) }\end{array}$ & $\begin{array}{l}\text { Thorium } \\
\text { (mg/kg) }\end{array}$ & $\begin{array}{c}\text { Tin } \\
(\mathrm{mg} / \mathrm{kg})\end{array}$ \\
\hline \multicolumn{11}{|c|}{ Group B (Near-mining sites; within 7.5 miles downstream from mining activity) } \\
\hline B1 & 2 & 34 & 100 & 14 & 8.6 & $<2$ & 49 & $<40$ & 14.0 & 18 \\
\hline B2 & 6.5 & 30 & 580 & $<4$ & 7.4 & $<2$ & 48 & $<40$ & 11.0 & 15 \\
\hline B3 & $<2$ & 27 & 140 & 10 & 6.1 & $<2$ & 52 & $<40$ & 9.1 & 6.8 \\
\hline B4 & $<2$ & 19 & 160 & 8 & 3 & .1 & 71 & $<40$ & 2.0 & 5 \\
\hline B5 & $<2$ & 32 & 140 & 9.3 & 6.6 & $<2$ & 48 & $<40$ & 11.0 & 16 \\
\hline \multirow[t]{2}{*}{ B6 } & $<2$ & 30 & 73 & 7 & 6 & .3 & 55 & $<40$ & 9.0 & 5 \\
\hline & $<2$ & 28 & 180 & $<4$ & 7 & $<2$ & 43 & $<40$ & 11.0 & 11 \\
\hline B7 & 2 & 36 & 76 & $<4$ & 7.6 & $<2$ & 42 & $<40$ & 9.5 & 18 \\
\hline B8 & 4.1 & 29 & 91 & $<7$ & 6.5 & $<4$ & 36 & $<70$ & 7.8 & 27 \\
\hline \multicolumn{11}{|c|}{ Group C (Distal-mining sites; more than 7.5 miles downstream from active mining) } \\
\hline $\mathrm{C} 1$ & $<2$ & 33 & 29 & 16 & 7.3 & $<2$ & 47 & $<40$ & 12.0 & 12 \\
\hline $\mathrm{C} 2$ & $<2$ & 30 & 30 & 14 & 6.4 & $<2$ & 48 & $<40$ & 11.0 & 18 \\
\hline $\mathrm{C} 3$ & $<2$ & 45 & 50 & 8 & 10 & .2 & 55 & $<40$ & 10.0 & 5 \\
\hline $\mathrm{C} 4$ & 2 & 40 & 78 & 18 & 9 & $<2$ & 42 & $<40$ & 12.0 & 19 \\
\hline C5 & $<2$ & 39 & 37 & 9 & 9 & .2 & 56 & $<40$ & 12.0 & $<5$ \\
\hline C6 & $<2$ & 29 & 31 & 7 & 5 & .2 & 61 & $<40$ & 8.0 & 5 \\
\hline \multirow[t]{2}{*}{$\mathrm{C} 7$} & $<2$ & 39 & 39 & 6 & 9 & .2 & 50 & $<40$ & 10.0 & 5 \\
\hline & $<2$ & 30 & 44 & 12 & 6.2 & $<2$ & 38 & $<40$ & 9.4 & 18 \\
\hline \multirow[t]{2}{*}{$\mathrm{C} 8$} & $<2$ & 34 & 29 & 6 & 7 & .2 & 51 & $<40$ & 9.0 & $<5$ \\
\hline & $<2$ & 37 & 42 & 18 & 8.5 & $<2$ & 43 & $<40$ & 12.0 & 11 \\
\hline C9 & $<3$ & 26 & 38 & $<6$ & 6.6 & $<3$ & 28 & $<60$ & 6.6 & 44 \\
\hline \multirow[t]{2}{*}{$\mathrm{C} 10$} & $<2$ & 42 & 36 & 13 & 8.9 & $<2$ & 45 & $<40$ & 11.3 & $<5$ \\
\hline & $<2$ & 34 & 47 & 12 & 7.9 & $<2$ & 57 & $<40$ & 9.1 & $<5$ \\
\hline $\mathrm{TEC}^{\mathrm{c}}$ & NA & NA & 22.7 & NA & NA & NA & NA & NA & NA & NA \\
\hline $\mathrm{PEC}^{\mathrm{c}}$ & NA & NA & 48.6 & NA & NA & NA & NA & NA & NA & NA \\
\hline
\end{tabular}


Table 1. Quantitative elemental analysis of the less than 63-micrometer fraction and bulk sample grain size distribution of streambed-sediment samples, threshold effects concentrations (TEC), and probable effects concentrations (PEC) for selected trace elements, 1992-2002. - Continued

[mm/dd/yyyy, month/day/year; \% wt, percent by weight; NA, not applicable; es, east side; ws, west side; mg/kg, milligrams per kilogram; <, less than; shaded cells indicate PEC exceedance; --, no data]

\begin{tabular}{|c|c|c|c|c|c|c|c|c|}
\hline \multirow{2}{*}{$\begin{array}{c}\text { Site } \\
\text { identifier }\end{array}$} & \multirow{2}{*}{$\begin{array}{l}\text { Uranium } \\
\text { (mg/kg) }\end{array}$} & \multirow{2}{*}{$\begin{array}{c}\text { Vanadium } \\
(\mathrm{mg} / \mathrm{kg})\end{array}$} & \multirow{2}{*}{$\begin{array}{c}\text { Ytterbium } \\
\text { (mg/kg) }\end{array}$} & \multirow{2}{*}{$\begin{array}{r}\text { Yttrium } \\
\text { (mg/kg) }\end{array}$} & \multirow{2}{*}{$\begin{array}{c}\text { Zinc } \\
(\mathrm{mg} / \mathrm{kg})\end{array}$} & \multicolumn{3}{|c|}{$\begin{array}{l}\text { Bulk grain size distribution } \\
\text { Percentage }\end{array}$} \\
\hline & & & & & & Coarse & Silt & Clay \\
\hline \multicolumn{9}{|c|}{ Group A (Non-mining sites) } \\
\hline A1 & 2.6 & 53 & 2.0 & 21 & 180 & -- & -- & -- \\
\hline \multirow[t]{2}{*}{ A2 } & 3.4 & 53 & 2.0 & 20 & 150 & -- & -- & -- \\
\hline & $<100$ & 58 & 2.2 & 20 & 100 & 96.8 & 2.5 & 0.6 \\
\hline A3 & $<100$ & 69 & 2.7 & 26 & 83 & 98.7 & 1.0 & .3 \\
\hline A4 & $<100$ & 73 & 3.3 & 35 & 280 & 99.3 & .4 & .3 \\
\hline A5 & 4.4 & 63 & 3.0 & 29 & 64 & -- & -- & -- \\
\hline A6 & $<100$ & 81 & 3.2 & 33 & 159 & 99.2 & .3 & .4 \\
\hline A7 & $<100$ & 81 & 3.0 & 30 & 81 & 97.7 & 1.1 & 1.2 \\
\hline A8 & $<100$ & 63 & 2.5 & 28 & 134 & 99.1 & .7 & .2 \\
\hline A9 & $<100$ & 70 & 3.0 & 28 & 74 & 98.5 & 1.0 & .6 \\
\hline \multirow[t]{2}{*}{ A10 } & $<100$ & 90 & 4.0 & 44 & 160 & 98.0 & 1.0 & 1.0 \\
\hline & $<100$ & 66 & 2.8 & 30 & 114 & 95.0 & 4.0 & 1.0 \\
\hline A11 & $<100$ & 62 & 3.0 & 32 & 110 & 99.1 & .4 & .4 \\
\hline A 12 & $<100$ & 68 & 3.0 & 28 & 87 & 98.6 & .8 & .6 \\
\hline A13 & $<100$ & 50 & 1.8 & 20 & 142 & 97.2 & 2.2 & .5 \\
\hline A14 & $<100$ & 80 & 2.4 & 27 & 166 & 98.8 & .8 & .3 \\
\hline A15 & $<100$ & 64 & 2.5 & 28 & 127 & 97.8 & 1.5 & .6 \\
\hline A16 & $<100$ & 72 & 3.0 & 32 & 152 & 91.6 & 7.0 & 1.4 \\
\hline A17 & $<100$ & 74 & 2.7 & 31 & 151 & 98.0 & 1.6 & .5 \\
\hline A18 & $<100$ & 51 & 2.4 & 27 & 128 & 98.0 & 1.6 & .3 \\
\hline A19 & $<100$ & 43 & 1.9 & 21 & 115 & 98.3 & 1.4 & .3 \\
\hline A20 & 4.8 & 87 & 3.0 & 31 & 54 & -- & -- & -- \\
\hline
\end{tabular}


Table 1. Quantitative elemental analysis of the less than 63-micrometer fraction and bulk sample grain size distribution of streambed-sediment samples, threshold effects concentrations (TEC), and probable effects concentrations (PEC) for selected trace elements, 1992-2002.-Continued

[mm/dd/yyyy, month/day/year; \% wt, percent by weight; NA, not applicable; es, east side; ws, west side; mg/kg, milligrams per kilogram; <, less than; shaded cells indicate PEC exceedance; --, no data]

\begin{tabular}{|c|c|c|c|c|c|c|c|c|}
\hline \multirow{2}{*}{$\begin{array}{c}\text { Site } \\
\text { identifier }\end{array}$} & \multirow{2}{*}{$\begin{array}{l}\text { Uranium } \\
\text { (mg/kg) }\end{array}$} & \multirow{2}{*}{$\begin{array}{l}\text { Vanadium } \\
\text { (mg/kg) }\end{array}$} & \multirow{2}{*}{$\begin{array}{l}\text { Ytterbium } \\
\text { (mg/kg) }\end{array}$} & \multirow{2}{*}{$\begin{array}{l}\text { Yttrium } \\
\text { (mg/kg) }\end{array}$} & \multirow{2}{*}{$\underset{(\mathrm{mg} / \mathrm{kg})}{\text { Zinc }}$} & \multicolumn{3}{|c|}{$\begin{array}{c}\text { Bulk grain size distribution } \\
\text { Percentage }\end{array}$} \\
\hline & & & & & & Coarse & Silt & Clay \\
\hline \multicolumn{9}{|c|}{ Group B (Near-mining sites; within 7.5 miles downstream from mining activity) } \\
\hline B1 & $<100$ & 97 & 2.7 & 24 & 850 & 98.5 & 1.1 & .4 \\
\hline B2 & $<100$ & 74 & 3.0 & 29 & 2,700 & 97.1 & 1.9 & 1.0 \\
\hline B3 & $<100$ & 63 & 2.0 & 18 & 810 & 97.3 & 1.7 & 1.0 \\
\hline B4 & 2.2 & 35 & 1.0 & 13 & 1,200 & -- & -- & -- \\
\hline B5 & $<100$ & 78 & 2.3 & 21 & 1,300 & 98.4 & .8 & .8 \\
\hline \multirow[t]{2}{*}{ B6 } & 3.6 & 63 & 2.0 & 24 & 460 & -- & -- & -- \\
\hline & $<100$ & 74 & 2.6 & 24 & 720 & 98.4 & 1.1 & .4 \\
\hline B7 & $<100$ & 70 & 3.4 & 33 & 200 & 98.5 & 1.2 & .3 \\
\hline B8 & $<200$ & 66 & 2.5 & 26 & 1,060 & 99.4 & .2 & .4 \\
\hline \multicolumn{9}{|c|}{ Group C (Distal-mining sites; more than 7.5 miles downstream from active mining) } \\
\hline $\mathrm{C} 1$ & $<100$ & 78 & 2.2 & 20 & 120 & 96.4 & 3.4 & .2 \\
\hline $\mathrm{C} 2$ & $<100$ & 74 & 2.1 & 19 & 210 & 99.4 & .4 & .2 \\
\hline $\mathrm{C} 3$ & 4 & 79 & 4.0 & 38 & 210 & -- & -- & -- \\
\hline $\mathrm{C} 4$ & $<100$ & 83 & 3.4 & 32 & 340 & 98.9 & .6 & .4 \\
\hline C5 & 4.1 & 69 & 28 & 3 & 150 & -- & -- & -- \\
\hline C6 & 2.7 & 52 & 2.0 & 22 & 200 & -- & -- & -- \\
\hline \multirow[t]{2}{*}{ C7 } & 3.7 & 74 & 3.0 & 33 & 120 & -- & -- & -- \\
\hline & $<100$ & 63 & 2.5 & 24 & 140 & 99.5 & .5 & $<0.1$ \\
\hline \multirow[t]{2}{*}{$\mathrm{C} 8$} & 3.9 & 56 & 2.0 & 24 & 91 & -- & -- & -- \\
\hline & $<100$ & 84 & 3.1 & 30 & 140 & 98.0 & 1.3 & .7 \\
\hline C9 & $<200$ & 69 & 2.1 & 22 & 130 & 99.5 & .1 & .4 \\
\hline \multirow[t]{2}{*}{$\mathrm{C} 10$} & $<100$ & 76 & 2.1 & 31 & 86 & 98.2 & .6 & 1.2 \\
\hline & $<100$ & 72 & 2.5 & 26 & 86 & 99.5 & .3 & .3 \\
\hline TEC $^{c}$ & NA & NA & NA & NA & 121 & NA & NA & NA \\
\hline $\mathrm{PEC}^{\mathrm{c}}$ & NA & NA & NA & NA & 459 & NA & NA & NA \\
\hline
\end{tabular}

${ }^{\text {a }}$ Sample collected as part of the National Water-Quality Assessment (NAWQA) Program.

${ }^{\mathrm{b}}$ Anthropogenic lead (lead shot) contaminated sample.

${ }^{\mathrm{c}}$ Concentrations from MacDonald and others, 2000. 
Table 2. Optical mineralogy of non-magnetic fraction (C3) of heavy mineral concentrate for streambed-sediment samples, $1995-2002$.

[All values are in percent; --, not detected; es, east side; ws, west side; contam, contaminated with man-made material]

\begin{tabular}{|c|c|c|c|c|c|c|c|c|c|c|c|c|c|}
\hline $\begin{array}{c}\text { Site } \\
\text { identifier } \\
\text { (fig. 1) }\end{array}$ & Sample name & Date & Dolomite & $\begin{array}{l}\text { Limonite } \\
\text { oxidized } \\
\text { rock } \\
\text { fragments }\end{array}$ & Zircon & Apatite & Rutile & Pyrite & Tourmaline & Anatase & Sphene & Barite & Cassiterite \\
\hline \multicolumn{14}{|c|}{ Group A (Non-mining sites) } \\
\hline$\overline{\mathrm{A} 2}$ & West Fork Black River near Greeley & $05 / 06 / 2002$ & 5 & 50 & -- & 5 & -- & 25 & -- & Trace & 5 & -- & -- \\
\hline A3 & Sinking Creek near Redford & 05/07/2002 & 30 & 40 & Trace & 25 & -- & -- & -- & 5 & -- & -- & -- \\
\hline A4 & Big Creek at Mauser Mill & $05 / 29 / 2002$ & 5 & 80 & 5 & 5 & -- & -- & -- & 5 & -- & -- & -- \\
\hline A6 & Blair Creek & $05 / 29 / 2002$ & 20 & 60 & Trace & 20 & -- & -- & -- & -- & -- & -- & -- \\
\hline A7 & Current River upstream from Blue Spring & 06/20/1995 & 10 & 10 & 50 & 2 & 20 & Trace & 5 & -- & -- & -- & -- \\
\hline A8 & Current River at Van Buren & $06 / 11 / 2002$ & 50 & -- & Trace & 40 & Trace & -- & -- & Trace & 10 & -- & -- \\
\hline A9 & Current River upstream from Big Spring & ${ }^{\mathrm{a}} 10 / 16 / 1997$ & -- & 5 & 1 & -- & 2 & 6 & 4 & -- & 3 & -- & 8 \\
\hline \multirow[t]{2}{*}{$\mathrm{A} 10$} & Big Spring orifice & ${ }^{\mathrm{a}} 10 / 16 / 1997$ & 6 & 4 & 1 & 8 & 2 & 7 & 3 & -- & -- & 5 & -- \\
\hline & & $06 / 11 / 2002$ & 99 & -- & -- & Trace & -- & -- & -- & -- & -- & -- & -- \\
\hline A11 & Big Spring branch & ${ }^{\mathrm{a}} 10 / 16 / 1997$ & 2 & 3 & 1 & -- & 7 & 5 & 4 & -- & -- & 6 & -- \\
\hline $\mathrm{A} 12$ & Current River downstream from Big Spring & ${ }^{\mathrm{a}} 10 / 16 / 1997$ & 7 & 4 & 1 & 11 & 2 & 6 & 3 & 10 & -- & 5 & -- \\
\hline A13 & Eleven Point River at Thomasville & 06/10/2002 & 35 & 30 & Trace & 35 & -- & -- & -- & -- & -- & -- & -- \\
\hline A14 & Spring Creek Tributary & $06 / 12 / 2002$ & 20 & 50 & 5 & 20 & -- & -- & -- & -- & -- & -- & -- \\
\hline A15 & Spring Creek & $06 / 25 / 2002$ & -- & 5 & -- & 90 & 2 & -- & -- & 3 & -- & -- & -- \\
\hline A16 & McCormack Spring & $06 / 12 / 2002$ & 90 & -- & -- & 10 & Trace & -- & -- & Trace & -- & -- & -- \\
\hline A17 & Greer Spring & $06 / 10 / 2002$ & 90 & 5 & -- & 5 & -- & -- & -- & -- & -- & -- & -- \\
\hline A18 & Hurricane Creek & $06 / 12 / 2002$ & 15 & -- & 2 & 80 & -- & -- & -- & -- & 2 & -- & -- \\
\hline A19 & Eleven Point River at Bardley & $06 / 10 / 2002$ & 90 & -- & Trace & Trace & Trace & -- & -- & Trace & -- & Trace & -- \\
\hline \multicolumn{14}{|c|}{ Group B (Near-mining sites; within 7.5 miles downstream from mining activity) } \\
\hline B1 & Courtois Creek near Courtois & $04 / 29 / 2002$ & 30 & 60 & -- & -- & -- & Trace & -- & -- & -- & -- & -- \\
\hline B2 & Strother Creek near Goodland & $04 / 30 / 2002$ & 90 & 10 & -- & -- & -- & -- & -- & -- & -- & -- & -- \\
\hline B3 & Neals Creek near Goodland & $04 / 30 / 2002$ & 70 & 25 & Trace & Trace & -- & Trace & -- & -- & -- & -- & -- \\
\hline B5 & Bills Creek near Greeley & 05/06/2002 & 20 & 75 & -- & Trace & -- & Trace & -- & Trace & -- & Trace & -- \\
\hline B6 & West Fork Black River at West Fork & ${ }^{\mathrm{a}} 05 / 06 / 2002$ & -- & -- & -- & 4 & 5 & 3 & -- & 2 & -- & -- & -- \\
\hline B7 & Bee Fork Black River near Reynolds & 05/06/2002 & 40 & 20 & 5 & 20 & -- & 10 & -- & Trace & Trace & -- & -- \\
\hline B8 & Logan Creek near Corridon & $05 / 29 / 2002$ & -- & 99 & -- & -- & -- & Trace & -- & -- & -- & -- & -- \\
\hline \multicolumn{14}{|c|}{ Group C (Distal-mining sites; more than 7.5 miles downstream from mining activity) } \\
\hline $\mathrm{C} 1$ & Huzzah Creek near Davisville & $04 / 29 / 2002$ & 30 & 60 & -- & 10 & -- & -- & -- & Trace & -- & -- & -- \\
\hline $\mathrm{C} 2$ & Huzzah Creek near Scotia & $04 / 29 / 2002$ & 20 & -- & -- & 60 & Trace & 5 & -- & 5 & 3 & -- & -- \\
\hline $\mathrm{C} 4$ & Middle Fork Black River near Black & $04 / 30 / 2002$ & 50 & 30 & 5 & 10 & -- & Trace & Trace & Trace & -- & -- & -- \\
\hline $\mathrm{C} 7$ & West Fork Black River at Centerville & 05/07/2002 & 20 & 75 & -- & Trace & -- & Trace & -- & Trace & -- & Trace & -- \\
\hline $\mathrm{C} 8$ & Black River near Lesterville & 05/07/2002 & 20 & 60 & -- & 20 & -- & -- & -- & -- & -- & -- & -- \\
\hline C9 & Logan Creek at Ellington & $05 / 29 / 2002$ & Trace & 98 & -- & Trace & -- & Trace & -- & -- & -- & -- & -- \\
\hline \multirow[t]{2}{*}{$\mathrm{C} 10$} & Current River downstream from Blue Spring_es & 06/20/1995 & 40 & 5 & 40 & 1 & 4 & Trace & 10 & -- & -- & Trace & Trace \\
\hline & Current River downstream from Blue Spring_ws & 06/20/1995 & 30 & 50 & 10 & 1 & 5 & Trace & -- & -- & -- & Trace & -- \\
\hline
\end{tabular}


Table 2. Optical mineralogy of non-magnetic fraction (C3) of heavy mineral concentrate for streambed-sediment samples,1995-2002.-Continued

[All values are in percent; --, not detected; es, east side; ws, west side; contam, contaminated with man-made material]

\begin{tabular}{|c|c|c|c|c|c|c|c|c|c|}
\hline $\begin{array}{c}\text { Site } \\
\text { identifier } \\
\text { (fig. 1) }\end{array}$ & Galena & Pyroxene & Hematite & Amphibole & Mica & $\begin{array}{l}\text { Lead } \\
\text { contam }\end{array}$ & $\begin{array}{c}\text { Zinc } \\
\text { (silvery } \\
\text { metal) }\end{array}$ & Brass & Glass \\
\hline \multicolumn{10}{|c|}{ Group A (Non-mining sites) } \\
\hline A2 & 5 & -- & -- & -- & -- & Trace & -- & -- & -- \\
\hline A3 & -- & -- & -- & -- & -- & -- & -- & -- & -- \\
\hline A4 & -- & -- & -- & -- & -- & -- & -- & -- & -- \\
\hline A6 & -- & -- & -- & -- & -- & -- & -- & -- & -- \\
\hline A7 & -- & -- & -- & -- & -- & -- & -- & -- & -- \\
\hline A8 & -- & -- & -- & -- & -- & -- & -- & -- & Trace \\
\hline A9 & -- & -- & -- & -- & -- & -- & 7 & -- & -- \\
\hline \multirow[t]{2}{*}{ A 10} & -- & -- & -- & -- & -- & 9 & 10 & -- & -- \\
\hline & -- & -- & Trace & -- & -- & -- & -- & -- & Trace \\
\hline A11 & -- & -- & -- & -- & -- & 8 & 9 & -- & -- \\
\hline A12 & -- & -- & -- & -- & -- & 8 & 9 & -- & -- \\
\hline A13 & -- & -- & Trace & -- & -- & -- & -- & -- & -- \\
\hline A14 & -- & -- & 5 & -- & -- & -- & -- & -- & -- \\
\hline A15 & -- & -- & -- & -- & -- & -- & -- & -- & Trace \\
\hline A16 & -- & -- & Trace & -- & -- & -- & Trace & -- & -- \\
\hline A17 & -- & -- & Trace & -- & -- & -- & -- & -- & -- \\
\hline A18 & -- & -- & 1 & -- & -- & -- & -- & -- & -- \\
\hline A19 & -- & 5 & -- & 5 & Trace & -- & -- & -- & -- \\
\hline \multicolumn{10}{|c|}{ Group B (Near-mining sites; within 7.5 miles downstream from mining activity) } \\
\hline B1 & -- & Trace & -- & -- & -- & -- & -- & -- & -- \\
\hline B2 & -- & -- & -- & -- & -- & -- & -- & -- & -- \\
\hline B3 & -- & -- & -- & -- & -- & -- & -- & -- & -- \\
\hline B5 & -- & -- & -- & -- & Trace & -- & -- & -- & -- \\
\hline B6 & -- & -- & -- & -- & 1 & -- & -- & -- & -- \\
\hline B7 & Trace & Trace & -- & -- & Trace & -- & -- & -- & -- \\
\hline B8 & -- & -- & -- & -- & -- & -- & -- & -- & Trace \\
\hline \multicolumn{10}{|c|}{ Group C (Distal-mining sites; more than 7.5 miles downstream from mining activity) } \\
\hline $\mathrm{C} 1$ & -- & -- & -- & -- & -- & -- & -- & -- & -- \\
\hline $\mathrm{C} 2$ & 5 & -- & -- & -- & -- & Trace & -- & -- & -- \\
\hline $\mathrm{C} 4$ & -- & -- & -- & -- & -- & -- & -- & -- & -- \\
\hline C7 & -- & -- & -- & -- & -- & -- & -- & -- & -- \\
\hline $\mathrm{C} 8$ & -- & -- & -- & -- & -- & Trace & -- & -- & -- \\
\hline C9 & -- & -- & -- & -- & -- & -- & -- & -- & -- \\
\hline $\mathrm{C} 10$ & Trace & -- & -- & -- & -- & -- & -- & -- & -- \\
\hline
\end{tabular}


Table 3. Summary statistics for Mississippi Valley Type-related metals and chromium in streambed-sediment samples, $1992-2002$.

[All concentrations in milligrams per kilogram; proximity-to-mining group-A, non-mining sites; B, near-mining sites (less than 7.5 miles downstream from mining activity); C, distal-mining sites (more than 7.5 miles downstream from mining activity); Q5, 5th percentile; Q25, 25th percentile; Q50, 50th percentile; Q75, 75th percentile; Q95, 95th percentile; Interquartile range, difference between Q75 and Q25]

\begin{tabular}{|c|c|c|c|c|c|c|c|c|c|c|c|c|c|c|c|c|}
\hline & \multicolumn{4}{|c|}{ Arsenic } & \multicolumn{4}{|c|}{ Cadmium } & \multicolumn{4}{|c|}{ Cobalt } & \multicolumn{4}{|c|}{ Copper } \\
\hline & \multirow{2}{*}{$\begin{array}{l}\text { All } \\
\text { sites }\end{array}$} & \multicolumn{3}{|c|}{$\begin{array}{c}\text { Proximity-to-mining } \\
\text { group }\end{array}$} & \multirow{2}{*}{$\begin{array}{c}\text { All } \\
\text { sites }\end{array}$} & \multicolumn{3}{|c|}{$\begin{array}{l}\text { Proximity-to-mining } \\
\text { group }\end{array}$} & \multirow{2}{*}{$\begin{array}{c}\text { All } \\
\text { sites }\end{array}$} & \multicolumn{3}{|c|}{$\begin{array}{c}\text { Proximity-to-mining } \\
\text { group }\end{array}$} & \multirow{2}{*}{$\begin{array}{l}\text { All } \\
\text { sites }\end{array}$} & \multicolumn{3}{|c|}{$\begin{array}{c}\text { Proximity-to-mining } \\
\text { group }\end{array}$} \\
\hline & & A & B & C & & A & B & C & & A & B & C & & A & B & C \\
\hline Number of samples & 44 & 22 & 9 & 13 & 44 & 22 & 9 & 13 & 44 & 22 & 9 & 13 & 44 & 22 & 9 & 13 \\
\hline Minimum & 4.1 & 4.1 & 7.2 & 5.5 & .2 & .2 & 2.0 & .6 & 9 & 9 & 43 & 18 & 15 & 15 & 31 & 21 \\
\hline Maximum & 35.0 & 18.0 & 35.0 & 20.0 & 9.9 & 2.0 & 9.9 & 3.0 & 1,200 & 38 & 1,200 & 49 & 240 & 166 & 240 & 140 \\
\hline Range & 30.9 & 13.9 & 27.8 & 14.5 & 9.7 & 1.8 & 7.9 & 2.4 & 1,191 & 29 & 1,157 & 31 & 225 & 151 & 209 & 119 \\
\hline Mean & 12.0 & 10.8 & 16.1 & 11.2 & 1.9 & 1.7 & 3.1 & 1.5 & 63 & 19 & 223 & 27 & 83 & 73 & 127 & 70 \\
\hline Standard Deviation & 5.4 & 3.5 & 8.7 & 4.2 & 1.4 & 0.6 & 2.6 & .7 & 179 & 8 & 369 & 9 & 54 & 46 & 69 & 45 \\
\hline Q5 & 5.3 & 4.6 & 7.2 & 5.6 & .5 & .3 & $<2$ & 6 & 10 & 10 & 43 & 18 & 18 & 16 & 31 & 21 \\
\hline Q25 & $<10$ & $<10$ & $<10$ & $<10$ & 1.6 & $<2$ & $<2$ & .8 & 18 & 14 & 72 & 20 & 32 & 29 & 83 & 31 \\
\hline Q50 median & $<10$ & $<10$ & 14.0 & $<10$ & $<2$ & $<2$ & $<2$ & 1.7 & 25 & 19 & 88 & 27 & 85 & 77 & 120 & 47 \\
\hline Q75 & 14.0 & 11.0 & 20.5 & 13.3 & $<2$ & $<2$ & 2.6 & 2.0 & 35 & 24 & 153 & 31 & 120 & 99 & 180 & 113 \\
\hline Q95 & 20.6 & 17.4 & 35.0 & 19.7 & 3.1 & 2.0 & 9.9 & 2.9 & 153 & 34 & 1,200 & 46 & 180 & 152 & 240 & 137 \\
\hline \multirow[t]{4}{*}{ Interquartile range } & 4.0 & 1.0 & 10.5 & 3.3 & .4 & 0 & .6 & 1.2 & 17 & 10 & 81 & 11 & 88 & 70 & 97 & 82 \\
\hline & \multicolumn{4}{|c|}{ Lead } & \multicolumn{4}{|c|}{ Nickel } & \multicolumn{4}{|c|}{ Zinc } & \multicolumn{4}{|c|}{ Chromium } \\
\hline & \multirow{2}{*}{$\begin{array}{l}\text { All } \\
\text { sites }\end{array}$} & \multicolumn{3}{|c|}{$\begin{array}{c}\text { Proximity-to-mining } \\
\text { group }\end{array}$} & \multirow{2}{*}{$\begin{array}{l}\text { All } \\
\text { sites }\end{array}$} & \multicolumn{3}{|c|}{$\begin{array}{c}\text { Proximity-to-mining } \\
\text { group }\end{array}$} & \multirow{2}{*}{$\begin{array}{l}\text { All } \\
\text { sites }\end{array}$} & \multicolumn{3}{|c|}{$\begin{array}{c}\text { Proximity-to-mining } \\
\text { group }\end{array}$} & \multirow{2}{*}{$\begin{array}{l}\text { All } \\
\text { sites }\end{array}$} & \multicolumn{3}{|c|}{$\begin{array}{c}\text { Proximity-to-mining } \\
\text { group }\end{array}$} \\
\hline & & A & B & C & & A & B & C & & A & B & C & & A & B & C \\
\hline Number of samples & 44 & 22 & 9 & 13 & 44 & 22 & 9 & 13 & 44 & 22 & 9 & 13 & 44 & 22 & 9 & 13 \\
\hline Minimum & 24 & 24 & 150 & 25 & 21 & 21 & 73 & 29 & 54 & 54 & 200 & 86 & 24 & 32 & 24 & 31 \\
\hline Maximum & 1,410 & 210 & 1,410 & 170 & 580 & 51 & 580 & 78 & 2,700 & 280 & 2,700 & 340 & 78 & 78 & 74 & 74 \\
\hline Range & 1,386 & 186 & 1,260 & 145 & 559 & 30 & 507 & 49 & 2,646 & 226 & 2,500 & 254 & 54 & 46 & 50 & 43 \\
\hline Mean & 190 & 63 & 630 & 100 & 64 & 33 & 171 & 41 & 321 & 128 & 1,033 & 156 & 54 & 56 & 50 & 54 \\
\hline Standard Deviation & 296 & 40 & 432 & 46 & 88 & 8 & 158 & 13 & 481 & 49 & 714 & 70 & 12 & 10 & 14 & 12 \\
\hline Q5 & 25 & 24 & 150 & 25 & 24 & 22 & 73 & 29 & 71 & 60 & 200 & 86 & 32 & 39 & 24 & 33 \\
\hline Q25 & 49 & 35 & 223 & 84 & 30 & 27 & 87 & 31 & 112 & 87 & 655 & 113 & 47 & 51 & 43 & 47 \\
\hline Q50 median & 84 & 53 & 510 & 100 & 37 & 34 & 140 & 38 & 146 & 128 & 850 & 140 & 54 & 57 & 50 & 53 \\
\hline Q75 & 165 & 79 & 943 & 115 & 51 & 37 & 165 & 45 & 210 & 152 & 1,225 & 203 & 61 & 62 & 60 & 60 \\
\hline Q95 & 943 & 142 & 1,410 & 170 & 166 & 47 & 580 & 74 & 1,230 & 220 & 2,700 & 321 & 74 & 75 & 74 & 74 \\
\hline Interquartile range & 116 & 44 & 720 & 31 & 21 & 10 & 78 & 14 & 98 & 65 & 570 & 90 & 14 & 11 & 17 & 13 \\
\hline
\end{tabular}




\section{Spatial and Temporal Patterns of Trace- Element Deposition in Bed Sediment from Clearwater Lake, Southeastern Missouri, 2002}

By Gary W. Krizanich

Chapter 4 of

Hydrologic Investigations Concerning Lead Mining Issues in Southeastern Missouri

Edited by Michael J. Kleeschulte

Scientific Investigations Report 2008-5140 


\section{Contents}

Abstract

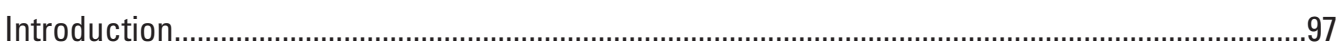

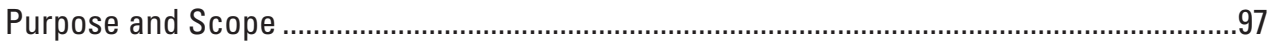

Study Area and Black River Basin Description ....................................................................98

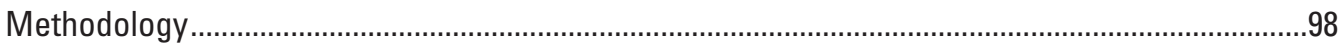

Spatial and Temporal Patterns of Trace-Element Deposition .....................................................100

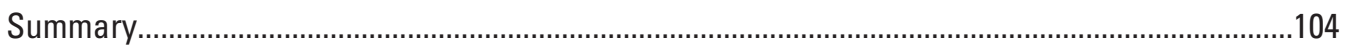

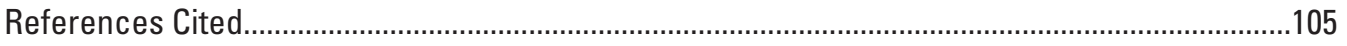

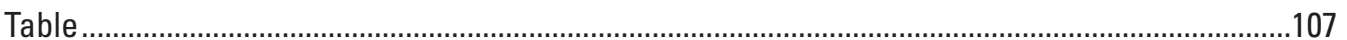

\section{Figures}

1. Map showing study area and streams draining the Viburnum Trend Subdistrict into Clearwater Lake ...................................................................................................99

2. Aerial photograph showing Clearwater Lake bed-sediment coring sites ......................100

3-4. Graphs showing-

3. Median concentrations of Mississippi Valley Type-related metals in Clearwater Lake bed sediment and range of lead and zinc concentrations in non-mining basins in southern Missouri.

4. Lead and zinc concentrations in Clearwater Lake bed sediments, 2002 ...............102

\section{Tables}

1. Quantitative elemental analyses of less than 63-micrometer fraction and lead-210 date of Clearwater Lake bed-sediment samples, 2002

2. Results of trend tests on lead and zinc concentrations in bed-sediment core samples collected from Clearwater Lake, Missouri, August 2002

3. Sediment-quality guidelines for trace elements (Mississippi Valley Type-related metals)

4. Exceedance of sediment-quality guidelines for trace elements (Mississippi Valley Type-related metals) at the Clearwater Lake sites 


\title{
Spatial and Temporal Patterns of Trace-Element Deposition in Bed Sediment from Clearwater Lake, Southeastern Missouri, 2002
}

\author{
By Gary W. Krizanich
}

\section{Abstract}

The dam for Clearwater Lake was completed in 1948 and reservoir filling began at that time. Lead and zinc mining operations along the Viburnum Trend Subdistrict began in the Black River Basin in 1966. Arsenic, cadmium, cobalt, copper, lead, nickel, and zinc are the major trace elements associated with the sulfide minerals of this Mississippi Valley Type ore deposit. Clearwater Lake may be a sink where these miningrelated trace elements accumulate and are stored.

Sediment cores were collected from six sites in Clearwater Lake to spatially and temporally characterize the concentration of trace elements in the bed sediment. The Clearwater Dam site core is the only core that is known, with certainty, to have penetrated to the pre-impoundment (pre-1948) surface. The core from Logan Creek probably penetrated the pre-mining (pre-1966) surface; the lower Black River core penetrated the pre-mining surface based on lead-210 isotopic analysis.

The non-parametric Spearman's rho correlation coefficient was computed for lead and zinc concentrations with core depth to determine the presence of temporal trends in lake bed-sediment samples at each coring site. Lead concentrations with depth indicated no trend occurred at the Webb Creek site (a basin where no mining occurs). Analyses of lead concentrations with depth at the other five coring sites indicated these five sites demonstrate significantly positive trends. Using zinc concentrations with depth was not a useful indicator to identify areas affected by mining.

The strongest correlation between lead concentrations and depth exists at the Logan Creek $\left(r_{s}=0.92\right)$ and Clearwater Dam $\left(r_{s}=0.91\right)$ sites. Samples from the Clearwater Dam site represent a composite for the fine sediments input into the lake from various tributaries. Age dating of the Clearwater Dam core by lead-210 isotopic methods indicates that the most abrupt increase in the lead concentration (from 43.9 to 77.9 milligrams per kilogram) occurred between 1965 and 1967 . The mean lead concentrations of the 1967 and later samples (78.9 milligrams per kilogram) were twice the mean lead concentrations of the 1965 and earlier samples (37.4 milligrams per kilogram).
The threshold effects concentration was used to assess the potential effects of sediment trace-element concentrations on freshwater ecosystems and was exceeded for lead at all coring sites except Webb Creek and exceeded for zinc at the lower Black River, Marina, and Clearwater Dam sites. However, all lead and zinc concentrations were well below the probable effects concentration at all core sites. The only probable effects concentration exceedance occurred for nickel in 7 percent of samples at the Marina and Clearwater Dam coring sites.

\section{Introduction}

Ten lead and zinc mines, six of which currently (2008) are active in the Black River Basin, are located along the Viburnum Trend Subdistrict (Viburnum Trend). Arsenic (As), cadmium $(\mathrm{Cd})$, cobalt $(\mathrm{Co})$, copper $(\mathrm{Cu})$, lead $(\mathrm{Pb})$, nickel $(\mathrm{Ni})$, and zinc $(\mathrm{Zn})$ are the major trace elements (all are metals) associated with the sulfide minerals of these Mississippi Valley Type (MVT) ores (Lopaka Lee, U.S. Geological Survey, written commun., 2006) and, therefore, are referred to as MVT-related metals in this chapter. Galena $(\mathrm{PbS})$ is the primary ore mineral in the Viburnum Trend, and sphalerite $(\mathrm{ZnS})$ is the second most common ore mineral. Both are predominant in the thick carbonate sequence of the Upper Cambrian Bonneterre Formation. Mining operations began along the Viburnum Trend in 1960; however, mines in the Black River Basin did not begin operations until 1966 (C.M. Seeger, Missouri Department of Natural Resources, Divison of Geology and Land Survey, written commun., 2008).

\section{Purpose and Scope}

The purpose of this chapter is to characterize the spatial and temporal concentration of select trace elements, referred to as MVT-related metals, in bed-sediment samples collected during August 2002 from six sites in Clearwater Lake. The 
focus of this study was on lead and zinc as they are the primary metals produced from the Viburnum Trend mines.

Clearwater Lake may be a sink where mining-related trace elements accumulate and are stored (Gale and others, 1976). Lake bed sediments can provide detailed chronostratigraphic histories of trace-element inputs in lakes and can be useful in assessing the effects of long-term activities, such as mining, within a basin. Understanding the transport and deposition of trace elements in an aquatic environment is important to the overall understanding of the effects of mining on the environment. This information can be used by Federal land managers to better assess potential risks to new areas being considered for future or expanded mine development.

\section{Study Area and Black River Basin Description}

The study area is Clearwater Lake, located in southeastern Missouri, which has a drainage area of approximately 898 square miles $\left(\mathrm{mi}^{2}\right)$ and a surface area of 1,600 acres (fig. 1). The dam was completed in 1948 as a flood control reservoir for the upper Black River and began filling at that time (Hauck and Harris, 2006).

Clearwater Lake actively receives stream sediment from the upper part of the Black River Basin, which is on the western flank of the St. Francois Mountains. Much of the area is within the boundaries of the Mark Twain National Forest (fig. 1) managed by the U.S. Department of Agriculture, Forest Service. The basin is predominantly uninhabited, hickory-oak forest with minor stands of conifers ( 91 percent) and pasture (7 percent) (Homer and others, 2007). The remaining 2 percent is a mixture of open water, wetlands, and urban. The basin includes scattered parcels of private property that contain either isolated homesteads or small communities. Most of the private property is concentrated along major roads or along stream valleys.

The upper Black River Basin lies within a large region of karst terrain that is characterized by the presence of caves, springs, sinkholes, and losing streams. Most of the springs in the basin are small, discharging 0.1 to 1.0 cubic foot per second ( $\mathrm{ft}^{3} / \mathrm{s}$; Vineyard and Feder, 1974). Losing streams have been identified in the basin (Harvey, 1980), and include the upper part of Logan Creek. The upper Black River Basin is characterized by deep, narrow valleys and narrow, steep-sided ridges that resulted from deep dissection of the carbonate bedrock by surface and karst drainage. More than 300 feet (ft) of relief is common between the ridge tops and the adjacent valleys.

\section{Methodology}

Lake bed-sediment cores were collected during August 2002 from six sites in Clearwater Lake (fig. 2). Sampling sites represent a mix of riverine, lacustrine, and intermediate environments. The cores were collected using a universal head gravity corer with a 2.8-inch (in.) inside diameter polycarbonate core barrel. Two collection methods were used. In shallow water areas, the core barrel was fitted with aluminum extension rods and pushed by hand from a boat. In deep water areas, cores were collected by divers with self-contained underwater breathing apparatus (SCUBA) by hand pushing the core barrel into the sediments until refusal. Because the core barrel was hand pushed into the sediments, core refusal occurred before the pre-impoundment (pre-1948) surface was penetrated at all sites except the Clearwater Dam site. Based on the observation of root hairs at the bottom of the Clearwater Dam core sample, which was $1.77 \mathrm{ft}$ long, the pre-impoundment soil surface was penetrated. The longest core sample was from Webb Creek (2.36 ft), a non-mining basin. This core did not penetrate the pre-1966 (pre-mining) lake bed surface. The upper Black River and Marina cores (both $2.00 \mathrm{ft}$ ) probably did not penetrate the pre-mining surface. The Logan Creek core $(0.92$ $\mathrm{ft}$ ) probably reached the pre-mining surface. The lower Black River core $(1.77 \mathrm{ft})$ also penetrated the pre-mining surface, based on isotopic age dating results.

After collection, both ends of the core tubes were capped with a polyethylene cover secured with electrical tape and transported to the University of Missouri-Rolla (now Missouri University of Science and Technology) geochemistry laboratory. Excess water was siphoned and the cores were allowed to air dry before longitudinally cutting in one-half. Air drying allowed the core sample to solidify before subsampling. One-half of the core was divided into 0.4-in. (1-centimeter) sections for geochemical analysis. The other one-half of the core was divided into 0.8-in. (2-centimeter) sections, and age dating was performed on the midpoint of each section.

Detailed geochemical analyses were performed on the less than 63 micrometer $(\mu \mathrm{m})$ fraction using inductively coupled plasma mass spectrometry (ICP-MS) methods (Taggart, 2002). The results for 37 elements, including major and trace elements, are listed in table 1 (at the back of this chapter). A marine sediment consisting of a fine-grained, gray-brown, clayey mud with low carbonate content (MAG-1) was used as the standard reference material with known elemental concentrations, and sample duplicates were used to assess quality assurance. The core samples were divided into 11 groups to be loaded and analyzed by the ICP-MS, with at least 1 duplicate sample analyzed with each group. Two MAG-1 sediment samples were included with each ICP-MS analytical run to assess the accuracy of the analysis. If there were a small number of samples in a group, the ICP-MS run may have contained more than one sample. The ICP-MS analysis of the standard reference material yielded an average percent recovery of the known concentrations that ranged from 94.5 percent for nickel (Ni) to 108.1 percent for aluminum (Al) with a median recovery of 101.6 percent. These results are a measure of the method performance. Precision of the ICP-MS analysis was calculated as relative standard deviation (RSD). The RSD was determined by dividing the standard deviation of each specific element concentration for all the MAG-1 analysis by the mean concentration for the same sample set, then multiplying by 100 


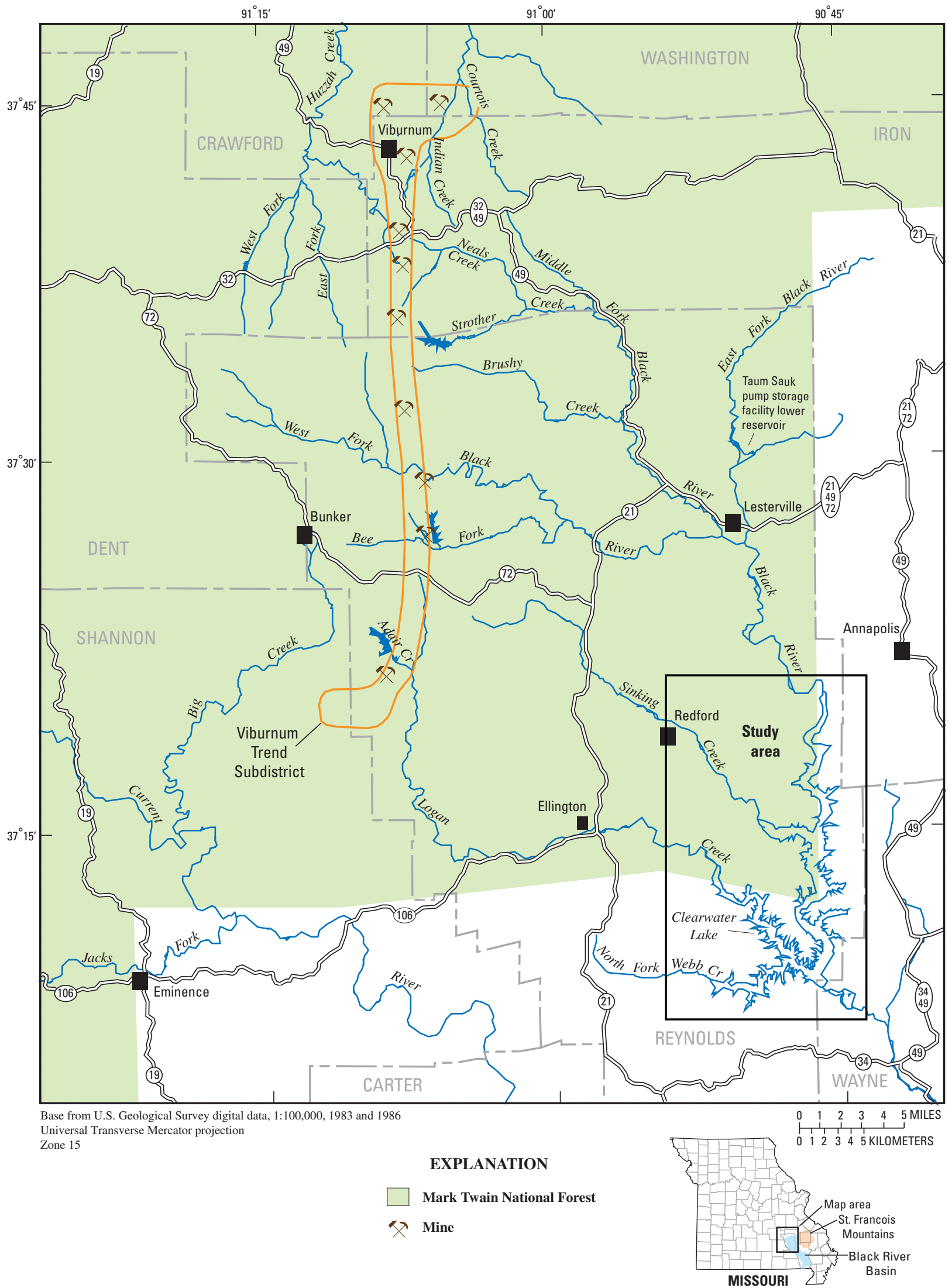

Figure 1. Study area and streams draining the Viburnum Trend Subdistrict into Clearwater Lake. 


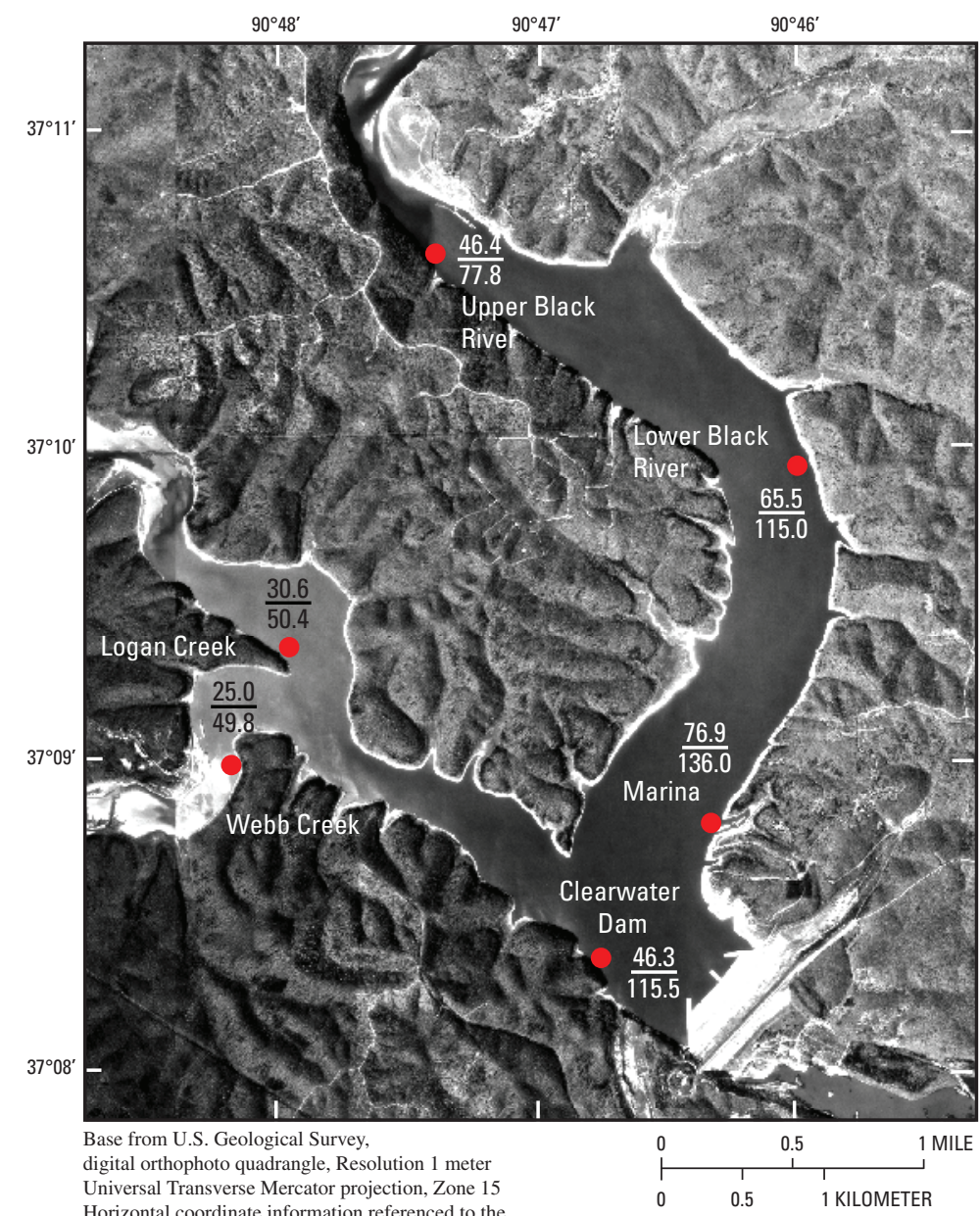

\section{EXPLANATION}

Marina Lake bed-sediment coring site and name

-

$$
\begin{array}{ll} 
& \begin{array}{c}
\text { Median concentration in core samples, } \\
\text { in milligrams per kilogram }
\end{array} \\
\mathbf{3 0 . 6} & \begin{array}{l}
\text { Lead } \\
50.4
\end{array} \quad \text { Zinc }
\end{array}
$$

Figure 2. Clearwater Lake bed-sediment coring sites.

The error for the ${ }^{210} \mathrm{~Pb}$ dating is estimated to be on the order of plus or minus 5 percent (J.R. Budahn, U.S. Geological Survey, written commun., 2007). The largest dating errors were associated with the samples dated from the 1950s, which were estimated at plus or minus 3 years.

The non-parametric Spearman's rho correlation coefficient $\left(\mathrm{r}_{\mathrm{s}}\right)$ ( Helsel and Hirsch, 1992) was computed for lead and zinc concentrations to determine the presence of temporal trends in lake bed-sediment samples from each coring site. A level of significance ( $\alpha$-value) less than 0.05 caused the rejection of the null hypotheses that states no temporal trends exist. The "attained significance level" ( $p$-value) is a probability value determined from the data (Helsel and Hirsch, 1992) that measures the "believability" of the null hypotheses. The larger the p-value, the weaker the evidence to reject the null hypothesis. Trends were determined to be significant if the probability (two-sided p-value) of rejecting the null hypothesis was less than or equal to 0.05 . A positive trend is indicated by increasing traceelement concentrations in younger sediments (top of core) and a negative trend would be decreasing trace-element concentrations in younger sediments.

$\mathrm{Au}$ ) had a RSD greater than 10 percent, probably because of a lack of optimization of the procedure for these elements or the elemental concentrations in the MAG-1 sample were near the detection limit of the instrument.

Sediment cores from the lower Black River and Clearwater Dam sites were dated to assess trace-element deposition with time using lead-210 $\left({ }^{210} \mathrm{~Pb}\right)$ and cesium-137 $\left({ }^{137} \mathrm{Cs}\right)$ isotopic methods (Zielinski and Budahn, 1998). Cesium-137 was used to assign a date marker to the core samples that are associated with the ${ }^{137} \mathrm{Cs}$ maximum concentration that occurred in 1964. Date markers also were assigned to the pre-impoundment surface (pre-1948) and the date of core collection (2002). Lead-210 dates were determined using the constant rate of supply model (Appleby and Oldfield, 1992). Variable mass accumulation rates were used to adjust ${ }^{210} \mathrm{~Pb}$ dates to known date markers within the cores (J.R. Budahn, U.S. Geological Survey, written commun., 2007). Concentrations of ${ }^{210} \mathrm{~Pb}$, radium-226 $\left({ }^{226} \mathrm{Ra}\right)$, lead-214 $\left({ }^{214} \mathrm{~Pb}\right)$, bismuth-214 $\left({ }^{214} \mathrm{Bi}\right)$, and ${ }^{137} \mathrm{Cs}$ were obtained using high precision gamma-ray spectrometry. Counts were made using a high-purity germanium semiconductor detector in the U.S. Geological Survey (USGS) mineralogic laboratory in Lakewood, Colorado.

\section{Spatial and Temporal Patterns of Trace-Element Deposition}

Median concentrations of the select trace elements, referred to as MVT-related metals, excluding cadmium, in sediments from Clearwater Lake are shown in figure 3. The largest median concentrations for all MVT-related metals, except cobalt, were detected at the Marina site. The largest median cobalt concentration was at the Clearwater Dam site (table 1, at the back of this chapter).

Lead and zinc concentrations in bed sediment at background sites (sites not considered to be affected by mining) in southern Missouri ranged from 15 to $28 \mathrm{mg} / \mathrm{kg}$ lead and 43 to $140 \mathrm{mg} / \mathrm{kg}$ zinc (Petersen and others, 1998). Webb Creek is the only site with a median lead concentration that is within the range defined by the background sites (fig. 3). Median concentrations in samples from the other five sites exceeded the 


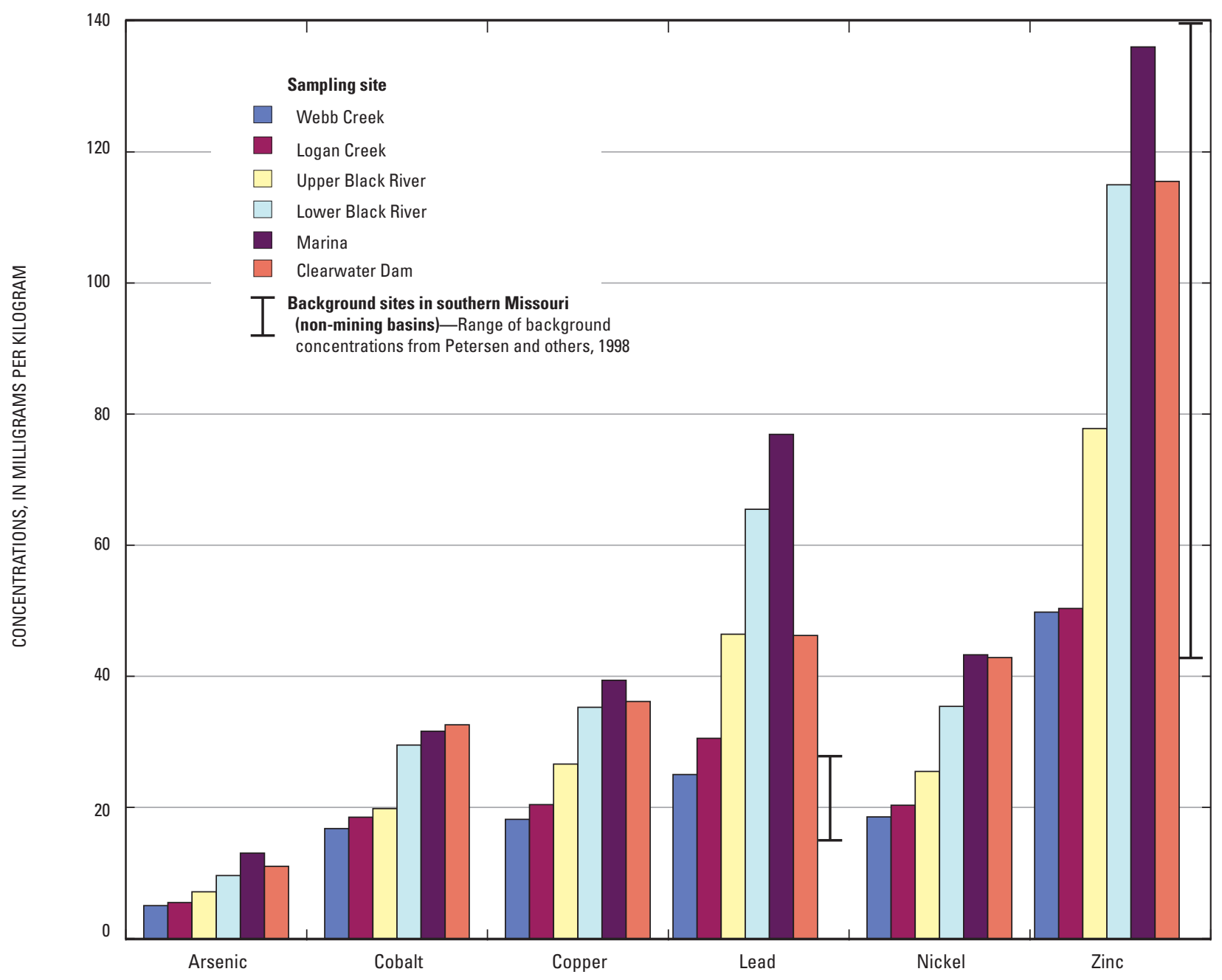

Figure 3. Median concentrations of Mississippi Valley Type-related metals in Clearwater Lake bed sediment and range of lead and zinc concentrations in non-mining basins in southern Missouri.

background lead concentration range. Although Webb Creek has the smallest median zinc concentration, the median zinc concentrations at all sites were within the background range.

Concentrations of lead and zinc at different depths (with time) were plotted (fig. 4) and used to determine if temporal trends existed. No trend was indicated by lead concentrations $(p$-value $=0.14)$ at the Webb Creek site (non-mining basin) where median lead and zinc concentrations were smallest (fig. 3) and changed little with depth (fig. 4). Analyses of lead concentrations with depth at the other five sites have $r_{s}$ ranging from 0.26 to 0.92 and p-values less than 0.05 , indicating all have significantly positive trends (table 2 ). Zinc concentrations at all six sites, including the background non-mining Webb Creek site, have positive trends ( $r_{s}$ ranging from 0.40 to 0.94 ) and p-values less than 0.05. Because of the positive trend at the background Webb Creek site and all core sites have median zinc concentrations within the background concentration, zinc concentrations probably are not a useful indicator to identify areas affected by mining.

A strong correlation $\left(r_{s}=0.92\right)$ exists for lead and zinc concentrations at the Logan Creek site. As the depth decreases, the concentration of both lead and zinc increases (except for the most recent sample). Whereas the Logan Creek core did not penetrate to the pre-impoundment surface, the core probably reached the pre-mining lake bed surface. This conclusion is based on the small concentrations of the seven MVT-related metals in the oldest (deepest) layers of the Logan Creek core. These concentrations are similar to those detected in samples from the non-mining Webb Creek site (fig. 4).

Although lead concentrations with decreasing depth showed a positive trend at the upper Black River site, this site had the weakest correlation $\left(r_{s}=0.26\right.$; table 2$)$ of the five sites that demonstrated a trend. The lower Black River site showed a slightly stronger correlation $\left(r_{s}=0.46\right)$ between lead concentrations with depth. Only the oldest section of the lower Black River core was age dated. Lead-210 analysis indicated the core barrel penetrated to the 1964 surface, which is before mining activity commenced in the Black River Basin part the Viburnum Trend.

Several possible explanations exist for the weak trend in lead concentrations with depth at the upper Black River site as compared to the lower Black River site. The lower Black River 


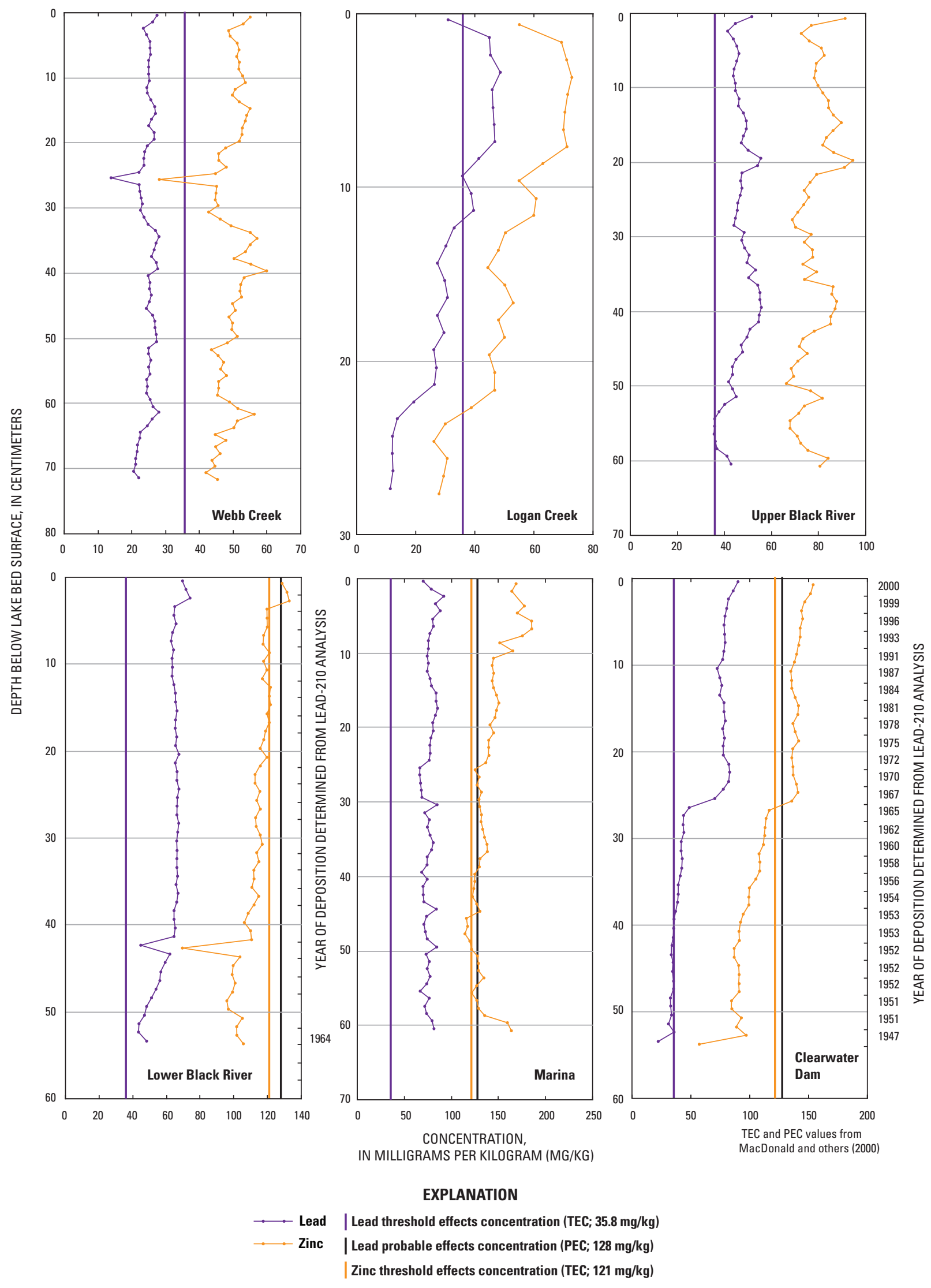

Figure 4. Lead and zinc concentrations in Clearwater Lake bed sediments, 2002. 
Table 2. Results of trend tests on lead and zinc concentrations in bed-sediment core samples collected from Clearwater Lake, Missouri, August 2002.

\begin{tabular}{lccl}
\hline \multicolumn{1}{c}{ Site } & Constituent & $\begin{array}{c}\text { Spearman's } \\
\text { rho }\end{array}$ & $\begin{array}{c}\text { Trend test at } \\
\text { a 0.05 level of } \\
\text { significance }\end{array}$ \\
\hline Webb Creek & Lead & 0.18 & No trend \\
& Zinc & .40 & Positive trend \\
Logan Creek & Lead & .92 & Positive trend \\
& Zinc & .92 & Positive trend \\
Upper Black River & Lead & .26 & Positive trend \\
& Zinc & .42 & Positive trend \\
Lower Black River & Lead & .46 & Positive trend \\
& Zinc & .92 & Positive trend \\
Marina & Lead & .33 & Positive trend \\
& Zinc & .73 & Positive trend \\
Clearwater Dam & Lead & .91 & Positive trend \\
& Zinc & .94 & Positive trend \\
\hline
\end{tabular}

site is farther into the lake than the upper Black River site and, consequently, is in a deeper, lower energy environment part of the lake. Lake-level fluctuations would have greater effect at the upper site, especially during low lake stages when this area becomes a riverine environment, resulting in more lake bed-sediment disturbance than at the lower site. These disturbances may have reworked the deposited sediments and obscured any chronological trends that may have been present. Also, the deeper, lower energy environment at the lower Black River site favored the accumulation of finer grained sediments (silt and clay size; less than $63 \mu \mathrm{m}$ ) as compared to the upper site. Because the material in the mine tailings ponds have been milled to primarily silt and clay size particles, these fine-grained, metal-rich particles have the potential to be transported farther into the lake where they would accumulate. Finally, the core from the upper Black River site likely did not penetrate the pre-mining lake bed surface to give a pre-mining baseline concentration for comparison. This conclusion is based on the lead and zinc concentrations at the upper Black River site, which are about twice the concentrations detected at the Webb Creek site.

A failure to penetrate the pre-mining lake bed surface also may explain the weak correlation $\left(r_{s}=0.33\right)$ between lead concentration and depth at the Marina site. Similar to the upper Black River site, lead concentrations at the Marina site were about twice the concentration at the Webb Creek site.

Analysis of pre-mining and mining concentrations in lake bed sediments from the Clearwater Dam site indicates a strong correlation between an increase in lead $\left(r_{s}=0.91\right)$ and zinc $\left(r_{s}=0.94\right)$ concentrations with a decrease in depth (fig. 4). Samples from this site represent a composite for the fine sediments input to the lake from the various tributaries. Age dating of the Clearwater Dam core indicates that the most abrupt increase in the lead concentration occurs in four consecutive 0.4-in. samples from 43.9 to $77.9 \mathrm{mg} / \mathrm{kg}$ (samples $25-28$, table 1) that correspond to the period between 1965 and 1967. Zinc concentrations increased from 114 to $141 \mathrm{mg} / \mathrm{kg}$ (samples $25-28$, table 1) during this same period. The mean lead concentrations of the 1967 and later samples $(78.9 \mathrm{mg} / \mathrm{kg})$ were twice the mean lead concentrations of the 1965 and earlier samples $(37.4 \mathrm{mg} / \mathrm{kg}$ ). Although probably not a reliable indicator of mining, an increase in mean zinc concentrations from 97 to 141 $\mathrm{mg} / \mathrm{kg}$ occurred when comparing the 1965 and earlier samples with the 1967 and later samples. Although not plotted, concentrations of arsenic, cobalt, copper, and nickel lack the abrupt increase observed in lead and zinc concentrations, but generally increase with time; however, cadmium concentrations show the same abrupt increase between 1965 and 1967 (0.42 to $0.58 \mathrm{mg} / \mathrm{kg}$; samples $25-28$, table 1) as lead concentrations.

Available consensus-based sediment-quality guidelines (MacDonald and others, 2000) were used to assess the potential effects of trace-element concentrations on freshwater ecosystems (table 3). The threshold effects concentration (TEC) is the level below which adverse effects to sediment dwelling organisms are not expected to occur; the probable effects concentration (PEC) is the level above which adverse effects are likely to occur. Adverse effects may occasionally occur in the range of concentrations between the TEC and PEC values. The TEC for nickel $(22.7 \mathrm{mg} / \mathrm{kg})$ was exceeded at all sites, and the TEC for lead $(35.8 \mathrm{mg} / \mathrm{kg})$ was exceeded at all sites except Webb Creek, whereas the TEC for arsenic, copper, and zinc was exceeded at the lower Black River, Marina, and Clearwater Dam sites (fig. 4; table 4). All of the lead and zinc concentrations were well below the PEC at all sites. The only PEC exceedance was for nickel at 7 percent of samples at both the Marina and Clearwater Dam core sites (table 4).

Table 3. Sediment-quality guidelines for trace elements (Mississippi Valley Type-related metals).

[TEC, threshold effects concentration; $\mathrm{mg} / \mathrm{kg}$, milligrams per kilogram; PEC, probable effects concentration; --, not applicable]

\begin{tabular}{lcc}
\hline \multicolumn{1}{c}{ Constituent } & $\begin{array}{c}\text { TEC } \\
(\mathbf{m g} / \mathbf{k g})\end{array}$ & $\begin{array}{c}\text { PEC }^{\mathbf{a}} \\
(\mathbf{m g} / \mathbf{k g})\end{array}$ \\
\hline Arsenic & 9.79 & 33.0 \\
Cadmium & .99 & 4.98 \\
Cobalt & -- & -- \\
Copper & 31.6 & 149 \\
Lead & 35.8 & 128 \\
Nickel & 22.7 & 48.6 \\
Zinc & 121 & 459 \\
\hline
\end{tabular}

${ }^{a}$ Concentrations from MacDonald and others (2000). 
Table 4. Exceedance of sediment-quality guidelines for trace elements (Mississippi Valley Type-related metals) at the Clearwater Lake sites.

[TEC, threshold effects contamination; PEC, probable effects concentration; No, guideline not exceeded; shaded cell, guideline exceeded with numbers indicating the percentage of samples exceeding the guideline concentration; sediment-quality guidelines from MacDonald and others, 2000]

\begin{tabular}{|c|c|c|c|c|c|c|c|c|c|c|c|c|}
\hline \multirow{2}{*}{ Site } & \multicolumn{2}{|c|}{ Arsenic } & \multicolumn{2}{|c|}{ Cadmium } & \multicolumn{2}{|c|}{ Copper } & \multicolumn{2}{|c|}{ Nickel } & \multicolumn{2}{|c|}{ Lead } & \multicolumn{2}{|c|}{ Zinc } \\
\hline & TEC & PEC & TEC & PEC & TEC & PEC & TEC & PEC & TEC & PEC & TEC & PEC \\
\hline Webb Creek & No & No & No & No & No & No & 1 & No & No & No & No & No \\
\hline Logan Creek & No & No & No & No & 4 & No & 32 & No & 36 & No & No & No \\
\hline Upper Black River & No & No & No & No & No & No & 80 & No & 98 & No & No & No \\
\hline Lower Black River & 32 & No & No & No & 94 & No & 100 & No & 100 & No & 9 & No \\
\hline Marina & 100 & No & 2 & No & 100 & No & 100 & 7 & 100 & No & 93 & No \\
\hline Clearwater Dam & 87 & No & No & No & 74 & No & 98 & 7 & 74 & No & 48 & No \\
\hline
\end{tabular}

\section{Summary}

The dam for Clearwater Lake was completed in 1948 and reservoir filling began at that time. Mining operations along the lead and zinc ore body known as the Viburnum Trend Subdistrict began in the Black River Basin part of the subdistrict in 1966. Arsenic, cadmium, cobalt, copper, lead, nickel, and zinc are the major trace elements (all metals) associated with the sulfide minerals of this Mississippi Valley Type ore deposit. Clearwater Lake may be a sink where these mining-related trace metals accumulate and are stored.

Sediment cores were collected from six sites in Clearwater Lake during August 2002 to spatially and temporally characterize the concentration of trace elements in the bed sediment. The cores represent a mix of riverine, lacustrine, and intermediate environments, and elemental analyses were performed using inductively coupled plasma mass spectrometry methods. The Clearwater Dam site core is the only core that is known, with certainty, to have penetrated to the pre-impoundment (pre-1948) surface. The core from Logan Creek probably penetrated the pre-mining (pre-1966) surface; the lower Black River core penetrated the pre-mining surface based on lead-210 isotopic analysis.

The non-parametric Spearman's rho correlation coefficient was computed for lead and zinc concentrations with core depth to determine the presence of temporal trends in lakebed sediment samples from each coring site. Median lead and zinc concentrations were the least and changed little with depth at the Webb Creek site (a basin where no mining occurs) and no trend (p-value $=0.14$ ) was indicated in lead concentrations. Analyses of lead concentrations with depth at the other five core sites indicated p-values less than 0.05 and correlation coefficients $\left(\mathrm{r}_{\mathrm{s}}\right)$ ranging from 0.26 to 0.92 , indicating these five sites had significantly positive trends. Median zinc concentrations at all six sites were within background concentrations for non-mining areas in southern Missouri; all six sites indicated significant positive trends, including the non-mining Webb Creek site. These two reasons indicate that zinc concentration probably was not a useful indicator to identify areas affected by mining.

The strongest correlation between lead concentrations and depth existed at the Logan Creek $\left(r_{s}=0.92\right)$ and Clearwater Dam $\left(r_{s}=0.91\right)$ sites. Based on the small concentrations of the seven select trace elements (Mississippi Valley Typerelated metals) in the deepest and presumably oldest layers of the Logan Creek core that were similar to the concentrations present in Webb Creek samples, it is probable that the core reached the pre-mining lakebed surface, but not the preimpoundment surface.

Samples from the Clearwater Dam site represent a composite for the fine sediments input into the lake from various tributaries. Age dating of the Clearwater Dam core by lead-210 isotopic methods shows that the most abrupt increase in the lead concentration (from 43.9 to 77.9 milligrams per kilogram) occurred in four consecutive 0.4-inch samples that correspond to the period between 1965 and 1967. The mean lead concentrations of the 1967 and later samples (78.9 milligrams per kilogram) were twice the mean lead concentrations of the 1965 and earlier samples (37.4 milligrams per kilogram).

The threshold effects concentration was used to assess the potential effects of sediment trace-element concentrations on freshwater ecosystems. The threshold effects concentration for nickel (22.7 milligrams per kilogram) was exceeded at all sites, and lead (35.8 milligrams per kilogram) was exceeded at all sites except Webb Creek, whereas the threshold effects concentrations for arsenic, copper, and zinc were exceeded at the lower Black River, Marina, and Clearwater Dam sites. All of the lead and zinc concentrations were well below the probable effects concentration at all sites. The only probable effects concentration exceedance was for nickel at 7 percent of samples at both the Marina and Clearwater Dam sites. 


\section{References Cited}

Appleby, P.G., and Oldfield, F., 1992, Application of lead-210 to sedimentation studies, in Ivanovich, M., and Harmon, R.S., eds. Uranium-series disequilibrium-Applications to earth, marine, and environmental sciences: Oxford, Clarendon Press, p. 731-778.

Gale, N.L., Bolter, Ernst., and Wixson, B.G., 1976, Investigation of Clearwater Lake as a potential sink for heavy metals from lead mining in southeast Missouri, in Hemphill, D.D., ed., Proceedings 10th Annual Conference on Trace Substances in Environmental Health: Columbia, University of Missouri, p. 187-196.

Harvey, E.J., 1980, Ground water in the Springfield-Salem Plateaus of southern Missouri and northern Arkansas: U.S. Geological Survey Water-Resources Investigations Report 80-101, 66 p.

Hauck, H.S., and Harris, T.E., 2006, Water resources dataMissouri water year 2005: U.S. Geological Survey WaterData Report MO-05-1, 724 p. (published annually).

Helsel, D.R., and Hirsch, R.M., 1995, Statistical methods in water resources, in Studies in Environmental Science 49: Amsterdam, The Netherlands, Elsevier Science, 529 p.

Homer, C., Dewitz, J., Fry, J., Coan, M., Hossain, N., Larson, C., Herold, N., McKerrow, A., VanDriel, J.N., and Wickham, J., 2007, Completion of the 2001 National land cover database for the conterminous United States: Photogrammetric Engineering and Remote Sensing, v. 73, no. 4, p. 337-341.

MacDonald, D.D., Ingersoll, C.G., and Berger, T.A., 2000, Development and evaluation of consensus-based sediment quality guidelines for freshwater ecosystems: Environmental Contamination and Toxicology, v. 39, p. 20-31.

Petersen, J.C., Adamski, J.C., Bell, R.W., Davis, J.V., Femmer, S.R., Freiwald, D.A., and Joseph, R.L., 1998, Water quality in the Ozark Plateaus-Arkansas, Kansas, Missouri, and Oklahoma, 1992-95: U.S. Geological Survey Circular 1158, $33 \mathrm{p}$.

Taggart, J.E., ed., 2002, Analytical methods for chemical analysis of geologic and other materials, U.S. Geological Survey: U.S. Geological Survey Open-File Report 02-223, $20 \mathrm{p}$.

Vineyard, J.D. and Feder, G.L., 1974, Springs of Missouri: Rolla, Missouri Department of Natural Resources, Division of Geology and Land Survey Water Resources Report Number 26, $266 \mathrm{p}$.
Zielinski, R.A., and Budahn, J.R., 1998, Radionuclides in fly ash and bottom ash: Improved characterization based on radiography and low energy gamma-ray spectrometry: Fuel, v. 77 , no. 4, p. 259-267. 

Table 
Table 1. Quantitative elemental analyses of less than 63-micrometer fraction and lead-210 date of Clearwater Lake bed-sediment samples, 2002.-Continued

[All concentrations in milligrams per kilogram; RSD, relative standard deviation; ${ }^{\circ}$, degrees; ', minutes; ", seconds; <, less than; shaded cells exceed threshold effects concentration; --, no data]

\begin{tabular}{|c|c|c|c|c|c|c|c|c|c|c|c|c|c|c|c|}
\hline $\begin{array}{l}\text { Depth below } \\
\text { lakebed } \\
\text { surface, } \\
\text { (centimeters) }\end{array}$ & Aluminum & Antimony & Arsenic & Barium & Beryllium & Bismuth & Cadmium & Calcium & Cerium & Cesium & Chromium & Cobalt & Copper & Gallium & Iron \\
\hline RSD (percent) & 3.99 & 9.92 & 6.64 & 2.58 & 3.87 & 5.27 & 11.26 & 3.52 & 6.28 & 3.09 & 2.72 & 2.65 & 2.84 & 2.14 & 2.87 \\
\hline \multicolumn{16}{|c|}{ Webb Creek (Latitude $37^{\circ} 08^{\prime} 56^{\prime \prime}$ Longitude $090^{\circ} 48^{\prime} 18^{\prime \prime}$ ) } \\
\hline 1 & 38,800 & 0.54 & 4.7 & 421 & 1.6 & 0.16 & 0.24 & 2,060 & 77.3 & 2.6 & 38.9 & 18.2 & 19.2 & 8.4 & 19,000 \\
\hline 2 & 38,100 & .56 & 3.9 & 406 & 1.5 & .15 & .24 & 2,010 & 78.4 & 2.5 & 41.0 & 15.6 & 18.3 & 8.3 & 16,000 \\
\hline 3 & 36,000 & .49 & 3.7 & 408 & 1.4 & .14 & .20 & 1,840 & 76.7 & 2.4 & 38.0 & 15.8 & 17.1 & 7.8 & 16,000 \\
\hline 4 & 36,200 & .49 & 4.3 & 405 & 1.5 & .14 & .22 & 1,740 & 74.9 & 2.4 & 44.7 & 16.5 & 18.4 & 7.9 & 18,000 \\
\hline 5 & 37,200 & .53 & 4.5 & 409 & 1.4 & .14 & .22 & 1,810 & 78.4 & 2.4 & 44.3 & 17.0 & 18.9 & 8.2 & 18,000 \\
\hline 6 & 36,000 & .46 & 3.8 & 397 & 1.5 & .15 & .24 & 1,910 & 79.0 & 2.4 & 41.7 & 16.4 & 18.6 & 7.8 & 17,000 \\
\hline 7 & 36,700 & .49 & 3.7 & 402 & 1.6 & .14 & .23 & 1,920 & 79.1 & 2.4 & 43.6 & 16.9 & 19.2 & 7.9 & 17,000 \\
\hline 8 & 36,500 & .51 & 3.8 & 393 & 1.4 & .15 & .23 & 2,000 & 78.0 & 2.4 & 42.4 & 16.1 & 17.8 & 7.9 & 16,000 \\
\hline 9 & 36,400 & .52 & 4.6 & 403 & 1.4 & .14 & .23 & 2,070 & 79.8 & 2.4 & 42.5 & 19.0 & 18.9 & 8.1 & 18,000 \\
\hline 10 & 36,700 & .58 & 5.1 & 401 & 1.6 & .15 & .25 & 2,260 & 85.0 & 2.4 & 43.0 & 20.4 & 19.6 & 8.2 & 18,000 \\
\hline 11 & 38,800 & .61 & 5.5 & 430 & 1.6 & .16 & .24 & 2,250 & 86.4 & 2.6 & 45.7 & 19.3 & 19.9 & 8.6 & 20,000 \\
\hline 12 & 37,400 & .55 & 4.7 & 418 & 1.5 & .15 & .22 & 2,140 & 81.6 & 2.5 & 44.7 & 17.2 & 17.8 & 8.3 & 18,000 \\
\hline 13 & 37,100 & .52 & 4.4 & 410 & 1.5 & .14 & .21 & 2,050 & 83.3 & 2.4 & 40.3 & 17.5 & 17.8 & 8.1 & 18,000 \\
\hline 14 & 38,300 & .57 & 4.2 & 419 & 1.6 & .14 & .22 & 1,990 & 84.7 & 2.6 & 41.8 & 18.5 & 18.1 & 8.4 & 17,000 \\
\hline 15 & 40,200 & .62 & 5.2 & 434 & 1.7 & .17 & .23 & 2,040 & 87.4 & 2.8 & 45.8 & 22.5 & 19.6 & 8.8 & 18,000 \\
\hline 16 & 39,100 & .60 & 5.2 & 430 & 1.6 & .16 & .22 & 2,070 & 87.3 & 2.7 & 46.5 & 19.4 & 19.6 & 8.7 & 19,000 \\
\hline 17 & 39,300 & .60 & 4.7 & 433 & 1.6 & .16 & .22 & 2,180 & 88.1 & 2.6 & 47.1 & 18.0 & 21.0 & 8.8 & 19,000 \\
\hline 18 & 39,500 & .55 & 4.3 & 440 & 1.5 & .15 & .22 & 2,020 & 85.3 & 2.6 & 42.1 & 17.0 & 19.0 & 8.7 & 18,000 \\
\hline 19 & 38,700 & .58 & 4.8 & 422 & 1.5 & .15 & .22 & 2,120 & 81.8 & 2.6 & 43.2 & 19.0 & 19.5 & 8.5 & 18,000 \\
\hline 20 & 38,000 & .54 & 4.7 & 423 & 1.6 & .14 & .21 & 2,140 & 81.8 & 2.6 & 42.9 & 18.0 & 20.7 & 8.4 & 18,000 \\
\hline 21 & 35,700 & .55 & 4.2 & 402 & 1.4 & .14 & .22 & 2,120 & 77.8 & 2.3 & 40.0 & 17.4 & 18.3 & 7.9 & 18,000 \\
\hline 22 & 34,200 & .50 & 3.7 & 393 & 1.4 & .15 & .20 & 2,100 & 78.4 & 2.2 & 38.2 & 16.5 & 16.7 & 7.6 & 17,000 \\
\hline 23 & 34,100 & .49 & 3.7 & 395 & 1.4 & .12 & .21 & 2,140 & 78.2 & 2.2 & 39.1 & 15.8 & 17.3 & 7.6 & 17,000 \\
\hline 24 & 33,200 & .46 & 3.7 & 391 & 1.3 & .14 & .22 & 2,020 & 75.0 & 2.1 & 39.1 & 17.2 & 24.6 & 7.3 & 16,000 \\
\hline 25 & 33,900 & .48 & 3.6 & 395 & 1.4 & .12 & .22 & 2,100 & 72.5 & 2.1 & 37.1 & 16.6 & 16.4 & 7.5 & 16,000 \\
\hline 26 & 20,400 & .28 & 2.0 & 250 & .7 & $<.1$ & .13 & 1,280 & 45.3 & 1.3 & 24.7 & 10.0 & 12.9 & 4.5 & 9,900 \\
\hline 27 & 33,500 & .51 & 3.7 & 394 & 1.4 & .12 & .20 & 2,000 & 75.8 & 2.2 & 39.4 & 18.0 & 16.9 & 7.4 & 16,000 \\
\hline 28 & 33,100 & .51 & 4.3 & 386 & 1.2 & .12 & .20 & 2,160 & 75.7 & 2.1 & 48.2 & 18.6 & 17.1 & 7.3 & 16,000 \\
\hline 29 & 31,400 & .54 & 6.6 & 394 & 1.4 & .12 & .22 & 2,470 & 79.4 & 2.0 & 37.8 & 22.3 & 18.4 & 7.0 & 16,000 \\
\hline 30 & 31,400 & .52 & 7.0 & 398 & 1.4 & .11 & .25 & 2,420 & 82.2 & 2.0 & 40.6 & 23.2 & 18.2 & 7.1 & 16,000 \\
\hline 31 & 30,600 & .44 & 6.2 & 381 & 1.3 & .10 & .23 & 2,240 & 71.6 & 2.0 & 34.6 & 19.9 & 16.8 & 6.9 & 15,000 \\
\hline 32 & 33,100 & .56 & 6.7 & 405 & 1.5 & .10 & .26 & 2,500 & 79.8 & 2.1 & 39.7 & 20.6 & 17.6 & 7.3 & 17,000 \\
\hline 33 & 36,200 & .54 & 6.0 & 410 & 1.6 & .11 & .22 & 2,240 & 79.6 & 2.4 & 41.3 & 18.4 & 18.4 & 8.1 & 17,000 \\
\hline 34 & 40,400 & .61 & 6.2 & 431 & 1.7 & .13 & .24 & 2,340 & 86.4 & 2.7 & 43.3 & 18.9 & 19.6 & 8.7 & 19,000 \\
\hline 35 & 41,700 & .61 & 6.5 & 442 & 1.8 & .13 & .22 & 2,120 & 85.4 & 2.8 & 46.2 & 19.8 & 20.9 & 9.2 & 20,000 \\
\hline 36 & 41,200 & .61 & 6.2 & 437 & 1.6 & .13 & .23 & 1,990 & 84.4 & 2.7 & 48.9 & 18.9 & 20.3 & 9.0 & 19,000 \\
\hline 37 & 39,900 & .67 & 6.0 & 443 & 1.5 & .12 & .22 & 2,020 & 86.1 & 2.6 & 43.6 & 17.9 & 19.9 & 8.7 & 18,000 \\
\hline 38 & 38,500 & .60 & 5.7 & 432 & 1.6 & .12 & .21 & 1,930 & 84.5 & 2.5 & 43.6 & 16.4 & 18.8 & 8.4 & 18,000 \\
\hline
\end{tabular}


Table 1. Quantitative elemental analyses of less than 63-micrometer fraction and lead-210 date of Clearwater Lake bed-sediment samples, 2002.-Continued

[All concentrations in milligrams per kilogram; RSD, relative standard deviation; ${ }^{\circ}$, degrees; ', minutes; ", seconds; <, less than; shaded cells exceed threshold effects concentration; --, no data]

\begin{tabular}{|c|c|c|c|c|c|c|c|c|c|c|c|c|c|c|c|}
\hline $\begin{array}{l}\text { Depth below } \\
\text { lakebed } \\
\text { surface, } \\
\text { (centimeters) }\end{array}$ & Aluminum & Antimony & Arsenic & Barium & Beryllium & Bismuth & Cadmium & Calcium & Cerium & Cesium & Chromium & Cobalt & Copper & Gallium & Iron \\
\hline RSD (percent) & 3.99 & 9.92 & 6.64 & 2.58 & 3.87 & 5.27 & 11.26 & 3.52 & 6.28 & 3.09 & 2.72 & 2.65 & 2.84 & 2.14 & 2.87 \\
\hline \multicolumn{16}{|c|}{ Webb Creek (Latitude $37^{\circ} 08^{\prime} 56^{\prime \prime}$ Longitude $090^{\circ} 48^{\prime} 18^{\prime \prime}$ )—Continued } \\
\hline 39 & 41,400 & 0.67 & 6.4 & 448 & 1.6 & 0.13 & 0.22 & 2,090 & 83.9 & 2.8 & 45.7 & 18.4 & 20.9 & 9.0 & 19,000 \\
\hline 40 & 44,300 & .69 & 6.4 & 475 & 1.7 & .14 & .21 & 2,010 & 83.6 & 3.0 & 51.4 & 18.2 & 23.2 & 9.6 & 20,000 \\
\hline 41 & 41,900 & 67 & 5.8 & 453 & 1.7 & .12 & .20 & 2,040 & 74.6 & 2.8 & 39.5 & 16.9 & 19.3 & 9.1 & 18,000 \\
\hline 42 & 38,800 & .61 & 5.6 & 430 & 1.6 & .12 & .22 & 2,220 & 73.2 & 2.6 & 38.4 & 17.4 & 19.6 & 8.6 & 18,000 \\
\hline 43 & 38,000 & .60 & 5.6 & 422 & 1.4 & .11 & .24 & 2,130 & 70.3 & 2.5 & 41.1 & 17.3 & 18.0 & 8.5 & 17,000 \\
\hline 44 & 37,400 & .62 & 5.4 & 417 & 1.4 & .11 & .24 & 2,240 & 72.1 & 2.4 & 43.1 & 17.0 & 19.0 & 8.4 & 17,000 \\
\hline 45 & 36,200 & .61 & 5.3 & 410 & 1.4 & .11 & .24 & 2,300 & 71.9 & 2.3 & 38.2 & 16.3 & 18.0 & 8.1 & 17,000 \\
\hline 46 & 35,600 & .58 & 5.0 & 408 & 1.4 & .10 & .22 & 2,280 & 70.9 & 2.3 & 36.9 & 15.3 & 18.8 & 7.9 & 16,000 \\
\hline 47 & 35,000 & .55 & 5.0 & 404 & 1.4 & .10 & .23 & 2,130 & 70.5 & 2.3 & 36.8 & 15.4 & 17.9 & 7.7 & 16,000 \\
\hline 48 & 35,900 & .56 & 5.1 & 412 & 1.3 & .10 & .24 & 2,090 & 68.7 & 2.3 & 41.4 & 16.1 & 18.1 & 7.7 & 17,000 \\
\hline 49 & 35,600 & .55 & 4.8 & 400 & 1.4 & .10 & .24 & 1,970 & 71.4 & 2.3 & 38.0 & 15.8 & 18.5 & 7.7 & 16,000 \\
\hline 50 & 37,000 & .57 & 5.0 & 409 & 1.4 & .10 & .23 & 1,980 & 73.3 & 2.4 & 38.4 & 16.4 & 18.8 & 8.0 & 16,000 \\
\hline 51 & 35,400 & .54 & 5.3 & 409 & 1.2 & .16 & .23 & 1,860 & 83.2 & 2.4 & 32.8 & 15.5 & 17.6 & 7.3 & 15,000 \\
\hline 52 & 32,500 & .45 & 5.2 & 390 & 1.2 & .14 & .20 & 1,960 & 84.4 & 2.2 & 32.6 & 13.7 & 15.4 & 7.2 & 16,000 \\
\hline 53 & 34,100 & .48 & 4.6 & 408 & 1.2 & .14 & .21 & 2,060 & 81.8 & 2.3 & 32.6 & 14.6 & 16.2 & 7.2 & 16,000 \\
\hline 54 & 35,200 & .46 & 4.8 & 408 & 1.1 & .14 & .22 & 2,110 & 82.5 & 2.4 & 33.2 & 15.0 & 16.6 & 7.4 & 16,000 \\
\hline 55 & 34,700 & .44 & 4.8 & 407 & 1.4 & .14 & .22 & 1,910 & 83.5 & 2.4 & 32.3 & 14.3 & 16.9 & 7.3 & 15,000 \\
\hline 56 & 35,100 & .45 & 5.3 & 415 & 1.4 & .15 & .22 & 2,040 & 85.7 & 2.4 & 37.9 & 14.5 & 17.4 & 7.4 & 15,000 \\
\hline 57 & 35,200 & .46 & 4.4 & 413 & 1.3 & .14 & .21 & 2,080 & 82.4 & 2.3 & 34.1 & 14.3 & 16.7 & 7.0 & 15,000 \\
\hline 58 & 34,800 & .47 & 5.0 & 404 & 1.4 & .15 & .22 & 2,100 & 86.1 & 2.3 & 37.0 & 13.6 & 16.8 & 7.3 & 15,000 \\
\hline 59 & 34,600 & .52 & 5.1 & 398 & 1.3 & .14 & .20 & 2,010 & 83.5 & 2.3 & 34.3 & 13.9 & 16.3 & 7.2 & 15,000 \\
\hline 60 & 37,600 & .48 & 5.3 & 426 & 1.4 & .15 & .22 & 2,010 & 86.4 & 2.5 & 38.4 & 15.8 & 16.8 & 7.6 & 16,000 \\
\hline 61 & 39,400 & .57 & 6.0 & 431 & 1.5 & .16 & .21 & 1,890 & 85.2 & 2.6 & 35.9 & 17.2 & 17.6 & 8.3 & 17,000 \\
\hline 62 & 41,600 & .60 & 6.7 & 429 & 1.4 & .18 & .24 & 1,820 & 90.2 & 2.9 & 39.0 & 18.0 & 18.8 & 8.9 & 17,000 \\
\hline 63 & 38,600 & .57 & 5.6 & 426 & 1.4 & .17 & .22 & 1,730 & 88.8 & 2.7 & 34.7 & 15.0 & 17.4 & 8.2 & 16,000 \\
\hline 64 & 38,800 & .55 & 5.6 & 440 & 1.4 & .16 & .23 & 1,900 & 86.5 & 2.7 & 36.8 & 14.7 & 18.1 & 8.0 & 16,000 \\
\hline 65 & 34,400 & .46 & 5.2 & 412 & 1.3 & .15 & .21 & 1,910 & 83.1 & 2.3 & 31.3 & 14.6 & 18.2 & 7.1 & 15,000 \\
\hline 66 & 35,000 & .43 & 4.5 & 415 & 1.2 & .15 & .20 & 2,150 & 88.4 & 2.3 & 34.7 & 15.8 & 16.4 & 7.2 & 14,000 \\
\hline 67 & 35,000 & .49 & 5.0 & 403 & 1.4 & .14 & .18 & 2,050 & 90.8 & 2.4 & 34.8 & 14.5 & 18.4 & 7.2 & 15,000 \\
\hline 68 & 35,000 & .49 & 5.0 & 414 & 1.2 & .15 & .19 & 2,370 & 91.5 & 2.4 & 37.0 & 14.4 & 16.2 & 7.3 & 15,000 \\
\hline 69 & 35,200 & .49 & 4.6 & 416 & 1.3 & .15 & .23 & 2,050 & 93.6 & 2.3 & 37.1 & 14.0 & 15.6 & 7.6 & 15,000 \\
\hline 70 & 36,300 & .48 & 5.0 & 429 & 1.2 & .14 & .18 & 2,080 & 85.4 & 2.4 & 32.9 & 16.0 & 16.2 & 7.3 & 15,000 \\
\hline 71 & 35,200 & .49 & 4.9 & 418 & 1.3 & .14 & .17 & 2,000 & 81.7 & 2.3 & 30.5 & 16.2 & 15.8 & 7.0 & 14,000 \\
\hline 72 & 36,600 & .50 & 4.5 & 421 & 1.4 & .15 & .10 & 2,070 & 85.8 & 2.4 & 34.4 & 14.2 & 17.2 & 7.6 & 15,000 \\
\hline Maximum & 44,300 & 0.69 & 7.0 & 475 & 1.8 & 0.18 & 0.26 & 2,500 & 93.6 & 3.0 & 51.4 & 23.2 & 24.6 & 9.6 & 20,000 \\
\hline Minimum & 20,400 & .28 & 2.0 & 250 & .7 & .10 & .10 & 1,280 & 45.3 & 1.3 & 24.7 & 10.0 & 12.9 & 4.5 & 9,900 \\
\hline Mean & 36,457 & .54 & 5.0 & 413 & 1.4 & .14 & .22 & 2,062 & 80.7 & 2.4 & 39.4 & 16.9 & 18.2 & 7.9 & 16,693 \\
\hline Median & 36,250 & .54 & 5.0 & 411 & 1.4 & .14 & .22 & 2,060 & 82.0 & 2.4 & 39.1 & 16.8 & 18.2 & 7.9 & 17,000 \\
\hline
\end{tabular}


Table 1. Quantitative elemental analyses of less than 63-micrometer fraction and lead-210 date of Clearwater Lake bed-sediment samples, 2002.-Continued

[All concentrations in milligrams per kilogram; RSD, relative standard deviation; ${ }^{\circ}$, degrees; ', minutes; ", seconds; <, less than; shaded cells exceed threshold effects concentration; --, no data]

\begin{tabular}{|c|c|c|c|c|c|c|c|c|c|c|c|c|c|c|c|}
\hline $\begin{array}{l}\text { Depth below } \\
\text { lakebed } \\
\text { surface, } \\
\text { (centimeters) }\end{array}$ & Aluminum & Antimony & Arsenic & Barium & Beryllium & Bismuth & Cadmium & Calcium & Cerium & Cesium & Chromium & Cobalt & Copper & Gallium & Iron \\
\hline RSD (percent) & 3.99 & 9.92 & 6.64 & 2.58 & 3.87 & 5.27 & 11.26 & 3.52 & 6.28 & 3.09 & 2.72 & 2.65 & 2.84 & 2.14 & 2.87 \\
\hline \multicolumn{16}{|c|}{ Logan Creek (Latitude $37^{\circ} 09^{\prime} 21^{\prime \prime}$ Longitude $090^{\circ} 48^{\prime} 07^{\prime \prime}$ ) } \\
\hline 1 & 42,700 & 0.64 & 6.8 & 382 & 1.5 & 0.18 & 0.24 & 2,100 & 72.0 & 2.8 & 49.6 & 18.0 & 23.2 & 9.2 & 19,000 \\
\hline 2 & 45,000 & .63 & 6.4 & 366 & 2.0 & .20 & .35 & 2,320 & 83.6 & 3.1 & 57.2 & 21.6 & 25.3 & 9.7 & 20,000 \\
\hline 3 & 45,300 & .64 & 6.6 & 370 & 1.8 & .22 & .37 & 2,320 & 75.3 & 3.2 & 64.7 & 22.6 & 25.5 & 9.8 & 21,000 \\
\hline 4 & 44,300 & .70 & 7.0 & 366 & 1.8 & .21 & .36 & 2,460 & 81.4 & 3.1 & 60.8 & 23.1 & 61.6 & 9.5 & 21,000 \\
\hline 5 & 45,600 & .67 & 6.9 & 383 & 1.8 & .20 & .36 & 2,300 & 76.2 & 3.2 & 46.0 & 23.2 & 25.1 & 9.9 & 23,000 \\
\hline 6 & 45,000 & .67 & 7.4 & 385 & 2.0 & .20 & .36 & 2,330 & 76.0 & 3.2 & 44.2 & 24.0 & 24.8 & 9.9 & 22,000 \\
\hline 7 & 44,200 & .65 & 7.2 & 377 & 1.8 & .22 & .36 & 2,320 & 82.9 & 3.2 & 44.6 & 23.4 & 25.0 & 9.7 & 22,000 \\
\hline 8 & 45,300 & .68 & 7.4 & 381 & 1.8 & .21 & .35 & 2,400 & 83.7 & 3.2 & 48.8 & 24.2 & 24.8 & 9.9 & 22,000 \\
\hline 9 & 39,600 & .60 & 6.7 & 329 & 1.7 & .18 & .31 & 2,160 & 68.9 & 2.8 & 41.6 & 20.5 & 25.2 & 8.5 & 19,000 \\
\hline 10 & 35,700 & .54 & 6.0 & 293 & 1.4 & .16 & .26 & 2,130 & 60.4 & 2.5 & 37.5 & 19.7 & 20.6 & 7.9 & 17,000 \\
\hline 11 & 40,000 & .59 & 6.9 & 326 & 1.6 & .18 & .30 & 2,080 & 67.1 & 2.9 & 41.4 & 21.2 & 22.0 & 8.8 & 19,000 \\
\hline 12 & 40,500 & .61 & 7.4 & 344 & 1.6 & .18 & .28 & 2,260 & 68.2 & 2.9 & 40.9 & 21.5 & 22.6 & 8.8 & 20,000 \\
\hline 13 & 35,700 & .52 & 5.4 & 312 & 1.4 & .16 & .25 & 2,040 & 68.0 & 2.4 & 36.5 & 18.6 & 20.9 & 7.7 & 16,000 \\
\hline 14 & 34,100 & .52 & 5.1 & 299 & 1.4 & .15 & .23 & 1,770 & 60.0 & 2.3 & 36.7 & 18.4 & 20.0 & 7.3 & 16,000 \\
\hline 15 & 31,900 & .46 & 5.3 & 286 & 1.3 & .13 & .21 & 1,740 & 55.6 & 2.2 & 34.7 & 17.5 & 18.1 & 6.9 & 16,000 \\
\hline 16 & 35,900 & .52 & 5.6 & 321 & 1.5 & .15 & .25 & 1,910 & 63.3 & 2.4 & 38.4 & 18.7 & 19.1 & 7.8 & 17,000 \\
\hline 17 & 38,100 & .53 & 6.1 & 332 & 1.6 & .16 & .26 & 1,950 & 64.2 & 2.6 & 39.4 & 19.9 & 23.5 & 8.2 & 18,000 \\
\hline 18 & 33,700 & .47 & 5.3 & 297 & 1.3 & .14 & .23 & 1,740 & 60.4 & 2.3 & 37.8 & 17.6 & 17.6 & 7.1 & 16,000 \\
\hline 19 & 35,900 & .50 & 5.0 & 316 & 1.5 & .15 & .24 & 1,840 & 63.6 & 2.5 & 39.4 & 18.2 & 19.4 & 7.7 & 17,000 \\
\hline 20 & 32,900 & .52 & 5.0 & 291 & 1.2 & .14 & .21 & 1,700 & 60.4 & 2.2 & 36.9 & 17.6 & 17.2 & 7.1 & 15,000 \\
\hline 21 & 33,900 & .47 & 5.1 & 293 & 1.4 & .14 & .22 & 1,700 & 57.4 & 2.3 & 38.7 & 16.8 & 20.2 & 7.4 & 15,000 \\
\hline 22 & 34,100 & .46 & 5.2 & 306 & 1.3 & .14 & .21 & 1,680 & 57.9 & 2.3 & 35.1 & 16.4 & 17.5 & 7.3 & 16,000 \\
\hline 23 & 32,400 & .48 & 4.3 & 306 & 1.2 & .16 & .15 & 1,570 & 55.5 & 2.2 & 41.6 & 13.9 & 16.7 & 6.9 & 13,000 \\
\hline 24 & 25,300 & .38 & 3.6 & 226 & .9 & .10 & .11 & 1,190 & 44.4 & 1.6 & 34.2 & 11.7 & 14.4 & 5.4 & 10,000 \\
\hline 25 & 23,800 & .35 & 3.0 & 209 & .9 & .09 & .07 & 1,050 & 41.9 & 1.5 & 29.4 & 10.1 & 17.2 & 5.2 & 9,300 \\
\hline 26 & 27,700 & .41 & 3.3 & 251 & 1.0 & .10 & .07 & 1,250 & 47.5 & 1.8 & 33.3 & 11.4 & 14.2 & 5.8 & 11,000 \\
\hline 27 & 28,600 & .36 & 3.3 & 257 & .9 & .11 & .10 & 1,260 & 47.5 & 1.8 & 32.9 & 11.0 & 13.9 & 6.0 & 10,000 \\
\hline 28 & 26,000 & .41 & 4.2 & 243 & .9 & .10 & .07 & 1,180 & 51.9 & 1.7 & 33.9 & 11.4 & 15.6 & 5.6 & 12,000 \\
\hline Maximum & 45,600 & 0.70 & 7.4 & 385 & 2.0 & 0.22 & 0.37 & 2,460 & 83.7 & 3.2 & 64.7 & 24.2 & 61.6 & 9.9 & 23,000 \\
\hline Minimum & 23,800 & .35 & 3.0 & 209 & .88 & .09 & .07 & 1,050 & 41.9 & 1.5 & 29.4 & 10.1 & 13.9 & 5.2 & 9,300 \\
\hline Mean & 36,543 & .54 & 5.6 & 316 & 1.4 & .16 & .24 & 1,884 & 64.1 & 2.5 & 41.3 & 18.3 & 21.8 & 7.9 & 16,868 \\
\hline Median & 35,800 & .52 & 5.5 & 314 & 1.5 & .16 & .25 & 1,930 & 63.5 & 2.5 & 39.1 & 18.5 & 20.4 & 7.8 & 17,000 \\
\hline
\end{tabular}


Table 1. Quantitative elemental analyses of less than 63-micrometer fraction and lead-210 date of Clearwater Lake bed-sediment samples, 2002.-Continued

[All concentrations in milligrams per kilogram; RSD, relative standard deviation; ${ }^{\circ}$, degrees; ', minutes; ", seconds; <, less than; shaded cells exceed threshold effects concentration; --, no data]

\begin{tabular}{|c|c|c|c|c|c|c|c|c|c|c|c|c|c|c|c|}
\hline $\begin{array}{l}\text { Depth below } \\
\text { lakebed } \\
\text { surface, } \\
\text { (centimeters) }\end{array}$ & Aluminum & Antimony & Arsenic & Barium & Beryllium & Bismuth & Cadmium & Calcium & Cerium & Cesium & Chromium & Cobalt & Copper & Gallium & Iron \\
\hline RSD (percent) & 3.99 & 9.92 & 6.64 & 2.58 & 3.87 & 5.27 & 11.26 & 3.52 & 6.28 & 3.09 & 2.72 & 2.65 & 2.84 & 2.14 & 2.87 \\
\hline \multicolumn{16}{|c|}{ Upper Black River (Latitude $37^{\circ} 10^{\prime} 38^{\prime \prime}$ Longitude $090^{\circ} 47^{\prime} 28^{\prime \prime}$ ) } \\
\hline 1 & 44,900 & 0.67 & 8.6 & 369 & 2.1 & 0.21 & 0.49 & 3,560 & 95.3 & 2.9 & 43.4 & 23.5 & 31.3 & 9.7 & 22,000 \\
\hline 2 & 40,200 & .56 & 6.4 & 349 & 1.7 & .19 & .44 & 3,340 & 88.8 & 2.5 & 41.3 & 18.4 & 26.3 & 8.6 & 18,000 \\
\hline 3 & 37,600 & .52 & 6.5 & 345 & 1.7 & .18 & .44 & 3,280 & 83.6 & 2.3 & 41.0 & 19.2 & 24.8 & 8.0 & 18,000 \\
\hline 4 & 39,800 & .55 & 6.8 & 355 & 1.9 & .19 & .46 & 3,190 & 89.4 & 2.4 & 40.0 & 19.2 & 26.8 & 8.5 & 19,000 \\
\hline 5 & 41,500 & .56 & 7.0 & 375 & 2.0 & .19 & .47 & 3,420 & 91.3 & 2.6 & 41.0 & 20.4 & 27.2 & 8.9 & 20,000 \\
\hline 6 & 42,200 & .58 & 7.1 & 368 & 1.9 & .20 & .50 & 3,430 & 92.4 & 2.6 & 41.7 & 20.8 & 27.4 & 9.0 & 20,000 \\
\hline 7 & 41,400 & .57 & 6.8 & 368 & 2.0 & .20 & .47 & 3,450 & 91.6 & 2.5 & 42.7 & 19.4 & 27.3 & 8.8 & 20,000 \\
\hline 8 & 41,400 & .52 & 6.7 & 356 & 1.9 & .19 & .45 & 3,320 & 89.3 & 2.5 & 43.4 & 19.9 & 26.6 & 8.8 & 19,000 \\
\hline 9 & 41,000 & .53 & 6.8 & 346 & 2.0 & .19 & .46 & 3,480 & 92.3 & 2.4 & 43.6 & 20.6 & 28.3 & 8.7 & 19,000 \\
\hline 10 & 41,000 & .59 & 7.6 & 341 & 1.9 & .20 & .44 & 3,450 & 94.0 & 2.5 & 44.0 & 22.5 & 27.8 & 8.9 & 19,000 \\
\hline 11 & 42,200 & .64 & 7.9 & 350 & 2.2 & .20 & .45 & 3,310 & 97.4 & 2.6 & 43.5 & 22.9 & 28.8 & 8.9 & 20,000 \\
\hline 12 & 43,800 & .64 & 8.1 & 360 & 2.1 & .21 & .46 & 3,350 & 101.0 & 2.7 & 47.5 & 24.2 & 29.9 & 9.6 & 21,000 \\
\hline 13 & 43,900 & .60 & 7.6 & 368 & 2.1 & .21 & .48 & 3,490 & 93.4 & 2.7 & 45.1 & 23.0 & 28.6 & 9.3 & 20,000 \\
\hline 14 & 43,200 & .60 & 7.2 & 362 & 2.1 & .20 & .52 & 3,640 & 90.9 & 2.6 & 43.7 & 21.6 & 27.8 & 9.1 & 20,000 \\
\hline 15 & 43,500 & .60 & 7.6 & 356 & 2.0 & .20 & .51 & 3,890 & 92.1 & 2.6 & 41.7 & 22.7 & 27.9 & 9.2 & 20,000 \\
\hline 16 & 42,500 & .60 & 7.4 & 354 & 1.8 & .19 & .49 & 3,740 & 91.2 & 2.6 & 43.4 & 21.7 & 27.9 & 8.9 & 20,000 \\
\hline 17 & 42,400 & .64 & 7.2 & 355 & 1.9 & .19 & .47 & 3,930 & 92.8 & 2.6 & 42.4 & 21.5 & 27.6 & 9.0 & 20,000 \\
\hline 18 & 41,700 & .54 & 6.5 & 345 & 1.9 & .20 & .49 & 4,630 & 90.4 & 2.5 & 44.6 & 20.0 & 26.6 & 8.6 & 18,000 \\
\hline 19 & 41,900 & .56 & 7.2 & 344 & 2.1 & .20 & .49 & 5,010 & 90.6 & 2.5 & 44.5 & 22.7 & 27.6 & 8.8 & 19,000 \\
\hline 20 & 44,300 & .60 & 7.8 & 351 & 2.1 & .21 & .51 & 4,610 & 95.3 & 2.8 & 47.8 & 24.0 & 29.4 & 9.6 & 20,000 \\
\hline 21 & 41,600 & .67 & 8.8 & 370 & 1.9 & .21 & .50 & 3,740 & 92.9 & 2.8 & 45.7 & 23.5 & 29.0 & 9.2 & 19,000 \\
\hline 22 & 37,800 & .54 & 7.2 & 356 & 1.9 & .18 & .46 & 5,010 & 85.4 & 2.6 & 40.1 & 19.8 & 25.7 & 8.4 & 18,000 \\
\hline 23 & 38,200 & .57 & 7.2 & 359 & 1.8 & .17 & .46 & 4,660 & 83.6 & 2.5 & 38.2 & 20.3 & 25.5 & 8.3 & 18,000 \\
\hline 24 & 37,100 & .58 & 7.2 & 359 & 1.7 & .17 & .45 & 4,200 & 83.9 & 2.4 & 38.9 & 19.8 & 25.3 & 8.0 & 17,000 \\
\hline 25 & 38,100 & .55 & 7.4 & 361 & 1.6 & .16 & .47 & 5,360 & 87.8 & 2.5 & 41.4 & 21.7 & 25.8 & 8.3 & 18,000 \\
\hline 26 & 38,200 & .54 & 7.2 & 358 & 1.9 & .17 & .45 & 4,400 & 87.8 & 2.6 & 39.9 & 18.5 & 26.6 & 8.6 & 18,000 \\
\hline 27 & 37,700 & .57 & 7.0 & 369 & 1.7 & .17 & .45 & 2,770 & 83.4 & 2.4 & 39.0 & 18.7 & 25.5 & 8.2 & 17,000 \\
\hline 28 & 37,500 & .57 & 6.7 & 364 & 1.7 & .17 & .41 & 2,620 & 84.0 & 2.4 & 40.8 & 17.8 & 25.1 & 8.1 & 17,000 \\
\hline 29 & 37,800 & .56 & 6.7 & 369 & 1.7 & .16 & .42 & 2,530 & 84.0 & 2.4 & 36.4 & 17.9 & 24.7 & 8.2 & 17,000 \\
\hline 30 & 40,100 & .57 & 7.1 & 377 & 1.6 & .17 & .47 & 2,720 & 87.0 & 2.6 & 40.2 & 19.0 & 25.8 & 8.6 & 18,000 \\
\hline 31 & 39,500 & .59 & 7.0 & 371 & 1.8 & .17 & .46 & 2,770 & 84.1 & 2.6 & 44.7 & 18.6 & 26.1 & 8.4 & 18,000 \\
\hline 32 & 41,200 & .59 & 7.1 & 378 & 1.8 & .17 & .49 & 2,800 & 92.6 & 2.7 & 46.2 & 20.0 & 25.6 & 8.7 & 18,000 \\
\hline 33 & 41,600 & .60 & 6.7 & 376 & 1.9 & .18 & .48 & 2,760 & 88.0 & 2.6 & 60.1 & 18.6 & 27.0 & 8.8 & 18,000 \\
\hline 34 & 40,200 & .58 & 6.2 & 393 & 1.8 & .16 & .49 & 2,650 & 85.1 & 2.6 & 42.4 & 17.3 & 25.6 & 8.4 & 17,000 \\
\hline 35 & 42,800 & .57 & 7.0 & 386 & 1.9 & .18 & .50 & 2,940 & 88.4 & 2.8 & 66.5 & 20.3 & 27.1 & 9.0 & 19,000 \\
\hline 36 & 40,000 & .52 & 6.8 & 364 & 1.9 & .17 & .43 & 2,750 & 88.3 & 2.6 & 69.7 & 18.6 & 26.7 & 8.4 & 18,000 \\
\hline 37 & 44,600 & .61 & 8.0 & 383 & 2.0 & .19 & .51 & 3,040 & 91.9 & 3.0 & 46.3 & 20.8 & 27.8 & 9.4 & 20,000 \\
\hline 38 & 46,600 & .61 & 7.9 & 397 & 2.0 & .20 & .54 & 3,090 & 98.6 & 3.1 & 52.1 & 21.8 & 29.0 & 9.6 & 20,000 \\
\hline
\end{tabular}


Table 1. Quantitative elemental analyses of less than 63-micrometer fraction and lead-210 date of Clearwater Lake bed-sediment samples, 2002.-Continued

[All concentrations in milligrams per kilogram; RSD, relative standard deviation; ${ }^{\circ}$, degrees; ', minutes; ", seconds; <, less than; shaded cells exceed threshold effects concentration; --, no data]

\begin{tabular}{|c|c|c|c|c|c|c|c|c|c|c|c|c|c|c|c|}
\hline $\begin{array}{l}\text { Depth below } \\
\text { lakebed } \\
\text { surface, } \\
\text { (centimeters) }\end{array}$ & Aluminum & Antimony & Arsenic & Barium & Beryllium & Bismuth & Cadmium & Calcium & Cerium & Cesium & Chromium & Cobalt & Copper & Gallium & Iron \\
\hline RSD (percent) & 3.99 & 9.92 & 6.64 & 2.58 & 3.87 & 5.27 & 11.26 & 3.52 & 6.28 & 3.09 & 2.72 & 2.65 & 2.84 & 2.14 & 2.87 \\
\hline \multicolumn{16}{|c|}{ Upper Black River (Latitude $37^{\circ} 10^{\prime} 38^{\prime \prime}$ Longitude $090^{\circ} 47^{\prime} 28^{\prime \prime}$ )—Continued } \\
\hline 39 & 46,700 & 0.62 & 7.9 & 386 & 2.0 & 0.20 & 0.56 & 3,440 & 93.0 & 3.2 & 48.7 & 22.0 & 28.9 & 10.0 & 21,000 \\
\hline 40 & 47,000 & .67 & 7.9 & 381 & 2.0 & .20 & .56 & 3,500 & 91.6 & 3.2 & 54.6 & 22.3 & 28.8 & 9.7 & 21,000 \\
\hline 41 & 46,900 & .63 & 8.0 & 388 & 1.9 & .22 & .54 & 2,960 & 91.1 & 3.2 & 44.3 & 22.4 & 28.7 & 9.6 & 21,000 \\
\hline 42 & 46,200 & .62 & 7.6 & 392 & 2.3 & .20 & .53 & 3,060 & 91.0 & 3.1 & 46.0 & 22.2 & 27.7 & 9.9 & 21,000 \\
\hline 43 & 43,500 & .57 & 7.1 & 390 & 1.9 & .18 & .54 & 3,010 & 85.0 & 2.9 & 42.0 & 19.8 & 26.4 & 9.4 & 19,000 \\
\hline 44 & 42,000 & .57 & 7.1 & 382 & 1.7 & .18 & .51 & 2,750 & 92.1 & 2.7 & 38.5 & 18.9 & 25.6 & 8.8 & 18,000 \\
\hline 45 & 41,400 & .61 & 6.8 & 380 & 1.7 & .17 & .47 & 2,820 & 85.1 & 2.6 & 38.2 & 18.2 & 25.1 & 8.8 & 18,000 \\
\hline 46 & 42,500 & .56 & 6.7 & 380 & 1.9 & .18 & .51 & 3,090 & 88.7 & 2.8 & 41.7 & 18.6 & 26.4 & 8.9 & 19,000 \\
\hline 47 & 41,700 & .57 & 7.0 & 383 & 1.8 & .17 & .50 & 3,200 & 87.1 & 2.7 & 38.8 & 17.8 & 25.6 & 8.5 & 18,000 \\
\hline 48 & 40,700 & .55 & 6.3 & 382 & 1.8 & .18 & .49 & 2,960 & 85.3 & 2.6 & 39.0 & 17.1 & 24.8 & 8.4 & 17,000 \\
\hline 49 & 40,400 & .57 & 6.4 & 383 & 1.8 & .17 & .48 & 2,900 & 92.4 & 2.5 & 37.8 & 17.8 & 24.4 & 8.6 & 17,000 \\
\hline 50 & 38,400 & .62 & 6.4 & 366 & 1.7 & .16 & .44 & 3,420 & 81.2 & 2.4 & 36.5 & 16.1 & 23.2 & 8.0 & 16,000 \\
\hline 51 & 41,900 & .67 & 7.7 & 374 & 1.6 & .18 & .48 & 3,570 & 73.9 & 2.6 & 41.2 & 17.8 & 26.6 & 9.0 & 18,000 \\
\hline 52 & 43,800 & .71 & 7.7 & 385 & 2.0 & .18 & .49 & 3,660 & 73.0 & 2.8 & 42.9 & 19.4 & 28.0 & 9.4 & 19,000 \\
\hline 53 & 41,000 & .67 & 7.3 & 380 & 1.7 & .16 & .47 & 3,840 & 74.1 & 2.6 & 40.6 & 19.2 & 26.3 & 8.9 & 18,000 \\
\hline 54 & 40,600 & .67 & 7.1 & 392 & 1.7 & .16 & .47 & 3,110 & 72.0 & 2.5 & 39.8 & 19.4 & 25.4 & 8.7 & 18,000 \\
\hline 55 & 39,500 & .65 & 6.9 & 386 & 1.6 & .15 & .45 & 2,840 & 73.1 & 2.4 & 39.2 & 16.6 & 25.2 & 8.5 & 16,000 \\
\hline 56 & 39,000 & .63 & 6.5 & 382 & 1.6 & .15 & .44 & 3,040 & 72.8 & 2.4 & 38.3 & 14.7 & 24.9 & 8.3 & 16,000 \\
\hline 57 & 40,200 & .67 & 7.2 & 382 & 1.5 & .15 & .44 & 3,210 & 72.6 & 2.5 & 40.7 & 16.7 & 25.0 & 8.7 & 17,000 \\
\hline 58 & 41,200 & .69 & 7.1 & 399 & 1.8 & .17 & .47 & 2,640 & 76.9 & 2.6 & 40.6 & 19.4 & 27.1 & 9.0 & 18,000 \\
\hline 59 & 42,600 & .68 & 7.4 & 400 & 1.7 & .18 & .48 & 2,800 & 76.5 & 2.7 & 40.5 & 20.0 & 26.4 & 9.4 & 18,000 \\
\hline 60 & 46,600 & .72 & 7.4 & 405 & 1.9 & .19 & .53 & 3,230 & 83.9 & 3.0 & 47.3 & 18.4 & 29.1 & 10.0 & 20,000 \\
\hline 61 & 43,500 & .71 & 6.9 & 385 & 1.7 & .18 & .51 & 3,290 & 73.2 & 2.8 & 44.8 & 18.0 & 28.3 & 9.6 & 19,000 \\
\hline Maximum & 47,000 & 0.72 & 8.8 & 405 & 2.3 & 0.22 & 0.56 & 5,360 & 101.0 & 3.2 & 69.7 & 24.2 & 31.3 & 10.0 & 22,000 \\
\hline Minimum & 37,100 & .52 & 6.2 & 341 & 1.5 & .15 & .41 & 2,530 & 72.0 & 2.3 & 36.4 & 14.7 & 23.2 & 8.0 & 16,000 \\
\hline Mean & 41,611 & .60 & 7.2 & 372 & 1.9 & .18 & .48 & 3,388 & 87.0 & 2.6 & 43.6 & 19.9 & 26.8 & 8.9 & 18,689 \\
\hline Median & 41,500 & .59 & 7.1 & 371 & 1.9 & .18 & .48 & 3,290 & 88.4 & 2.6 & 42.0 & 19.8 & 26.6 & 8.8 & 18,000 \\
\hline
\end{tabular}


Table 1. Quantitative elemental analyses of less than 63-micrometer fraction and lead-210 date of Clearwater Lake bed-sediment samples, 2002.-Continued

[All concentrations in milligrams per kilogram; RSD, relative standard deviation; ${ }^{\circ}$, degrees; ', minutes; ”, seconds; <, less than; shaded cells exceed threshold effects concentration; --, no data]

\begin{tabular}{|c|c|c|c|c|c|c|c|c|c|c|c|c|c|c|c|}
\hline $\begin{array}{l}\text { Depth below } \\
\text { lakebed } \\
\text { surface, } \\
\text { (centimeters) }\end{array}$ & Aluminum & Antimony & Arsenic & Barium & Beryllium & Bismuth & Cadmium & Calcium & Cerium & Cesium & Chromium & Cobalt & Copper & Gallium & Iron \\
\hline RSD (percent) & 3.99 & 9.92 & 6.64 & 2.58 & 3.87 & 5.27 & 11.26 & 3.52 & 6.28 & 3.09 & 2.72 & 2.65 & 2.84 & 2.14 & 2.87 \\
\hline \multicolumn{16}{|c|}{ Lower Black River (Latitude $37^{\circ} 09^{\prime} 54^{\prime \prime}$ Longitude $090^{\circ} 45^{\prime} 58^{\prime \prime}$ ) } \\
\hline 1 & 61,400 & 0.82 & 13.0 & 428 & 2.9 & 0.25 & 0.62 & 3,920 & 93.3 & 4.4 & 55.8 & 32.5 & 39.9 & 14.0 & 33,000 \\
\hline 2 & 62,900 & .84 & 11.0 & 419 & 2.7 & .27 & .63 & 3,320 & 94.8 & 4.4 & 57.3 & 32.6 & 42.7 & 14.0 & 30,000 \\
\hline 3 & 64,100 & .92 & 12.0 & 412 & 2.7 & .27 & .58 & 2,870 & 94.6 & 4.5 & 51.8 & 34.7 & 40.1 & 14.0 & 31,000 \\
\hline 4 & 58,800 & .71 & 10.0 & 422 & 2.3 & .25 & .60 & 2,820 & 93.2 & 4.0 & 55.1 & 30.4 & 37.2 & 13.0 & 29,000 \\
\hline 5 & 59,200 & .74 & 10.0 & 417 & 2.6 & .25 & .60 & 2,840 & 93.3 & 4.0 & 52.1 & 30.5 & 36.5 & 13.0 & 29,000 \\
\hline 6 & 58,800 & .76 & 11.0 & 421 & 2.2 & .25 & .58 & 2,870 & 93.3 & 4.0 & 50.1 & 30.7 & 36.5 & 13.0 & 30,000 \\
\hline 7 & 57,600 & .73 & 9.4 & 409 & 2.4 & .24 & .60 & 2,940 & 93.3 & 3.9 & 52.0 & 29.2 & 35.8 & 12.0 & 28,000 \\
\hline 8 & 58,300 & .76 & 9.9 & 417 & 2.4 & .24 & .60 & 3,100 & 93.2 & 3.9 & 62.6 & 29.1 & 36.4 & 12.0 & 29,000 \\
\hline 9 & 60,100 & .74 & 10.0 & 412 & 2.5 & .25 & .62 & 3,030 & 94.8 & 4.0 & 54.4 & 30.2 & 37.6 & 13.0 & 30,000 \\
\hline 10 & 58,000 & .71 & 9.6 & 408 & 2.5 & .24 & .59 & 3,010 & 94.6 & 3.9 & 49.3 & 29.5 & 37.4 & 12.0 & 29,000 \\
\hline 11 & 59,300 & .74 & 9.8 & 410 & 2.4 & .25 & .62 & 3,110 & 94.6 & 4.0 & 57.4 & 30.0 & 37.3 & 12.0 & 29,000 \\
\hline 12 & 58,800 & .70 & 9.7 & 409 & 2.5 & .24 & .61 & 3,010 & 93.8 & 3.9 & 56.8 & 30.0 & 35.4 & 12.0 & 28,000 \\
\hline 13 & 60,200 & .64 & 9.7 & 407 & 2.5 & .25 & .62 & 3,120 & 94.7 & 4.0 & 50.1 & 30.6 & 37.5 & 13.0 & 29,000 \\
\hline 14 & 60,300 & .66 & 9.9 & 411 & 2.7 & .25 & .61 & 3,160 & 96.8 & 4.0 & 48.2 & 31.0 & 37.0 & 13.0 & 30,000 \\
\hline 15 & 60,000 & .76 & 9.8 & 404 & 2.3 & .25 & .62 & 3,120 & 94.8 & 4.0 & 54.9 & 31.0 & 36.6 & 13.0 & 29,000 \\
\hline 16 & 58,700 & .76 & 9.9 & 404 & 2.6 & .25 & .60 & 3,060 & 94.5 & 3.9 & 56.9 & 30.9 & 35.7 & 12.0 & 29,000 \\
\hline 17 & 59,200 & .73 & 9.6 & 405 & 2.5 & .24 & .60 & 3,090 & 93.0 & 3.9 & 47.4 & 30.8 & 36.4 & 12.0 & 28,000 \\
\hline 18 & 58,600 & .79 & 9.6 & 412 & 2.4 & .23 & .58 & 3,040 & 92.6 & 3.8 & 47.1 & 29.3 & 35.9 & 12.0 & 28,000 \\
\hline 19 & 58,700 & .76 & 9.7 & 405 & 2.3 & .24 & .57 & 3,030 & 95.5 & 3.8 & 49.8 & 31.9 & 35.7 & 12.0 & 28,000 \\
\hline 20 & 58,100 & .68 & 9.4 & 411 & 2.3 & .24 & .59 & 3,060 & 93.8 & 3.8 & 49.1 & 30.9 & 35.6 & 12.0 & 28,000 \\
\hline 21 & 60,300 & .80 & 10.0 & 412 & 2.4 & .24 & .61 & 3,100 & 95.2 & 4.0 & 55.2 & 31.0 & 36.7 & 13.0 & 29,000 \\
\hline 22 & 58,200 & .77 & 9.8 & 413 & 2.5 & .23 & .57 & 3,000 & 92.6 & 3.8 & 49.6 & 30.1 & 35.5 & 12.0 & 28,000 \\
\hline 23 & 55,900 & .75 & 9.9 & 420 & 2.5 & .28 & .59 & 2,880 & 93.2 & 4.0 & 48.1 & 29.8 & 35.4 & 12.0 & 27,000 \\
\hline 24 & 56,200 & .72 & 10.0 & 422 & 2.5 & .27 & .59 & 2,950 & 93.4 & 4.0 & 48.0 & 29.3 & 34.8 & 12.0 & 28,000 \\
\hline 25 & 58,700 & .80 & 9.6 & 423 & 2.5 & .28 & .61 & 2,960 & 96.7 & 4.2 & 52.6 & 30.0 & 35.2 & 13.0 & 28,000 \\
\hline 26 & 57,500 & .76 & 9.6 & 426 & 2.5 & .28 & .61 & 2,940 & 94.1 & 4.2 & 53.0 & 29.3 & 35.0 & 13.0 & 28,000 \\
\hline 27 & 57,500 & .72 & 9.4 & 428 & 2.3 & .28 & .61 & 2,890 & 95.8 & 4.1 & 51.6 & 29.9 & 35.3 & 13.0 & 28,000 \\
\hline 28 & 57,400 & .73 & 9.6 & 430 & 2.7 & .27 & .60 & 2,900 & 95.4 & 4.1 & 50.6 & 29.3 & 35.0 & 13.0 & 28,000 \\
\hline 29 & 57,500 & .79 & 9.4 & 415 & 2.6 & .28 & .62 & 2,880 & 97.2 & 4.1 & 52.1 & 28.8 & 35.1 & 13.0 & 28,000 \\
\hline 30 & 58,100 & .76 & 9.5 & 425 & 2.6 & .28 & .62 & 2,860 & 96.3 & 4.2 & 55.2 & 29.5 & 34.9 & 13.0 & 29,000 \\
\hline 31 & 58,800 & .80 & 9.1 & 424 & 2.5 & .27 & .61 & 2,890 & 92.3 & 4.2 & 55.7 & 29.7 & 35.0 & 13.0 & 28,000 \\
\hline 32 & 58,600 & .80 & 9.7 & 429 & 2.6 & .28 & .62 & 2,900 & 92.0 & 4.2 & 54.6 & 29.6 & 34.8 & 13.0 & 29,000 \\
\hline 33 & 58,800 & .83 & 9.6 & 420 & 2.6 & .27 & .62 & 2,820 & 91.3 & 4.2 & 53.2 & 29.6 & 35.6 & 13.0 & 28,000 \\
\hline 34 & 57,500 & .83 & 9.0 & 421 & 2.6 & .27 & .60 & 2,840 & 93.0 & 4.2 & 52.9 & 29.1 & 35.4 & 13.0 & 27,000 \\
\hline 35 & 57,700 & .84 & 9.2 & 422 & 2.5 & .27 & .60 & 2,780 & 91.3 & 4.2 & 53.4 & 29.0 & 35.2 & 13.0 & 27,000 \\
\hline 36 & 57,600 & .83 & 9.3 & 425 & 2.5 & .27 & .59 & 2,840 & 90.8 & 4.3 & 56.8 & 29.4 & 34.6 & 13.0 & 28,000 \\
\hline 37 & 59,400 & .87 & 9.4 & 426 & 2.6 & .28 & .60 & 2,840 & 91.9 & 4.4 & 52.9 & 30.4 & 35.1 & 13.0 & 29,000 \\
\hline 38 & 58,500 & .81 & 9.6 & 428 & 2.5 & .27 & .58 & 2,810 & 90.8 & 4.3 & 52.3 & 29.2 & 34.6 & 13.0 & 28,000 \\
\hline
\end{tabular}


Table 1. Quantitative elemental analyses of less than 63-micrometer fraction and lead-210 date of Clearwater Lake bed-sediment samples, 2002.-Continued

[All concentrations in milligrams per kilogram; RSD, relative standard deviation; ${ }^{\circ}$, degrees; ', minutes; ", seconds; <, less than; shaded cells exceed threshold effects concentration; --, no data]

\begin{tabular}{|c|c|c|c|c|c|c|c|c|c|c|c|c|c|c|c|}
\hline $\begin{array}{c}\text { Depth below } \\
\text { lakebed } \\
\text { surface, } \\
\text { (centimeters) } \\
\end{array}$ & Aluminum & Antimony & Arsenic & Barium & Beryllium & Bismuth & Cadmium & Calcium & Cerium & Cesium & Chromium & Cobalt & Copper & Gallium & Iron \\
\hline RSD (percent) & 3.99 & 9.92 & 6.64 & 2.58 & 3.87 & 5.27 & 11.26 & 3.52 & 6.28 & 3.09 & 2.72 & 2.65 & 2.84 & 2.14 & 2.87 \\
\hline \multicolumn{16}{|c|}{ Lower Black River (Latitude $37^{\circ} 09^{\prime} 54^{\prime \prime}$ Longitude $090^{\circ} 45^{\prime} 58^{\prime \prime}$ )—Continued } \\
\hline 39 & 57,600 & 0.84 & 9.1 & 425 & 2.4 & 0.27 & 0.58 & 2,750 & 91.5 & 4.2 & 53.4 & 28.3 & 33.3 & 13.0 & 28,000 \\
\hline 40 & 57,000 & .82 & 9.1 & 428 & 2.4 & .27 & .58 & 2,740 & 91.2 & 4.1 & 54.6 & 28.0 & 33.3 & 12.0 & 27,000 \\
\hline 41 & 57,500 & .84 & 8.8 & 414 & 2.5 & .28 & .58 & 2,700 & 89.7 & 4.2 & 51.3 & 28.0 & 33.3 & 13.0 & 28,000 \\
\hline 42 & 57,900 & .86 & 9.3 & 419 & 2.3 & .28 & .57 & 2,720 & 90.9 & 4.2 & 52.2 & 28.6 & 34.0 & 13.0 & 28,000 \\
\hline 43 & 45,200 & .63 & 6.6 & 367 & 1.8 & .20 & .36 & 2,270 & 79.1 & 3.2 & 53.9 & 21.3 & 25.1 & 9.7 & 20,000 \\
\hline 44 & 55,600 & .80 & 9.1 & 420 & 2.5 & .27 & .57 & 2,710 & 91.4 & 4.0 & 53.7 & 28.0 & 32.4 & 12.0 & 26,000 \\
\hline 45 & 53,400 & .79 & 9.9 & 408 & 2.3 & .26 & .54 & 2,780 & 81.2 & 3.8 & 50.5 & 29.8 & 31.8 & 12.0 & 25,000 \\
\hline 46 & 52,800 & .81 & 9.3 & 417 & 2.3 & .26 & .56 & 2,830 & 81.8 & 3.6 & 50.8 & 27.0 & 31.0 & 12.0 & 24,000 \\
\hline 47 & 54,200 & .79 & 8.4 & 413 & 2.4 & .25 & .56 & 2,790 & 89.4 & 3.8 & 52.1 & 25.5 & 33.0 & 12.0 & 24,000 \\
\hline 48 & 54,000 & .79 & 9.1 & 422 & 2.3 & .26 & .54 & 2,740 & 91.5 & 3.8 & 53.4 & 25.5 & 32.8 & 12.0 & 25,000 \\
\hline 49 & 54,300 & .82 & 9.2 & 418 & 2.2 & .26 & .50 & 2,770 & 90.6 & 3.8 & 51.3 & 26.3 & 31.6 & 12.0 & 25,000 \\
\hline 50 & 54,700 & .84 & 8.5 & 431 & 2.4 & .26 & .53 & 2,760 & 91.1 & 3.9 & 51.5 & 25.2 & 31.8 & 12.0 & 26,000 \\
\hline 51 & 56,900 & .85 & 9.4 & 437 & 2.4 & .24 & .55 & 2,970 & 91.2 & 4.0 & 50.2 & 26.4 & 35.4 & 13.0 & 27,000 \\
\hline 52 & 56,100 & .83 & 9.4 & 430 & 2.4 & .24 & .54 & 2,940 & 91.6 & 3.9 & 49.5 & 25.9 & 33.6 & 13.0 & 26,000 \\
\hline 53 & 57,600 & .81 & 9.5 & 431 & 2.4 & .25 & .53 & 2,940 & 93.7 & 4.1 & 53.0 & 27.3 & 33.6 & 13.0 & 26,000 \\
\hline 54 & 57,600 & .80 & 9.0 & 416 & 2.3 & .25 & .55 & 3,260 & 91.0 & 4.1 & 50.5 & 25.3 & 33.4 & 13.0 & 25,000 \\
\hline Maximum & 64,100 & 0.92 & 13.0 & 437 & 2.9 & 0.28 & 0.63 & 3,920 & 97.2 & 4.5 & 62.6 & 34.7 & 42.7 & 14.0 & 33,000 \\
\hline Minimum & 45,200 & .63 & 6.6 & 367 & 1.8 & .20 & .36 & 2,270 & 79.1 & 3.2 & 47.1 & 21.3 & 25.1 & 9.7 & 20,000 \\
\hline Mean & 57,735 & .78 & 9.6 & 418 & 2.5 & .26 & .58 & 2,931 & 92.5 & 4.0 & 52.6 & 29.2 & 35.1 & 12.6 & 27,759 \\
\hline Median & 58,050 & .79 & 9.6 & 419 & 2.5 & .26 & .60 & 2,895 & 93.2 & 4.0 & 52.3 & 29.5 & 35.3 & 13.0 & 28,000 \\
\hline
\end{tabular}


Table 1. Quantitative elemental analyses of less than 63-micrometer fraction and lead-210 date of Clearwater Lake bed-sediment samples, 2002.-Continued

[All concentrations in milligrams per kilogram; RSD, relative standard deviation; ${ }^{\circ}$, degrees; ', minutes; ”, seconds; <, less than; shaded cells exceed threshold effects concentration; --, no data]

\begin{tabular}{|c|c|c|c|c|c|c|c|c|c|c|c|c|c|c|c|}
\hline $\begin{array}{l}\text { Depth below } \\
\text { lakebed } \\
\text { surface, } \\
\text { (centimeters) }\end{array}$ & Aluminum & Antimony & Arsenic & Barium & Beryllium & Bismuth & Cadmium & Calcium & Cerium & Cesium & Chromium & Cobalt & Copper & Gallium & Iron \\
\hline RSD (percent) & 3.99 & 9.92 & 6.64 & 2.58 & 3.87 & 5.27 & 11.26 & 3.52 & 6.28 & 3.09 & 2.72 & 2.65 & 2.84 & 2.14 & 2.87 \\
\hline \multicolumn{16}{|c|}{ Marina (Latitude $37^{\circ} 08^{\prime} 27^{\prime \prime}$ Longitude $090^{\circ} 46^{\prime} 14^{\prime \prime}$ ) } \\
\hline 1 & 63,000 & 1.50 & 13.0 & 482 & 2.4 & 0.30 & 0.82 & 12,000 & 96.7 & 4.6 & 53.6 & 28.9 & 42.8 & 15.0 & 30,000 \\
\hline 2 & 65,800 & 1.80 & 13.0 & 470 & 2.7 & .27 & .68 & 5,850 & 105.0 & 5.5 & 55.1 & 33.5 & 41.2 & 16.0 & 35,000 \\
\hline 3 & 67,600 & 19.20 & 14.0 & 467 & 2.9 & .36 & .38 & 7,380 & 114.0 & 5.6 & 65.3 & 33.8 & 72.0 & 17.0 & 39,000 \\
\hline 4 & 67,600 & 1.80 & 12.0 & 449 & 2.8 & .29 & 1.70 & 7,460 & 109.0 & 5.7 & 62.0 & 33.1 & 47.9 & 17.0 & 35,000 \\
\hline 5 & 69,900 & 1.70 & 13.0 & 444 & 2.9 & .30 & .71 & 5,050 & 111.0 & 6.0 & 72.1 & 34.6 & 46.3 & 17.0 & 37,000 \\
\hline 6 & 73,100 & .82 & 14.0 & 437 & 2.8 & .30 & .67 & 4,180 & 104.0 & 5.6 & 64.6 & 34.3 & 43.8 & 17.0 & 36,000 \\
\hline 7 & 70,000 & .70 & 14.0 & 425 & 2.9 & .30 & .65 & 5,980 & 104.0 & 5.5 & 73.1 & 33.4 & 42.8 & 16.0 & 36,000 \\
\hline 8 & 68,900 & .63 & 14.0 & 424 & 2.8 & .29 & .70 & 5,180 & 104.0 & 5.4 & 59.4 & 34.0 & 40.6 & 16.0 & 35,000 \\
\hline 9 & 67,800 & .58 & 14.0 & 417 & 2.7 & .30 & .61 & 5,470 & 103.0 & 5.3 & 57.2 & 32.1 & 41.2 & 16.0 & 35,000 \\
\hline 10 & 67,100 & .57 & 13.0 & 422 & 2.8 & .30 & .77 & 5,330 & 105.0 & 5.2 & 57.4 & 32.1 & 39.6 & 16.0 & 35,000 \\
\hline 11 & 66,300 & .54 & 13.0 & 410 & 2.8 & .30 & .62 & 3,420 & 103.0 & 5.2 & 60.0 & 31.3 & 37.9 & 15.0 & 34,000 \\
\hline 12 & 67,500 & .56 & 13.0 & 420 & 2.8 & .30 & .65 & 3,570 & 101.0 & 5.3 & 57.0 & 32.0 & 38.3 & 16.0 & 35,000 \\
\hline 13 & 67,700 & .56 & 14.0 & 419 & 2.7 & .31 & .67 & 3,500 & 98.7 & 5.2 & 55.0 & 32.8 & 40.1 & 16.0 & 35,000 \\
\hline 14 & 69,200 & .56 & 14.0 & 442 & 3.0 & .32 & .66 & 3,490 & 103.0 & 5.2 & 56.0 & 32.5 & 41.5 & 16.0 & 36,000 \\
\hline 15 & 69,000 & .56 & 14.0 & 423 & 2.8 & .33 & .66 & 3,330 & 104.0 & 5.3 & 60.0 & 33.7 & 40.0 & 16.0 & 36,000 \\
\hline 16 & 71,100 & .57 & 14.0 & 430 & 2.8 & .32 & .65 & 3,280 & 104.0 & 5.5 & 60.8 & 35.2 & 42.6 & 16.0 & 36,000 \\
\hline 17 & 72,200 & .57 & 14.0 & 434 & 2.9 & .32 & .68 & 3,230 & 105.0 & 5.6 & 58.9 & 36.0 & 42.8 & 17.0 & 37,000 \\
\hline 18 & 71,300 & .57 & 14.0 & 436 & 2.9 & .33 & .64 & 3,350 & 106.0 & 5.6 & 59.7 & 35.2 & 40.7 & 16.0 & 36,000 \\
\hline 19 & 71,200 & .58 & 14.0 & 431 & 2.9 & .33 & .63 & 3,220 & 106.0 & 5.6 & 61.9 & 35.5 & 40.8 & 16.0 & 36,000 \\
\hline 20 & 70,400 & .54 & 14.0 & 451 & 2.8 & .32 & .63 & 3,180 & 101.0 & 5.4 & 58.9 & 35.8 & 40.3 & 16.0 & 36,000 \\
\hline 21 & 71,900 & .54 & 14.0 & 436 & 2.8 & .33 & .63 & 3,080 & 103.0 & 5.5 & 59.0 & 35.2 & 41.6 & 16.0 & 36,000 \\
\hline 22 & 69,500 & .55 & 14.0 & 446 & 2.8 & .31 & .64 & 3,070 & 99.4 & 5.4 & 56.8 & 33.5 & 41.2 & 16.0 & 35,000 \\
\hline 23 & 68,900 & .53 & 13.0 & 425 & 2.8 & .31 & .64 & 3,010 & 99.1 & 5.3 & 59.2 & 32.8 & 39.2 & 16.0 & 35,000 \\
\hline 24 & 69,600 & .54 & 13.0 & 439 & 2.7 & .32 & .64 & 3,060 & 99.2 & 5.4 & 62.8 & 33.1 & 39.3 & 17.0 & 35,000 \\
\hline 25 & 68,800 & .52 & 13.0 & 424 & 2.8 & .31 & .56 & 2,940 & 98.3 & 5.3 & 56.6 & 31.8 & 39.4 & 16.0 & 35,000 \\
\hline 26 & 62,100 & .95 & 12.0 & 428 & 2.6 & .29 & .58 & 2,910 & 99.1 & 5.0 & 49.8 & 28.8 & 36.1 & 15.0 & 32,000 \\
\hline 27 & 63,600 & 1.00 & 13.0 & 418 & 2.8 & .30 & .61 & 2,890 & 96.8 & 5.1 & 51.6 & 29.4 & 36.6 & 15.0 & 32,000 \\
\hline 28 & 63,700 & .92 & 12.0 & 426 & 2.7 & .29 & .59 & 2,960 & 98.2 & 5.1 & 54.1 & 29.3 & 36.3 & 15.0 & 32,000 \\
\hline 29 & 66,100 & 1.10 & 13.0 & 430 & 2.8 & .29 & .47 & 2,900 & 95.4 & 5.2 & 52.7 & 30.4 & 40.2 & 15.0 & 33,000 \\
\hline 30 & 65,400 & .94 & 13.0 & 437 & 2.9 & .30 & .58 & 2,980 & 100.0 & 5.2 & 52.8 & 30.5 & 36.9 & 15.0 & 33,000 \\
\hline 31 & 65,200 & .93 & 13.0 & 419 & 2.8 & .29 & .60 & 2,900 & 97.3 & 5.2 & 53.5 & 30.2 & 37.7 & 16.0 & 33,000 \\
\hline 32 & 67,000 & .92 & 14.0 & 435 & 2.7 & .29 & .61 & 2,960 & 103.0 & 5.2 & 56.2 & 31.7 & 38.9 & 16.0 & 34,000 \\
\hline 33 & 68,600 & .95 & 14.0 & 427 & 3.0 & .31 & .40 & 2,970 & 104.0 & 5.3 & 56.1 & 32.4 & 40.4 & 16.0 & 33,000 \\
\hline 34 & 69,500 & .96 & 14.0 & 446 & 3.0 & .31 & .61 & 2,900 & 106.0 & 5.4 & 57.5 & 32.1 & 39.0 & 16.0 & 34,000 \\
\hline 35 & 69,200 & 1.00 & 14.0 & 444 & 2.8 & .31 & .58 & 2,790 & 108.0 & 5.6 & 56.3 & 33.0 & 39.8 & 16.0 & 34,000 \\
\hline 36 & 70,200 & 1.00 & 14.0 & 475 & 3.1 & .31 & .60 & 2,820 & 112.0 & 5.5 & 59.2 & 32.2 & 41.2 & 16.0 & 34,000 \\
\hline 37 & 71,300 & 1.00 & 14.0 & 441 & 2.9 & .32 & .53 & 2,850 & 109.0 & 5.7 & 63.0 & 31.2 & 41.6 & 17.0 & 34,000 \\
\hline 38 & 70,800 & .95 & 14.0 & 463 & 2.8 & .31 & .54 & 2,890 & 104.0 & 5.4 & 56.1 & 31.5 & 38.0 & 16.0 & 33,000 \\
\hline
\end{tabular}


Table 1. Quantitative elemental analyses of less than 63-micrometer fraction and lead-210 date of Clearwater Lake bed-sediment samples, 2002.-Continued

[All concentrations in milligrams per kilogram; RSD, relative standard deviation; ${ }^{\circ}$, degrees; ', minutes; ", seconds; <, less than; shaded cells exceed threshold effects concentration; --, no data]

\begin{tabular}{|c|c|c|c|c|c|c|c|c|c|c|c|c|c|c|c|}
\hline $\begin{array}{l}\text { Depth below } \\
\text { lakebed } \\
\text { surface, } \\
\text { (centimeters) }\end{array}$ & Aluminum & Antimony & Arsenic & Barium & Beryllium & Bismuth & Cadmium & Calcium & Cerium & Cesium & Chromium & Cobalt & Copper & Gallium & Iron \\
\hline RSD (percent) & 3.99 & 9.92 & 6.64 & 2.58 & 3.87 & 5.27 & 11.26 & 3.52 & 6.28 & 3.09 & 2.72 & 2.65 & 2.84 & 2.14 & 2.87 \\
\hline \multicolumn{16}{|c|}{ Marina (Latitude $37^{\circ} 08^{\prime} 27^{\prime \prime}$ Longitude $090^{\circ} 46^{\prime} 14^{\prime \prime}$ )—Continued } \\
\hline 39 & 70,000 & 0.92 & 14.0 & 431 & 2.8 & 0.32 & 0.54 & 2,870 & 106.0 & 5.4 & 56.7 & 32.0 & 38.0 & 16.0 & 34,000 \\
\hline 40 & 69,800 & .91 & 13.0 & 450 & 2.8 & .31 & .52 & 2,840 & 105.0 & 5.2 & 56.4 & 28.6 & 36.2 & 16.0 & 32,000 \\
\hline 41 & 69,900 & .92 & 13.0 & 435 & 2.8 & .30 & .44 & 2,790 & 104.0 & 5.3 & 57.3 & 29.2 & 38.6 & 16.0 & 33,000 \\
\hline 42 & 70,200 & .92 & 13.0 & 451 & 2.7 & .30 & .52 & 2,770 & 108.0 & 5.4 & 55.8 & 29.1 & 37.0 & 16.0 & 32,000 \\
\hline 43 & 68,700 & .92 & 13.0 & 442 & 2.8 & .31 & .51 & 2,620 & 106.0 & 5.3 & 56.0 & 29.8 & 37.1 & 16.0 & 33,000 \\
\hline 44 & 69,400 & .92 & 13.0 & 450 & 2.8 & .30 & .54 & 2,860 & 106.0 & 5.3 & 58.2 & 29.2 & 37.2 & 16.0 & 33,000 \\
\hline 45 & 67,600 & 1.20 & 12.0 & 417 & 2.7 & .30 & .62 & 3,040 & 103.0 & 5.0 & 58.3 & 28.2 & 38.2 & 16.0 & 33,000 \\
\hline 46 & 61,900 & .90 & 12.0 & 417 & 2.7 & .28 & .54 & 2,910 & 101.0 & 4.7 & 53.3 & 26.6 & 35.1 & 14.0 & 30,000 \\
\hline 47 & 62,700 & .94 & 12.0 & 406 & 2.7 & .29 & .59 & 3,000 & 101.0 & 4.8 & 57.5 & 26.6 & 36.4 & 15.0 & 31,000 \\
\hline 48 & 61,800 & .82 & 12.0 & 409 & 2.8 & .28 & .57 & 3,020 & 97.5 & 4.6 & 53.8 & 27.1 & 34.9 & 14.0 & 30,000 \\
\hline 49 & 65,900 & .87 & 13.0 & 408 & 2.7 & .30 & .59 & 2,900 & 101.0 & 4.9 & 57.7 & 27.3 & 37.0 & 15.0 & 32,000 \\
\hline 50 & 66,000 & .87 & 13.0 & 413 & 2.8 & .30 & .60 & 2,880 & 103.0 & 5.0 & 55.9 & 27.4 & 36.1 & 15.0 & 32,000 \\
\hline 51 & 66,900 & .92 & 13.0 & 407 & 2.8 & .26 & .62 & 2,920 & 85.1 & 5.0 & 61.1 & 29.8 & 40.3 & 16.0 & 33,000 \\
\hline 52 & 66,000 & .94 & 13.0 & 407 & 2.9 & .26 & .62 & 2,900 & 87.2 & 5.0 & 60.6 & 29.2 & 38.9 & 16.0 & 33,000 \\
\hline 53 & 66,100 & .97 & 13.0 & 410 & 2.9 & .26 & .63 & 2,850 & 84.1 & 5.1 & 57.1 & 30.5 & 38.6 & 16.0 & 33,000 \\
\hline 54 & 68,300 & 1.00 & 14.0 & 423 & 2.8 & .27 & .62 & 2,910 & 88.5 & 5.3 & 59.5 & 30.8 & 40.0 & 16.0 & 34,000 \\
\hline 55 & 64,200 & .98 & 13.0 & 416 & 2.9 & .26 & .59 & 2,760 & 85.8 & 5.0 & 57.0 & 30.2 & 50.8 & 16.0 & 32,000 \\
\hline 56 & 61,300 & .91 & 12.0 & 395 & 2.6 & .25 & .58 & 2,840 & 82.0 & 4.7 & 54.6 & 28.5 & 36.6 & 15.0 & 30,000 \\
\hline 57 & 65,000 & .98 & 12.0 & 409 & 2.8 & .27 & .61 & 2,940 & 89.0 & 5.0 & 57.6 & 29.2 & 38.2 & 16.0 & 32,000 \\
\hline 58 & 66,000 & .96 & 12.0 & 411 & 2.9 & .27 & .63 & 3,040 & 89.6 & 5.0 & 59.0 & 29.2 & 38.6 & 16.0 & 31,000 \\
\hline 59 & 66,100 & .97 & 12.0 & 400 & 2.9 & .28 & .64 & 3,080 & 90.1 & 5.1 & 60.9 & 28.2 & 38.5 & 16.0 & 31,000 \\
\hline 60 & 70,000 & 1.00 & 14.0 & 409 & 3.0 & .29 & .67 & 3,130 & 91.1 & 5.4 & 66.8 & 31.6 & 42.8 & 17.0 & 35,000 \\
\hline 61 & 72,800 & 1.10 & 15.0 & 416 & 3.2 & .29 & .63 & 3,180 & 91.1 & 5.8 & 66.0 & 36.1 & 45.0 & 18.0 & 37,000 \\
\hline Maximum & 73,100 & 19.20 & 15.0 & 482 & 3.2 & 0.36 & 1.70 & 12,000 & 114.0 & 6.0 & 73.1 & 36.1 & 72.0 & 18.0 & 39,000 \\
\hline Minimum & 61,300 & .52 & 12.0 & 395 & 2.4 & .25 & .38 & 2,620 & 82.0 & 4.6 & 49.8 & 26.6 & 34.9 & 14.0 & 30,000 \\
\hline Mean & 67,782 & 1.18 & 13.3 & 430 & 2.8 & .30 & .62 & 3,564 & 100.4 & 5.3 & 58.3 & 31.4 & 40.3 & 15.9 & 33,820 \\
\hline Median & 68,300 & .92 & 13.0 & 427 & 2.8 & .30 & .62 & 3,000 & 103.0 & 5.3 & 57.5 & 31.6 & 39.4 & 16.0 & 34,000 \\
\hline
\end{tabular}


Table 1. Quantitative elemental analyses of less than 63-micrometer fraction and lead-210 date of Clearwater Lake bed-sediment samples, 2002.-Continued

[All concentrations in milligrams per kilogram; RSD, relative standard deviation; ${ }^{\circ}$, degrees; ', minutes; ”, seconds; <, less than; shaded cells exceed threshold effects concentration; --, no data]

\begin{tabular}{|c|c|c|c|c|c|c|c|c|c|c|c|c|c|c|c|}
\hline $\begin{array}{l}\text { Depth below } \\
\text { lakebed } \\
\text { surface, } \\
\text { (centimeters) }\end{array}$ & Aluminum & Antimony & Arsenic & Barium & Beryllium & Bismuth & Cadmium & Calcium & Cerium & Cesium & Chromium & Cobalt & Copper & Gallium & Iron \\
\hline RSD (percent) & 3.99 & 9.92 & 6.64 & 2.58 & 3.87 & 5.27 & 11.26 & 3.52 & 6.28 & 3.09 & 2.72 & 2.65 & 2.84 & 2.14 & 2.87 \\
\hline \multicolumn{16}{|c|}{ Clearwater Dam (Latitude $37^{\circ} 08^{\prime} 19^{\prime \prime}$ Longitude $090^{\circ} 46^{\prime} 47^{\prime \prime}$ ) } \\
\hline 1 & 77,100 & 1.00 & 13.0 & 433 & 3.1 & 0.31 & 0.60 & 2,950 & 99.4 & 6.0 & 69.3 & 39.0 & 43.3 & 17.0 & 39,000 \\
\hline 2 & 78,100 & 1.00 & 12.0 & 432 & 3.2 & .30 & .60 & 3,360 & 100.0 & 6.1 & 80.5 & 37.4 & 45.8 & 18.0 & 39,000 \\
\hline 3 & 78,800 & 1.00 & 12.0 & 438 & 3.2 & .31 & .56 & 3,160 & 100.0 & 6.0 & 66.2 & 37.0 & 42.7 & 18.0 & 40,000 \\
\hline 4 & 76,500 & .96 & 12.0 & 438 & 3.3 & .28 & .56 & 3,130 & 99.6 & 6.0 & 66.9 & 37.4 & 41.3 & 17.0 & 40,000 \\
\hline 5 & 77,600 & .95 & 11.0 & 435 & 3.1 & .26 & .56 & 3,250 & 101.0 & 6.0 & 74.4 & 36.7 & 43.6 & 17.0 & 40,000 \\
\hline 6 & 77,700 & .98 & 11.0 & 432 & 3.2 & .28 & .56 & 3,230 & 100.0 & 5.9 & 82.1 & 36.5 & 42.3 & 17.0 & 39,000 \\
\hline 7 & 77,100 & .95 & 11.0 & 437 & 3.2 & .24 & .58 & 3,300 & 100.0 & 5.9 & 66.0 & 35.6 & 41.6 & 17.0 & 40,000 \\
\hline 8 & 76,200 & .96 & 11.0 & 437 & 3.2 & .24 & .56 & 3,310 & 101.0 & 5.9 & 64.6 & 35.7 & 40.3 & 17.0 & 39,000 \\
\hline 9 & 74,100 & .94 & 11.0 & 430 & 3.0 & .26 & .56 & 3,210 & 99.4 & 5.8 & 66.8 & 35.9 & 39.6 & 17.0 & 38,000 \\
\hline 10 & 75,000 & .96 & 11.0 & 440 & 3.0 & .28 & .53 & 3,160 & 101.0 & 5.9 & 69.2 & 37.4 & 40.9 & 17.0 & 38,000 \\
\hline 11 & 74,300 & 1.10 & 12.0 & 446 & 3.1 & .29 & .54 & 3,130 & 99.6 & 5.7 & 66.1 & 38.6 & 41.3 & 17.0 & 37,000 \\
\hline 12 & 75,200 & 1.00 & 11.0 & 447 & 3.1 & .30 & .54 & 3,200 & 101.0 & 5.8 & 65.9 & 34.8 & 40.2 & 17.0 & 38,000 \\
\hline 13 & 75,900 & 1.00 & 11.0 & 450 & 3.2 & .30 & .52 & 3,210 & 102.0 & 5.9 & 64.1 & 34.9 & 39.9 & 17.0 & 39,000 \\
\hline 14 & 77,600 & 1.00 & 17.0 & 452 & 3.2 & .27 & .55 & 3,360 & 102.0 & 6.0 & 67.1 & 35.4 & 40.5 & 17.0 & 38,000 \\
\hline 15 & 79,200 & 1.10 & 12.0 & 458 & 3.4 & .31 & .54 & 3,440 & 105.0 & 6.0 & 67.5 & 37.7 & 41.7 & 18.0 & 40,000 \\
\hline 16 & 77,400 & 1.00 & 11.0 & 450 & 3.1 & .29 & .53 & 3,360 & 103.0 & 6.0 & 66.9 & 36.7 & 41.1 & 18.0 & 39,000 \\
\hline 17 & 76,000 & .93 & 11.0 & 445 & 3.3 & .26 & .54 & 3,330 & 104.0 & 5.9 & 64.8 & 35.6 & 40.2 & 17.0 & 38,000 \\
\hline 18 & 76,400 & .99 & 11.0 & 447 & 3.1 & .29 & .54 & 3,340 & 103.0 & 6.0 & 66.6 & 36.1 & 40.9 & 18.0 & 38,000 \\
\hline 19 & 77,400 & 1.00 & 11.0 & 457 & 3.2 & .31 & .54 & 3,300 & 104.0 & 6.1 & 65.8 & 36.8 & 46.3 & 18.0 & 38,000 \\
\hline 20 & 76,000 & 1.00 & 11.0 & 450 & 3.2 & .31 & .54 & 3,160 & 103.0 & 6.0 & 65.6 & 35.6 & 40.7 & 17.0 & 38,000 \\
\hline 21 & 76,100 & 1.10 & 10.0 & 451 & 3.2 & .30 & .55 & 3,280 & 104.0 & 5.9 & 65.8 & 34.8 & 41.0 & 17.0 & 37,000 \\
\hline 22 & 75,000 & .99 & 10.0 & 454 & 3.2 & .31 & .58 & 4,210 & 100.0 & 5.9 & 66.2 & 36.1 & 41.3 & 17.0 & 38,000 \\
\hline 23 & 76,500 & .99 & 11.0 & 457 & 3.2 & .31 & .59 & 3,440 & 101.0 & 5.8 & 64.4 & 35.3 & 40.8 & 17.0 & 38,000 \\
\hline 24 & 76,700 & 1.00 & 11.0 & 458 & 3.1 & .32 & .59 & 3,340 & 103.0 & 6.0 & 65.5 & 34.7 & 41.5 & 17.0 & 37,000 \\
\hline 25 & 78,900 & 1.10 & 12.0 & 466 & 3.2 & .31 & .58 & 3,390 & 104.0 & 6.1 & 67.0 & 35.2 & 43.0 & 18.0 & 38,000 \\
\hline 26 & 76,600 & 1.00 & 11.0 & 468 & 3.3 & .30 & .56 & 3,490 & 105.0 & 5.9 & 68.3 & 33.8 & 41.1 & 18.0 & 38,000 \\
\hline 27 & 72,500 & .96 & 13.0 & 466 & 3.0 & .30 & .47 & 3,180 & 105.0 & 5.5 & 59.3 & 32.3 & 36.4 & 16.0 & 34,000 \\
\hline 28 & 71,500 & .98 & 13.0 & 469 & 3.0 & .30 & .42 & 2,930 & 105.0 & 5.5 & 59.2 & 32.4 & 35.9 & 16.0 & 34,000 \\
\hline 29 & 71,300 & .98 & 13.0 & 472 & 2.8 & .28 & .42 & 3,680 & 103.0 & 5.5 & 58.9 & 31.2 & 35.5 & 16.0 & 34,000 \\
\hline 30 & 70,000 & .92 & 12.0 & 467 & 3.0 & .30 & .42 & 2,960 & 102.0 & 5.5 & 62.0 & 32.8 & 35.7 & 16.0 & 33,000 \\
\hline 31 & 70,200 & 1.00 & 12.0 & 464 & 2.7 & .31 & .41 & 2,840 & 96.6 & 5.4 & 63.5 & 32.2 & 35.6 & 16.0 & 33,000 \\
\hline 32 & 67,800 & .94 & 12.0 & 461 & 2.9 & .30 & .40 & 2,890 & 96.2 & 5.2 & 66.7 & 31.5 & 35.1 & 15.0 & 32,000 \\
\hline 33 & 68,100 & .96 & 11.0 & 470 & 2.9 & .29 & .40 & 2,910 & 95.8 & 5.2 & 61.7 & 31.2 & 34.3 & 15.0 & 31,000 \\
\hline 34 & 68,100 & .96 & 11.0 & 475 & 2.8 & .30 & .41 & 2,920 & 97.0 & 5.2 & 68.0 & 31.4 & 35.2 & 15.0 & 31,000 \\
\hline 35 & 66,900 & .98 & 11.0 & 470 & 2.7 & .29 & .39 & 2,760 & 97.4 & 5.1 & 60.0 & 31.5 & 34.6 & 15.0 & 30,000 \\
\hline 36 & 65,000 & .88 & 10.0 & 470 & 2.5 & .29 & .38 & 2,720 & 95.6 & 4.9 & 56.7 & 29.1 & 32.3 & 14.0 & 30,000 \\
\hline 37 & 64,900 & .86 & 10.0 & 476 & 2.6 & .28 & .38 & 2,840 & 95.9 & 4.9 & 58.0 & 29.6 & 32.0 & 14.0 & 30,000 \\
\hline 38 & 64,800 & .88 & 11.0 & 483 & 2.8 & .28 & .38 & 2,900 & 95.9 & 4.9 & 57.4 & 30.3 & 32.4 & 14.0 & 30,000 \\
\hline
\end{tabular}


Table 1. Quantitative elemental analyses of less than 63-micrometer fraction and lead-210 date of Clearwater Lake bed-sediment samples, 2002.-Continued

[All concentrations in milligrams per kilogram; RSD, relative standard deviation; ${ }^{\circ}$, degrees; ', minutes; ", seconds; <, less than; shaded cells exceed threshold effects concentration; --, no data]

\begin{tabular}{|c|c|c|c|c|c|c|c|c|c|c|c|c|c|c|c|}
\hline $\begin{array}{c}\text { Depth below } \\
\text { lakebed } \\
\text { surface, } \\
\text { (centimeters) }\end{array}$ & Aluminum & Antimony & Arsenic & Barium & Beryllium & Bismuth & Cadmium & Calcium & Cerium & Cesium & Chromium & Cobalt & Copper & Gallium & Iron \\
\hline RSD (percent) & 3.99 & 9.92 & 6.64 & 2.58 & 3.87 & 5.27 & 11.26 & 3.52 & 6.28 & 3.09 & 2.72 & 2.65 & 2.84 & 2.14 & 2.87 \\
\hline \multicolumn{16}{|c|}{ Clearwater Dam (Latitude $37^{\circ} 08^{\prime} 19^{\prime \prime}$ Longitude $090^{\circ} 46^{\prime} 47^{\prime \prime}$ )—Continued } \\
\hline 39 & 62,300 & 0.89 & 9.9 & 467 & 2.6 & 0.26 & 0.36 & 2,980 & 95.2 & 4.6 & 55.4 & 31.0 & 31.4 & 14.0 & 28,000 \\
\hline 40 & 60,600 & .87 & 9.9 & 465 & 2.4 & .26 & .36 & 3,210 & 93.2 & 4.5 & 52.3 & 29.8 & 30.7 & 14.0 & 28,000 \\
\hline 41 & 60,200 & .92 & 9.9 & 458 & 2.4 & .26 & .34 & 3,360 & 92.8 & 4.6 & 55.6 & 29.9 & 31.2 & 13.0 & 28,000 \\
\hline 42 & 60,400 & .86 & 9.7 & 462 & 2.4 & .25 & .35 & 3,240 & 91.1 & 4.5 & 50.4 & 28.9 & 32.3 & 13.0 & 28,000 \\
\hline 43 & 58,200 & .82 & 9.6 & 464 & 2.4 & .26 & .33 & 2,850 & 89.4 & 4.2 & 50.6 & 27.2 & 29.9 & 13.0 & 26,000 \\
\hline 44 & 58,300 & .82 & 9.1 & 459 & 2.3 & .24 & .34 & 2,700 & 88.2 & 4.3 & 50.4 & 28.4 & 30.7 & 13.0 & 26,000 \\
\hline 45 & 60,500 & .87 & 9.9 & 472 & 2.3 & .26 & .33 & 2,700 & 90.2 & 4.5 & 52.9 & 29.6 & 31.4 & 13.0 & 27,000 \\
\hline 46 & 60,800 & 90 & 10.0 & 473 & 2.5 & .26 & .34 & 2,770 & 91.3 & 4.5 & 53.9 & 29.2 & 30.9 & 13.0 & 27,000 \\
\hline 47 & 61,300 & .86 & 10.0 & 477 & 2.6 & .26 & .36 & 2,800 & 91.8 & 4.5 & 54.6 & 30.3 & 30.7 & 14.0 & 28,000 \\
\hline 48 & 61,200 & .86 & 9.7 & 479 & 2.5 & .26 & .35 & 2,810 & 92.8 & 4.5 & 53.3 & 28.6 & 31.4 & 14.0 & 28,000 \\
\hline 49 & 56,000 & .84 & 9.2 & 469 & 2.3 & .24 & .32 & 2,620 & 90.0 & 4.1 & 50.2 & 28.3 & 29.6 & 13.0 & 25,000 \\
\hline 50 & 58,100 & .82 & 9.4 & 474 & 2.4 & .24 & .31 & 2,610 & 91.3 & 4.3 & 49.4 & 28.9 & 30.1 & 13.0 & 26,000 \\
\hline 51 & 60,700 & .90 & 11.0 & 511 & 2.4 & .25 & .35 & 3,030 & 89.3 & 4.6 & 50.0 & 31.7 & 31.4 & 14.0 & 28,000 \\
\hline 52 & 61,600 & 1.50 & 10.0 & 518 & 2.3 & .25 & .31 & 2,800 & 90.0 & 4.7 & 48.2 & 29.3 & 31.2 & 14.0 & 27,000 \\
\hline 53 & 60,700 & .89 & 9.8 & 511 & 2.3 & .24 & .38 & 2,790 & 87.9 & 4.5 & 49.8 & 27.8 & 33.7 & 14.0 & 26,000 \\
\hline 54 & 37,600 & .60 & 6.4 & 388 & 1.5 & .15 & .25 & 2,420 & 60.8 & 2.6 & 32.0 & 17.7 & 19.6 & 8.3 & 16,000 \\
\hline Maximum & 79,200 & 1.50 & 17.0 & 518 & 3.4 & 0.32 & 0.60 & 4,210 & 105.0 & 6.1 & 82.1 & 39.0 & 46.3 & 18.0 & 40,000 \\
\hline Minimum & 37,600 & .60 & 6.4 & 388 & 1.5 & .15 & .25 & 2,420 & 60.8 & 2.6 & 32.0 & 17.7 & 19.6 & 8.3 & 16,000 \\
\hline Mean & 69,500 & .95 & 10.9 & 459 & 2.9 & .28 & .46 & 3,097 & 97.3 & 5.3 & 61.2 & 32.8 & 36.7 & 15.6 & 33,426 \\
\hline Median & 72,000 & 96 & 11.0 & 460 & 3.0 & .28 & .45 & 3,160 & 99.6 & 5.5 & 64.3 & 32.6 & 36.2 & 16.0 & 34,000 \\
\hline
\end{tabular}


Table 1. Quantitative elemental analyses of less than 63-micrometer fraction and lead-210 date of Clearwater Lake bed-sediment samples-Continued

[All concentrations in milligrams per kilogram; RSD, relative standard deviation; ${ }^{\circ}$, degrees; ', minutes; ”, seconds; <, less than; shaded cells exceed threshold effects concentration; --, no data]

\begin{tabular}{|c|c|c|c|c|c|c|c|c|c|c|c|c|c|c|c|}
\hline $\begin{array}{l}\text { Depth below } \\
\text { lakebed } \\
\text { surface, } \\
\text { (centimeters) }\end{array}$ & Lanthanum & Lead & Lithium & Magnesium & Manganese & Molybdenum & Nickel & Niobium & Phosphorous & Potassium & Rubidium & Scandium & Silver & Sodium & Strontium \\
\hline RSD (percent) & 6.64 & 3.83 & 2.48 & 2.70 & 2.52 & 10.69 & 3.35 & 23.19 & 2.84 & 2.86 & 2.65 & 3.57 & 41.48 & 2.44 & 3.74 \\
\hline \multicolumn{16}{|c|}{ Webb Creek (Latitude $37^{\circ} 08^{\prime} 56^{\prime \prime}$ Longitude $090^{\circ} 48^{\prime} 18^{\prime \prime}$ ) } \\
\hline 1 & 36.9 & 27.4 & 22.0 & 2,250 & 1,080 & 1.1 & 19.4 & 8.8 & 420 & 11,300 & 53.7 & 6.3 & $<0.02$ & 2,770 & 49.0 \\
\hline 2 & 37.8 & 26.3 & 21.9 & 2,120 & 435 & 1.4 & 19.9 & 10.0 & 400 & 11,200 & 53.3 & 6.4 & $<.02$ & 2,780 & 49.0 \\
\hline 3 & 37.6 & 23.4 & 20.6 & 1,970 & 682 & 1.2 & 17.8 & 8.6 & 370 & 11,200 & 51.2 & 5.7 & $<.02$ & 2,900 & 49.2 \\
\hline 4 & 37.7 & 24.5 & 20.7 & 1,960 & 922 & 1.9 & 22.2 & 8.2 & 390 & 11,300 & 51.2 & 5.9 & $<.02$ & 2,850 & 48.9 \\
\hline 5 & 37.9 & 25.4 & 21.1 & 2,020 & 969 & 1.2 & 19.3 & 10.0 & 400 & 11,400 & 52.2 & 6.0 & $<.02$ & 2,900 & 49.6 \\
\hline 6 & 38.4 & 25.5 & 21.1 & 2,050 & 735 & 1.2 & 19.0 & 8.8 & 360 & 11,100 & 50.9 & 5.9 & $<.02$ & 2,860 & 48.4 \\
\hline 7 & 38.1 & 25.6 & 21.1 & 2,040 & 706 & 1.4 & 20.1 & 9.4 & 370 & 11,300 & 51.6 & 6.1 & $<.02$ & 2,860 & 49.1 \\
\hline 8 & 37.7 & 25.0 & 20.8 & 2,060 & 547 & 1.6 & 21.0 & 10.0 & 370 & 11,000 & 50.5 & 5.9 & $<.02$ & 2,770 & 47.2 \\
\hline 9 & 38.3 & 25.0 & 21.1 & 2,040 & 886 & 1.2 & 18.8 & 10.0 & 390 & 10,900 & 51.3 & 6.1 & $<.02$ & 2,690 & 48.3 \\
\hline 10 & 41.4 & 25.0 & 21.3 & 2,110 & 1,190 & 1.7 & 21.9 & 11.0 & 450 & 10,600 & 50.9 & 6.3 & $<.02$ & 2,400 & 46.1 \\
\hline 11 & 41.4 & 25.2 & 22.4 & 2,200 & 1,670 & 1.6 & 21.3 & 12.0 & 480 & 11,100 & 54.4 & 6.6 & $<.02$ & 2,500 & 48.0 \\
\hline 12 & 39.3 & 24.5 & 21.6 & 2,090 & 1,270 & 1.7 & 21.7 & 8.8 & 420 & 11,100 & 52.9 & 6.3 & $<.02$ & 2,570 & 47.8 \\
\hline 13 & 39.6 & 24.6 & 20.8 & 2,040 & 1,090 & 1.2 & 19.2 & 7.5 & 400 & 10,700 & 52.0 & 6.1 & $<.02$ & 2,540 & 47.2 \\
\hline 14 & 41.2 & 25.7 & 22.0 & 2,090 & 940 & 1.3 & 19.6 & 10.0 & 410 & 11,200 & 53.9 & 6.5 & $<.02$ & 2,590 & 47.9 \\
\hline 15 & 42.5 & 26.9 & 23.1 & 2,230 & 1,190 & 1.7 & 22.4 & 10.0 & 460 & 11,200 & 56.6 & 7.0 & $<.02$ & 2,500 & 48.8 \\
\hline 16 & 43.3 & 27.0 & 22.7 & 2,160 & 1,350 & 1.6 & 21.7 & 9.7 & 450 & 11,100 & 54.7 & 6.8 & $<.02$ & 2,520 & 48.4 \\
\hline 17 & 42.6 & 25.9 & 22.3 & 2,120 & 1,770 & 1.4 & 20.9 & 10.0 & 440 & 11,100 & 55.3 & 6.8 & $<.02$ & 2,500 & 49.0 \\
\hline 18 & 41.4 & 25.0 & 22.4 & 2,090 & 1,450 & 1.6 & 20.2 & 8.5 & 420 & 11,400 & 56.6 & 6.7 & $<.02$ & 2,610 & 50.0 \\
\hline 19 & 39.2 & 26.6 & 22.0 & 2,100 & 1,340 & 1.6 & 21.2 & 11.0 & 430 & 10,900 & 53.9 & 6.5 & $<.02$ & 2,550 & 48.5 \\
\hline 20 & 39.0 & 26.7 & 21.8 & 2,090 & 1,290 & 1.6 & 20.9 & 11.0 & 420 & 11,000 & 53.4 & 6.4 & $<.02$ & 2,590 & 49.1 \\
\hline 21 & 37.6 & 24.6 & 20.1 & 1,930 & 1,280 & 1.2 & 18.0 & 11.0 & 390 & 10,500 & 49.5 & 5.8 & $<.02$ & 2,540 & 47.2 \\
\hline 22 & 37.7 & 23.9 & 19.3 & 1,840 & 1,340 & 1.3 & 17.7 & 8.2 & 370 & 10,400 & 49.0 & 5.6 & $<.02$ & 2,500 & 46.0 \\
\hline 23 & 37.9 & 23.6 & 19.3 & 1,860 & 1,350 & 1.3 & 17.7 & 5.0 & 380 & 10,500 & 49.1 & 5.6 & $<.02$ & 2,520 & 46.7 \\
\hline 24 & 36.5 & 23.7 & 18.2 & 1,800 & 1,120 & 1.5 & 19.5 & 5.2 & 360 & 10,200 & 47.6 & 5.4 & $<.02$ & 2,560 & 46.7 \\
\hline 25 & 34.8 & 22.2 & 18.7 & 1,800 & 1,120 & 1.1 & 17.3 & 5.7 & 370 & 10,400 & 47.9 & 5.5 & $<.02$ & 2,640 & 47.4 \\
\hline 26 & 22.3 & 14.0 & 11.5 & 1,120 & 651 & 1.0 & 11.6 & $<2$ & 220 & 6,270 & 28.8 & 3.3 & $<.02$ & 1,540 & 29.7 \\
\hline 27 & 37.1 & 22.2 & 18.8 & 1,840 & 877 & 1.2 & 17.4 & 9.6 & 360 & 10,400 & 48.3 & 5.5 & $<.02$ & 2,580 & 47.5 \\
\hline 28 & 37.4 & 22.4 & 18.4 & 1,830 & 888 & 2.2 & 22.1 & 9.2 & 370 & 10,000 & 46.7 & 5.4 & $<.02$ & 2,450 & 45.9 \\
\hline 29 & 40.4 & 22.7 & 18.1 & 1,910 & 770 & 1.4 & 17.5 & 7.1 & 380 & 9,620 & 43.2 & 5.3 & .68 & 2,340 & 43.4 \\
\hline 30 & 40.8 & 23.2 & 18.1 & 1,900 & 654 & 1.7 & 18.7 & 6.8 & 380 & 9,640 & 43.9 & 5.3 & .59 & 2,320 & 44.0 \\
\hline 31 & 36.6 & 22.5 & 17.5 & 1,800 & 676 & 1.4 & 16.8 & 4.0 & 380 & 9,500 & 42.8 & 5.1 & .39 & 2,240 & 42.0 \\
\hline 32 & 41.5 & 23.7 & 18.5 & 1,960 & 855 & 1.8 & 19.8 & 8.2 & 410 & 9,870 & 45.1 & 5.5 & .50 & 2,280 & 43.5 \\
\hline 33 & 39.5 & 24.8 & 20.5 & 2,040 & 1,020 & 1.3 & 18.7 & 10.0 & 400 & 10,800 & 50.6 & 6.1 & .64 & 2,530 & 47.6 \\
\hline 34 & 42.2 & 27.2 & 22.8 & 2,210 & 1,300 & 1.6 & 20.6 & 13.0 & 450 & 11,600 & 56.1 & 6.9 & .73 & 2,610 & 50.0 \\
\hline 35 & 41.6 & 28.1 & 23.7 & 2,220 & 1,230 & 1.6 & 22.0 & 13.0 & 450 & 11,800 & 57.8 & 7.1 & .67 & 2,640 & 50.2 \\
\hline 36 & 41.1 & 27.3 & 23.1 & 2,150 & 1,030 & 2.0 & 22.5 & 14.0 & 440 & 11,600 & 57.0 & 7.0 & .75 & 2,640 & 49.8 \\
\hline 37 & 42.0 & 26.7 & 22.4 & 2,070 & 1,020 & 1.5 & 20.4 & 17.0 & 430 & 11,700 & 56.1 & 6.8 & .85 & 2,720 & 51.3 \\
\hline 38 & 41.5 & 25.8 & 21.8 & 1,990 & 1,000 & 1.3 & 18.4 & 12.0 & 420 & 11,600 & 54.1 & 6.7 & .65 & 2,720 & 50.4 \\
\hline
\end{tabular}


Table 1. Quantitative elemental analyses of less than 63-micrometer fraction and lead-210 date of Clearwater Lake bed-sediment samples-Continued

[All concentrations in milligrams per kilogram; RSD, relative standard deviation; ${ }^{\circ}$, degrees; ', minutes; ", seconds; <, less than; shaded cells exceed threshold effects concentration; --, no data]

\begin{tabular}{|c|c|c|c|c|c|c|c|c|c|c|c|c|c|c|c|}
\hline $\begin{array}{l}\text { Depth below } \\
\text { lakebed } \\
\text { surface, } \\
\text { (centimeters) }\end{array}$ & Lanthanum & Lead & Lithium & Magnesium & Manganese & Molybdenum & Nickel & Niobium & Phosphorous & Potassium & Rubidium & Scandium & Silver & Sodium & Strontium \\
\hline RSD (percent) & 6.64 & 3.83 & 2.48 & 2.70 & 2.52 & 10.69 & 3.35 & 23.19 & 2.84 & 2.86 & 2.65 & 3.57 & 41.48 & 2.44 & 3.74 \\
\hline \multicolumn{16}{|c|}{ Webb Creek (Latitude $37^{\circ} 08^{\prime} 56^{\prime \prime}$ Longitude $090^{\circ} 48^{\prime} 18^{\prime \prime}$ ) —Continued } \\
\hline 39 & 41.0 & 27.2 & 23.7 & 2,180 & 1,190 & 1.9 & 21.4 & 14.0 & 470 & 11,900 & 58.4 & 7.1 & 0.68 & 2,720 & 51.0 \\
\hline 40 & 41.1 & 27.7 & 25.2 & 2,250 & 1,170 & 2.4 & 25.1 & 15.0 & 500 & 12,500 & 62.3 & 7.6 & .78 & 2,800 & 53.2 \\
\hline 41 & 36.9 & 24.8 & 23.2 & 2,100 & 1,280 & 1.6 & 20.0 & 17.0 & 470 & 12,200 & 59.0 & 7.0 & .84 & 2,770 & 52.0 \\
\hline 42 & 35.8 & 25.5 & 21.8 & 2,120 & 1,190 & 1.5 & 19.6 & 15.0 & 430 & 11,500 & 54.8 & 6.5 & .75 & 2,680 & 49.6 \\
\hline 43 & 34.0 & 25.3 & 21.5 & 2,120 & 981 & 1.8 & 20.2 & 15.0 & 400 & 11,100 & 53.9 & 6.4 & .77 & 2,580 & 48.8 \\
\hline 44 & 35.6 & 25.8 & 21.2 & 2,140 & 901 & 1.9 & 21.5 & 16.0 & 410 & 11,100 & 52.9 & 6.4 & .82 & 2,610 & 48.8 \\
\hline 45 & 34.8 & 25.3 & 20.3 & 2,100 & 980 & 1.3 & 18.0 & 16.0 & 390 & 10,900 & 50.9 & 6.1 & .78 & 2,590 & 48.6 \\
\hline 46 & 35.6 & 24.4 & 20.3 & 2,070 & 777 & 1.4 & 17.7 & 14.0 & 380 & 10,800 & 50.5 & 6.1 & .67 & 2,560 & 48.2 \\
\hline 47 & 34.9 & 26.2 & 19.9 & 1,920 & 999 & 1.4 & 17.5 & 15.0 & 360 & 10,900 & 50.0 & 5.9 & .77 & 2,660 & 48.2 \\
\hline 48 & 33.5 & 26.8 & 19.6 & 1,880 & 958 & 1.7 & 19.7 & 15.0 & 360 & 11,100 & 51.2 & 6.0 & .74 & 2,720 & 49.3 \\
\hline 49 & 35.0 & 26.8 & 20.2 & 1,860 & 834 & 1.2 & 17.6 & 15.0 & 350 & 11,000 & 50.3 & 6.0 & .74 & 2,630 & 48.8 \\
\hline 50 & 35.5 & 27.2 & 21.0 & 1,920 & 757 & 1.4 & 18.1 & 17.0 & 360 & 11,200 & 51.9 & 6.2 & .83 & 2,660 & 49.9 \\
\hline 51 & 35.5 & 27.2 & 18.3 & 1,870 & 680 & .9 & 16.7 & 9.9 & 360 & 10,500 & 50.1 & 6.0 & .68 & 2,620 & 44.1 \\
\hline 52 & 36.8 & 25.0 & 17.1 & 1,820 & 1,190 & .8 & 15.3 & 5.2 & 360 & 10,400 & 46.5 & 5.4 & .42 & 2,640 & 41.7 \\
\hline 53 & 35.6 & 25.0 & 17.7 & 1,870 & 1,100 & .8 & 15.9 & 5.2 & 350 & 10,600 & 47.8 & 5.5 & .39 & 2,720 & 43.1 \\
\hline 54 & 35.4 & 25.6 & 18.7 & 1,980 & 887 & .9 & 16.6 & 4.7 & 350 & 10,800 & 49.8 & 5.5 & .37 & 2,780 & 44.4 \\
\hline 55 & 36.4 & 25.0 & 18.4 & 1,950 & 825 & .7 & 16.0 & 4.6 & 330 & 10,900 & 49.7 & 5.8 & .39 & 2,770 & 44.2 \\
\hline 56 & 37.8 & 25.5 & 17.9 & 2,010 & 764 & .8 & 16.4 & 5.8 & 350 & 11,000 & 48.8 & 5.6 & .37 & 2,750 & 45.1 \\
\hline 57 & 36.0 & 24.4 & 18.0 & 1,960 & 736 & .8 & 16.3 & 4.8 & 350 & 10,800 & 48.8 & 5.5 & .34 & 2,700 & 45.3 \\
\hline 58 & 37.1 & 24.6 & 18.4 & 2,040 & 744 & .9 & 16.4 & 5.2 & 360 & 10,500 & 48.0 & 5.7 & .41 & 2,720 & 42.4 \\
\hline 59 & 36.3 & 24.4 & 18.8 & 2,010 & 682 & .8 & 15.6 & 6.2 & 350 & 10,600 & 47.0 & 5.6 & .46 & 2,710 & 42.0 \\
\hline 60 & 37.6 & 25.5 & 18.9 & 2,020 & 931 & .8 & 17.6 & 6.1 & 380 & 11,000 & 51.6 & 6.1 & .37 & 2,750 & 44.9 \\
\hline 61 & 36.4 & 26.3 & 20.0 & 2,060 & 681 & .9 & 18.2 & 7.8 & 410 & 11,300 & 54.6 & 6.2 & .48 & 2,670 & 44.4 \\
\hline 62 & 38.7 & 28.1 & 22.1 & 2,220 & 558 & 1.1 & 20.1 & 8.2 & 440 & 11,200 & 56.8 & 6.9 & .51 & 2,600 & 44.5 \\
\hline 63 & 38.0 & 26.0 & 20.8 & 2,030 & 729 & .8 & 18.1 & 7.8 & 390 & 11,200 & 52.6 & 6.5 & .46 & 2,750 & 43.7 \\
\hline 64 & 37.4 & 24.7 & 20.1 & 1,980 & 851 & .9 & 17.3 & 6.6 & 400 & 11,500 & 54.1 & 6.2 & .43 & 2,800 & 47.1 \\
\hline 65 & 36.5 & 22.6 & 18.3 & 1,760 & 944 & .8 & 15.8 & 5.4 & 340 & 10,700 & 47.8 & 5.6 & .31 & 2,750 & 43.4 \\
\hline 66 & 38.6 & 22.4 & 18.2 & 1,790 & 815 & 1.0 & 15.9 & 3.4 & 330 & 11,000 & 47.3 & 5.6 & .28 & 2,820 & 43.1 \\
\hline 67 & 39.2 & 21.8 & 18.2 & 1,830 & 1,010 & .9 & 15.5 & 4.9 & 380 & 10,300 & 47.9 & 5.6 & .33 & 2,520 & 41.4 \\
\hline 68 & 39.8 & 21.6 & 18.4 & 1,750 & 1,340 & .9 & 16.8 & 5.7 & 380 & 10,500 & 47.8 & 5.9 & .39 & 2,520 & 42.0 \\
\hline 69 & 38.2 & 21.1 & 18.9 & 1,720 & 1,350 & .8 & 16.0 & 5.6 & 360 & 10,700 & 48.7 & 5.7 & .36 & 2,650 & 43.0 \\
\hline 70 & 37.4 & 21.1 & 17.8 & 1,710 & 1,290 & .8 & 16.6 & 4.8 & 360 & 11,000 & 48.2 & 5.4 & .33 & 2,730 & 44.4 \\
\hline 71 & 35.3 & 20.6 & 17.7 & 1,720 & 1,320 & .8 & 16.0 & 5.0 & 350 & 10,700 & 47.3 & 5.5 & .34 & 2,690 & 41.8 \\
\hline 72 & 37.8 & 22.0 & 18.0 & 1,830 & 1,230 & .8 & 16.6 & 5.7 & 360 & 11,000 & 49.4 & 5.8 & .36 & 2,710 & 44.1 \\
\hline Maximum & 43.3 & 28.1 & 25.2 & 2,250 & 1,770 & 2.4 & 25.1 & 17.0 & 500 & 12,500 & 62.3 & 7.6 & 0.85 & 2,900 & 53.2 \\
\hline Minimum & 22.3 & 14.0 & 11.5 & 1,120 & 435 & .7 & 11.6 & 3.4 & 220 & 6,270 & 28.8 & 3.3 & .28 & 1,540 & 29.7 \\
\hline Mean & 37.9 & 24.8 & 20.2 & 1,982 & 1,005 & 1.3 & 18.7 & 9.4 & 391 & 10,874 & 51.0 & 6.0 & .55 & 2,625 & 46.6 \\
\hline Median & 37.8 & 25.0 & 20.3 & 2,015 & 981 & 1.3 & 18.6 & 8.8 & 380 & 11,000 & 50.9 & 6.0 & .51 & 2,640 & 47.5 \\
\hline
\end{tabular}


Table 1. Quantitative elemental analyses of less than 63-micrometer fraction and lead-210 date of Clearwater Lake bed-sediment samples-Continued

[All concentrations in milligrams per kilogram; RSD, relative standard deviation; ${ }^{\circ}$, degrees; ', minutes; ”, seconds; <, less than; shaded cells exceed threshold effects concentration; --, no data]

\begin{tabular}{|c|c|c|c|c|c|c|c|c|c|c|c|c|c|c|c|}
\hline $\begin{array}{c}\text { Depth below } \\
\text { lakebed } \\
\text { surface, } \\
\text { (centimeters) }\end{array}$ & Lanthanum & Lead & Lithium & Magnesium & Manganese & Molybdenum & Nickel & Niobium & Phosphorous & Potassium & Rubidium & Scandium & Silver & Sodium & Strontium \\
\hline RSD (percent) & 6.64 & 3.83 & 2.48 & 2.70 & 2.52 & 10.69 & 3.35 & 23.19 & 2.84 & 2.86 & 2.65 & 3.57 & 41.48 & 2.44 & 3.74 \\
\hline \multicolumn{16}{|c|}{ Logan Creek (Latitude $37^{\circ} 09^{\prime} 21^{\prime \prime}$ Longitude $090^{\circ} 48^{\prime} 07^{\prime \prime}$ ) } \\
\hline 1 & 35.6 & 30.9 & 22.8 & 2,280 & 1,120 & 1.6 & 23.9 & 16.0 & 450 & 10,500 & 52.2 & 7.2 & 0.45 & 2,450 & 41.4 \\
\hline 2 & 38.1 & 44.9 & 25.1 & 2,610 & 771 & 2.5 & 29.8 & 16.0 & 480 & 10,400 & 54.8 & 7.8 & .42 & 2,190 & 39.6 \\
\hline 3 & 36.8 & 45.1 & 25.1 & 2,650 & 944 & 4.3 & 36.4 & 17.0 & 480 & 10,400 & 55.6 & 8.2 & .45 & 2,190 & 39.7 \\
\hline 4 & 37.1 & 48.5 & 25.3 & 2,690 & 757 & 3.5 & 33.9 & 18.0 & 470 & 10,200 & 54.5 & 7.9 & .43 & 2,130 & 39.1 \\
\hline 5 & 37.3 & 45.8 & 25.4 & 2,660 & 1,220 & 1.4 & 24.6 & 18.0 & 520 & 10,600 & 56.0 & 8.2 & .47 & 2,180 & 40.8 \\
\hline 6 & 37.2 & 46.1 & 26.0 & 2,620 & 1,260 & 1.4 & 25.6 & 17.0 & 540 & 10,500 & 55.8 & 8.2 & .41 & 2,190 & 40.4 \\
\hline 7 & 37.9 & 46.4 & 25.5 & 2,620 & 1,260 & 1.5 & 24.9 & 17.0 & 500 & 10,300 & 55.1 & 7.9 & .44 & 2,140 & 39.8 \\
\hline 8 & 38.2 & 46.7 & 25.7 & 2,680 & 1,310 & 1.8 & 26.9 & 18.0 & 500 & 10,400 & 55.9 & 8.2 & .46 & 2,120 & 40.4 \\
\hline 9 & 33.8 & 41.3 & 22.8 & 2,360 & 1,150 & 1.2 & 21.7 & 14.0 & 450 & 8,980 & 47.9 & 7.1 & .36 & 1,800 & 34.6 \\
\hline 10 & 29.2 & 35.8 & 20.8 & 2,220 & 1,010 & 1.2 & 20.9 & 14.0 & 410 & 7,820 & 42.3 & 6.2 & .34 & 1,520 & 29.9 \\
\hline 11 & 32.5 & 38.8 & 22.9 & 2,340 & 1,090 & 1.5 & 23.1 & 15.0 & 460 & 8,810 & 48.5 & 7.3 & .37 & 1,680 & 33.3 \\
\hline 12 & 33.0 & 39.5 & 22.8 & 2,400 & 1,470 & 1.4 & 22.6 & 14.0 & 490 & 9,090 & 49.8 & 7.2 & .37 & 1,760 & 34.6 \\
\hline 13 & 30.7 & 33.1 & 20.3 & 2,020 & 1,390 & 1.1 & 19.3 & 12.0 & 400 & 8,380 & 43.2 & 6.3 & .32 & 1,760 & 32.2 \\
\hline 14 & 29.6 & 30.3 & 19.5 & 1,860 & 1,320 & 1.1 & 19.3 & 12.0 & 370 & 7,980 & 41.4 & 5.9 & .30 & 1,680 & 30.8 \\
\hline 15 & 28.0 & 27.1 & 18.1 & 1,730 & 1,630 & 1.2 & 18.3 & 11.0 & 370 & 7,500 & 38.4 & 5.5 & .25 & 1,570 & 29.0 \\
\hline 16 & 31.6 & 29.7 & 20.3 & 1,940 & 1,610 & 1.3 & 20.5 & 12.0 & 380 & 8,500 & 43.9 & 6.3 & .27 & 1,810 & 32.6 \\
\hline 17 & 31.6 & 30.8 & 20.8 & 2,020 & 1,630 & 1.2 & 21.0 & 14.0 & 420 & 8,860 & 46.2 & 6.5 & .32 & 1,810 & 33.4 \\
\hline 18 & 30.3 & 27.2 & 19.2 & 1,800 & 1,340 & 1.2 & 18.9 & 11.0 & 370 & 7,830 & 40.4 & 5.8 & .29 & 1,650 & 30.5 \\
\hline 19 & 31.2 & 29.5 & 20.4 & 1,910 & 1,290 & 1.3 & 20.2 & 10.0 & 390 & 8,420 & 43.7 & 6.3 & .26 & 1,750 & 32.4 \\
\hline 20 & 27.6 & 26.0 & 18.9 & 1,750 & 1,080 & 1.3 & 19.1 & 12.0 & 370 & 7,580 & 39.5 & 5.6 & .28 & 1,570 & 29.0 \\
\hline 21 & 28.6 & 26.9 & 18.8 & 1,780 & 959 & 1.0 & 19.0 & 11.0 & 370 & 7,650 & 40.2 & 5.7 & .27 & 1,550 & 29.1 \\
\hline 22 & 28.6 & 26.3 & 19.2 & 1,810 & 1,070 & 1.1 & 18.4 & 11.0 & 380 & 7,920 & 41.4 & 5.8 & .26 & 1,640 & 30.2 \\
\hline 23 & 28.6 & 19.3 & 18.3 & 1,630 & 568 & 1.1 & 16.8 & 11.0 & 340 & 8,300 & 40.3 & 5.5 & .28 & 1,910 & 32.4 \\
\hline 24 & 22.3 & 13.7 & 14.3 & 1,260 & 312 & 1.5 & 16.3 & 8.6 & 280 & 6,020 & 29.5 & 4.4 & .23 & 1,280 & 23.6 \\
\hline 25 & 21.1 & 12.0 & 13.2 & 1,160 & 212 & .9 & 13.0 & 6.9 & 260 & 5,540 & 26.8 & 3.8 & .16 & 1,230 & 21.8 \\
\hline 26 & 23.8 & 12.2 & 15.1 & 1,330 & 198 & 1.1 & 15.0 & 8.2 & 300 & 6,830 & 32.0 & 4.6 & .19 & 1,570 & 26.7 \\
\hline 27 & 24.0 & 12.3 & 15.6 & 1,370 & 165 & 1.0 & 14.8 & 7.3 & 290 & 7,040 & 33.3 & 4.5 & .18 & 1,650 & 27.3 \\
\hline 28 & 23.6 & 11.4 & 14.6 & 1,250 & 399 & 1.2 & 15.2 & 7.9 & 300 & 6,360 & 30.6 & 4.3 & .18 & 1,460 & 25.5 \\
\hline Maximum & 38.2 & 48.5 & 26.0 & 2,690 & 1,630 & 4.3 & 36.4 & 18.0 & 540 & 10,600 & 56.0 & 8.2 & 0.47 & 2,450 & 41.4 \\
\hline Minimum & 21.1 & 11.4 & 13.2 & 1,160 & 165 & .9 & 13.0 & 6.9 & 260 & 5,540 & 26.8 & 3.8 & .16 & 1,230 & 21.8 \\
\hline Mean & 31.0 & 31.3 & 20.6 & 2,027 & 1,019 & 1.5 & 21.4 & 12.9 & 405 & 8,525 & 44.3 & 6.4 & .32 & 1,794 & 32.9 \\
\hline Median & 31.0 & 30.6 & 20.4 & 1,980 & 1,105 & 1.3 & 20.4 & 12.0 & 395 & 8,400 & 43.5 & 6.3 & .31 & 1,755 & 32.4 \\
\hline
\end{tabular}


Table 1. Quantitative elemental analyses of less than 63-micrometer fraction and lead-210 date of Clearwater Lake bed-sediment samples-Continued

[All concentrations in milligrams per kilogram; RSD, relative standard deviation; ${ }^{\circ}$, degrees; ', minutes; ”, seconds; <, less than; shaded cells exceed threshold effects concentration; --, no data]

\begin{tabular}{|c|c|c|c|c|c|c|c|c|c|c|c|c|c|c|c|}
\hline $\begin{array}{l}\text { Depth below } \\
\text { lakebed } \\
\text { surface, } \\
\text { (centimeters) }\end{array}$ & Lanthanum & Lead & Lithium & Magnesium & Manganese & Molybdenum & Nickel & Niobium & Phosphorous & Potassium & Rubidium & Scandium & Silver & Sodium & Strontium \\
\hline RSD (percent) & 6.64 & 3.83 & 2.48 & 2.70 & 2.52 & 10.69 & 3.35 & 23.19 & 2.84 & 2.86 & 2.65 & 3.57 & 41.48 & 2.44 & 3.74 \\
\hline \multicolumn{16}{|c|}{ Upper Black River (Latitude $37^{\circ} 10^{\prime} 38^{\prime \prime}$ Longitude $090^{\circ} 47^{\prime} 28^{\prime \prime}$ ) } \\
\hline 1 & 40.2 & 51.7 & 23.7 & 3,300 & 832 & 0.9 & 29.0 & 8.1 & 530 & 13,000 & 56.7 & 8.0 & 0.65 & 2,810 & 49.2 \\
\hline 2 & 37.2 & 44.9 & 20.2 & 2,820 & 414 & 1.2 & 26.0 & 5.8 & 430 & 12,500 & 51.7 & 6.9 & .52 & 3,060 & 49.2 \\
\hline 3 & 35.4 & 41.4 & 18.8 & 2,640 & 701 & 1.4 & 25.6 & 5.5 & 400 & 12,400 & 49.2 & 6.2 & .48 & 3,080 & 48.2 \\
\hline 4 & 37.4 & 43.9 & 20.0 & 2,680 & 667 & 1.0 & 24.6 & 6.0 & 420 & 12,700 & 51.1 & 6.9 & .50 & 3,120 & 49.9 \\
\hline 5 & 38.8 & 45.3 & 21.4 & 2,880 & 739 & .9 & 25.2 & 5.7 & 480 & 13,100 & 52.8 & 7.4 & .50 & 3,260 & 50.9 \\
\hline 6 & 39.0 & 46.2 & 21.7 & 2,940 & 783 & .9 & 25.6 & 6.0 & 460 & 13,300 & 53.5 & 7.3 & .58 & 3,140 & 51.5 \\
\hline 7 & 38.6 & 45.3 & 21.2 & 2,970 & 584 & 1.1 & 25.9 & 6.0 & 460 & 13,300 & 53.0 & 7.4 & .52 & 3,170 & 51.3 \\
\hline 8 & 38.2 & 44.3 & 21.3 & 2,950 & 627 & 1.3 & 26.6 & 4.5 & 440 & 13,300 & 52.6 & 7.1 & .41 & 3,160 & 49.7 \\
\hline 9 & 39.0 & 44.0 & 21.4 & 3,080 & 579 & 1.1 & 26.9 & 6.0 & 450 & 13,200 & 51.4 & 7.0 & .52 & 3,070 & 48.1 \\
\hline 10 & 39.5 & 44.8 & 21.7 & 3,040 & 703 & 1.4 & 28.1 & 5.7 & 470 & 12,400 & 51.5 & 7.4 & .49 & 2,780 & 46.0 \\
\hline 11 & 39.4 & 44.8 & 22.0 & 2,940 & 739 & 1.6 & 29.1 & 8.2 & 500 & 12,400 & 52.8 & 7.5 & .62 & 2,660 & 46.1 \\
\hline 12 & 41.9 & 46.4 & 23.6 & 3,120 & 824 & 1.5 & 31.2 & 7.4 & 540 & 12,700 & 53.9 & 8.0 & .58 & 2,780 & 47.1 \\
\hline 13 & 39.5 & 46.0 & 22.2 & 3,060 & 761 & 1.2 & 29.4 & 6.9 & 500 & 13,100 & 53.7 & 7.6 & .54 & 3,020 & 49.1 \\
\hline 14 & 38.1 & 48.2 & 22.4 & 3,240 & 655 & 1.2 & 27.6 & 7.1 & 470 & 13,300 & 53.9 & 7.6 & .57 & 3,100 & 50.0 \\
\hline 15 & 38.4 & 49.4 & 22.2 & 3,360 & 642 & 1.1 & 27.6 & 5.8 & 480 & 13,500 & 54.3 & 7.4 & .45 & 3,050 & 50.1 \\
\hline 16 & 38.4 & 49.5 & 22.2 & 3,390 & 676 & 1.2 & 28.1 & 6.2 & 470 & 13,100 & 52.9 & 7.3 & .49 & 3,120 & 49.7 \\
\hline 17 & 38.5 & 48.3 & 22.0 & 3,420 & 699 & .9 & 27.3 & 6.8 & 480 & 13,100 & 52.6 & 7.2 & .55 & 3,080 & 50.2 \\
\hline 18 & 37.6 & 47.2 & 20.5 & 3,780 & 502 & 1.4 & 28.4 & 4.8 & 440 & 12,900 & 51.9 & 7.1 & .43 & 2,990 & 48.6 \\
\hline 19 & 37.8 & 50.0 & 21.6 & 3,970 & 677 & 1.5 & 29.2 & 4.8 & 460 & 12,900 & 52.3 & 7.2 & .41 & 2,860 & 47.4 \\
\hline 20 & 39.8 & 55.5 & 22.4 & 3,860 & 955 & 1.6 & 32.6 & 5.6 & 530 & 13,100 & 54.5 & 7.8 & .48 & 2,850 & 49.1 \\
\hline 21 & 40.0 & 54.4 & 22.1 & 3,200 & 942 & 1.1 & 29.5 & 7.6 & 490 & 11,900 & 53.6 & 7.9 & .71 & 2,710 & 47.7 \\
\hline 22 & 37.1 & 47.5 & 19.8 & 4,080 & 861 & 1.1 & 25.5 & 4.7 & 430 & 11,500 & 49.8 & 6.6 & .46 & 2,840 & 45.9 \\
\hline 23 & 36.1 & 46.9 & 19.3 & 3,710 & 658 & 1.0 & 24.5 & 5.9 & 420 & 11,700 & 49.9 & 6.7 & .50 & 2,840 & 46.9 \\
\hline 24 & 35.9 & 47.6 & 19.0 & 3,450 & 596 & 1.2 & 24.9 & 4.9 & 410 & 11,500 & 49.9 & 6.4 & .44 & 2,980 & 48.0 \\
\hline 25 & 37.4 & 46.9 & 19.8 & 4,100 & 724 & .9 & 24.8 & 4.2 & 420 & 11,800 & 49.2 & 6.7 & .38 & 2,900 & 47.5 \\
\hline 26 & 38.0 & 45.6 & 19.9 & 3,600 & 972 & 1.0 & 23.4 & 4.6 & 420 & 11,400 & 50.2 & 6.9 & .39 & 2,860 & 46.2 \\
\hline 27 & 36.2 & 45.6 & 19.4 & 2,500 & 714 & 1.0 & 24.1 & 5.1 & 410 & 12,100 & 49.4 & 6.6 & .42 & 3,030 & 48.2 \\
\hline 28 & 36.4 & 44.7 & 19.2 & 2,400 & 581 & 1.3 & 24.3 & 5.4 & 410 & 11,800 & 49.2 & 6.6 & .43 & 3,010 & 47.9 \\
\hline 29 & 36.4 & 44.3 & 19.3 & 2,430 & 526 & .8 & 23.1 & 3.9 & 400 & 12,000 & 49.4 & 6.5 & .33 & 3,080 & 47.3 \\
\hline 30 & 37.5 & 48.4 & 20.5 & 2,670 & 614 & 1.0 & 23.8 & 6.5 & 440 & 12,200 & 51.7 & 7.0 & .47 & 3,090 & 48.7 \\
\hline 31 & 36.3 & 47.6 & 19.9 & 2,630 & 566 & 1.6 & 26.5 & 9.3 & 420 & 12,400 & 50.9 & 6.7 & .61 & 3,110 & 47.9 \\
\hline 32 & 37.6 & 48.9 & 20.3 & 2,720 & 630 & 1.5 & 28.1 & 6.8 & 450 & 12,400 & 52.9 & 7.2 & .48 & 3,040 & 48.6 \\
\hline 33 & 37.8 & 50.5 & 20.3 & 2,630 & 610 & 1.5 & 33.2 & 6.4 & 440 & 12,400 & 52.1 & 7.0 & .45 & 3,090 & 48.8 \\
\hline 34 & 36.7 & 49.7 & 19.3 & 2,480 & 494 & 1.2 & 25.0 & 5.8 & 410 & 13,000 & 51.8 & 6.9 & .43 & 3,370 & 50.8 \\
\hline 35 & 38.2 & 53.4 & 21.3 & 2,840 & 631 & 3.8 & 38.7 & 6.3 & 470 & 12,800 & 53.7 & 7.3 & .44 & 3,140 & 49.3 \\
\hline 36 & 36.0 & 50.2 & 19.5 & 2,720 & 610 & 4.0 & 40.0 & 5.0 & 440 & 11,900 & 49.4 & 6.4 & .38 & 2,840 & 45.0 \\
\hline 37 & 39.3 & 54.2 & 21.9 & 3,070 & 606 & 1.2 & 27.5 & 7.0 & 490 & 13,100 & 55.3 & 7.6 & .53 & 3,000 & 48.7 \\
\hline 38 & 39.8 & 55.2 & 22.4 & 3,130 & 622 & 1.2 & 28.6 & 6.9 & 500 & 13,100 & 56.4 & 7.9 & .45 & 3,000 & 50.1 \\
\hline
\end{tabular}


Table 1. Quantitative elemental analyses of less than 63-micrometer fraction and lead-210 date of Clearwater Lake bed-sediment samples-Continued

[All concentrations in milligrams per kilogram; RSD, relative standard deviation; ${ }^{\circ}$, degrees; ', minutes; ”, seconds; <, less than; shaded cells exceed threshold effects concentration; --, no data]

\begin{tabular}{|c|c|c|c|c|c|c|c|c|c|c|c|c|c|c|c|}
\hline $\begin{array}{c}\text { Depth below } \\
\text { lakebed } \\
\text { surface, } \\
\text { (centimeters) }\end{array}$ & Lanthanum & Lead & Lithium & Magnesium & Manganese & Molybdenum & Nickel & Niobium & Phosphorous & Potassium & Rubidium & Scandium & Silver & Sodium & Strontium \\
\hline RSD (percent) & 6.64 & 3.83 & 2.48 & 2.70 & 2.52 & 10.69 & 3.35 & 23.19 & 2.84 & 2.86 & 2.65 & 3.57 & 41.48 & 2.44 & 3.74 \\
\hline \multicolumn{16}{|c|}{ Upper Black River (Latitude $37^{\circ} 10^{\prime} 38^{\prime \prime}$ Longitude $090^{\circ} 47^{\prime} 28^{\prime \prime}$ ) } \\
\hline 39 & 40.2 & 55.3 & 23.8 & 3,470 & 701 & 1.2 & 28.0 & 7.4 & 510 & 13,000 & 57.7 & 8.2 & 0.50 & 2,970 & 48.8 \\
\hline 40 & 39.4 & 55.7 & 23.9 & 3,590 & 661 & 1.0 & 28.4 & 7.8 & 500 & 12,600 & 57.3 & 8.2 & .48 & 2,900 & 48.3 \\
\hline 41 & 39.7 & 55.0 & 23.4 & 3,250 & 713 & 1.1 & 27.3 & 8.4 & 510 & 12,400 & 58.3 & 8.1 & .58 & 2,950 & 48.3 \\
\hline 42 & 39.6 & 54.5 & 23.5 & 3,220 & 786 & 1.2 & 27.0 & 8.2 & 510 & 12,900 & 58.3 & 8.0 & .54 & 3,010 & 49.5 \\
\hline 43 & 37.1 & 50.9 & 21.2 & 3,020 & 601 & 1.0 & 24.6 & 7.1 & 460 & 12,800 & 55.1 & 7.5 & .46 & 3,180 & 49.0 \\
\hline 44 & 37.1 & 49.8 & 21.1 & 2,890 & 562 & .8 & 21.9 & 7.6 & 430 & 12,600 & 53.5 & 7.1 & .51 & 3,280 & 49.2 \\
\hline 45 & 37.0 & 47.4 & 20.4 & 2,910 & 585 & .7 & 21.2 & 7.5 & 430 & 12,500 & 53.2 & 7.1 & .45 & 3,280 & 49.9 \\
\hline 46 & 38.4 & 47.9 & 20.8 & 3,060 & 633 & 1.0 & 23.6 & 7.6 & 450 & 12,400 & 55.2 & 7.3 & .51 & 3,150 & 49.8 \\
\hline 47 & 38.3 & 45.2 & 20.4 & 3,030 & 636 & .9 & 22.2 & 7.4 & 440 & 12,900 & 53.5 & 7.0 & .53 & 3,220 & 49.7 \\
\hline 48 & 37.0 & 43.5 & 20.6 & 2,880 & 583 & .8 & 21.4 & 7.6 & 420 & 12,800 & 52.5 & 6.8 & .54 & 3,310 & 49.8 \\
\hline 49 & 37.5 & 43.7 & 20.2 & 2,900 & 540 & .7 & 21.4 & 7.5 & 410 & 13,000 & 52.1 & 6.9 & .53 & 3,350 & 49.7 \\
\hline 50 & 35.4 & 41.9 & 19.7 & 3,160 & 518 & .7 & 20.2 & 9.0 & 400 & 12,200 & 48.8 & 6.7 & .58 & 3,170 & 47.1 \\
\hline 51 & 35.9 & 43.6 & 21.3 & 3,110 & 590 & 1.2 & 22.2 & 13.0 & 440 & 12,800 & 53.8 & 7.2 & .88 & 3,050 & 53.6 \\
\hline 52 & 36.3 & 45.2 & 22.2 & 3,260 & 616 & 1.3 & 24.3 & 14.0 & 460 & 13,200 & 56.7 & 7.8 & .94 & 3,080 & 55.4 \\
\hline 53 & 36.2 & 40.2 & 20.8 & 3,210 & 654 & 1.4 & 23.0 & 13.0 & 420 & 13,000 & 54.4 & 7.0 & .81 & 3,180 & 55.2 \\
\hline 54 & 35.6 & 37.9 & 20.4 & 2,690 & 588 & 1.3 & 21.6 & 12.0 & 390 & 13,500 & 55.0 & 7.0 & .74 & 3,430 & 57.4 \\
\hline 55 & 35.6 & 35.9 & 19.7 & 2,520 & 541 & 1.1 & 20.7 & 15.0 & 370 & 13,500 & 53.2 & 6.6 & .98 & 3,470 & 56.6 \\
\hline 56 & 36.2 & 35.9 & 19.2 & 2,610 & 489 & 1.1 & 19.6 & 14.0 & 370 & 13,300 & 52.5 & 6.5 & .85 & 3,400 & 56.2 \\
\hline 57 & 35.8 & 35.7 & 20.0 & 2,770 & 565 & 1.3 & 21.2 & 14.0 & 390 & 13,400 & 54.0 & 6.8 & .79 & 3,320 & 56.5 \\
\hline 58 & 37.8 & 36.4 & 20.8 & 2,470 & 661 & 1.3 & 22.7 & 14.0 & 390 & 13,700 & 56.0 & 7.3 & .83 & 3,480 & 58.3 \\
\hline 59 & 37.5 & 36.9 & 21.4 & 2,590 & 821 & 1.3 & 23.3 & 14.0 & 420 & 13,800 & 57.7 & 7.5 & .83 & 3,430 & 57.8 \\
\hline 60 & 41.1 & 40.8 & 23.3 & 3,020 & 994 & 1.2 & 24.7 & 15.0 & 450 & 14,200 & 61.9 & 8.4 & .81 & 3,240 & 58.4 \\
\hline 61 & 36.1 & 42.9 & 22.1 & 2,930 & 712 & 1.3 & 24.6 & 13.0 & 420 & 13,300 & 57.5 & 7.6 & .80 & 3,110 & 56.1 \\
\hline Maximum & 41.9 & 55.7 & 23.9 & 4,100 & 994 & 4.0 & 40.0 & 15.0 & 540 & 14,200 & 61.9 & 8.4 & 0.98 & 3,480 & 58.4 \\
\hline Minimum & 35.4 & 35.7 & 18.8 & 2,400 & 414 & .7 & 19.6 & 3.9 & 370 & 11,400 & 48.8 & 6.2 & .33 & 2,660 & 45.0 \\
\hline Mean & 37.8 & 46.6 & 21.1 & 3,055 & 663 & 1.2 & 26.0 & 7.8 & 447 & 12,756 & 53.3 & 7.2 & .56 & 3,083 & 50.1 \\
\hline Median & 37.6 & 46.4 & 21.1 & 3,020 & 636 & 1.2 & 25.5 & 6.9 & 440 & 12,900 & 53.0 & 7.2 & .51 & 3,080 & 49.2 \\
\hline
\end{tabular}


Table 1. Quantitative elemental analyses of less than 63-micrometer fraction and lead-210 date of Clearwater Lake bed-sediment samples-Continued

[All concentrations in milligrams per kilogram; RSD, relative standard deviation; ${ }^{\circ}$, degrees; ', minutes; ", seconds; <, less than; shaded cells exceed threshold effects concentration; --, no data]

\begin{tabular}{|c|c|c|c|c|c|c|c|c|c|c|c|c|c|c|c|}
\hline $\begin{array}{l}\text { Depth below } \\
\text { lakebed } \\
\text { surface, } \\
\text { (centimeters) }\end{array}$ & Lanthanum & Lead & Lithium & Magnesium & Manganese & Molybdenum & Nickel & Niobium & Phosphorous & Potassium & Rubidium & Scandium & Silver & Sodium & Strontium \\
\hline RSD (percent) & 6.64 & 3.83 & 2.48 & 2.70 & 2.52 & 10.69 & 3.35 & 23.19 & 2.84 & 2.86 & 2.65 & 3.57 & 41.48 & 2.44 & 3.74 \\
\hline \multicolumn{16}{|c|}{ Lower Black River (Latitude $37^{\circ} 09^{\prime} 54^{\prime \prime}$ Longitude $090^{\circ} 45^{\prime} 58^{\prime \prime}$ ) } \\
\hline 1 & 46.3 & 69.7 & 32.2 & 4,380 & 2,250 & 1.4 & 38.7 & 18.0 & 780 & 14,000 & 72.4 & 11.2 & 0.12 & 2,280 & 50.5 \\
\hline 2 & 45.6 & 71.6 & 33.1 & 4,280 & 970 & 1.7 & 40.3 & 18.0 & 730 & 14,100 & 73.1 & 11.2 & .12 & 2,330 & 50.8 \\
\hline 3 & 46.5 & 74.0 & 33.4 & 4,150 & 904 & 2.0 & 42.2 & 16.0 & 750 & 14,200 & 74.1 & 11.6 & .06 & 2,200 & 48.7 \\
\hline 4 & 45.6 & 65.2 & 30.6 & 3,740 & 975 & 2.3 & 40.5 & 15.0 & 680 & 14,200 & 68.7 & 10.5 & $<.02$ & 2,550 & 50.7 \\
\hline 5 & 45.8 & 64.8 & 30.2 & 3,700 & 1,060 & 1.6 & 37.9 & 17.0 & 690 & 14,200 & 69.1 & 10.4 & .05 & 2,580 & 50.8 \\
\hline 6 & 45.5 & 65.9 & 30.7 & 3,710 & 1,100 & 1.5 & 36.6 & 18.0 & 700 & 14,200 & 68.6 & 10.5 & .08 & 2,530 & 50.4 \\
\hline 7 & 45.3 & 63.8 & 29.7 & 3,610 & 1,120 & 1.8 & 37.1 & 16.0 & 640 & 14,200 & 67.4 & 10.3 & .04 & 2,600 & 50.6 \\
\hline 8 & 45.8 & 62.9 & 29.4 & 3,670 & 1,140 & 2.5 & 40.1 & 18.0 & 650 & 14,500 & 68.4 & 10.4 & .10 & 2,670 & 51.3 \\
\hline 9 & 46.6 & 64.4 & 30.4 & 3,780 & 1,110 & 1.4 & 36.8 & 18.0 & 670 & 14,600 & 69.8 & 10.6 & .10 & 2,660 & 51.6 \\
\hline 10 & 46.0 & 63.4 & 30.0 & 3,680 & 1,100 & 1.4 & 35.0 & 16.0 & 650 & 14,200 & 68.1 & 10.3 & .04 & 2,590 & 50.8 \\
\hline 11 & 46.4 & 64.0 & 30.7 & 3,720 & 1,080 & 1.9 & 38.1 & 18.0 & 670 & 14,500 & 68.9 & 10.6 & .09 & 2,580 & 50.7 \\
\hline 12 & 45.5 & 63.3 & 30.2 & 3,740 & 1,080 & 2.0 & 38.0 & 16.0 & 640 & 14,300 & 68.1 & 10.4 & .02 & 2,610 & 49.9 \\
\hline 13 & 46.1 & 64.8 & 30.5 & 3,810 & 1,060 & 1.4 & 36.5 & 16.0 & 650 & 14,500 & 69.7 & 10.8 & .02 & 2,550 & 50.4 \\
\hline 14 & 47.3 & 65.7 & 30.6 & 3,850 & 1,030 & 1.4 & 36.7 & 14.0 & 660 & 14,700 & 70.3 & 10.7 & $<.02$ & 2,600 & 51.0 \\
\hline 15 & 45.4 & 65.6 & 30.5 & 3,780 & 952 & 1.6 & 37.8 & 15.0 & 650 & 14,500 & 69.2 & 10.7 & .02 & 2,550 & 50.4 \\
\hline 16 & 45.9 & 66.5 & 30.7 & 3,760 & 934 & 1.8 & 39.1 & 18.0 & 640 & 14,200 & 67.8 & 10.5 & .04 & 2,550 & 49.7 \\
\hline 17 & 45.3 & 65.5 & 30.4 & 3,660 & 908 & 1.4 & 36.4 & 15.0 & 640 & 14,500 & 68.4 & 10.5 & .03 & 2,590 & 50.8 \\
\hline 18 & 44.8 & 65.5 & 29.8 & 3,670 & 880 & 1.3 & 35.5 & 15.0 & 650 & 14,800 & 67.6 & 10.6 & $<.02$ & 2,710 & 50.9 \\
\hline 19 & 45.6 & 66.5 & 30.1 & 3,670 & 954 & 1.7 & 37.0 & 16.0 & 650 & 14,700 & 68.0 & 10.5 & .02 & 2,640 & 50.2 \\
\hline 20 & 45.0 & 65.4 & 29.6 & 3,600 & 951 & 2.1 & 38.8 & 15.0 & 630 & 14,600 & 67.4 & 10.5 & .02 & 2,590 & 51.5 \\
\hline 21 & 45.7 & 67.4 & 30.9 & 3,710 & 951 & 1.4 & 36.7 & 18.0 & 720 & 14,500 & 69.2 & 10.8 & .09 & 2,690 & 50.5 \\
\hline 22 & 44.6 & 65.3 & 29.7 & 3,610 & 940 & 1.4 & 35.8 & 16.0 & 680 & 14,500 & 67.1 & 10.3 & .05 & 2,740 & 49.7 \\
\hline 23 & 44.4 & 66.4 & 30.2 & 3,560 & 938 & 1.8 & 35.3 & 15.0 & 630 & 13,600 & 69.2 & 10.4 & .52 & 2,430 & 49.4 \\
\hline 24 & 45.1 & 66.4 & 30.6 & 3,670 & 998 & 2.0 & 35.5 & 15.0 & 620 & 13,500 & 68.8 & 10.4 & .46 & 2,440 & 49.2 \\
\hline 25 & 45.6 & 67.6 & 31.6 & 3,780 & 1,090 & 1.5 & 35.3 & 17.0 & 630 & 13,800 & 70.7 & 10.8 & .50 & 2,440 & 49.2 \\
\hline 26 & 45.2 & 66.7 & 31.4 & 3,750 & 1,080 & 1.5 & 34.1 & 17.0 & 610 & 13,700 & 70.8 & 10.6 & .50 & 2,480 & 49.5 \\
\hline 27 & 45.7 & 66.8 & 31.2 & 3,710 & 981 & 2.0 & 36.8 & 15.0 & 600 & 13,700 & 70.8 & 10.7 & .46 & 2,420 & 49.9 \\
\hline 28 & 45.5 & 66.4 & 31.1 & 3,690 & 1,030 & 1.9 & 36.1 & 16.0 & 580 & 13,600 & 69.5 & 10.5 & .48 & 2,480 & 49.6 \\
\hline 29 & 46.4 & 67.4 & 31.9 & 3,750 & 991 & 1.4 & 34.3 & 16.0 & 590 & 13,500 & 69.4 & 10.5 & .48 & 2,460 & 49.1 \\
\hline 30 & 45.6 & 67.0 & 31.7 & 3,700 & 1,020 & 1.5 & 34.8 & 16.0 & 590 & 13,700 & 70.6 & 10.7 & .46 & 2,430 & 50.1 \\
\hline 31 & 43.7 & 66.2 & 31.9 & 3,730 & 996 & 1.8 & 36.1 & 18.0 & 610 & 13,900 & 71.3 & 10.7 & .50 & 2,470 & 49.2 \\
\hline 32 & 43.3 & 66.4 & 31.6 & 3,680 & 997 & 1.7 & 35.7 & 17.0 & 600 & 14,000 & 70.8 & 10.6 & .47 & 2,430 & 49.2 \\
\hline 33 & 43.1 & 66.4 & 31.6 & 3,650 & 940 & 1.5 & 34.0 & 18.0 & 600 & 13,800 & 70.7 & 10.7 & .48 & 2,380 & 49.0 \\
\hline 34 & 43.7 & 66.4 & 32.0 & 3,610 & 936 & 1.4 & 33.5 & 19.0 & 580 & 13,900 & 69.9 & 10.5 & .49 & 2,460 & 49.6 \\
\hline 35 & 43.3 & 66.6 & 31.6 & 3,630 & 936 & 1.6 & 34.7 & 18.0 & 580 & 13,700 & 71.0 & 10.4 & .51 & 2,420 & 49.1 \\
\hline 36 & 42.7 & 65.8 & 31.8 & 3,690 & 973 & 1.9 & 36.1 & 18.0 & 580 & 13,700 & 71.6 & 10.6 & .50 & 2,420 & 49.5 \\
\hline 37 & 43.1 & 67.3 & 32.2 & 3,710 & 1,030 & 1.6 & 34.8 & 19.0 & 590 & 13,700 & 73.1 & 10.7 & .50 & 2,290 & 49.0 \\
\hline 38 & 42.8 & 66.3 & 31.8 & 3,650 & 1,030 & 1.6 & 33.8 & 18.0 & 580 & 13,500 & 71.8 & 10.6 & .50 & 2,330 & 48.9 \\
\hline
\end{tabular}


Table 1. Quantitative elemental analyses of less than 63-micrometer fraction and lead-210 date of Clearwater Lake bed-sediment samples-Continued

[All concentrations in milligrams per kilogram; RSD, relative standard deviation; ${ }^{\circ}$, degrees; ', minutes; ”, seconds; <, less than; shaded cells exceed threshold effects concentration; --, no data]

\begin{tabular}{|c|c|c|c|c|c|c|c|c|c|c|c|c|c|c|c|}
\hline $\begin{array}{c}\text { Depth below } \\
\text { lakebed } \\
\text { surface, } \\
\text { (centimeters) }\end{array}$ & Lanthanum & Lead & Lithium & Magnesium & Manganese & Molybdenum & Nickel & Niobium & Phosphorous & Potassium & Rubidium & Scandium & Silver & Sodium & Strontium \\
\hline RSD (percent) & 6.64 & 3.83 & 2.48 & 2.70 & 2.52 & 10.69 & 3.35 & 23.19 & 2.84 & 2.86 & 2.65 & 3.57 & 41.48 & 2.44 & 3.74 \\
\hline \multicolumn{16}{|c|}{ Lower Black River (Latitude $37^{\circ} 09^{\prime} 54^{\prime \prime}$ Longitude $090^{\circ} 45^{\prime} 58^{\prime \prime}$ )—Continued } \\
\hline 39 & 43.4 & 64.7 & 31.3 & 3,560 & 972 & 1.6 & 33.5 & 19.0 & 570 & 13,600 & 71.4 & 10.4 & 0.51 & 2,390 & 49.7 \\
\hline 40 & 43.3 & 64.8 & 31.1 & 3,560 & 945 & 1.8 & 33.4 & 18.0 & 550 & 13,500 & 70.2 & 10.3 & .50 & 2,400 & 49.2 \\
\hline 41 & 42.0 & 65.5 & 31.8 & 3,600 & 975 & 1.6 & 32.2 & 20.0 & 570 & 13,300 & 70.2 & 10.5 & .55 & 2,330 & 47.6 \\
\hline 42 & 42.6 & 64.8 & 31.7 & 3,600 & 1,010 & 1.5 & 32.7 & 19.0 & 580 & 13,500 & 71.1 & 10.5 & .51 & 2,340 & 48.3 \\
\hline 43 & 36.6 & 45.1 & 25.3 & 2,570 & 781 & 2.5 & 29.4 & 16.0 & 470 & 10,300 & 54.9 & 7.7 & .44 & 2,140 & 40.1 \\
\hline 44 & 42.7 & 62.2 & 30.0 & 3,410 & 953 & 1.9 & 32.5 & 18.0 & 540 & 13,300 & 68.6 & 10.0 & .50 & 2,420 & 48.3 \\
\hline 45 & 41.0 & 59.2 & 28.7 & 3,300 & 951 & 1.6 & 29.7 & 19.0 & 520 & 13,000 & 65.7 & 9.6 & .49 & 2,440 & 47.5 \\
\hline 46 & 40.8 & 56.8 & 27.9 & 3,240 & 944 & 1.5 & 29.2 & 18.0 & 510 & 13,400 & 65.6 & 9.5 & .48 & 2,560 & 49.2 \\
\hline 47 & 42.6 & 55.9 & 28.6 & 3,340 & 946 & 1.7 & 30.8 & 18.0 & 520 & 13,700 & 67.2 & 9.7 & .50 & 2,560 & 49.3 \\
\hline 48 & 43.4 & 54.0 & 28.8 & 3,320 & 982 & 1.7 & 30.9 & 19.0 & 530 & 13,700 & 67.3 & 9.6 & .51 & 2,590 & 49.5 \\
\hline 49 & 42.8 & 51.1 & 28.3 & 3,360 & 1,030 & 1.4 & 29.4 & 19.0 & 510 & 13,700 & 67.3 & 9.6 & .52 & 2,600 & 49.2 \\
\hline 50 & 43.6 & 48.2 & 28.7 & 3,300 & 963 & 1.5 & 30.6 & 21.0 & 520 & 14,200 & 69.0 & 9.6 & .53 & 2,640 & 50.8 \\
\hline 51 & 45.0 & 47.2 & 29.2 & 3,680 & 1,030 & 1.4 & 31.7 & 18.0 & 550 & 14,400 & 73.1 & 10.4 & 1.10 & 2,690 & 57.7 \\
\hline 52 & 45.4 & 43.9 & 29.2 & 3,630 & 979 & 1.4 & 30.4 & 18.0 & 550 & 14,400 & 72.0 & 10.3 & 1.00 & 2,760 & 57.2 \\
\hline 53 & 46.3 & 43.2 & 29.3 & 3,650 & 917 & 1.5 & 31.5 & 16.0 & 550 & 14,600 & 73.3 & 10.6 & .95 & 2,690 & 56.5 \\
\hline 54 & 44.6 & 48.3 & 29.2 & 3,820 & 824 & 1.4 & 32.1 & 16.0 & 540 & 14,400 & 72.6 & 10.4 & .96 & 2,640 & 56.1 \\
\hline Maximum & 47.3 & 74.0 & 33.4 & 4,380 & 2,250 & 2.5 & 42.2 & 21.0 & 780 & 14,800 & 74.1 & 11.6 & 1.10 & 2,760 & 57.7 \\
\hline Minimum & 36.6 & 43.2 & 25.3 & 2,570 & 781 & 1.3 & 29.2 & 14.0 & 470 & 10,300 & 54.9 & 7.7 & .02 & 2,140 & 40.1 \\
\hline Mean & 44.5 & 62.8 & 30.5 & 3,651 & 1,011 & 1.7 & 35.0 & 17.1 & 611 & 13,944 & 69.5 & 10.4 & .37 & 2,507 & 50.1 \\
\hline Median & 45.2 & 65.5 & 30.6 & 3,675 & 977 & 1.6 & 35.4 & 18.0 & 605 & 14,000 & 69.5 & 10.5 & .48 & 2,540 & 49.7 \\
\hline
\end{tabular}


Table 1. Quantitative elemental analyses of less than 63-micrometer fraction and lead-210 date of Clearwater Lake bed-sediment samples-Continued

[All concentrations in milligrams per kilogram; RSD, relative standard deviation; ${ }^{\circ}$, degrees; ', minutes; ", seconds; <, less than; shaded cells exceed threshold effects concentration; --, no data]

\begin{tabular}{|c|c|c|c|c|c|c|c|c|c|c|c|c|c|c|c|}
\hline $\begin{array}{l}\text { Depth below } \\
\text { lakebed } \\
\text { surface, } \\
\text { (centimeters) }\end{array}$ & Lanthanum & Lead & Lithium & Magnesium & Manganese & Molybdenum & Nickel & Niobium & Phosphorous & Potassium & Rubidium & Scandium & Silver & Sodium & Strontium \\
\hline RSD (percent) & 6.64 & 3.83 & 2.48 & 2.70 & 2.52 & 10.69 & 3.35 & 23.19 & 2.84 & 2.86 & 2.65 & 3.57 & 41.48 & 2.44 & 3.74 \\
\hline \multicolumn{16}{|c|}{ Marina (Latitude $37^{\circ} 08^{\prime} 27^{\prime \prime}$ Longitude $090^{\circ} 46^{\prime} 14^{\prime \prime}$ ) } \\
\hline 1 & 42.8 & 70.8 & 36.6 & 4,780 & 1,430 & 2.8 & 40.0 & 11.0 & 3,600 & 11,700 & 69.1 & 10.7 & 0.81 & 3,590 & 62.7 \\
\hline 2 & 44.7 & 79.1 & 39.1 & 4,900 & 1,350 & 2.2 & 44.8 & 17.0 & 1,700 & 12,100 & 79.6 & 12.2 & .66 & 1,860 & 51.5 \\
\hline 3 & 49.0 & 92.4 & 39.6 & 5,690 & 1,420 & 2.7 & 51.2 & 19.0 & 1,900 & 12,300 & 82.5 & 12.9 & .58 & 1,920 & 69.2 \\
\hline 4 & 46.3 & 83.8 & 39.0 & 4,900 & 1,490 & 2.4 & 47.4 & 17.0 & 2,200 & 12,200 & 82.2 & 12.6 & .48 & 1,720 & 57.0 \\
\hline 5 & 46.4 & 88.5 & 40.7 & 4,770 & 1,580 & 2.9 & 53.5 & 18.0 & 1,700 & 12,200 & 86.5 & 13.0 & .46 & 1,350 & 49.6 \\
\hline 6 & 45.0 & 81.0 & 41.8 & 5,060 & 1,740 & 2.5 & 49.9 & 18.0 & 1,200 & 12,700 & 81.2 & 13.1 & .65 & 2,090 & 48.0 \\
\hline 7 & 45.2 & 81.4 & 39.9 & 4,800 & 1,720 & 2.5 & 53.8 & 17.0 & 2,100 & 12,300 & 81.2 & 12.7 & .62 & 1,500 & 47.4 \\
\hline 8 & 44.8 & 77.6 & 38.3 & 4,760 & 1,960 & 2.2 & 45.6 & 17.0 & 1,600 & 12,400 & 79.7 & 12.4 & .57 & 1,540 & 48.0 \\
\hline 9 & 45.0 & 76.6 & 37.4 & 4,680 & 1,700 & 2.0 & 44.1 & 17.0 & 1,800 & 12,200 & 79.1 & 12.4 & .52 & 1,450 & 46.4 \\
\hline 10 & 44.9 & 76.6 & 37.1 & 4,670 & 1,700 & 2.2 & 44.3 & 16.0 & 1,700 & 12,200 & 78.8 & 12.2 & .49 & 1,520 & 48.4 \\
\hline 11 & 45.2 & 75.2 & 36.6 & 4,510 & 1,550 & 1.9 & 43.9 & 17.0 & 800 & 11,900 & 78.2 & 12.1 & .50 & 1,410 & 46.0 \\
\hline 12 & 43.3 & 76.1 & 36.7 & 4,450 & 1,620 & 2.2 & 45.1 & 17.0 & 850 & 12,000 & 81.1 & 12.4 & .47 & 1,400 & 47.5 \\
\hline 13 & 43.0 & 74.7 & 36.6 & 4,350 & 1,540 & 2.0 & 43.9 & 17.0 & 860 & 11,900 & 80.7 & 12.2 & .47 & 1,390 & 46.8 \\
\hline 14 & 43.8 & 77.4 & 37.2 & 4,420 & 1,520 & 2.0 & 44.2 & 17.0 & 800 & 12,100 & 81.8 & 12.6 & .47 & 1,430 & 49.1 \\
\hline 15 & 44.3 & 79.4 & 38.0 & 4,490 & 1,470 & 2.3 & 47.0 & 18.0 & 820 & 12,300 & 83.4 & 12.8 & .45 & 1,380 & 47.8 \\
\hline 16 & 44.8 & 84.0 & 38.6 & 4,530 & 1,460 & 2.3 & 47.8 & 18.0 & 810 & 12,400 & 85.1 & 13.1 & .43 & 1,350 & 48.2 \\
\hline 17 & 44.5 & 84.2 & 39.1 & 4,520 & 1,430 & 2.1 & 47.1 & 18.0 & 840 & 12,500 & 86.5 & 13.0 & .46 & 1,330 & 49.0 \\
\hline 18 & 45.6 & 85.6 & 39.0 & 4,530 & 1,380 & 2.2 & 47.1 & 17.0 & 790 & 12,600 & 86.1 & 13.3 & .44 & 1,350 & 49.5 \\
\hline 19 & 45.4 & 83.3 & 39.0 & 4,470 & 1,420 & 2.3 & 48.1 & 18.0 & 800 & 12,600 & 85.9 & 13.1 & .45 & 1,340 & 49.4 \\
\hline 20 & 44.0 & 80.5 & 38.3 & 4,390 & 1,530 & 2.2 & 46.4 & 21.0 & 770 & 12,600 & 85.2 & 12.9 & .54 & 1,360 & 49.8 \\
\hline 21 & 43.8 & 81.4 & 38.4 & 4,360 & 1,560 & 2.1 & 46.2 & 18.0 & 790 & 12,500 & 85.4 & 13.1 & .46 & 1,330 & 50.2 \\
\hline 22 & 42.2 & 78.8 & 37.8 & 4,220 & 1,460 & 2.2 & 45.1 & 18.0 & 760 & 12,600 & 84.7 & 12.7 & .45 & 1,380 & 50.6 \\
\hline 23 & 42.0 & 77.8 & 37.4 & 4,140 & 1,500 & 2.2 & 45.8 & 17.0 & 760 & 12,200 & 84.4 & 12.8 & .45 & 1,320 & 49.0 \\
\hline 24 & 42.1 & 77.9 & 37.9 & 4,230 & 1,670 & 2.4 & 47.7 & 17.0 & 760 & 12,400 & 84.9 & 12.7 & .44 & 1,360 & 49.9 \\
\hline 25 & 42.2 & 77.0 & 37.1 & 4,110 & 1,600 & 1.9 & 43.8 & 17.0 & 740 & 12,100 & 83.4 & 12.7 & .43 & 1,330 & 48.2 \\
\hline 26 & 42.5 & 66.6 & 37.5 & 4,360 & 1,270 & 1.8 & 39.5 & 17.0 & 680 & 11,700 & 76.2 & 11.3 & .53 & 1,530 & 45.1 \\
\hline 27 & 41.6 & 66.5 & 38.0 & 4,390 & 1,310 & 1.8 & 40.4 & 17.0 & 670 & 11,800 & 77.7 & 11.6 & .51 & 1,470 & 45.0 \\
\hline 28 & 41.6 & 67.3 & 37.7 & 4,320 & 1,280 & 1.9 & 40.7 & 17.0 & 680 & 11,700 & 78.7 & 12.0 & .48 & 1,440 & 46.1 \\
\hline 29 & 41.2 & 68.2 & 38.3 & 4,360 & 1,340 & 2.0 & 41.3 & 18.0 & 690 & 11,900 & 80.0 & 12.0 & .50 & 1,400 & 46.2 \\
\hline 30 & 43.2 & 69.4 & 38.2 & 4,340 & 1,380 & 1.8 & 41.3 & 17.0 & 690 & 12,000 & 80.6 & 12.1 & .50 & 1,430 & 46.9 \\
\hline 31 & 42.2 & 84.8 & 37.8 & 4,260 & 1,370 & 1.9 & 41.1 & 18.0 & 690 & 11,900 & 80.1 & 12.0 & .48 & 1,380 & 46.1 \\
\hline 32 & 44.8 & 71.8 & 37.5 & 4,400 & 1,640 & 2.2 & 43.0 & 17.0 & 730 & 12,200 & 79.6 & 12.1 & .49 & 1,470 & 46.6 \\
\hline 33 & 44.8 & 77.2 & 38.6 & 4,410 & 1,780 & 2.0 & 43.3 & 17.0 & 710 & 12,300 & 81.9 & 12.6 & .49 & 1,410 & 47.1 \\
\hline 34 & 44.4 & 75.2 & 40.0 & 4,460 & 1,800 & 2.1 & 44.1 & 18.0 & 730 & 12,700 & 83.1 & 12.8 & .48 & 1,450 & 48.8 \\
\hline 35 & 45.3 & 77.9 & 40.0 & 4,420 & 1,870 & 2.1 & 43.6 & 18.0 & 740 & 12,900 & 85.1 & 12.9 & .48 & 1,450 & 48.9 \\
\hline 36 & 45.9 & 81.2 & 40.6 & 4,470 & 1,560 & 2.0 & 44.4 & 18.0 & 760 & 13,300 & 85.8 & 13.2 & .48 & 1,520 & 51.1 \\
\hline 37 & 45.6 & 79.4 & 40.2 & 4,470 & 1,800 & 2.1 & 45.9 & 18.0 & 750 & 12,800 & 86.0 & 13.2 & .50 & 1,360 & 48.9 \\
\hline 38 & 44.7 & 74.7 & 40.1 & 4,440 & 1,900 & 2.1 & 42.1 & 18.0 & 710 & 12,200 & 83.4 & 12.8 & .48 & 1,360 & 48.5 \\
\hline
\end{tabular}


Table 1. Quantitative elemental analyses of less than 63-micrometer fraction and lead-210 date of Clearwater Lake bed-sediment samples-Continued

[All concentrations in milligrams per kilogram; RSD, relative standard deviation; ${ }^{\circ}$, degrees; ', minutes; ”, seconds; <, less than; shaded cells exceed threshold effects concentration; --, no data]

\begin{tabular}{|c|c|c|c|c|c|c|c|c|c|c|c|c|c|c|c|}
\hline $\begin{array}{c}\text { Depth below } \\
\text { lakebed } \\
\text { surface, } \\
\text { (centimeters) }\end{array}$ & Lanthanum & Lead & Lithium & Magnesium & Manganese & Molybdenum & Nickel & Niobium & Phosphorous & Potassium & Rubidium & Scandium & Silver & Sodium & Strontium \\
\hline RSD (percent) & 6.64 & 3.83 & 2.48 & 2.70 & 2.52 & 10.69 & 3.35 & 23.19 & 2.84 & 2.86 & 2.65 & 3.57 & 41.48 & 2.44 & 3.74 \\
\hline \multicolumn{16}{|c|}{ Marina (Latitude $37^{\circ} 08^{\prime} 27^{\prime \prime}$ Longitude $090^{\circ} 46^{\prime} 14^{\prime \prime}$ )-Continued } \\
\hline 39 & 45.2 & 74.6 & 39.2 & 4,380 & 2,060 & 2.1 & 42.3 & 17.0 & 720 & 12,200 & 84.2 & 12.5 & 0.44 & 1,320 & 47.3 \\
\hline 40 & 44.6 & 69.4 & 38.2 & 4,210 & 1,840 & 2.0 & 40.4 & 17.0 & 700 & 12,200 & 82.2 & 12.3 & .46 & 1,400 & 48.2 \\
\hline 41 & 44.7 & 74.7 & 38.6 & 4,100 & 1,800 & 2.0 & 40.4 & 18.0 & 720 & 12,400 & 84.4 & 12.6 & .47 & 1,380 & 48.3 \\
\hline 42 & 45.3 & 70.6 & 38.6 & 4,140 & 1,290 & 2.1 & 40.0 & 18.0 & 720 & 12,400 & 84.3 & 12.5 & .49 & 1,400 & 49.4 \\
\hline 43 & 45.1 & 70.4 & 39.1 & 4,080 & 1,480 & 2.1 & 40.0 & 18.0 & 730 & 12,400 & 85.2 & 12.4 & .48 & 1,400 & 49.3 \\
\hline 44 & 44.5 & 71.0 & 38.3 & 4,090 & 1,770 & 1.9 & 40.6 & 18.0 & 740 & 12,500 & 85.8 & 12.3 & .49 & 1,390 & 49.7 \\
\hline 45 & 44.8 & 84.5 & 36.3 & 4,110 & 1,470 & 1.7 & 39.5 & 16.0 & 680 & 12,400 & 82.0 & 12.1 & .42 & 1,420 & 49.0 \\
\hline 46 & 43.9 & 74.4 & 35.1 & 4,280 & 1,240 & 1.9 & 37.0 & 16.0 & 650 & 12,100 & 74.3 & 11.0 & .46 & 1,590 & 44.2 \\
\hline 47 & 44.4 & 71.0 & 35.7 & 4,280 & 1,280 & 2.1 & 39.4 & 16.0 & 660 & 12,100 & 75.7 & 11.3 & .48 & 1,550 & 44.5 \\
\hline 48 & 42.9 & 72.5 & 34.6 & 4,170 & 1,380 & 1.7 & 36.7 & 14.0 & 640 & 11,900 & 74.3 & 11.1 & .42 & 1,530 & 44.5 \\
\hline 49 & 45.1 & 74.7 & 35.4 & 4,260 & 1,750 & 1.8 & 38.6 & 17.0 & 680 & 12,100 & 76.8 & 11.5 & .51 & 1,510 & 44.8 \\
\hline 50 & 45.4 & 85.3 & 36.6 & 4,280 & 1,720 & 1.8 & 39.4 & 17.0 & 700 & 12,300 & 78.5 & 11.6 & .49 & 1,500 & 45.3 \\
\hline 51 & 42.4 & 73.4 & 36.4 & 4,170 & 1,590 & 1.9 & 39.8 & 20.0 & 710 & 12,300 & 78.0 & 12.1 & 1.00 & 1,420 & 45.1 \\
\hline 52 & 42.5 & 76.9 & 36.5 & 4,170 & 1,540 & 2.0 & 42.5 & 18.0 & 720 & 12,300 & 78.7 & 12.2 & .87 & 1,430 & 45.6 \\
\hline 53 & 41.1 & 75.2 & 37.2 & 4,200 & 1,560 & 1.9 & 39.7 & 18.0 & 710 & 12,200 & 78.4 & 12.4 & .95 & 1,390 & 45.8 \\
\hline 54 & 43.4 & 78.1 & 38.2 & 4,270 & 1,630 & 2.0 & 40.2 & 20.0 & 740 & 12,600 & 82.0 & 12.6 & 1.00 & 1,430 & 46.8 \\
\hline 55 & 41.6 & 73.8 & 37.5 & 4,090 & 1,610 & 1.9 & 38.9 & 18.0 & 700 & 12,200 & 78.4 & 12.0 & .88 & 1,430 & 46.1 \\
\hline 56 & 39.8 & 67.6 & 34.9 & 3,900 & 1,690 & 1.8 & 36.4 & 18.0 & 660 & 11,600 & 74.8 & 11.5 & .90 & 1,380 & 43.7 \\
\hline 57 & 43.4 & 76.9 & 36.7 & 4,160 & 1,620 & 1.9 & 38.5 & 19.0 & 680 & 12,400 & 78.6 & 12.1 & .96 & 1,520 & 46.9 \\
\hline 58 & 43.6 & 71.8 & 36.6 & 4,200 & 1,640 & 1.8 & 39.6 & 19.0 & 660 & 12,700 & 80.3 & 12.2 & .99 & 1,560 & 48.0 \\
\hline 59 & 44.2 & 74.1 & 37.1 & 4,220 & 1,590 & 2.0 & 40.2 & 20.0 & 670 & 12,400 & 80.0 & 12.7 & .96 & 1,430 & 46.8 \\
\hline 60 & 43.9 & 79.8 & 40.3 & 4,560 & 1,490 & 2.5 & 47.1 & 20.0 & 850 & 12,500 & 82.0 & 13.4 & 1.00 & 1,320 & 46.8 \\
\hline 61 & 44.0 & 82.1 & 41.6 & 4,600 & 1,780 & 2.4 & 46.4 & 19.0 & 1,200 & 12,500 & 85.0 & 13.8 & .99 & 1,210 & 46.6 \\
\hline Maximum & 49.0 & 92.4 & 41.8 & 5,690 & 2,060 & 2.9 & 53.8 & 21.0 & 3,600 & 13,300 & 86.5 & 13.8 & 1.00 & 3,590 & 69.2 \\
\hline Minimum & 39.8 & 66.5 & 34.6 & 3,900 & 1,240 & 1.7 & 36.4 & 11.0 & 640 & 11,600 & 69.1 & 10.7 & .42 & 1,210 & 43.7 \\
\hline Mean & 44.0 & 76.8 & 38.0 & 4,406 & 1,572 & 2.1 & 43.2 & 17.6 & 940 & 12,280 & 81.3 & 12.4 & .58 & 1,485 & 48.3 \\
\hline Median & 44.4 & 76.9 & 38.0 & 4,360 & 1,560 & 2.1 & 43.3 & 18.0 & 740 & 12,300 & 81.8 & 12.4 & .49 & 1,420 & 48.0 \\
\hline
\end{tabular}


Table 1. Quantitative elemental analyses of less than 63-micrometer fraction and lead-210 date of Clearwater Lake bed-sediment samples-Continued

[All concentrations in milligrams per kilogram; RSD, relative standard deviation; ${ }^{\circ}$, degrees; ', minutes; ", seconds; <, less than; shaded cells exceed threshold effects concentration; --, no data]

\begin{tabular}{|c|c|c|c|c|c|c|c|c|c|c|c|c|c|c|c|}
\hline $\begin{array}{l}\text { Depth below } \\
\text { lakebed } \\
\text { surface, } \\
\text { (centimeters) }\end{array}$ & Lanthanum & Lead & Lithium & Magnesium & Manganese & Molybdenum & Nickel & Niobium & Phosphorous & Potassium & Rubidium & Scandium & Silver & Sodium & Strontium \\
\hline RSD (percent) & 6.64 & 3.83 & 2.48 & 2.70 & 2.52 & 10.69 & 3.35 & 23.19 & 2.84 & 2.86 & 2.65 & 3.57 & 41.48 & 2.44 & 3.74 \\
\hline \multicolumn{16}{|c|}{ Clearwater Dam (Latitude $37^{\circ} 08^{\prime} 19^{\prime \prime}$ Longitude $090^{\circ} 46^{\prime} 47^{\prime \prime}$ ) } \\
\hline 1 & 47.6 & 89.8 & 45.4 & 5,220 & 1,260 & 3.0 & 51.0 & 23.0 & 920 & 13,200 & 89.0 & 14.4 & 0.37 & 1,220 & 45.5 \\
\hline 2 & 48.3 & 85.6 & 45.0 & 5,290 & 1,490 & 4.5 & 56.3 & 22.0 & 900 & 13,400 & 89.3 & 14.5 & .32 & 1,270 & 46.3 \\
\hline 3 & 47.8 & 82.0 & 44.6 & 5,100 & 1,440 & 2.1 & 47.1 & 23.0 & 920 & 13,400 & 89.1 & 14.1 & .36 & 1,310 & 46.9 \\
\hline 4 & 48.2 & 80.3 & 44.4 & 4,990 & 1,600 & 2.5 & 47.1 & 21.0 & 900 & 13,200 & 88.7 & 14.2 & .35 & 1,330 & 46.1 \\
\hline 5 & 48.4 & 79.0 & 44.2 & 5,070 & 1,580 & 3.4 & 50.8 & 21.0 & 900 & 13,400 & 89.3 & 14.2 & .34 & 1,340 & 46.2 \\
\hline 6 & 49.1 & 78.3 & 45.2 & 5,030 & 1,520 & 4.6 & 55.0 & 23.0 & 900 & 13,200 & 88.3 & 14.0 & .40 & 1,330 & 46.7 \\
\hline 7 & 49.7 & 78.6 & 44.2 & 5,120 & 1,580 & 2.0 & 45.9 & 23.0 & 890 & 13,300 & 88.4 & 14.3 & .38 & 1,360 & 46.4 \\
\hline 8 & 49.0 & 79.1 & 44.4 & 5,070 & 1,540 & 1.9 & 45.1 & 21.0 & 870 & 13,300 & 88.2 & 14.1 & .32 & 1,350 & 46.1 \\
\hline 9 & 47.8 & 78.3 & 44.1 & 4,980 & 1,500 & 2.6 & 46.9 & 21.0 & 850 & 12,900 & 86.5 & 13.9 & .35 & 1,320 & 45.6 \\
\hline 10 & 48.2 & 77.1 & 43.8 & 4,980 & 1,500 & 3.0 & 47.9 & 20.0 & 850 & 12,900 & 87.9 & 13.9 & .31 & 1,340 & 46.2 \\
\hline 11 & 46.4 & 72.6 & 43.0 & 4,790 & 1,460 & 2.5 & 45.2 & 24.0 & 830 & 13,200 & 87.0 & 13.8 & .43 & 1,480 & 47.8 \\
\hline 12 & 47.3 & 74.9 & 44.2 & 4,970 & 1,460 & 2.4 & 44.7 & 23.0 & 850 & 13,400 & 89.1 & 14.0 & .40 & 1,470 & 46.4 \\
\hline 13 & 51.1 & 76.2 & 45.0 & 5,070 & 1,470 & 2.3 & 44.8 & 24.0 & 830 & 13,200 & 89.3 & 13.9 & .43 & 1,370 & 46.3 \\
\hline 14 & 49.1 & 74.9 & 45.4 & 5,160 & 1,500 & 2.5 & 46.9 & 24.0 & 860 & 13,400 & 90.8 & 14.5 & .41 & 1,380 & 47.4 \\
\hline 15 & 50.1 & 78.0 & 45.6 & 5,280 & 1,640 & 2.3 & 46.3 & 26.0 & 860 & 13,600 & 91.4 & 14.5 & .42 & 1,390 & 47.9 \\
\hline 16 & 49.4 & 78.3 & 45.8 & 5,200 & 1,560 & 2.4 & 45.4 & 23.0 & 850 & 13,400 & 90.5 & 14.6 & .38 & 1,380 & 47.2 \\
\hline 17 & 49.2 & 79.7 & 45.4 & 5,200 & 1,520 & 2.3 & 44.9 & 19.0 & 810 & 13,300 & 89.1 & 14.1 & .26 & 1,390 & 46.6 \\
\hline 18 & 50.5 & 77.0 & 45.5 & 5,140 & 1,520 & 2.5 & 45.6 & 22.0 & 830 & 13,400 & 89.7 & 14.5 & .37 & 1,400 & 47.4 \\
\hline 19 & 51.3 & 78.9 & 45.4 & 5,120 & 1,530 & 2.5 & 45.7 & 24.0 & 860 & 13,500 & 91.4 & 14.3 & .41 & 1,410 & 48.1 \\
\hline 20 & 49.1 & 77.8 & 45.1 & 5,090 & 1,470 & 2.3 & 44.5 & 24.0 & 820 & 13,200 & 89.5 & 14.2 & .44 & 1,400 & 47.1 \\
\hline 21 & 49.4 & 77.9 & 45.0 & 5,080 & 1,420 & 2.2 & 43.4 & 25.0 & 810 & 13,200 & 89.4 & 14.5 & .49 & 1,450 & 47.4 \\
\hline 22 & 48.4 & 82.2 & 45.2 & 5,140 & 1,430 & 2.7 & 45.4 & 23.0 & 820 & 13,300 & 89.8 & 14.0 & .40 & 1,470 & 47.2 \\
\hline 23 & 48.6 & 82.9 & 44.5 & 5,160 & 1,420 & 2.2 & 42.7 & 23.0 & 820 & 13,500 & 89.7 & 14.2 & .37 & 1,500 & 47.8 \\
\hline 24 & 49.6 & 82.4 & 44.9 & 5,170 & 1,450 & 2.4 & 44.1 & 23.0 & 830 & 13,700 & 90.7 & 14.2 & .43 & 1,480 & 47.1 \\
\hline 25 & 50.4 & 77.9 & 45.3 & 5,200 & 1,590 & 2.5 & 44.8 & 24.0 & 880 & 14,000 & 93.5 & 14.5 & .45 & 1,460 & 48.9 \\
\hline 26 & 51.0 & 70.6 & 44.6 & 5,210 & 1,710 & 2.4 & 44.6 & 23.0 & 840 & 14,000 & 91.6 & 14.3 & .45 & 1,570 & 48.4 \\
\hline 27 & 51.8 & 48.6 & 40.1 & 4,770 & 1,590 & 2.0 & 39.3 & 20.0 & 780 & 13,500 & 86.4 & 13.0 & .69 & 1,690 & 47.5 \\
\hline 28 & 52.1 & 43.9 & 40.0 & 4,620 & 1,710 & 2.2 & 39.2 & 20.0 & 780 & 13,800 & 87.1 & 13.1 & .66 & 1,730 & 47.7 \\
\hline 29 & 51.4 & 43.8 & 39.7 & 4,620 & 1,860 & 2.0 & 38.5 & 22.0 & 770 & 13,600 & 86.6 & 12.9 & .67 & 1,740 & 47.5 \\
\hline 30 & 51.4 & 43.9 & 39.0 & 4,600 & 2,180 & 2.2 & 40.3 & 20.0 & 760 & 13,800 & 86.1 & 13.1 & .60 & 1,760 & 47.2 \\
\hline 31 & 47.3 & 42.0 & 39.4 & 4,520 & 1,950 & 2.1 & 40.7 & 22.0 & 760 & 13,300 & 84.0 & 12.9 & .68 & 1,710 & 46.2 \\
\hline 32 & 46.7 & 41.8 & 38.8 & 4,370 & 2,240 & 3.3 & 43.0 & 22.0 & 740 & 13,500 & 82.5 & 12.5 & .62 & 1,760 & 46.1 \\
\hline 33 & 48.4 & 42.8 & 38.7 & 4,370 & 2,250 & 2.7 & 40.7 & 21.0 & 740 & 13,600 & 82.9 & 12.3 & .61 & 1,820 & 47.0 \\
\hline 34 & 47.8 & 42.6 & 38.6 & 4,390 & 2,150 & 3.4 & 43.2 & 22.0 & 740 & 13,400 & 83.4 & 12.5 & .62 & 1,790 & 49.9 \\
\hline 35 & 47.9 & 41.2 & 37.5 & 4,190 & 2,300 & 2.0 & 36.9 & 22.0 & 710 & 13,300 & 81.6 & 12.4 & .61 & 1,820 & 46.9 \\
\hline 36 & 47.3 & 39.4 & 36.8 & 4,070 & 2,370 & 2.0 & 36.2 & 20.0 & 700 & 13,100 & 80.2 & 11.8 & .60 & 1,880 & 46.5 \\
\hline 37 & 47.1 & 39.2 & 36.6 & 4,070 & 2,670 & 2.4 & 37.6 & 20.0 & 680 & 13,200 & 80.4 & 11.6 & .58 & 1,900 & 47.0 \\
\hline 38 & 47.8 & 38.6 & 36.0 & 3,990 & 2,730 & 2.3 & 36.7 & 21.0 & 690 & 13,400 & 80.7 & 11.6 & .60 & 1,970 & 47.8 \\
\hline
\end{tabular}


Table 1. Quantitative elemental analyses of less than 63-micrometer fraction and lead-210 date of Clearwater Lake bed-sediment samples-Continued

[All concentrations in milligrams per kilogram; RSD, relative standard deviation; ${ }^{\circ}$, degrees; ', minutes; ”, seconds; <, less than; shaded cells exceed threshold effects concentration; --, no data]

\begin{tabular}{|c|c|c|c|c|c|c|c|c|c|c|c|c|c|c|c|}
\hline $\begin{array}{l}\text { Depth below } \\
\text { lakebed } \\
\text { surface, } \\
\text { (centimeters) }\end{array}$ & Lanthanum & Lead & Lithium & Magnesium & Manganese & Molybdenum & Nickel & Niobium & Phosphorous & Potassium & Rubidium & Scandium & Silver & Sodium & Strontium \\
\hline RSD (percent) & 6.64 & 3.83 & 2.48 & 2.70 & 2.52 & 10.69 & 3.35 & 23.19 & 2.84 & 2.86 & 2.65 & 3.57 & 41.48 & 2.44 & 3.74 \\
\hline \multicolumn{16}{|c|}{ Clearwater Dam (Latitude $37^{\circ} 08^{\prime} 19^{\prime \prime}$ Longitude $090^{\circ} 46^{\prime} 47^{\prime \prime}$ )—Continued } \\
\hline 39 & 46.0 & 37.0 & 34.7 & 3,770 & 3,480 & 1.9 & 34.8 & 21.0 & 660 & 13,100 & 76.1 & 11.1 & 0.60 & 2,070 & 47.2 \\
\hline 40 & 45.2 & 35.9 & 33.8 & 3,660 & 3,790 & 1.7 & 32.6 & 21.0 & 670 & 13,000 & 74.5 & 10.7 & .59 & 2,110 & 47.6 \\
\hline 41 & 44.8 & 35.2 & 33.6 & 3,640 & 4,140 & 2.4 & 35.0 & 23.0 & 670 & 12,700 & 73.6 & 10.9 & .67 & 2,040 & 46.5 \\
\hline 42 & 44.1 & 35.4 & 33.8 & 3,600 & 4,140 & 2.0 & 33.5 & 20.0 & 670 & 12,800 & 74.0 & 10.8 & .57 & 2,040 & 46.4 \\
\hline 43 & 43.7 & 33.9 & 31.8 & 3,420 & 3,900 & 1.6 & 30.7 & 19.0 & 640 & 13,000 & 72.3 & 10.3 & .54 & 2,160 & 47.2 \\
\hline 44 & 43.1 & 33.5 & 32.0 & 3,450 & 4,020 & 2.0 & 32.8 & 19.0 & 630 & 12,900 & 71.6 & 10.4 & .53 & 2,160 & 46.5 \\
\hline 45 & 44.2 & 34.7 & 32.8 & 3,580 & 3,970 & 2.0 & 33.7 & 20.0 & 660 & 13,200 & 74.4 & 10.6 & .58 & 2,100 & 46.8 \\
\hline 46 & 44.5 & 34.5 & 33.7 & 3,580 & 4,590 & 2.1 & 34.0 & 20.0 & 680 & 13,100 & 75.6 & 10.9 & .61 & 2,080 & 46.9 \\
\hline 47 & 44.8 & 35.1 & 33.6 & 3,610 & 5,040 & 1.8 & 33.4 & 20.0 & 680 & 13,000 & 75.1 & 10.9 & .57 & 2,060 & 47.0 \\
\hline 48 & 44.7 & 35.3 & 33.3 & 3,590 & 5,130 & 1.8 & 33.3 & 19.0 & 680 & 13,100 & 74.7 & 10.8 & .53 & 2,040 & 46.6 \\
\hline 49 & 43.7 & 32.9 & 31.5 & 3,310 & 4,400 & 1.8 & 31.4 & 21.0 & 640 & 12,900 & 71.2 & 10.0 & .59 & 2,220 & 46.5 \\
\hline 50 & 44.2 & 32.8 & 31.7 & 3,300 & 4,540 & 1.8 & 31.4 & 24.0 & 660 & 12,900 & 72.7 & 10.4 & .57 & 2,170 & 46.4 \\
\hline 51 & 42.7 & 33.6 & 33.8 & 3,570 & 5,280 & 1.8 & 33.3 & 20.0 & 690 & 13,300 & 78.5 & 11.3 & 1.00 & 2,060 & 51.9 \\
\hline 52 & 42.4 & 31.3 & 34.6 & 3,580 & 3,970 & 1.9 & 32.3 & 18.0 & 690 & 13,000 & 80.3 & 11.4 & 1.10 & 2,010 & 50.4 \\
\hline 53 & 42.2 & 35.6 & 33.1 & 3,560 & 2,470 & 1.6 & 32.9 & 18.0 & 690 & 14,100 & 79.3 & 11.3 & 1.10 & 2,140 & 52.7 \\
\hline 54 & 30.3 & 22.3 & 20.3 & 2,150 & 1,420 & 1.2 & 19.3 & 12.0 & 420 & 10,700 & 51.4 & 6.5 & .70 & 2,220 & 43.7 \\
\hline Maximum & 52.1 & 89.8 & 45.8 & 5,290 & 5,280 & 4.6 & 56.3 & 26.0 & 920 & 14,100 & 93.5 & 14.6 & 1.10 & 2,220 & 52.7 \\
\hline Minimum & 30.3 & 22.3 & 20.3 & 2,150 & 1,260 & 1.2 & 19.3 & 12.0 & 420 & 10,700 & 51.4 & 6.5 & .26 & 1,220 & 43.7 \\
\hline Mean & 47.3 & 57.4 & 39.7 & 4,459 & 2,397 & 2.3 & 40.7 & 21.5 & 770 & 13,256 & 83.4 & 12.7 & .53 & 1,693 & 47.2 \\
\hline Median & 47.9 & 46.3 & 40.1 & 4,695 & 1,675 & 2.3 & 42.9 & 21.5 & 780 & 13,300 & 86.6 & 13.1 & .53 & 1,700 & 47.0 \\
\hline
\end{tabular}


Table 1. Quantitative elemental analyses of less than 63-micrometer fraction and lead-210 date of Clearwater Lake bed-sediment samples.-Continued

[All concentrations in milligrams per kilogram; RSD, relative standard deviation; ${ }^{\circ}$, degrees; ', minutes; ", seconds; <, less than; shaded cells exceed threshold effects concentration; --, no data]

\begin{tabular}{|c|c|c|c|c|c|c|c|c|}
\hline $\begin{array}{l}\text { Depth below } \\
\text { lakebed surface, } \\
\text { (centimeters) }\end{array}$ & Thallium & Thorium & Titanium & Uranium & Vanadium & Yttrium & Zinc & $\begin{array}{c}\text { Lead-210 } \\
\text { date }\end{array}$ \\
\hline RSD (percent) & 5.74 & 6.48 & 8.58 & 6.62 & 2.62 & 3.28 & 2.58 & -- \\
\hline \multicolumn{9}{|c|}{ Webb Creek (Latitude $37^{\circ} 08^{\prime} 56^{\prime \prime}$ Longitude $090^{\circ} 48^{\prime} 18^{\prime \prime}$ ) } \\
\hline 1 & 0.5 & 9.6 & 3,000 & 2.7 & 55.6 & 29.1 & 55.0 & -- \\
\hline 2 & .5 & 9.8 & 3,200 & 2.8 & 55.2 & 30.6 & 53.0 & \\
\hline 3 & .5 & 9.8 & 3,100 & 2.6 & 50.3 & 27.8 & 48.9 & -- \\
\hline 4 & .5 & 9.5 & 3,000 & 2.5 & 51.0 & 28.5 & 49.3 & \\
\hline 5 & .5 & 9.8 & 3,300 & 2.5 & 52.8 & 30.1 & 51.2 & -- \\
\hline 6 & .5 & 9.6 & 3,200 & 2.6 & 51.9 & 30.1 & 51.8 & \\
\hline 7 & .5 & 9.7 & 3,300 & 2.7 & 52.3 & 30.4 & 51.1 & -- \\
\hline 8 & .5 & 9.7 & 3,200 & 2.6 & 51.6 & 29.4 & 51.9 & \\
\hline 9 & .5 & 9.9 & 3,300 & 2.8 & 54.0 & 32.9 & 51.7 & -- \\
\hline 10 & .5 & 10.4 & 3,400 & 3.1 & 56.7 & 32.7 & 52.9 & \\
\hline 11 & .5 & 10.7 & 3,600 & 3.2 & 59.2 & 33.2 & 53.7 & -- \\
\hline 12 & .5 & 9.9 & 3,200 & 2.9 & 56.2 & 31.8 & 50.6 & \\
\hline 13 & .5 & 10.0 & 3,100 & 2.9 & 54.8 & 31.5 & 49.8 & -- \\
\hline 14 & .5 & 10.5 & 3,400 & 3.1 & 56.4 & 32.3 & 51.9 & \\
\hline 15 & .6 & 10.6 & 3,500 & 3.6 & 61.0 & 33.7 & 55.1 & -- \\
\hline 16 & .6 & 10.8 & 3,400 & 3.4 & 57.8 & 34.1 & 54.1 & \\
\hline 17 & .6 & 10.8 & 3,500 & 3.5 & 58.0 & 35.1 & 53.6 & -- \\
\hline 18 & .6 & 10.5 & 3,400 & 3.4 & 57.9 & 33.2 & 52.9 & \\
\hline 19 & .5 & 10.1 & 3,400 & 3.2 & 55.7 & 31.3 & 52.6 & -- \\
\hline 20 & .5 & 10.0 & 3,500 & 3.1 & 56.5 & 31.6 & 51.8 & \\
\hline 21 & .5 & 9.6 & 3,200 & 2.9 & 52.5 & 30.7 & 47.8 & -- \\
\hline 22 & .5 & 9.6 & 3,000 & 2.8 & 50.2 & 29.8 & 45.7 & \\
\hline 23 & .5 & 9.6 & 2,900 & 2.8 & 51.3 & 30.3 & 45.7 & -- \\
\hline 24 & .5 & 9.1 & 3,000 & 2.7 & 47.9 & 29.7 & 48.0 & \\
\hline 25 & .5 & 9.0 & 3,000 & 2.7 & 48.9 & 30.0 & 44.8 & -- \\
\hline 26 & .3 & 5.6 & 1,900 & 1.8 & 28.3 & 19.0 & 28.3 & \\
\hline 27 & .5 & 9.4 & 3,100 & 2.8 & 48.8 & 30.1 & 45.1 & -- \\
\hline 28 & .5 & 9.6 & 3,000 & 2.9 & 48.8 & 30.9 & 45.0 & \\
\hline 29 & .5 & 8.9 & 3,100 & 2.8 & 50.5 & 33.4 & 44.8 & -- \\
\hline 30 & .5 & 9.3 & 3,300 & 3.0 & 50.3 & 33.5 & 45.6 & \\
\hline 31 & .5 & 8.0 & 2,900 & 2.8 & 49.3 & 30.1 & 42.8 & -- \\
\hline 32 & .5 & 8.9 & 3,000 & 2.8 & 51.2 & 35.2 & 46.1 & \\
\hline 33 & .5 & 9.6 & 3,200 & 2.7 & 53.7 & 31.7 & 49.5 & -- \\
\hline 34 & .6 & 10.6 & 3,600 & 2.9 & 60.2 & 34.2 & 55.0 & \\
\hline 35 & .6 & 10.5 & 3,600 & 2.9 & 61.4 & 33.3 & 57.0 & -- \\
\hline 36 & .6 & 10.4 & 3,500 & 3.0 & 60.6 & 32.9 & 55.1 & \\
\hline 37 & .6 & 10.4 & 3,700 & 3.0 & 59.2 & 34.0 & 53.6 & -- \\
\hline 38 & .6 & 10.2 & 3,600 & 3.0 & 57.2 & 35.1 & 50.2 & \\
\hline
\end{tabular}


Table 1. Quantitative elemental analyses of less than 63-micrometer fraction and lead-210 date of Clearwater Lake bed-sediment samples.—Continued

[All concentrations in milligrams per kilogram; RSD, relative standard deviation; ${ }^{\circ}$, degrees; ', minutes; ”, seconds; <, less than; shaded cells exceed threshold effects concentration; --, no data]

\begin{tabular}{|c|c|c|c|c|c|c|c|c|}
\hline $\begin{array}{l}\text { Depth below } \\
\text { lakebed surface, } \\
\text { (centimeters) }\end{array}$ & Thallium & Thorium & Titanium & Uranium & Vanadium & Yttrium & Zinc & $\begin{array}{c}\text { Lead-210 } \\
\text { date }\end{array}$ \\
\hline RSD (percent) & 5.74 & 6.48 & 8.58 & 6.62 & 2.62 & 3.28 & 2.58 & -- \\
\hline \multicolumn{9}{|c|}{ Webb Creek (Latitude $37^{\circ} 08^{\prime} 56^{\prime \prime}$ Longitude $090^{\circ} 48^{\prime} 18^{\prime \prime}$ )—Continued } \\
\hline 39 & 0.6 & 10.2 & 3,700 & 3.1 & 61.0 & 33.9 & 55.2 & -- \\
\hline 40 & .6 & 10.4 & 3,900 & 3.3 & 64.4 & 35.3 & 60.0 & \\
\hline 41 & .6 & 9.4 & 3,500 & 3.0 & 60.1 & 32.5 & 53.3 & -- \\
\hline 42 & .5 & 9.2 & 3,400 & 2.8 & 56.2 & 31.4 & 52.2 & \\
\hline 43 & .5 & 8.9 & 3,200 & 2.6 & 55.2 & 30.0 & 52.1 & -- \\
\hline 44 & .5 & 9.4 & 3,500 & 2.7 & 54.5 & 31.2 & 52.4 & \\
\hline 45 & .5 & 9.1 & 3,600 & 2.8 & 53.0 & 30.7 & 49.8 & -- \\
\hline 46 & .5 & 9.0 & 3,500 & 2.8 & 52.9 & 30.1 & 50.7 & \\
\hline 47 & .5 & 9.1 & 3,400 & 2.5 & 50.2 & 28.3 & 48.9 & -- \\
\hline 48 & .5 & 8.9 & 3,300 & 2.4 & 50.7 & 28.7 & 49.8 & \\
\hline 49 & .5 & 9.2 & 3,400 & 2.5 & 50.6 & 29.1 & 49.7 & -- \\
\hline 50 & .5 & 9.2 & 3,700 & 2.6 & 53.0 & 30.0 & 51.2 & \\
\hline 51 & .5 & 9.0 & 4,100 & 2.6 & 50.0 & 29.3 & 48.2 & -- \\
\hline 52 & .5 & 9.1 & 4,100 & 2.4 & 47.7 & 27.7 & 43.6 & \\
\hline 53 & .5 & 9.1 & 4,100 & 2.5 & 47.6 & 29.1 & 45.5 & -- \\
\hline 54 & .5 & 9.2 & 4,100 & 2.5 & 48.1 & 28.9 & 47.2 & \\
\hline 55 & .5 & 9.2 & 4,100 & 2.4 & 49.1 & 28.9 & 46.4 & -- \\
\hline 56 & .5 & 9.5 & 4,100 & 2.5 & 50.7 & 30.0 & 48.1 & \\
\hline 57 & .5 & 9.3 & 4,100 & 2.7 & 48.3 & 29.3 & 45.7 & -- \\
\hline 58 & .5 & 9.6 & 4,100 & 2.7 & 49.8 & 28.8 & 45.7 & \\
\hline 59 & .5 & 9.5 & 4,100 & 2.7 & 48.8 & 29.2 & 45.4 & -- \\
\hline 60 & .5 & 9.3 & 4,100 & 2.4 & 52.6 & 29.4 & 49.1 & \\
\hline 61 & .5 & 9.5 & 4,100 & 2.6 & 54.8 & 30.6 & 51.4 & -- \\
\hline 62 & .6 & 9.8 & 4,100 & 3.0 & 60.3 & 31.9 & 56.3 & \\
\hline 63 & .5 & 9.8 & 4,100 & 2.9 & 55.9 & 31.6 & 51.2 & -- \\
\hline 64 & .5 & 9.7 & 4,100 & 2.8 & 53.8 & 31.2 & 50.3 & \\
\hline 65 & .5 & 9.3 & 4,100 & 2.6 & 49.6 & 30.0 & 44.7 & -- \\
\hline 66 & .5 & 9.7 & 4,100 & 2.7 & 49.3 & 29.8 & 47.8 & \\
\hline 67 & .5 & 10.0 & 4,100 & 3.0 & 50.4 & 31.1 & 44.7 & -- \\
\hline 68 & .5 & 10.1 & 4,100 & 3.1 & 50.8 & 31.7 & 46.2 & \\
\hline 69 & .5 & 9.9 & 4,100 & 2.9 & 49.8 & 36.0 & 43.7 & -- \\
\hline 70 & .5 & 9.7 & 4,100 & 2.5 & 49.4 & 30.1 & 44.5 & \\
\hline 71 & .5 & 9.1 & 4,100 & 2.7 & 47.1 & 28.4 & 42.0 & -- \\
\hline 72 & .5 & 9.7 & 4,100 & 2.8 & 51.6 & 30.0 & 45.3 & \\
\hline Maximum & 0.6 & 10.8 & 4,100 & 3.6 & 64.4 & 36.0 & 60.0 & \\
\hline Minimum & .3 & 5.6 & 1,900 & 1.8 & 28.3 & 19.0 & 28.3 & \\
\hline Mean & .5 & 9.6 & 3,540 & 2.8 & 53.0 & 30.9 & 49.3 & \\
\hline Median & .5 & 9.6 & 3,500 & 2.8 & 52.4 & 30.6 & 49.8 & \\
\hline
\end{tabular}


Table 1. Quantitative elemental analyses of less than 63-micrometer fraction and lead-210 date of Clearwater Lake bed-sediment samples.-Continued

[All concentrations in milligrams per kilogram; RSD, relative standard deviation; ${ }^{\circ}$, degrees; ', minutes; ", seconds; <, less than; shaded cells exceed threshold effects concentration; --, no data]

\begin{tabular}{|c|c|c|c|c|c|c|c|c|}
\hline $\begin{array}{l}\text { Depth below } \\
\text { lakebed surface, } \\
\text { (centimeters) }\end{array}$ & Thallium & Thorium & Titanium & Uranium & Vanadium & Yttrium & Zinc & $\begin{array}{c}\text { Lead-210 } \\
\text { date }\end{array}$ \\
\hline RSD (percent) & 5.74 & 6.48 & 8.58 & 6.62 & 2.62 & 3.28 & 2.58 & -- \\
\hline \multicolumn{9}{|c|}{ Logan Creek (Latitude $37^{\circ} 09^{\prime} 21^{\prime \prime}$ Longitude $090^{\circ} 48^{\prime} 07^{\prime \prime}$ ) } \\
\hline 1 & 0.5 & 9.2 & 3,100 & 2.6 & 57.1 & 29.9 & 55.0 & -- \\
\hline 2 & .6 & 9.7 & 3,100 & 2.3 & 62.7 & 31.1 & 69.4 & \\
\hline 3 & .6 & 9.6 & 3,300 & 2.4 & 63.8 & 31.7 & 71.1 & -- \\
\hline 4 & .6 & 9.6 & 3,500 & 2.4 & 63.1 & 31.5 & 72.9 & \\
\hline 5 & .6 & 9.8 & 3,500 & 2.5 & 65.3 & 32.3 & 71.5 & -- \\
\hline 6 & .6 & 9.6 & 3,400 & 2.5 & 64.4 & 31.8 & 70.4 & \\
\hline 7 & .6 & 9.9 & 3,400 & 2.6 & 63.6 & 31.1 & 70.0 & -- \\
\hline 8 & .6 & 9.9 & 4,000 & 2.6 & 65.8 & 32.2 & 71.2 & \\
\hline 9 & .5 & 8.8 & 3,200 & 2.4 & 57.4 & 29.1 & 63.0 & -- \\
\hline 10 & .5 & 7.7 & 2,700 & 2.2 & 51.7 & 26.0 & 55.0 & \\
\hline 11 & .5 & 8.5 & 2,800 & 2.3 & 58.2 & 28.4 & 60.7 & -- \\
\hline 12 & .5 & 8.6 & 2,900 & 2.4 & 57.8 & 28.5 & 60.1 & \\
\hline 13 & .5 & 8.0 & 2,700 & 2.2 & 49.7 & 26.3 & 50.5 & -- \\
\hline 14 & .4 & 7.9 & 2,700 & 2.2 & 48.1 & 25.4 & 48.0 & \\
\hline 15 & .4 & 7.2 & 2,500 & 2.0 & 44.7 & 23.9 & 44.4 & -- \\
\hline 16 & .5 & 8.2 & 2,800 & 2.1 & 50.5 & 26.3 & 50.2 & \\
\hline 17 & .5 & 8.2 & 2,800 & 2.2 & 53.6 & 27.5 & 53.1 & -- \\
\hline 18 & .4 & 7.8 & 2,700 & 2.1 & 47.3 & 25.5 & 47.8 & \\
\hline 19 & .5 & 8.2 & 2,900 & 2.2 & 50.7 & 26.7 & 50.0 & -- \\
\hline 20 & .4 & 7.4 & 2,600 & 2.0 & 46.6 & 24.5 & 44.9 & \\
\hline 21 & .4 & 7.4 & 2,400 & 2.0 & 47.0 & 24.1 & 46.7 & -- \\
\hline 22 & .4 & 7.4 & 2,400 & 2.1 & 46.9 & 24.6 & 46.8 & \\
\hline 23 & .4 & 7.6 & 2,600 & 2.3 & 47.8 & 24.7 & 38.8 & -- \\
\hline 24 & .3 & 6.0 & 2,100 & 2.0 & 36.7 & 20.3 & 30.0 & \\
\hline 25 & .3 & 5.5 & 1,900 & 1.9 & 34.2 & 18.9 & 26.0 & -- \\
\hline 26 & .3 & 6.5 & 2,200 & 2.2 & 37.8 & 21.1 & 30.6 & \\
\hline 27 & .3 & 6.4 & 2,000 & 2.0 & 39.5 & 21.3 & 29.4 & -- \\
\hline 28 & .3 & 6.2 & 2,200 & 2.2 & 36.9 & 21.0 & 27.9 & \\
\hline Maximum & 0.6 & 9.9 & 4,000 & 2.6 & 65.8 & 32.3 & 72.9 & \\
\hline Minimum & .3 & 5.5 & 1,900 & 1.9 & 34.2 & 18.9 & 26.0 & \\
\hline Mean & .5 & 8.1 & 2,800 & 2.2 & 51.7 & 26.6 & 52.0 & \\
\hline Median & .5 & 8.1 & 2,750 & 2.2 & 50.6 & 26.3 & 50.4 & \\
\hline
\end{tabular}


Table 1. Quantitative elemental analyses of less than 63-micrometer fraction and lead-210 date of Clearwater Lake bed-sediment samples.—Continued

[All concentrations in milligrams per kilogram; RSD, relative standard deviation; ${ }^{\circ}$, degrees; ', minutes; ”, seconds; <, less than; shaded cells exceed threshold effects concentration; --, no data]

\begin{tabular}{|c|c|c|c|c|c|c|c|c|}
\hline $\begin{array}{c}\text { Depth below } \\
\text { lakebed surface, } \\
\text { (centimeters) }\end{array}$ & Thallium & Thorium & Titanium & Uranium & Vanadium & Yttrium & Zinc & $\begin{array}{c}\text { Lead-210 } \\
\text { date }\end{array}$ \\
\hline RSD (percent) & 5.74 & 6.48 & 8.58 & 6.62 & 2.62 & 3.28 & 2.58 & -- \\
\hline \multicolumn{9}{|c|}{ Upper Black River (Latitude $37^{\circ} 10^{\prime} 38^{\prime \prime}$ Longitude $090^{\circ} 47^{\prime} 28^{\prime \prime}$ ) } \\
\hline 1 & 0.5 & 10.1 & 4,100 & 2.8 & 63.5 & 37.4 & 91.4 & -- \\
\hline 2 & .5 & 9.0 & 4,000 & 2.3 & 52.2 & 33.4 & 77.0 & \\
\hline 3 & .4 & 8.6 & 4,000 & 2.2 & 48.3 & 35.4 & 72.8 & -- \\
\hline 4 & .5 & 9.4 & 4,100 & 2.4 & 51.2 & 33.7 & 76.2 & \\
\hline 5 & .5 & 9.5 & 4,100 & 2.5 & 54.9 & 34.8 & 81.3 & -- \\
\hline 6 & .5 & 9.7 & 4,100 & 2.6 & 55.4 & 35.3 & 82.5 & \\
\hline 7 & .5 & 9.4 & 4,100 & 2.4 & 54.8 & 34.7 & 79.4 & -- \\
\hline 8 & .5 & 9.2 & 4,000 & 2.3 & 53.6 & 34.2 & 79.0 & \\
\hline 9 & .5 & 9.4 & 4,100 & 2.5 & 53.6 & 38.4 & 78.4 & -- \\
\hline 10 & .5 & 9.5 & 4,100 & 2.7 & 55.3 & 35.1 & 79.9 & \\
\hline 11 & .5 & 9.6 & 4,100 & 2.8 & 58.2 & 36.4 & 82.0 & -- \\
\hline 12 & .6 & 10.0 & 4,100 & 2.9 & 61.2 & 37.7 & 84.6 & \\
\hline 13 & .5 & 9.6 & 4,100 & 2.6 & 57.1 & 35.8 & 84.5 & -- \\
\hline 14 & .5 & 9.5 & 4,100 & 2.5 & 56.5 & 35.7 & 86.7 & \\
\hline 15 & .5 & 9.5 & 4,100 & 2.5 & 56.1 & 35.7 & 89.8 & -- \\
\hline 16 & .5 & 9.7 & 4,100 & 2.6 & 56.1 & 34.5 & 86.4 & \\
\hline 17 & .5 & 9.7 & 4,100 & 2.6 & 54.4 & 35.2 & 83.5 & -- \\
\hline 18 & .5 & 9.2 & 4,000 & 2.5 & 53.2 & 34.2 & 82.1 & \\
\hline 19 & .5 & 9.2 & 4,000 & 2.5 & 54.7 & 34.6 & 86.5 & -- \\
\hline 20 & .5 & 9.8 & 4,100 & 2.7 & 58.0 & 36.5 & 94.8 & \\
\hline 21 & .5 & 9.4 & 4,100 & 2.7 & 57.9 & 38.0 & 91.3 & -- \\
\hline 22 & .5 & 8.9 & 4,000 & 2.3 & 52.0 & 33.3 & 79.4 & \\
\hline 23 & .5 & 8.9 & 4,000 & 2.3 & 50.1 & 33.7 & 76.6 & -- \\
\hline 24 & .5 & 8.7 & 4,000 & 2.4 & 48.4 & 33.4 & 74.1 & \\
\hline 25 & .5 & 8.9 & 4,000 & 2.4 & 51.3 & 34.5 & 75.8 & -- \\
\hline 26 & .5 & 9.4 & 4,100 & 2.5 & 53.4 & 34.6 & 73.7 & \\
\hline 27 & .4 & 9.0 & 4,100 & 2.4 & 50.0 & 34.3 & 71.2 & -- \\
\hline 28 & .4 & 9.0 & 4,100 & 2.4 & 49.6 & 33.7 & 69.0 & \\
\hline 29 & .5 & 9.0 & 4,000 & 2.4 & 50.1 & 33.1 & 70.4 & -- \\
\hline 30 & .5 & 9.4 & 4,100 & 2.5 & 52.1 & 35.2 & 77.1 & \\
\hline 31 & .5 & 9.3 & 4,100 & 2.4 & 51.3 & 33.0 & 74.2 & -- \\
\hline 32 & .5 & 9.3 & 4,000 & 2.4 & 53.8 & 34.2 & 77.5 & \\
\hline 33 & .5 & 9.4 & 4,000 & 2.3 & 50.9 & 34.5 & 77.8 & -- \\
\hline 34 & .5 & 9.0 & 4,000 & 2.3 & 51.6 & 33.8 & 73.5 & \\
\hline 35 & .5 & 9.4 & 4,000 & 2.3 & 54.9 & 34.7 & 79.2 & -- \\
\hline 36 & .5 & 8.9 & 4,000 & 2.2 & 51.1 & 32.5 & 74.2 & \\
\hline 37 & .5 & 9.9 & 4,000 & 2.4 & 58.1 & 36.8 & 86.4 & -- \\
\hline 38 & .5 & 10.0 & 4,000 & 2.4 & 59.7 & 37.1 & 85.8 & \\
\hline
\end{tabular}


Table 1. Quantitative elemental analyses of less than 63-micrometer fraction and lead-210 date of Clearwater Lake bed-sediment samples.-Continued

[All concentrations in milligrams per kilogram; RSD, relative standard deviation; ${ }^{\circ}$, degrees; ', minutes; ", seconds; <, less than; shaded cells exceed threshold effects concentration; --, no data]

\begin{tabular}{|c|c|c|c|c|c|c|c|c|}
\hline $\begin{array}{l}\text { Depth below } \\
\text { lakebed surface, } \\
\text { (centimeters) }\end{array}$ & Thallium & Thorium & Titanium & Uranium & Vanadium & Yttrium & Zinc & $\begin{array}{l}\text { Lead-210 } \\
\text { date }\end{array}$ \\
\hline RSD (percent) & 5.74 & 6.48 & 8.58 & 6.62 & 2.62 & 3.28 & 2.58 & -- \\
\hline \multicolumn{9}{|c|}{ Upper Black River (Latitude $37^{\circ} 10^{\prime} 38^{\prime \prime}$ Longitude $090^{\circ} 47^{\prime} 28^{\prime \prime}$ ) } \\
\hline 39 & 0.5 & 10.2 & 4,100 & 2.4 & 61.6 & 37.0 & 87.9 & -- \\
\hline 40 & .6 & 10.0 & 4,100 & 2.5 & 59.5 & 37.5 & 87.2 & \\
\hline 41 & .6 & 10.1 & 4,100 & 2.4 & 61.8 & 36.7 & 85.3 & -- \\
\hline 42 & .6 & 10.1 & 4,100 & 2.4 & 60.4 & 36.4 & 85.5 & \\
\hline 43 & .5 & 9.3 & 4,000 & 2.3 & 55.6 & 34.2 & 78.3 & -- \\
\hline 44 & .5 & 9.4 & 4,100 & 2.4 & 53.3 & 33.8 & 73.4 & \\
\hline 45 & .5 & 9.2 & 4,100 & 2.5 & 52.4 & 33.7 & 71.8 & -- \\
\hline 46 & .5 & 9.5 & 4,100 & 2.4 & 55.4 & 35.1 & 75.2 & \\
\hline 47 & .5 & 9.5 & 4,100 & 2.5 & 50.9 & 34.6 & 71.2 & -- \\
\hline 48 & .5 & 9.3 & 4,100 & 2.4 & 51.3 & 33.4 & 68.6 & \\
\hline 49 & .5 & 9.3 & 4,100 & 2.4 & 51.5 & 33.7 & 69.6 & -- \\
\hline 50 & .4 & 9.0 & 4,100 & 2.6 & 49.0 & 33.5 & 66.6 & \\
\hline 51 & .5 & 9.1 & 3,200 & 2.4 & 54.9 & 34.4 & 76.9 & -- \\
\hline 52 & .5 & 9.3 & 3,400 & 2.6 & 58.2 & 36.6 & 81.6 & \\
\hline 53 & .5 & 9.1 & 3,200 & 2.6 & 53.8 & 35.8 & 74.0 & -- \\
\hline 54 & .5 & 9.1 & 3,300 & 2.5 & 52.5 & 34.9 & 71.6 & \\
\hline 55 & .5 & 8.8 & 3,100 & 2.4 & 50.1 & 34.1 & 68.0 & -- \\
\hline 56 & .5 & 9.1 & 3,100 & 2.4 & 49.7 & 32.6 & 67.9 & \\
\hline 57 & .5 & 8.9 & 3,200 & 2.6 & 52.0 & 34.2 & 70.9 & -- \\
\hline 58 & .5 & 9.4 & 3,400 & 2.5 & 53.1 & 35.6 & 72.7 & \\
\hline 59 & .5 & 9.3 & 3,100 & 2.5 & 54.8 & 36.3 & 75.5 & -- \\
\hline 60 & .6 & 10.2 & 3,300 & 2.5 & 61.9 & 39.5 & 84.0 & \\
\hline 61 & .5 & 9.4 & 3,200 & 2.5 & 58.2 & 36.1 & 80.9 & -- \\
\hline Maximum & 0.6 & 10.2 & 4,100 & 2.9 & 63.5 & 39.5 & 94.8 & \\
\hline Minimum & .4 & 8.6 & 3,100 & 2.2 & 48.3 & 32.5 & 66.6 & \\
\hline Mean & .5 & 9.4 & 3,913 & 2.5 & 54.4 & 35.1 & 78.5 & \\
\hline Median & .5 & 9.4 & 4,100 & 2.4 & 53.8 & 34.7 & 77.8 & \\
\hline
\end{tabular}


Table 1. Quantitative elemental analyses of less than 63-micrometer fraction and lead-210 date of Clearwater Lake bed-sediment samples.—Continued

[All concentrations in milligrams per kilogram; RSD, relative standard deviation; ${ }^{\circ}$, degrees; ', minutes; ”, seconds; <, less than; shaded cells exceed threshold effects concentration; --, no data]

\begin{tabular}{|c|c|c|c|c|c|c|c|c|}
\hline $\begin{array}{c}\text { Depth below } \\
\text { lakebed surface, } \\
\text { (centimeters) }\end{array}$ & Thallium & Thorium & Titanium & Uranium & Vanadium & Yttrium & Zinc & $\begin{array}{c}\text { Lead-210 } \\
\text { date }\end{array}$ \\
\hline RSD (percent) & 5.74 & 6.48 & 8.58 & 6.62 & 2.62 & 3.28 & 2.58 & -- \\
\hline \multicolumn{9}{|c|}{ Lower Black River (Latitude $37^{\circ} 09^{\prime} 54^{\prime \prime}$ Longitude $090^{\circ} 45^{\prime} 58^{\prime \prime}$ ) } \\
\hline 1 & 0.7 & 11.5 & 3,700 & 2.7 & 86.7 & 43.4 & 129 & -- \\
\hline 2 & .7 & 11.4 & 3,700 & 2.8 & 87.4 & 43.5 & 132 & \\
\hline 3 & .7 & 11.5 & 3,400 & 2.9 & 90.6 & 43.7 & 133 & -- \\
\hline 4 & .7 & 11.2 & 3,300 & 2.8 & 81.3 & 42.5 & 120 & \\
\hline 5 & .7 & 11.3 & 3,400 & 2.6 & 80.9 & 42.2 & 120 & -- \\
\hline 6 & .7 & 11.3 & 3,600 & 2.8 & 80.9 & 42.1 & 120 & \\
\hline 7 & .7 & 11.1 & 3,500 & 2.8 & 79.4 & 41.5 & 118 & -- \\
\hline 8 & .7 & 11.2 & 3,500 & 2.6 & 79.4 & 41.6 & 117 & \\
\hline 9 & .7 & 11.2 & 3,600 & 2.6 & 81.5 & 42.3 & 121 & -- \\
\hline 10 & .7 & 11.2 & 3,600 & 2.6 & 79.2 & 41.7 & 118 & \\
\hline 11 & .7 & 11.3 & 3,600 & 2.7 & 81.0 & 42.3 & 120 & -- \\
\hline 12 & .7 & 11.1 & 3,400 & 2.5 & 80.6 & 41.3 & 117 & \\
\hline 13 & .7 & 11.2 & 3,400 & 2.6 & 82.0 & 42.4 & 122 & -- \\
\hline 14 & .7 & 11.4 & 3,400 & 2.6 & 82.6 & 42.4 & 121 & \\
\hline 15 & .7 & 11.1 & 3,400 & 2.6 & 83.6 & 42.1 & 122 & -- \\
\hline 16 & .7 & 11.3 & 3,400 & 2.6 & 81.9 & 41.7 & 120 & \\
\hline 17 & .7 & 11.1 & 3,500 & 2.7 & 80.7 & 41.6 & 121 & -- \\
\hline 18 & .7 & 11.0 & 3,500 & 2.7 & 80.6 & 41.1 & 119 & \\
\hline 19 & .7 & 11.2 & 3,800 & 2.8 & 81.2 & 41.4 & 118 & -- \\
\hline 20 & .7 & 11.0 & 3,500 & 2.7 & 80.6 & 41.5 & 116 & \\
\hline 21 & .7 & 11.3 & 3,500 & 2.7 & 82.3 & 42.5 & 120 & -- \\
\hline 22 & .7 & 10.9 & 3,200 & 2.7 & 80.0 & 41.6 & 116 & \\
\hline 23 & .7 & 11.0 & 3,300 & 2.8 & 79.1 & 41.4 & 113 & -- \\
\hline 24 & .7 & 11.0 & 3,200 & 2.8 & 79.4 & 41.6 & 113 & \\
\hline 25 & .7 & 11.5 & 3,400 & 2.8 & 81.3 & 42.2 & 116 & -- \\
\hline 26 & .7 & 11.4 & 3,400 & 2.7 & 80.8 & 41.7 & 114 & \\
\hline 27 & .7 & 11.3 & 3,500 & 2.8 & 81.3 & 42.2 & 116 & -- \\
\hline 28 & .7 & 11.4 & 3,500 & 2.8 & 79.0 & 41.7 & 113 & \\
\hline 29 & .7 & 11.4 & 3,600 & 2.8 & 80.3 & 41.9 & 114 & -- \\
\hline 30 & .7 & 11.6 & 3,700 & 2.8 & 81.8 & 42.3 & 116 & \\
\hline 31 & .7 & 11.1 & 3,400 & 2.6 & 82.6 & 41.6 & 117 & -- \\
\hline 32 & .7 & 11.1 & 3,200 & 2.6 & 80.6 & 40.8 & 114 & \\
\hline 33 & .7 & 11.1 & 3,400 & 2.7 & 82.0 & 41.2 & 115 & -- \\
\hline 34 & .7 & 11.4 & 3,400 & 2.7 & 82.1 & 41.5 & 112 & \\
\hline 35 & .7 & 11.1 & 3,700 & 2.8 & 81.2 & 41.3 & 112 & -- \\
\hline 36 & .7 & 11.1 & 3,300 & 2.7 & 82.0 & 40.9 & 111 & \\
\hline 37 & .7 & 11.2 & 3,500 & 2.8 & 83.8 & 41.8 & 115 & -- \\
\hline 38 & .7 & 11.0 & 3,400 & 2.8 & 83.4 & 41.2 & 112 & \\
\hline
\end{tabular}


Table 1. Quantitative elemental analyses of less than 63-micrometer fraction and lead-210 date of Clearwater Lake bed-sediment samples.-Continued

[All concentrations in milligrams per kilogram; RSD, relative standard deviation; ${ }^{\circ}$, degrees; ', minutes; ", seconds; <, less than; shaded cells exceed threshold effects concentration; --, no data]

\begin{tabular}{|c|c|c|c|c|c|c|c|c|}
\hline $\begin{array}{c}\text { Depth below } \\
\text { lakebed surface, } \\
\text { (centimeters) }\end{array}$ & Thallium & Thorium & Titanium & Uranium & Vanadium & Yttrium & Zinc & $\begin{array}{c}\text { Lead-210 } \\
\text { date }\end{array}$ \\
\hline RSD (percent) & 5.74 & 6.48 & 8.58 & 6.62 & 2.62 & 3.28 & 2.58 & -- \\
\hline \multicolumn{9}{|c|}{ Lower Black River (Latitude $37^{\circ} 09^{\prime} 54^{\prime \prime}$ Longitude $090^{\circ} 45^{\prime} 58^{\prime \prime}$ )—Continued } \\
\hline 39 & 0.7 & 11.2 & 3,500 & 2.8 & 80.7 & 41.1 & 109 & -- \\
\hline 40 & .7 & 11.2 & 3,500 & 2.8 & 79.7 & 40.2 & 107 & \\
\hline 41 & .7 & 11.0 & 3,600 & 2.7 & 80.9 & 40.6 & 110 & -- \\
\hline 42 & .7 & 11.1 & 3,700 & 2.7 & 80.6 & 40.6 & 111 & \\
\hline 43 & .6 & 9.5 & 3,100 & 2.3 & 62.9 & 30.6 & 69.5 & \\
\hline 44 & .7 & 10.8 & 3,400 & 2.6 & 76.5 & 39.1 & 104 & \\
\hline 45 & .7 & 10.4 & 3,400 & 2.6 & 73.4 & 38.6 & 99.9 & \\
\hline 46 & .6 & 10.5 & 3,400 & 2.6 & 71.8 & 38.5 & 99.2 & \\
\hline 47 & .6 & 10.6 & 3,800 & 2.6 & 74.0 & 39.4 & 101 & -- \\
\hline 48 & .7 & 10.6 & 3,500 & 2.7 & 73.6 & 39.7 & 99.6 & \\
\hline 49 & .7 & 10.9 & 4,000 & 2.7 & 73.1 & 39.3 & 96.4 & -- \\
\hline 50 & .6 & 10.7 & 3,800 & 2.7 & 74.6 & 40.2 & 97.0 & \\
\hline 51 & .7 & 11.2 & 3,900 & 2.8 & 79.0 & 46.6 & 105 & -- \\
\hline 52 & .7 & 11.2 & 3,800 & 2.8 & 77.2 & 48.9 & 102 & \\
\hline 53 & .7 & 11.2 & 3,700 & 2.8 & 78.3 & 45.5 & 102 & 1964 \\
\hline 54 & .7 & 11.1 & 3,400 & 2.7 & 79.2 & 44.5 & 106 & \\
\hline Maximum & 0.7 & 11.6 & 4,000 & 2.9 & 90.6 & 48.9 & 133 & \\
\hline Minimum & .6 & 9.5 & 3,100 & 2.3 & 62.9 & 30.6 & 69.5 & \\
\hline Mean & .7 & 11.1 & 3,506 & 2.7 & 80.0 & 41.6 & 113 & \\
\hline Median & .7 & 11.2 & 3,500 & 2.7 & 80.7 & 41.6 & 115 & \\
\hline
\end{tabular}


Table 1. Quantitative elemental analyses of less than 63-micrometer fraction and lead-210 date of Clearwater Lake bed-sediment samples.—Continued

[All concentrations in milligrams per kilogram; RSD, relative standard deviation; ${ }^{\circ}$, degrees; ', minutes; ”, seconds; <, less than; shaded cells exceed threshold effects concentration; --, no data]

\begin{tabular}{|c|c|c|c|c|c|c|c|c|}
\hline $\begin{array}{l}\text { Depth below } \\
\text { lakebed surface, } \\
\text { (centimeters) }\end{array}$ & Thallium & Thorium & Titanium & Uranium & Vanadium & Yttrium & Zinc & $\begin{array}{c}\text { Lead-210 } \\
\text { date }\end{array}$ \\
\hline RSD (percent) & 5.74 & 6.48 & 8.58 & 6.62 & 2.62 & 3.28 & 2.58 & -- \\
\hline \multicolumn{9}{|c|}{ Marina (Latitude $37^{\circ} 08^{\prime} 27^{\prime \prime}$ Longitude $090^{\circ} 46^{\prime} 14^{\prime \prime}$ ) } \\
\hline 1 & 0.7 & 12.5 & 3,800 & 3.0 & 89.1 & 45.4 & 169 & -- \\
\hline 2 & .8 & 12.5 & 4,100 & 3.0 & 99.6 & 51.7 & 165 & \\
\hline 3 & .8 & 12.7 & 4,200 & 3.5 & 105.0 & 54.4 & 232 & -- \\
\hline 4 & .8 & 12.6 & 4,100 & 3.5 & 104.0 & 53.8 & 178 & \\
\hline 5 & .9 & 13.2 & 4,200 & 3.4 & 109.0 & 57.1 & 171 & -- \\
\hline 6 & .8 & 12.7 & 4,200 & 3.3 & 105.0 & 53.0 & 186 & \\
\hline 7 & .8 & 12.7 & 4,200 & 3.2 & 102.0 & 54.6 & 186 & -- \\
\hline 8 & .8 & 12.6 & 4,200 & 3.2 & 100.0 & 53.5 & 176 & \\
\hline 9 & .8 & 12.6 & 4,100 & 3.1 & 98.2 & 53.6 & 152 & -- \\
\hline 10 & .8 & 12.6 & 4,100 & 3.0 & 97.4 & 52.7 & 166 & \\
\hline 11 & .8 & 12.6 & 4,200 & 3.1 & 97.5 & 52.4 & 145 & -- \\
\hline 12 & .8 & 12.4 & 4,100 & 3.1 & 98.3 & 53.9 & 144 & \\
\hline 13 & .8 & 12.6 & 4,100 & 3.0 & 98.5 & 53.5 & 145 & -- \\
\hline 14 & .8 & 12.8 & 4,200 & 3.1 & 100.0 & 54.3 & 144 & \\
\hline 15 & .9 & 13.1 & 4,200 & 3.2 & 102.0 & 55.2 & 145 & -- \\
\hline 16 & .9 & 13.2 & 4,200 & 3.3 & 105.0 & 55.5 & 148 & \\
\hline 17 & .9 & 13.0 & 4,200 & 3.3 & 106.0 & 57.1 & 151 & -- \\
\hline 18 & .9 & 13.7 & 4,200 & 3.4 & 105.0 & 56.5 & 148 & \\
\hline 19 & .9 & 13.4 & 4,200 & 3.4 & 104.0 & 56.2 & 147 & -- \\
\hline 20 & .9 & 13.3 & 4,200 & 3.3 & 104.0 & 56.1 & 142 & \\
\hline 21 & .9 & 13.3 & 4,200 & 3.4 & 106.0 & 57.2 & 145 & -- \\
\hline 22 & .9 & 12.9 & 4,200 & 3.3 & 103.0 & 55.3 & 140 & \\
\hline 23 & .9 & 13.0 & 4,200 & 3.3 & 102.0 & 55.6 & 140 & -- \\
\hline 24 & .9 & 13.0 & 4,200 & 3.4 & 103.0 & 55.8 & 140 & \\
\hline 25 & .9 & 13.0 & 4,100 & 3.4 & 101.0 & 54.9 & 137 & -- \\
\hline 26 & .8 & 11.3 & 4,000 & 2.8 & 91.7 & 48.6 & 126 & \\
\hline 27 & .8 & 11.5 & 4,000 & 2.8 & 94.5 & 49.5 & 130 & -- \\
\hline 28 & .8 & 11.7 & 4,100 & 2.9 & 96.0 & 50.0 & 128 & \\
\hline 29 & .8 & 11.7 & 4,100 & 2.9 & 96.2 & 51.1 & 132 & -- \\
\hline 30 & .8 & 12.3 & 4,100 & 3.1 & 97.5 & 52.2 & 130 & \\
\hline 31 & .8 & 12.1 & 4,100 & 3.0 & 97.0 & 52.4 & 131 & -- \\
\hline 32 & .8 & 12.3 & 4,100 & 3.0 & 96.6 & 52.5 & 132 & \\
\hline 33 & .9 & 12.5 & 4,100 & 3.3 & 99.1 & 52.1 & 132 & -- \\
\hline 34 & .9 & 12.5 & 4,100 & 3.3 & 102.0 & 53.1 & 134 & \\
\hline 35 & .9 & 12.7 & 4,200 & 3.4 & 102.0 & 54.5 & 136 & -- \\
\hline 36 & .9 & 12.9 & 4,200 & 3.6 & 103.0 & 55.9 & 139 & \\
\hline 37 & .9 & 13.1 & 4,200 & 3.5 & 104.0 & 55.6 & 139 & -- \\
\hline 38 & .9 & 13.0 & 4,200 & 3.4 & 102.0 & 52.3 & 131 & \\
\hline
\end{tabular}


Table 1. Quantitative elemental analyses of less than 63-micrometer fraction and lead-210 date of Clearwater Lake bed-sediment samples.-Continued

[All concentrations in milligrams per kilogram; RSD, relative standard deviation; ${ }^{\circ}$, degrees; ', minutes; ", seconds; <, less than; shaded cells exceed threshold effects concentration; --, no data]

\begin{tabular}{|c|c|c|c|c|c|c|c|c|}
\hline $\begin{array}{l}\text { Depth below } \\
\text { lakebed surface, } \\
\text { (centimeters) }\end{array}$ & Thallium & Thorium & Titanium & Uranium & Vanadium & Yttrium & Zinc & $\begin{array}{c}\text { Lead-210 } \\
\text { date }\end{array}$ \\
\hline RSD (percent) & 5.74 & 6.48 & 8.58 & 6.62 & 2.62 & 3.28 & 2.58 & -- \\
\hline \multicolumn{9}{|c|}{ Marina (Latitude $37^{\circ} 08^{\prime} 27^{\prime \prime}$ Longitude $090^{\circ} 46^{\prime} 14^{\prime \prime}$ )-Continued } \\
\hline 39 & 0.9 & 12.9 & 4,100 & 3.4 & 103.0 & 51.7 & 130 & -- \\
\hline 40 & .9 & 12.7 & 4,200 & 3.3 & 101.0 & 50.6 & 126 & \\
\hline 41 & .9 & 12.7 & 4,200 & 3.4 & 101.0 & 51.0 & 126 & -- \\
\hline 42 & .9 & 13.1 & 4,200 & 3.5 & 101.0 & 51.4 & 124 & \\
\hline 43 & .9 & 12.8 & 4,200 & 3.5 & 101.0 & 51.5 & 123 & -- \\
\hline 44 & .9 & 12.8 & 4,200 & 3.4 & 100.0 & 53.5 & 127 & \\
\hline 45 & .8 & 12.4 & 4,100 & 3.2 & 96.2 & 52.2 & 131 & -- \\
\hline 46 & .8 & 11.4 & 4,100 & 3.1 & 89.2 & 46.5 & 116 & \\
\hline 47 & .8 & 11.6 & 4,200 & 3.0 & 91.3 & 47.8 & 118 & -- \\
\hline 48 & .8 & 11.4 & 4,100 & 2.9 & 89.2 & 46.8 & 115 & \\
\hline 49 & .8 & 12.0 & 4,100 & 2.9 & 93.4 & 49.1 & 120 & -- \\
\hline 50 & .8 & 12.0 & 4,100 & 3.0 & 95.3 & 49.4 & 122 & \\
\hline 51 & .8 & 11.6 & 3,500 & 2.8 & 98.9 & 44.0 & 128 & -- \\
\hline 52 & .8 & 11.7 & 3,600 & 2.9 & 99.4 & 45.1 & 129 & \\
\hline 53 & .8 & 11.5 & 3,600 & 3.0 & 101.0 & 47.0 & 129 & -- \\
\hline 54 & .8 & 11.9 & 3,900 & 3.0 & 103.0 & 49.0 & 135 & \\
\hline 55 & .8 & 11.5 & 3,800 & 3.0 & 99.2 & 45.1 & 127 & -- \\
\hline 56 & .8 & 10.9 & 3,500 & 2.8 & 93.3 & 43.0 & 122 & \\
\hline 57 & .8 & 11.9 & 3,600 & 3.0 & 97.1 & 45.8 & 127 & -- \\
\hline 58 & .8 & 12.0 & 3,800 & 3.0 & 98.8 & 46.4 & 129 & \\
\hline 59 & .8 & 12.2 & 3,800 & 3.1 & 102.0 & 45.5 & 136 & -- \\
\hline 60 & .9 & 12.3 & 3,900 & 3.2 & 109.0 & 46.9 & 160 & \\
\hline 61 & .9 & 12.4 & 3,700 & 3.3 & 111.0 & 48.0 & 164 & -- \\
\hline Maximum & 0.9 & 13.7 & 4,200 & 3.6 & 111.0 & 57.2 & 232 & \\
\hline Minimum & .7 & 10.9 & 3,500 & 2.8 & 89.1 & 43.0 & 115 & \\
\hline Mean & .8 & 12.4 & 4,064 & 3.2 & 100.0 & 51.6 & 141 & \\
\hline Median & .8 & 12.6 & 4,100 & 3.2 & 101.0 & 52.3 & 136 & \\
\hline
\end{tabular}


Table 1. Quantitative elemental analyses of less than 63-micrometer fraction and lead-210 date of Clearwater Lake bed-sediment samples.—Continued

[All concentrations in milligrams per kilogram; RSD, relative standard deviation; ${ }^{\circ}$, degrees; ', minutes; ”, seconds; <, less than; shaded cells exceed threshold effects concentration; --, no data]

\begin{tabular}{|c|c|c|c|c|c|c|c|c|}
\hline $\begin{array}{l}\text { Depth below } \\
\text { lakebed surface, } \\
\text { (centimeters) }\end{array}$ & Thallium & Thorium & Titanium & Uranium & Vanadium & Yttrium & Zinc & $\begin{array}{c}\text { Lead-210 } \\
\text { date }\end{array}$ \\
\hline RSD (percent) & 5.74 & 6.48 & 8.58 & 6.62 & 2.62 & 3.28 & 2.58 & -- \\
\hline \multicolumn{9}{|c|}{ Clearwater Dam (Latitude $37^{\circ} 08^{\prime} 19^{\prime \prime}$ Longitude $090^{\circ} 46^{\prime} 47^{\prime \prime}$ ) } \\
\hline 1 & 0.9 & 13.1 & 4,000 & 3.3 & 115.0 & 45.3 & 154 & 2000 \\
\hline 2 & .9 & 13.1 & 3,900 & 3.2 & 114.0 & 46.2 & 152 & \\
\hline 3 & .9 & 13.0 & 4,100 & 3.1 & 113.0 & 46.4 & 147 & 1999 \\
\hline 4 & .9 & 13.0 & 4,100 & 3.1 & 112.0 & 45.3 & 144 & \\
\hline 5 & .9 & 13.2 & 4,200 & 3.1 & 112.0 & 46.9 & 145 & 1996 \\
\hline 6 & .9 & 13.1 & 4,300 & 3.1 & 112.0 & 46.4 & 143 & \\
\hline 7 & .9 & 13.2 & 4,000 & 3.1 & 111.0 & 46.2 & 143 & 1993 \\
\hline 8 & .9 & 13.2 & 3,900 & 3.1 & 112.0 & 45.5 & 142 & \\
\hline 9 & .9 & 13.1 & 3,900 & 3.1 & 111.0 & 45.0 & 140 & 1991 \\
\hline 10 & .9 & 13.2 & 4,000 & 3.2 & 113.0 & 44.6 & 138 & \\
\hline 11 & .9 & 13.1 & 4,000 & 3.2 & 110.0 & 44.2 & 135 & 1987 \\
\hline 12 & .9 & 13.1 & 4,000 & 3.3 & 112.0 & 45.1 & 136 & \\
\hline 13 & .9 & 13.5 & 4,400 & 3.4 & 113.0 & 45.3 & 136 & 1984 \\
\hline 14 & .9 & 13.5 & 4,200 & 3.4 & 115.0 & 46.8 & 139 & \\
\hline 15 & .9 & 13.9 & 4,200 & 3.5 & 118.0 & 46.8 & 142 & 1981 \\
\hline 16 & 1.0 & 13.7 & 4,200 & 3.4 & 117.0 & 47.0 & 141 & \\
\hline 17 & .9 & 13.5 & 4,100 & 3.4 & 113.0 & 45.2 & 137 & 1978 \\
\hline 18 & 1.0 & 13.6 & 4,100 & 3.4 & 116.0 & 45.6 & 139 & \\
\hline 19 & 1.0 & 14.0 & 4,300 & 3.6 & 116.0 & 47.0 & 142 & 1975 \\
\hline 20 & 1.0 & 13.7 & 4,200 & 3.5 & 115.0 & 46.9 & 137 & \\
\hline 21 & .9 & 13.8 & 4,600 & 3.6 & 113.0 & 47.2 & 136 & 1972 \\
\hline 22 & 1.0 & 13.4 & 4,000 & 3.2 & 113.0 & 45.4 & 137 & \\
\hline 23 & 1.0 & 13.6 & 4,000 & 3.2 & 113.0 & 45.3 & 137 & 1970 \\
\hline 24 & 1.0 & 13.7 & 4,000 & 3.5 & 114.0 & 46.1 & 140 & \\
\hline 25 & 1.0 & 13.7 & 4,100 & 3.5 & 116.0 & 47.8 & 141 & 1967 \\
\hline 26 & 1.0 & 13.6 & 4,200 & 3.4 & 113.0 & 47.2 & 136 & \\
\hline 27 & .9 & 12.6 & 4,000 & 3.2 & 104.0 & 51.4 & 117 & 1965 \\
\hline 28 & .9 & 12.7 & 4,200 & 3.3 & 105.0 & 51.2 & 114 & \\
\hline 29 & .9 & 12.6 & 4,300 & 3.2 & 103.0 & 51.5 & 113 & 1962 \\
\hline 30 & .9 & 12.9 & 4,300 & 3.3 & 104.0 & 50.9 & 113 & \\
\hline 31 & .9 & 12.3 & 4,100 & 3.2 & 103.0 & 50.3 & 112 & 1960 \\
\hline 32 & .9 & 12.2 & 4,100 & 3.1 & 100.0 & 48.6 & 108 & \\
\hline 33 & .9 & 12.0 & 3,800 & 3.1 & 98.4 & 48.9 & 109 & 1958 \\
\hline 34 & .9 & 12.3 & 4,100 & 3.2 & 101.0 & 49.5 & 109 & \\
\hline 35 & .9 & 12.2 & 4,200 & 3.2 & 98.2 & 48.7 & 105 & 1956 \\
\hline 36 & .8 & 12.0 & 3,800 & 3.0 & 94.5 & 50.9 & 100 & \\
\hline 37 & .8 & 11.9 & 3,800 & 3.0 & 93.6 & 47.0 & 99.6 & 1954 \\
\hline 38 & .8 & 11.9 & 4,200 & 3.1 & 94.6 & 47.7 & 99.7 & \\
\hline
\end{tabular}


Table 1. Quantitative elemental analyses of less than 63-micrometer fraction and lead-210 date of Clearwater Lake bed-sediment samples.-Continued

[All concentrations in milligrams per kilogram; RSD, relative standard deviation; ${ }^{\circ}$, degrees; ', minutes; ", seconds; <, less than; shaded cells exceed threshold effects concentration; --, no data]

\begin{tabular}{|c|c|c|c|c|c|c|c|c|}
\hline $\begin{array}{l}\text { Depth below } \\
\text { lakebed surface, } \\
\text { (centimeters) }\end{array}$ & Thallium & Thorium & Titanium & Uranium & Vanadium & Yttrium & Zinc & $\begin{array}{c}\text { Lead-210 } \\
\text { date }\end{array}$ \\
\hline RSD (percent) & 5.74 & 6.48 & 8.58 & 6.62 & 2.62 & 3.28 & 2.58 & -- \\
\hline \multicolumn{9}{|c|}{ Clearwater Dam (Latitude $37^{\circ} 08^{\prime} 19^{\prime \prime}$ Longitude $090^{\circ} 46^{\prime} 47^{\prime \prime}$ )—Continued } \\
\hline 39 & .8 & 11.8 & 4,200 & 3.0 & 89.7 & 46.0 & 94.6 & 1953 \\
\hline 40 & .7 & 11.5 & 4,100 & 3.0 & 87.3 & 46.3 & 92.3 & \\
\hline 41 & .8 & 11.6 & 4,400 & 3.2 & 86.9 & 46.0 & 91.4 & 1953 \\
\hline 42 & .8 & 11.2 & 4,000 & 2.9 & 87.2 & 43.3 & 91.3 & \\
\hline 43 & .7 & 11.1 & 3,600 & 2.9 & 83.8 & 47.4 & 87.3 & 1952 \\
\hline 44 & .7 & 11.0 & 3,600 & 2.8 & 82.5 & 42.9 & 87.0 & \\
\hline 45 & .7 & 11.3 & 4,000 & 3.0 & 85.7 & 48.2 & 90.6 & 1952 \\
\hline 46 & .8 & 11.4 & 4,000 & 3.0 & 87.1 & 45.4 & 91.4 & \\
\hline 47 & .8 & 11.2 & 3,800 & 3.1 & 87.2 & 45.0 & 91.2 & 1952 \\
\hline 48 & .8 & 11.4 & 3,700 & 3.0 & 86.3 & 45.0 & 90.9 & \\
\hline 49 & .7 & 11.0 & 3,700 & 3.1 & 81.3 & 42.3 & 84.8 & 1951 \\
\hline 50 & .7 & 11.0 & 3,900 & 3.0 & 83.1 & 43.6 & 84.7 & \\
\hline 51 & .8 & 11.3 & 4,100 & 3.0 & 91.1 & 45.6 & 93.2 & 1951 \\
\hline 52 & .8 & 11.3 & 3,800 & 3.3 & 90.7 & 44.6 & 88.6 & \\
\hline 53 & .8 & 11.3 & 3,800 & 3.2 & 89.6 & 45.7 & 97.1 & 1947 \\
\hline 54 & .5 & 7.9 & 2,800 & 2.5 & 54.1 & 29.8 & 57.3 & \\
\hline Maximum & 1.0 & 14.0 & 4,600 & 3.6 & 118.0 & 51.5 & 154 & \\
\hline Minimum & .5 & 7.9 & 2,800 & 2.5 & 54.1 & 29.8 & 57.3 & \\
\hline Mean & .9 & 12.5 & 4,026 & 3.2 & 101.9 & 46.3 & 118 & \\
\hline Median & .9 & 12.8 & 4,050 & 3.2 & 104.5 & 46.2 & 116 & \\
\hline
\end{tabular}




\section{National Water-Quality Assessment (NAWQA) Program Black River Synoptic Study, Southeastern Missouri, 1993 and 1995}

By Suzanne R. Femmer

Chapter 5 of

Hydrologic Investigations Concerning Lead Mining Issues in Southeastern Missouri

Edited by Michael J. Kleeschulte

Scientific Investigations Report 2008-5140 


\section{Contents}

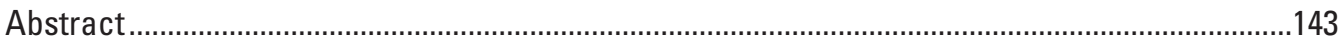

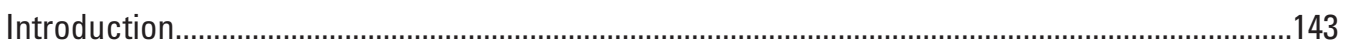

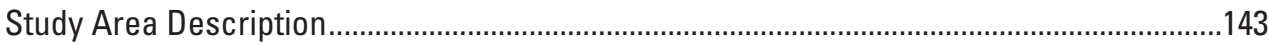

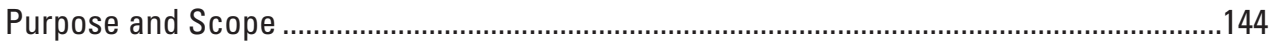

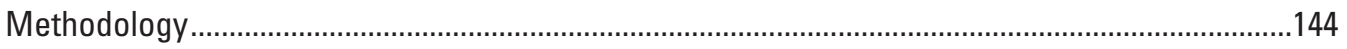

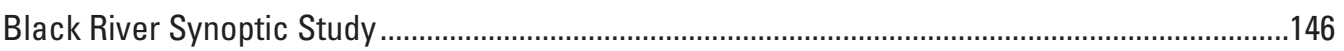

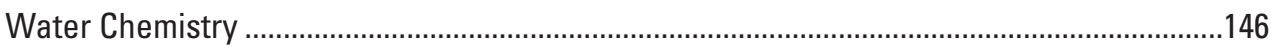

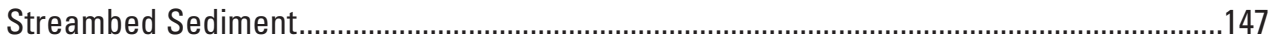

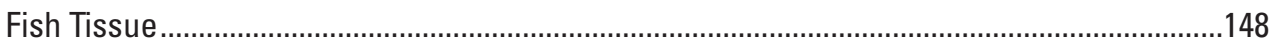

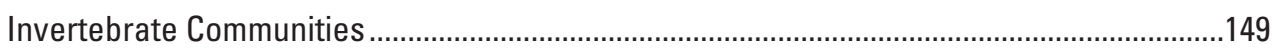

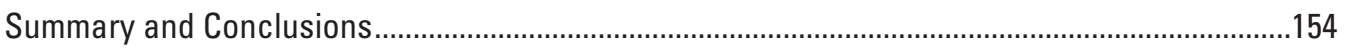

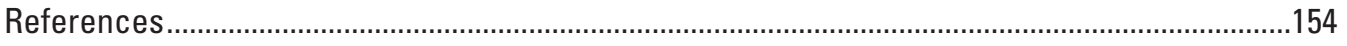

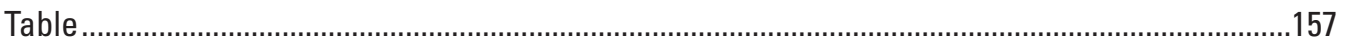

\section{Figures}

1. Map showing study area and sampling sites on streams draining the Viburnum Trend Subdistrict.

2-3. Graphs showing-

2. Cadmium, lead, and zinc concentrations in streambed sediment at West Fork Black River sites and downstream

3. Cadmium, lead, and zinc concentrations in fish tissue at West Fork Black River sites and downstream

\section{Tables}

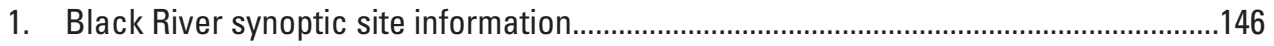

2. Water-quality data from the National Water-Quality Assessment Program Black River synoptic study, August and September 1995.

3. Streambed sediment and fish tissue data from the National Water-Quality Assessment Program Black River synoptic study.

4. Selected metrics applied to invertebrate data collected for the National Water-Quality Assessment Program Black River synoptic study. 


\title{
National Water-Quality Assessment (NAW0A) Program Black River Synoptic Study, Southeastern Missouri, 1993 and 1995
}

\author{
By Suzanne R. Femmer
}

\section{Abstract}

Water chemistry, streambed sediment, fish tissue, and invertebrate samples were collected from 14 sites to evaluate the effects of lead and zinc mining and related activities on streams in the Viburnum Trend Subdistrict (Viburnum Trend). All samples were collected during August to September 1995, except for streambed sediment and fish tissue collected at Black River near Lesterville during October 1993. The metalsulfide deposits in the Viburnum Trend are known as Mississippi Valley Type deposits and include the trace-elements (all metals) arsenic, cadmium, cobalt, copper, lead, nickel, and zinc. The sampled sites were grouped according to their proximity to mining activity: non-mining sites, sites upstream from mining activity or mine tailings, or sites where no mining activity is present in the basin; near-mining sites, mining activity or mine tailings ponds within 7.5 miles upstream from the site; and distal-mining sites, mining activity greater than 7.5 miles upstream from the site.

The water-quality samples from non-mining sites had small concentrations of Mississippi Valley Type-related metals, but at the near-mining sites, all of these metals were detected above the method reporting level. Only dissolved copper, nickel, and zinc were detected above the method reporting level at distal-mining sites. Dissolved lead, nickel, and zinc concentrations were substantially larger at the nearmining sites than at the non-mining or distal-mining sites. The Mississippi Valley Type-related metals detected at near-mining sites decreased in concentration downstream at the distalmining sites.

In streambed-sediment and fish-tissue samples, the largest concentrations for each of the Mississippi Valley Typerelated metals, except arsenic and copper, were detected at near-mining sites. The largest mean concentration for the Mississippi Valley Type-related metals, except for copper, occurred at the near-mining sites, followed by the distalmining sites; the smallest mean concentrations were for the non-mining sites. Arsenic and cobalt concentrations gradually increased and cadmium, lead, and zinc decreased as the distance downstream from mining activity increased.
Invertebrate samples were collected at 13 sites for community structure analysis. These data indicated that there were disturbances in invertebrate community structure near mining activities. Quantitative and qualitative samples were collected at each site. The results of multiple metrics applied to the invertebrate community data consistently indicated effects to the community near mining activities. There was apparent recovery in community structure metrics as the distance downstream from mining activities increased.

The commonality through all the water chemistry, streambed sediment, fish tissue, and invertebrate data is that where mining activity is occurring in the vicinity of sampling sites, these stream reaches exhibit characteristics of an affected stream. The data also support the lessening effect of these mining activities as the distance downstream from the mining activity increased.

\section{Introduction}

During 1993 and 1995, water chemistry, streambed sediment, fish tissue, and invertebrate data were collected from streams in southeastern Missouri for the National Water-Quality Assessment (NAWQA) program (for more information access http://water.usgs.gov/nawqa/). This synoptic sampling was performed as part of a reconnaissance investigation to evaluate, by using these multiple lines of evidence, the effects of lead and zinc mining and related activities on area streams in the Viburnum Trend Subdistrict (Viburnum Trend).

\section{Study Area Description}

The Black River synoptic study was located in an area of well-developed karst terrain in southeastern Missouri. This study area is in the Salem Plateau physiographic region and has deep narrow valleys with steep-sided ridges. Relief can be as much as 300 feet (ft) (Fenneman, 1938). The study lies entirely within the Ozark Highlands ecoregion as designated by Omernik and Gallant (1987). In the Ozark Highlands, land use typically is forested (oak-hickory) with occasional stands 
of pine. Typically, less than one-quarter of the land use is cropland or pasture; the cropland and pasture available is located mainly in river valleys.

\section{Purpose and Scope}

The purpose of this chapter is to present data collected as part of the NAWQA program Black River synoptic study with emphasis on trace elements, although other constituents were analyzed. Sampling efforts were concentrated in the northern part of the upper Black River Basin where several lead and zinc mining operations are located (fig. 1). The metal-sulfide deposits in the Viburnum Trend subdistrict are a class known as Mississippi Valley Type (MVT) deposits and include the trace elements (all metals) arsenic, cadmium, cobalt, copper, lead, nickel, and zinc.

\section{Methodology}

Eleven sites in the Black River Basin and three sites outside of the basin (fig. 1) were selected for this analysis and grouped according to their proximity-to-mining activity. Non-mining sites include sampling sites upstream from mining activity or mine tailings or sites in basins where no mining activity is present. Non-mining sites outside of the basin were represented by Jacks Fork at Alley Spring (site N-1), Paddy Creek above Slabtown Spring (site N-2), and Big Creek near Rat (site N-3) and were selected because of similar geology, basin size, and land use (except for mining activity) to selected sites in the Black River Basin. Non-mining sites in the Black River Basin included West Fork Black River near Greeley (site N-4) and Middle Fork Black River at Redmondville (site N-5). Near-mining sites are those where mining activity or mine tailings ponds are within 7.5 miles (mi) upstream from the site. Near-mining sites are represented by the West Fork Black River at West Fork (site NR-1), Strother Creek near Oates (site NR-2), Neals Creek near Goodland (site NR-3), and Strother Creek near Redmondville (site NR-4). Distal-mining sites are those where mining activity is more than $7.5 \mathrm{mi}$ upstream from the site. These sites are represented by the West Fork Black River near Centerville (site D-1), West Fork Black River at Centerville (site D-2), Middle Fork Black River at Black (site D-3), and the Black River near Lesterville (site D-4). Waterchemistry data from the Black River near Annapolis (site D-5, 8 mi downstream from Lesterville) was used as a supplement to data from the Black River near Lesterville. Water-quality data were not collected at the Lesterville site because of limited access during high flows.

All 14 sites were sampled during August or September, 1995. Streambed sediment and fish tissue were collected at the Black River near Lesterville (site D-4) during October 1993 (table 1). Collection and processing methods for all samples were performed according to protocols developed for the NAWQA program (U.S. Geological Survey, 2003). Water properties determined at each location included discharge, dissolved oxygen concentration, $\mathrm{pH}$, specific conductance, water and air temperature, barometric pressure, alkalinity, and fecal indicator bacteria density [Escherichia coli (E. coli), fecal coliform, and fecal streptococci]. Water samples were sent to the USGS National Water Quality Laboratory (NWQL) in Lakewood, Colorado, to be analyzed for major ions, nutrients, organic carbon content, and trace elements using the methods described in Fishman (1993) and in Fishman and Friedman, (1989). Suspended-sediment samples were sent to the USGS sediment laboratory in Rolla, Missouri, for sediment concentration analysis using standard methods (M.N. Barr, U.S. Geological Survey, written commun., 2007). Qualitycontrol samples were collected for quality assurance. Duplicate or split samples were collected at 10 percent of the sites. All duplicate or split samples were comparable to original samples. No quality-assurance data were collected for the fish tissue or bed sediment samples in 1993 or 1995.

Streambed-sediment samples were collected at 10 sites, 8 of which are in the Black River system (fig. 1). Depositional areas of fine sediment were targeted for sampling at these sites, because the targeted analytes have an affinity for silt- and claysized particles (Rickert and others, 1977; Wilber and Hunter, 1979). The fine fraction [less than 63-micrometer $(\mu \mathrm{m})]$ of the sediment was used for analysis. Streambed-sediment samples were collected by compositing subsamples at each site so as to obtain a representative sample of available material. Individual subsamples were obtained from a 1.5-inch (in.) Teflon cup hand sampler. The sampling cup was used to collect sediment from the top $0.6 \mathrm{in}$. of the streambed and the collected material was deposited into a glass container. The composited sample was sieved to obtain at least 10 grams (g) dry weight of the fine fraction. After the sample had settled, excess water was decanted. The samples were chilled and then shipped to the NWQL for analysis. Total elemental abundances of samples were analyzed using inductively coupled plasma-atomic emission spectrometry (ICP-AES) (Garbarino and Struzeski, 1998).

Fish tissue (livers) samples were collected at eight sites, six of which are in the Black River Basin (fig. 1). An attempt was made to target the same fish species at each site to eliminate species variability, but this was not possible. Centrachids (sunfish) were collected at all sites except for Strother Creek near Redmondville (site NR-4) where the hogsucker, Hypentelium nigricans, was collected. The hogsucker is a bottom dwelling fish, unlike the centrarchids, and may have greater exposure to trace-metal laden sediment. The long-eared sunfish, Lepomis megalotis, was collected at four sites [Jacks Fork River at Alley Spring (site N-1), West Fork Black River near Centerville (site D-1), West Fork Black River at Centerville (site D-2), and the Black River near Lesterville (site D-4)]. The small mouth bass, Micropterus dolomieu, was collected at the West Fork Black River near Greeley (N-4) and West Fork Black River at West Fork (NR-1). The hogsucker, Hypentelium nigricans, was collected at Strother Creek near Redmondville (site NR-4), and the shadow bass, Ambloplites ariommus, was collected at Paddy Creek above Slabtown Spring (site 


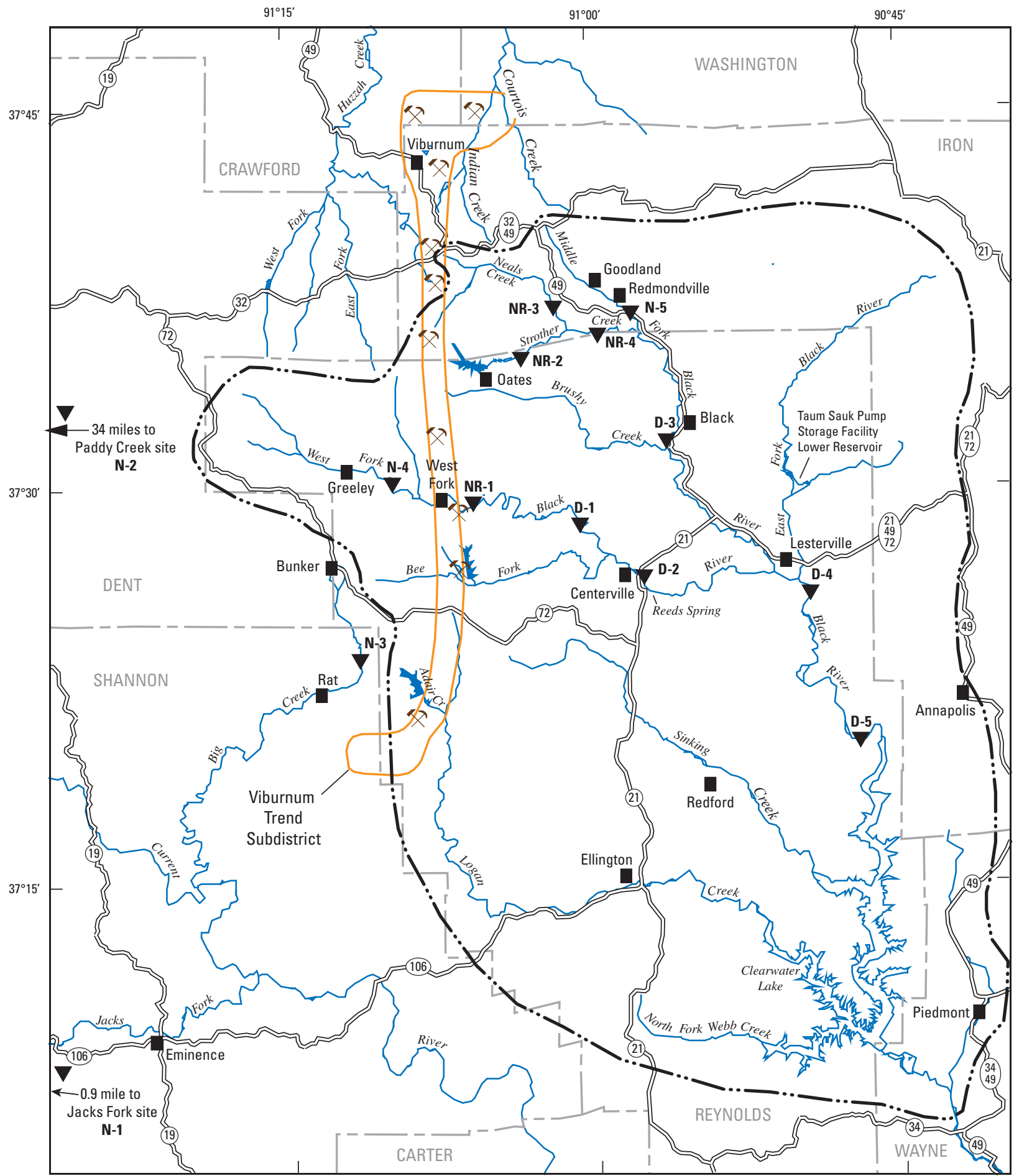

Base from U.S. Geological Survey digital data, 1:100,000, 1983 and 1986 Universal Transverse Mercator projection Zone 15

EXPLANATION

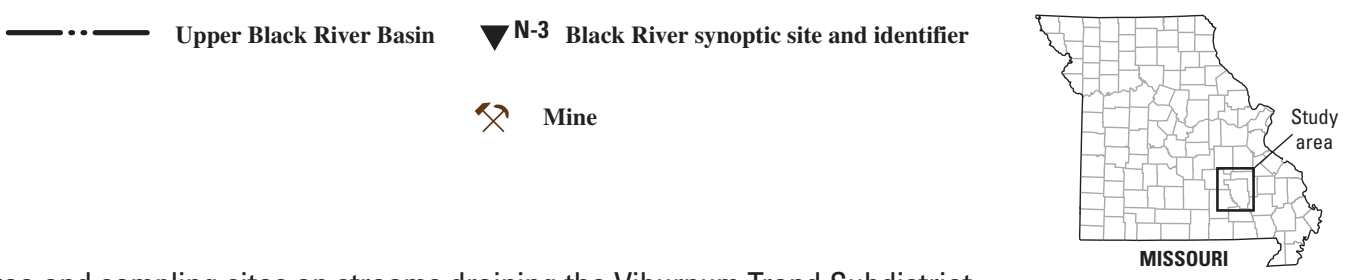

Figure 1. Study area and sampling sites on streams draining the Viburnum Trend Subdistrict.

\begin{tabular}{llllll}
0 & 1 & 2 & 3 & 4 & 5 \\
\hline & MILES & &
\end{tabular}

$\begin{array}{lllllllll}0 & 1 & 1 & 1 & 1 & 1 & 1 & & \\ 0 & 1 & 2 & 3 & 4 & 5 & \text { KILOMETERS }\end{array}$

MISSOURI 3 
Table 1. Black River synoptic site information.

[X, collected; NC, not collected]

\begin{tabular}{|c|c|c|c|c|c|}
\hline \multirow{2}{*}{ Site name and identifier } & \multirow{2}{*}{$\begin{array}{l}\text { Sampling } \\
\text { date }\end{array}$} & \multicolumn{4}{|c|}{ Samples collected } \\
\hline & & Water quality & Streambed sediment & Fish tissue & Invertebrates \\
\hline \multicolumn{6}{|c|}{ Non-mining sites } \\
\hline Jacks Fork River at Alley Spring (N-1) & 08/09/1995 & $\mathrm{X}$ & $\mathrm{X}$ & $\mathrm{X}$ & $\mathrm{X}$ \\
\hline Paddy Creek above Slabtown Spring (N-2) & 08/11/1995 & $\mathrm{x}$ & $\mathrm{x}$ & $\mathrm{X}$ & $\mathrm{X}$ \\
\hline Big Creek near Rat (N-3) & 09/11/1995 & $\mathrm{x}$ & $\mathrm{NC}$ & $\mathrm{NC}$ & $\mathrm{X}$ \\
\hline West Fork Black River near Greeley (N-4) & $09 / 12 / 1995$ & $\mathrm{x}$ & $\mathrm{x}$ & $\mathrm{X}$ & $\mathrm{X}$ \\
\hline Middle Fork Black River at Redmondville (N-5) & 09/14/1995 & $\mathrm{X}$ & $\mathrm{X}$ & $\mathrm{NC}$ & $\mathrm{X}$ \\
\hline \multicolumn{6}{|c|}{ Near-mining sites } \\
\hline West Fork Black River at West Fork (NR-1) & $09 / 11 / 1995$ & $\mathrm{X}$ & $\mathrm{X}$ & $\mathrm{X}$ & $\mathrm{X}$ \\
\hline Strother Creek near Oates (NR-2) & $09 / 19 / 1995$ & $\mathrm{X}$ & $\mathrm{NC}$ & $\mathrm{NC}$ & $\mathrm{X}$ \\
\hline Neals Creek near Goodland (NR-3) & 09/19/1995 & $\mathrm{X}$ & $\mathrm{NC}$ & $\mathrm{NC}$ & $\mathrm{X}$ \\
\hline Strother Creek near Redmondville (NR-4) & 09/18/1995 & $\mathrm{X}$ & $\mathrm{X}$ & $\mathrm{X}$ & $\mathrm{X}$ \\
\hline \multicolumn{6}{|c|}{ Distal-mining sites } \\
\hline West Fork Black River near Centerville (D-1) & 09/13/1995 & $\mathrm{X}$ & $\mathrm{X}$ & $\mathrm{X}$ & $\mathrm{X}$ \\
\hline West Fork Black River at Centerville (D-2) & 09/13/1995 & $\mathrm{X}$ & $\mathrm{X}$ & $\mathrm{X}$ & $\mathrm{X}$ \\
\hline Middle Fork Black River at Black (D-3) & $09 / 14 / 1995$ & $\mathrm{X}$ & $\mathrm{X}$ & $\mathrm{NC}$ & $\mathrm{X}$ \\
\hline Black River near Lesterville (D-4) & $10 / 26 / 1993$ & $\mathrm{NC}$ & $\mathrm{X}$ & $\mathrm{X}$ & $\mathrm{NC}$ \\
\hline Black River near Lesterville (D-4) & $08 / 22 / 1995$ & $\mathrm{NC}$ & $\mathrm{NC}$ & $\mathrm{NC}$ & $\mathrm{X}$ \\
\hline Black River below Annapolis (D-5) & 09/15/1995 & $\mathrm{X}$ & $\mathrm{NC}$ & $\mathrm{NC}$ & $\mathrm{NC}$ \\
\hline
\end{tabular}

$\mathrm{N}-2$ ). Livers were removed from fish at each sampled site and composited until a total mass of $5 \mathrm{~g}$ (ranged from 4 to 12 fish) was attained. Each composited sample was sent to the NWQL for analysis.

Quantitative and qualitative invertebrate community samples were collected at 13 sites. For the quantitative sample, five sampling points were selected at each site in the riffle habitat, which is the most likely to produce the richest community of invertebrates (U.S. Geological Survey, 2003). The samples collected at the five sampling points were combined into one composite sample for analysis at the NWQL. The qualitative invertebrate sample was collected by sampling every available habitat at the sites for 30 minutes. This method is used in an attempt to collect a sample of all present taxa at the site.

\section{Black River Synoptic Study}

Water chemistry, streambed sediment, fish tissue, and invertebrate data were collected at 14 sites in the Salem Plateau physiographic region. These data were analyzed in order to compare and assess mining, non-mining, and near-mining activities on biotic conditions.

\section{Water Chemistry}

Physical properties were determined onsite at 13 sampling locations (table 2, at the end of this chapter). During time of sampling, discharge ranged from 1.5 to 174 cubic feet per second $\left(\mathrm{ft}^{3} / \mathrm{s}\right)$; dissolved oxygen ranged from 4.2 to 9.6 milligrams per liter (mg/L); pH ranged from 7.6 to 8.2 standard $\mathrm{pH}$ units; specific conductance ranged from 109 to 729 microsiemens per centimeter at 25 degrees Celsius $(\mu \mathrm{S} / \mathrm{cm})$; and water temperature ranged from 16.3 to 25.4 degrees Celsius $\left({ }^{\circ} \mathrm{C}\right)$, which tended to be related to air temperature at the time of sampling. E. coli and fecal coliform densities generally were larger at the non-mining sites than at the near-mining or distal-mining sites. Fecal streptococci densities generally were larger at the near-mining sites.

Nutrient analyses included several dissolved and total nitrogen and phosphorus species. Most nutrient concentrations were less than the method reporting level (MRL). The largest concentrations of nitrogen species were detected at the near-mining sites. Dissolved nitrate plus nitrite concentrations ranged from less than 0.05 to $0.25 \mathrm{mg} / \mathrm{L}$ as nitrogen at the non-mining sites, from 0.07 to $0.74 \mathrm{mg} / \mathrm{L}$ at the near-mining sites, and from 0.06 to $0.10 \mathrm{mg} / \mathrm{L}$ at the distal-mining sites. The concentrations above the MRL for dissolved phosphorus were at near-mining or distal-mining sites and for total phosphorus were at non-mining sites (table 2). Although nutrient 
concentrations varied throughout the study area, no concentrations were at levels of concern by state or national standards [Missouri Department of Natural Resources (2005), U.S. Environmental Protection Agency (2004)]. No substantial differences were noted in dissolved or particulate organic carbon concentrations among the sites.

Trace-element analyses included those metals associated with MVT ore deposits and local mining activities. Of the MVT-related metals, only nickel and zinc were detected at concentrations above MRL at the non-mining sites, but at near-mining sites, all seven metals were detected above the MRL at one or more sites. Dissolved copper, nickel, and zinc were detected above the MRL at one or more distal-mining sites. Dissolved lead, nickel, and zinc concentrations were substantially larger at some of the near-mining sites than at the non-mining or distal-mining sites. The MVT-related metals detected at near-mining sites decreased in concentration downstream from the mining activities (distal-mining sites).

Analyses of the non-MVT related elements indicated dissolved aluminum and barium concentrations were detected above the MRL at all sites. Dissolved manganese concentrations were detected at substantially larger concentrations at two near-mining sites NR-1 [141 micrograms per liter $(\mu \mathrm{g} / \mathrm{L})]$ and NR-2 $(57 \mu \mathrm{g} / \mathrm{L})$ than at non-mining or distal-mining sites.

\section{Streambed Sediment}

Streambed-sediment samples were collected at 10 sites, 8 of which are in the Black River Basin, during this synoptic study (fig. 1; table 3). The following discussion emphasizes the MVT-related metals (arsenic, cadmium, cobalt, copper, lead, nickel, and zinc) data. The results for the complete suite of analyzed elements are in chapter 3 of this report.

A couple of trends were observed after grouping the streambed-sediment data into the three proximity-to-mining related categories. The largest concentrations for each of the seven MVT-related metals, except arsenic and copper, were detected at near-mining sites when compared to the non-mining and distal-mining sites (table 3). The largest arsenic and copper concentrations were at the distal-mining Middle Fork Black River at Black (D-3) site, which is downstream from several tributaries with mining activities. The more obvious differences in concentrations at near-mining sites when compared to the other two groups were for cobalt [Strother Creek near Redmondville (site NR-4); 150 milligrams per kilogram $(\mathrm{mg} / \mathrm{kg})$ ], lead [West Fork Black River at West Fork (site NR-1); 950 mg/kg], nickel [Strother Creek near Redmondville (site NR-4); $160 \mathrm{mg} / \mathrm{kg}$ ], and zinc [Strother Creek near Redmondville (site NR-4); 1,200 mg/kg]. The largest mean concentration for each of the seven MVT-related metals occurred at the near-mining sites, followed by the distalmining sites. The smallest mean concentration was for the non-mining sites.
Although the sample set was small, data from streambedsediment samples collected downstream from the West Fork Black River at West Fork (site NR-1) exhibited a continual decrease in cadmium, lead, and zinc concentrations as the distance downstream from mining activity increased (fig. 2). However, arsenic and cobalt concentrations reversed this trend and the concentrations gradually increased as the distance downstream from mining activity increased, possibly because of tributary contributions.

Sediment-quality guidelines were exceeded several times (MacDonald and others, 2000). These guidelines are used to predict concentrations that can cause probable harm to sediment-dwelling organisms in freshwater ecosystems. These guidelines include threshold effect concentrations (TEC) and probable effects concentrations (PEC). TECs are concentrations below which harmful effects are not expected and PECs are concentrations above which harmful effects are expected. For arsenic, cadmium, and copper, no concentrations were above the PEC and most all were below the TEC (table 3). None of the non-mining sites had arsenic, cadmium,
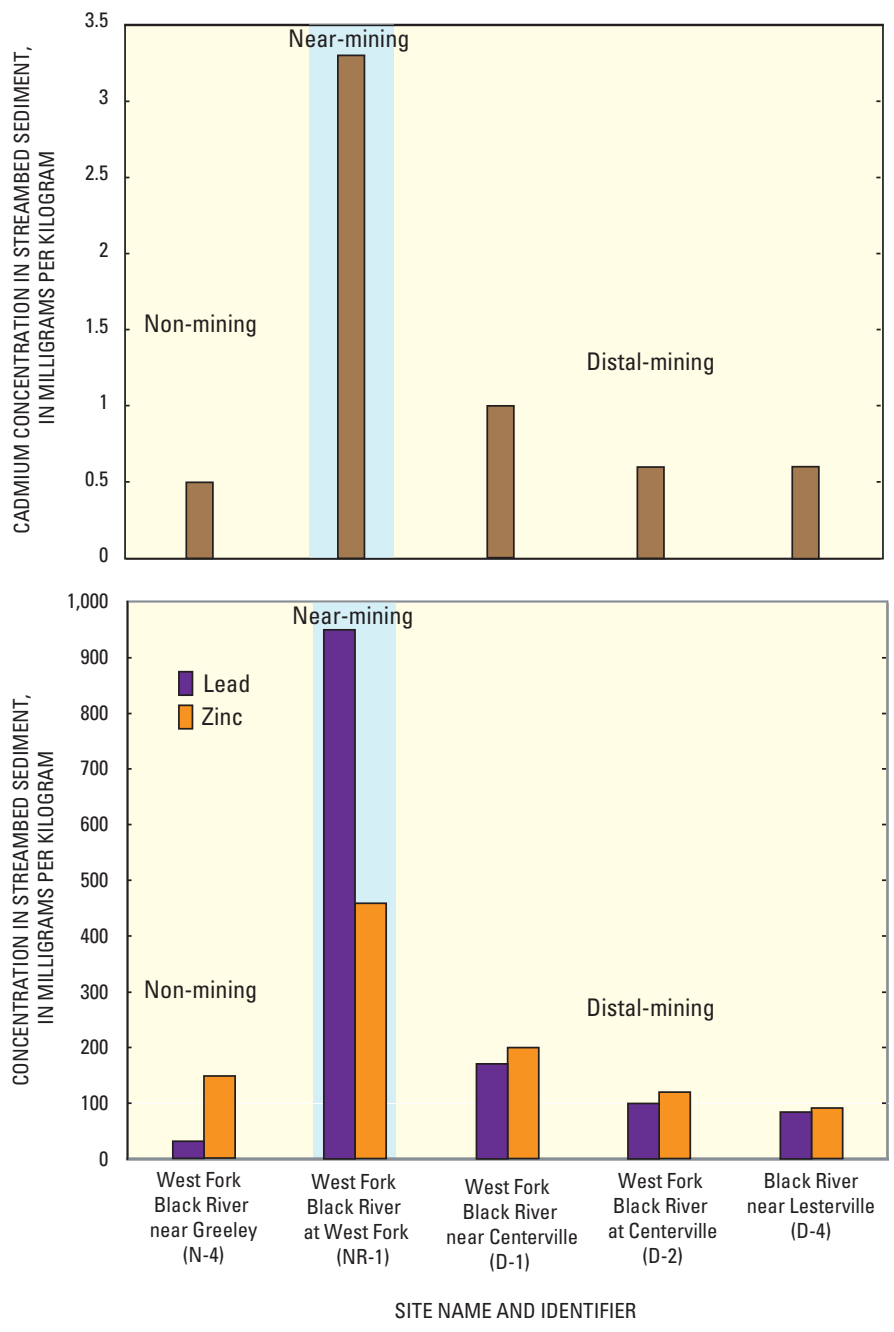

Figure 2. Cadmium, lead, and zinc concentrations in streambed sediment at West Fork Black River sites and downstream. 
Table 3. Streambed sediment and fish tissue data from the National Water-Quality Assessment Program Black River synoptic study.

[mg/kg, milligram per kilogram; (N), non-mining site; (NR), near-mining site; (D), distal-mining site; NA, not available; $\mu \mathrm{g} / \mathrm{g}$, microgram per gram; <, less than]

\begin{tabular}{|c|c|c|c|c|c|c|c|}
\hline \multirow{2}{*}{$\begin{array}{c}\text { Site name and identifier } \\
\text { (fig. 1) }\end{array}$} & \multicolumn{7}{|c|}{ Streambed sediment (mg/kg) } \\
\hline & Arsenic & Cadmium & Cobalt & Copper & Lead & Nickel & Zinc \\
\hline \multicolumn{8}{|c|}{ Non-mining sites } \\
\hline Jacks Fork at Alley Spring (N-1) & 8.4 & 0.4 & 16 & 19 & 24 & 30 & 64 \\
\hline Paddy Creek above Slabtown Spring (N-2) & 4.1 & .2 & 9 & 15 & 27 & 23 & 54 \\
\hline West Fork Black River near Greeley (N-4) & 4.9 & .5 & 16 & 17 & 33 & 21 & 150 \\
\hline Middle Fork Black River at Redmondville (N-5) & 6.9 & .8 & 15 & 26 & 80 & 28 & 180 \\
\hline \multicolumn{8}{|c|}{ Near-mining sites } \\
\hline West Fork Black River at West Fork (NR-1) & 10 & 3.3 & 43 & 36 & 950 & 73 & 460 \\
\hline Strother Creek near Redmondville (NR-4) & 7.2 & 2.2 & 150 & 31 & 200 & 160 & 1,200 \\
\hline \multicolumn{8}{|c|}{ Distal-mining sites } \\
\hline West Fork Black River near Centerville (D-1) & 5.5 & 1.0 & 18 & 22 & 170 & 31 & 200 \\
\hline West Fork Black River at Centerville (D-2) & 6.1 & .6 & 20 & 29 & 100 & 39 & 120 \\
\hline Middle Fork Black River at Black (D-3) & 14 & .8 & 31 & 37 & 100 & 50 & 210 \\
\hline Black River near Lesterville (D-4) & 7.6 & .6 & 25 & 21 & 84 & 29 & 91 \\
\hline Probable effects concentration (PEC) & 33 & 4.98 & NA & 149 & 128 & 48.6 & 459 \\
\hline Threshold effects concentration (TEC) & 9.79 & .99 & NA & 31.6 & 35.8 & 22.7 & 121 \\
\hline \multirow{2}{*}{$\begin{array}{c}\text { Site name and identifier } \\
\text { (fig. 1) }\end{array}$} & \multicolumn{7}{|c|}{ Fish tissue $(\mu \mathrm{g} / \mathrm{g})$} \\
\hline & Arsenic & Cadmium & Cobalt & Copper & Lead & Nickel & Zinc \\
\hline \multicolumn{8}{|c|}{ Non-mining sites } \\
\hline Jacks Fork at Alley Spring (N-1) & 0.5 & 2.2 & 0.8 & 7.2 & $<0.2$ & 0.3 & 71 \\
\hline Paddy Creek above Slabtown Spring (N-2) & .4 & .5 & .5 & 17 & $<.2$ & .2 & 71 \\
\hline West Fork Black River near Greeley (N-4) & .4 & 1.0 & 1.2 & 8.9 & $<.2$ & $<.2$ & 70 \\
\hline \multicolumn{8}{|c|}{ Near-mining sites } \\
\hline West Fork Black River at West Fork (NR-1) & 0.9 & 6.7 & 3.0 & 25 & 8.3 & 2.6 & 86 \\
\hline Strother Creek near Redmondville (NR-4) & .8 & 1.4 & 3.8 & 56 & .7 & 1.2 & 140 \\
\hline \multicolumn{8}{|c|}{ Distal-mining sites } \\
\hline West Fork Black River near Centerville (D-1) & 1.2 & 6.1 & 1.5 & 150 & 0.9 & 1.1 & 110 \\
\hline West Fork Black River at Centerville (D-2) & .6 & 1.6 & 1.0 & 9.5 & .5 & $<.2$ & 70 \\
\hline Black River near Lesterville (D-4) & .3 & 2.4 & .9 & 8.6 & $<.3$ & .4 & 77 \\
\hline
\end{tabular}

and copper concentrations above the TEC. Most non-mining sites had concentrations above the TEC for lead, nickel, and zinc, especially for nickel. The Middle Fork Black River at Redmondville ( site N-5) had above the TEC concentrations for lead, nickel, and zinc, although the concentrations were well below the PEC concentrations. All of the near-mining sites had concentrations of lead, nickel, and zinc above the PEC. All of the distal-mining sites had concentrations above the TEC and several concentrations were above the PEC.

\section{Fish Tissue}

Fish tissue samples (livers) were collected at eight sites, six of which are in the Black River Basin (fig. 1). In this chapter, emphasis is placed only on the analyses for the MVTrelated metals (arsenic, cadmium, cobalt, copper, lead, nickel, and zinc). The results for the complete suite of analyzed elements can be accessed on the internet at (http://waterdata. usgs.gov/nwis). 
The results of the fish tissue sampling were similar to those of the streambed sediment in that the largest concentrations for each of the seven MVT-related metals, except arsenic and copper, were detected at near-mining sites (table 3 ). In general, the largest mean concentration for each of the seven metals, except for copper, was at the near-mining sites, followed by the distal-mining sites; the smallest mean value was for the non-mining sites.

Whereas there appears to be a general trend that cadmium, lead, and zinc concentrations in fish tissue decrease as the stream distance from mining activity increases (fig. 3), the trend is more obscure than the streambed-sediment data. The data from West Fork Black River sites (NR-1, D-1, and D-2) and the Black River near Lesterville site (D-4) indicate cadmium, cobalt, lead, and nickel concentrations were less at the distal-mining sites than at the upstream near-mining sites. However, cadmium and nickel concentrations did not decrease continually downstream from mining activity. In addition, larger arsenic, copper, and zinc concentrations were detected downstream at a distal-mining site (D-1) than at the upstream near-mining site (NR-1).
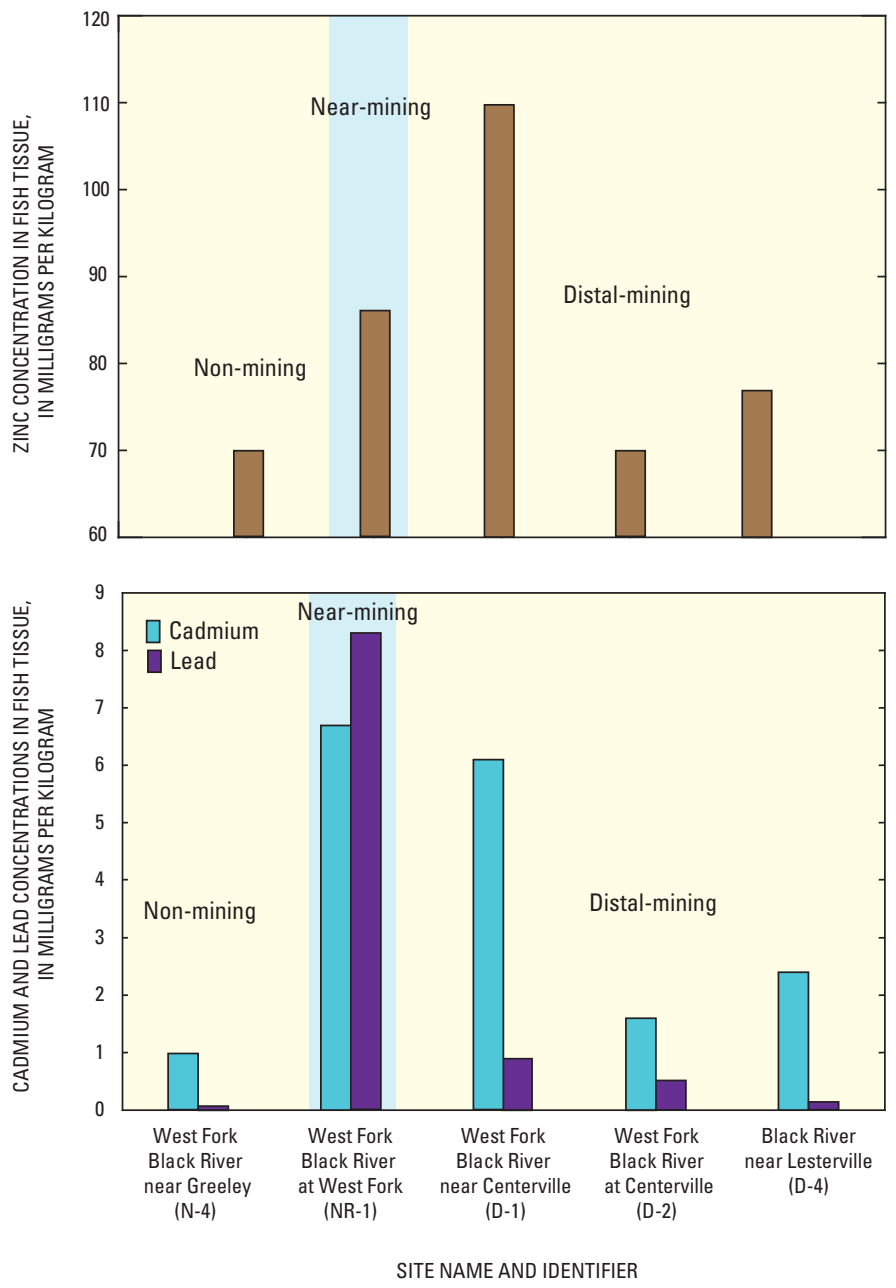

Figure 3. Cadmium, lead, and zinc concentrations in fish tissue at West Fork Black River sites and downstream.

\section{Invertebrate Communities}

Invertebrate samples were collected at 13 sites for community structure analysis. Quantitative and qualitative collections were made (data can be found at http://infotrek.er.usgs. gov/traverself? $p=N A W Q A: H O M E: 3152244205107217)$. A series of metrics were applied to the data to determine community similarities and differences (table 4) (Plafkin and others, 1989; Kerans and Karr, 1994). The metrics applied to the quantitative data include percent EPT (Ephemeroptera, Plecoptera, and Tricoptera) index, taxa richness, family taxa richness, percent dominant taxa, abundance, percent intolerant taxa, and percent sensitive EPT. A taxa richness metric also was applied to the qualitative data (table 4). With the exception of taxa richness, data at the family taxonomic level were used.

The percent EPT index metric, commonly used for stream assessment, is the percentage of the sample that is composed of the invertebrates of these three taxonomic groups [Ephemeroptera, Plecoptera, and Tricoptera (Bode and Novak, 1995)]. Generally, these three taxonomic groups are relatively intolerant of contaminants, so a large EPT score for a sample is an indicator of good stream condition. The percent EPT index metric for this study ranged from 21 (poor score) at Strother Creek near Redmondville (site NR-4) to 71 (better score) at the West Fork Black River near Greeley (site N-4). The mean percent EPT index metric score for this data set was 55 for non-mining sites, 28 for near-mining sites, and 58 for distal-mining sites. A definite reduction of the intolerant species at sites near mining activities existed.

Taxa richness is the number of invertebrate genera that were in each sample. In general, the larger the number of genera, or species diversity, in a sample, the better the stream conditions at the site. The taxa richness ranged from 27 at Strother Creek near Oates (site NR-2) to 72 at Big Creek near Rat (site N-3). The mean taxa richness score was 59 at nonmining sites, 43 at near-mining sites, and 54 at distal-mining sites. The number of invertebrate genera notably decreased at the near-mining sites when compared to the other site categories.

Family taxa richness is similar to taxa richness except that the invertebrates are identified only to the family level. The family taxa richness supported the taxa richness data and verified that species diversity was lower at the sites near mining activities, with recovery occurring downstream.

The percent dominant taxa metric indicates the percent of the most common or dominant taxa of the invertebrate population. It is an indication of imbalance if a single type of invertebrate dominates the community. The percent dominant taxa ranged from 12 at the West Fork Black River at Centerville (site D-2) to 56 at Strother Creek near Redmondville (site NR-4). The mean score was 19 (median of 19) for non-mining sites, 32 (median of 28) for near-mining sites, and 17 (median of 15) for distal-mining sites.

The abundance score is the number of individuals present in each sample. In general, the more individuals, the better the 
Table 4. Selected metrics applied to invertebrate data collected for the National Water-Quality Assessment Program Black River synoptic study.

[EPT, Ephemeroptera, Plecoptera, and Trichoptera; (N), non-mining site; NA, not available; (NR), near-mining site; (D), distal-mining site]

\begin{tabular}{|c|c|c|c|c|c|c|c|c|}
\hline $\begin{array}{l}\text { Site name and identifier } \\
\text { (fig. 1) }\end{array}$ & $\begin{array}{l}\text { Percent } \\
\text { EPT } \\
\text { index }\end{array}$ & $\begin{array}{l}\text { Taxa } \\
\text { rich- } \\
\text { ness }\end{array}$ & $\begin{array}{l}\text { Family } \\
\text { taxa } \\
\text { richness }\end{array}$ & $\begin{array}{c}\text { Percent } \\
\text { dominant } \\
\text { taxa }\end{array}$ & Abundance & $\begin{array}{c}\text { Percent } \\
\text { intolerant } \\
\text { taxa }\end{array}$ & $\begin{array}{l}\text { Percent } \\
\text { sensitive } \\
\text { EPT }\end{array}$ & $\begin{array}{c}\text { Qualitative } \\
\text { taxa } \\
\text { richness }\end{array}$ \\
\hline \multicolumn{9}{|c|}{ Non-mining sites } \\
\hline Jacks Fork River at Alley Spring (N-1) & 59 & 60 & 32 & 15 & 8,681 & 11 & 13 & NA \\
\hline Paddy Creek above Slabtown Spring (N-2) & 34 & 57 & 33 & 23 & 8,319 & 32 & 29 & NA \\
\hline Big Creek near Rat (N-3) & 52 & 72 & 32 & 13 & 9,740 & 7 & 12 & 76 \\
\hline West Fork Black River near Greeley (N-4) & 71 & 62 & 29 & 19 & 9,976 & 18 & 19 & 65 \\
\hline Middle Fork Black River at Redmondville (N-5) & 58 & 42 & 24 & 25 & 19,277 & 30 & 28 & 53 \\
\hline \multicolumn{9}{|c|}{ Near-mining sites } \\
\hline West Fork Black River at West Fork (NR-1) & 40 & 51 & 25 & 24 & 1,900 & 10 & 5 & 49 \\
\hline Strother Creek near Oates (NR-2) & 25 & 27 & 16 & 32 & 3,510 & 1 & 16 & 26 \\
\hline Neals Creek near Goodland (NR-3) & 28 & 43 & 22 & 19 & 9,106 & 11 & 10 & 53 \\
\hline Strother Creek near Redmondville (NR-4) & 21 & 51 & 23 & 56 & 20,075 & 9 & 12 & 62 \\
\hline \multicolumn{9}{|c|}{ Distal-mining sites } \\
\hline West Fork Black River near Centerville (D-1) & 50 & 65 & 33 & 16 & 16,901 & 13 & 15 & 66 \\
\hline West Fork Black River at Centerville (D-2) & 52 & 60 & 27 & 12 & 18,372 & 11 & 11 & 84 \\
\hline Middle Fork Black River at Black (D-3) & 67 & 52 & 30 & 14 & 31,483 & 12 & 13 & 79 \\
\hline Black River near Lesterville (D-4) & 62 & 40 & 19 & 26 & 7,477 & 28 & 27 & 87 \\
\hline
\end{tabular}

stream conditions, although at some sites, an imbalance in the invertebrate population can lead to an excessive number of a single taxa, which skews the metric. In this study, the abundance ranged from 1,900 individuals at West Fork Black River at West Fork (site NR-1) to 31,483 individuals at the Middle Fork Black River at Black (site D-3). The mean abundance score was 11,199 (median of 9,740) at non-mining sites, 8,648 (median of 6,308) at near-mining sites, and 18,558 (median of 17,636) at distal-mining sites.

Tolerance scores have been developed to rank species by the degree of tolerance of the taxon to contaminants. Most of these tolerance scores have been developed specifically for organic contaminants. The tolerance scores used in this study are modified from Hilsenhoff (1987) and data from other streams have been taken into account (Plafkin and others, 1989; Bode and others, 1996; and Mackie, 2001). The tolerance values used in this study have not been specifically developed for the stream conditions in the Black River and are used only for a general comparison. Using these scores, the percent of the sampled taxa that are intolerant to contaminants in the system can be determined. The scores of 0 to 2 were used as intolerant metrics for this study. The type of invertebrates with these low tolerance scores are able to live in contaminated streams. Large scores for the percent intolerant taxa metric indicate better stream conditions. The percent intolerant scores ranged from 1 at Strother Creek near Oates (site NR-2) to 32 at Paddy Creek above Slabtown Spring (site N-2). The largest mean percent intolerant score was for non-mining sites (20), followed by distal-mining sites (16), and the lowest score was for near-mining sites (8). The percent intolerant index scores indicate recovery as the distance downstream from mining increases.

The percent sensitive EPT metric is the percentage of these three taxon with tolerance scores of 0 to 3 . These genera are the most contaminant sensitive species of the EPT community. As with the other metrics, the percent sensitive EPT metric has a smaller mean score at near-mining sites (11) and slightly larger scores for the non-mining (20) and distalmining sites (16). There also is the trend of recovery as the distance downstream from mining activity increases.

The qualitative taxa richness is the number of taxa present in each sample. Because multiple habitats are sampled for a qualitative sample, a greater number of taxa would be expected than in the quantitative sample (taxa richness) where only one type of habitat is sampled. A greater number of qualitative taxa than quantitative taxa (taxa richness) were found at 9 of the 11 sites with available data. Qualitative taxa richness scores ranged from 26 at Strother Creek near Oates (NR-2) to 87 at Black River near Lesterville (D-4). As with the quantitative taxa richness data, the near-mining sites have a smaller mean score (48) than the non-mining (65) and distal-mining sites (79).

The invertebrate community relative abundance data for these sites are illustrated in figure 4. Each part of the 


\section{Non-mining sites}
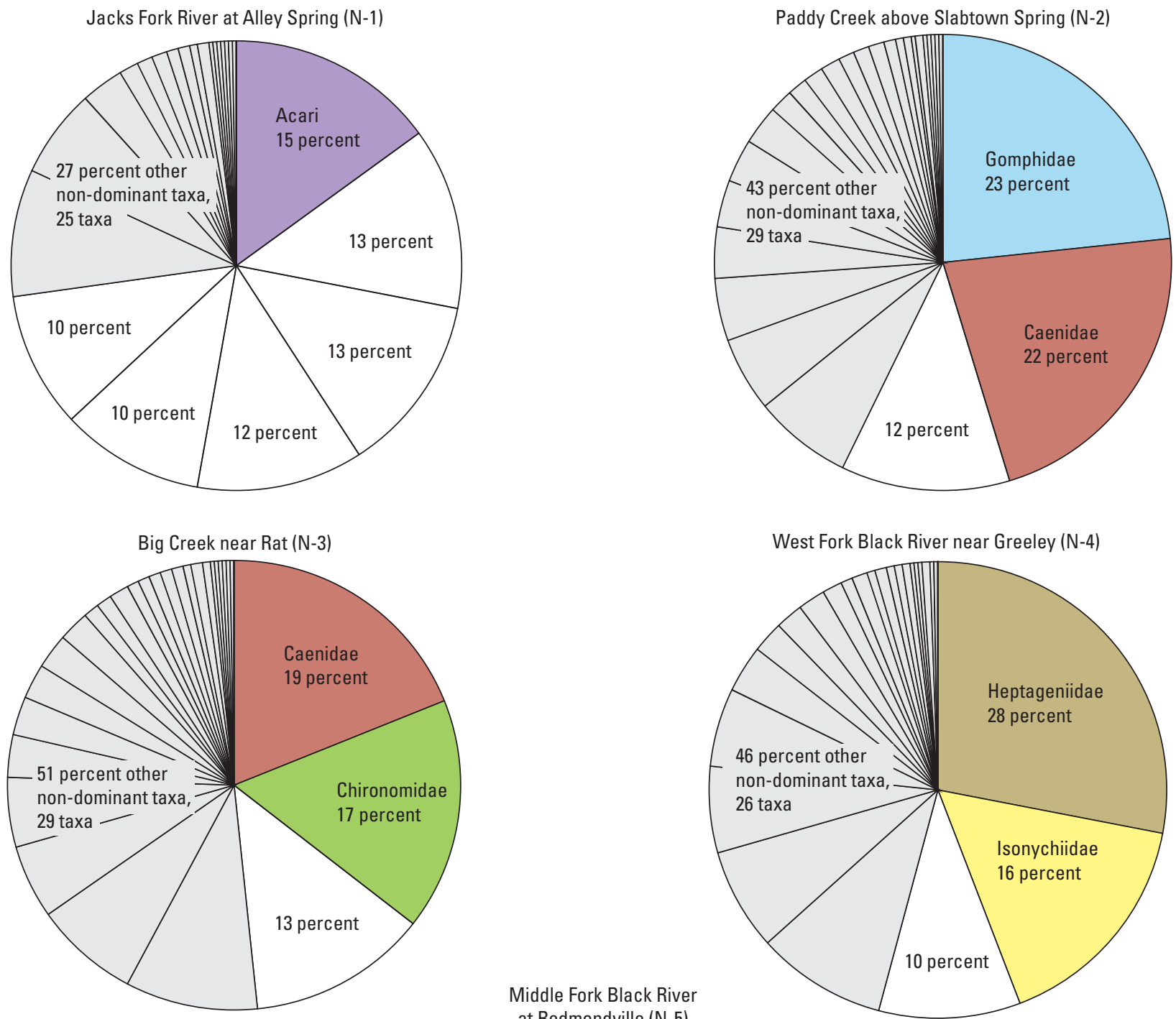

Middle Fork Black River at Redmondville (N-5)

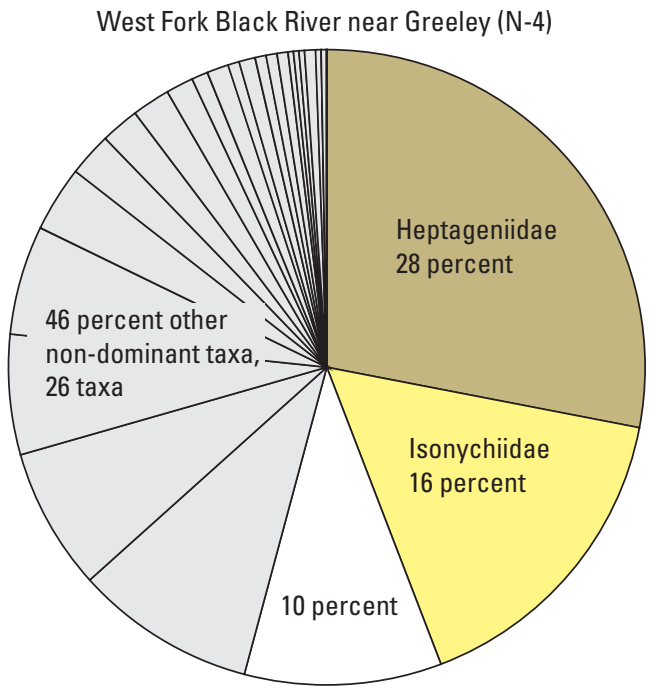

Note: Taxa and relative abundance values presented in table 4. Dominant taxa (greater than 15 percent of total population) shown in color. Non-dominant taxa (10 to 15 percent) shown in white. Non-dominant taxa (less than 10 percent) shown in gray and percentage not listed.

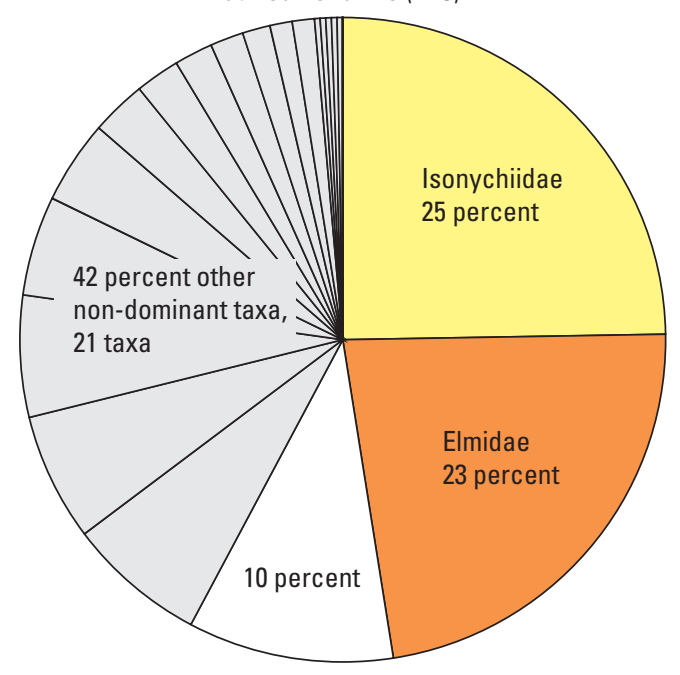

Figure 4. Invertebrate community relative abundance data,1995, showing dominant taxa at each site in relation to community structure. 


\section{Distal-mining sites}

West Fork Black River near Centerville (D-1)

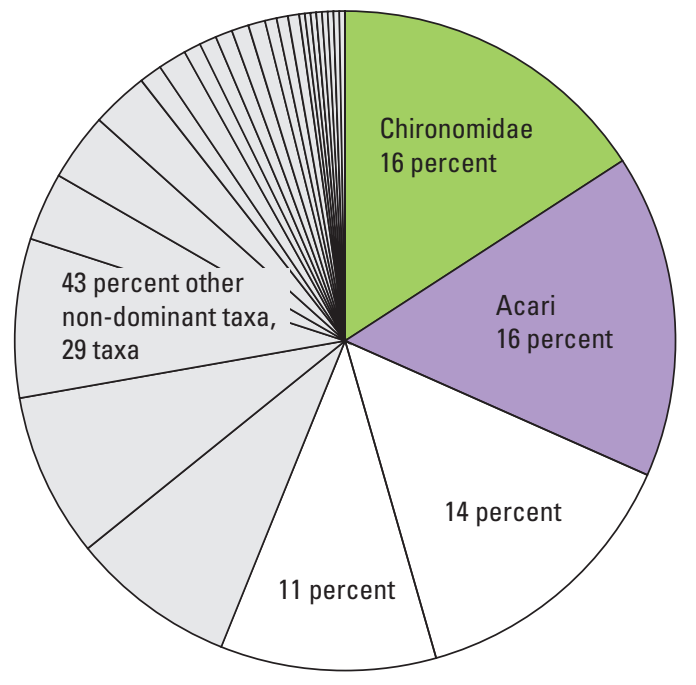

Middle Fork Black River at Black (D-3)

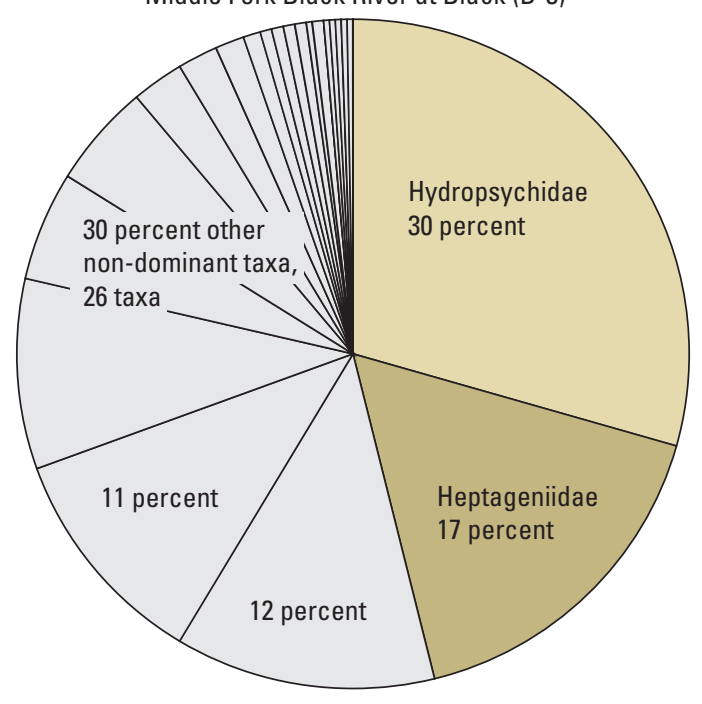

West Fork Black River at Centerville (D-2)

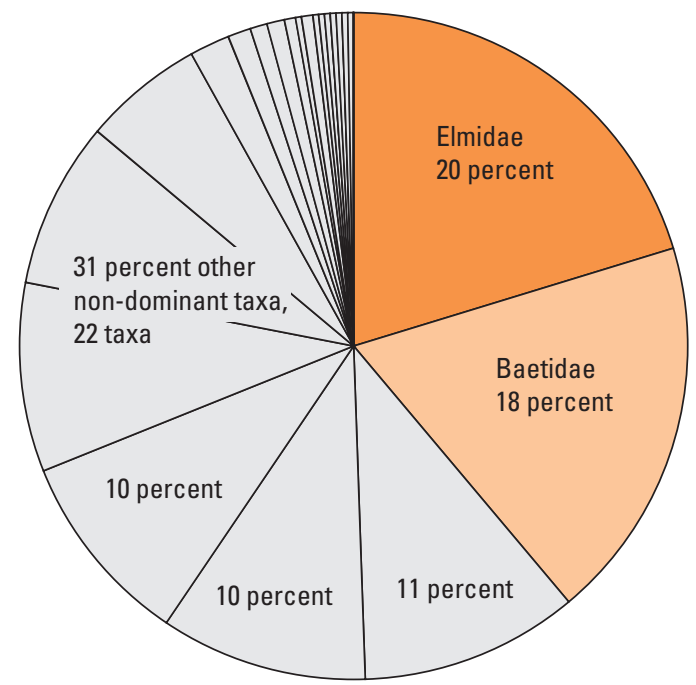

Black River near Lesterville (D-4)

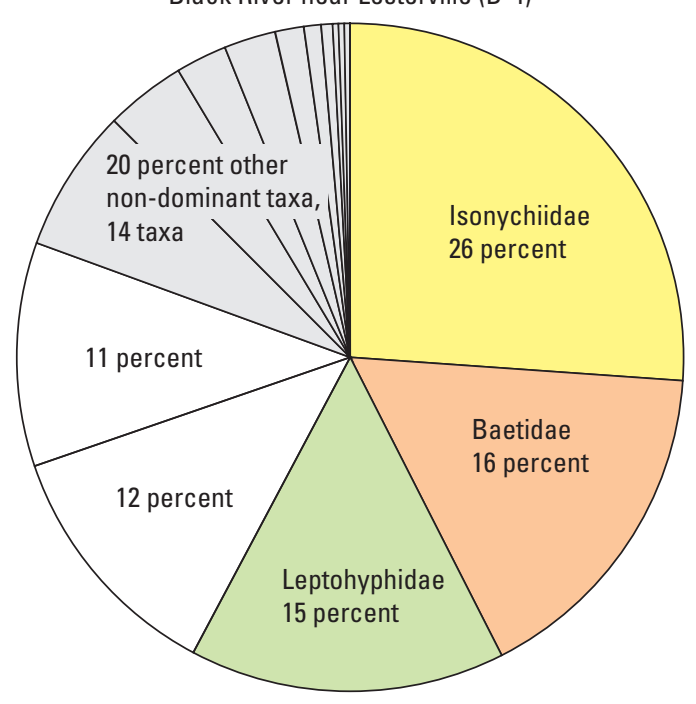

Note: Taxa and relative abundance values presented in table 4. Dominant taxa (greater than 15 percent of total population) shown in color. Non-dominant taxa (10 to 15 percent) shown in white. Non-dominant taxa (less than 10 percent) shown in gray and percentage not listed.

Figure 4 Invertebrate community relative abundance data,1995, showing dominant taxa at each site in relation to community structure.-Continued 


\section{Near-mining sites}

West Fork Black River at West Fork (NR-1)

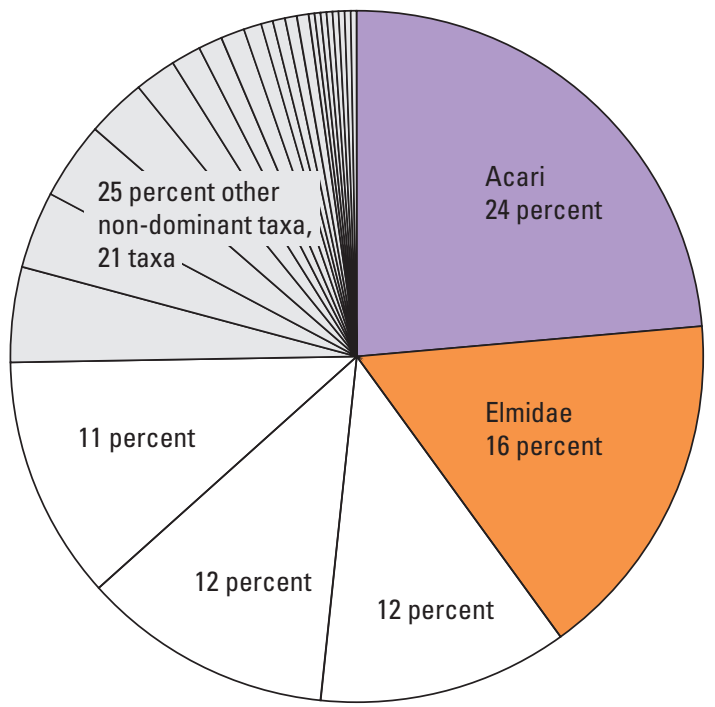

Neals Creek near Goodland (NR-3)

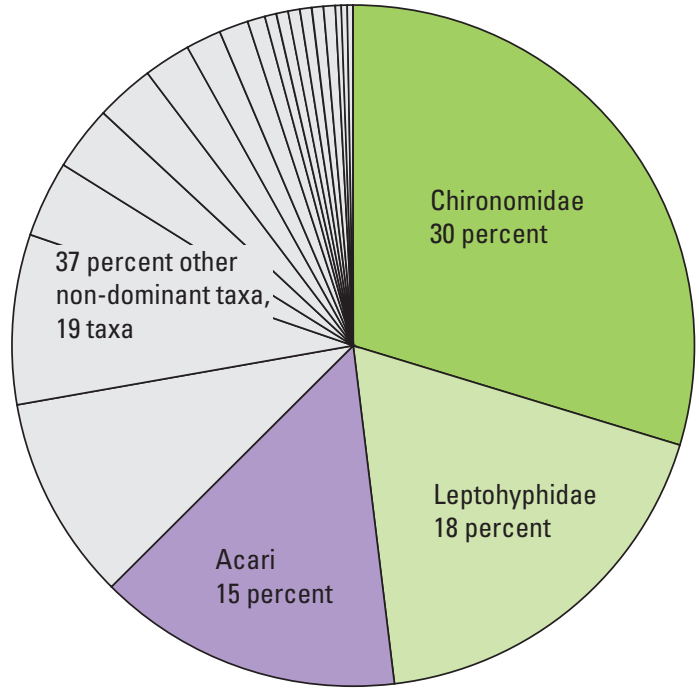

Strother Creek near Oates (NR-2)

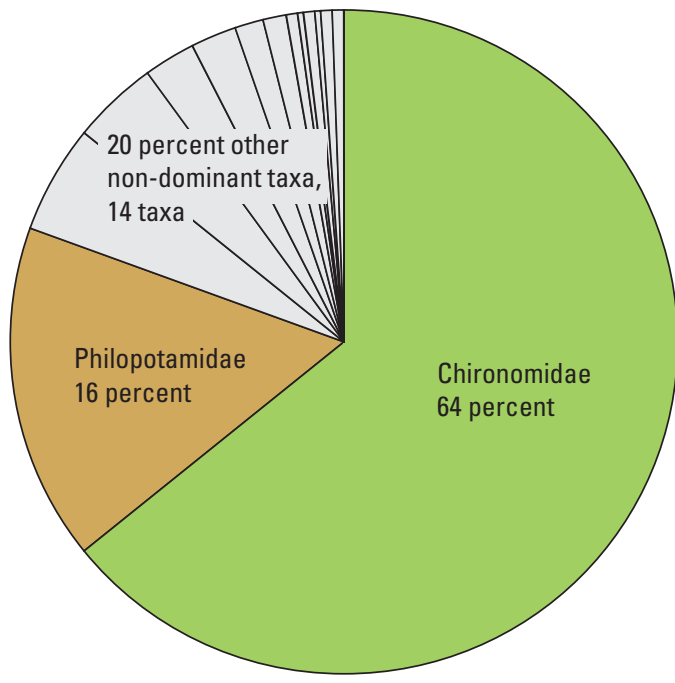

Strother Creek near Redmondville (NR-4)

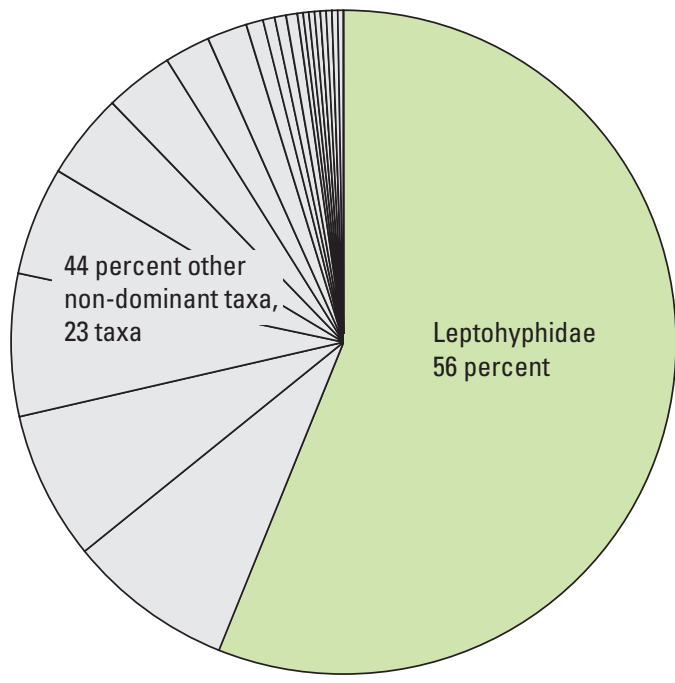

Note: Taxa and relative abundance values presented in table 4. Dominant taxa (greater than 15 percent of total population) shown in color. Non-dominant taxa (10 to 15 percent) shown in white. Non-dominant taxa (less than 10 percent) shown in gray and percentage not listed.

Figure 4 Invertebrate community relative abundance data,1995, showing dominant taxa at each site in relation to community structure.-Continued 
pie-shaped graph represents the percentage of the population of each family collected. For instance, a wedge equaling 25 percent of the pie represents a family that comprised 25 percent of the sampled population. The two near-mining Strother Creek sites (NR-2 and NR-4) have a single family that dominates the community structure. These families also are invertebrates that are tolerant of contaminants in their habitats. The distal-mining and non-mining sites generally did not have an overwhelmingly dominant family present and had a more heterogeneous population.

\section{Summary and Conclusions}

Water chemistry, streambed sediment, fish tissue, and invertebrate data were collected to evaluate the effects of lead and zinc mining and related activities on area streams in the Viburnum Trend Subdistrict. All samples were collected during August to September 1995, except for streambed sediment and fish tissue collected at Black River near Lesterville during October 1993. The metal-sulfide deposits in the Viburnum Trend Subdistrict are a class known as Mississippi Valley Type deposits and include the trace elements (all metals) arsenic, cadmium, cobalt, copper, lead, nickel, and zinc. Eleven sites in the Black River Basin and three sites outside of the basin were grouped according to their proximity to mining activity: non-mining sites, sites upstream from mining activity or mine tailings, or sites where no mining activity is present in the basin; near-mining sites, mining activity or mine tailings ponds within 7.5 miles upstream from the site; and distalmining sites, mining activity greater than 7.5 miles upstream from the site.

The water-quality samples from non-mining sites had small concentrations of Mississippi Valley Type-related metals. With the exception of zinc, the Mississippi Valley Type-related metal concentrations typically were less than the method reporting levels at the non-mining sites. At nearmining sites, Mississippi Valley Type-related metal concentrations frequently were larger than the method reporting levels; however, only dissolved copper, nickel, and zinc were detected at concentrations larger than the method reporting levels at distal-mining sites. Dissolved lead, nickel, and zinc concentrations were substantially larger at the near-mining sites than at the non-mining or distal-mining sites. The Mississippi Valley Type-related metals detected at near-mining sites decreased in concentration downstream from the mining activities (distalmining sites).

Streambed-sediment samples were collected at 10 sites, 8 of which are in the Black River Basin, during this synoptic study. The largest concentrations for each of the seven Mississippi Valley Type-related metals, except arsenic and copper, were detected at near-mining sites. The largest mean concentration for the Mississippi Valley Type-related metals occurred at the near-mining sites, followed by the distal-mining sites; the smallest mean concentration was for the non-mining sites. Generally, arsenic and cobalt concentrations gradually increased as the distance downstream from mining activity increased. Cadmium, lead, and zinc concentrations decreased as the distance downstream from mining activities increased.

Fish tissue samples (livers) were collected at eight sites, six of which are in the Black River Basin. Results were similar to those of the streambed sediment in that the largest concentrations for each of the seven Mississippi Valley Type-related metals, except arsenic and copper, were detected at near-mining sites. The largest mean concentration for each of the seven metals, except for copper, is at the near-mining sites, followed by the distal-mining sites, and the smallest mean value is for the non-mining sites.

Invertebrate samples were collected for community structure analysis. These data indicated a definite reduction of the intolerant species at sites near mining activities.

Invertebrate genera also decreased noticeably at the nearmining sites when compared to the other site categories. The family taxa richness supported these conclusions and verified that species diversity was lower at the sites near mining activities, with recovery occurring downstream. The percent dominant taxa metric had fewer individuals at the sites near mining activities, indicating an imbalance in the invertebrate community. The percent intolerant index scores indicated recovery as the distance downstream from mining increased. As with the other metrics, the percent Ephemeroptera, Plecoptera, and Tricoptera scores indicated a trend of recovery for these three taxonomic groups as the distance from the mining activity increased. The invertebrate community relative abundance data indicated the two near-mining Strother Creek sites have a single family that dominates the community structure. These families are invertebrates that are tolerant of contaminants in their habitats. The distal-mining and non-mining sites generally did not have an overwhelmingly dominant family present and had a more heterogeneous population.

The commonality through all the water chemistry, streambed sediment, fish tissue, and invertebrate data analyses is that where mining activity is occurring in the vicinity of sampling sites, the stream reaches exhibit characteristics of an affected stream. The data also support the lessening effects of these mining activities as the distance downstream from the mine increased.

\section{References}

Barbour, M.T., Gerritsen, J., Snyder, B.D., and Stribling, J.B., 1999, Rapid bioassessment protocols for use in streams and wadeable rivers-Periphyton, benthic macroinvertebrates and fish (2d ed.): Washington, D.C., U.S. Environmental Protection Agency, Office of Water, EPA 841-B-99-002, $339 \mathrm{p}$. 
Bode, R.W., and Novak, M.A., 1995, Development and application of biological impairment criteria for rivers and streams in New York State, in Davis, W.S. and Simon, T.P., eds., Biological assessment and criteria-Tools for water resources planning and decision making: Lewis Publishers, chap. 8, p. 97-107.

Bode, R.W., Novak, M.A., and Abele, L.E., 1996, Quality assurance work plan for biological stream monitoring in New York State: New York State Department of Environmental Conservation, 89 p.

Fenneman, N.M., 1938, Physiography of eastern United States: New York, McGraw-Hill Book Co., 689 p.

Fishman, M.J., ed., 1993, Methods of analysis by the U.S. Geological Survey National Water Quality LaboratoryDetermination of inorganic and organic constituents in water and fluvial sediments: U.S. Geological Survey OpenFile Report 93-125, 217 p.

Fishman, M.J., and Friedman, L.C., 1989, Methods for determination of inorganic substances in water and fluvial sediments: U.S. Geological Survey Techniques of WaterResources Investigations, book 5, chap. A1, 545 p.

Garbarino, J.R., and Struzeski, T.M., 1998, Methods of analysis by the U.S. Geological Survey National Water Quality Laboratory-Determination of elements in whole-water digests using inductively coupled plasma-optical emission spectrometry and inductively coupled plasma-mass spectrometry: U.S. Geological Survey Open-File Report 98-165, $101 \mathrm{p}$.

Hilsenhoff, W.L., 1987, An improved biotic index of organic stream pollution: Great Lakes Entomology, v. 20, p. 31-39.

Kerans, B.L., and Karr, J.R., 1994, A benthic index of integrity (B-IBI) for rivers of the Tennessee Valley: Ecological Applications, v. 4, no. 4, p. 768-785.

MacDonald, D.D., Ingersoll, T.A., and Berger, T.A., 2000, Development and evaluation of consensus-based sediment quality guidelines for freshwater ecosystems, Archives of Environmental Contamination and Toxicology, v. 39, p. 20-31.

Mackie, G.L., 2001, Applied aquatic ecosystem concepts: Dubuque, Iowa, Kendall/Hunt Publishing Co., 744 p.

Missouri Department of Natural Resources, 2005, Missouri water quality standards-Chapter 7, Water quality: Jefferson City, Mo., Clean Water Commission, 136 p.

Omernik, J.M., and Gallant, A.L., 1987, Ecoregions of the south central United States: U.S. Environmental Protection Agency, scale 1:2,500,000.
Plafkin, J.L., Barbour, M.T., Porter, K.D., Gross, S.K., and Hughes, R.M., 1989, Rapid bioassessment protocols for use in streams and rivers-Benthic macroinvertebrates and fish: U.S. Environmental Protection Agency, EPA 440/4-4-89/001, 340 p.

Rickert, D.A., Kennedy, V.C., McKenzie, S.W., and Hines, W.G., 1977, A synoptic survey of trace metals in bottom sediments of the Willamette River, Oregon: U.S. Geological Survey Circular 715-F, 27 p.

U.S. Environmental Protection Agency, 2004, National recommended water quality criteria: Washington, D.C., Office of Water, Office of Science and Technology, EPA/4304T, 22 p.

U.S. Geological Survey, 2003, National Water-Quality Assessment (NAWQA) protocols, accessed March 8, 2007, at URL http://water.usgs.gov/nawqa/protocols/doc_list.html

Wilber, W.G., and Hunter, J.V., 1979, The impact of urbanization on the distribution of heavy metals in bottom sediments of the Saddle River: Water Resources Bulletin, v. 15, no. 3, p. 790-800. 

Table 
Table 2. Water-quality data from the National Water-Quality Assessment Program Black River synoptic study, August and September 1995.

[ft 3 s, cubic feet per second; mg/L, milligrams per liter; $\mu \mathrm{S} / \mathrm{cm}$, microsiemens per centimeter at $25^{\circ} \mathrm{Celsius} ;{ }^{\circ} \mathrm{C}$, degree Celsius; mm, millimeters; $\mathrm{Hg}, \mathrm{mercury} ;(\mathrm{N})$, non-mining site; --, not available; $(\mathrm{NR})$, near-mining site; (D), distal-mining site; $\mathrm{N}$, nitrogen; <, less than; col/100 mL, colonies per 100 milliliters; mL, milliliter; E, estimated; $\mu \mathrm{g} / \mathrm{L}$, micrograms per liter]

\begin{tabular}{|c|c|c|c|c|c|c|c|c|c|c|}
\hline $\begin{array}{c}\text { Site name and identifier } \\
\text { (fig. 1) }\end{array}$ & $\begin{array}{l}\text { Sample } \\
\text { date }\end{array}$ & $\begin{array}{c}\text { Discharge } \\
\left(\mathrm{ft}^{3} / \mathrm{s}\right)\end{array}$ & $\begin{array}{c}\text { Dissolved } \\
\text { oxygen } \\
\text { (mg/L) }\end{array}$ & $\begin{array}{c}\mathrm{pH} \\
\text { (standard } \\
\text { units) }\end{array}$ & $\begin{array}{c}\text { Specific } \\
\text { conductance } \\
(\mu \mathrm{S} / \mathrm{cm})\end{array}$ & $\begin{array}{c}\text { Temperature, } \\
\text { water } \\
\left({ }^{\circ} \mathrm{C}\right)\end{array}$ & $\begin{array}{c}\text { Temperature, } \\
\text { air } \\
\left({ }^{\circ} \mathrm{C}\right)\end{array}$ & $\begin{array}{c}\text { Barometric } \\
\text { pressure } \\
(\mathrm{mm} \text { of } \mathrm{Hg})\end{array}$ & $\begin{array}{c}\text { Calcium, } \\
\text { dissolved } \\
\text { (mg/L) }\end{array}$ & $\begin{array}{c}\text { Magnesium, } \\
\text { dissolved } \\
\text { (mg/L) }\end{array}$ \\
\hline & \multicolumn{10}{|c|}{ Non-mining sites } \\
\hline Jacks Fork River at Alley Spring (N-1) & $08 / 09 / 1995$ & 124.0 & 8.1 & 8.2 & 306 & 25.4 & 33.0 & 770 & 32 & 20.0 \\
\hline Paddy Creek upstream from Slabtown (N-2) & 08/11/1995 & 1.5 & 6.4 & 8.0 & 295 & 22.3 & 32.0 & 762 & 32 & 18.0 \\
\hline Big Creek near Rat (N-3) & 09/11/1995 & 1.6 & 4.2 & 7.6 & 109 & 21.0 & 22.2 & 745 & 11 & 5.6 \\
\hline West Fork Black River near Greeley (N-4) & 09/12/1995 & 10.0 & 8.9 & 8.1 & 343 & 17.3 & -- & 761 & 37 & 22.0 \\
\hline \multirow[t]{2}{*}{ Middle Fork Black River at Redmondville (N-5) } & 09/14/1995 & 5.1 & 9.6 & 8.2 & 319 & 17.7 & 17.5 & 767 & 35 & 20.0 \\
\hline & \multicolumn{10}{|c|}{ Near-mining sites } \\
\hline West Fork Black River at West Fork (NR-1) & $09 / 11 / 1995$ & 23.0 & 8.8 & 8.2 & 416 & 23.1 & 24.3 & 746 & 39 & 25.0 \\
\hline Strother Creek near Oates (NR-2) & 09/19/1995 & 9.9 & 8.6 & 8.1 & 729 & 19.7 & 20.0 & 759 & 64 & 44.0 \\
\hline Neals Creek near Goodland (NR-3) & 09/19/1995 & 4.1 & 8.4 & 8.1 & 407 & 16.3 & 14.5 & 761 & 43 & 27.0 \\
\hline \multirow{2}{*}{ Strother Creek near Redmondville (NR-4) } & 09/18/1995 & 18.0 & 9.4 & 8.0 & 558 & 18.6 & 16.0 & 766 & 51 & 34.0 \\
\hline & \multicolumn{10}{|c|}{ Distal-mining sites } \\
\hline West Fork Black River near Centerville (D-1) & $09 / 13 / 1995$ & 33.0 & 8.8 & 8.2 & 365 & 18.2 & 20.5 & 766 & 36 & 22.0 \\
\hline West Fork Black River at Centerville (D-2) & 09/13/1995 & 54.0 & 8.2 & 8.2 & 345 & 20.5 & 21.1 & 750 & 34 & 21.0 \\
\hline Middle Fork Black River at Black (D-3) & 09/14/1995 & 33.0 & 7.8 & 7.9 & 403 & 21.2 & 20.3 & 752 & 39 & 24.0 \\
\hline \multirow{3}{*}{$\begin{array}{c}\text { Black River near Annapolis (D-5) } \\
\text { Site name and identifier } \\
\text { (fig. 1) }\end{array}$} & $09 / 15 / 1995$ & 174.0 & 9.3 & 8.0 & 295 & 21.3 & 22.8 & 757 & 30 & 18.0 \\
\hline & $\begin{array}{l}\text { Potas- } \\
\text { sium, } \\
\text { dissolved } \\
\text { (mg/L) }\end{array}$ & $\begin{array}{c}\text { Sodium, } \\
\text { dissolved } \\
\text { (mg/L) }\end{array}$ & $\begin{array}{c}\text { Alkalinity } \\
\text { (mg/L) }\end{array}$ & $\begin{array}{l}\text { Chloride } \\
\text { dissolved } \\
\text { (mg/L) }\end{array}$ & $\begin{array}{l}\text { Fluoride } \\
\text { dissolved } \\
\text { (mg/L) }\end{array}$ & $\begin{array}{c}\text { Silica, } \\
\text { dissolved } \\
\text { (mg/L) }\end{array}$ & $\begin{array}{c}\text { Sulfate, } \\
\text { dissolved } \\
\text { (mg/L) }\end{array}$ & $\begin{array}{l}\text { Ammonia } \\
\text { plus organics } \\
\text { dissolved } \\
\text { (mg/L as } \mathrm{N} \text { ) }\end{array}$ & $\begin{array}{l}\text { Ammonia } \\
\text { plus } \\
\text { organics, } \\
\text { total } \\
\text { (mg/L as } \mathrm{N} \text { ) }\end{array}$ & $\begin{array}{c}\text { Ammonia, } \\
\text { dissolved } \\
\text { (mg/L as N) }\end{array}$ \\
\hline & \multicolumn{10}{|c|}{ Non-mining sites } \\
\hline Jacks Fork River at Alley Spring (N-1) & 1.7 & 1.3 & 159 & 2.0 & $<0.1$ & 9.4 & 2.4 & $<0.02$ & $<0.02$ & $<0.01$ \\
\hline Paddy Creek upstream from Slabtown (N-2) & 1.2 & 1.4 & 154 & 1.5 & $<.1$ & 10.0 & 2.7 & $<.02$ & $<.02$ & $<.01$ \\
\hline Big Creek near Rat (N-3) & 1.3 & 1.4 & 46 & 1.5 & .1 & 6.6 & 2.7 & $<.02$ & $<.02$ & .05 \\
\hline West Fork Black River near Greeley (N-4) & 0.8 & 1.5 & 185 & 1.8 & $<.1$ & 8.0 & 2.0 & $<.02$ & $<.02$ & $<.01$ \\
\hline \multirow{2}{*}{ Middle Fork Black River at Redmondville (N-5) } & 0.9 & 1.3 & 160 & 1.4 & $<.1$ & 9.3 & 2.7 & $<.02$ & $<.02$ & .02 \\
\hline & \multicolumn{10}{|c|}{ Near-mining sites } \\
\hline West Fork Black River at West Fork (NR-1) & 1.9 & 8.2 & 160 & 10 & 0.2 & 7.9 & 38 & $<0.02$ & $<0.02$ & 0.05 \\
\hline Strother Creek near Oates (NR-2) & 4.8 & 17.0 & 136 & 24 & .2 & 6.2 & 200 & $<.02$ & .20 & .04 \\
\hline Neals Creek near Goodland (NR-3) & 1.0 & 1.9 & 197 & 1.9 & $<.1$ & 8.0 & 14 & $<.02$ & $<.02$ & $<.01$ \\
\hline \multirow[t]{2}{*}{ Strother Creek near Redmondville (NR-4) } & 3.0 & 10.0 & 175 & 13 & .1 & 7.0 & 120 & $<.02$ & $<.02$ & .01 \\
\hline & \multicolumn{10}{|c|}{ Distal-mining sites } \\
\hline West Fork Black River near Centerville (D-1) & 1.3 & 5.1 & 151 & 6.6 & $<0.1$ & 7.2 & 23 & $<0.02$ & $<0.02$ & $<0.01$ \\
\hline West Fork Black River at Centerville (D-2) & 1.3 & 5.0 & 151 & 5.7 & .1 & 7.3 & 20 & $<.02$ & $<.02$ & $<.01$ \\
\hline Middle Fork Black River at Black (D-3) & 1.9 & 5.2 & 137 & 7.3 & $<.1$ & 8.1 & 58 & $<.02$ & $<.02$ & $<.01$ \\
\hline Black River near Annapolis (D-5) & 1.2 & 2.7 & 131 & 3.6 & $<.1$ & 7.3 & 14 & $<.02$ & $<.02$ & $<.01$ \\
\hline
\end{tabular}


Table 2. Water-quality data from the National Water Quality Assessment program Black River synoptic study, August and September 1995.—Continued

$\left[\mathrm{ft}^{3} / \mathrm{s}\right.$, cubic feet per second; mg/L, milligrams per liter; $\mu \mathrm{S} / \mathrm{cm}$, microsiemens per centimeter at $25^{\circ} \mathrm{Celsius} ;{ }^{\circ} \mathrm{C}$, degree Celsius; mm, millimeters; $\mathrm{Hg}$, mercury; (N), non-mining site; --, not available; (NR), near-mining site; (D), distal-mining site; $\mathrm{N}$, nitrogen; <, less than; col/100 mL, colonies per 100 milliliters; mL, milliliter; E, estimated; $\mu \mathrm{g} / \mathrm{L}$, micrograms per liter]

\begin{tabular}{|c|c|c|c|c|c|c|c|c|c|c|}
\hline $\begin{array}{c}\text { Site name and identifier } \\
\text { (fig. 1) }\end{array}$ & $\begin{array}{l}\text { Nitrite plus } \\
\text { nitrate } \\
\text { dissolved } \\
\text { (mg/L as } \mathrm{N} \text { ) }\end{array}$ & $\begin{array}{c}\text { Nitrite, } \\
\text { dissolved } \\
\text { (mg/L as } N \text { ) }\end{array}$ & $\begin{array}{l}\text { Ortho- } \\
\text { phosphate, } \\
\text { dissolved } \\
\text { (mg/L) }\end{array}$ & $\begin{array}{l}\text { Phosphorus, } \\
\text { dissolved } \\
\text { (mg/L) }\end{array}$ & $\begin{array}{c}\text { Phosphorus, } \\
\text { total } \\
\text { (mg/L) }\end{array}$ & $\begin{array}{l}\text { Carbon, } \\
\text { organic, } \\
\text { dissolved } \\
\text { (mg/L) }\end{array}$ & $\begin{array}{l}\text { Carbon, } \\
\text { organic, } \\
\text { particulate, } \\
\text { total }(\mathrm{mg} / \mathrm{L})\end{array}$ & $\begin{array}{l}\text { Escherichia } \\
\text { coli } \\
\text { (col/100 mL) }\end{array}$ & $\begin{array}{c}\text { Fecal } \\
\text { coliform } \\
\text { (col/100 mL) }\end{array}$ & $\begin{array}{c}\text { Fecal } \\
\text { streptococci } \\
\text { (col/100 mL) }\end{array}$ \\
\hline & \multicolumn{10}{|c|}{ Non-mining sites } \\
\hline Jacks Fork River at Alley Spring (N-1) & 0.17 & $<0.01$ & $<0.01$ & $<0.01$ & 0.02 & 1.0 & 0.1 & 37 & 42 & 17 \\
\hline Paddy Creek upstream from Slabtown (N-2) & $<.05$ & $<.01$ & $<.01$ & $<.01$ & .02 & .9 & .1 & 27 & 20 & 41 \\
\hline Big Creek near Rat (N-3) & .25 & $<.01$ & $<.01$ & $<.01$ & $<.01$ & .7 & .1 & 120 & 86 & 37 \\
\hline West Fork Black River near Greeley (N-4) & $<.05$ & $<.01$ & $<.01$ & $<.01$ & .02 & .4 & .2 & 37 & 54 & 61 \\
\hline \multirow[t]{2}{*}{ Middle Fork Black River at Redmondville (N-5) } & .06 & $<.01$ & $<.01$ & $<.01$ & $<.01$ & .5 & .2 & 44 & 34 & 50 \\
\hline & \multicolumn{10}{|c|}{ Near-mining sites } \\
\hline West Fork Black River at West Fork (NR-1) & 0.25 & 0.01 & $<0.01$ & $<0.01$ & $<0.01$ & 0.5 & 0.2 & 8 & 7 & 22 \\
\hline Strother Creek near Oates (NR-2) & .74 & .01 & $<.01$ & .02 & $<.01$ & 1.1 & .3 & 11 & 25 & 85 \\
\hline Neals Creek near Goodland (NR-3) & .07 & $<.01$ & $<.01$ & $<.01$ & $<.01$ & .5 & .1 & 18 & 14 & 120 \\
\hline \multirow{2}{*}{ Strother Creek near Redmondville (NR-4) } & .17 & .01 & $<.01$ & .01 & $<.01$ & $<.5$ & $<.1$ & $<10$ & $<14$ & E48 \\
\hline & \multicolumn{10}{|c|}{ Distal-mining sites } \\
\hline West Fork Black River near Centerville (D-1) & 0.09 & $<0.01$ & $<0.01$ & $<0.01$ & $<0.01$ & 0.5 & 0.2 & 12 & 24 & 42 \\
\hline West Fork Black River at Centerville (D-2) & .08 & $<.01$ & $<.01$ & .02 & $<.01$ & .4 & .1 & 15 & 5 & 14 \\
\hline Middle Fork Black River at Black (D-3) & .10 & $<.01$ & $<.01$ & $<.01$ & $<.01$ & .4 & .1 & 10 & 9 & 22 \\
\hline Black River near Annapolis (D-5) & .06 & $<.01$ & $<.01$ & $<.01$ & $<.01$ & .4 & .1 & E10 & E4 & 77 \\
\hline \multirow[t]{2}{*}{$\begin{array}{c}\text { Site name and identifier } \\
\text { (fig. 1) }\end{array}$} & $\begin{array}{l}\text { Antimony, } \\
\text { dissolved } \\
(\mu \mathrm{g} / \mathrm{L})\end{array}$ & $\begin{array}{l}\text { Aluminum, } \\
\text { dissolved } \\
(\mu \mathrm{g} / \mathrm{L})\end{array}$ & $\begin{array}{l}\text { Arsenica, } \\
\text { dissolved } \\
(\mu \mathrm{g} / \mathrm{L})\end{array}$ & $\begin{array}{c}\text { Barium, } \\
\text { dissolved } \\
(\mu \mathrm{g} / \mathrm{L})\end{array}$ & $\begin{array}{l}\text { Beryllium, } \\
\text { dissolved } \\
(\mu \mathrm{g} / \mathrm{L})\end{array}$ & $\begin{array}{c}\text { Cadmiuma, } \\
\text { dissolved } \\
(\mu \mathrm{g} / \mathrm{L})\end{array}$ & $\begin{array}{l}\text { Chromium, } \\
\text { dissolved } \\
(\mu \mathrm{g} / \mathrm{L})\end{array}$ & $\begin{array}{c}\text { Cobalt }{ }^{\mathrm{a}} \text {, } \\
\text { dissolved } \\
(\mu \mathrm{g} / \mathrm{L})\end{array}$ & $\begin{array}{c}\text { Copper }^{\mathrm{a}} \\
\text { dissolved } \\
(\mu \mathrm{g} / \mathrm{L})\end{array}$ & $\begin{array}{c}\text { Iron, } \\
\text { dissolved } \\
(\mu \mathrm{g} / \mathrm{L})\end{array}$ \\
\hline & \multicolumn{10}{|c|}{ Non-mining sites } \\
\hline Jacks Fork River at Alley Spring (N-1) & $<1$ & 6 & $<1$ & 35 & $<1$ & $<1$ & 1 & $<1$ & $<1$ & $<3$ \\
\hline Paddy Creek upstream from Slabtown (N-2) & $<1$ & 5 & $<1$ & 54 & $<1$ & $<1$ & 1 & $<1$ & $<1$ & 7 \\
\hline Big Creek near Rat (N-3) & $<1$ & 12 & $<1$ & 40 & $<1$ & $<1$ & $<1$ & $<1$ & $<1$ & 10 \\
\hline West Fork Black River near Greeley (N-4) & $<1$ & 5 & $<1$ & 40 & $<1$ & $<1$ & 2 & $<1$ & $<1$ & $<3$ \\
\hline \multirow[t]{2}{*}{ Middle Fork Black River at Redmondville (N-5) } & $<1$ & 4 & $<1$ & 43 & $<1$ & $<1$ & 1 & $<1$ & $<1$ & $<3$ \\
\hline & \multicolumn{10}{|c|}{ Near-mining sites } \\
\hline West Fork Black River at West Fork (NR-1) & $<1$ & 8 & 3 & 41 & $<1$ & $<1$ & 2 & 6 & 1 & 28 \\
\hline Strother Creek near Oates (NR-2) & 2 & 6 & 2 & 31 & $<1$ & $<1$ & 2 & 31 & 2 & 19 \\
\hline Neals Creek near Goodland (NR-3) & $<1$ & 5 & $<1$ & 30 & $<1$ & $<1$ & $<1$ & $<1$ & $<1$ & $<3$ \\
\hline \multirow[t]{2}{*}{ Strother Creek near Redmondville (NR-4) } & $<1$ & 6 & 1 & 46 & $<1$ & 1 & $<1$ & $<1$ & $<1$ & $<3$ \\
\hline & \multicolumn{10}{|c|}{ Distal-mining sites } \\
\hline West Fork Black River near Centerville (D-1) & $<1$ & 5 & $<1$ & 39 & $<1$ & $<1$ & 1 & $<1$ & $<1$ & $<3$ \\
\hline West Fork Black River at Centerville (D-2) & $<1$ & 5 & $<1$ & 39 & $<1$ & $<1$ & 1 & $<1$ & 1 & $<3$ \\
\hline Middle Fork Black River at Black (D-3) & $<1$ & 5 & $<1$ & 50 & $<1$ & $<1$ & 1 & $<1$ & 1 & $<3$ \\
\hline Black River near Annapolis (D-5) & $<1$ & 6 & $<1$ & 39 & $<1$ & $<1$ & 2 & $<1$ & 1 & $<3$ \\
\hline
\end{tabular}


Table 2. Water-quality data from the National Water Quality Assessment program Black River synoptic study, August and September 1995.—Continued

$\left[\mathrm{ft} \mathrm{t}^{3} \mathrm{~s}\right.$, cubic feet per second; $\mathrm{mg} / \mathrm{L}$, milligrams per liter; $\mu \mathrm{S} / \mathrm{cm}$, microsiemens per centimeter at $25^{\circ} \mathrm{Celsius;}{ }^{\circ} \mathrm{C}$, degree Celsius; $\mathrm{mm}$, millimeters; $\mathrm{Hg}$, mercury; (N), non-mining site; --, not available; (NR), near-mining site; (D), distal-mining site; $\mathrm{N}$, nitrogen; <, less than; col/100 mL, colonies per 100 milliliters; $\mathrm{mL}$, milliliter; $\mathrm{E}$, estimated; $\mu \mathrm{g} / \mathrm{L}$, micrograms per liter]

\begin{tabular}{|c|c|c|c|c|c|c|c|c|c|}
\hline $\begin{array}{c}\text { Site and identifier } \\
\text { (fig. 1) }\end{array}$ & $\begin{array}{c}\text { Lead }^{\mathrm{a}}, \\
\text { dissolved } \\
(\mu \mathrm{g} / \mathrm{L})\end{array}$ & $\begin{array}{c}\text { Manganese, } \\
\text { dissolved } \\
\text { ( } \mu \mathrm{g} / \mathrm{L})\end{array}$ & $\begin{array}{l}\text { Molybdenum, } \\
\text { dissolved } \\
\text { ( } \mu \mathrm{g} / \mathrm{L})\end{array}$ & $\begin{array}{c}\text { Nickel }^{\mathrm{a}}, \\
\text { dissolved } \\
(\mu \mathrm{g} / \mathrm{L})\end{array}$ & $\begin{array}{c}\text { Selenium, } \\
\text { dissolved } \\
(\mu \mathrm{g} / \mathrm{L})\end{array}$ & $\begin{array}{c}\text { Silver, } \\
\text { dissolved } \\
(\mu \mathrm{g} / \mathrm{L})\end{array}$ & $\begin{array}{l}\text { Uranium, } \\
\text { dissolved } \\
(\mu \mathrm{g} / \mathrm{L})\end{array}$ & $\begin{array}{c}\text { Zinc }^{a}, \\
\text { dissolved } \\
\text { ( } \mu \mathrm{g} / \mathrm{L})\end{array}$ & $\begin{array}{l}\text { Suspended } \\
\text { sediment } \\
\text { (mg/L) }\end{array}$ \\
\hline & \multicolumn{9}{|c|}{ Non-mining sites } \\
\hline Jacks Fork River at Alley Spring (N-1) & $<1$ & 2 & $<1$ & 2 & $<1$ & $<1$ & -- & 1 & 298 \\
\hline Paddy Creek upstream from Slabtown (N-2) & $<1$ & 8 & $<1$ & 2 & $<1$ & $<1$ & -- & 1 & -- \\
\hline Big Creek near Rat (N-3) & $<1$ & 12 & $<1$ & $<1$ & $<1$ & $<1$ & $<1$ & 8 & 15 \\
\hline West Fork Black River near Greeley (N-4) & $<1$ & 2 & $<1$ & $<1$ & $<1$ & $<1$ & $<1$ & 13 & 29 \\
\hline \multirow[t]{2}{*}{ Middle Fork Black River at Redmondville (N-5) } & $<1$ & 2 & $<1$ & $<1$ & $<1$ & $<1$ & $<1$ & 7 & 22 \\
\hline & \multicolumn{9}{|c|}{ Near-mining sites } \\
\hline West Fork Black River at West Fork (NR-1) & 11 & 141 & 3 & 12 & $<1$ & $<1$ & $<1$ & 33 & 20 \\
\hline Strother Creek near Oates (NR-2) & 3 & 57 & 13 & 114 & $<1$ & $<1$ & $<1$ & 148 & 51 \\
\hline Neals Creek near Goodland (NR-3) & $<1$ & $<1$ & 1 & 7 & $<1$ & $<1$ & $<1$ & 47 & 20 \\
\hline \multirow[t]{2}{*}{ Strother Creek near Redmondville (NR-4) } & 1 & $<1$ & 3 & 10 & $<1$ & 1 & 1 & 33 & 42 \\
\hline & \multicolumn{9}{|c|}{ Distal-mining sites } \\
\hline West Fork Black River near Centerville (D-1) & $<1$ & 2 & 1 & $<1$ & $<1$ & $<1$ & $<1$ & 13 & 19 \\
\hline West Fork Black River at Centerville (D-2) & $<1$ & 2 & 1 & $<1$ & $<1$ & $<1$ & $<1$ & 2 & 36 \\
\hline Middle Fork Black River at Black (D-3) & $<1$ & 1 & 2 & 1 & $<1$ & $<1$ & $<1$ & 3 & 40 \\
\hline Black River near Annapolis (D-5) & $<1$ & 3 & $<1$ & $<1$ & $<1$ & $<1$ & $<1$ & 2 & 25 \\
\hline
\end{tabular}

${ }^{a}$ Mississippi Valley Type-related metals 


\section{Water Quality of the Viburnum Trend Subdistrict, Exploration Area, and Strother Creek, Southeastern Missouri, 1964-2006}

By Michael J. Kleeschulte

Chapter 6 of

Hydrologic Investigations Concerning Lead Mining Issues in

Southeastern Missouri

Edited by Michael J. Kleeschulte

Scientific Investigations Report 2008-5140 


\section{Contents}

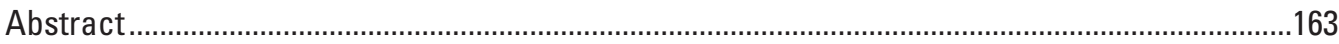

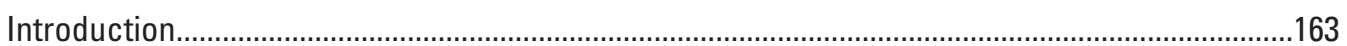

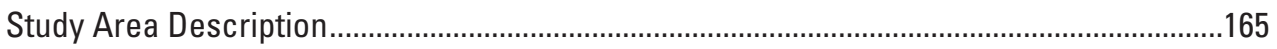

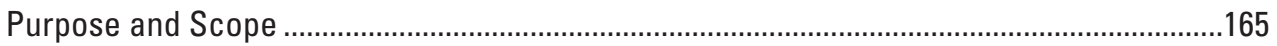

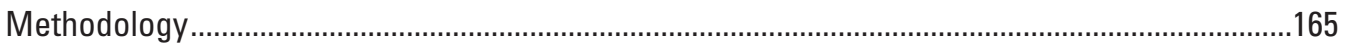

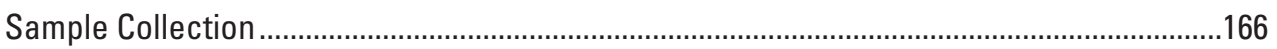

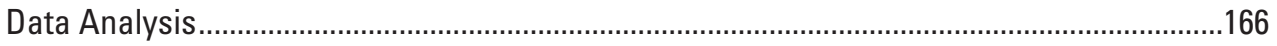

Water Quality of the Viburnum Trend and Exploration Area..........................................................167

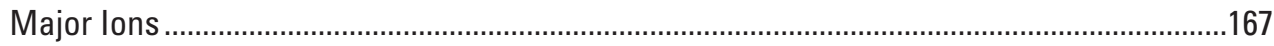

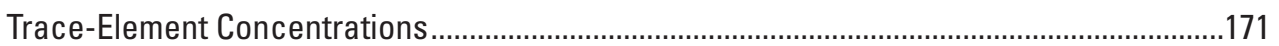

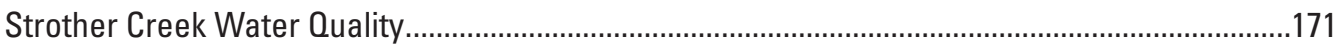

Summary

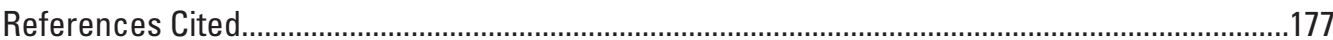

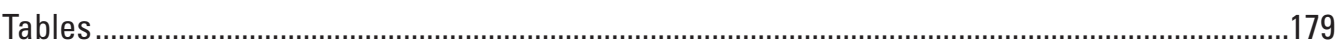

\section{Figures}

1. Map showing study area and water-quality sampling sites .........................................164

2. Trilinear diagram showing general water-quality samples from proximity-to mining groups, April through June 2002

3. Stiff diamgrams showing general water quality from near- and distal-mining sites and the West Fork Black River near Greeley (non-mining site), April through May 2002

4. Boxplots showing general water-quality and trace-element concentrations for the proximity-to-mining groups, April through June 2002.

5. Map showing water-quality sampling sites in Strother Creek and specific conductance values, August 10-11, 2006

6. Trilinear diagram showing general surface-water quality of Strother Creek, 1964-2005

7. Boxplots showing major chemical constituents and trace-element concentrations for Strother Creek near Oates and Goodland, 1964-2005.

\section{Tables}

1. Water-quality data for the Viburnum Trend and exploration area, April-June 2002

2. Water-quality summary statistics for major anions and cations and trace elements, April-June 2002

3. Surface-water quality data for Strother Creek near Oates and Goodland, 1964-2005 


\title{
Water Quality of the Viburnum Trend Subdistrict, Exploration Area, and Strother Creek, Southeastern Missouri, 1964-2006
}

\author{
By Michael J. Kleeschulte
}

\section{Abstract}

The lead ore deposits of the Viburnum Trend Subdistrict (Viburnum Trend), southeastern Missouri are a part of the largest known lead reserve within the United States. Mining in the Viburnum Trend began in 1960 and has continued uninterrupted since; presently (2008), six mines are active. Total ore production from individual mines ranges from 20 to more than 50 million tons, and ore contains as much as 8 percent lead and 3 percent zinc.

Water-quality data collected from streams and springs draining lead and zinc mining areas along the Viburnum Trend Subdistrict and non-mining areas to the south in the Current River and Eleven Point River Basins from April through June 2002 were compared to quantify changes in water quality spatially and with distance downstream from active mining. Data from 37 historical water-quality samples collected from 1964 to 2005 downstream from the Buick Mine in Strother Creek also were analyzed to document water-quality changes in this stream with time.

Three groups were used for comparison: non-mining sites which are sites upstream from mining activity or mine tailings or sites where no mining activity is present in the basin; nearmining sites where there is mining activity or mine tailings ponds within 7.5 miles upstream from the site; and distalmining sites where mining activity is conducted more than 7.5 miles upstream from the site.

Samples collected from non-mining sites had little variability in general chemical composition, but samples from near-mining and distal-mining sites had differing sodium, chloride, and sulfate concentrations. Most surface- and springwater samples collected during this study were calciummagnesium-bicarbonate water type, except for Strother Creek near Goodland, which was calcium-magnesium-sulfate type. When compared to non-mining sites, most near-mining and distal-mining sites had elevated sulfate and chloride concentrations. Sodium, chloride, and sulfate concentrations peaked at near-mining sites, then decreased downstream at distal-mining sites; however, concentrations at distal-mining sites did not decrease to the lower concentrations detected at non-mining sites.

Trace-element concentrations also were different at the three site groups. The copper, nickel, lead, zinc, lithium, and strontium concentrations also peaked at near-mining sites, but decreased downstream at distal-mining sites to concentrations statistically similar to those observed at non-mining sites. Cadmium concentrations were similar at near-mining and distalmining sites, but were significantly smaller at non-mining sites. Cobalt concentrations peaked at near-mining sites, and then decreased at the distal-mining sites; however, even though the concentrations significantly decreased downstream from mining activity, they did not achieve the lesser concentrations measured at the non-mining sites.

Historical water-quality data from 1964 through 2005 from two near-mining sites on Strother Creek downstream from the Buick Mine indicated surface-water quality changes with time. The data were separated into four periods and statistically analyzed. Sodium, chloride, sulfate, and potassium concentrations significantly increased after 1967 (pre-mining period is considered 1964-67) in Strother Creek Basin, and remained elevated through the most recent sampling period (1995 through 2005). Sulfate concentrations continued to significantly increase during each successive period sampled. Calcium and magnesium concentrations also increased with time. The concentrations of copper, manganese, and zinc were similar during the pre-mining and most recent periods.

\section{Introduction}

The lead ore deposits of the Viburnum Trend Subdistrict (Viburnum Trend), southeastern Missouri, are a part of the largest known lead reserve within the United States (U.S. Geological Survey, 2008a). Mining in the Viburnum Trend began in 1960 and has continued uninterrupted since. As many as 10 mines have operated in the Viburnum Trend, and presently (2008), six mines are active (fig. 1). Total ore production from individual mines ranges from 20 to more than 50 million tons, and ore contains as much as 8 percent 


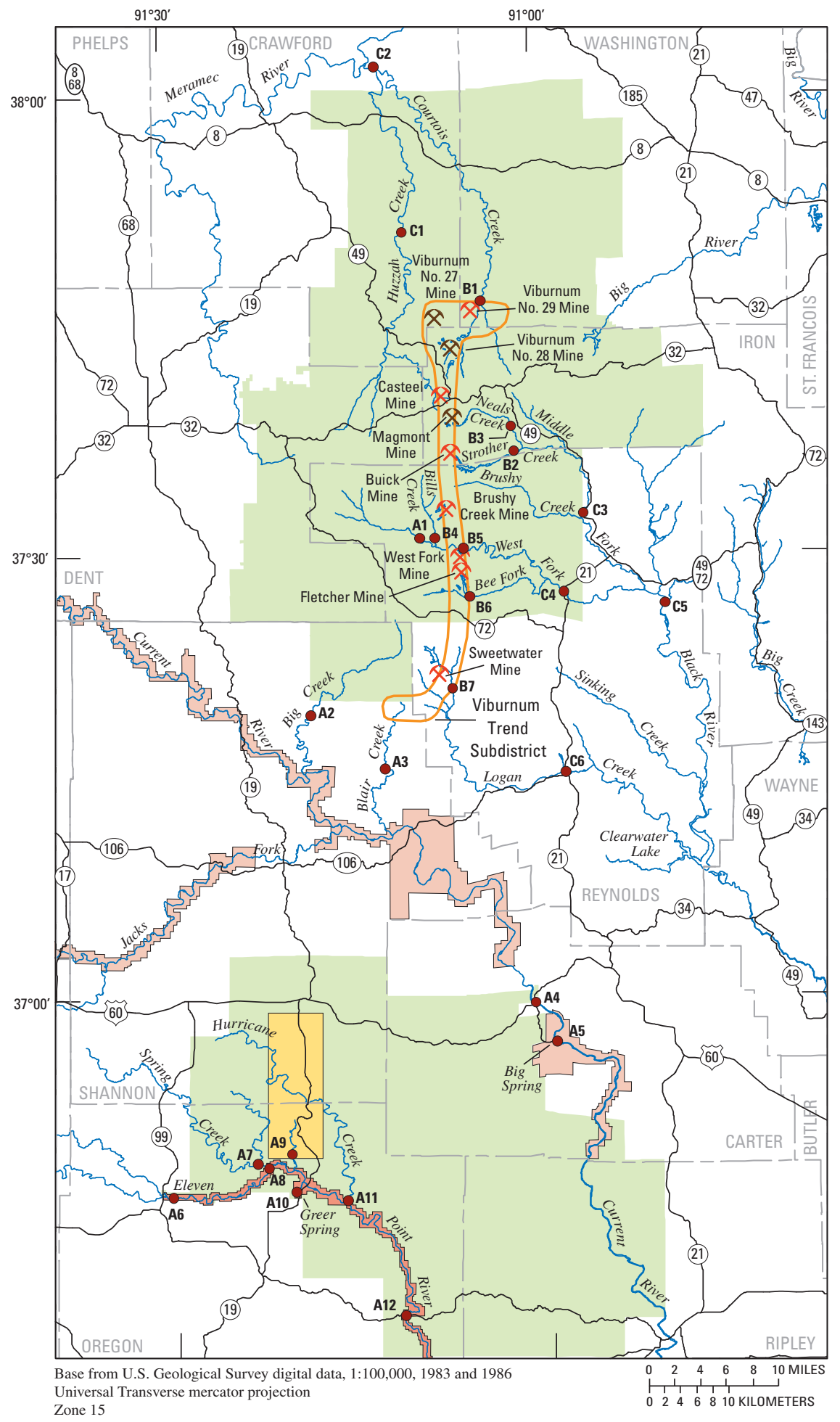

EXPLANATION

Mark Twain National Forest

Ozark National Scenic Riverways

Eleven Point National Wild and Scenic River

Exploration area
A9 Water-quality sampling site and identifier (table 1)

x Mine-Red color indicates active mine (2008)

Note: West Fork Mine is currently (2008) considered part of Fletcher Mine.

Figure 1. Study area and water-quality sampling sites.

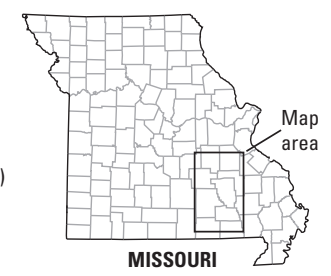


lead and 3 percent zinc (C.M. Seeger, Missouri Department of Natural Resources, Division of Geology and Land Survey, written commun., 2008). Ore mined underground is brought to the surface and milled at several of the mines. Tailings (waste rock) from the milling process are pumped as slurry to large surface impoundments that fill headwater stream valleys. After ore mineral concentrates have been prepared for shipment, they are transported by truck directly to a smelter, or in the case of copper and zinc, by truck to a rail system for shipment to a smelter.

As ore reserves are being depleted in the Viburnum Trend, exploration for new deposits is expanding farther south in part of the Mark Twain National Forest (fig. 1). Two federally designated streams occur in this region, the Ozark National Scenic Riverways, administered by the National Park Service and the Eleven Point National Wild and Scenic River managed by the U.S. Department of Agriculture, Forest Service. This area of mature karst terrain contains the two largest springs in Missouri-Big Spring and Greer Spring (Vineyard and Feder, 1982).

The potential expansion of mining activities into these federally designated areas has prompted a series of long-term environmental studies that are characterizing the effects of lead and zinc mining in southeastern Missouri.

\section{Study Area Description}

The study area includes streams that drain the Viburnum Trend, and streams draining non-mining areas to the south (fig. 1). The area lies within a large region of well-developed karst terrain that is characterized by the presence of caves, springs, sinkholes, and gaining and losing streams. The topography is characterized by deep, narrow valleys and narrow, steep-sided ridges that resulted from deep dissection of the carbonate bedrock by surface and karst drainage. More than 300 feet ( $\mathrm{ft}$ ) of relief is common between the ridge top and the adjacent valley.

\section{Purpose and Scope}

Water samples were collected by the U.S. Geological Survey (USGS) from streams and springs in the Huzzah Creek and Black River Basins that drain the Viburnum Trend and from the non-mining Current River and Eleven Point River Basins to the south. Twenty-five samples were collected during high-base flow from April through June 2002 to quantify water-quality changes spatially and with distance downstream from active mining. Data from 37 historical water-quality samples collected from 1964 to 2005 downstream from the Buick Mine in Strother Creek also were analyzed to document water-quality changes in this stream with time.

\section{Methodology}

The Huzzah Creek and Black River Basin sampling sites (fig. 1) were selected based on their proximity to active mining sites and tailings ponds. Sampling sites that were upstream from known mining activity or in stream basins where no known mining activity has occurred were used as baseline water-quality sites. In the Current River and Eleven Point River Basins, stream and spring sites were selected that have an increased probability of being affected if future mining activities would occur in the exploration area. This determination was based on proximity of the site to the exploration area and results of dye-tracing tests performed in the Mark Twain National Forest that delineated spring recharge areas (data on file at the USGS, Rolla, Missouri, and the Missouri Department of Natural Resources, Division of Geology and Land Survey, Rolla, Missouri). Base flow occurs during periods of minimum streamflow and minimum daily fluctuations when discharge is sustained by diffuse ground water and spring inflow, not by surface runoff. Base flow rates change seasonally and generally are higher during spring or early summer than during the late summer and fall. This sample collection effort was suspended at one point because intense rainfall caused runoff to occur and the base-flow conditions, required for sampling, to cease.

Springs are excellent water-quality monitoring sites because they are points at which ground-water flow converges and typically are representative of the water in the entire recharge area for the spring. Spring recharge areas can contain ground-water flow from less than one to hundreds of square miles, and typically, the size of the recharge area is reflected by the volume of the spring discharge.

Sample sites were classified into proximity-to-mining groups based on the location of the site to the nearest mining activities or tailings ponds. The classification contains three groups: non-mining sites, which are sampling sites upstream from mining activity or tailings ponds, or sites where no mining activity is present in the basin; near-mining sites, where mining activity or tailings ponds occur within 7.5 miles (mi) upstream from sampling site; and distal-mining sites, where mining activity occurs upstream from the sampling sites at distances greater than $7.5 \mathrm{mi}$.

Because water in Strother Creek had a large sulfate concentration and elevated calcium, magnesium, potassium, sodium, and chloride concentrations as compared to water from non-mining sites, and water-quality data that preceded mining in the basin were available, a time-trend analysis was performed. These historical data were collected for different studies with different objectives; consequently, different suites of constituents were analyzed and reported. Concentrations for major anions and cations were available for most samples, but some trace-element data also are available. Some nutrient data also are available for Strother Creek and are on file at the USGS office in Rolla, Missouri. 


\section{Sample Collection}

The 1995 and later samples were collected from cross sections in the stream or spring branch where water was moving with sufficient velocity to allow mixing of the water. These samples were collected using the equal-width increment (EWI) method (Wilde and others, 1999). This method consists of dividing the stream into 10 to 15 sampling verticals of equal width. At each sampling vertical, a water sample was collected using a hand-held, depth integrating sampler (DH-81) constructed of polypropylene and nylon with a Teflon container. The sampler was raised and lowered using the same transit rate at each vertical, and the amount of water collected in the sample bottle was a function of velocity and depth of water at the vertical. The water sample from each vertical was composited into a polyethylene churn splitter using the techniques described by Wilde and others (1999). Water samples collected before 1976 used methods and procedures that were established and acceptable at the time. The historical methods and procedures that were used to collect the water samples are described in publications of the National Water Quality Laboratory (NWQL) (USGS, 2008b).

The aliquot to be analyzed was dispensed from the composite water sample in the churn splitter. The chemical constituents to be analyzed in the "dissolved" phase were filtered onsite through a 0.45 -micrometer $(\mu \mathrm{m})$ cellulosenitrate membrane filter supported between Lucite plates, using a peristaltic pump as the pressure source. After onsite filtration of the water and rinsing of the sample bottle, the samples were placed into polyethylene bottles. Cation and trace-element samples were preserved with ultrapure nitric acid to a $\mathrm{pH}$ less than 2; samples for dissolved anions were not acidified.

Stream or spring discharge, specific conductance, $\mathrm{pH}$, and water temperature were measured onsite, and alkalinity was determined at all sampling locations (Wilde and Radtke, 1998). At sites where no USGS gaging station was present, discharge measurements were made with a current meter using the general methods described by Rantz and others (1982). At sites near gaging stations, stage readings were converted to discharge using the stage-discharge relation developed for the site (Rantz and others, 1982). Specific conductance values were measured using a portable conductivity meter with temperature compensation designed to express readings in microsiemens per centimeter at $25^{\circ} \mathrm{C}(\mu \mathrm{S} / \mathrm{cm})$. The $\mathrm{pH}$ was measured with a portable $\mathrm{pH}$ meter. Water temperature was measured with a thermistor to the nearest $0.1^{\circ} \mathrm{C}$. The acid neutralizing capacity (ANC) was determined by incremental titration past the bicarbonate-carbonic acid inflection point using 0.1600 normal $(\mathrm{N})$ sulfuric acid.

All water-quality samples collected from 1995 and later were analyzed by the USGS NWQL in Lakewood, Colorado. Samples collected before 1976 were analyzed using standard procedures that were acceptable at the time. Samples were analyzed for inorganic constituents according to methods described by Taggart (2002). When chemical constituents were detected at concentrations that were less than concentra- tions deemed reliable for reporting as numerical values, they were considered to be less than the reporting level. In the water-quality tables at the back of this chapter, these data are indicated by a less than $(<)$ symbol in front of the reporting level value. Suspended-sediment samples were collected using the EWI method from about 10 verticals across the stream with a depth-integrating sampler. All suspended sediment concentrations were determined in the USGS sediment laboratory in Rolla, Missouri, using the evaporation method (Guy, 1969). The historical Strother Creek data were analyzed according to acceptable procedures available at the time of collection. There were no duplicate or split samples for comparison or quality control purposes collected with the 2002 sample set or the historical Strother Creek sample set.

\section{Data Analysis}

The water-sample composition described in this chapter can be diagrammatically represented in terms of major dissolved ionic constituents. The relation among these major constituents can be displayed by grouping ions with similar chemical properties on trilinear diagrams. Units on trilinear diagrams generally are expressed as percentages of the total milliequivalents per liter for each ion in a sample, and the assumption is made that the sum of these percentages is equal to 100 .

Trilinear diagrams approximate the general composition of the water by showing proportions of the water-quality constituents relative to three cationic and anionic groups. On trilinear diagrams, there are three separate areas where each sample is plotted. The central diamond is used to show the overall chemical character of the water. The position of each point in the diamond indicates the composition of the sample in terms of the cation-anion groups that correspond to the four vertices of the diamond. The triangle in the lower left is used to display the relations of the three groups of cations in the sample and the triangle in the lower right is used to display the relations of the three groups of anions (Hem, 1985).

Stiff diagrams are another method used to display the major chemical composition of water samples. This method uses a distinctive pattern or polygon to represent general water composition. The diagram uses three parallel horizontal axes extending on each side of a vertical axis that represents zero. The milliequivalent concentration of cations is plotted to the left of zero and anions are plotted to the right of zero. All ions are plotted on the diagram using the same scale, and on all diagrams each ion is plotted in the same order (Hem, 1985).

Boxplots provide a visual summary of the 25 th, 50th, and 75th percentiles and any extreme values in the distribution. The boxplot consists of the median value (50th percentile) plotted as a horizontal line, and a box is drawn from the 25 th percentile to the 75th percentile. The box length is the interquartile range (IQR) and represents one-half of the data values. The IQR is insensitive to the presence of extreme values in the distribution. If the median value does not divide 
the box into two equal parts, it indicates asymmetry in the data distribution. Adjacent values are located outside the box and within 1.5 times the IQR. They are shown as whiskers connected to and drawn vertically outward from the 25 th and 75 th percentile lines. The individual data points represented by adjacent values are not plotted along the whisker lines. The length of the whisker connected to the 75th percentile represents the value of the largest adjacent point; the length of the whisker connected to the 25 th percentile represents the smallest adjacent value. Values more extreme in either direction than the adjacent values are plotted individually. The values equal to 1.5 to 3.0 times the IQR are called "far-out values" and are represented by an asterisk (D.R. Helsel, U.S. Geological Suurvey, written commun., 1989). Values greater than "far-out values" are represented by a circle.

Statistical analyses can be used to examine the distribution of values for an individual variable or for comparing two or more groups of data. The computer software SYSTAT (SYSTAT Software Inc., 2002) was used for statistical hypothesis tests, summary statistics, and the boxplot preparation. A level of significance ( $\alpha$-value) less than 0.05 caused rejection of the null hypothesis that states the water-quality concentrations for compared groups were similar. The "attained significance level" ( $p$-value) is a probability value determined from the data (Helsel and Hirsch, 1995) and measures the "believability" of the null hypothesis. The larger the p-value, the more likely is the observed test statistic when the null hypothesis is true and the weaker the evidence to reject the null hypothesis.

The summary statistics, including the minimum, maximum, range, mean, standard deviation, the 25th, median, 75 th percentiles, and IQR were calculated for the water samples collected as a result of this study. When a constituent concentration was less than the reporting level, the reporting level value was substituted for the "less than" value during the summary statistics calculation. A "less than" symbol $(<)$ was inserted before the appropriate calculated value listed in the summary statistics table.

\section{Water Quality of the Viburnum Trend and Exploration Area}

The concentrations of major anions and cations and trace elements from samples collected from the Viburnum Trend, exploration area, and Strother Creek were compared graphically and statistically. Trilinear diagrams, stiff diagrams, and boxplots can be used to show differences in chemical composition, whereas statistical analyses indicate if those differences are significant. The samples collected in 2002 were analyzed to determine if concentrations changed with distance downstream from mining activity. The historical data from Strother Creek were analyzed to determine if concentrations changed with time.

\section{Major lons}

The general chemical composition of most water samples collected during this study was a calcium-magnesium bicarbonate type (table 1, at the back of this chapter). Non-mining sites had little variability (fig. 2) and generally plotted within a "tight" cluster represented by the blue areas on the trilinear diagram. These data plotted approximately at the left vertex of the central diamond field that represents the 100 percent calcium plus magnesium and carbonate plus bicarbonate water type. The data extended over a larger area in the lower left triangle, but are centered on approximately an equal distribution of calcium and magnesium. The anion distribution is near the vertex of 100 percent bicarbonate plus carbonate distribution.

Water-quality data from the near-mining sites and the distal-mining sites had much more variability in general chemical composition than the non-mining sites. Much of the variability was caused by differing percentages of sodium, chloride, and sulfate that were much larger in samples from near-mining sites than in samples from non-mining or distal-mining sites (table 1). Concentrations of these three constituents in samples from distal-mining sites generally were intermediate to concentrations present in non-mining and near-mining sites.

Applications for trilinear diagrams include assessing water-analysis groups to determine if a specific water can be a mixture of other waters for which analyses are available. The analysis of a water sample that is a result of a mixture of two different water types ( $\mathrm{Y}$ and $\mathrm{Z}$ ) will plot on the straight line $\mathrm{YZ}$ on the central diamond field of the trilinear diagram if the ions do not react chemically as a result of mixing (Hem, 1985).

Water samples from individual basins along the Viburnum Trend can be analyzed to indicate obvious mixing scenarios. The three northern-most sampling sites (B1, C1, and C2) are on Courtois Creek and Huzzah Creek (fig. 1). The headwaters of Courtois Creek upstream from the Courtois site (B1) drain tailings piles from Viburnum No. 28 and No. 29 Mines, and the headwaters of Huzzah Creek upstream from the Davisville site (C1) drain waters from the Casteel Mine. The Huzzah Creek near Scotia (site C2) is downstream and receives flow from sites $\mathrm{B} 1$ and $\mathrm{C} 1$. The central diamond on figure 2 indicates that the waters from these three sites have different relative proportions of major ions than non-mining samples and do not plot in a straight line. Instead, C2, the most downstream site on Huzzah Creek (near Scotia sample), plots within a triangle formed by the two upstream samples and the shaded area representing non-mining samples in the central diamond and the lower left cation triangle. The analysis indicates that the water at the Scotia site $\mathrm{C} 2$ is not a simple mixing of the two tributaries, but also contains a third component. Because the sample from site C2 plots off-line defined by the other two samples and plots closer to the area represented by the non-mining sites (baseline for indigenous waters), the third component appears to be inflow of indigenous waters upstream from site $\mathrm{C} 2$. 


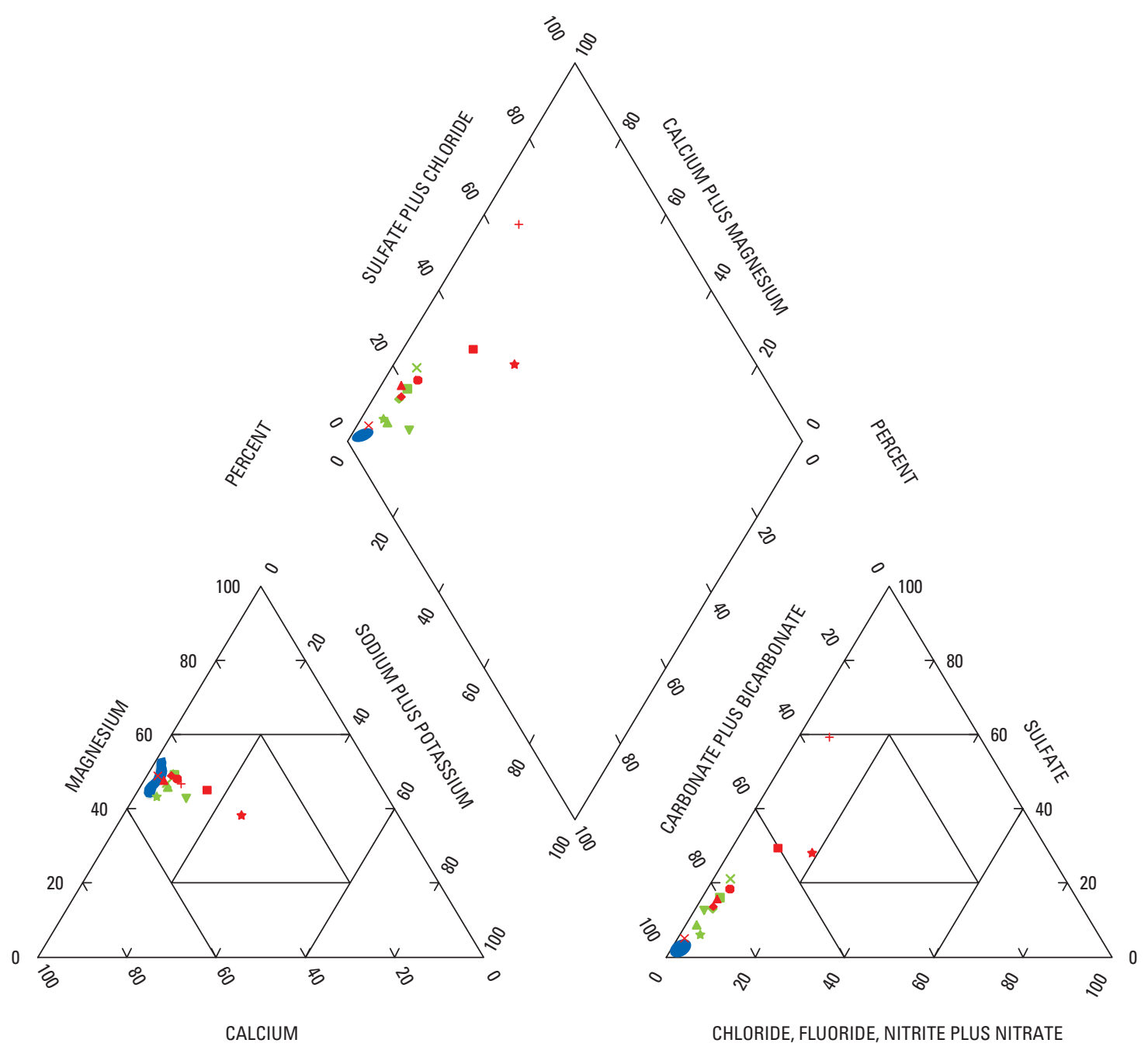

PERCENT

EXPLANATION

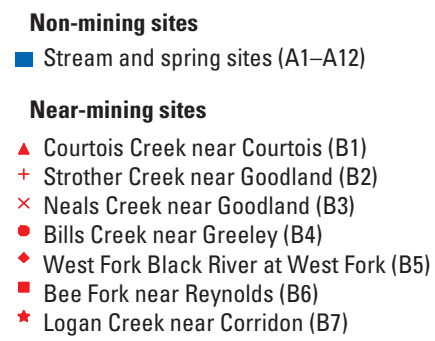

- Stream and spring sites (A1-A12)

Near-mining sites

- Courtois Creek near Courtois (B1)

+ Strother Creek near Goodland (B2)

$\times$ Neals Creek near Goodland (B3)

- Bills Creek near Greeley (B4)

- West Fork Black River at West Fork (B5)

- Bee Fork near Reynolds (B6)

* Logan Creek near Corridon (B7)

Distal-mining sites

$\checkmark$ Huzzah Creek near Davisville (C1)

- Huzzah Creek near Scotia (C2)

$\times$ Middle Fork Black River near Black (C3)

- West Fork Black River at Centerville (C4)

- Black River near Lesterville (C5)

* Logan Creek at Ellington (C6)

Figure 2. General water-quality samples from proximity-to-mining groups, April through June 2002. 
The northern-most sampling sites in the Black River Basin include three sites (B2, B3, and C3) in the Middle Fork Black River (Middle Fork) Basin. Strother Creek near Goodland (site B2) drains the Buick Mine tailings pile (fig. 1), Neals Creek near Goodland (site B3) drains the inactive Magmont Mine tailings pile that has been reclaimed, and Middle Fork near Black (site C3) is downstream from the other two sites. The samples from Neals Creek site B3 plot in or near all the shaded areas on figure 2 that are considered typical for indigenous waters (baseline non-mining sites), and appears to be on a mixing line that uses the sample from Strother Creek site B2 as one end point and the non-mining sites as the other. Strother Creek site B2 had a large sulfate concentration (table 1), but a closer inspection indicates the site also had elevated concentrations of calcium, magnesium, potassium, sodium, and chloride as compared to the samples from Neals Creek site $\mathrm{B} 3$ or the non-mining sites. The Middle Fork site C3 is downstream from sites B2 and B3 and the sample plots along a mixing line between these two upstream sites.

Five sites were sampled in the West Fork Black River Basin. The most upstream site is West Fork Black River near Greeley (site A1), which plotted in the shaded area that is considered baseline for indigenous waters. Downstream, other West Fork Black River sites included Bills Creek near Greeley (site B4, downstream from the Brushy Creek Mine tailings pile), West Fork Black River at West Fork (site B5, downstream from the West Fork Mine), Bee Fork near Reynolds (site B6, downstream from the Fletcher Mine tailings pile), and the most downstream site West Fork Black River at Centerville (site C4). Samples from the three near-mining sites and the distal-mining site had greatly elevated sulfate concentrations [16 to $40 \mathrm{mg} / \mathrm{L}$ (milligrams per liter)] and elevated sodium concentrations (2.99 to $9.28 \mathrm{mg} / \mathrm{L}$ ) when compared to the range of sulfate concentrations $(2.2$ to $4.1 \mathrm{mg} / \mathrm{L})$ and sodium concentrations $(0.70$ to $1.45 \mathrm{mg} / \mathrm{L})$ from non-mining sites (table 1). Chloride concentrations were slightly elevated $(6.8 \mathrm{mg} / \mathrm{L})$ in the sample from Bills Creek site B4. Samples from the two West Fork Black River sites, West Fork Black River at West Fork (site B5) and West Fork Black River at Centerville (site C4), plot at about the same location on figure 2. These samples appear to be the result of simple mixing of waters that have Bills Creek site B4 and indigenous waters (baseline non-mining sites) as possible end members even though Bee Fork contributes flow between the two sites. The sample from Bee Fork site B6 had the largest concentrations of sodium $(9.28 \mathrm{mg} / \mathrm{L})$, chloride $(10 \mathrm{mg} / \mathrm{L})$, and sulfate $(40$ $\mathrm{mg} / \mathrm{L}$ ) of the five West Fork Black River Basin samples.

The Black River at Lesterville (site C5) site is the most downstream sampling site in the mainstem of the Black River Basin and included waters from Middle Fork near Black (site C3) and West Fork Black River at Centerville (site C4), along with other tributaries that flowed into the river upstream from the sampling site. The general water composition of the Lesterville sample (site $\mathrm{C} 5$ ) indicates an elevated sulfate concentration $(15 \mathrm{mg} / \mathrm{L})$ and slightly elevated sodium $(2.34 \mathrm{mg} / \mathrm{L})$ and chloride $(2.6 \mathrm{mg} / \mathrm{L})$ concentrations as compared to con- centrations from samples collected at non-mining sites (table 1). The Lesterville sample (site C5) appears to be the result of the mixing of waters from the downstream-most samples collected from the West Fork Black River Basin (site C4) and the Middle Fork Basin (site C3) and plots on the mixing line defined with these two sites being the end members (fig. 2).

Two sites were sampled in Logan Creek, one was downstream from the Sweetwater Mine tailings pond near Corridon (site B7) and the other was in a lower reach of the basin at Ellington (site C6). Downstream from Corridon site B7, the entire flow in Logan Creek is lost through the streambed and the creek remains dry for several miles until flow begins again a few miles upstream from the Ellington site C6. The Corridon site B7 has the largest concentrations of sodium $(17.2 \mathrm{mg} / \mathrm{L})$ and chloride $(19 \mathrm{mg} / \mathrm{L})$ of all the sampled sites, and elevated sulfate $(39 \mathrm{mg} / \mathrm{L})$ concentrations (table 1$)$, whereas the water sampled at Ellington site C6 had slightly elevated sulfate (5.4 $\mathrm{mg} / \mathrm{L})$ and nitrate $(2.3 \mathrm{mg} / \mathrm{L})$ concentrations. The Ellington site C6 sample plots on the mixing line with the Corridon site B7 and the shaded area representing the non-mining sites being the end members (fig. 2).

Stiff diagrams also were used to graphically represent the general chemical composition of the individual water samples from the near- and distal-mining sites and one representative sample from the non-mining sites. Unlike the trilinear diagram that plots percentages of major cations and anions, stiff diagrams give an indication of the concentration of plotted constituents by plotting the milliequivalents of each constituent. The sites on figure 3 are shown in downstream order for each basin, and all diagrams use the same unit length on the horizontal axis. An increase in the overall width of the diagram indicates a greater concentration of the various constituents plotted.

All non-mining sites have similar general water chemistry; therefore, their stiff diagrams have a similar appearance and all sites were represented by the single analysis from West Fork Black River near Greeley (site A1; fig. 3). The cation side of the stiff diagram (left side of the vertical axis) for this sample shows the sodium plus potassium concentration was negligible. The magnesium and calcium concentrations were about equal, which is represented by the nearly vertical line connecting these two horizontal axes. On the anion side (right side of the vertical axis), the chloride plus fluoride and sulfate concentrations also were negligible and the bicarbonate plus carbonate concentration was dominant.

Stiff diagrams for most of near- and distal-mining water samples had a shape similar to the non-mining samples, with slight variations to the basic pattern and width. On the cation side of the vertical axis, the most consistent variation was the noticeable increase on the sodium plus potassium horizontal axis at several sites. On the anion side of the vertical axis, the bicarbonate plus carbonate horizontal axis remained the dominant component for nearly all samples. With the exception of the Strother Creek near Goodland (site B2) sample, which was a calcium-magnesium-sulfate water, the general chemical composition of most water samples collected during this study 
Huzzah Basin

Courtois Creek near Courtois (B1)

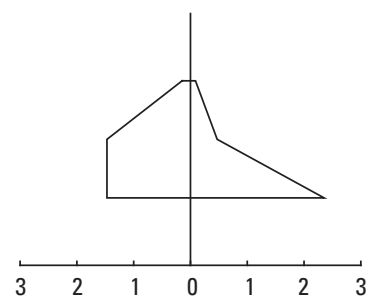

Strother Creek near Goodland (B2)

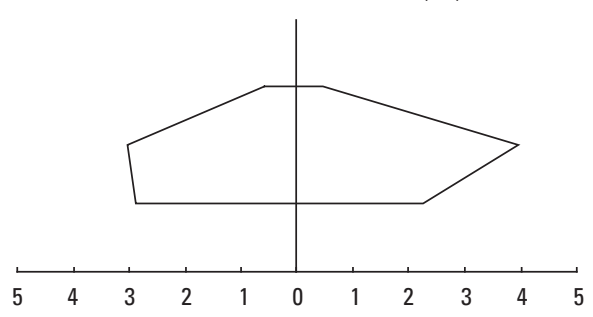

Huzzah Creek near Davisville (C1)

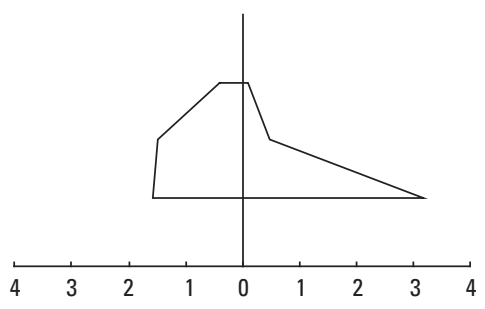

Middle Fork Black River Basin

Neals Creek near Goodland (B3)

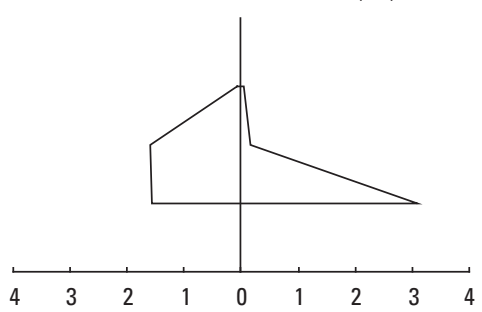

Huzzah Creek near Scotia (C2)

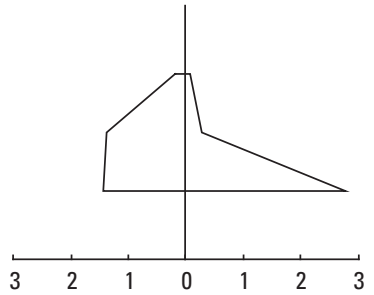

Middle Fork Black River near Black (C3)

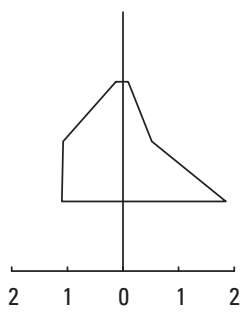

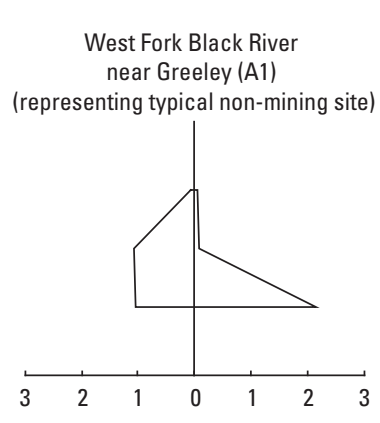

West Fork Black River Basin

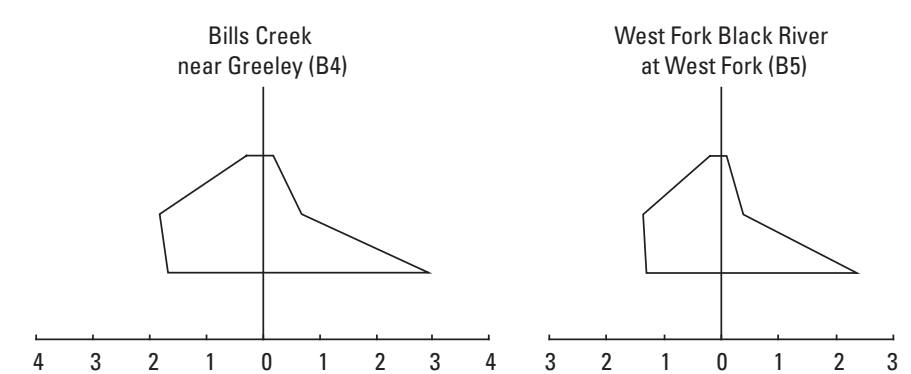

Bee Fork near Reynolds (B6)

West Fork Black River Basin-continued

West Fork Black River at Centerville (C4)

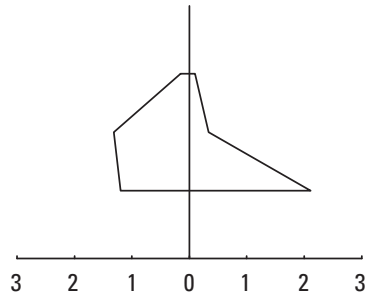

Logan Creek Basin

Logan Creek near Corridon (B7)

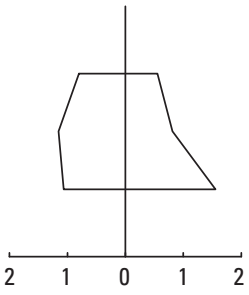

Black River Basin

Black River near Lesterville (C5)

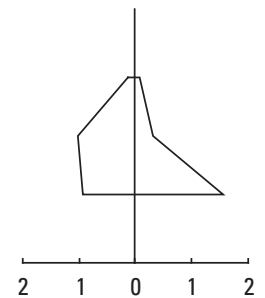

Logan Creek at Ellington (C6)

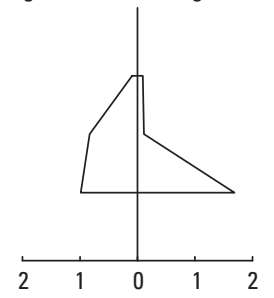

EXPLANATION

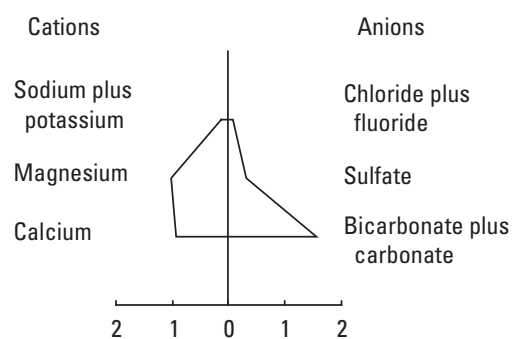

Concentrations, in milliequivalents per liter

Note: B1-B7 are near-mining sites. C1-C6 are distal-mining sites.

Figure 3. General water quality from near- and distal-mining sites and the West Fork Black River near Greeley (non-mining site), April through May 2002. 
was a calcium-magnesium-bicarbonate water. However, sulfate and chloride concentrations were elevated in several samples from near- and distal-mining sites compared to concentrations that were typically detected at non-mining sites (table 1; figs. $2,3)$.

The general chemical composition of the water samples in the three groups was statistically compared using the nonparametric Kruskal-Wallis statistical test (Helsel and Hirsch, 1995; SYSTAT Software Inc., 2002). Significant differences (p-values less than 0.05) were detected for sodium, chloride, and sulfate concentrations between the three groups. A Tukey's multiple comparison test (SYSTAT Software Inc., 2002) was applied to the ranked concentration data of these three constituents to identify which specific proximity-tomining groups were statistically different. This analysis indicated that the sodium, chloride, and sulfate concentrations were statistically similar in near- and distal-mining sites, but these concentrations in non-mining sites were significantly smaller than the other two groups. These differences can be seen graphically in the boxplots on figure 4, which show that sodium, chloride, and sulfate concentrations increased from non-mining sites, peaked at near-mining sites, and then decreased downstream at distal-mining sites. Whereas concentrations decreased between near- and distal-mining sites, this decline did not reach the smaller concentrations detected in samples from non-mining sites.

\section{Trace-Element Concentrations}

Arsenic, cadmium, cobalt, copper, lead, nickel, and zinc are the primary elements associated with the sulfide minerals of Mississippi Valley Type (MVT) ores and, therefore, are referred to as MVT-related metals in this chapter. The concentrations of these metals and of barium, chromium, lithium, manganese, rubidium, and strontium were analyzed by proximity-to-mining group (table 2 , at the back of this chapter; fig. 4). Because all but one arsenic concentration was less than the reporting level (table 1), this element was omitted from further analysis. The same statistical approach used to analyze the general chemical composition was used for these trace elements. Using the Kruskal-Wallis statistical test, all the MVT-related metals (except arsenic), lithium, and strontium had $p$-values less than 0.05 , which indicated that a significant difference existed in concentrations between the three groups. Applying the Tukey's multiple comparison test (SYSTAT Software Inc., 2002) to the ranked concentration data of these eight elements indicated that the distribution of the MVTrelated metals copper, nickel, lead, and zinc, and the trace elements lithium and strontium in non-mining sites and distalmining sites were statistically similar ( $\mathrm{p}$-value greater than 0.05). However, the elevated concentrations of these elements in near-mining sites were significantly larger ( $p$-value less than 0.05) than the other two groups. The concentrations for these trace elements peaked at near-mining sites (fig. 4), but decreased downstream at distal-mining sites to concentrations similar to those observed at the non-mining sites. Cadmium concentrations were similar at the near- and distal-mining sites, but were significantly smaller at non-mining sites. This indicates that cadmium concentrations peaked at near-mining sites (fig. 4), remained persistent in the stream and did not significantly decrease downstream at the distal-mining sites. Cobalt concentrations were significantly different for all three site types. Cobalt concentrations peaked at near-mining sites (fig. 4) and then decreased at the distal-mining sites. Even though the concentrations significantly decreased downstream, they did not achieve the lower concentrations detected at the non-mining sites.

\section{Strother Creek Water Quality}

Historical water-quality data collected by the USGS from 1964 through 2005 are available from two Strother Creek sampling sites downstream from the Buick Mine. Water-quality data were collected from 1964 through 1975 at varying intervals ranging from once a year to four times a year at Strother Creek near Oates (ST1), which is about 1 mi upstream from the Strother Creek near Goodland (site B2) sampling site discussed previously (B2; figs. 1, 5). Little Creek flows into Strother Creek between these two sampling sites. No waterquality analyses were available for Little Creek; however, a specific conductance value of $409 \mu \mathrm{S} / \mathrm{cm}$ was recorded during a seepage run on Strother Creek in August 2006 (chapter 2 of this report). Specific conductance measurements ranged from 708 to $1,155 \mu \mathrm{S} / \mathrm{cm}$ in the mainstem of Strother Creek downstream from mining activities. In several streams draining non-mining areas, specific conductance values from about 400 to $450 \mu \mathrm{S} / \mathrm{cm}$ were measured during the same seepage run. Based on these values, Little Creek is assumed to discharge water unaffected by mining and, therefore, water samples from the two Strother Creek sites are assumed to be comparable for this analysis. After 1975, the next water sample collected was in 1995 near Oates (site ST1). The two water-quality samples collected in 2002 and 2005 from the Goodland site (site B2) were added to the larger sample set from the Oates site.

Both Strother Creek sampling sites are upstream from the junction with Neals Creek and both receive water from the Buick Mine, which began production in 1969 (chapter 1 of this report) and currently (2008) is producing ore. Mine development and site preparation were occurring at the Buick Mine before ore production began. A tailings dam was constructed in 1966 and a railroad line to the mine was completed in 1967 as the mine was being developed (chapter 1 of this report).

The water-quality data from the two Strother Creek sampling sites were separated into four periods based on sampling date and statistically analyzed to determine if certain constituent concentrations had changed with time. The time frame for the pre-mining samples (period T1) was determined to be 1964 through 1967. This pre-mining period was not extended to the beginning of ore production in 1969. The effects of pre-ore 
Major chemical constituents
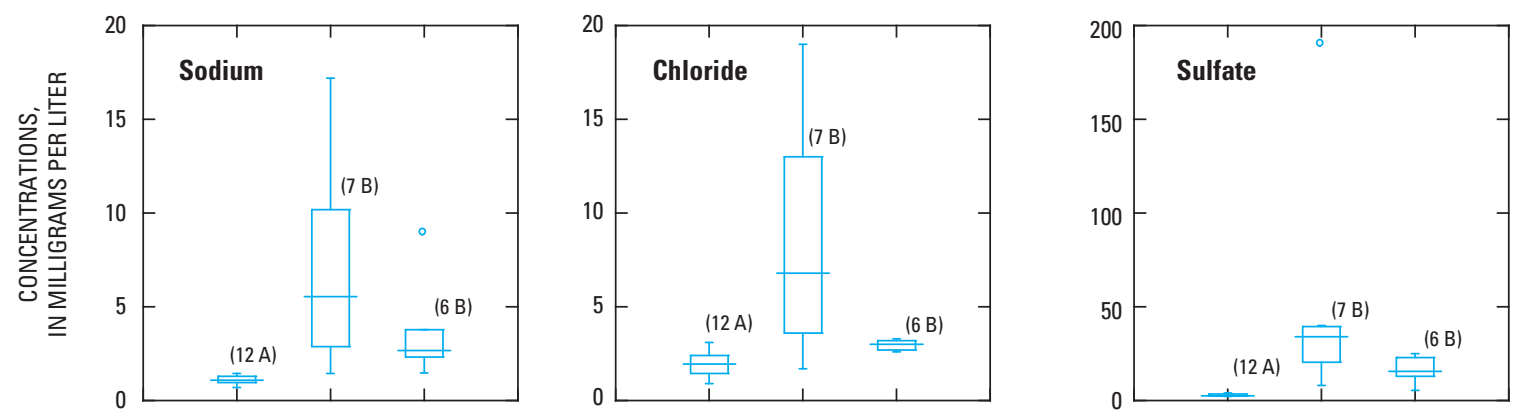

Trace elements
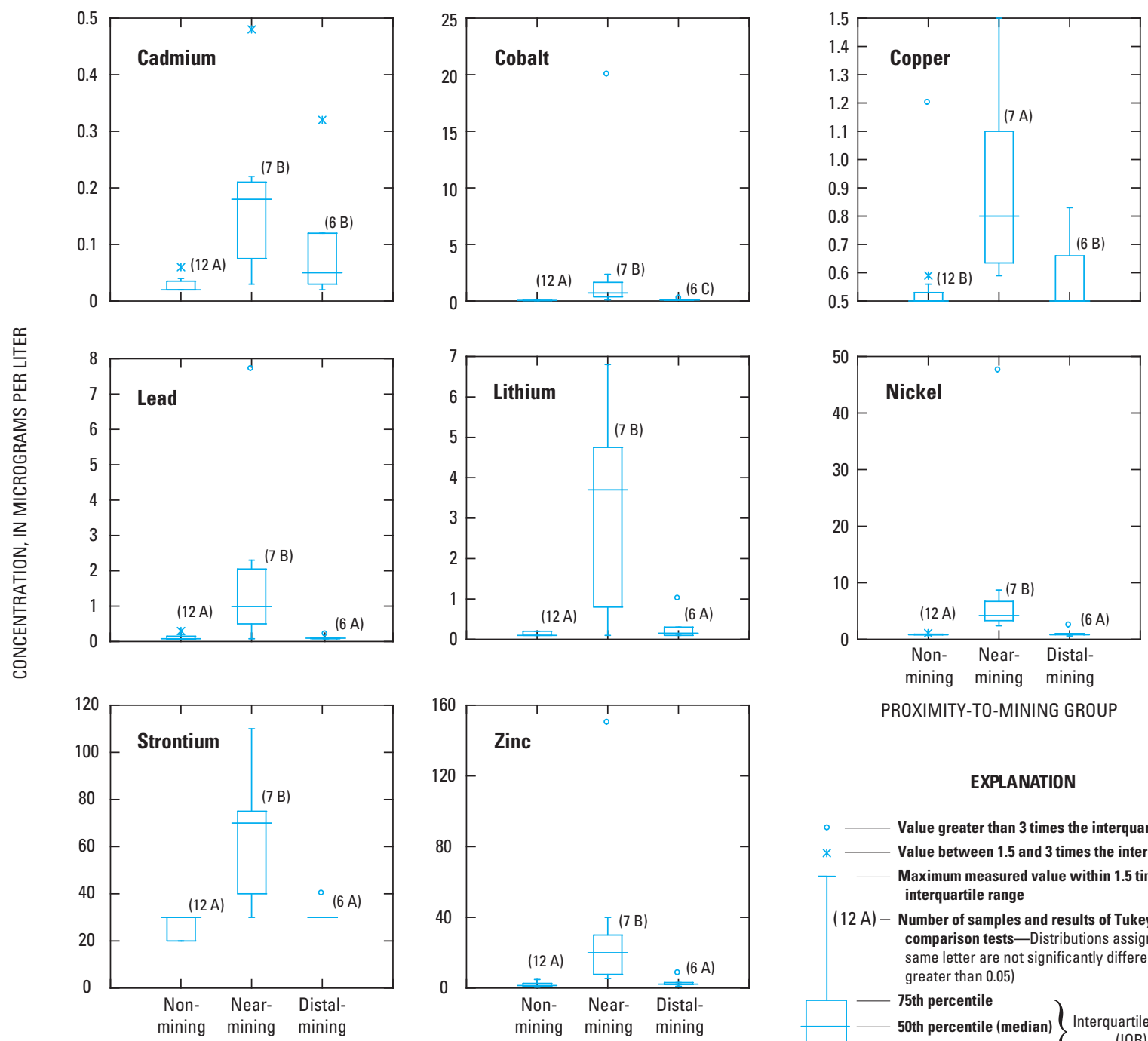

PROXIMITY-TO-MINING GROUP

\section{EXPLANATION}

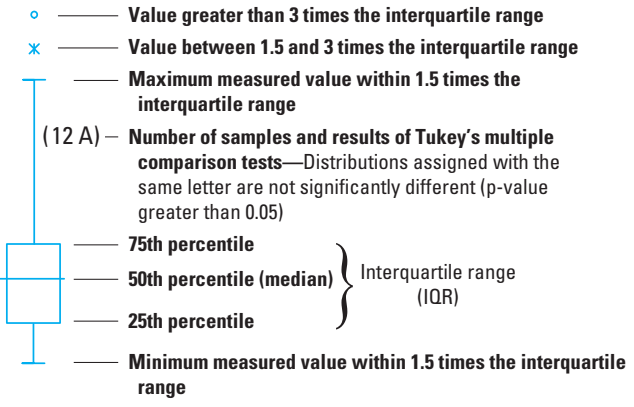

Figure 4. General water-quality and trace-element concentrations for the proximity-to-mining groups, April through June 2002. 


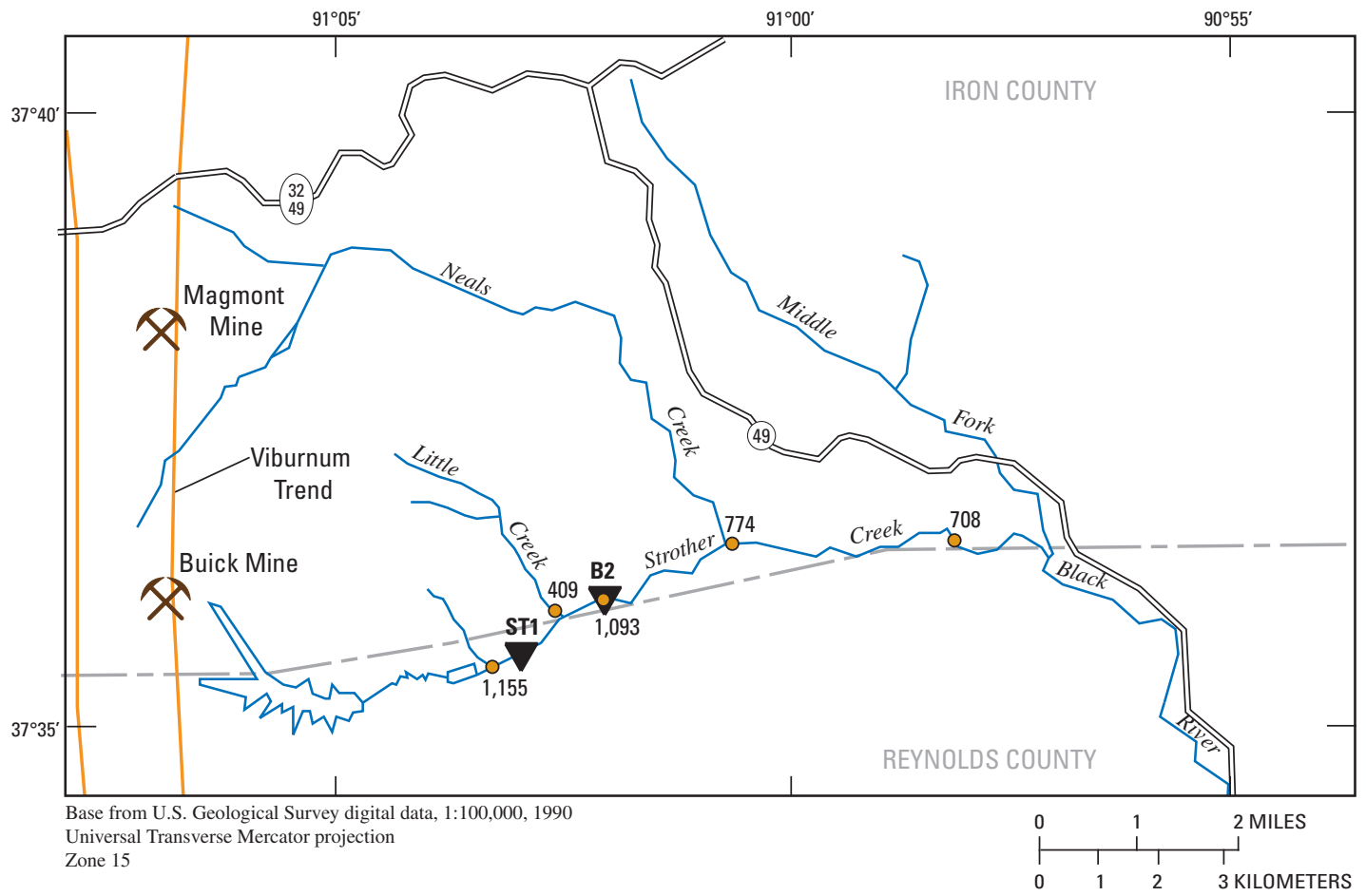

EXPLANATION

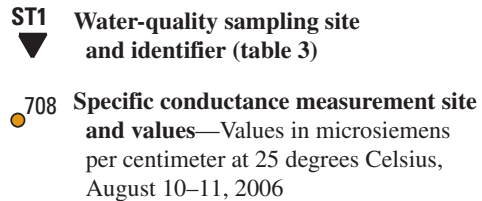

Figure 5. Water-quality sampling sites in Strother Creek and specific conductance values, August 10-11, 2006.

removal construction activities at the mine on the water quality of Strother Creek are uncertain. However, because the waterquality sample collected in 1967 plotted differently than the previous samples, this infers water-quality changes apparently were occurring in Strother Creek before ore removal (fig. 6). A much larger data set existed for samples collected from 1968 to 1975 . These data arbitrarily were divided into the smaller periods T2 (1968 through 1970) and T3 (1971 through 1975). Period T4 (1995 through 2005) represents the most recent data collected after the more frequent sampling ended in 1975. Two of the 1974 and all of the 1975 samples were not plotted because no calcium or magnesium analyses were performed.

Water quality in Strother Creek (fig. 6; table 3, at the back of this chapter) changed during 1964 to 2005 as indicated by the sodium, chloride, and sulfate concentrations that increased steadily with time. The non-parametric KruskalWallis statistical test (Helsel and Hirsch, 1995; SYSTAT Software Inc., 2002) was performed on the grouped concentration data from Strother Creek to determine if there was an equality of distribution among the four groups. The results indicated that there was a statistical difference in the distribution of the major cations and anions and the trace elements. The MVT- related metals, arsenic, cadmium, and lead, had incomplete data sets and could not be statistically analyzed by groups.

The Tukey's method for multiple comparisons (SYSTAT Software Inc., 2002) was applied to the ranked concentration data of those constituents that have a statistical difference in distribution (p-values less than 0.05). Results need to be used with caution because of the small number of samples available for the statistical test from some groups. Concentrations of the major cations and anions (sodium, chloride, sulfate, calcium, magnesium, and potassium) during the most recent period (T4; 1995 through 2005) were significantly larger than the premining period (T1; 1964 through 1967) (fig. 7). The sodium, chloride, sulfate, and potassium concentrations significantly increased after 1967 and remained elevated through the most recent sampling period (T4; 1995 through 2005). Sulfate concentrations continued to significantly increase during each successive period. Calcium and magnesium concentrations also have significantly increased with time (fig. 7).

Trace-element concentration data for copper, cobalt, manganese, nickel, and zinc were sufficient to perform a statistical analysis with time. Because many period T1 and T2 concentrations were reported as "not detected" the reporting level value at the time the analysis was performed was 


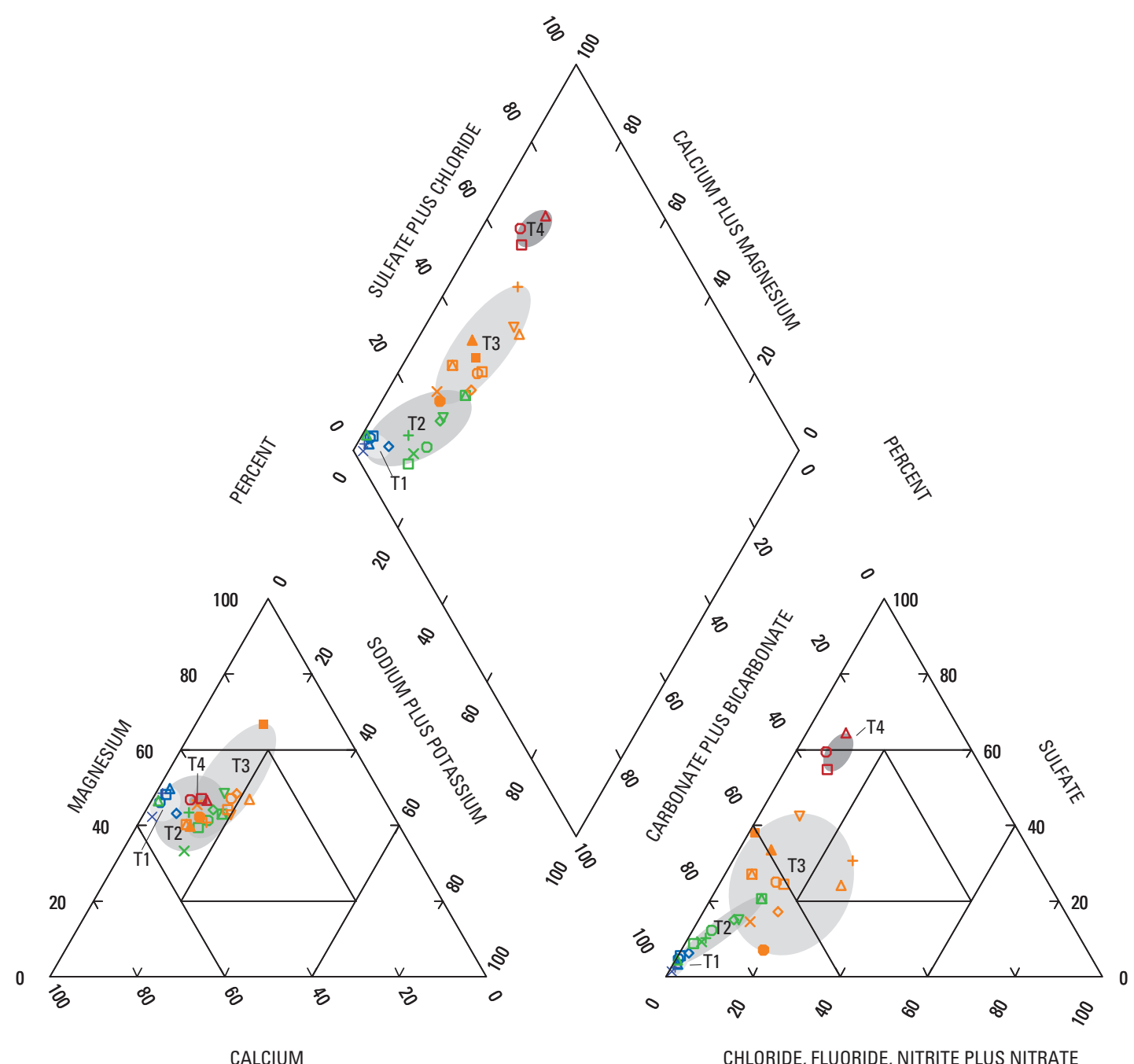

CALCIUM

$\begin{array}{cl}\text { PERCENT } & \text { Note: All samples from Strother Creek near Oates } \\ \text { (site ST1) except for those samples collected in 2002 } \\ \text { and 2005 [Strother Creek near Goodland (site B2)]. } \\ \text { Shaded area encompasses the range of samples } \\ \text { from each group. }\end{array}$

\begin{tabular}{|c|c|c|c|}
\hline $\begin{array}{l}\text { Group T1 } \\
\text { (1964-67) }\end{array}$ & $\begin{array}{l}\text { Group T2 } \\
(1968-70)\end{array}$ & $\begin{array}{l}\text { Group T3 } \\
\text { (1971-74) }\end{array}$ & $\begin{array}{c}\text { Group T4 } \\
\text { (1995-2005) }\end{array}$ \\
\hline 口 03/16/1964 & 口 10/17/1968 & 口 01/27/1971 & 口 09/19/1995 \\
\hline ○ 06/08/1965 & ○ 01/16/1969 & ○ 04/20/1971 & ○ 04/30/2002 \\
\hline$\Delta$ 09/30/1965 & $\Delta 04 / 09 / 1969$ & $\Delta 07 / 12 / 1971$ & $\Delta 07 / 06 / 2005$ \\
\hline$\times 08 / 10 / 1966$ & $\times 07 / 15 / 1969$ & $\times 02 / 01 / 1972$ & \\
\hline \multirow[t]{7}{*}{$\diamond 10 / 24 / 1967$} & $\diamond 10 / 29 / 1969$ & $\diamond 08 / 21 / 1972$ & \\
\hline & $\nabla$ 01/26/1970 & $\nabla$ 10/17/1972 & \\
\hline & $+04 / 30 / 1970$ & $+01 / 30 / 1973$ & \\
\hline & ه 11/03/1970 & ه 05/15/1973 & \\
\hline & & — 07/31/1973 & \\
\hline & & - $03 / 05 / 1974$ & \\
\hline & & $\Delta 05 / 14 / 1974$ & \\
\hline
\end{tabular}

Figure 6. General surface-water quality of Strother Creek, 1964-2005. 
Major chemical constituents
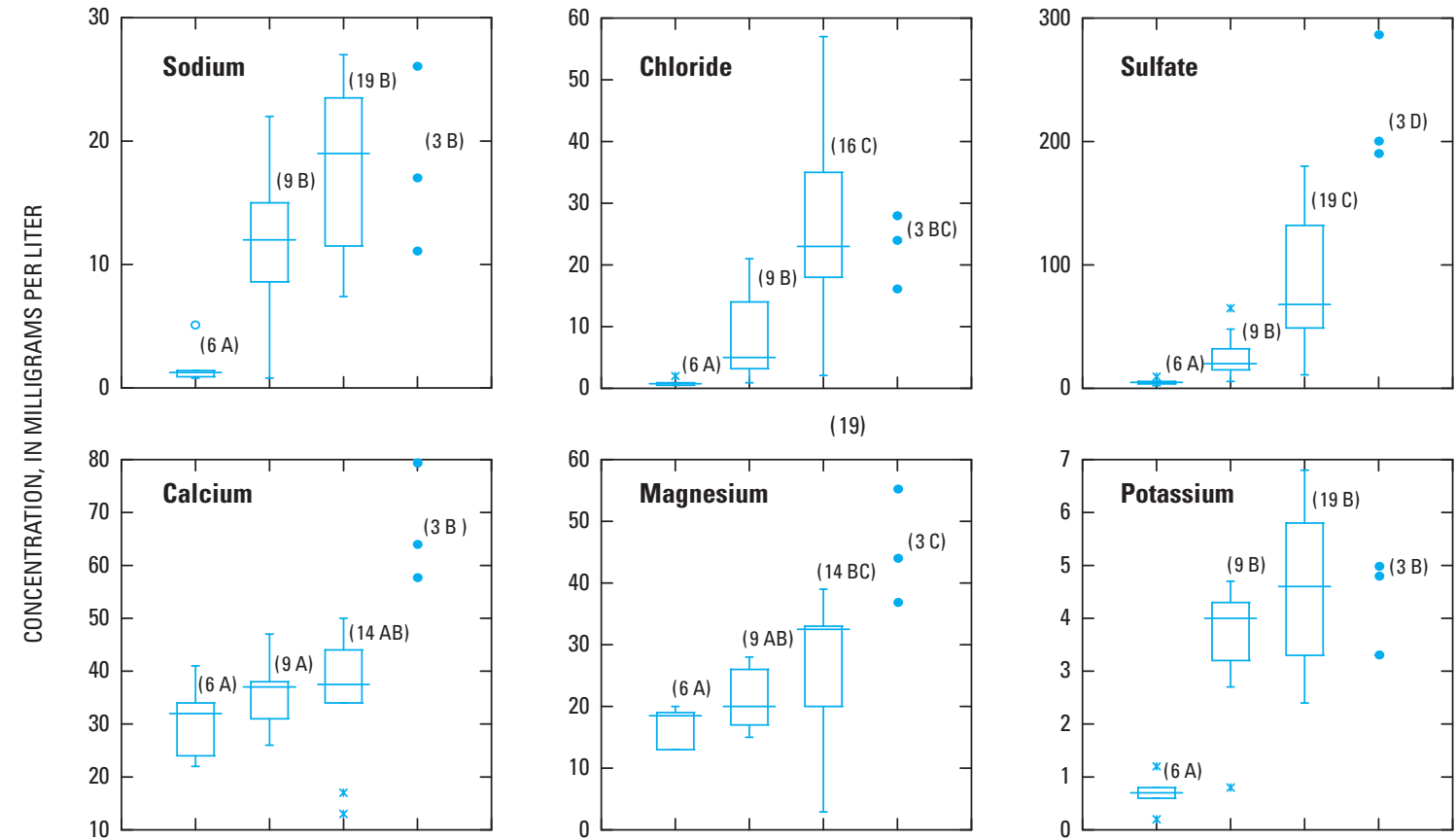

$(19)$
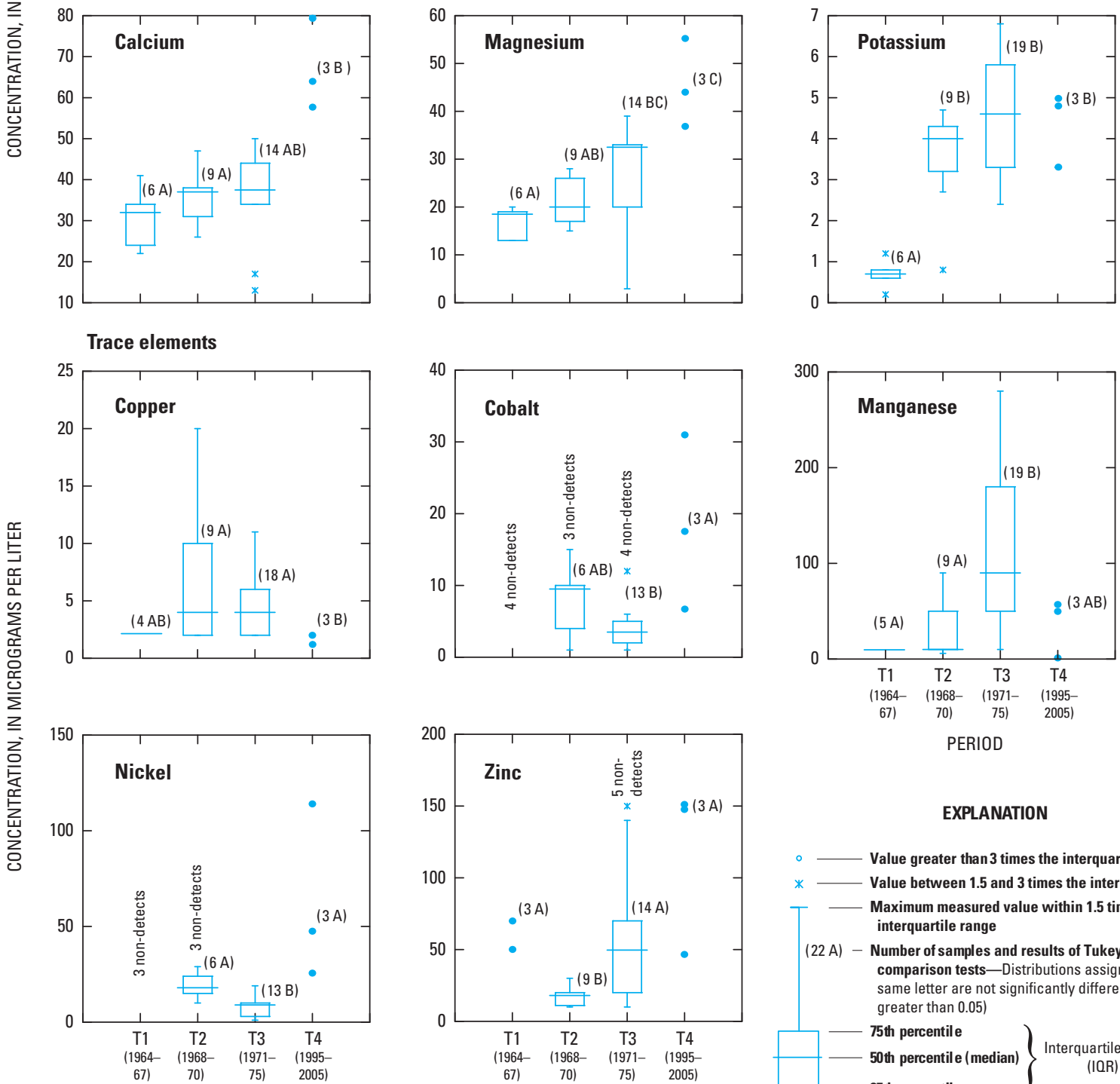

PERIOD

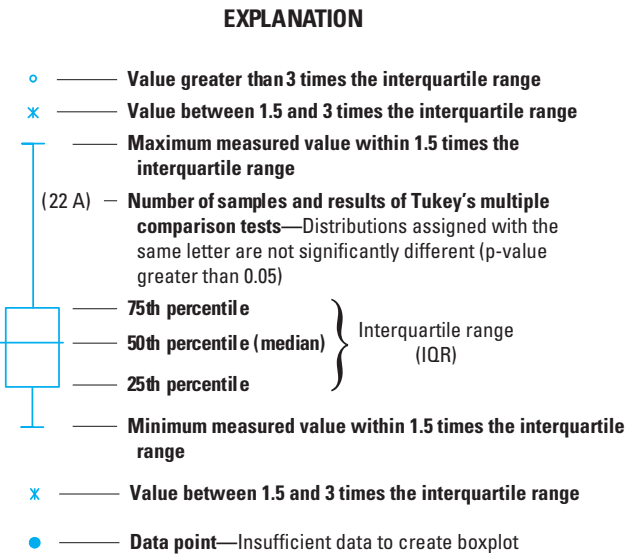

Figure 7. Major chemical constituents and trace-element concentrations for Strother Creek near Oates and Goodland, 1964-2005. 
substituted for each specific constituent concentration. If the reporting level for an analysis could not be determined or was many times greater than later analysis, no concentration was substituted. Consequently, no concentrations were substituted for the reported non-detected concentrations of cobalt and nickel during period $\mathrm{T} 1$, and no values were substituted for the five non-detected concentrations for zinc in period T3.

The trace-element concentrations (copper, manganese, and zinc) for periods $\mathrm{T} 1$ and $\mathrm{T} 4$ were statistically similar during both of these time periods. However, manganese concentrations indicated a significant increase during period $\mathrm{T} 3$, and zinc concentrations significantly decreased during period $\mathrm{T} 2$ when compared to the concentrations from period T1. Copper concentrations during periods T2 and T3 (1968 through 1975) increased compared to the pre-mining period, but the increase was not statistically significant.

No pre-mining cobalt and nickel concentration data were available to document conditions during the pre-mining period $\mathrm{T} 1$; however, a comparison of data for these two constituent concentrations can be made between the early mining period (T2) and the most current (T4) samples. Cobalt concentrations did not change significantly from period $\mathrm{T} 2$ to $\mathrm{T} 4$, but nickel concentrations decreased significantly during period T3, only to increase again during period $\mathrm{T} 4$.

\section{Summary}

The lead ore deposits of the Viburnum Trend Subdistrict (Viburnum Trend), in southeastern Missouri are a part of the largest known lead reserve within the United States. Mining in the Viburnum Trend began in 1960 and has continued uninterrupted since. As many as 10 mines have operated in the Viburnum Trend, and presently (2008), six mines are active. Total ore production from individual mines ranges from 20 to more than 50 million tons.

Water-quality data collected from April through June 2002 from streams and springs draining lead and zinc mining areas along the Viburnum Trend and non-mining areas to the south in the Current River and Eleven Point River Basins were compared to quantify water-quality changes spatially and with distance downstream from active mining. Data from 37 historical water-quality samples collected from 1964 to 2005 downstream from the Buick Mine in Strother Creek also were analyzed to document water-quality changes in this stream with time.

Three groups, based on the location of the sampling site to the nearest mining activities or tailings ponds, were used to compare the general chemical composition and trace-element concentrations in surface- and spring-water samples collected from April through June 2002. The three groups include: nonmining sites, sites upstream from mining activity or mine tailings, or sites where no mining activity is present in the basin; near-mining sites, mining activity or mine tailings ponds within 7.5 miles upstream from the sampling site; and distal- mining sites, mining activity distally upstream from sampling site at distances greater than 7.5 miles.

Samples collected from the non-mining sites were calcium-magnesium bicarbonate type, which had little variability and plotted within a "tight" cluster in all three fields of the trilinear diagram. Water-quality data from the near-mining and distal-mining sites had more variability, which generally was caused by differing percentages of sodium, chloride, and sulfate. The upstream-most sites on Courtois Creek and Huzzah Creek drain mining areas, and each have different relative proportions of major ions than non-mining sites. The Huzzah Creek near Scotia site receives water from these two upstream sites and appears to be a mixture of the two upstream sites and ground-water inflow of indigenous water.

Water-sample analysis for the downstream-most Middle Fork Black River site appears to be the product of simple mixing of the waters sampled from the upstream Strother Creek and Neals Creek sites. The Neals Creek site is downstream from the Magmont Mine tailings pile that has been reclaimed; the water-quality analysis plots on the trilinear diagram near the area considered typical for indigenous waters. The Strother Creek site is downstream from the Buick Mine and had a large sulfate concentration and elevated calcium, magnesium, potassium, sodium, and chloride concentrations as compared to samples from Neals Creek and non-mining sites.

Five sites in the West Fork Black River Basin were sampled-one of which is a non-mining site that plotted on the trilinear diagram in the area considered baseline for indigenous waters. The other four sites had greatly elevated sulfate concentrations [16 to $40 \mathrm{mg} / \mathrm{L}$ (milligrams per liter)] and elevated sodium concentrations ( 2.99 to $9.28 \mathrm{mg} / \mathrm{L}$ ) when compared to the range of sulfate concentrations ( 2.2 to $4.1 \mathrm{mg} / \mathrm{L}$ ) and sodium concentrations $(0.7$ to $1.45 \mathrm{mg} / \mathrm{L})$ from non-mining sites. The Bee Fork sample had the largest concentrations of sodium $(9.28 \mathrm{mg} / \mathrm{L})$, chloride $(10 \mathrm{mg} / \mathrm{L})$, and sulfate $(40 \mathrm{mg} / \mathrm{L})$ of the five West Fork Black River Basin samples.

The downstream-most site in the Black River Basin at Lesterville had elevated sulfate $(15 \mathrm{mg} / \mathrm{L})$ concentrations and slightly elevated sodium $(2.34 \mathrm{mg} / \mathrm{L})$ and chloride $(2.6 \mathrm{mg} / \mathrm{L})$ concentrations as compared to samples from non-mining sites. The upstream-most sampling site in Logan Creek, which drains the Sweetwater Mine tailings pond, had the largest concentrations of sodium (17.2 mg/L) and chloride (19 mg/L) of all the sampled sites, and elevated sulfate $(39 \mathrm{mg} / \mathrm{L})$ concentrations compared to non-mining sites. Further downstream at the Ellington site, the water sample had slightly elevated sulfate $(5.4 \mathrm{mg} / \mathrm{L})$ and nitrate $(2.3 \mathrm{mg} / \mathrm{L})$ concentrations and plots on the mixing line with the upstream Logan Creek site and non-mining sites.

The general chemical composition of most water samples collected during this study is a calcium-magnesiumbicarbonate water type, except for Strother Creek, which is a calcium-magnesium-sulfate type. When compared to nonmining samples, most near-mining and distal-mining sites had elevated sulfate and chloride concentrations. 
Sodium, chloride, and sulfate concentrations peak at near-mining sites, then decrease at distal-mining sites, but do not reach the lower concentrations detected at non-mining sites. The copper, nickel, lead, zinc, lithium, and strontium concentrations in non-mining sites and distal-mining sites were similar, but were significantly larger at near-mining sites when compared to the other two groups. These trace-element concentrations peaked at near-mining sites, but decreased downstream at distal-mining sites to concentrations similar to those detected at non-mining sites. Cadmium concentrations were similar at near-mining and distal-mining sites, but were significantly smaller at non-mining sites. Cobalt concentrations peaked at near-mining sites and then decreased at the distal-mining sites. However, even though the concentrations significantly decreased downstream from mining activity, they did not achieve the lesser concentrations detected at the nonmining sites.

Historical water-quality data collected from 1964 through 2005 for two near-mining sites on Strother Creek downstream from the Buick Mine indicated surface-water quality changes with time. The data were separated into four periods and statistically analyzed; period $\mathrm{T} 1$ represents pre-mining data (1964 through 1967), period T2 includes 1968 through 1970 data, period T3 includes 1971 through 1975 data, and period T4 includes 1995 through 2005 data.

Sodium, chloride, sulfate, and potassium concentrations significantly increased after 1967 in Strother Creek Basin and remained elevated through the most recent sampled period (1995 through 2005). Sulfate concentrations continued to significantly increase during each successive period. Calcium and magnesium concentrations also have increased with time. The copper, manganese, and zinc concentrations were similar during the pre-mining and most recent periods. However, manganese concentrations significantly increased during 1971 through 1975, and zinc concentrations decreased during 1968 through 1970 as compared to the pre-mining period.

\section{References Cited}

Guy, H.P., 1969, Laboratory theory and methods for sediment analysis: U.S. Geological Survey Techniques of WaterResources Investigations, book 5, chap. C1, 58 p.

Helsel, D.R., and Hirsch, R.M., 1995, Statistical methods in water resources, in Studies in Environmental Science 49: Amsterdam, The Netherlands, Elsevier Science, 529 p.

Hem, J.D., 1985, Study and interpretation of the chemical characteristics of natural water (3d ed.): U.S. Geological Survey Water-Supply Paper 2254, 263 p.

SYSTAT Software Inc., 2002, SYSTAT user's guide-Statistics I and II: Richmond, Calif., version 10.2, 1,376 p.
Rantz, S.E., and others, 1982, Measurement and computation of streamflow-Volume 1. Measurement of stage and discharge: U.S. Geological Survey Water-Supply Paper 2175, $284 \mathrm{p}$.

Taggart, J.E., Jr., 2002, Analytical methods for chemical analysis of geologic and other materials, U.S. Geological Survey: U.S. Geological Survey Open-File Report 02-223, $20 \mathrm{p}$.

U.S. Geological Survey, 2008a, Mineral commodity summary, accessed January 18, 2008, at URL http://minerals.usgs. gov/minerals/pubs/commodity/statistical_summary/

U.S. Geological Survey, 2008b, National Water Quality Laboratory publications, accessed June 17, 2008, at URL http:// wwwnwql.cr.usgs.gov/USGS/pubs-only.html

Vineyard, J.D., and Feder, G.L., 1982, Springs of Missouri: Rolla, Missouri Department of Natural Resources, Division of Geology and Land Survey Water Resources Report 29, $212 \mathrm{p}$.

Wilde, F.D., and Radtke, D.B., 1998, National field manual for the collection of water-quality data-Field measurements: U.S. Geological Survey Techniques of Water-Resources Investigations, book 9, chap. A6, 238 p.

Wilde, F.D., Radtke, D.B., Gibs, Jacob, and Iwatsubo, R.T., 1999, National field manual for the collection of waterquality data-Collection of water samples: U.S. Geological Survey Techniques of Water-Resources Investigations, book 9, chap. A4, 103 p. with appendices. 

Tables 
Table 1. Water-quality data for the Viburnum Trend and exploration area, April-June 2002.

[mm/dd/yyyy, month, day, year; $\mathrm{ft}^{3} / \mathrm{s}$, cubic feet per second; $\mu \mathrm{S} / \mathrm{cm}$, microsiemens per centimeter at 25 degrees Celsius; ${ }^{\circ} \mathrm{C}$, degrees Celsius; mg/L, milligrams per liter; $\mathrm{NA}$, not applicable; ANC, acid neutralizing capacity; <, less than; $\mu \mathrm{g} / \mathrm{L}$, micrograms per liter]

\begin{tabular}{|c|c|c|c|c|c|c|c|c|c|}
\hline $\begin{array}{c}\text { Site } \\
\text { number } \\
\text { (fig. 1) }\end{array}$ & $\begin{array}{c}\text { Distance } \\
\text { to mining } \\
\text { (miles) }\end{array}$ & Site & $\begin{array}{c}\text { Date } \\
\text { (mm/dd/yyyy) }\end{array}$ & Time & $\begin{array}{c}\text { Discharge } \\
\left(\mathrm{ft}^{3} / \mathbf{s}\right)\end{array}$ & $\begin{array}{c}\text { pH } \\
\text { (standard } \\
\text { units) }\end{array}$ & 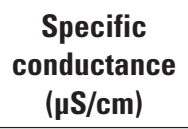 & $\begin{array}{c}\text { Temperature } \\
\left({ }^{\circ} \mathrm{C}\right)\end{array}$ & $\begin{array}{c}\text { Calcium } \\
\text { (mg/L) }\end{array}$ \\
\hline \multicolumn{10}{|c|}{ Non-mining sites } \\
\hline A1 & NA & West Fork Black River near Greeley & $05 / 06 / 2002$ & 1300 & 61 & 8.2 & 220 & 18.8 & 21.0 \\
\hline $\mathrm{A} 2$ & NA & Big Creek at Mauser Mill & $05 / 29 / 2002$ & 1100 & 37 & 7.9 & 247 & 17.5 & 28.3 \\
\hline A3 & NA & Blair Creek & $05 / 29 / 2002$ & 1300 & 16 & 7.9 & 226 & 17.5 & 24.9 \\
\hline A4 & NA & Current River at Van Buren & $06 / 11 / 2002$ & 1030 & 1,910 & 7.7 & 279 & 20.0 & 26.2 \\
\hline A5 & NA & Big Spring near Van Buren & $06 / 11 / 2002$ & 0900 & 697 & 7.0 & 271 & 13.8 & 26.2 \\
\hline A6 & NA & Eleven Point River at Thomasville & $06 / 10 / 2002$ & 1200 & 93 & 7.8 & 294 & 17.7 & 29.7 \\
\hline A7 & NA & Spring Creek Tributary & $06 / 12 / 2002$ & 1500 & 17 & 7.5 & 305 & 15.7 & 29.3 \\
\hline A8 & NA & Spring Creek & $06 / 25 / 2002$ & 1400 & 74 & 8.2 & 305 & 19.2 & 32.2 \\
\hline A9 & NA & McCormack Spring & $06 / 12 / 2002$ & 1715 & 4.6 & 7.5 & 379 & 13.7 & 36.6 \\
\hline A10 & NA & Greer Spring & $06 / 10 / 2002$ & 1700 & 580 & 7.4 & 260 & 13.8 & 26.2 \\
\hline A11 & NA & Hurricane Creek & $06 / 12 / 2002$ & 1130 & 44 & 7.8 & 305 & 18.1 & 29.2 \\
\hline $\mathrm{A} 12$ & NA & Eleven Point River at Bardley & 06/10/2002 & 1500 & 1,200 & 8.2 & 288 & 17.6 & 28.7 \\
\hline \multicolumn{10}{|c|}{ Near-mining sites } \\
\hline B1 & 4.0 & Courtois Creek near Courtois & $04 / 29 / 2002$ & 1300 & 90 & 8.3 & 294 & 16.4 & 29.5 \\
\hline B2 & 6.4 & Strother Creek near Goodland & $04 / 30 / 2002$ & 1100 & 31 & 8.1 & 641 & 17.2 & 57.6 \\
\hline B3 & 7.5 & Neals Creek near Goodland & $04 / 30 / 2002$ & 0830 & 19 & 8.1 & 299 & 13.3 & 31.5 \\
\hline B4 & 3.7 & Bills Creek near Greeley & $05 / 06 / 2002$ & 1430 & 19 & 8.2 & 370 & 18.5 & 33.3 \\
\hline B5 & .9 & West Fork Black River at West Fork & $05 / 06 / 2002$ & 1600 & 107 & 8.2 & 282 & 19.0 & 25.6 \\
\hline B6 & .6 & Bee Fork near Reynolds & 05/06/2002 & 1800 & 22 & 7.7 & 155 & 19.9 & 22.6 \\
\hline B7 & 4.3 & Logan Creek near Corridon & $05 / 29 / 2002$ & 1530 & 16 & 7.7 & 313 & 19.6 & 21.3 \\
\hline \multicolumn{10}{|c|}{ Distal-mining sites } \\
\hline $\mathrm{C} 1$ & 33.6 & Huzzah Creek near Davisville & $04 / 29 / 2002$ & 1545 & 207 & 8.6 & 326 & 18.5 & 31.4 \\
\hline $\mathrm{C} 2$ & 51.9 & Huzzah Creek near Scotia & $04 / 29 / 2002$ & 1845 & 821 & 8.5 & 286 & 17.8 & 28.9 \\
\hline $\mathrm{C} 3$ & 25.2 & Middle Fork Black River near Black & 04/30/2002 & 1400 & 268 & 8.1 & 238 & 16.1 & 22.0 \\
\hline $\mathrm{C} 4$ & 17.5 & West Fork Black River at Centerville & $05 / 07 / 2002$ & 1000 & 176 & 8.2 & 265 & 17.6 & 24.0 \\
\hline C5 & 34.5 & Black River near Lesterville & $05 / 07 / 2002$ & 1100 & 642 & 8.0 & 210 & 16.9 & 18.6 \\
\hline C6 & 35.1 & Logan Creek at Ellington & $05 / 29 / 2002$ & 1800 & 41 & 7.6 & 200 & 18.7 & 19.8 \\
\hline
\end{tabular}


Table 1. Water-quality data for the Viburnum Trend and exploration area, April-June 2002.—Continued

$\left[\mathrm{mm} / \mathrm{dd} / \mathrm{yyyy}\right.$, month, day, year; ft ${ }^{3} / \mathrm{s}$, cubic feet per second; $\mu \mathrm{S} / \mathrm{cm}$, microsiemens per centimeter at 25 degrees Celsius; ${ }^{\circ} \mathrm{C}$, degrees Celsius; mg/L, milligrams per liter; $\mathrm{NA}$, not applicable; ANC, acid neutralizing capacity; <, less than; $\mu \mathrm{g} / \mathrm{L}$, micrograms per liter]

\begin{tabular}{|c|c|c|c|c|c|c|c|c|c|c|c|}
\hline $\begin{array}{c}\text { Site } \\
\text { number } \\
\text { (fig. 1) }\end{array}$ & $\begin{array}{l}\text { Magnesium } \\
\text { (mg/L) }\end{array}$ & $\begin{array}{l}\text { Potassium } \\
\text { (mg/L) }\end{array}$ & $\begin{array}{l}\text { Sodium } \\
\text { (mg/L) }\end{array}$ & $\begin{array}{l}\text { ANC } \\
(\mathrm{mg} / \mathrm{L})\end{array}$ & $\begin{array}{l}\text { Bicarbonate } \\
\text { (mg/L) }\end{array}$ & $\begin{array}{l}\text { Carbonate } \\
\text { (mg/L) }\end{array}$ & $\begin{array}{l}\text { Chloride } \\
\text { (mg/L) }\end{array}$ & $\begin{array}{l}\text { Fluoride } \\
\text { (mg/L) }\end{array}$ & $\begin{array}{l}\text { Silica } \\
\text { (mg/L) }\end{array}$ & $\begin{array}{l}\text { Sulfate } \\
\text { (mg/L) }\end{array}$ & $\begin{array}{l}\text { Nitrate } \\
\text { (mg/L) }\end{array}$ \\
\hline \multicolumn{12}{|c|}{ Non-mining sites } \\
\hline $\mathrm{A} 1$ & 13.0 & 0.8 & 1.17 & 109 & 132 & $<1$ & 1.3 & $<0.08$ & 3.6 & 4.1 & $<0.08$ \\
\hline $\mathrm{A} 2$ & 14.3 & 1.0 & 1.04 & 130 & 158 & $<1$ & 1.5 & $<.08$ & 3.4 & 3.9 & .5 \\
\hline A3 & 12.7 & 1.3 & .87 & 105 & 128 & $<1$ & 1.6 & $<.08$ & 3.6 & 3.7 & $<.08$ \\
\hline A4 & 17.2 & .9 & 1.45 & 135 & 165 & $<1$ & 2.5 & $<.08$ & 2.7 & 3.4 & 1.2 \\
\hline A5 & 17.0 & .9 & 1.27 & 131 & 160 & $<1$ & 2.2 & $<.08$ & 3.5 & 2.3 & 2.5 \\
\hline A6 & 17.9 & 1.2 & 1.11 & 148 & 181 & $<1$ & 2.3 & $<.08$ & 3.2 & 2.4 & 2.5 \\
\hline A7 & 19.9 & .5 & .70 & 151 & 185 & $<1$ & .9 & $<.08$ & 3.4 & 2.5 & $<.08$ \\
\hline A8 & 17.0 & 1.1 & 1.05 & 149 & 182 & $<1$ & 2.3 & $<.08$ & 3.8 & 2.5 & 2.6 \\
\hline A9 & 25.3 & .5 & .96 & 191 & 233 & $<1$ & 1.4 & $<.08$ & 3.7 & 2.2 & $<.08$ \\
\hline A10 & 16.3 & 1.4 & 1.44 & 124 & 151 & $<1$ & 3.1 & $<.08$ & 3.9 & 2.4 & 4.3 \\
\hline A11 & 19.6 & .8 & .97 & 144 & 176 & $<1$ & 1.7 & $<.08$ & 3.3 & 2.8 & .9 \\
\hline $\mathrm{A} 12$ & 17.5 & 1.2 & 1.31 & 136 & 166 & $<1$ & 2.7 & $<.08$ & 3.2 & 2.4 & 2.3 \\
\hline \multicolumn{12}{|c|}{ Near-mining sites } \\
\hline B1 & 17.8 & 0.8 & 2.62 & 118 & 144 & $<1$ & 3.5 & 0.08 & 4.0 & 22 & $<0.08$ \\
\hline B2 & 36.7 & 3.3 & 11.1 & 112 & 137 & $<1$ & 16 & .2 & 2.8 & 190 & 2.5 \\
\hline B3 & 19.2 & .7 & 1.44 & 154 & 188 & $<1$ & 1.7 & $<.08$ & 3.5 & 8.1 & $<.08$ \\
\hline B4 & 21.7 & 1.2 & 5.55 & 147 & 180 & $<1$ & 6.8 & .1 & 3.9 & 34 & 2.1 \\
\hline B5 & 16.4 & 1.0 & 3.13 & 120 & 146 & $<1$ & 3.7 & .09 & 3.6 & 19 & .4 \\
\hline B6 & 15.6 & 1.4 & 9.28 & 85 & 104 & $<1$ & 10 & .2 & 3.2 & 40 & 1.2 \\
\hline B7 & 14.0 & 2.0 & 17.2 & 78 & 95 & $<1$ & 19 & .3 & 2.8 & 39 & .5 \\
\hline \multicolumn{12}{|c|}{ Distal-mining sites } \\
\hline $\mathrm{C} 1$ & 18.0 & 1.0 & 8.92 & 159 & 191 & $<1$ & 2.9 & 0.08 & 3.6 & 23 & 0.2 \\
\hline $\mathrm{C} 2$ & 16.8 & .9 & 3.78 & 140 & 170 & $<1$ & 2.7 & $<.08$ & 3.8 & 13 & .2 \\
\hline $\mathrm{C} 3$ & 13.1 & 1.0 & 2.32 & 92 & 113 & $<1$ & 3.2 & $<.08$ & 3.7 & 25 & .5 \\
\hline $\mathrm{C} 4$ & 16.0 & .9 & 2.99 & 106 & 129 & $<1$ & 3.3 & .09 & 3.4 & 16 & .4 \\
\hline $\mathrm{C} 5$ & 12.4 & 1.0 & 2.34 & 78 & 95 & $<1$ & 2.6 & $<.08$ & 3.9 & 15 & .3 \\
\hline C6 & 10.1 & 1.2 & 1.47 & 84 & 103 & $<1$ & 3.1 & $<.08$ & 3.3 & 5.4 & 2.3 \\
\hline
\end{tabular}


Table 1. Water-quality data for the Viburnum Trend and exploration area, April-June 2002.—Continued

[mm/dd/yyyy, month, day, year; $\mathrm{ft}^{3} / \mathrm{s}$, cubic feet per second; $\mu \mathrm{S} / \mathrm{cm}$, microsiemens per centimeter at 25 degrees Celsius; ${ }^{\circ} \mathrm{C}$, degrees Celsius; mg/L, milligrams per liter; $\mathrm{NA}$, not applicable; ANC, acid neutralizing capacity; $<$, less than; $\mu \mathrm{g} / \mathrm{L}$, micrograms per liter]

\begin{tabular}{|c|c|c|c|c|c|c|c|c|c|c|c|c|}
\hline $\begin{array}{c}\text { Site } \\
\text { number } \\
\text { (fig. 1) }\end{array}$ & $\begin{array}{l}\text { Phosphorus } \\
\text { (mg/L) }\end{array}$ & $\begin{array}{c}\text { Aluminum } \\
(\mu \mathrm{g} / \mathrm{L})\end{array}$ & $\begin{array}{c}\text { Antimony } \\
(\mu \mathrm{g} / \mathrm{L})\end{array}$ & $\begin{array}{c}\text { Arsenic } \\
\text { ( } \mu \mathrm{g} / \mathrm{L})\end{array}$ & $\begin{array}{c}\text { Barium } \\
\text { ( } \mu \mathrm{g} / \mathrm{L})\end{array}$ & $\begin{array}{l}\text { Beryllium } \\
\text { ( } \mu \mathrm{g} / \mathrm{L})\end{array}$ & $\begin{array}{l}\text { Bismuth } \\
\text { ( } \mu \mathrm{g} / \mathrm{L})\end{array}$ & $\begin{array}{l}\text { Cadmium } \\
\text { ( } \mu \mathrm{g} / \mathrm{L})\end{array}$ & $\begin{array}{c}\text { Cesium } \\
\text { ( } \mu \mathrm{g} / \mathrm{L})\end{array}$ & $\begin{array}{c}\text { Chromium } \\
(\mu \mathrm{g} / \mathrm{L})\end{array}$ & $\begin{array}{l}\text { Cobalt } \\
(\mu \mathrm{g} / \mathrm{L})\end{array}$ & $\begin{array}{c}\text { Copper } \\
\text { ( } \mu \mathrm{g} / \mathrm{L})\end{array}$ \\
\hline \multicolumn{13}{|c|}{ Non-mining sites } \\
\hline A1 & $<0.01$ & 2 & $<0.1$ & $<1.0$ & 27.4 & $<0.05$ & $<0.005$ & 0.06 & $<0.01$ & 2.2 & 0.08 & 1.2 \\
\hline A2 & $<.01$ & 3 & $<.1$ & $<1.0$ & 31.9 & $<.05$ & $<.005$ & .02 & $<.01$ & 1.7 & .06 & .56 \\
\hline A3 & $<.01$ & 8 & $<.1$ & $<1.0$ & 26.1 & $<.05$ & $<.005$ & .04 & .03 & 1.1 & .06 & .59 \\
\hline A4 & $<.01$ & 1 & $<.1$ & $<1.0$ & 32.8 & $<.05$ & $<.005$ & $<.02$ & $<.01$ & 1.1 & .06 & $<.5$ \\
\hline A5 & $<.01$ & 5 & $<.1$ & $<1.0$ & 29.3 & $<.05$ & $<.005$ & .03 & .02 & 1.4 & .04 & $<.5$ \\
\hline A6 & $<.01$ & 1 & $<.1$ & $<1.0$ & 33.1 & $<.05$ & $<.005$ & $<.02$ & $<.01$ & 2.2 & .07 & $<.5$ \\
\hline A7 & $<.01$ & .3 & $<.1$ & $<1.0$ & 19.1 & $<.05$ & $<.005$ & $<.02$ & $<.01$ & 1.1 & .04 & $<.5$ \\
\hline A8 & .01 & 5 & $<.3$ & $<1.0$ & 30.9 & $<.05$ & $<.2$ & .04 & $<.02$ & $<1$ & .05 & $<.5$ \\
\hline A9 & $<.01$ & 6 & $<.1$ & $<1.0$ & 24.3 & $<.05$ & $<.005$ & .02 & $<.01$ & 1.4 & .06 & $<.5$ \\
\hline A 10 & $<.01$ & 5 & $<.1$ & $<1.0$ & 32.5 & $<.05$ & $<.005$ & $<.02$ & .02 & 2.0 & .04 & $<.5$ \\
\hline A11 & $<.01$ & .7 & $<.1$ & $<1.0$ & 27.2 & $<.05$ & $<.005$ & $<.02$ & $<.01$ & $<1$ & .06 & $<.5$ \\
\hline A12 & $<.01$ & 1 & $<.1$ & $<1.0$ & 31.0 & $<.05$ & $<.005$ & $<.02$ & $<.01$ & 2.2 & .06 & $<.5$ \\
\hline \multicolumn{13}{|c|}{ Near-mining sites } \\
\hline B1 & $<0.01$ & 2 & 0.1 & $<1.0$ & 31.7 & $<0.05$ & $<0.005$ & 0.48 & $<0.01$ & 1.7 & 0.23 & 1.5 \\
\hline B2 & $<.01$ & 2 & 1 & 2 & 30.8 & $<.05$ & $<.005$ & .22 & .06 & 2.0 & 20 & 1.2 \\
\hline B3 & $<.01$ & 1 & .1 & $<1.0$ & 31.4 & $<.05$ & $<.005$ & .20 & $<.01$ & 2.3 & .10 & .66 \\
\hline B4 & $<.01$ & 2 & .3 & $<1.0$ & 30.7 & $<.05$ & $<.005$ & .18 & $<.01$ & 2.8 & 2.4 & 1.0 \\
\hline B5 & $<.01$ & 3 & .1 & $<1.0$ & 29.2 & $<.05$ & $<.005$ & .03 & $<.01$ & 2.5 & .93 & .61 \\
\hline B6 & $<.01$ & 3 & .3 & $<1.0$ & 35.5 & $<.05$ & $<.005$ & .05 & .02 & 2.0 & .50 & .59 \\
\hline B7 & $<.01$ & 3 & .1 & $<1.0$ & 50.3 & $<.05$ & $<.005$ & .10 & $<.01$ & 1.1 & .71 & .80 \\
\hline \multicolumn{13}{|c|}{ Distal-mining sites } \\
\hline $\mathrm{C} 1$ & $<0.01$ & 2 & 0.8 & $<1.0$ & 35.2 & $<0.05$ & $<0.005$ & 0.32 & $<0.01$ & 3.0 & 0.08 & 0.83 \\
\hline $\mathrm{C} 2$ & $<.01$ & 3 & .2 & $<1.0$ & 87.7 & $<.05$ & $<.005$ & .12 & .02 & 2.6 & .09 & .66 \\
\hline $\mathrm{C} 3$ & $<.01$ & 2 & .2 & $<1.0$ & 28.4 & $<.05$ & $<.005$ & .07 & $<.01$ & 1.6 & .21 & $<.5$ \\
\hline $\mathrm{C} 4$ & $<.01$ & 1 & .1 & $<1.0$ & 29.6 & $<.05$ & $<.005$ & .03 & $<.01$ & 1.1 & .09 & $<.5$ \\
\hline C5 & $<.01$ & 5 & $<.1$ & $<1.0$ & 31.6 & $<.05$ & $<.005$ & $<.02$ & .02 & $<1$ & .08 & $<.5$ \\
\hline C6 & $<.01$ & 2 & $<.1$ & $<1.0$ & 39.0 & $<.05$ & $<.005$ & .03 & $<.01$ & 1.2 & .07 & $<.5$ \\
\hline
\end{tabular}


Table 1. Water-quality data for the Viburnum Trend and exploration area, April-June 2002.—Continued

$\left[\mathrm{mm} / \mathrm{dd} / \mathrm{yyyy}\right.$, month, day, year; ft ${ }^{3} / \mathrm{s}$, cubic feet per second; $\mu \mathrm{S} / \mathrm{cm}$, microsiemens per centimeter at 25 degrees Celsius; ${ }^{\circ} \mathrm{C}$, degrees Celsius; mg/L, milligrams per liter; $\mathrm{NA}$, not applicable; ANC, acid neutralizing capacity; $<$, less than; $\mu \mathrm{g} / \mathrm{L}$, micrograms per liter]

\begin{tabular}{|c|c|c|c|c|c|c|c|c|c|c|c|c|}
\hline $\begin{array}{c}\text { Site } \\
\text { number } \\
\text { (fig. 1) }\end{array}$ & $\begin{array}{c}\text { Gallium } \\
\text { ( } \mu \mathrm{g} / \mathrm{L})\end{array}$ & $\begin{array}{c}\text { Germanium } \\
(\mu \mathrm{g} / \mathrm{L})\end{array}$ & $\begin{array}{l}\text { Iron } \\
(\mu \mathrm{g} / \mathrm{L})\end{array}$ & $\begin{array}{l}\text { Lanthanum } \\
(\mu \mathrm{g} / \mathrm{L})\end{array}$ & $\begin{array}{l}\text { Lead } \\
\text { ( } \mu \mathrm{g} / \mathrm{L})\end{array}$ & $\begin{array}{l}\text { Lithium } \\
\text { ( } \mu \mathrm{g} / \mathrm{L} \text { ) }\end{array}$ & $\begin{array}{c}\text { Manganese } \\
(\mu \mathrm{g} / \mathrm{L})\end{array}$ & $\begin{array}{c}\text { Molybdenum } \\
(\mu \mathrm{g} / \mathrm{L})\end{array}$ & $\begin{array}{c}\text { Nickel } \\
(\mu \mathrm{g} / \mathrm{L})\end{array}$ & $\begin{array}{c}\text { Niobium } \\
(\mu \mathrm{g} / \mathrm{L})\end{array}$ & $\begin{array}{c}\text { Rubidium } \\
\text { ( } \mu \mathrm{g} / \mathrm{L})\end{array}$ & $\begin{array}{c}\text { Scandium } \\
(\mu \mathrm{g} / \mathrm{L})\end{array}$ \\
\hline \multicolumn{13}{|c|}{ Non-mining sites } \\
\hline $\mathrm{A} 1$ & $<0.02$ & $<0.02$ & $<50$ & $<0.01$ & 0.2 & $<0.1$ & 2.9 & $<0.2$ & 0.7 & 0.04 & 0.66 & 0.7 \\
\hline $\mathrm{A} 2$ & $<.02$ & $<.02$ & $<50$ & .02 & .1 & $<.1$ & 6.0 & $<.2$ & .8 & .04 & .92 & 1.2 \\
\hline A3 & $<.02$ & $<.02$ & $<50$ & .03 & .3 & .2 & 3.9 & $<.2$ & .8 & .03 & .77 & 1.1 \\
\hline A4 & $<.02$ & $<.02$ & $<50$ & .01 & .05 & $<.1$ & 4.5 & $<.2$ & .8 & $<.02$ & .87 & .8 \\
\hline A5 & $<.02$ & $<.02$ & $<50$ & .05 & .07 & .1 & 0.4 & .23 & .9 & $<.02$ & 1 & .9 \\
\hline A6 & $<.02$ & $<.02$ & $<50$ & .02 & .05 & .2 & 10 & .32 & .8 & .04 & .95 & .9 \\
\hline A7 & $<.02$ & $<.02$ & $<50$ & .02 & .07 & $<.1$ & 1.1 & .29 & .8 & $<.02$ & .56 & 1 \\
\hline A8 & $<.05$ & $<.05$ & $<50$ & .02 & .2 & .2 & 3.5 & $<2$ & .9 & $<.20$ & 1 & 1 \\
\hline A9 & $<.02$ & $<.02$ & $<50$ & .10 & .1 & $<.1$ & 1.3 & $<.2$ & 1.1 & $<.02$ & .68 & 1 \\
\hline $\mathrm{A} 10$ & $<.02$ & $<.02$ & $<50$ & .06 & .05 & .1 & .2 & .32 & .8 & .02 & 1 & 1 \\
\hline A11 & $<.02$ & $<.02$ & $<50$ & .01 & .09 & $<.1$ & 7.4 & .20 & .9 & $<.02$ & .84 & .9 \\
\hline $\mathrm{A} 12$ & $<.02$ & $<.02$ & $<50$ & .02 & .06 & .2 & 6.0 & .31 & .9 & $<.02$ & 1 & .9 \\
\hline \multicolumn{13}{|c|}{ Near-mining sites } \\
\hline B1 & $<0.02$ & $<0.02$ & $<50$ & 0.01 & 0.4 & 0.3 & 2.6 & 0.43 & 3.6 & 0.06 & 0.80 & 1 \\
\hline B2 & $<.02$ & .04 & $<50$ & .01 & 2.3 & 3.7 & 50 & 6 & 47.5 & .06 & 5 & .6 \\
\hline B3 & $<.02$ & $<.02$ & $<50$ & .01 & .08 & $<.1$ & 1.9 & .34 & 2.4 & .05 & .62 & .8 \\
\hline B4 & $<.02$ & .03 & $<50$ & .01 & 7.7 & 4.4 & 4.3 & 1 & 8.7 & .03 & 1 & .8 \\
\hline B5 & $<.02$ & $<.02$ & $<50$ & $<.01$ & 1.0 & 1.3 & 30 & .64 & 4.2 & .04 & .99 & .7 \\
\hline B6 & $<.02$ & $<.02$ & $<50$ & .01 & 1.8 & 5.1 & 6.7 & 2 & 4.7 & .04 & 2 & .7 \\
\hline B7 & $<.02$ & $<.02$ & $<50$ & $<.01$ & .6 & 6.8 & 260 & 2 & 3.0 & .03 & 3 & .8 \\
\hline \multicolumn{13}{|c|}{ Distal-mining sites } \\
\hline $\mathrm{C} 1$ & $<0.02$ & $<0.02$ & $<50$ & 0.02 & 0.1 & $<0.1$ & 3.5 & 0.40 & 1.0 & 0.06 & 0.86 & 0.8 \\
\hline $\mathrm{C} 2$ & $<.02$ & $<.02$ & $<50$ & .02 & .09 & $<.1$ & 8.0 & .29 & 1.0 & .04 & .71 & .8 \\
\hline $\mathrm{C} 3$ & $<.02$ & $<.02$ & $<50$ & .01 & .08 & .2 & 1.9 & .91 & 2.4 & .05 & .98 & .9 \\
\hline $\mathrm{C} 4$ & $<.02$ & $<.02$ & $<50$ & $<.01$ & .2 & 1 & 1.6 & .55 & 1.0 & .02 & .89 & .7 \\
\hline $\mathrm{C} 5$ & $<.02$ & $<.02$ & $<50$ & .02 & .08 & .3 & 3.3 & .38 & .8 & .02 & .86 & .7 \\
\hline C6 & $<.02$ & $<.02$ & $<50$ & .01 & .1 & $<.1$ & 7.5 & .24 & .6 & .02 & .91 & .8 \\
\hline
\end{tabular}


Table 1. Water-quality data for the Viburnum Trend and exploration area, April-June 2002.—Continued

[mm/dd/yyyy, month, day, year; $\mathrm{ft}^{3} / \mathrm{s}$, cubic feet per second; $\mu \mathrm{S} / \mathrm{cm}$, microsiemens per centimeter at 25 degrees Celsius; ${ }^{\circ} \mathrm{C}$, degrees Celsius; mg/L, milligrams per liter; $\mathrm{NA}$, not applicable; ANC, acid neutralizing capacity; $<$, less than; $\mu \mathrm{g} / \mathrm{L}$, micrograms per liter]

\begin{tabular}{|c|c|c|c|c|c|c|c|c|c|c|c|c|}
\hline $\begin{array}{c}\text { Site } \\
\text { number } \\
\text { (fig. 1) }\end{array}$ & $\begin{array}{l}\text { Selenium } \\
(\mu \mathrm{g} / \mathrm{L})\end{array}$ & $\begin{array}{l}\text { Silver } \\
\text { ( } \mu \mathrm{g} / \mathrm{L})\end{array}$ & $\begin{array}{c}\text { Strontium } \\
(\mu \mathrm{g} / \mathrm{L})\end{array}$ & $\begin{array}{l}\text { Thallium } \\
\text { ( } \mu \mathrm{g} / \mathrm{L})\end{array}$ & $\begin{array}{c}\text { Thorium } \\
\text { ( } \mu \mathrm{g} / \mathrm{L} \text { ) }\end{array}$ & $\begin{array}{c}\text { Titanium } \\
(\mu \mathrm{g} / \mathrm{L})\end{array}$ & $\begin{array}{l}\text { Vanadium } \\
\text { ( } \mu \mathrm{g} / \mathrm{L})\end{array}$ & $\begin{array}{c}\text { Yttrium } \\
\text { ( } \mu \mathrm{g} / \mathrm{L})\end{array}$ & $\begin{array}{c}\text { Zinc } \\
(\mu \mathrm{g} / \mathrm{L})\end{array}$ & $\begin{array}{l}\text { Zirconium } \\
\quad(\mu \mathrm{g} / \mathrm{L})\end{array}$ & $\begin{array}{c}\text { Uranium } \\
(\mu \mathrm{g} / \mathrm{L})\end{array}$ & $\begin{array}{c}\text { Suspended } \\
\text { sediment } \\
\text { (mg/L) }\end{array}$ \\
\hline \multicolumn{13}{|c|}{ Non-mining sites } \\
\hline A1 & $<1$ & $<3$ & 20 & $<0.1$ & 0.005 & $<0.1$ & 1.0 & 0.02 & 2.6 & $<0.05$ & 0.1 & 14 \\
\hline $\mathrm{A} 2$ & $<1$ & $<3$ & 30 & $<.1$ & $<.005$ & $<.1$ & .8 & .03 & 2.0 & $<.05$ & .1 & 13 \\
\hline $\mathrm{A} 3$ & $<1$ & $<3$ & 20 & $<.1$ & $<.005$ & $<.1$ & .5 & .04 & 4.9 & $<.05$ & .1 & 16 \\
\hline A4 & $<1$ & $<3$ & 30 & $<.1$ & $<.005$ & $<.1$ & .7 & .04 & 0.8 & $<.05$ & .2 & 17 \\
\hline A5 & $<1$ & $<3$ & 20 & $<.1$ & $<.005$ & .3 & .7 & .09 & 2.8 & $<.05$ & .2 & 31 \\
\hline A6 & $<1$ & $<3$ & 30 & $<.1$ & .005 & $<.1$ & 1.0 & .03 & 1.0 & $<.05$ & .2 & 13 \\
\hline A7 & $<1$ & $<3$ & 20 & $<.1$ & $<.005$ & $<.1$ & .6 & .02 & .6 & $<.05$ & .1 & 18 \\
\hline A8 & $<1$ & $<3$ & 30 & $<.1$ & $<.200$ & $<.5$ & $<.5$ & .04 & 3.7 & $<.2$ & .2 & 18 \\
\hline A9 & $<1$ & $<3$ & 30 & $<.1$ & $<.005$ & $<.1$ & .6 & .11 & 2.0 & $<.05$ & .2 & 22 \\
\hline $\mathrm{A} 10$ & $<1$ & $<3$ & 30 & $<.1$ & $<.005$ & $<.1$ & .8 & .08 & 1.0 & $<.05$ & .2 & 15 \\
\hline A11 & $<1$ & $<3$ & 20 & $<.1$ & $<.005$ & $<.1$ & .5 & .03 & 1.0 & $<.05$ & .1 & 23 \\
\hline A12 & $<1$ & $<3$ & 30 & $<.1$ & $<.005$ & $<.1$ & 1.0 & .04 & .9 & $<.05$ & .2 & 18 \\
\hline \multicolumn{13}{|c|}{ Near-mining sites } \\
\hline $\mathrm{B} 1$ & $<1$ & $<3$ & 40 & $<.1$ & .020 & $<.1$ & .7 & .03 & 20 & $<.05$ & .1 & 27 \\
\hline B2 & $<1$ & $<3$ & 110 & $<.1$ & .020 & 2.0 & .7 & .03 & 150 & $<.05$ & .2 & 59 \\
\hline B3 & $<1$ & $<3$ & 30 & $<.1$ & .007 & $<.1$ & 1.0 & .02 & 5.4 & $<.05$ & .1 & 23 \\
\hline B4 & $<1$ & $<3$ & 70 & $<.1$ & .009 & .2 & 1.1 & .03 & 40 & $<.05$ & .1 & 18 \\
\hline B5 & $<1$ & $<3$ & 40 & $<.1$ & .007 & $<.1$ & 1.1 & .02 & 10 & $<.05$ & .1 & 14 \\
\hline B6 & $<1$ & $<3$ & 70 & $<.1$ & .008 & .3 & .8 & .02 & 5.5 & $<.05$ & .2 & 19 \\
\hline B7 & $<1$ & $<3$ & 80 & $<.1$ & $<.005$ & .4 & .4 & .02 & 20 & $<.05$ & .1 & 19 \\
\hline \multicolumn{13}{|c|}{ Distal-mining sites } \\
\hline $\mathrm{C} 1$ & $<1$ & $<3$ & 30 & $<.1$ & .009 & .1 & 1.4 & .03 & 2.4 & $<.05$ & .2 & 21 \\
\hline $\mathrm{C} 2$ & $<1$ & $<3$ & 30 & $<.1$ & .006 & $<.1$ & 1.1 & .04 & 2.0 & $<.05$ & .1 & 19 \\
\hline $\mathrm{C} 3$ & $<1$ & $<3$ & 30 & $<.1$ & .008 & .2 & .8 & .02 & 8.3 & $<.05$ & .1 & 14 \\
\hline $\mathrm{C} 4$ & $<1$ & $<3$ & 40 & $<.1$ & .006 & $<.1$ & .7 & .02 & 2.0 & $<.05$ & .1 & 14 \\
\hline C5 & $<1$ & $<3$ & 30 & $<.1$ & $<.005$ & $<.1$ & .6 & .04 & 1.0 & $<.05$ & .1 & 12 \\
\hline C6 & $<1$ & $<3$ & 30 & $<.1$ & $<.005$ & $<.1$ & .5 & .02 & 3.1 & $<.05$ & .1 & 9 \\
\hline
\end{tabular}


Table 2. Water-quality summary statistics for major anions and cations and trace elements, April-June 2002.

[mg/L, milligrams per liter; proximity-to-mining category-A, non-mining sites; B, near-mining sites, mining activities within 7.5 miles upstream; C, distal-mining sites, mining acitivity greater than 7.5 miles upstream; Q25, 25th percentile; Q50, 50th percentile; Q75, 75th percentile; interquartile range, difference between Q75 and Q25; $\mu \mathrm{g} / \mathrm{L}$, micrograms per liter; <, less than; --, not computed]

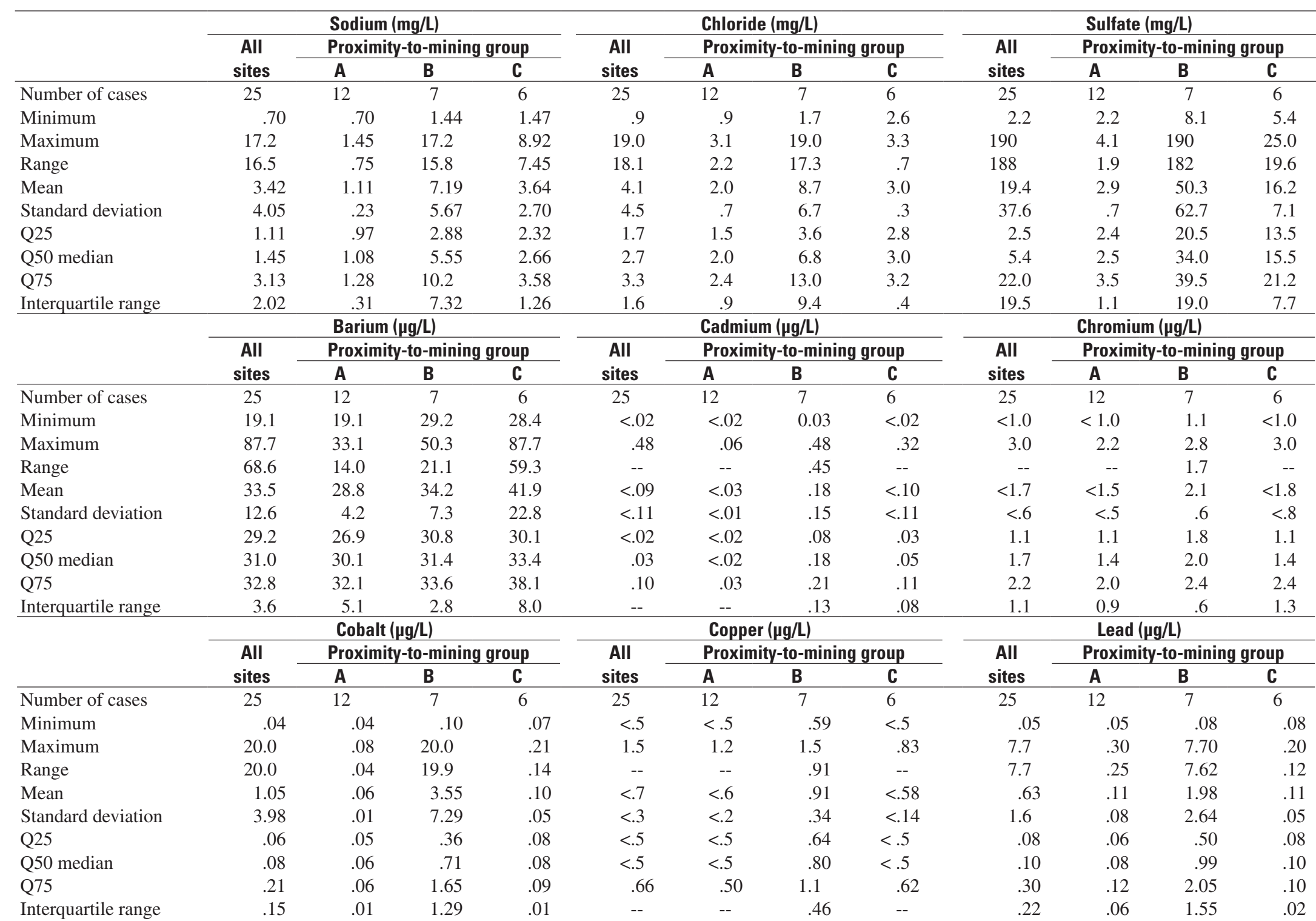


Table 2. Water-quality summary statistics for major anions and cations and trace elements, April-June 2002.—Continued

[mg/L, milligrams per liter; proximity-to-mining category—A, non-mining site; B, near-mining sites, mining activities within 7.5 miles upstream; C, distal-mining sites, mining acitivity greater than 7.5 miles upstream; Q25, 25th percentile; Q50, 50th percentile; Q75, 75th percentile; interquartile range, difference between Q75 and Q25; $\mu \mathrm{g} / \mathrm{L}$, micrograms per liter; <, less than; --, not computed]

\begin{tabular}{|c|c|c|c|c|c|c|c|c|c|c|c|c|}
\hline & \multicolumn{4}{|c|}{ Lithium ( $\mu \mathrm{g} / \mathrm{L})$} & \multicolumn{4}{|c|}{ Manganese ( $\mu \mathrm{g} / \mathrm{L})$} & \multicolumn{4}{|c|}{ Nickel ( $\mu \mathrm{g} / \mathrm{L})$} \\
\hline & \multirow{2}{*}{$\begin{array}{c}\text { All } \\
\text { sites }\end{array}$} & \multicolumn{3}{|c|}{ Proximity-to-mining group } & \multirow{2}{*}{$\begin{array}{c}\text { All } \\
\text { sites }\end{array}$} & \multicolumn{3}{|c|}{ Proximity-to-mining group } & \multirow{2}{*}{$\begin{array}{c}\text { All } \\
\text { sites }\end{array}$} & \multicolumn{3}{|c|}{ Proximity-to-mining group } \\
\hline & & A & B & C & & A & B & C & & A & B & C \\
\hline Number of cases & 25 & 12 & 7 & 6 & 25 & 12 & 7 & 6 & 25 & 12 & 7 & 6 \\
\hline Minimum & $<.1$ & $<.1$ & $<.1$ & $<.1$ & .2 & .2 & 1.9 & 1.6 & .6 & .7 & 2.4 & .6 \\
\hline Maximum & 6.8 & .2 & 6.8 & 1.0 & 260 & 10 & 260 & 8.0 & 47.5 & 1.1 & 47.5 & 2.4 \\
\hline Range & -- & -- & -- & -- & 260 & 9.8 & 258 & 6.4 & 46.9 & .4 & 45.1 & 1.8 \\
\hline Mean & $<1.0$ & $<.1$ & $<3.1$ & $<.3$ & 17 & 3.9 & 51 & 4.3 & 3.6 & .8 & 10.6 & 1.1 \\
\hline Standard deviation & $<1.9$ & $<.05$ & $<2.6$ & $<.4$ & 52 & 3.0 & 94 & 2.8 & 9.3 & .1 & 16.4 & .6 \\
\hline Q25 & $<.1$ & $<.1$ & .8 & $<.1$ & 1.9 & 1.2 & 3.4 & 2.2 & .8 & .8 & 3.3 & .8 \\
\hline Q50 median & .2 & .1 & 3.7 & .2 & 3.9 & 3.7 & 6.7 & 3.4 & .9 & .8 & 4.2 & 1.0 \\
\hline Q75 & .3 & .2 & 4.8 & .3 & 7.4 & 6.0 & 40 & 6.5 & 2.4 & .9 & 6.7 & 1.0 \\
\hline \multirow[t]{4}{*}{ Interquartile range } & -- & -- & 4.0 & -- & 5.5 & 4.8 & 36.6 & 4.3 & 1.6 & .1 & 3.4 & .2 \\
\hline & \multicolumn{4}{|c|}{ Rubidium ( $\mu \mathrm{g} / \mathrm{L}$ ) } & \multicolumn{4}{|c|}{ Strontium ( $\mu \mathrm{g} / \mathrm{L}$ ) } & \multicolumn{4}{|c|}{ Zinc $(\mu \mathrm{g} / \mathrm{L})$} \\
\hline & \multirow{2}{*}{$\begin{array}{c}\text { All } \\
\text { sites }\end{array}$} & \multicolumn{3}{|c|}{ Proximity-to-mining group } & All & \multicolumn{3}{|c|}{ Proximity-to-mining group } & All & \multicolumn{3}{|c|}{ Proximity-to-mining group } \\
\hline & & A & B & C & sites & A & B & C & sites & A & B & C \\
\hline Number of cases & 25 & 12 & 7 & 6 & 25 & 12 & 7 & 6 & 25 & 12 & 7 & 6 \\
\hline Minimum & .56 & .56 & .62 & .71 & 20 & 20 & 30 & 30 & 6 & 6 & 5.4 & 1.0 \\
\hline Maximum & 5.00 & 1.00 & 5.00 & .98 & 110 & 30 & 110 & 40 & 150 & 4.9 & 150 & 8.3 \\
\hline Range & 4.44 & .44 & 4.38 & .27 & 90 & 10 & 80 & 10 & 149.4 & 4.3 & 144.6 & 7.3 \\
\hline Mean & 1.15 & .85 & 1.92 & .87 & 38 & 26 & 63 & 32 & 11.7 & 1.9 & 35.8 & 3.1 \\
\hline Standard deviation & .94 & .15 & 1.60 & .09 & 22 & 5 & 28 & 4 & 30.1 & 1.3 & 51.7 & 2.6 \\
\hline Q25 & .80 & .75 & .90 & .86 & 30 & 20 & 40 & 30 & 1.0 & 1.0 & 7.8 & 2.0 \\
\hline Q50 median & .91 & .90 & 1.00 & .88 & 30 & 30 & 70 & 30 & 2.6 & 1.5 & 20.0 & 2.2 \\
\hline Q75 & 1.00 & 1.00 & 2.50 & .90 & 40 & 30 & 75 & 30 & 5.5 & 2.7 & 30.0 & 2.9 \\
\hline Interquartile range & .20 & .25 & 1.6 & .04 & 10 & 10 & 35 & 0 & 4.5 & 1.7 & 22.2 & .9 \\
\hline
\end{tabular}


Table 3. Surface-water quality data for Strother Creek near Oates and Goodland, 1964-2005.

[All constituents dissolved unless otherwise indicated as total; $\mathrm{mm} / \mathrm{dd} / \mathrm{yyyy}$, month, day, year; $\mathrm{ft} / \mathrm{s}$, cubic feet per second; $\mu \mathrm{S} / \mathrm{cm}$, microsiemens per centimeter at $25 \mathrm{degrees}$ Celsius; ${ }^{\circ} \mathrm{C}$, degrees Celsius; mg/L, milligrams per liter; $\mathrm{ANC}$, acid neutralizing capacity; $\mathrm{CaCO}_{3}$, calcium carbonate; --, no data; $\mathrm{N}$, nitrogen; $\mu \mathrm{g} / \mathrm{L}$, micrograms per liter; $<$, less than; $\mathrm{ND}$, not detected]

\begin{tabular}{|c|c|c|c|c|c|c|c|c|c|c|}
\hline Group & $\begin{array}{c}\text { Date } \\
\text { (mm/dd/yyyy) }\end{array}$ & $\begin{array}{c}\text { Discharge } \\
\left(\mathrm{ft}^{3} / \mathrm{s}\right)\end{array}$ & $\begin{array}{c}\text { pH } \\
\text { (standard } \\
\text { units) }\end{array}$ & $\begin{array}{c}\text { Specific } \\
\text { conductance } \\
(\mu \mathrm{S} / \mathrm{cm})\end{array}$ & $\begin{array}{c}\text { Temperature } \\
\left({ }^{\circ} \mathrm{C}\right)\end{array}$ & $\begin{array}{c}\text { Calcium } \\
\text { (mg/L) }\end{array}$ & $\begin{array}{l}\text { Magnesium } \\
\text { (mg/L) }\end{array}$ & $\begin{array}{l}\text { Potassium } \\
\text { (mg/L) }\end{array}$ & $\begin{array}{c}\text { Sodium } \\
\text { (mg/L) }\end{array}$ & $\begin{array}{c}\text { ANC } \\
\text { total } \\
\left(\mathrm{mg} / \mathrm{L} \text { as } \mathrm{CaCO}_{3}\right)\end{array}$ \\
\hline \multicolumn{11}{|c|}{ Strother Creek near Oates (ST1) } \\
\hline $\mathrm{T} 1$ & $03 / 16 / 1964$ & -- & 8.2 & 207 & -- & 22 & 13 & 0.6 & 0.9 & -- \\
\hline \multirow[t]{5}{*}{$(1964-1967)$} & 06/08/1965 & -- & 8.0 & 220 & -- & 24 & 13 & .6 & .8 & -- \\
\hline & 09/30/1965 & 3.1 & 7.9 & 225 & -- & 30 & 19 & .8 & 1.4 & -- \\
\hline & $01 / 26 / 1966$ & 1.5 & 8.1 & 250 & -- & 34 & 20 & .2 & 1.1 & 154 \\
\hline & 08/10/1966 & 1.5 & 8.1 & 300 & -- & 41 & 19 & .8 & 1.4 & 170 \\
\hline & $10 / 24 / 1967$ & 2.0 & 7.9 & 286 & 15 & 34 & 18 & 1.2 & 5.1 & 143 \\
\hline $\mathrm{T} 2$ & $10 / 17 / 1968$ & 12 & 8.0 & 300 & 20 & 31 & 16 & 3.9 & 8.6 & 158 \\
\hline \multirow[t]{8}{*}{$(1968-1970)$} & $01 / 16 / 1969$ & 13 & 8.0 & 260 & 6 & 26 & 15 & 3.2 & 9.1 & 106 \\
\hline & $04 / 09 / 1969$ & 13 & 8.2 & 270 & 15 & 37 & 20 & .8 & .8 & 146 \\
\hline & 07/15/1969 & 11 & 8.4 & 360 & 27 & 47 & 18 & 4.0 & 12 & 198 \\
\hline & $10 / 29 / 1969$ & 9.1 & 7.8 & 405 & 12 & 38 & 25 & 4.3 & 14 & 170 \\
\hline & 01/26/1970 & 15 & 8.0 & 412 & 7 & 34 & 28 & 4.1 & 15 & 170 \\
\hline & 04/30/1970 & 3.9 & 8.0 & 304 & 18 & 30 & 17 & 2.7 & 6.1 & 140 \\
\hline & 08/20/1970 & 10 & 9.2 & 486 & 27 & 37 & 26 & 4.7 & 22 & -- \\
\hline & $11 / 03 / 1970$ & 14 & 8.4 & 500 & 12 & 39 & 26 & 4.4 & 18 & 166 \\
\hline $\mathrm{T} 3$ & $01 / 27 / 1971$ & 10 & 8.6 & 550 & 1 & 46 & 33 & 5.6 & 23 & 162 \\
\hline \multirow[t]{9}{*}{ (1971-1975) } & 04/20/1971 & 12 & 8.4 & 540 & 17 & 39 & 32 & 5.0 & 20 & 166 \\
\hline & $07 / 12 / 1971$ & 10 & 8.3 & 570 & 28 & 36 & 33 & 6.5 & 26 & 138 \\
\hline & $11 / 08 / 1971$ & 12 & 7.6 & 650 & 10 & 48 & 33 & 6.0 & 26 & 158 \\
\hline & 02/01/1972 & 1.3 & 7.3 & 420 & 3 & 38 & 24 & 2.8 & 9.5 & 174 \\
\hline & 06/06/1972 & 10 & 7.4 & 550 & 24 & 42 & 33 & 5.1 & 21 & 168 \\
\hline & 08/21/1972 & 9.9 & 7.8 & 600 & 32 & 44 & 39 & 6.0 & 25 & 168 \\
\hline & $10 / 17 / 1972$ & 14 & 8.1 & 650 & 14 & 50 & 35 & 6.6 & 27 & 158 \\
\hline & 01/30/1973 & 14 & 8.0 & 425 & 6 & 37 & 21 & 3.9 & 13 & 118 \\
\hline & 05/15/1973 & 16 & 8.2 & 375 & 18 & 34 & 17 & 2.7 & 7.4 & 126 \\
\hline
\end{tabular}


Table 3. Surface-water quality data for Strother Creek near Oates and Goodland, 1964-2005.-Continued

[All constituents dissolved unless otherwise indicated as total; mm/dd/yyyy, month, day, year; $\mathrm{ft} / \mathrm{s}$, cubic feet per second; $\mu \mathrm{S} / \mathrm{cm}$, microsiemens per centimeter at $25 \mathrm{degrees} \mathrm{Celsius;}{ }^{\circ} \mathrm{C}$, degrees Celsius; mg/L, milligrams per liter; $\mathrm{ANC}$, acid neutralizing capacity; $\mathrm{CaCO}_{3}$, calcium carbonate; --, no data; $\mathrm{N}$, nitrogen; $\mu \mathrm{g} / \mathrm{L}$, micrograms per liter; <, less than; $\mathrm{ND}$, not detected]

\begin{tabular}{|c|c|c|c|c|c|c|c|c|c|c|}
\hline Group & $\begin{array}{c}\text { Date } \\
\text { (mm/dd/yyyy) }\end{array}$ & $\begin{array}{c}\text { Discharge } \\
\left(\mathrm{ft}^{3} / \mathrm{s}\right)\end{array}$ & $\begin{array}{c}\text { pH } \\
\text { (standard } \\
\text { units) }\end{array}$ & $\begin{array}{c}\text { Specific } \\
\text { conductance } \\
(\mu \mathrm{S} / \mathrm{cm})\end{array}$ & $\begin{array}{c}\text { Temperature } \\
\left({ }^{\circ} \mathrm{C}\right)\end{array}$ & $\begin{array}{c}\text { Calcium } \\
\text { (mg/L) }\end{array}$ & $\begin{array}{l}\text { Magnesium } \\
\text { (mg/L) }\end{array}$ & $\begin{array}{l}\text { Potassium } \\
\text { (mg/L) }\end{array}$ & $\begin{array}{c}\text { Sodium } \\
\text { (mg/L) }\end{array}$ & $\begin{array}{c}\text { ANC } \\
\text { total } \\
\left(\mathrm{mg} / \mathrm{L} \mathrm{as} \mathrm{CaCO}_{3}\right)\end{array}$ \\
\hline \multicolumn{11}{|c|}{ Strother Creek near Oates (ST1)_-Continued } \\
\hline $\mathrm{T} 3$ & $07 / 31 / 1973$ & 8.7 & 7.5 & 460 & 26 & 17 & 39 & 3.8 & 15 & 126 \\
\hline \multirow[t]{8}{*}{$(1971-1975)$} & $11 / 27 / 1973$ & 107 & 7.6 & 340 & 12 & 13 & 2.9 & 2.7 & 8.3 & 80 \\
\hline & 03/05/1974 & 22 & 7.9 & 360 & 14 & 35 & 20 & 2.9 & 10 & 124 \\
\hline & 05/14/1974 & 23 & 8.3 & 370 & 18 & 34 & 17 & 2.4 & 8.6 & 126 \\
\hline & 08/13/1974 & 7.1 & 8.3 & 625 & 27 & -- & -- & 6.8 & 24 & 106 \\
\hline & $10 / 30 / 1974$ & 12 & 8.3 & 700 & 18 & -- & -- & 5.2 & 23 & 136 \\
\hline & 01/07/1975 & 14 & 7.8 & 600 & 4 & -- & -- & 3.8 & 19 & 150 \\
\hline & $04 / 22 / 1975$ & 14.2 & 8.3 & 500 & 15 & -- & -- & 3.7 & 14 & 130 \\
\hline & $07 / 29 / 1975$ & 13 & 8.0 & 560 & 27 & -- & -- & 4.6 & 18 & 162 \\
\hline \multirow{4}{*}{$\begin{array}{l}\text { T4 } \\
(1995-2005) \\
\end{array}$} & 09/19/1995 & 9.9 & 8.1 & 729 & 19.7 & 64 & 44 & 4.8 & 17 & 142 \\
\hline & \multicolumn{10}{|c|}{ Strother Creek near Goodland (B2) } \\
\hline & $04 / 30 / 2002$ & 30.6 & 8.1 & 641 & 17.2 & 58 & 37 & 3.3 & 11 & 111 \\
\hline & $07 / 06 / 2005$ & 8.5 & 7.9 & 864 & 25.9 & 80 & 55 & 5.0 & 26 & ${ }^{\mathrm{a}} 123$ \\
\hline
\end{tabular}


Table 3. Surface-water quality data for Strother Creek near Oates and Goodland, 1964-2005._Continued

[All constituents dissolved unless otherwise indicated as total; $\mathrm{mm} / \mathrm{dd} / \mathrm{yyyy}$, month, day, year; $\mathrm{ft} / \mathrm{s}$, cubic feet per second; $\mu \mathrm{S} / \mathrm{cm}$, microsiemens per centimeter at 25 degrees Celsius; ${ }^{\circ} \mathrm{C}$, degrees Celsius; $\mathrm{mg} / \mathrm{L}$, milligrams per liter; $\mathrm{ANC}$, acid neutralizing capacity; $\mathrm{CaCO}_{3}$, calcium carbonate; --, no data; $\mathrm{N}$, nitrogen; $\mu \mathrm{g} / \mathrm{L}$, micrograms per liter; <, less than; $\mathrm{ND}$, not detected

\begin{tabular}{|c|c|c|c|c|c|c|c|c|c|c|c|}
\hline Group & $\begin{array}{c}\text { Date } \\
\text { (mm/dd/yyyy) }\end{array}$ & $\begin{array}{c}\text { Bicarbonate, } \\
\text { total } \\
(\mathrm{mg} / \mathrm{L})\end{array}$ & $\begin{array}{c}\text { Carbonate, } \\
\text { total } \\
(\mathrm{mg} / \mathrm{L})\end{array}$ & $\begin{array}{l}\text { Chloride } \\
\text { (mg/L) }\end{array}$ & $\begin{array}{c}\text { Fluoride } \\
\text { (mg/L) }\end{array}$ & $\begin{array}{l}\text { Silica } \\
\text { (mg/L) }\end{array}$ & $\begin{array}{l}\text { Sulfate } \\
\text { (mg/L) }\end{array}$ & $\begin{array}{c}\text { Nitrate } \\
\text { (mg/L as } N)\end{array}$ & $\begin{array}{c}\text { Barium } \\
(\mu \mathrm{g} / \mathrm{L})\end{array}$ & $\begin{array}{l}\text { Boron } \\
\text { ( } \mu \mathrm{g} / \mathrm{L})\end{array}$ & $\begin{array}{c}\text { Cadmium } \\
\text { ( } \mu \mathrm{g} / \mathrm{L})\end{array}$ \\
\hline \multicolumn{12}{|c|}{ Strother Creek near Oates (ST1) } \\
\hline $\mathrm{T} 1$ & $03 / 16 / 1964$ & 122 & $<1$ & 0.5 & ND & 6.5 & 5.6 & 0.2 & -- & -- & ND \\
\hline \multirow[t]{5}{*}{ (1964-1967) } & 06/08/1965 & 138 & $<1$ & .5 & ND & 8.6 & 5.4 & .1 & 30 & -- & -- \\
\hline & 09/30/1965 & 165 & $<1$ & .9 & 0.1 & 8.4 & 4.4 & .1 & 30 & -- & -- \\
\hline & $01 / 26 / 1966$ & 188 & $<1$ & .9 & .1 & 6.2 & 3.6 & ND & -- & -- & -- \\
\hline & 08/10/1966 & 207 & $<1$ & 6 & .2 & 9.7 & 2.2 & -- & ${ }^{\mathrm{b}} .06$ & -- & -- \\
\hline & $10 / 24 / 1967$ & 174 & $<1$ & 2.0 & .2 & 7.4 & 9.4 & ND & 40 & 10 & -- \\
\hline $\mathrm{T} 2$ & $10 / 17 / 1968$ & 193 & $<1$ & 2.4 & .2 & 8.0 & 14 & .1 & 90 & ND & ND \\
\hline \multirow[t]{8}{*}{ (1968-1970) } & 01/16/1969 & 129 & $<1$ & 3.2 & .4 & 10.0 & 15 & .4 & 1,600 & ND & ND \\
\hline & 04/09/1969 & 178 & $<1$ & .9 & ND & 6.6 & 5.6 & .1 & ND & 200 & ND \\
\hline & 07/15/1969 & 233 & 4 & 5.0 & .6 & 8.8 & 20 & -- & ND & ND & ND \\
\hline & $10 / 29 / 1969$ & 207 & $<1$ & 12 & .3 & 9.0 & 32 & -- & ND & 63 & 3 \\
\hline & 01/26/1970 & 207 & $<1$ & 14 & .4 & 6.2 & 32 & -- & 300 & ND & ND \\
\hline & 04/30/1970 & 171 & $<1$ & 4.7 & ND & 7.3 & 16 & .3 & ND & ND & ND \\
\hline & 08/20/1970 & -- & -- & 21 & .5 & 6.7 & 65 & .3 & ND & 140 & ND \\
\hline & $11 / 03 / 1970$ & 197 & 2 & 20 & .1 & 7.0 & 48 & .3 & 15,000 & -- & ND \\
\hline T3 & 01/27/1971 & 193 & 2 & 25 & 1.4 & 6.0 & 62 & .4 & ND & -- & ND \\
\hline \multirow[t]{9}{*}{ (1971-1975) } & 04/20/1971 & 200 & 1 & 24 & .1 & 6 & 64 & 1.2 & ND & -- & ND \\
\hline & 07/12/1971 & 163 & 2 & 57 & .1 & .8 & 66 & .9 & ND & -- & ND \\
\hline & 11/08/1971 & 193 & $<1$ & -- & -- & 7.2 & 13 & c 1.7 & ND & -- & ND \\
\hline & 02/01/1972 & 212 & $<1$ & 20 & $<.1$ & -- & 33 & c. 7 & ND & -- & ND \\
\hline & 06/06/1972 & 205 & $<1$ & -- & -- & 8.6 & 75 & ${ }^{\mathrm{c}} .5$ & ND & -- & ND \\
\hline & 08/21/1972 & 205 & $<1$ & 30 & .6 & 6.0 & 42 & ${ }^{\mathrm{c}}<.1$ & ND & -- & ND \\
\hline & $10 / 17 / 1972$ & 193 & $<1$ & 22 & $<.1$ & 4.2 & 134 & ${ }^{\mathrm{c}}<.1$ & $<100$ & -- & ND \\
\hline & 01/30/1973 & 144 & $<1$ & 55 & .1 & 6.9 & 83 & c .5 & $<100$ & -- & ND \\
\hline & 05/15/1973 & 154 & $<1$ & 7.5 & .5 & 8.0 & 49 & c. 1 & $<100$ & -- & ND \\
\hline
\end{tabular}


Table 3. Surface-water quality data for Strother Creek near Oates and Goodland, 1964-2005.-Continued

[All constituents dissolved unless otherwise indicated as total; mm/dd/yyyy, month, day, year; $\mathrm{ft} / \mathrm{s}$, cubic feet per second; $\mu \mathrm{S} / \mathrm{cm}$, microsiemens per centimeter at 25 degrees Celsius; ${ }^{\circ} \mathrm{C}$, degrees Celsius; $\mathrm{mg} / \mathrm{L}$, milligrams per liter; $\mathrm{ANC}$, acid neutralizing capacity; $\mathrm{CaCO}_{3}$, calcium carbonate; --, no data; $\mathrm{N}$, nitrogen; $\mu \mathrm{g} / \mathrm{L}$, micrograms per liter; <, less than; $\mathrm{ND}$, not detected]

\begin{tabular}{|c|c|c|c|c|c|c|c|c|c|c|c|}
\hline Group & $\begin{array}{c}\text { Date } \\
\text { (mm/dd/yyyy) }\end{array}$ & $\begin{array}{c}\text { Bicarbonate, } \\
\text { total } \\
\text { (mg/L) }\end{array}$ & $\begin{array}{c}\text { Carbonate, } \\
\text { total } \\
\text { (mg/L) }\end{array}$ & $\begin{array}{c}\text { Chloride } \\
\text { (mg/L) }\end{array}$ & $\begin{array}{c}\text { Fluoride } \\
\text { (mg/L) }\end{array}$ & $\begin{array}{l}\text { Silica } \\
\text { (mg/L) }\end{array}$ & $\begin{array}{l}\text { Sulfate } \\
\text { (mg/L) }\end{array}$ & $\begin{array}{c}\text { Nitrate } \\
\text { (mg/L as } N)\end{array}$ & $\begin{array}{c}\text { Barium } \\
(\mu \mathrm{g} / \mathrm{L})\end{array}$ & $\begin{array}{l}\text { Boron } \\
(\mu g / L)\end{array}$ & $\begin{array}{l}\text { Cadmium } \\
\text { ( } \mu \mathrm{g} / \mathrm{L})\end{array}$ \\
\hline \multicolumn{12}{|c|}{ Strother Creek near Oates (ST1)_-Continued } \\
\hline $\mathrm{T} 3$ & $07 / 31 / 1973$ & 154 & $<1$ & 2.1 & 0.1 & 5.0 & 76 & ${ }^{\mathrm{c}}<0.1$ & 10 & -- & ND \\
\hline \multirow[t]{8}{*}{$(1971-1975)$} & $11 / 27 / 1973$ & 98 & $<1$ & -- & .1 & -- & 49 & c. 3 & $<100$ & -- & ND \\
\hline & 03/05/1974 & 151 & $<1$ & 22 & .3 & -- & 11 & ${ }^{\mathrm{c}} .6$ & $<100$ & -- & ND \\
\hline & 05/14/1974 & 154 & $<1$ & 11 & .2 & -- & 68 & c. 5 & $<100$ & -- & $<2$ \\
\hline & 08/13/1974 & -- & -- & 40 & -- & -- & 130 & ${ }^{\mathrm{c}} 1.3$ & -- & -- & ND \\
\hline & $10 / 30 / 1974$ & -- & -- & 50 & -- & -- & 180 & ${ }^{\mathrm{c}} 1.9$ & -- & -- & ND \\
\hline & 01/07/1975 & -- & -- & 28 & -- & -- & 135 & ${ }^{\mathrm{c}} 1.6$ & -- & -- & $<2$ \\
\hline & $04 / 22 / 1975$ & -- & -- & 17 & -- & -- & 140 & ${ }^{\mathrm{c}} .8$ & -- & -- & ND \\
\hline & $07 / 29 / 1975$ & -- & -- & 19 & -- & -- & 140 & ${ }^{\mathrm{c}}<.1$ & -- & -- & $<2$ \\
\hline \multirow{4}{*}{$\begin{array}{l}\text { T4 } \\
(1995-2005)\end{array}$} & 09/19/1995 & 165 & $<1$ & 24 & .2 & 6.2 & 200 & .73 & 31 & -- & $<1$ \\
\hline & & \multicolumn{10}{|c|}{ Strother Creek near Goodland (B2) } \\
\hline & $04 / 30 / 2002$ & 137 & $<1$ & 16 & 0.2 & 2.8 & 190 & 2.5 & 31 & -- & 0.2 \\
\hline & $07 / 06 / 2005$ & 150 & $<1$ & 28 & .2 & 6.7 & 287 & -- & 46 & 82 & .1 \\
\hline
\end{tabular}


Table 3. Surface-water quality data for Strother Creek near Oates and Goodland, 1964-2005._Continued

[All constituents dissolved unless otherwise indicated as total; $\mathrm{mm} / \mathrm{dd} / \mathrm{yyyy}$, month, day, year; $\mathrm{ft} / \mathrm{s}$, cubic feet per second; $\mu \mathrm{S} / \mathrm{cm}$, microsiemens per centimeter at 25 degrees Celsius; ${ }^{\circ} \mathrm{C}$, degrees Celsius; $\mathrm{mg} / \mathrm{L}$, milligrams per liter; $\mathrm{ANC}$, acid neutralizing capacity; $\mathrm{CaCO}_{3}$, calcium carbonate; --, no data; $\mathrm{N}$, nitrogen; $\mu \mathrm{g} / \mathrm{L}$, micrograms per liter; <, less than; $\mathrm{ND}$, not detected]

\begin{tabular}{|c|c|c|c|c|c|c|c|c|c|c|c|c|}
\hline Group & $\begin{array}{c}\text { Date } \\
\text { (mm/dd/ } \\
\text { yyyy) }\end{array}$ & $\begin{array}{c}\text { Chromium } \\
(\mu \mathrm{g} / \mathrm{L})\end{array}$ & $\begin{array}{l}\text { Cobalt } \\
(\mu \mathrm{g} / \mathrm{L})\end{array}$ & $\begin{array}{c}\text { Copper } \\
(\mu \mathrm{g} / \mathrm{L})\end{array}$ & $\begin{array}{l}\text { Iron } \\
\text { ( } \mu \mathrm{g} / \mathrm{L})\end{array}$ & $\begin{array}{l}\text { Lead } \\
(\mu \mathrm{g} / \mathrm{L})\end{array}$ & $\begin{array}{c}\text { Lithium } \\
\text { ( } \mu \mathrm{g} / \mathrm{L})\end{array}$ & $\begin{array}{c}\text { Manganese } \\
(\mu \mathrm{g} / \mathrm{L})\end{array}$ & $\begin{array}{c}\text { Nickel } \\
\text { ( } \mu \mathrm{g} / \mathrm{L})\end{array}$ & $\begin{array}{l}\text { Silver } \\
\text { ( } \mu \mathrm{g} / \mathrm{L})\end{array}$ & $\begin{array}{c}\text { Strontium } \\
(\mu \mathrm{g} / \mathrm{L})\end{array}$ & $\begin{array}{c}\text { Zinc } \\
\text { ( } \mu \mathrm{g} / \mathrm{L})\end{array}$ \\
\hline \multicolumn{13}{|c|}{ Strother Creek near Oates (ST1) } \\
\hline $\mathrm{T} 1$ & $03 / 16 / 1964$ & ND & ND & ${ }^{\mathrm{b}} 0.01$ & -- & 20 & -- & ND & -- & -- & -- & 50 \\
\hline \multirow[t]{5}{*}{$(1964-1967)$} & $06 / 08 / 1965$ & ND & ND & ND & -- & ND & -- & ND & ND & -- & -- & 70 \\
\hline & 09/30/1965 & ND & ND & ND & -- & ND & -- & ND & ND & -- & -- & 70 \\
\hline & $01 / 26 / 1966$ & -- & -- & -- & -- & -- & -- & ND & -- & -- & -- & -- \\
\hline & 08/10/1966 & -- & -- & -- & -- & -- & ND & ND & -- & -- & ${ }^{\mathrm{b}} 0.03$ & -- \\
\hline & $10 / 24 / 1967$ & ND & ND & ND & -- & ND & ND & -- & ND & -- & 20 & -- \\
\hline $\mathrm{T} 2$ & $10 / 17 / 1968$ & ND & ND & ND & -- & ND & 20 & 10 & ND & -- & 140 & 10 \\
\hline \multirow[t]{8}{*}{ (1968-1970) } & $01 / 16 / 1969$ & ND & 10 & ND & -- & ND & ND & 10 & ND & -- & 60 & 10 \\
\hline & 04/09/1969 & ND & ND & ND & -- & 10 & ND & ND & 20 & -- & 20 & 30 \\
\hline & $07 / 15 / 1969$ & ND & 10 & 20 & -- & ND & ND & 20 & 10 & -- & 220 & 20 \\
\hline & $10 / 29 / 1969$ & 5 & 4 & 4 & 6 & ND & ND & 50 & 29 & 8 & 210 & 13 \\
\hline & $01 / 26 / 1970$ & 5 & 1 & 10 & 33 & 25 & 13 & 54 & 16 & ND & 200 & 21 \\
\hline & 04/30/1970 & 5 & 15 & 10 & 22 & 6 & ND & 6 & 15 & ND & 200 & 11 \\
\hline & 08/20/1970 & 7 & ND & 10 & 17 & ND & 6 & 10 & ND & ND & -- & 18 \\
\hline & $11 / 03 / 1970$ & 14 & 9 & 4 & 40 & 60 & -- & 90 & 24 & 20 & -- & 20 \\
\hline $\mathrm{T} 3$ & $01 / 27 / 1971$ & 6 & 12 & 4 & 100 & 32 & -- & 70 & ND & ND & -- & 40 \\
\hline \multirow[t]{9}{*}{ (1971-1975) } & 04/20/1971 & 1 & ND & 2 & ND & 23 & -- & 25 & 14 & ND & -- & 10 \\
\hline & 07/12/1971 & 4 & 2 & 5 & 20 & 20 & -- & 60 & 2 & ND & -- & 30 \\
\hline & $11 / 08 / 1971$ & 1 & ND & 11 & ND & 4 & -- & 30 & 1 & ND & -- & 20 \\
\hline & $02 / 01 / 1972$ & 1 & 1 & 4 & ND & 8 & -- & ND & 6 & 2 & -- & ND \\
\hline & 06/06/1972 & ND & ND & -- & ND & 4 & -- & 140 & 19 & ND & -- & 70 \\
\hline & 08/21/1972 & ND & 3 & 2 & 10 & 2 & -- & 40 & 1 & ND & -- & ND \\
\hline & $10 / 17 / 1972$ & ND & ND & 3 & $<10$ & ND & -- & 40 & 10 & ND & -- & 60 \\
\hline & $01 / 30 / 1973$ & $<20$ & 4 & 8 & $<10$ & ND & -- & 210 & 3 & ND & -- & 140 \\
\hline & $05 / 15 / 1973$ & $<20$ & 5 & 4 & $<10$ & ND & -- & 180 & 12 & ND & -- & 70 \\
\hline
\end{tabular}


Table 3. Surface-water quality data for Strother Creek near Oates and Goodland, 1964-2005.-Continued

[All constituents dissolved unless otherwise indicated as total; mm/dd/yyyy, month, day, year; $\mathrm{ft} / \mathrm{s}$, cubic feet per second; $\mu \mathrm{S} / \mathrm{cm}$, microsiemens per centimeter at 25 degrees Celsius; ${ }^{\circ} \mathrm{C}$, degrees Celsius; $\mathrm{mg} / \mathrm{L}$, milligrams per liter; $\mathrm{ANC}$, acid neutralizing capacity; $\mathrm{CaCO}_{3}$, calcium carbonate; --, no data; $\mathrm{N}$, nitrogen; $\mu \mathrm{g} / \mathrm{L}$, micrograms per liter; <, less than; $\mathrm{ND}$, not detected]

\begin{tabular}{|c|c|c|c|c|c|c|c|c|c|c|c|c|}
\hline Group & $\begin{array}{c}\text { Date } \\
\text { (mm/dd/ } \\
\text { yyyy) }\end{array}$ & $\begin{array}{l}\text { Chromium } \\
(\mu \mathrm{g} / \mathrm{L})\end{array}$ & $\begin{array}{l}\text { Cobalt } \\
\text { ( } \mu \mathrm{g} / \mathrm{L})\end{array}$ & $\begin{array}{c}\text { Copper } \\
\text { ( } \mu \mathrm{g} / \mathrm{L} \text { ) }\end{array}$ & 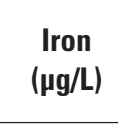 & $\begin{array}{l}\text { Lead } \\
\text { ( } \mu \mathrm{g} / \mathrm{L})\end{array}$ & $\begin{array}{l}\text { Lithium } \\
\text { ( } \mu \mathrm{g} / \mathrm{L})\end{array}$ & $\begin{array}{c}\text { Manganese } \\
(\mu \mathrm{g} / \mathrm{L})\end{array}$ & $\begin{array}{c}\text { Nickel } \\
\text { ( } \mu \mathrm{g} / \mathrm{L})\end{array}$ & $\begin{array}{l}\text { Silver } \\
\text { ( } \mu \mathrm{g} / \mathrm{L})\end{array}$ & $\begin{array}{c}\text { Strontium } \\
(\mu \mathrm{g} / \mathrm{L})\end{array}$ & $\begin{array}{l}\text { Zinc } \\
(\mu \mathrm{g} / \mathrm{L})\end{array}$ \\
\hline \multicolumn{13}{|c|}{ Strother Creek near Oates (ST1)_Continued } \\
\hline $\mathrm{T} 3$ & $07 / 31 / 1973$ & ND & $<2$ & 5 & 18.75 & $<2$ & -- & 90 & 9 & ND & -- & 20 \\
\hline \multirow[t]{8}{*}{$(1971-1975)$} & $11 / 27 / 1973$ & ND & 6 & 6 & 40 & 65 & -- & 210 & 10 & ND & -- & 90 \\
\hline & 03/05/1974 & ND & 5 & 11 & 30 & 12 & -- & 280 & 10 & ND & -- & 60 \\
\hline & 05/14/1974 & ND & $<2$ & 3 & 50 & 4 & -- & 120 & 6 & ND & -- & $<20$ \\
\hline & 08/13/1974 & -- & -- & 2 & -- & 5 & -- & 80 & -- & -- & -- & ND \\
\hline & $10 / 30 / 1974$ & -- & -- & $<2$ & -- & 2 & -- & 140 & -- & -- & -- & 40 \\
\hline & 01/07/1975 & -- & -- & 7 & -- & 4 & -- & 200 & -- & -- & -- & 150 \\
\hline & $04 / 22 / 1975$ & -- & -- & 4 & -- & 4 & -- & 180 & -- & -- & -- & ND \\
\hline & 07/29/1975 & -- & -- & $<2$ & -- & 10 & -- & 60 & -- & -- & -- & ND \\
\hline $\begin{array}{l}\text { T4 } \\
(1995-2005)\end{array}$ & 09/19/1995 & 2 & 31 & 2 & 19 & 3 & -- & 57 & 114 & $<1$ & -- & 148 \\
\hline \multicolumn{13}{|c|}{ Strother Creek near Goodland (B2) } \\
\hline & $04 / 30 / 2002$ & 2 & 18 & 1 & $<50$ & 2 & 4 & 50 & 48 & $<3$ & 106 & 151 \\
\hline & 07/06/2005 & $<.8$ & 7 & 2 & $<6$ & 1 & 6 & 1.4 & 26 & $<.2$ & 160 & 46 \\
\hline
\end{tabular}

${ }^{a}$ Dissolved concentration.

${ }^{\mathrm{b}}$ Suspicious value may have been entered in data base as milligrams per liter.

${ }^{c}$ Total concentration. 


\section{Water-Quality Trends and Effects of Lead and Zinc Mining on Upper Logan Creek and Blue Spring, Southeastern Missouri, 1925-2006}

By John G. Schumacher

\section{Chapter 7 of}

Hydrologic Investigations Concerning Lead Mining Issues in Southeastern Missouri

Edited by Michael J. Kleeschulte

Scientific Investigations Report 2008-5140 


\section{Contents}

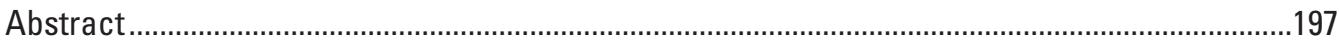

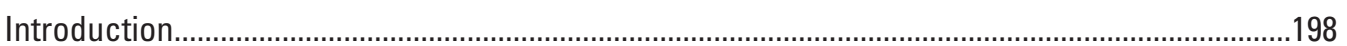

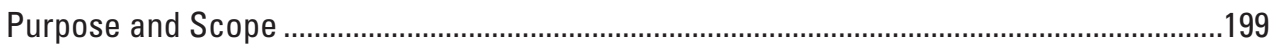

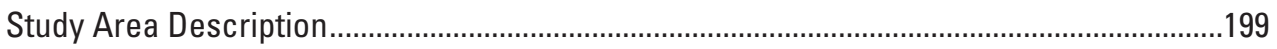

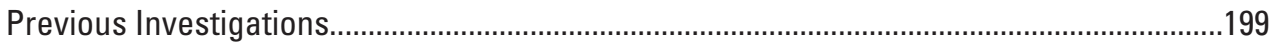

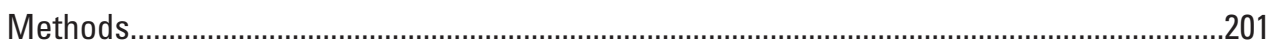

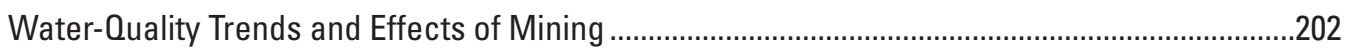

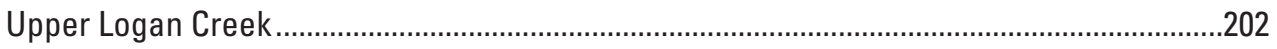

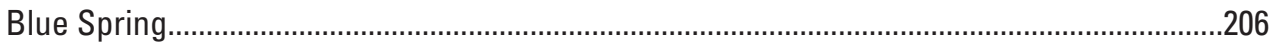

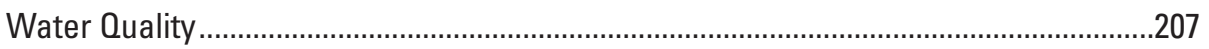

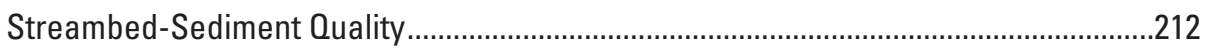

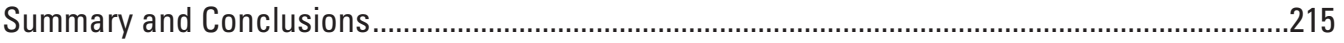

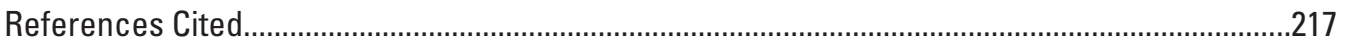

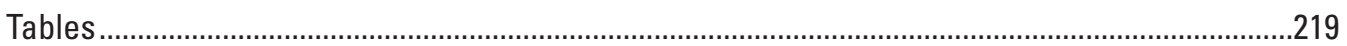

\section{Figures}

1-2. Maps showing-

1. Location of Logan Creek, Blue Spring, and the Ozark National Scenic Riverways.

2. Historical water-quality sampling sites and seepage-run measurements made at high base flow (March 7, 2006) and low base flow (August 15, 2006) along the upper reach of Logan Creek.

3. Trilinear diagram of water samples collected from upper Logan Creek, 1965-2006....203

4. Graphs showing discharge, specific conductance values, and concentrations of selected inorganic constituents in water samples collected from Logan Creek, 1969-1975.

5. Trilinear diagram of water samples collected from Blue Spring (1925-2006) and water samples collected from upper Logan Creek during 2006

6. Box plots showing concentrations of selected major ions and nitrate plus nitrate as nitrogen in water samples from Blue Spring, Big Spring, and Greer Spring and results of analysis of variance and multiple comparison tests on rank ordered data

7-9. Graphs showing-

7. Discharge, specific conductance, and concentrations of selected chemical constituents with time in water samples from Blue Spring, 1925-2006

8. Concentrations of Mississippi Valley Type (MVT)-related metals in the fine (less than 0.063-millimeter size) fraction of streambed-sediment samples from Blue Spring and baseline concentrations from Big and Greer Springs, 1995-2002

9. Maximum concentrations of Mississippi Valley Type (MVT)-related metals in the fine (less than 0.063 -millimeter size) fraction by inductively coupled liquid plasma-atomic emmission spectrometry (ICP-AES) and semi-quantitative concentrations in the non-magnetic heavy mineral fraction by emmission spectrometry in streambed-sediment samples from Blue Spring and the Current River, 1995-2002. 


\section{Tables}

1. Physical properties and concentrations of inorganic chemical constituents in water samples from upper Logan Creek, 1965-2006

2. Water-quality samples from Blue Spring analyzed for major and trace inorganic constituents, 1925-2006.

3. Measured and simulated concentrations of selected constituents in water samples collected from Blue Spring in March and August 2006.

4. Grain-size, semi-quantitative bulk mineralogy, and optical mineralogy of the non-magnetic heavy mineral (C3) fraction of streambed-sediment samples from Blue Spring, Big Spring, and Greer Spring, 1995-2002.

5. Concentrations of major and trace elements in the fine (less than 0.063-millimeter size) fraction of streambed-sediment samples from Blue Spring, Current River, Big Spring, and Greer Spring, 1995-2002.

6. Semi-quantitative concentrations of major and trace elements in the nonmagnetic heavy mineral (C3) fraction of streambed-sediment samples from Blue Spring and the Current River near Blue Spring, 1995. 



\title{
Water-Quality Trends and Effects of Lead and Zinc Mining on Upper Logan Creek and Blue Spring, Southeastern Missouri, 1925-2006
}

\author{
By John G. Schumacher
}

\section{Abstract}

Dye-tracing investigations have indicated a subsurface connection between a losing reach of Logan Creek downstream from an active lead mine (Sweetwater Mine) and Blue Spring, the seventh largest spring in Missouri. Blue Spring is an important source of water to the Current River within the Ozark National Scenic Riverways. Analyses of historical water-quality samples from Logan Creek (1965-2006) and Blue Spring (1925-2006) and streambed-sediment samples (1995-2002) were performed to characterize changes in water quality in the upper Logan Creek Basin resulting from a lead and zinc mine and the effects of these changes on the water quality of Blue Spring. These multiple lines of evidence from previous dye-trace investigations, water-quality sampling, and trace element and mineralogic composition of streambed-sediment samples indicate likely effects of mining on the water quality of Blue Spring.

Water samples from upper Logan Creek ranged from calcium-magnesium-bicarbonate type, typical of the Ozark region, to mixed sodium-magnesium-calcium-bicarbonatesulfate type, indicating increased concentrations of sodium, chloride, and sulfate resulting from mining operations. After mine development in the 1960s, sulfate and chloride typically comprised more than 20 percent of the major anions in samples from Logan Creek as compared to less than 5 percent in baseline surface- and ground-water samples from the region. Concentrations of Mississippi Valley Type-related dissolved trace elements (arsenic, cadmium, cobalt, copper, lead, nickel, and zinc) in water samples collected from Logan Creek generally were small and near the reporting level. Zinc was the most commonly detected dissolved trace element and was detected above the reporting level in 30 of 68 samples collected; the maximum concentration of 90 micrograms per liter was detected from a sample collected during 1972. Depending on the quantity of runoff from the mine tailings impoundment and streamflow conditions, discharge from the mine complex and tailings impoundment comprised as much as 68 percent of the discharge in upper Logan Creek, which is lost into the subsurface and resurges at Blue Spring. Concentrations of major and trace constituents in Logan Creek decreased with increasing distance downstream from Adair Creek; however, concentrations of sodium, sulfate, chloride, and boron remained substantially greater than baseline concentrations as flow was lost into the subsurface.

Mining in the Logan Creek Basin has resulted in changes in major ion chemistry of water discharged from Blue Spring. Mining also may have resulted in subtle changes in the mineralogy and concentrations of several trace elements in streambed-sediment samples from Blue Spring and the east side of the Current River downstream from the spring. Generally, water samples from Blue Spring had significantly larger sodium, sulfate, and chloride concentrations as compared to baseline concentrations represented by samples from Big Spring and Greer Spring. Results of a simple mixing model indicate that base-flow concentrations of major ions in an August 2006 sample from Blue Spring can be reasonably approximated by mixing of flow lost from Logan Creek with a baseline ground-water component. Mining effects at Blue Spring also were expressed as spikes in magnesium, sodium, and chloride concentrations and molar ratios of calcium to magnesium in samples collected between 1965 and 1974, and a generally steady increase in sulfate concentrations beginning in 1965. The spike in constituent concentrations and calcium to magnesium ratios coincides with the opening of the Sweetwater Mine exploration shaft during 1962 and initial development of the mine and corresponding increase in concentrations of these constituents in samples from Logan Creek during the same time.

Larger than baseline concentrations of cobalt and nickel (averages of 34 and 65 milligrams per kilogram in the fine (less than 0.063-millimeter size) fraction of streambed-sediment samples from Blue Spring may indicate subtle effects from mine tailings releases into Logan Creek during several breaches of a tailings impoundment at the mine during the 1970s. Mine tailings releases into Logan Creek also may be the source of lead and lead-ore minerals detected in the nonmagnetic heavy-mineral (C3) fraction of streambed-sediment samples from Blue Spring and the east side of the Current River downstream from Blue Spring. 


\section{Introduction}

Blue Spring is an important source of water to the Current River, which is part of the Ozark National Scenic Riverways (fig. 1). Dye traces have confirmed a subsurface connection between Blue Spring and a losing reach of Logan Creek about 10 miles (mi) northeast of the spring orifice (Feder and Barks, 1972; Maxwell, 1974; Aley and Aley, 1987). Although near the Current River, much of the recharge area for Blue Spring is to the northeast along Logan Creek and within the Black River Basin (fig. 1). Whereas most of the Logan Creek
Basin is sparsely populated and predominantly rural and forested, a lead and zinc mine known as the Sweetwater Mine is located along two tributaries in the upper reach of Logan Creek (fig. 1).

Blue Spring has an average discharge of about 130 cubic feet per second $\left(\mathrm{ft}^{3} / \mathrm{s}\right)$ based on 115 discharge measurements [data on file at the U.S. Geological Survey (USGS) office in Rolla, Missouri] making it the seventh largest spring in Missouri (Miller and Vandike, 1997). The spring rises from a deep pool at the base of a dolostone bluff in Shannon County, Missouri, and flows along a branch about 0.25 mile (mi) to the

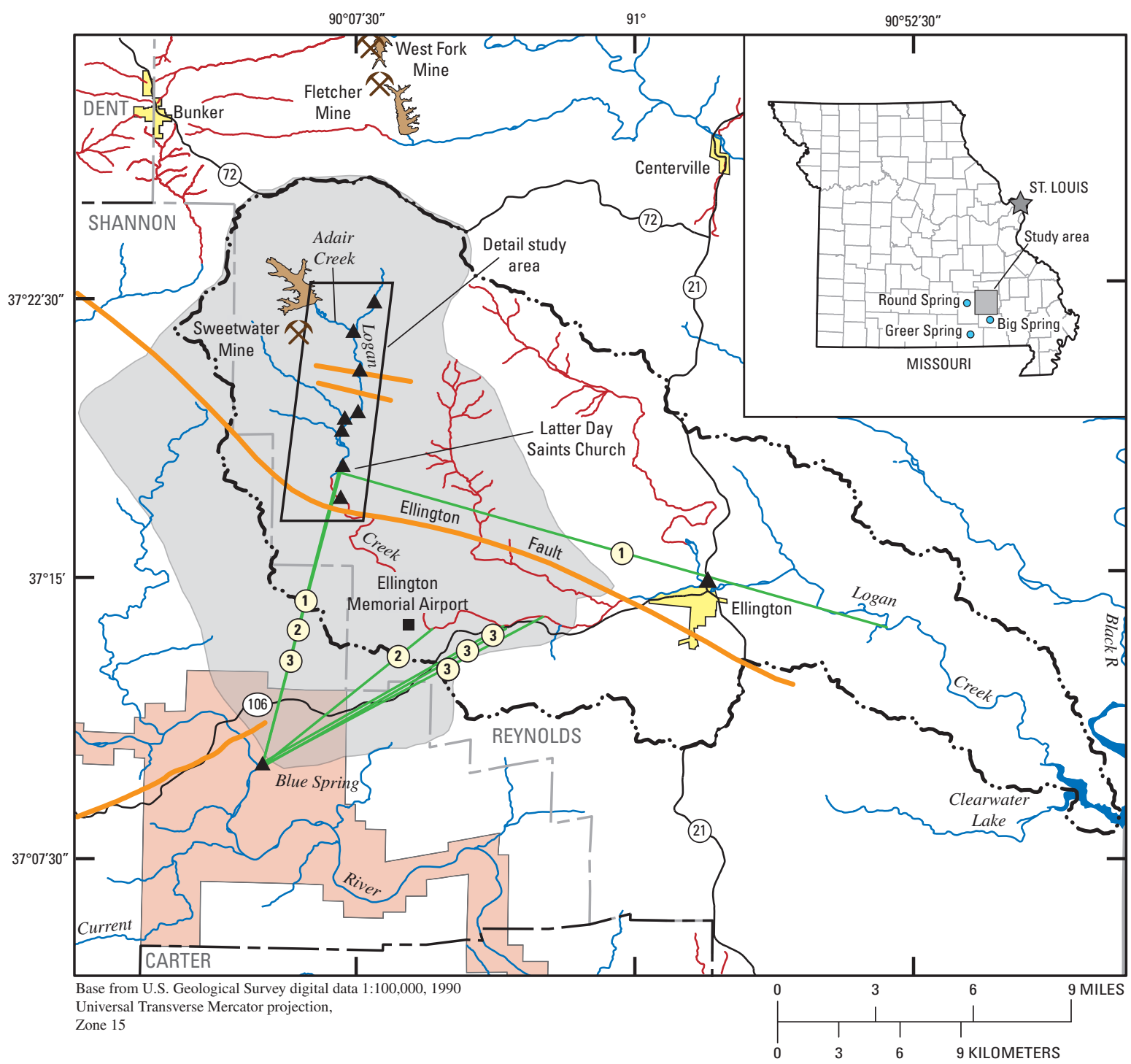

EXPLANATION

Approximate recharge area for Blue Spring (Aley and Aley, 1987)

Mine tailings pile

Ozark National Scenic Riverways

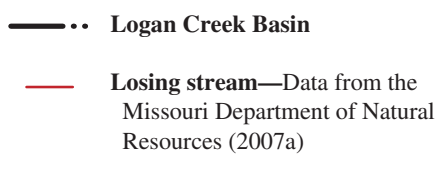

Major fault

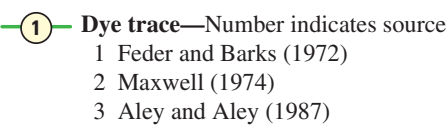

A Water-quality sampling site

Figure 1. Location of Logan Creek, Blue Spring, and the Ozark National Scenic Riverways. 
Current River. The spring orifice is supplied by a near vertical tubular conduit that has been mapped to more than 300 feet (ft) deep, making it the deepest known spring in southern Missouri (Vineyard and Feder, 1982). A resort lodge previously was located at Blue Spring, and the site was a popular retreat until the 1960s when the spring and surrounding land were purchased by the Missouri Department of Conservation (MDC). The spring and adjacent 17 acres were designated the Blue Spring Nature Area in 1972 (Missouri Department of Conservation, 2008).

The Sweetwater Mine is the southernmost mine in the Viburnum Trend, a world-class lead and zinc ore producing subdistrict. The Viburnum Trend was opened in the early 1960s and became the largest producer of lead in the world by 1972 , accounting for 15 percent of world production with more than 1 billion tons of reserves (Rueff, 1970). The initial discovery hole at the Sweetwater Mine was drilled in 1962 and production began at what was then called the Ozark Lead Company Mine in 1968 (Mouat and Clendenin, 1977) and later referred to as the "Adair Creek Mine" and eventually the Sweetwater Mine. Waste rock, called tailings, from the mining and milling of lead and zinc ore at the mine and effluent from the mill are discharged to an impoundment in the headwaters of Adair Creek where the tailings settle and excess water is discharged through a control structure and constructed wetlands into Adair Creek (fig. 2). The tailings impoundment was created by building a dam across Adair Creek, behind which mine tailings are placed using a slurry process. During 1977 and 1978, part of the Sweetwater Mine tailings pile was breached on three separate occasions, causing mine tailings to be released into Adair Creek and Logan Creek (Duchrow and others, 1980). Increased turbidity in response to the tailings releases extended more than 40 mi downstream in Logan Creek; Blue Spring also reportedly became turbid (Duchrow and others, 1980).

\section{Purpose and Scope}

This chapter discusses the results of an analysis of water-quality trends and effects of lead and zinc mining based on water-quality sampling along Logan Creek, a tributary to the Black River, and at Blue Spring, an important source of water to the Current River within the Ozark National Scenic Riverways (ONSR). The analysis of historical (1925-2006) water-quality samples was done in an effort to characterize changes in water quality as a result of lead and zinc mining in the Logan Creek Basin and the effect of these changes on the water quality of Blue Spring. This report is part of a larger multi-discipline investigation of the effects of lead mining in the Viburnum Trend on ground- and surface-water quality and biota conducted by the USGS from 2000 through 2006. Results are presented from more than 60 water-quality samples collected by the USGS from two sites on Logan Creek from 1965 to 2002, base-flow water-quality sampling (6 samples) and a reconnaissance seepage run along upper Logan
Creek during 2006, and more than 70 water-quality samples collected from Blue Spring by various agencies from 1925 through 2006. A discussion of results from five streambedsediment samples collected from Blue Spring and the Current River upstream and downstream from Blue Spring also is presented, along with a summary of dye-tracing activities at Blue Spring.

\section{Study Area Description}

The study area focuses on a 7-mi reach of Logan Creek near the Sweetwater Mine and Blue Spring, about $10 \mathrm{mi}$ to the southwest in the Current River Basin (fig. 1). Although Logan Creek flows near the Sweetwater Mine, all flow in Logan Creek is lost to the subsurface in the vicinity of the Ellington Fault (except during extreme runoff), which the creek crosses several miles downstream from the mine. The entire Logan Creek Basin has a surface area of about 200 square miles $\left(\mathrm{mi}^{2}\right)$ and the creek is about $50 \mathrm{mi}$ long from its headwaters to its mouth with the Black River in Clearwater Lake (fig. 1). Logan Creek crosses the Ellington Fault twice, and the creek is a losing stream with flow seldom continuous along the 13-mi reach between these two fault crossings. The amount of flow lost along the reach between the two fault crossings can be substantial, such as during January 1969, when an estimated 200 $\mathrm{ft}^{3} / \mathrm{s}$ of flow was lost to the subsurface along a 2-mi reach of the creek downstream from the upper Ellington Fault crossing (Feder and Barks, 1972). Topography is steep in the study area and land-surface altitudes range from about 1,250 ft in upland areas near the Sweetwater Mine to about $565 \mathrm{ft}$ at the orifice of Blue Spring along the Current River (fig. 1). The land-surface altitude is about $865 \mathrm{ft}$ in the streambed of Logan Creek in the vicinity of the upper Ellington Fault crossing. Most of the area is forested with scattered pasture along the creek valleys. The area contains small towns, such as Ellington, and the Sweetwater Mine complex. The area is underlain by dolostones of Cambrian and Ordovician age. Bedrock is exposed along the Current River and Blue Spring area and across much of the region; however, few exposures of bedrock occur in the losing part of the Logan Creek Basin because it is covered by a thick blanket of permeable cherty residuum.

\section{Previous Investigations}

From 1965 through 1975, the USGS collected waterquality samples from a network of stream monitoring sites in the vicinity of mines throughout the Viburnum Trend. Two sites in this historical network were along Logan Creek near the Sweetwater Mine-Logan Creek upstream from Sweetwater Creek (site 3, USGS station number 07061810) and Logan Creek below Sweetwater Creek (site 5, USGS station number 07061840) in the vicinity of the Sweetwater Mine (fig. 2). Monitoring at most historical sites was reduced or had ceased until the present USGS lead-mining-issues study began in 2000, when sampling resumed at several sites. 


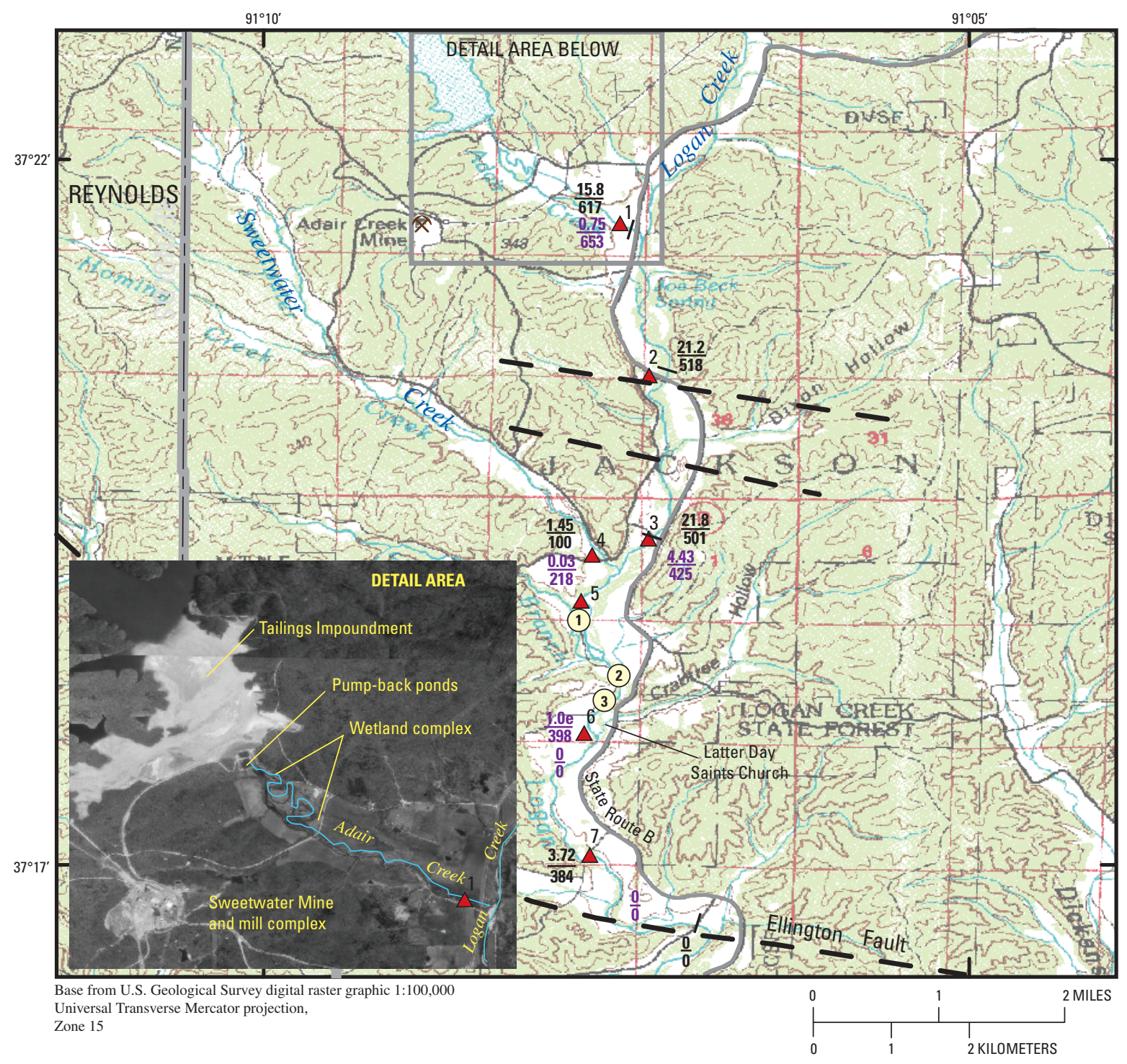

EXPLANATION

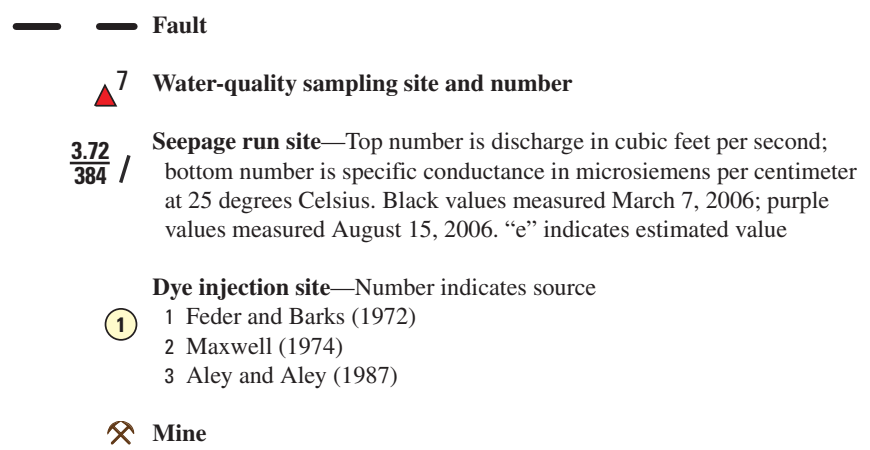

Figure 2. Historical water-quality sampling sites and seepage-run measurements made at high base flow (March 7, 2006) and low base flow (August 15, 2006) along the upper reach of Logan Creek. 
The USGS has made occasional discharge measurements at Blue Spring since 1923 and collected occasional water-quality samples since 1925. During 1970 and 1971, a continuous streamflow gaging station was installed at Blue Spring; however, because of frequent periods of backwater from the Current River, the record was rated poor, and the data were never published. During October 1969, Feder and Barks (1972) conducted a fluorescent dye trace from Logan Creek near the Latter Day Saints (LDS) Church to verify a suspected subsurface connection between Logan Creek and Blue Spring (Bridge, 1930). Dye recovery points included two locations along Logan Creek that were 17 and 22 mi downstream from the injection point and downstream from the town of Ellington and Powder Mill Spring and Blue Spring, both in the Current River Basin. Dye was recovered at Blue Spring and at the downstream-most site in Logan Creek between 3 and 10 days after injection (fig. 1).

Maxwell (1974) identified several sinkholes and caves along Logan Creek in the vicinity of the LDS Church where dye previously had been injected and recovered at Blue Spring during 1969 (Feder and Barks, 1972). Two small (about $6 \mathrm{ft}$ wide, $20 \mathrm{ft}$ long, and $3 \mathrm{ft}$ deep) sinkholes were located in the channel of Logan Creek about $700 \mathrm{ft}$ downstream from the LDS Church, as well as a sinkhole about $40 \mathrm{ft}$ wide and $25 \mathrm{ft}$ deep on the floodplain west of the Logan Creek channel and about 2,100 ft southwest of the LDS Church (Maxwell, 1974). The sinkhole had a 5-ft high cave entrance, referred to as "Latter Day cave" that extended about $50 \mathrm{ft}$ below the surface of the floodplain. A small cave on the west bank of Logan Creek about 3,000 ft downstream from the LDS Church also was noted. Maxwell (1974) reported that during 1972, a sinkhole about $12 \mathrm{ft}$ in diameter and $8 \mathrm{ft}$ deep developed over 6 months in the sandy floor of the small cave. During a storm on April 11, 1972, Maxwell (1974) injected dye into Logan Creek about 0.25 -mi upstream from the LDS Church and a second dye into a sinkhole near the Ellington Memorial Airport about $7 \mathrm{mi}$ further downstream. Both dyes were recovered at Blue Spring (fig. 1) within 12 days.

Aley and Aley (1987) reported four successful dye traces to Blue Spring as part of a study to delineate recharge areas for several major springs in the ONSR (fig. 1). This study also provided a compilation of all dye-tracing activities to springs in the ONSR and mapping of potential water-quality hazards to springs in the ONSR. Of the four reported successful dye traces to Blue Spring, one was a confirmation of the previous 1969 Feder and Barks (1972) trace from the upper reach of Logan Creek; the other three traces were from a reach of Logan Creek or mouths of tributaries about 3 mi downstream from the Maxwell (1974) trace immediately south of Ellington Memorial Airport (fig. 1).

From 1965 through 1971, the USGS collected six waterquality samples from Blue Spring as part of a network of spring and surface water monitoring along the Current River (data on file at the USGS office in Rolla, Missouri). Barks (1978) collected four water-quality samples from Blue Spring during 1973 and 1974 and water-quality samples from several other springs and wells as part of a water-quality assessment of the Current River. Barks noted that springs contained larger concentrations of dissolved nitrite plus nitrate as nitrogen; $\left[\mathrm{NO}_{2}+\mathrm{NO}_{3}\right.$; average of 0.42 milligram per liter $\left.(\mathrm{mg} / \mathrm{L})\right]$ compared to well samples (average of $0.22 \mathrm{mg} / \mathrm{L}$ ). Barks (1978) also noted that springs in the upper Current River and Jacks Fork Basins contained larger $\mathrm{NO}_{2}+\mathrm{NO}_{3}$ concentrations as compared to springs along the middle and lower part of the Current River Basin (Blue Spring, Big Spring, and Round Spring) (fig. 1), presumably because of differing land use in the spring recharge basins.

The MDC conducted an assessment of the effects of tailings releases from the Ozark Lead Company mine tailings pond (presently Sweetwater Mine) in 1978 (Duchrow and others, 1980). The MDC concluded that the tailings releases severely degraded water quality in Logan Creek as evidenced by decreased densities and diversity of invertebrates. They attributed the degradation to a combination of physical and chemical effects of the tailings in streambed sediments, although concentrations of heavy metals, except lead in one streambed-sediment sample, were less than sediment-quality guidelines established for aquatic life and concentrations of dissolved cadmium, copper, lead, and zinc in surface-water samples were small.

\section{Methods}

Data described in the following discussion include the analyses of water-quality samples collected over more than 80 years. The methods used to collect and analyze these samples varied with time; however, methods used were consistent with standard acceptable practices at the time of collection. Discharge, specific conductance, $\mathrm{pH}$, temperature, and dissolved oxygen concentrations were determined onsite at the time of sampling. Samples collected during the 1920s were collected and analyzed by the Missouri Geological Survey [presently the Missouri Division of Geology and Land Survey (DGLS)]. Water-quality samples collected during the 1950s and later were analyzed by USGS laboratories. Samples collected by the USGS during the 1960s and 1970s were collected according to the methods described in Guy and Norman (1970) with subsequent samples collected according to the methods described in Ward and Harr (1990) and Wilde and others (1999). Specific sample collection and analytical methods used during 1973 and 1974 are discussed in Barks (1978).

A detailed description of methods used for the collection and analysis of streambed sediment samples is presented in chapter 3 of this report describing trace-metal concentrations and sulfide-mineral occurrence in streambed sediment of the Viburnum Trend Subdistrict and non-mining areas in southeastern Missouri. Generally, composite streambed-sediment samples were collected along cross sections on spring or stream channels. A plastic scoop was used to collect about 20 subsamples from the upper 2 inches (in.) of streambed sediment along each cross section. Because of high-water veloci- 
ties and gravel and cobble bottoms, sampling usually was targeted to depositional areas downstream from large rocks or debris. All streambed-sediment samples were analyzed by the USGS mineralogy laboratory in Lakewood, Colorado, for grain-size distribution-coarse [(greater than 0.063 millimeter (mm)], silt (0.002 to $0.063 \mathrm{~mm}$ ), and clay (less than 0.002 $\mathrm{mm})$. Samples also were analyzed for bulk mineralogy of the less than $2 \mathrm{~mm}$-size fraction using X-ray diffraction, inorganic chemical content of the fine (less than $0.063 \mathrm{~mm}$-size) fraction by inductively coupled plasma atomic emission spectroscopy (ICP-AES) and mineralogy of the non-magnetic heavy mineral concentrate (hereinafter referred to as the $\mathrm{C} 3$-fraction) by bromoform separation and optical mineralogy. In addition, semiquantitative emission spectrometry analysis of the $\mathrm{C} 3$ fraction was performed on the 1995 streambed-sediment samples collected from Blue Spring and the Current River.

The relations among major ionic constituents in water samples can be graphically displayed by grouping ions with similar chemical properties on a trilinear diagram (Hem, 1985). Major ions are expressed as percentages of milliequivalents per liter of major cations calcium, magnesium, sodium, and potassium and the major anions sulfate, bicarbonate, chloride, fluoride, and $\mathrm{NO}_{2}+\mathrm{NO}_{3}$. Trilinear diagrams are useful in examining simple mixtures of solutions or dissolution or precipitation of simple salts. Because mine waters and infiltration percolating through mine tailings piles often produce chemical reactions that involve oxidation and subsequent dissolution of sulfide and carbonate minerals that affects concentrations of major ions, trilinear diagrams were used to examine waterquality changes and evolution of water chemistry in mining areas. Arsenic, cadmium, cobalt, copper, lead, nickel, and zinc are trace elements commonly associated with Mississippi Valley Type ore deposits and are referred to as MVT-related metals in this chapter.

Statistical analyses of water-quality data including summary statistics, distribution tests, correlations, box plots, and scatter plots were performed using the computer software SYSTAT (SYSTAT Software, Inc., 2002). A Shapiro-Wilk test was used to test distributions for normality. Hypothesis testing was done using the non-parametric Kruskal-Wallis test with the null hypothesis that no difference exists between the central tendency of groups being compared, and rejection is at a significance level (p-value) of 0.05 or less. For "censored" data where constituent concentrations were less than the laboratory reporting level and the concentration was reported as "less than the reporting level", the reporting level was substituted for the censored value. This method was appropriate for most data sets examined because only one reporting level was listed. Insufficient data for statistical analysis were available for concentrations of dissolved trace elements at Blue Spring (only three samples). Statistical comparisons were made on concentrations of total lead and total zinc in water samples from Blue Spring to baseline concentrations represented by samples from the non-mining affected Big Spring and Greer Spring. About 50 percent of the values reported were censored and multiple reporting levels were listed; some of which were too large to be a practical value for statistical analysis. The multiple censoring levels generally were the result of changing analytical methods. For example, in the combined data set for Blue Spring, Big Spring, and Greer Spring, reporting levels for total lead (numbers in parenthesis are number of values in data set) were less than $(<) 0.06$ microgram per liter $(\mu \mathrm{g} / \mathrm{L}, 4)$, $<1 \mu \mathrm{g} / \mathrm{L}(72),<2 \mu \mathrm{g} / \mathrm{L}(2),<5 \mu \mathrm{g} / \mathrm{L}(5),<50 \mu \mathrm{g} / \mathrm{L}$ (1) and $<200$ $\mu \mathrm{g} / \mathrm{L}$ (4). Censored values of $<5,<50$, and $<200 \mu \mathrm{g} / \mathrm{L}$ were removed from the data set and censored values of $<0.06,<1$, and $<2 \mu \mathrm{g} / \mathrm{L}$ were all set to a threshold of $2 \mu \mathrm{g} / \mathrm{L}$ for statistical analysis. A similar process was performed for total zinc concentrations where the minimum threshold value was $<10 \mu \mathrm{g} / \mathrm{L}$.

\section{Water-Quality Trends and Effects of Mining}

Because much of the recharge area for Blue Spring lies within the upper part of the Logan Creek Basin and flow lost within Logan Creek emerges at Blue Spring, a discussion of the water-quality of Logan Creek is presented followed by a discussion of the water-quality trends in Blue Spring. During March and August 2006, a reconnaissance seepage run and water-quality sampling effort was conducted along Logan Creek downstream from Adair Creek to provide additional information on the effect of mine water discharge on the more recent (2006) water quality of Blue Spring.

\section{Upper Logan Creek}

The establishment of monitoring sites on Logan Creek upstream (site 3; fig. 2) and downstream (site 5) from Sweetwater Creek during the 1965 to 1975 USGS water-quality monitoring of Black River Basin indicates that at the time of the initial sampling, Sweetwater Creek was the receiving stream for effluent from early operations at the Sweetwater Mine complex (fig. 2). However, at the time of the initial USGS sampling during 1965, the Sweetwater Mine complex had been in development for nearly 3 years and early samples may not represent "pre-mining conditions."

Water samples from sites 3 and 5 have a mixture of major ions ranging from calcium-magnesium-bicarbonate (typical of Ozark streams and ground water) to sodium-magnesium-calcium-bicarbonate-sulfate and magnesium-calcium-bicarbonate-chloride, indicating disproportional amounts of sodium, sulfate, and chloride in some of the samples (fig. 3). The increased sodium, sulfate, and chloride in some surface-water samples is consistent with effects from the Sweetwater Mine as seep samples from mine faces within the Sweetwater Mine contained large proportions of sodium, sulfate, and chloride relative to calcium, magnesium, and bicarbonate (Lopaka Lee, U.S. Geological Survey written commun., 2006). In addition, oxidation of sulfide minerals within the mine tailings piles contributes to increased sulfate concentrations in seeps from 


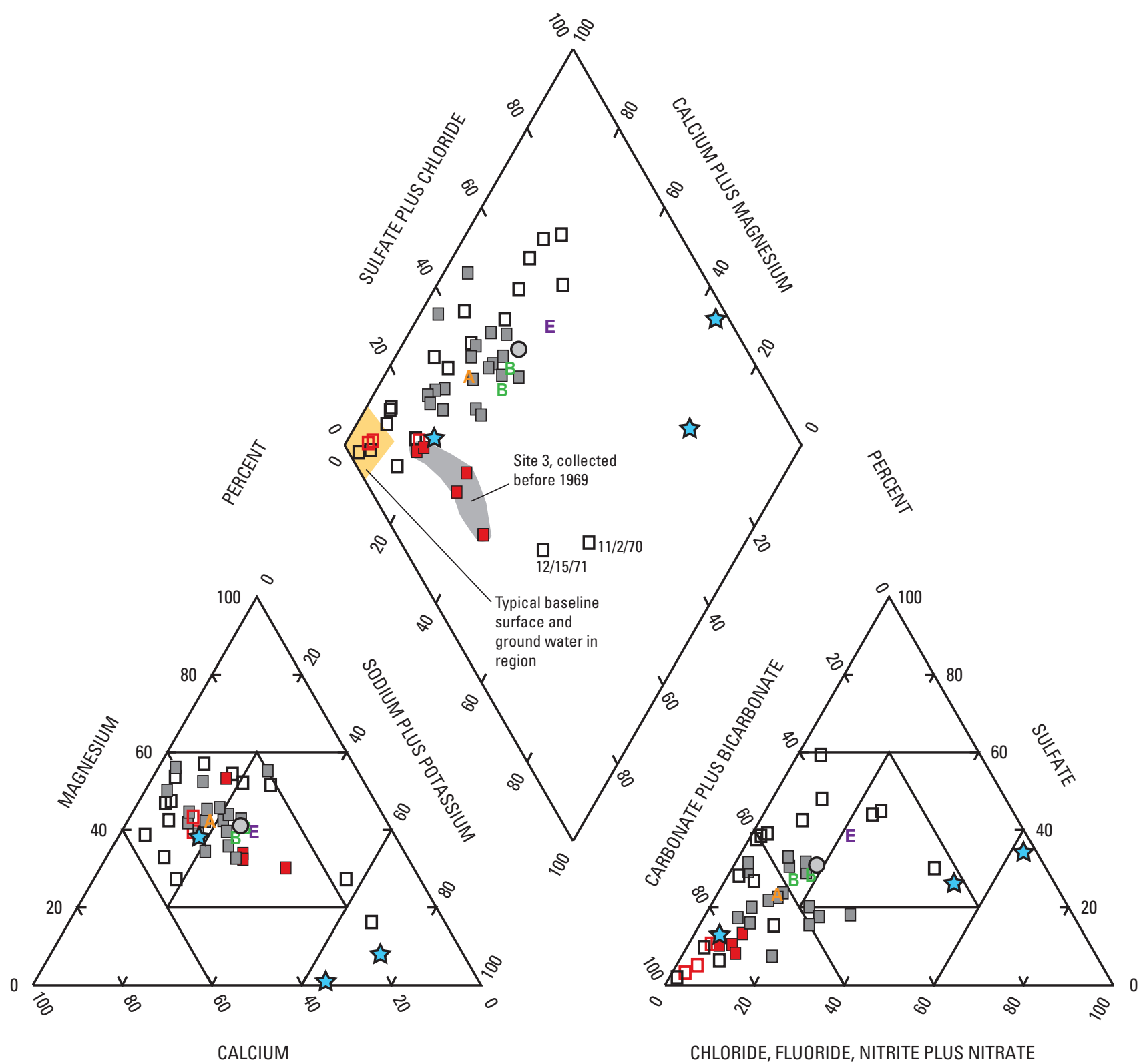

PERCENT

\section{EXPLANATION}

E Site 1, Adair Creek (3/7/06)

B Site 2, Logan Creek at State Route B downstream from Adair Creek (5/9/02-3/7/06)

ㄷ Site 3, Logan Creek upstream from Sweetwater Creek (9/30/65-7/29/75), red outline indicates collected before 1969

$\square$ Site 5, Logan Creek downstream from Sweetwater Creek (9/30/65-7/29/75), red shade indicates collected before 1969

A Site 6, Logan Creek at Latter Day Saints Church (8/15/06)

Site 7, Logan Creek at County Road 776 (3/7/06)

Sweetwater Mine seep sample

Figure 3. Trilinear diagram of water samples collected from upper Logan Creek, 1965-2006. 
the piles (Schumacher and Hockanson, 1996). The presence of increased proportions of sodium, sulfate, and chloride in some of the surface-water samples indicates likely effects of mining operations on the water quality in upper Logan Creek as early as 1965. All samples from site 3 (upstream from Sweetwater Creek) collected before 1969 plot near the calcium plus magnesium-bicarbonate vertex typical of baseline surface and ground water in the region. However, samples collected from site 3 during 1969 and later typically plot trending toward the upper right of figure 3 with sulfate plus chloride generally comprising more than 20 percent of the major anions compared to less than 5 percent in baseline samples, indicating effects from mining operations. Unlike site 3, no samples from site 5 (downstream from Sweetwater Creek) collected before 1969 plot within the baseline range of regional surface and ground water, but plot in a band with increased sodium plus potassium and slightly increased proportions of sulfate and chloride (fig. 3). Samples collected from site 5 during and after 1969 trend toward increased proportions of sulfate plus chloride (greater than 20 percent of the major anions) as compared to earlier samples (fig. 3).

The differences in major ion chemistry between the upstream site (site 3) and downstream site (site 5) on Logan Creek before 1969, but similar chemistry after 1969 indicates changes in the composition of effluent from the mining operation or the location of the effluent discharge, or both. For mines typical of the period, the two major sources of discharge water were water pumped to dewater the mine workings and effluent from the mill complex. Both sources were initially derived from water pumped from the mine workings. Typically, mill effluent is used to slurry waste rock to the tailings impoundment where the tailings settle, and the water is partially recycled through the mill. At the Sweetwater Mine, excess water in the tailings impoundment and storm runoff is discharged through an overflow structure and flows through a constructed wetland meander system into Adair Creek. Based on the initial selection of the sampling site 3 and site 5 , in 1965 most discharges during development of the mine shaft and mill complex probably went into Sweetwater Creek. This supposition is supported by the baseline distribution of major ions in the pre-1969 samples from site 3 and increased proportion of sodium, sulfate, and chloride in pre-1969 samples from site 5 (fig. 3). The trend of samples from site 3 away from the calcium plus magnesium-carbonate plus bicarbonate vertex toward more sulfate and chloride-rich water during and after 1969 probably is the result of discharges from the mine complex shifting to the mine-tailings pond on Adair Creek about $2.5 \mathrm{mi}$ upstream from the sampling site 3 (fig. 2). However, apparently not all discharges from the facility had shifted to Adair Creek after production began because specific conductance values and concentrations of calcium, magnesium (not shown), sodium, chloride, sulfate, and alkalinity in post-1969 samples from site 5 were significantly larger ( $\mathrm{p}$-value $<0.05$ using the non-parametric Mann-Whitney test) than those in site 3 samples (fig. 4). By 1987 when National Pollution Discharge Elimination System (NPDES) permits were issued for the facility (chapter 1 of this report), all discharges from the facility apparently were to Adair Creek.

Although changes in major ion chemistry attributed to mining operations were evident as early as 1965 in upper Logan Creek, little, if any, effects were observed on concentrations of dissolved trace elements during sampling from 1965 through 1975. Most water samples from the two sites on upper Logan Creek (sites 3 and 5) were analyzed for a variety of trace elements such as barium, cadmium, chromium, cobalt, cupper, iron, lead, manganese, nickel, silver, and zinc. Several samples collected before September 1970 also were analyzed for boron, lithium, and strontium (table 1, at the back of this chapter). Concentrations of MVT-related metals and other trace elements in water samples collected from upper Logan Creek during 1965 through 1975 generally were small and near reporting levels in the 63 samples collected from sites 3 and 5. Zinc was the most commonly reported trace element, detected above reporting levels in 25 of the 54 samples in which it was analyzed, with a maximum concentration of 90 $\mu \mathrm{g} / \mathrm{L}$ in two samples collected during 1972 (table 1). Cadmium and lead concentrations were less than the reporting level of $2 \mu \mathrm{g} / \mathrm{L}$ in all samples, chromium was detected in 4 samples (maximum concentration of $20 \mu \mathrm{g} / \mathrm{L}$ ), cobalt was detected in 2 samples (maximum concentration of $40 \mu \mathrm{g} / \mathrm{L}$ ), and copper was detected in 7 samples (maximum concentration of $20 \mu \mathrm{g} / \mathrm{L}$ ) (table 1). The Mann-Whitney test indicated no significant differences in trace-element concentrations between site 3 and site 5 . Only 4 of the 54 samples analyzed for zinc concentrations from these two upper Logan Creek sites indicated zinc concentrations that exceeded the current Missouri chronic standard based on water hardness for the protection of aquatic life (Missouri Department of Natural Resources, 2007b). These samples were collected from sites 3 and 5 during June and October 1972. Several samples from sites 3 and 5 contained unusually small values of specific conductance [less than 100 microsiemens per centimeter at 25 degrees Celsius $(\mu \mathrm{S} / \mathrm{cm})]$ and concentrations of calcium and magnesium less than $10 \mathrm{mg} / \mathrm{L}$ (table 1 ).

Results of the March and August 2006 seepage run and water-quality sampling effort along the upper reach of Logan Creek indicated that, depending on the quantity of runoff from the mine tailings impoundment and streamflow conditions, discharge from the mine complex and tailings impoundment comprised between about 17 to 68 percent of the discharge in upper Logan Creek that is lost into the subsurface. During high base-flow conditions on March 7, 2006, discharge and specific conductance were measured or estimated at six sites and water-quality samples were collected from three sites along Logan Creek, in addition to Blue Spring. Precipitation records from Ellington indicated that 0.2 in. of precipitation occurred in the area on March 5 and 6, 2006, and 0.15 in. of precipitation was recorded on March 7, 2006 (National Oceanic and Atmospheric Administration, 2007). Whereas no runoff was noted when discharge measurements and waterquality samples were collected on March 7, 2006, and the 

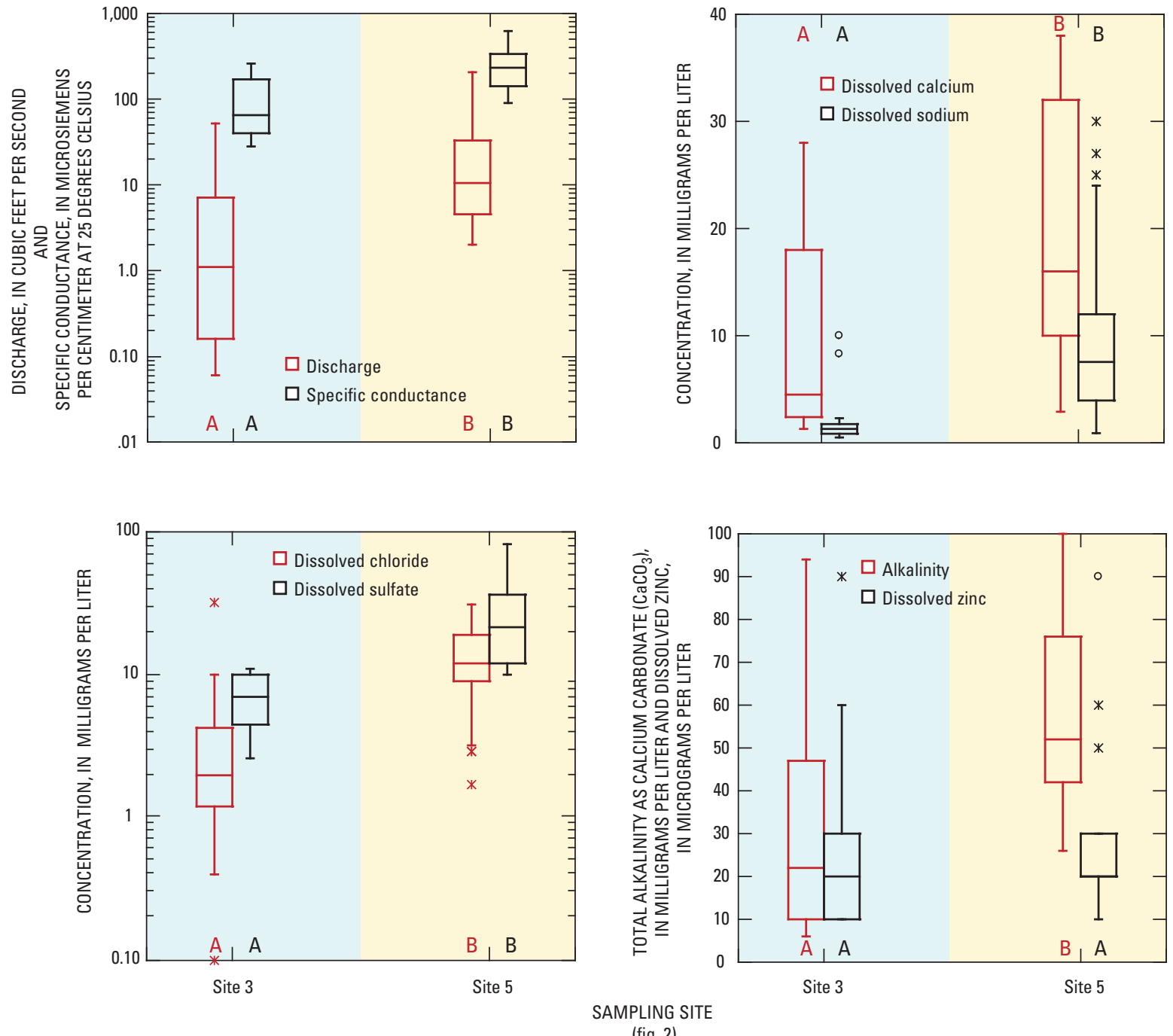

Note: Mine production began during 1968

(fig. 2)

EXPLANATION

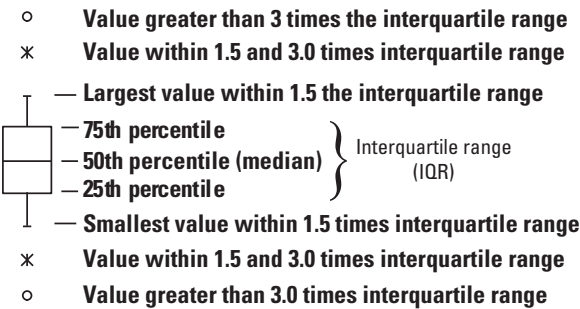

A LETTER - Results of Kruskal-Wallis and Tukey's multiple comparison tests. Distributions associated with the same letter are not significantly different ( $\mathrm{p}$-value greater than 0.05$)$. Order of letter designation is from smallest distribution to largest such that " $A$ " is the smallest

Figure 4. Discharge, specific conductance values, and concentrations of selected inorganic constituents in water samples collected from Logan Creek, 1969-1975. 
streams were clear, puddles were observed along road sides and ditches, confirming recent rainfall in the area.

On March 7, 2006, the discharge from the mine complex and tailings impoundment of $15.8 \mathrm{ft}^{3} / \mathrm{s}$ was measured at site 1 at the mouth of Adair Creek (fig. 2). The discharges of Logan Creek downstream from Adair Creek at sites 2 and 3 were similar at 21.2 and $21.8 \mathrm{ft}^{3} / \mathrm{s}$, indicating a maximum increase of $6 \mathrm{ft}^{3} / \mathrm{s}$, most of which was inflow from several springs (such as Joe Beck Spring) upstream from site 2 and unmeasured flow in Logan Creek upstream from Adair Creek (fig. 2). Discharge decreased to $3.72 \mathrm{ft}^{3} / \mathrm{s}$ at site 7 , indicating more than 80 percent $\left(19.5 \mathrm{ft}^{3} / \mathrm{s}\right)$ of the discharge in Logan Creek was lost along the 3-mi reach between sites 3 and 7. This loss included a $1.45 \mathrm{ft}^{3} / \mathrm{s}$ inflow from Sweetwater Creek measured at site 4. No flow was observed at an unnamed gravel-road crossing about $1 \mathrm{mi}$ downstream from site 7 , indicating a total measured loss of $23.3 \mathrm{ft}^{3} / \mathrm{s}$, of which about 70 percent $\left(15.8 \mathrm{ft}^{3} / \mathrm{s}\right)$ was discharge from the mine complex and tailings impoundment. A discharge of $98.6 \mathrm{ft}^{3} / \mathrm{s}$ was measured at Blue Spring on March 7, 2006. Assuming that previous dye traces indicate that all flow lost in Logan Creek resurges at Blue Spring and that flow conditions in Adair Creek and Logan Creek were stable for at least the 10- to 12-day subsurface travel time from Logan Creek to Blue Spring, then flow lost in Logan Creek comprised about $23.3 \mathrm{ft}^{3} / \mathrm{s}$ of the $98.6 \mathrm{ft}^{3} / \mathrm{s}$, or about 24 percent of the discharge measured at Blue Spring.

During low base-flow conditions on August 15, 2006, discharge was measured or estimated at five sites (including Blue Spring) and a water-quality sample was collected from Logan Creek at site 6 immediately upstream from where all flow was lost to the subsurface (fig. 2). Whereas 0.7 in. of precipitation was recorded at Ellington on August 15, 2006 (National Oceanic and Atmospheric Administration, 2007), no evidence of precipitation, such as damp ground, pools, or puddles along roads, was noted along upper Logan Creek. The discharge of Adair Creek at site 1 was $0.75 \mathrm{ft}^{3} / \mathrm{s}$ on August 15, 2006 - considerably smaller than the $15.8 \mathrm{ft}^{3} / \mathrm{s}$ measured on March 7, 2006. The maximum discharge along Logan Creek was $4.43 \mathrm{ft}^{3} / \mathrm{s}$ at site 3 . Most of this discharge, including an estimated $0.03 \mathrm{ft}^{3} / \mathrm{s}$ inflow from Sweetwater Creek, was lost into the subsurface by site 6 , where a discharge of $1.0 \mathrm{ft}^{3} / \mathrm{s}$ was estimated, and all flow was lost within a few hundred feet downstream from site 6 (fig. 2). The $4.46 \mathrm{ft}^{3} / \mathrm{s}$ of flow lost along Logan Creek on August 15, 2006 (about 17 percent of which was from Adair Creek and mostly mine related), represents about 5 percent of the $88.4 \mathrm{ft}^{3} / \mathrm{s}$ measured at Blue Spring that same day.

Water samples collected during 2006 from Adair Creek (site 1) and three sites on Logan Creek at State Route B (site 2), near the LDS Church (site 6), and County Road 776 downstream from the LDS Church (site 7), all contained increased proportions of sodium plus potassium and sulfate plus chloride and plot within the range of samples collected during the 1960s and 1970s (fig. 3). During the March 7, 2006, sampling event, most of the $15.8 \mathrm{ft}^{3} / \mathrm{s}$ discharge from Adair Creek at site 1 probably was from the mine or mine tailings impoundment.
The specific conductance value of $617 \mu \mathrm{S} / \mathrm{cm}$ and concentrations of sodium (40.2 mg/L), sulfate (114 mg/L), and chloride $(49.1 \mathrm{mg} / \mathrm{L})$ in the Adair Creek sample greatly exceeded baseline concentrations of sodium $(1.5 \mathrm{mg} / \mathrm{L})$, sulfate $(4 \mathrm{mg} / \mathrm{L})$, and chloride $(3 \mathrm{mg} / \mathrm{L})$ reported for area streams. The baseline concentrations were determined from the maximum values for non-mining sites in table 2, chapter 6 of this report. Concentrations of several MVT-related metals such as cobalt $(2 \mu \mathrm{g} / \mathrm{L})$, copper $(3.2 \mu \mathrm{g} / \mathrm{L})$, lead $(11.7 \mu \mathrm{g} / \mathrm{L})$, nickel $(8.5 \mu \mathrm{g} / \mathrm{L})$, and zinc $(34 \mu \mathrm{g} / \mathrm{L})$ in addition to manganese $(330 \mu \mathrm{g} / \mathrm{L})$ in Adair Creek (table 1) also exceeded baseline non-mining concentrations (table 2, chapter 6). The Adair Creek sample also contained a large concentration of boron at $125 \mu \mathrm{g} / \mathrm{L}$ compared to typical concentrations of 10 to $20 \mu \mathrm{g} / \mathrm{L}$ in surface and ground water in southern Missouri (Schumacher and Hockanson, 1996). Adair Creek comprised about 68 percent of the flow in Logan Creek during the March 7, 2006, sampling. Dilution and possibly geochemical reactions such as precipitation, cation exchange, or sorption resulted in smaller concentrations of sodium, sulfate, chloride, and trace elements at site 2, but concentrations remained substantially above baseline values (table 1). Concentrations of most MVT-related metals decreased to baseline concentrations at site 7; however, effects from mining were evident in the above-baseline concentrations of sodium, sulfate, chloride, and boron in the sample from site 7 .

Concentrations of sodium (15.6 mg/L), sulfate (42.4 $\mathrm{mg} / \mathrm{L})$, chloride $(18.5 \mathrm{mg} / \mathrm{L})$, and boron $(62 \mu \mathrm{g} / \mathrm{L})$ in flow lost to the subsurface during low base-flow conditions approximated by the sample from Logan Creek at site 6 on August 15, 2006, were much smaller than during high base-flow conditions approximated by the sample from site 7 on March 7 , 2006 (table 1). The smaller major ion concentrations during August 2006 were the result of the much smaller discharge from Adair Creek (0.75 ft $3 / \mathrm{s})$ measured on August 15, 2006, compared to the $15.8 \mathrm{ft}^{3} / \mathrm{s}$ measured during high base-flow conditions in March 2006. A water-quality sample was not collected from Adair Creek during the August 2006 seepage study, but the specific conductance of $653 \mu \mathrm{S} / \mathrm{cm}$ measured on August 15, 2006, is similar to the $617 \mu \mathrm{S} / \mathrm{cm}$ measured on March 7, 2006, when a water-quality sample was collected, indicating that concentrations of major ions probably were of similar magnitude.

\section{Blue Spring}

A difficulty in documenting long-term effects of mining on the water quality of Blue Spring is the lack of a consistent sampling program for mining-related constituents. For example, although 77 water-quality samples have been collected from Blue Spring since 1925 (U.S. Geological Survey, 2007), these samples were collected for various agencies and generally focused on the recreational quality of the water (emphasis on nutrients and fecal bacteria) rather than an assessment of lead and zinc mining effects. Of the 77 water-quality samples collected from Blue Spring, 13 were analyzed for a complete 
set of major ions (table 2, at the back of this chapter), 3 were analyzed for a comprehensive suite of trace elements, and none were analyzed for organic compounds. Of the 13 samples analyzed for major ions, 2 samples (June 1925 and May 1952) were collected before development of the Sweetwater Mine began in 1962, 8 samples were collected from 1965 to 1973 during the early production years of the Sweetwater Mine, and 3 samples were collected after 2000 (May 2002, March 2006, and August 2006).

The following sections examine water-quality changes at Blue Spring and compares water and sediment data collected from Blue Spring to baseline spring sites in the region-Big Spring about 20 mi downstream along the Current River and Greer Spring about $30 \mathrm{mi}$ southwest along the Eleven Point River. A meaningful comparison of trace-element data from Blue Spring with Big Spring and Greer Spring could not be performed because most trace-element data from Blue Spring was from unfiltered samples, with total concentrations reported in contrast to filtered or dissolved trace-element concentrations at Big Spring and Greer Spring. Although the discharges of Big Spring and Greer Spring (about 445 and $342 \mathrm{ft}^{3} / \mathrm{s}$ mean annual discharge; Hauck and Harris, 2006) are larger than Blue Spring, they have similar geologic settings to Blue Spring. Big Spring and Greer Spring have no lead and zinc mining in their known recharge areas. However, exploration for lead and zinc deposits has occurred within their recharge areas (Imes and others, 2007). Water-quality changes at Blue Spring, in response to mining, may provide insight to potential water-quality changes to Big Spring and Greer Spring if mining processes similar to those used in the Viburnum Trend were to occur in their respective recharge basins.

\section{Water Quality}

The measured discharge of Blue Spring ranged from 59.3 to $290 \mathrm{ft}^{3} / \mathrm{s}$ (115 measurements from 1923 through 2006, data on file at the USGS office in Rolla, Missouri) with a median of $108 \mathrm{ft}^{3} / \mathrm{s}$ and mean of $127 \mathrm{ft}^{3} / \mathrm{s}$. Similar to most springs in southern Missouri, water samples collected from Blue Spring have moderate specific conductance values (118 to $350 \mu \mathrm{S} / \mathrm{cm}$ ), near neutral $\mathrm{pH}$ values (6.9 to 8.2) and calcium, magnesium, and bicarbonate as the predominant ions (data on file at the USGS office in Rolla, Missouri). Five of the 13 samples from Blue Spring plot outside the baseline range for regional spring water caused by increased concentrations of sodium, sulfate, and chloride relative to calcium plus magnesium and bicarbonate (fig. 5). All five samples plotting outside the baseline range were collected after 1969 and after the Sweetwater Mine began operation within the Blue Spring recharge area (fig. 1). These five samples plot along a trend between baseline spring water samples and samples from mining-affected sites along upper Logan Creek. The increased concentrations of sodium, sulfate, and chloride in these samples are consistent with previous dye traces (Feder and Barks, 1972; Maxwell 1974; Aley and Aley, 1987), indicating that flow lost in Logan
Creek downstream from the Sweetwater Mine emerges at Blue Spring.

To provide additional evidence that the increased sodium, sulfate, and chloride, in water samples from Blue Spring might be related to operations at the Sweetwater Mine, a simple twocomponent mixing model was constructed to simulate major ion concentrations in Blue Spring samples collected during the 2006 seepage-run study. The simple model was constructed using the following relations:

$$
\begin{gathered}
\mathrm{Qt}^{*} \mathrm{Ct}=\left(\mathrm{Qb}^{*} \mathrm{Cb}\right)+(\mathrm{QL} * \mathrm{CL}) \\
\mathrm{Qt}=\mathrm{Qb}+\mathrm{QL}
\end{gathered}
$$

where,

$$
\begin{gathered}
\text { Qt is the measured discharge, in cubic feet per } \\
\text { second at Blue Spring; } \\
\mathrm{Ct} \quad \text { is the measured constituent concentration, in } \\
\text { milligrams per liter at Blue Spring; } \\
\mathrm{Qb} \quad \text { is the base flow (non-Logan Creek flow) } \\
\text { component of discharge, in cubic feet per } \\
\text { second of Blue Spring; } \\
\mathrm{Cb} \quad \text { is the baseline base-flow constituent } \\
\text { concentration, in milligrams per liter in } \\
\text { Blue Spring (approximated by median } \\
\text { constituent concentrations in samples from } \\
\text { Big Spring and Greer Spring); } \\
\text { is the total measured discharge lost, in cubic } \\
\text { feet per second in Logan Creek between } \\
\text { sites } 3 \text { and the unnamed gravel-road } \\
\text { crossing downstream from site } 7 \text { (fig. 2); } \\
\text { and } \\
\text { is the constituent concentration, in milligrams } \\
\text { per liter in flow lost in Logan Creek } \\
\text { (approximated using 2006 sample results } \\
\text { from sites 6 and 7). }
\end{gathered}
$$

Equation 2 was solved for $\mathrm{Ct}$ for each constituent of interest. Initially, all flow lost in Logan Creek was assumed to contribute to the flow measured at Blue Spring. This assumption implies steady-state conditions of discharge and waterquality in Logan Creek and Blue Spring for at least the 10- to 12-day subsurface travel time between Logan Creek to Blue Spring estimated from previous dye traces.

Results of the simple mixing model indicated that concentrations of major ions measured in the March and August 2006 samples from Blue Spring can be reasonably approximated by the simple mixing of flow lost along Logan Creek with a baseline ground-water component (table 3). At low base-flow conditions in August 2006, this assumption resulted in reliably predicted concentrations of chloride and other constituents (table 3). Differences between measured and simulated major constituent concentrations generally were within 15 percent - an acceptable error based on an assumed 5 percent error in measured constituent concentrations and 5 to 8 percent error in discharge measurements. However, the initial 


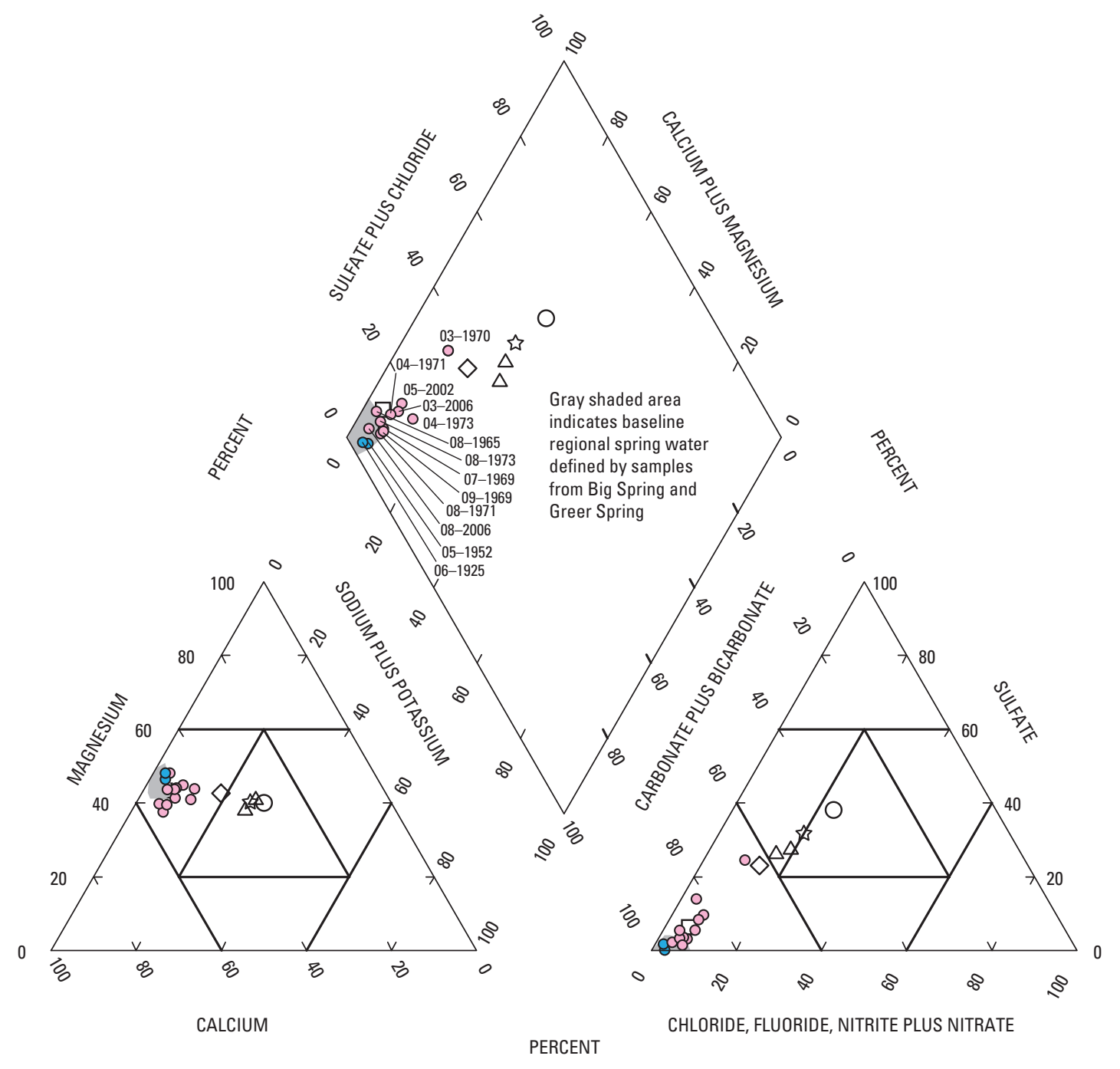

EXPLANATION
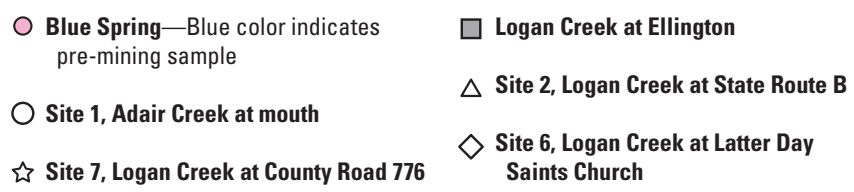

Figure 5. Trilinear diagram of water samples collected from Blue Spring (1925-2006) and water samples collected from upper Logan Creek during 2006.

simulated concentrations of chloride and other constituents in the March 2006 high base-flow simulation were greatly overestimated and the discharge of the Logan Creek component had to be reduced 23 percent from $23.3 \mathrm{ft}^{3} / \mathrm{s}$ (total discharge lost) to $17.9 \mathrm{ft}^{3} / \mathrm{s}$ to balance the chloride concentration at Blue Spring. This reduction in discharge required in the high baseflow simulation probably indicates non-steady state conditions existed along upper Logan Creek-likely the result of precipitation and runoff affecting the discharge, water-quality, or both before the March 7, 2006, seepage run.
Significant differences ( $\mathrm{p}$-value $<0.05$ ) in discharge, specific conductance, and concentrations of several major inorganic constituents were detected between Blue Spring and baseline concentrations represented by Big Spring and Greer Spring (fig. 6). Samples from Blue Spring had significantly smaller specific conductance values and dissolved concentrations of calcium and magnesium, but significantly larger concentrations of sodium, sulfate, and chloride as compared to Big Spring and Greer Spring (fig. 6). The significantly larger concentrations of sodium, sulfate, and chloride in Blue Spring 
Table 3. Measured and simulated concentrations of selected constituents in water samples collected from Blue Spring in March and August 2006.

[ft ${ }^{3} / \mathrm{s}$, cubic feet per second; --, no data; $\mathrm{Ca}$, calcium; $\mathrm{Mg}$, magnesium; $\mathrm{Na}$, sodium; $\mathrm{K}$, potassium; $\mathrm{Cl}$, chloride; $\mathrm{SO}_{4}$, sulfate; $\mathrm{HCO}_{3}$, total bicarbonate; $\mathrm{B}$, boron; $\mu \mathrm{g} / \mathrm{L}$, micrograms per liter; all concentrations are dissolved in milligrams per liter unless noted otherwise; shade indicates percent difference greater than 15 percent]

\begin{tabular}{|c|c|c|c|c|c|c|c|c|c|}
\hline \multirow[b]{2}{*}{ Constituent } & \multirow{2}{*}{$\begin{array}{c}\text { Base-flow } \\
\text { component }\end{array}$} & \multicolumn{2}{|c|}{ Logan Creek component } & \multicolumn{3}{|c|}{$\begin{array}{l}\text { Blue Spring high base flow } \\
(03 / 07 / 06)\end{array}$} & \multicolumn{3}{|c|}{$\begin{array}{c}\text { Blue Spring low base flow } \\
(08 / 15 / 06)\end{array}$} \\
\hline & & $\begin{array}{c}\text { High base- } \\
\text { flow site } 7 \\
(03 / 07 / 06)\end{array}$ & $\begin{array}{l}\text { Low base- } \\
\text { flow site } 6 \\
(08 / 15 / 06)\end{array}$ & Measured & $\begin{array}{c}\text { Simulated } \\
\text { (simulated } \\
\text { with adjusted } \\
\text { discharge) }\end{array}$ & $\begin{array}{c}\text { Percent } \\
\text { difference } \\
\text { (with adjusted } \\
\text { discharge) }\end{array}$ & Measured & Simulated & $\begin{array}{c}\text { Percent } \\
\text { difference }\end{array}$ \\
\hline $\begin{array}{l}\text { Discharge } \\
\left(\mathrm{ft}^{3} / \mathrm{s}\right)\end{array}$ & -- & $\begin{array}{c}23.3 \\
\mathrm{a}(17.9)\end{array}$ & b4.46 & 98.6 & -- & -- & 88.4 & -- & -- \\
\hline $\mathrm{Ca}$ & 35 & 29.2 & 30.2 & 29.8 & $\begin{array}{c}33.6 \\
(33.9)\end{array}$ & $\begin{array}{c}12.9 \\
(13.9)\end{array}$ & 32.4 & 34.8 & 7.3 \\
\hline $\mathrm{Mg}$ & 20 & 21.7 & 20.1 & 18.1 & $\begin{array}{c}20.4 \\
(20.3)\end{array}$ & $\begin{array}{c}12.7 \\
(12.2)\end{array}$ & 18.7 & 20.0 & 7.0 \\
\hline $\mathrm{Na}$ & 1.6 & 25.3 & 15.6 & 6.3 & $\begin{array}{c}7.2 \\
(5.9)\end{array}$ & $\begin{array}{l}14.9 \\
(-6.3)\end{array}$ & 3.1 & 2.3 & -26.8 \\
\hline $\mathrm{K}$ & .9 & 2.08 & 1.83 & 0.9 & $\begin{array}{c}1.1 \\
(1.0)\end{array}$ & $\begin{array}{c}22.5 \\
(14.7)\end{array}$ & .7 & .9 & 21.7 \\
\hline $\mathrm{SO}_{4}$ & 2.4 & 73.5 & 42.4 & 16.0 & $\begin{array}{c}19.2 \\
(15.3)\end{array}$ & $\begin{array}{l}20.0 \\
(-4.3)\end{array}$ & 4.8 & 4.4 & -8.0 \\
\hline $\mathrm{Cl}$ & 2.5 & 31.4 & 18.5 & 7.7 & $\begin{array}{c}9.3 \\
\mathrm{a}(7.7)\end{array}$ & $\begin{array}{l}20.7 \\
(0)\end{array}$ & 3.4 & 3.3 & -4.1 \\
\hline $\mathrm{HCO}_{3}$ & 200 & 141 & 245 & 189 & $\begin{array}{c}186 \\
(189)\end{array}$ & $\begin{array}{l}-1.6 \\
(0.2)\end{array}$ & 185 & 197 & 9.3 \\
\hline $\mathrm{B}(\mu \mathrm{g} / \mathrm{L})$ & 8 & 72 & 62 & 17.3 & $\begin{array}{c}23.2 \\
(19.7)\end{array}$ & $\begin{array}{c}36.4 \\
(15.7)\end{array}$ & 11.1 & 10.7 & -3.5 \\
\hline
\end{tabular}

a Total discharge lost was $23.3 \mathrm{ft}^{3} / \mathrm{s}\left(21.8 \mathrm{ft}^{3} / \mathrm{s}\right.$ measured at site 3 plus $1.45 \mathrm{ft}^{3} / \mathrm{s}$ measured at site 4$)$; Logan Creek adjusted component reduced 23 percent to $17.9 \mathrm{ft}^{3} / \mathrm{s}$ to address mass balance of chloride at Blue Spring.

b Total discharge lost was $4.43 \mathrm{ft}^{3} / \mathrm{s}$ measured at site 3 plus an estimated $0.03 \mathrm{ft}^{3} / \mathrm{s}$ loss at site 4 (Sweetwater Creek).

likely are the result of water from the Sweetwater Mine and tailings impoundment that discharges into upper Logan Creek and emerges at Blue Spring. Samples of mine seeps within the Sweetwater Mine confirm large concentrations of sodium, sulfate, and chloride in water pumped from the mine (data on file at the USGS office in Rolla, Missouri). Concentrations of $\mathrm{NO}_{2}+\mathrm{NO}_{3}$ in Blue Spring were similar to those in Big Spring, but significantly smaller than those in Greer Spring. It is noteworthy that concentrations of sodium, sulfate, chloride, $\mathrm{NO}_{2}+\mathrm{NO}_{3}$, and fecal coliform densities (not shown) in Greer Spring were significantly larger than those in Big Spring (fig. 6). Although a sufficient number of samples from Big Spring and Greer Spring were analyzed for dissolved trace elements to establish baseline trace-element concentrations, only three samples from Blue Spring have been analyzed for dissolved trace elements, and no statistical comparison of these values to baseline concentrations was made. A Kruskal-Wallis non-parametric test indicated that concentrations of total lead and total zinc in water samples from Blue Spring were not significantly larger than baseline concentrations.
Because of the paucity of samples from Blue Spring for complete chemical analysis, especially before mining began in the Logan Creek Basin, insufficient data are available for detailed trend analysis. A Lowess smooth of historical data from Blue Spring using the software package SYSTAT (SYSTAT Software Inc., 2002) indicated that discharge tended to increase from the mid-1960s through the mid-1990s, then decreased thereafter (fig. 7). Specific conductance values, while variable for the period of record, exhibited no time trends. The period between 1965 and 1974 had noticeable spikes in concentrations of magnesium, sodium, chloride, and a relatively steady increase in sulfate concentrations (fig. 7). Concentrations of total dissolved solids (TDS) and molar ratios of calcium to magnesium $(\mathrm{Ca}: \mathrm{Mg})$ also tended to increase during this period (table 2). The spike in constituent concentrations and $\mathrm{Ca}: \mathrm{Mg}$ ratios coincides with the opening of the exploration shaft during 1962 and initial development of the Sweetwater Mine complex and corresponding increase in concentrations of these constituents in samples from upper Logan Creek. Sampling density also may contribute to the 

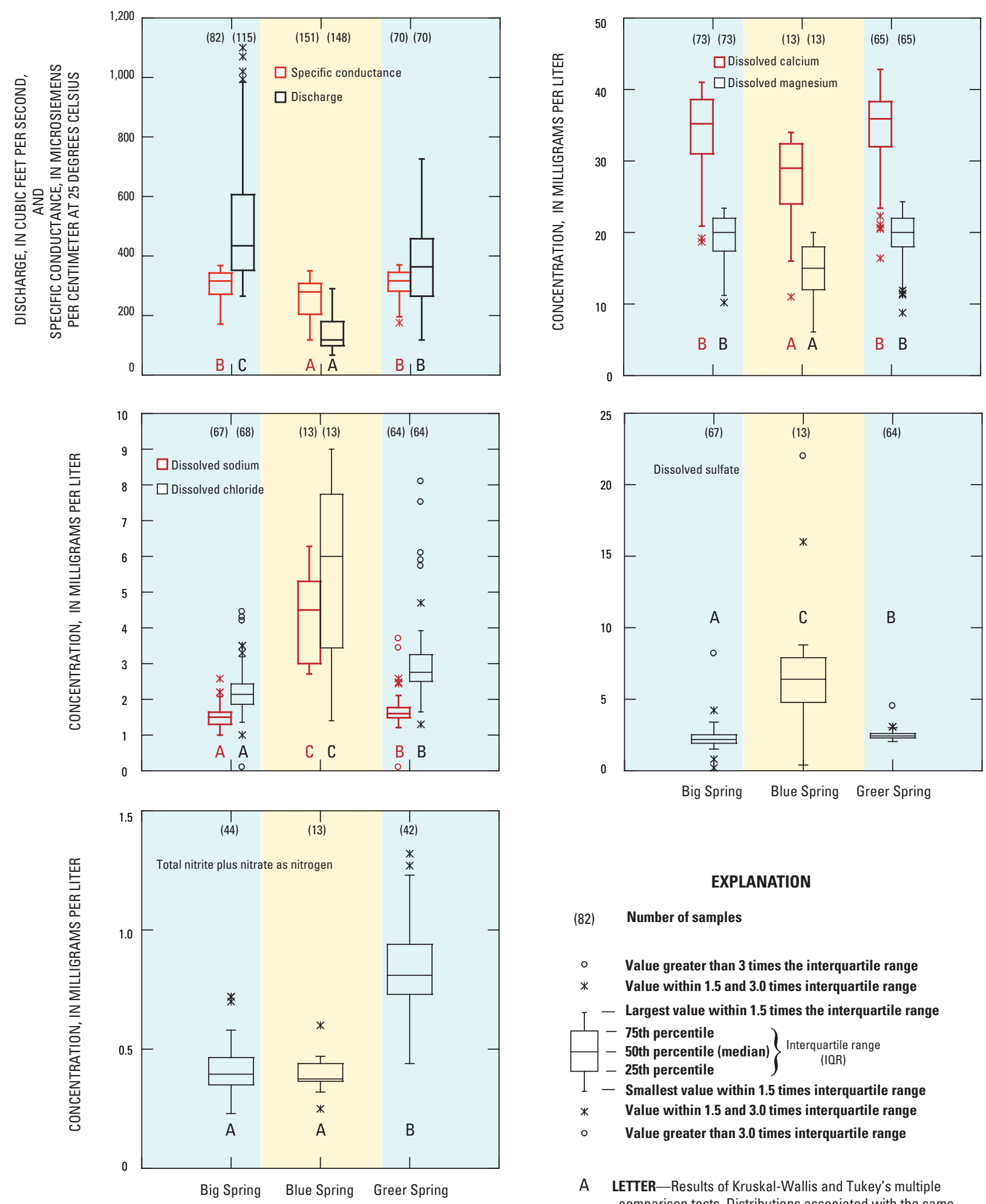

EXPLANATION

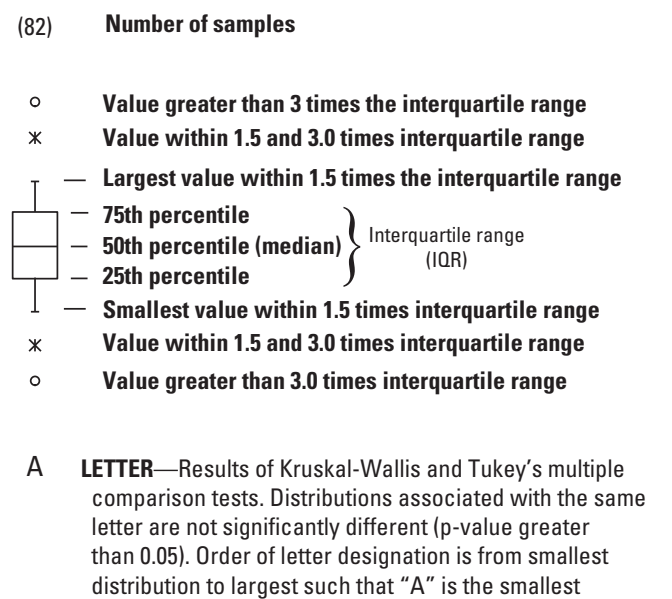

Figure 6. Concentrations of selected major ions and nitrate plus nitrate as nitrogen in water samples from Blue Spring, Big Spring, and Greer Spring and results of analysis of variance and multiple comparison tests on rank ordered data. 

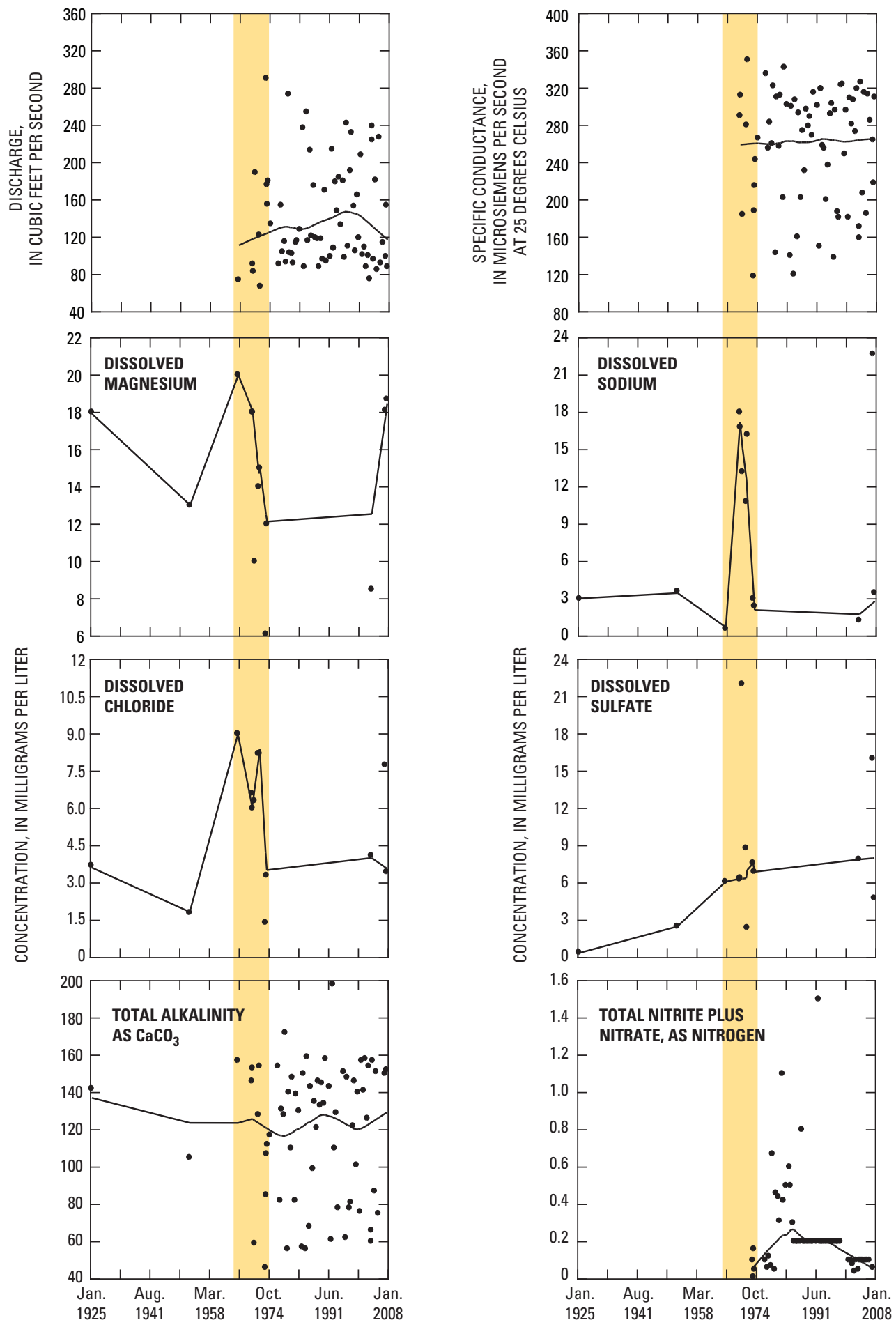

EXPLANATION

Beginning of mine development in 1962 through 1974

Lowess smooth function (SYSTAT Inc., 2002)

Figure 7. Discharge, specific conductance, and concentrations of selected chemical constituents with time in water samples from Blue Spring, 1925-2006. 
observed spike in concentrations because more samples from Blue Spring were analyzed for these constituents during the 1960s and early 1970s than at any other time.

\section{Streambed-Sediment Quality}

Many mining-related constituents, such as lead, are considered insoluble and usually are not detected in the dissolved phase in surface water. Streambed-sediment samples were collected to determine if mine tailings releases into Logan Creek have resulted in increased concentrations of mining-related minerals and trace elements in streambed sediment from Blue Spring or the Current River downstream from Blue Spring.

During 1995, five composite streambed-sediment samples were collected; two from cross sections along Blue Spring branch (one about $20 \mathrm{ft}$ downstream from the orifice referred to as the orifice and the other about $150 \mathrm{ft}$ upstream from the spring branch mouth, referred to as the branch), one from a cross section on the Current River about $900 \mathrm{ft}$ upstream from Blue Spring, and one each from the east and west sides of the Current River along a cross section about $100 \mathrm{ft}$ downstream from Blue Spring. The Current River was about $250 \mathrm{ft}$ wide at each cross section. The Blue Spring branch enters the Current River from the east bank. The section across the Current River downstream from Blue Spring branch was divided into an east one-half and a west one-half sample to document differences in mineralogy or chemistry of the streambed sediment. Streambed-sediment samples from 0 to $50 \mathrm{ft}$ from each bank were collected as described in chapter 3 of this report and samples from the middle $150 \mathrm{ft}$ of the channel were collected from a boat using a USGS BM-60 bed-sediment sampler. During 2002, an additional streambed-sediment sample was collected from a cross section about $20 \mathrm{ft}$ downstream from the spring orifice as part of a larger streambed-sediment sampling effort along the Black River and adjacent streams described in chapter 3 in this report.

Concentrations of trace elements in streambed-sediment samples from Blue Spring were compared to results of streambed-sediment samples collected during 1997 and 2002 by the USGS from the baseline sites at Big Spring and Greer Spring (chapter 3). Comparisons focused on the MVT-related metals (arsenic, cadmium, cobalt, copper, lead, nickel, and zinc) in the fine fraction (less than $0.063-\mathrm{mm}$ size) and the presence of lead-ore minerals such as galena $(\mathrm{PbS})$ and cerrusite $\left(\mathrm{PbCO}_{3}\right)$. Because of the inherent heterogeneity in streambed sediment and difficulty in collecting representative samples, comparisons between single samples often are equivocal; comparisons are best made between groups of samples.

Streambed-sediment samples from Blue Spring, the Current River upstream and downstream from Blue Spring, and the baseline sites (Big Spring and Greer Spring) generally were coarse grained [more than about 90 percent sand-size (greater than $0.063 \mathrm{~mm}$ ) or greater] and composed primarily of quartz and dolomite, with smaller amounts of amorphous silicates and oxides and other minerals (table 4 , at the back of this chapter). Baseline sites tended to have larger quartz con- tents (74 to 93.1 percent by weight) and have less amorphous silicates and oxides and other minerals than samples from Blue Spring, which were composed of 59 to 69 percent quartz by weight. Dolomite generally was the predominant mineral in the $\mathrm{C} 3$-fraction at all sites and is present as a contaminant because its density [ 2.85 grams per cubic centimeter $(\mathrm{gm} /$ $\left.\mathrm{cm}^{3}\right)$ ] is similar to that of the bromoform $\left(2.87 \mathrm{gm} / \mathrm{cm}^{3}\right)$ used to concentrate the heavy mineral fraction. Samples from Blue Spring and the Current River upstream and downstream from Blue Spring tended to have larger amounts of limonite and oxidized rock fragments (5 to 50 percent of the $\mathrm{C} 3$-fraction) compared to baseline sites that had 5 percent or less limonite and oxidized rock fragments (table 4).

Subtle effects from mine tailings releases into upper Logan Creek during the 1970s may be the source of significantly larger than baseline cobalt and nickel concentrations and the lead-ore minerals galena and cerrusite detected in streambed-sediment samples from Blue Spring. Concentrations of cobalt and nickel averaged 34 and 65 milligrams per kilogram $(\mathrm{mg} / \mathrm{kg})$ in the fine fraction of streambed sediments from the Blue Spring orifice or spring branch and were significantly larger ( $p$-value $<0.05$ using a Kruskal-Wallis test) than concentrations at Big Spring and Greer Spring, which had averages of 21 and $41 \mathrm{mg} / \mathrm{kg}$ (fig. 8; table 5, at the back of this chapter). The increased cobalt and nickel concentrations may be related to mine tailings releases into Logan Creek during the 1970s because cobalt- and nickel-rich minerals, such as siegenite $\left[(\mathrm{Co}, \mathrm{Ni})_{3} \mathrm{~S}_{4}\right]$ and millerite $(\mathrm{NiS})$, are present within the Viburnum Trend ore deposits and, as described in chapter 3 of this report, cobalt and nickel are known to occur in increased concentrations in streambed-sediment samples downstream from Viburnum Trend mining areas. Concentrations of other MVT-related metals in Blue Spring streambed-sediment samples were not significantly larger than baseline concentrations and several, such as arsenic (all less than reporting level), lead, and zinc, were slightly smaller than baseline concentrations (fig. 8). The absence of elevated lead in the fine fraction of Blue Spring samples is unexpected considering that trace amounts of the lead-ore minerals galena and cerrusite were detected in the $\mathrm{C} 3$ fraction from three of the four samples from Blue Spring and on the east side of the Current River downstream from Blue Spring (table 4). The absence of elevated lead concentrations in the fine fraction of streambed-sediment samples from Blue Spring may indicate that much of the lead in this fraction is associated with minerals or particles other than the galena or cerrusite identified in the $\mathrm{C} 3$ fraction. Anthropogenic contamination in the $\mathrm{C} 3$ fraction was noted by the presence of metallic lead and zinc in the 1997 samples from Big Spring orifice and Big Spring branch, and trace amounts of glass were detected in a Big Spring orifice sample (table 4). This contamination may account for the significantly larger lead and zinc concentrations in baseline sites compared to Blue Spring. Brass contamination also was detected in the sample from the west side of the Current River sample downstream from Blue Spring. 

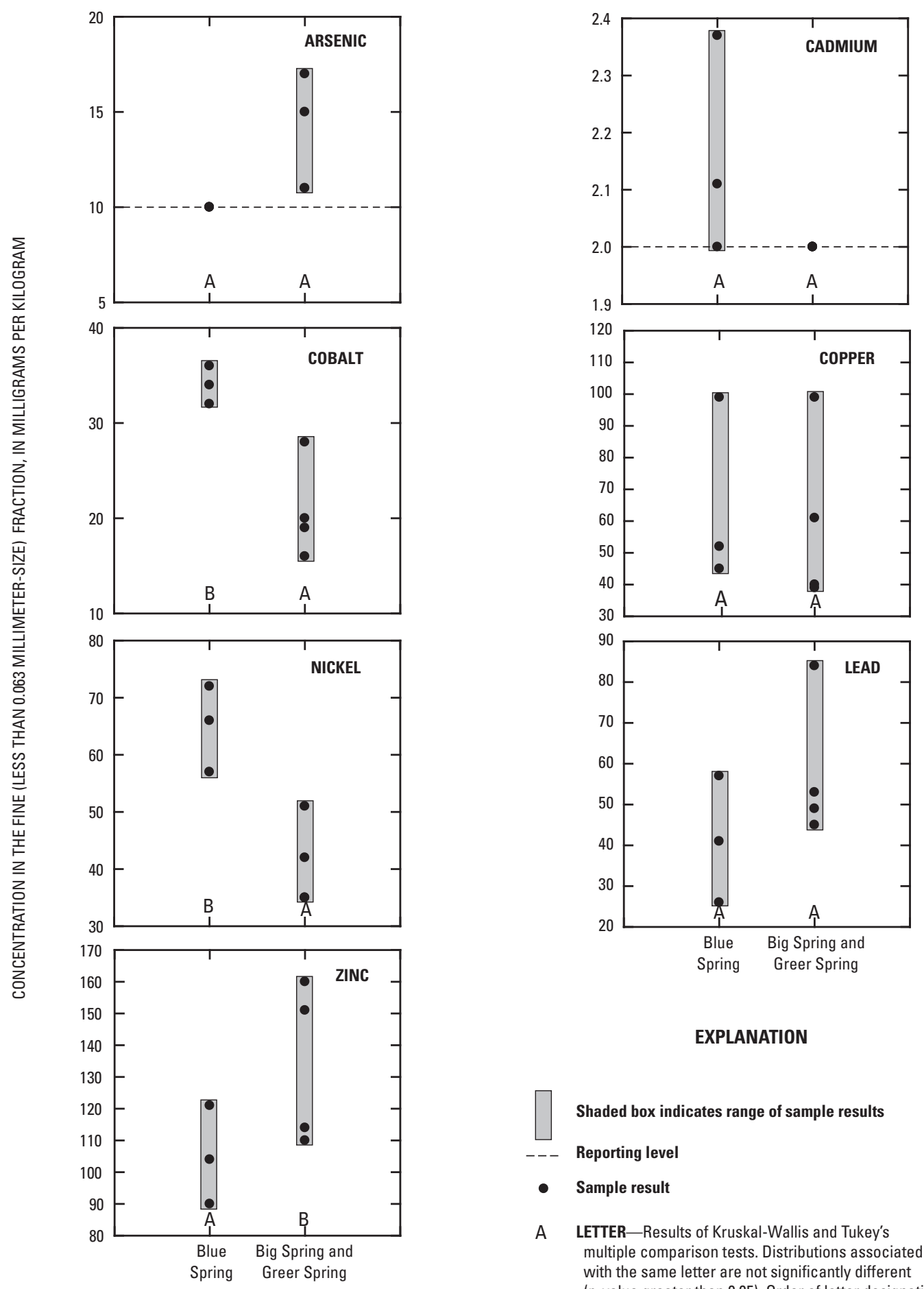

EXPLANATION

Shaded box indicates range of sample results

Reporting level

- Sample result

A LETTER - Results of Kruskal-Wallis and Tukey's multiple comparison tests. Distributions associated with the same letter are not significantly different ( $p$-value greater than 0.05 ). Order of letter designation is from smallest distribution to larger such that " $A$ " is the smallest

Figure 8. Concentrations of Mississippi Valley Type (MVT)-related metals in the fine (less than 0.063-millimeter size) fraction of streambed-sediment samples from Blue Spring and baseline concentrations from Big and Greer Springs, 1995-2002. 
Comparison of MVT-related metals in the fine fraction in samples from Blue Spring and the Current River collected during 1995 and 2002 indicated generally larger trace element concentrations in Blue Spring (fig. 9). Using a Kruskal-Wallis non-parametric test and grouping the 1995 and 2002 samples from Blue Spring orifice and the 1995 spring branch sample together and comparing these against the three 1995 Current River samples indicated concentrations of cadmium, cobalt, nickel, and zinc in the fine fraction of streambed sediment from Blue Spring (fig. 9) were significantly larger (p-value $<0.05$ ) than concentrations in the Current River streambed sediment. However, larger trace-element (copper, nickel, lead,
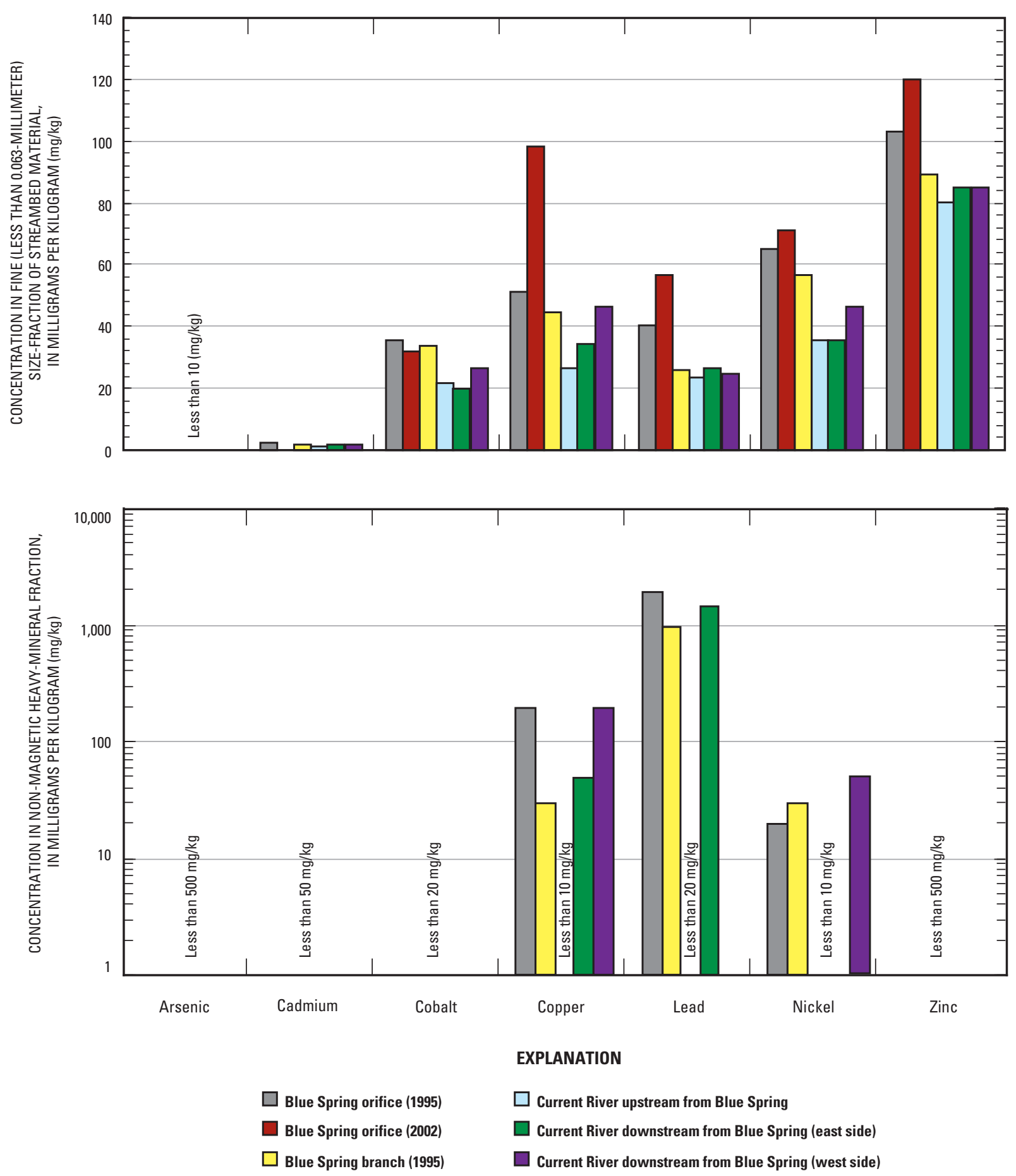

Figure 9. Maximum concentrations of Mississippi Valley Type (MVT)-related metals in the fine (less than 0.063millimeter size) fraction by inductively coupled liquid plasma-atomic emmission spectrometry (ICP-AES) and semiquantitative concentrations in the non-magnetic heavy mineral fraction by emmission spectrometry in streambedsediment samples from Blue Spring and the Current River, 1995-2002. 
and zinc) concentrations also were detected in samples from Big Spring and its spring branch as compared to the Current River upstream and downstream from Big Spring (table 5). The cause of the generally larger trace-element concentrations in streambed sediment in Blue Spring and Big Spring as compared to samples from the Current River is not known, but may be related to differences in geology of the spring recharge basins compared to that of the Current River. Both Blue Spring and Big Spring orifices have developed in the lower part of the Eminence Dolomite (Vineyard and Feder, 1982), and presumably streambed sediment in their orifices and spring branches are effected mostly by the Eminence Dolomite or underlying Potosi Dolomite. In contrast, streambed-sediment samples from the Current River are derived from rocks exposed upstream from either spring that include (from oldest to youngest) the Eminence Dolomite, Gasconade Dolomite, Roubidoux Formation, and Jefferson City Dolomite. In addition, streambed sediment in the Current River presumably has been transported further from its source and subjected to more weathering than streambed sediment in the spring orifices or spring branches. Indeed, streambed-sediment samples from the spring orifices and spring branches have larger quantities of dolomite and other carbonate material and much smaller quantities of quartz as compared to the Current River samples (table 4). Although significant differences were detected, results should be carefully considered, given the small sample sizes for the Blue Spring orifice and spring branch (three samples), the Big Spring orifice and spring branch, and Greer Spring (four samples) and the large variability in concentrations of some constituents between samples (fig. 9). A more important observation is that concentrations of MVT-related metals in the fine fraction of streambed sediment from the east side of the Current River downstream from Blue Spring were similar to those in the Current River sample upstream from Blue Spring (fig. 9), indicating no measurable effect from the 1970s mine tailings releases to Logan Creek on trace-element concentrations in the fine fraction of streambed sediment in the Current River.

Semi-quantitative concentrations of MVT-related metals in the C3 fraction of streambed-sediment samples from Blue Spring and the Current River also indicate subtle effects from historical mine tailings releases into Logan Creek. Because the $\mathrm{C} 3$ fraction tends to concentrate ore minerals and other mineral enriched in trace elements, trace-element concentrations in this fraction typically are larger than those in the fine fraction. The semi-quantitative trace-element concentrations in the C3 fraction are order-of-magnitude estimates and cannot be compared directly with concentrations in the fine fraction that were determined by quantitative ICP-AES. The mouth of Blue Spring branch is on the east bank of the Current River. Semi-quantitative concentrations of lead in the C3 fraction of streambed-sediment samples from Blue Spring (orifice and spring branch) and the east side of the Current River downstream from Blue Spring were estimated at about 1,000 to $2,000 \mathrm{mg} / \mathrm{kg}$ as compared to less than detection in samples from the Current River upstream from Blue Spring and on the west side of the Current River downstream from Blue Spring (fig. 9; table 6, at the back of this chapter). The large semi-quantitative concentrations of lead in the $\mathrm{C} 3$ fraction of streambed-sediment samples from Blue Spring and the east side of the Current River downstream from Blue Spring is consistent with the presence of the lead-ore minerals galena and cerrusite in the $\mathrm{C} 3$ fraction from these three sites.

\section{Summary and Conclusions}

Water-tracing investigations using fluorescent dye have indicated a subsurface connection between a losing reach of Logan Creek downstream from an active lead mine and Blue Spring, the seventh largest spring in Missouri. Blue Spring is about 10 miles southwest of Logan Creek and an important source of water to the Current River within the Ozark National Scenic Riverways. Logan Creek typically is dry several miles downstream from the mine, and previous investigations have indicated that as much as 200 cubic feet per second $\left(\mathrm{ft}^{3} / \mathrm{s}\right)$ of discharge can be lost into the subsurface along this reach. An analysis of historical water-quality samples from upper Logan Creek and Blue Spring and streambed-sediment samples was performed to characterize changes in water- and streambedsediment quality as a result of lead and zinc mining in the Logan Creek Basin and how those changes affect the water quality of Blue Spring. Multiple lines of evidence from results of dye-trace investigations, water-quality sampling, and traceelement and mineralogic composition of streambed-sediment samples indicate likely effects of mining on the water quality of Blue Spring.

Analysis of more than 70 water-quality samples collected by the U.S. Geological Survey from 1965 to 2006 along Logan Creek indicate effects from mining activities on major ion concentrations in Logan Creek. Water samples from Logan Creek ranged from calcium-magnesium-bicarbonate type, typical of the Ozark region, to mixed sodium-magnesium-calcium bicarbonate-sulfate type, indicating increased concentrations of sodium, sulfate, and chloride likely resulting from mining operations. Development of the Sweetwater Mine began in 1962, 3 years before the start of water-quality sampling along Logan Creek. Initial water-quality samples collected from a downstream site on Logan Creek contained increased proportions of sodium, sulfate, and chloride relative to other major ions. After the mine began production during 1968, the distribution of major ions at the upstream sampling site (site 3, Logan Creek above Sweetwater Creek) trended from the calcium-magnesium-bicarbonate vertex on a trilinear diagram toward increasingly sulfate and chloride-rich water as mill tailings began to be discharged to an impoundment on Adair Creek, a tributary to Logan Creek upstream from site 3. After mining began, sulfate and chloride typically comprised more than 20 percent of the major anions in Logan Creek compared to less than 5 percent in baseline surface- and ground-water samples from the region. Little, if any, effects of mine dis- 
charges were observed on concentrations of dissolved trace elements in Logan Creek. Historically, concentrations of Mississippi Valley Type (MVT)-related dissolved metals (arsenic, cadmium, cobalt, copper, lead, nickel, and zinc) and other dissolved trace elements in water samples collected from upper Logan Creek generally were small and near reporting levels in the 63 samples collected from sites 3 and 5 . Dissolved zinc was the most commonly reported trace element being detected above reporting levels in 25 of the 54 samples in which it was analyzed, with a maximum concentration of 90 micrograms per liter $(\mu \mathrm{g} / \mathrm{L})$ detected in a sample collected during 1972. Dissolved zinc concentrations in four water samples (all collected during 1972) exceeded the current (2008) Missouri Department of Natural Resources chronic standard for the protection of aquatic life.

Results of a March and August 2006 stream seepage-run and water-quality investigation along the upper reach of Logan Creek indicated that, depending on the quantity of runoff from the mine tailings impoundment and streamflow conditions, discharge from the mine complex and tailings impoundment along Adair Creek comprised as much as 68 percent of the discharge in upper Logan Creek that is lost into the subsurface and resurges at Blue Spring. This finding is significant because at times, nearly all the flow in Adair Creek is from mining-related sources. On March 7, 2006, Logan Creek lost $23.3 \mathrm{ft}^{3} / \mathrm{s}$ of discharge downstream from the Sweetwater Mine in comparison to a discharge of $98.6 \mathrm{ft}^{3} / \mathrm{s}$ measured at Blue Spring that same day. On August 15, 2006, the discharge along Logan Creek was smaller, and about $4.46 \mathrm{ft}^{3} / \mathrm{s}$ of flow was lost (about 17 percent of which was from Adair Creek), which represented about 5 percent of the $88.4 \mathrm{ft}^{3} / \mathrm{s}$ measured at Blue Spring that same day. Water-quality samples collected during 2006 from Adair Creek and Logan Creek downstream from the mine all contained increased proportions of sodium, sulfate, and chloride as compared to other major ions. A March 7, 2006, sample from Adair Creek had a specific conductance value of 617 microsiemens per centimeter at 25 degrees Celsius $(\mu \mathrm{S} / \mathrm{cm})$ and concentrations of sodium [40.2 milligrams per liter $(\mathrm{mg} / \mathrm{L})$ ], sulfate $(114 \mathrm{mg} / \mathrm{L})$, and chloride $(49.1 \mathrm{mg} / \mathrm{L})$ that exceeded baseline concentrations. This sample also contained above baseline concentrations of several MVT-related metals such as cobalt $(2 \mu \mathrm{g} / \mathrm{L})$, copper $(3.2 \mu \mathrm{g} / \mathrm{L})$, lead $(11.7 \mu \mathrm{g} / \mathrm{L})$, nickel $(8.5 \mu \mathrm{g} / \mathrm{L})$, and zinc (34 $\mu \mathrm{g} / \mathrm{L})$ and the trace elements boron $(125 \mu \mathrm{g} / \mathrm{L})$ and manganese $(330 \mu \mathrm{g} / \mathrm{L})$. Concentrations of major and trace constituents in Logan Creek decreased with increasing distance downstream from Adair Creek; however, concentrations of sodium, sulfate, chloride, and boron remained above baseline values until flow was lost into the subsurface.

Similar to water samples from most springs in southern Missouri, water samples collected from Blue Spring have moderate specific conductance values (118 to $350 \mu \mathrm{S} / \mathrm{cm}$ ), near neutral $\mathrm{pH}$ values (6.9 to 8.2) and calcium, magnesium, and bicarbonate as the predominant ions. Effects of mining in the Logan Creek Basin have resulted in changes in major ion chemistry of the water discharged from Blue Spring and possibly subtle changes in the trace mineralogy and concentrations of several trace elements in streambed-sediment samples from the spring and east side of the Current River downstream from the spring. Although water samples from Blue Spring have calcium, magnesium, and bicarbonate as the predominant ions, 5 of the 13 samples from Blue Spring for which complete water-quality analyses are available plot outside the baseline range for area springs on a trilinear diagram and contain increased concentrations of sulfate, chloride, and sodium relative to bicarbonate and calcium plus magnesium. All five samples plotting outside the baseline range were collected after the Sweetwater Mine began operation, and they plot along a trend between baseline spring water samples and samples from Logan Creek downstream from the mine. The increased sulfate, chloride, and sodium concentrations in these samples are consistent with previous dye traces, indicating that flow lost in upper Logan Creek downstream from the mine emerges at Blue Spring. Results of a simple mixing model indicated that concentrations of major ions measured in the March and August 2006 samples from Blue Spring can be reasonably approximated by the mixing of flow lost along upper Logan Creek with a baseline ground-water component. The simple mixing model also is consistent with results from pervious dye-trace investigations.

Samples from Blue Spring had significantly smaller specific conductance values and calcium and magnesium concentrations, but significantly larger sodium, sulfate, and chloride concentrations as compared to baseline concentrations represented by samples from Big Spring and Greer Spring. The significantly larger sodium, chloride, and sulfate concentrations in Blue Spring likely are the result of loss of discharge from the mine complex into the subsurface along upper Logan Creek that resurges at Blue Spring. Samples of mine seeps within the Sweetwater Mine confirmed large concentrations of sodium, sulfate, and chloride in water pumped from the mine. Insufficient data were available for statistical comparisons of dissolved trace-element concentrations in Blue Spring to baseline concentrations; however, concentrations of total lead and total zinc in water samples from Blue Spring were not significantly larger than baseline concentrations. Samples collected from Blue Spring from 1965 to 1974 had spikes in concentrations of magnesium, sodium, chloride, and molar ratios of calcium to magnesium and a relatively steady increase in sulfate concentrations. The spike in constituent concentrations and calcium to magnesium ratios coincides with the opening of the exploration shaft during 1962 and initial development of the mine and corresponding increase in concentrations of these constituents in water samples from Logan Creek during the same period.

Mine tailings releases into Logan Creek from breaches in the mine tailings impoundment that occurred during the 1970s appear to be the source of significantly larger than baseline cobalt and nickel concentrations and the presence of the lead-ore minerals galena and cerrusite in streambed-sediment samples from Blue Spring. Concentrations of cobalt and nickel averaged 34 and 65 milligrams per kilogram $(\mathrm{mg} / \mathrm{kg})$ 
in the fine (less than 0.063 millimeter) fraction of streambed sediments from Blue Spring and its spring branch and were significantly larger (p-values less than 0.05 using a KruskalWallis test) than in sediment from baseline sites that had average cobalt and nickel concentrations of 21 and $41 \mathrm{mg} / \mathrm{kg}$. Cobalt and nickel-rich minerals, such as siegenite and millerite, are present within ore deposits in the Viburnum Trend and are a possible source of the increased cobalt and nickel concentrations in streambed-sediment samples from Blue Spring. Concentrations of MVT-related metals in streambed-sediment samples from the Current River downstream from Blue Spring were not significantly larger than baseline, indicating no measurable effect from the 1970s mine tailings releases into upper Logan Creek on trace-element concentrations in the fine fraction of streambed sediment in the Current River. However, mine tailings releases into Logan Creek may be the source of lead and lead-ore minerals detected in the non-magnetic heavy-mineral (C3) fraction of streambed-sediment samples from Blue Spring and the east side of the Current River downstream from Blue Spring.

\section{References Cited}

Aley, T.J., and Aley, C., 1987, Groundwater study-Ozarks National Scenic Riverways: Protem, Mo., Ozark Underground Laboratory, National Park Service Contract CX 6000-4-0083, 222 p.

Barks, J.H., 1978, Water quality of the Ozark National Scenic Riverways, Missouri: U.S. Geological Survey Water-Supply Paper 2048, 57 p.

Bridge, Josiah, 1930, Geology of the Eminence and Cardareva quadrangles: Missouri Bureau of Geology and Mines, $2 \mathrm{~d}$. series, v. 24, p 30.

Duchrow, R.M., Robinson-Wilson, E., and Trial, Linden, 1980, The effects of lead mine tailings on the water quality of Logan Creek, Reynolds County, Missouri: Missouri Department of Conservation, 29 p.

Feder, G.L., and Barks, J.H., 1972, A losing drainage basin in the Missouri Ozarks identified by side-looking radar imagery, in Geological Survey Research 1972: U.S. Geological Survey Professional Paper 800-C, p. C249-C252.

Guy H.P., and Norman, V.W., 1970, Field methods for measurement of fluvial sediments: U.S. Geological Survey Techniques of Water-Resources Investigations, book 3 , chap. C2, 59 p.

Hauck, H.S., and Harris, T.H., 2006, Water resources data, Missouri, water year 2005: U.S. Geological Survey WaterData Report MO-05-1, 724 p. (published annually).
Hem, J.D., 1985, Study and interpretation of the chemical characteristics of natural water ( $3 \mathrm{~d}$ ed.): U.S. Geological Survey Water-Supply Paper 2254, 263 p.

Imes, J.L., Plummer, L.N., Kleeschulte, M.J., and Schumacher, J.G., 2007, Recharge area, base-flow and quickflow discharge rates and ages, and general water quality of Big Spring in Carter County, Missouri, 2000-04: U.S. Geological Survey Scientific Investigations Report 2007-5049, $79 \mathrm{p}$.

Maxwell, J.C., 1974, Water resources of the Current River Basin, Missouri: Rolla, University of Missouri-Rolla, Water Resources Research Center, 134 p.

Miller, D.E., and Vandike, J.E., 1997, Groundwater resources of Missouri: Jefferson City, Missouri State Water Plan, v. II, Missouri Department of Natural Resources Water Resources Report Number 46, 210 p.

Missouri Department of Conservation, 2008, Directory of Missouri natural areas, accessed June 27, 2008, at http:// mdc.mo.gov/areas/natareas/p124-2.htm

Missouri Department of Natural Resources, 2007a, Missouri environmental geology atlas: Rolla, Division of Geology and Land Survey, CD_ROM.

Missouri Department of Natural Resources, 2007b, Code of State Regulations Title 10, Division 20, Chapter 7, Water Quality Standards, accessed October 5, 2007, at URL http:// www.sos.mo.gov/adrules/csr/current/10csr/10c20-7a.pdf

Mouat, M.M., and Clendenin, C.W., 1977, Geology of the Ozark Lead Company Mine, Viburnum Trend, southeast Missouri: Economic Geology, v. 72, p. 398-407.

National Oceanic and Atmospheric Administration, 2007, National Climate Data Center, accessed August 15, 2007, at URL http://www.ncdc.noaa.gov/oa/climate/stationlocator. html

Rueff, Ardell, 1970, Milestones in Missouri mining: Missouri Mineral Industry News: Rolla, Missouri Geological Survey and Water Resources, v. 10, p. 58-65.

Schumacher, J.G., and Hockanson, E.A., 1996, Hydrogeology and water quality at the St. Francois County landfill and vicinity, southeastern Missouri: U.S. Geological Survey Water-Resources Investigations Report 96-4022, 138 p.

SYSTAT Software Inc., 2002, SYSTAT user's guide-Statistics I and II: Richmond, Calif., version 10.2, 1,376 p.

U.S. Geological Survey, 2007, Water-quality data for Missouri: accessed June 2007 at URL http://waterdata.usgs. gov/mo/nwis/gw 
Vineyard, J.D., and Feder, G.L., 1982, Springs of Missouri: Rolla, Missouri Department of Natural Resources, Division of Geology and Land Survey Water Resources Report 29, $212 \mathrm{p}$.

Ward, J.R., and Harr, A., eds, 1990, Methods for collection and processing of surface-water and bed-materials samples for physical and chemical analyses: U.S. Geological Survey Open-File Report 90-140, 71 p.

Wilde, F.D., Radtke, D.B., Gibs, Jacob, and Iwatsubo, R.T., eds., 1999, National field manual for the collection of waterquality data_Processing of water samples: U.S. Geological Survey Techniques of Water-Resources Investigations, book 9, chap. A5, 128 p. 
Tables 
Table 1. Physical properties and concentrations of inorganic chemical constituents in water samples from upper Logan Creek, 1965-2006.

[All concentrations are dissolved and in milligrams per liter unless noted otherwise; $\mathrm{ft}^{3} / \mathrm{s}$, cubic feet per second; Cond, specific conductance in microsiemens per centimeter at $25 \mathrm{degrees}$ Celsius; Temp, temperature in degrees Celsius; USGS, U.S. Geological Survey; --, no data; <, less than; $\mathrm{CaCO}_{3}$, calcium carbonate; N, nitrogen; P, phosphorus; ND, not detected; E, estimated value; $\mu \mathrm{g} / \mathrm{L}$, micrograms per liter]

\begin{tabular}{|c|c|c|c|c|c|c|c|c|c|c|}
\hline Date & Time & $\begin{array}{c}\text { Discharge } \\
\left(\mathrm{ft}^{3} / \mathrm{s}\right)\end{array}$ & Cond & Temp & $\mathrm{pH}$ & $\begin{array}{c}\text { Dissolved } \\
\text { oxygen }\end{array}$ & Calcium & Magnesium & Sodium & Potassium \\
\hline \multicolumn{11}{|c|}{ Site 1, Adair Creek at mouth (USGS station number 372128091072201) } \\
\hline 03/07/2006 & 0930 & 15.8 & 617 & 7.4 & 7.6 & -- & 37 & 30 & 40 & 3.1 \\
\hline \multicolumn{11}{|c|}{ Site 2, Logan Creek at State Route B (USGS station number 372026091071101) } \\
\hline $05 / 29 / 2002$ & 1530 & 16 & 313 & 19.6 & 7.7 & -- & 21 & 14 & 17 & 2.0 \\
\hline 03/07/2006 & 1030 & 21.2 & 518 & 7.7 & 7.7 & -- & 33 & 26 & 31 & 2.6 \\
\hline \multicolumn{11}{|c|}{ Site 3, Logan Creek above Sweetwater Creek (USGS station number 07061810) } \\
\hline $09 / 30 / 1965$ & -- & 21 & 95 & -- & 7.3 & 7.8 & 9.2 & 14 & 4.8 & 0.8 \\
\hline 01/26/1966 & -- & 7.1 & 100 & -- & 7.5 & 13.9 & 11 & 6.1 & 4.1 & .3 \\
\hline 08/10/1966 & -- & 3.8 & 160 & -- & 7.5 & 7.0 & 16 & 10 & 5.2 & 1 \\
\hline $10 / 24 / 1967$ & 1615 & .30 & 168 & 15 & 7.5 & 8.4 & 19 & 11 & 1.6 & 1.1 \\
\hline $10 / 17 / 1968$ & 0930 & .17 & 180 & 18 & 7.2 & 6.5 & 20 & 12 & 1.5 & 1.1 \\
\hline $01 / 14 / 1969$ & 1530 & 2.5 & 30 & 10 & 7.1 & 12.5 & 4.0 & 1.5 & .7 & .5 \\
\hline 07/15/1969 & 1600 & .11 & 150 & 34 & 8.3 & 8.0 & 18 & 11 & 1.7 & 1 \\
\hline $10 / 29 / 1969$ & 1400 & .15 & 237 & 11 & 7.4 & 10.3 & 27 & 22 & 2.3 & .7 \\
\hline 01/27/1970 & -- & .62 & 105 & 7 & 7.8 & 11.4 & 11 & 7.0 & 1.1 & .8 \\
\hline 04/29/1970 & -- & 13 & 28 & 18 & 6.8 & 9.2 & 2.4 & 1.5 & .5 & .9 \\
\hline 08/19/1970 & -- & .15 & 204 & 27 & 8.2 & 7.6 & 23 & 12 & 1.8 & .7 \\
\hline $11 / 02 / 1970$ & -- & 10 & 43 & 12 & 7.0 & 10.0 & 2.4 & 1.5 & 10 & 2.1 \\
\hline $01 / 26 / 1971$ & -- & 3.5 & 40 & 1 & 7.8 & 12.7 & 2.4 & 2.4 & 8.3 & 1.3 \\
\hline 04/21/1971 & -- & 3.6 & 40 & 16 & 7.3 & 10.2 & 3.2 & 2.4 & 1.4 & .7 \\
\hline 07/14/1971 & -- & .06 & 110 & 23 & 7.5 & 6.4 & 11 & 6 & 1.4 & 1 \\
\hline $11 / 09 / 1971$ & -- & .10 & 260 & 10 & 7.0 & 10.4 & 28 & 17 & 2.3 & .8 \\
\hline 02/01/1972 & -- & .85 & 70 & 5 & 7.9 & 12.1 & 5.6 & 5.8 & 1.0 & 1 \\
\hline 06/06/1972 & -- & 1.1 & 65 & 25 & 6.8 & 9.4 & 5.0 & 6.0 & 1.0 & .7 \\
\hline 08/22/1972 & -- & .28 & 170 & 25 & 6.7 & 7.7 & 18 & 7.8 & 1.3 & 1. \\
\hline 10/17/1972 & -- & .13 & 210 & 16 & 7.5 & 8.2 & 18 & 11 & 1.5 & .8 \\
\hline $01 / 31 / 1973$ & -- & 10 & 45 & 6 & 6.3 & 12.4 & 1.6 & 1.9 & .7 & .6 \\
\hline 05/15/1973 & -- & 6 & 38 & 17 & 6.8 & 9.4 & 3.2 & 1.0 & .7 & .8 \\
\hline
\end{tabular}


Table 1. Physical properties and concentrations of inorganic chemical constituents in water samples from upper Logan Creek, 1965-2006.-Continued

[All concentrations are dissolved and in milligrams per liter unless noted otherwise; $\mathrm{ft}^{3} / \mathrm{s}$, cubic feet per second; Cond, specific conductance in microsiemens per centimeter at 25 degrees Celsius; Temp, temperature in degrees Celsius; USGS, U.S. Geological Survey; --, no data; <, less than; $\mathrm{CaCO}_{3}$, calcium carbonate; N, nitrogen; $\mathrm{P}$, phosphorus; ND, not detected; E, estimated value; $\mu \mathrm{g} / \mathrm{L}$, micrograms per liter]

\begin{tabular}{|c|c|c|c|c|c|c|c|c|c|c|}
\hline Date & Time & $\begin{array}{c}\text { Discharge } \\
\left(\mathrm{ft}^{3} / \mathrm{s}\right)\end{array}$ & Cond & Temp & pH & $\begin{array}{c}\text { Dissolved } \\
\text { oxygen }\end{array}$ & Calcium & Magnesium & Sodium & Potassium \\
\hline \multicolumn{11}{|c|}{ Site 3, Logan Creek above Sweetwater Creek (USGS station number 07061810)—Continued } \\
\hline 07/31/1973 & -- & 3.3 & 46 & 24 & 7.4 & 8.7 & 1.3 & 4.1 & 1 & .8 \\
\hline $11 / 27 / 1973$ & 1515 & 52 & 40 & 12 & 6.3 & 9.6 & 1.3 & .12 & .7 & .6 \\
\hline 03/05/1974 & 1725 & 9.7 & 32 & 12 & 6.3 & 11.2 & 1.6 & 1.9 & .9 & .7 \\
\hline $05 / 14 / 1974$ & 1725 & 13 & 30 & 15 & 5.8 & 9.0 & 1.6 & 2.4 & 1.8 & .8 \\
\hline 08/13/1974 & -- & .86 & 170 & 23 & 7.8 & 8.6 & -- & -- & 1.5 & .8 \\
\hline $10 / 30 / 1974$ & 0815 & .57 & 98 & 15 & 7.0 & 8.1 & -- & -- & 1.2 & .6 \\
\hline 01/07/1975 & 1440 & 8.4 & 38 & 6 & 7.0 & 11.8 & -- & -- & .6 & 1 \\
\hline $04 / 22 / 1975$ & -- & 2 & 55 & 15 & 7.0 & 10.4 & -- & -- & .8 & .8 \\
\hline 07/29/1975 & 1445 & .13 & 200 & 27 & 7.7 & 7.6 & -- & -- & 1.8 & .8 \\
\hline \multicolumn{11}{|c|}{ Site 5, Logan Creek below Sweetwater Creek (USGS station number 07061840) } \\
\hline $09 / 30 / 1965$ & -- & 26 & 100 & -- & 7.6 & 8.3 & 10 & 11 & 5.6 & .8 \\
\hline 01/26/1966 & -- & 12 & 140 & -- & 7.6 & 13.4 & 12 & 6.8 & 11 & .3 \\
\hline 08/10/1966 & -- & 6.6 & 210 & -- & 7.6 & 7.2 & 15 & 10 & 24 & 1.3 \\
\hline $10 / 24 / 1967$ & 1715 & 10 & 248 & 15 & 7.8 & 9.2 & 20 & 11 & 18 & 1.1 \\
\hline $10 / 17 / 1968$ & 0830 & 4.9 & 240 & 19 & 7.5 & 6.9 & 21 & 15 & 9.8 & 1.2 \\
\hline $01 / 14 / 1969$ & 1600 & 14 & 90 & 5 & 7.5 & 12.0 & 10 & 9.2 & 3.2 & .8 \\
\hline $04 / 08 / 1969$ & 1200 & 36 & 105 & 16 & 7.7 & 9.8 & 9.6 & 6.3 & 3.2 & .9 \\
\hline 07/16/1969 & 0915 & 26 & 420 & 25 & 7.7 & 7.1 & 38 & 21 & 30 & 4.9 \\
\hline $10 / 29 / 1969$ & 1430 & 5.1 & 622 & 12 & 7.4 & 9.8 & 38 & 25 & 25 & 2.7 \\
\hline $01 / 27 / 1970$ & -- & 9 & 342 & 6 & 8.0 & 11.8 & 30 & 21 & 14 & 1.7 \\
\hline 04/30/1970 & -- & 9.2 & 143 & 18 & 7.3 & 8.8 & 10 & 7.3 & 5.9 & 1.5 \\
\hline 08/19/1970 & -- & 3.7 & 439 & 27 & 8.0 & 6.9 & 34 & 20 & 24 & 2.6 \\
\hline $11 / 02 / 1970$ & -- & 48 & 225 & 13 & 7.6 & 9.9 & 14 & 9.7 & 1.1 & .8 \\
\hline $01 / 26 / 1971$ & -- & 19 & 210 & 4 & 8.2 & 12.8 & 14 & 12 & .9 & .5 \\
\hline 04/21/1971 & -- & 12 & 225 & 18 & 8.0 & 8.9 & 16 & 12 & 8.6 & 1.5 \\
\hline 07/14/1971 & -- & 2.9 & 250 & 25 & 7.8 & 5.0 & 22 & 14 & 6.3 & 1.7 \\
\hline $11 / 09 / 1971$ & -- & 2 & 320 & 12 & 7.0 & 9.5 & 35 & 16 & 7.6 & 1.5 \\
\hline 02/01/1972 & -- & 7 & 250 & 5 & 7.0 & 12.5 & 18 & 13 & 7.5 & 1.6 \\
\hline
\end{tabular}


Table 1. Physical properties and concentrations of inorganic chemical constituents in water samples from upper Logan Creek, 1965-2006.-Continued

[All concentrations are dissolved and in milligrams per liter unless noted otherwise; $\mathrm{ft}^{3} / \mathrm{s}$, cubic feet per second; Cond, specific conductance in microsiemens per centimeter at $25 \mathrm{degrees}$ Celsius; Temp, temperature in degrees Celsius; USGS, U.S. Geological Survey; --, no data; <, less than; $\mathrm{CaCO}_{3}$, calcium carbonate; N, nitrogen; P, phosphorus; ND, not detected; E, estimated value; $\mu \mathrm{g} / \mathrm{L}$, micrograms per liter]

\begin{tabular}{|c|c|c|c|c|c|c|c|c|c|c|}
\hline Date & Time & $\begin{array}{c}\text { Discharge } \\
\left(\mathrm{ft}^{3} / \mathrm{s}\right)\end{array}$ & Cond & Temp & $\mathrm{pH}$ & $\begin{array}{c}\text { Dissolved } \\
\text { oxygen }\end{array}$ & Calcium & Magnesium & Sodium & Potassium \\
\hline \multicolumn{11}{|c|}{ Site 5, Logan Creek below Sweetwater Creek (USGS station number 07061840)—Continued } \\
\hline 06/06/1972 & -- & 4.2 & 240 & 27 & 7.2 & 8.2 & 16 & 12 & 7.5 & 1.8 \\
\hline 08/23/1972 & -- & 3.4 & 350 & 24 & 7.9 & 6.1 & 34 & 19 & 13 & 2.4 \\
\hline $10 / 17 / 1972$ & -- & 6.9 & 550 & 16 & 7.7 & 8.6 & 34 & 28 & 27 & 4.3 \\
\hline $01 / 31 / 1973$ & -- & 46 & 150 & 5 & 7.3 & 12.4 & 12 & 5.8 & 5.6 & 1.4 \\
\hline 05/15/1973 & -- & 39 & 102 & 19 & 7.5 & 8.7 & 9.6 & 5.8 & 2.6 & 1.2 \\
\hline 07/31/1973 & -- & 25 & 218 & 26 & 7.5 & 7.4 & 7.6 & 13 & 9.1 & 2.1 \\
\hline $11 / 27 / 1973$ & 1600 & 206 & 112 & 12 & 7.1 & 9.2 & 2.9 & 1.4 & 4.5 & 1.5 \\
\hline 03/05/1974 & 1805 & 39 & 128 & 13 & 7.5 & 9.9 & 10 & 7.7 & 6.1 & 1.5 \\
\hline 05/14/1974 & 1810 & 81 & 125 & 16 & 7.0 & 8.8 & 9.6 & 6.2 & 3.4 & 1.2 \\
\hline 08/13/1974 & -- & 2.4 & 280 & 26 & 8.2 & 8.3 & -- & -- & 8.1 & 2.2 \\
\hline $10 / 30 / 1974$ & 0740 & 7.1 & 330 & 15 & 7.5 & 8.7 & -- & -- & 11 & 1.9 \\
\hline 01/07/1975 & 1520 & 30 & 140 & 6 & 6.7 & 12.0 & -- & -- & 3.4 & 1.1 \\
\hline $04 / 22 / 1975$ & -- & 20 & 210 & 17 & 7.5 & 9.5 & -- & -- & 8.1 & 2 \\
\hline $07 / 29 / 1975$ & 1510 & 3 & 420 & 27 & 7.5 & 8.3 & -- & -- & 18 & 3.3 \\
\hline \multicolumn{11}{|c|}{ Site 6, Logan Creek at Latter Day Saints (LDS) Church (USGS station number 371754091073901) } \\
\hline $08 / 15 / 2006$ & 1140 & E1.0 & 398 & 26.6 & 8.3 & -- & 30.2 & 20.1 & 15.6 & 1.8 \\
\hline \multicolumn{11}{|c|}{ Site 7, Logan Creek at County Road 776 (USGS station number 371703091074201) } \\
\hline 03/07/2006 & 1355 & 3.72 & 384 & 9 & 7.9 & -- & 29.2 & 21.7 & 25.3 & 2.08 \\
\hline
\end{tabular}


Table 1. Physical properties and concentrations of inorganic chemical constituents in water samples from upper Logan Creek, 1965-2006-Continued.

[All concentrations are dissolved and in milligrams per liter unless noted otherwise; $\mathrm{ft}$ /s, cubic feet per second; Cond, specific conductance in microsiemens per centimeter at 25 degrees Celsius; Temp, temperature in degrees Celsius; USGS, U.S. Geological Survey; --, no data; <, less than; $\mathrm{CaCO}_{3}$, calcium carbonate; N, nitrogen; $\mathrm{P}$, phosphorus; ND, not detected; E, estimated value; $\mu \mathrm{g} / \mathrm{L}$, micrograms per liter]

\begin{tabular}{|c|c|c|c|c|c|c|c|c|c|}
\hline $\begin{array}{l}\text { Alkalinity, total } \\
\text { as } \mathrm{CaCO}_{3}\end{array}$ & $\begin{array}{l}\text { Bicarbonate } \\
\text { (total) }\end{array}$ & Sulfate & Chloride & Fluoride & Silica & $\begin{array}{l}\text { Nitrite plus } \\
\text { nitrate, total } \\
\text { as } \mathbf{N}\end{array}$ & $\begin{array}{c}\text { Ammonia, total, } \\
\text { as N }\end{array}$ & $\begin{array}{l}\text { Phosphorus, } \\
\text { dissolved as P }\end{array}$ & $\begin{array}{c}\text { Orthophosphorus, } \\
\text { as } \mathbf{P}\end{array}$ \\
\hline \multicolumn{10}{|c|}{ Site 1, Adair Creek at mouth (USGS station number 372128091072201) } \\
\hline${ }^{\mathrm{a}} 120$ & ${ }^{\mathrm{a}} 144$ & 114 & 49.1 & 0.5 & 2.7 & 0.82 & 0.14 & $<0.04$ & $<0.02$ \\
\hline \multicolumn{10}{|c|}{ Site 2, Logan Creek at State Route B (USGS station number 372026091071101) } \\
\hline 77 & 95 & 39 & 19 & 0.3 & 2.8 & -- & -- & $<0.01$ & -- \\
\hline a200 & a245 & 89.2 & 37.7 & .38 & 3.5 & 0.65 & 0.06 & $<.04$ & $<0.02$ \\
\hline \multicolumn{10}{|c|}{ Site 3, Logan Creek above Sweetwater Creek (USGS station number 07061810) } \\
\hline-- & -- & 5.4 & 1.6 & 0.1 & 6.7 & -- & 1.1 & -- & -- \\
\hline 48 & -- & 5.6 & 2.6 & .2 & 5.0 & -- & 1.4 & -- & -- \\
\hline 80 & -- & 4.6 & 2.4 & .1 & 7.4 & -- & .9 & -- & -- \\
\hline 84 & -- & 4.0 & 1.6 & ND & 6.2 & -- & -- & -- & -- \\
\hline 94 & -- & 3.2 & 1.7 & 1.6 & 6.3 & -- & -- & -- & -- \\
\hline 14 & -- & 5.4 & .8 & .2 & 4.5 & -- & -- & -- & -- \\
\hline 92 & -- & 3.4 & 1.3 & .3 & 6.6 & -- & -- & -- & -- \\
\hline 134 & -- & 2.6 & 1.1 & ND & 6.8 & -- & -- & -- & -- \\
\hline 48 & -- & 5.0 & 1.4 & ND & 4.9 & -- & -- & -- & -- \\
\hline 6 & -- & 9.6 & .5 & ND & 5.8 & -- & -- & -- & -- \\
\hline -- & -- & 3.2 & 1.4 & ND & 6.8 & -- & -- & -- & -- \\
\hline 10 & -- & 6 & .1 & .1 & 5.0 & -- & .1 & -- & -- \\
\hline 26 & -- & 10 & .5 & .1 & 6.0 & -- & .3 & -- & -- \\
\hline 12 & -- & 10 & 1.5 & .1 & 0.8 & -- & -- & -- & -- \\
\hline 46 & -- & 10 & 7.4 & .1 & 1.0 & -- & -- & -- & -- \\
\hline 118 & -- & $<10$ & -- & -- & 9.6 & -- & -- & -- & -- \\
\hline 22 & -- & $<10$ & 4.5 & $<.1$ & -- & -- & -- & -- & -- \\
\hline 32 & -- & $<10$ & -- & -- & 7.0 & -- & -- & -- & -- \\
\hline 72 & -- & 5.0 & 5.0 & .1 & 8.6 & -- & -- & -- & -- \\
\hline 86 & -- & $<10$ & 3.4 & .1 & 8.1 & -- & -- & -- & -- \\
\hline 7 & -- & $<10$ & 4.0 & .1 & 6.2 & -- & -- & -- & -- \\
\hline 10 & -- & 11 & 1.7 & .28 & 5.8 & -- & -- & -- & -- \\
\hline
\end{tabular}


Table 1. Physical properties and concentrations of inorganic chemical constituents in water samples from upper Logan Creek, 1965-2006-Continued.

[All concentrations are dissolved and in milligrams per liter unless noted otherwise; $\mathrm{ft}^{3} / \mathrm{s}$, cubic feet per second; Cond, specific conductance in microsiemens per centimeter at 25 degrees Celsius; Temp, temperature in degrees Celsius; USGS, U.S. Geological Survey; --, no data; <, less than; $\mathrm{CaCO}_{3}$, calcium carbonate; N, nitrogen; P, phosphorus; ND, not detected; E, estimated value; $\mu \mathrm{g} / \mathrm{L}$, micrograms per liter]

\begin{tabular}{|c|c|c|c|c|c|c|c|c|c|}
\hline $\begin{array}{l}\text { Alkalinity, total } \\
\text { as } \mathrm{CaCO}_{3}\end{array}$ & $\begin{array}{c}\text { Bicarbonate } \\
\text { (total) }\end{array}$ & Sulfate & Chloride & Fluoride & Silica & $\begin{array}{c}\text { Nitrite plus } \\
\text { nitrate, total } \\
\text { as } \mathbf{N}\end{array}$ & $\begin{array}{c}\text { Ammonia, total, } \\
\text { as N }\end{array}$ & $\begin{array}{l}\text { Phosphorus, } \\
\text { dissolved as P }\end{array}$ & $\begin{array}{c}\text { Orthophosphorus, } \\
\text { as } \mathrm{P}\end{array}$ \\
\hline \multicolumn{10}{|c|}{ Site 3, Logan Creek above Sweetwater Creek (USGS station number 07061810)—Continued } \\
\hline 16 & -- & $<10$ & 0.4 & 0.1 & 6.9 & -- & -- & -- & -- \\
\hline 6 & -- & 3.8 & -- & .03 & -- & -- & -- & -- & -- \\
\hline 8 & -- & 9.0 & 9.7 & .15 & -- & -- & -- & -- & -- \\
\hline 6 & -- & 8.0 & 3.1 & .04 & -- & -- & -- & -- & -- \\
\hline 92 & -- & 7.0 & 4.0 & -- & -- & -- & -- & -- & -- \\
\hline 40 & -- & 7.0 & 32 & -- & -- & -- & -- & -- & -- \\
\hline 10 & -- & 4.0 & 10 & -- & -- & -- & -- & -- & -- \\
\hline 22 & -- & 5.0 & 2.3 & -- & -- & -- & -- & -- & -- \\
\hline 102 & -- & 4.0 & 3.1 & -- & -- & -- & -- & -- & -- \\
\hline \multicolumn{10}{|c|}{ Site 5, Logan Creek below Sweetwater Creek (USGS station number 07061840) } \\
\hline 46 & -- & 5.8 & 1.9 & 0.1 & 6.6 & -- & 1.2 & -- & -- \\
\hline 57 & -- & 9.6 & 5.4 & .4 & 5.0 & -- & 1.4 & -- & -- \\
\hline 93 & -- & 10 & 8.4 & .5 & 7.0 & -- & .8 & -- & -- \\
\hline 103 & -- & 13 & 7.7 & .4 & 5.7 & -- & -- & -- & -- \\
\hline 110 & -- & 14 & 6.2 & .2 & 7.9 & -- & -- & -- & -- \\
\hline 44 & -- & 10 & 2.9 & .2 & 4.4 & -- & -- & -- & -- \\
\hline 36 & -- & 10 & 3.2 & .1 & 4.4 & -- & -- & -- & -- \\
\hline 147 & -- & 81 & 31 & 6 & 4.5 & -- & -- & -- & -- \\
\hline 132 & -- & 69 & 29 & ND & 8.7 & -- & -- & -- & -- \\
\hline 90 & -- & 51 & 18 & .2 & 3.7 & -- & -- & -- & -- \\
\hline 40 & -- & 21 & 5.6 & ND & 6.2 & -- & -- & -- & -- \\
\hline 218 & -- & 70 & 27 & .3 & 5.9 & -- & -- & -- & -- \\
\hline 50 & -- & 30 & 12 & .1 & 4.0 & -- & .1 & -- & -- \\
\hline 58 & -- & 21 & 9.0 & .2 & 5.0 & -- & .6 & -- & -- \\
\hline 66 & -- & 23 & 10 & .1 & 0.6 & -- & -- & -- & -- \\
\hline 100 & -- & 10 & 19 & ND & 1.2 & -- & -- & -- & -- \\
\hline 122 & -- & 12 & -- & -- & 7.5 & -- & -- & -- & -- \\
\hline 72 & -- & 18 & 20 & .1 & -- & -- & -- & -- & -- \\
\hline
\end{tabular}


Table 1. Physical properties and concentrations of inorganic chemical constituents in water samples from upper Logan Creek, 1965-2006-Continued.

[All concentrations are dissolved and in milligrams per liter unless noted otherwise; $\mathrm{ft}$ /s, cubic feet per second; Cond, specific conductance in microsiemens per centimeter at 25 degrees Celsius; Temp, temperature in degrees Celsius; USGS, U.S. Geological Survey; --, no data; <, less than; $\mathrm{CaCO}_{3}$, calcium carbonate; N, nitrogen; P, phosphorus; ND, not detected; E, estimated value; $\mu \mathrm{g} / \mathrm{L}$, micrograms per liter]

\begin{tabular}{|c|c|c|c|c|c|c|c|c|c|}
\hline $\begin{array}{l}\text { Alkalinity, total } \\
\text { as } \mathrm{CaCO}_{3}\end{array}$ & $\begin{array}{c}\text { Bicarbonate } \\
\text { (total) }\end{array}$ & Sulfate & Chloride & Fluoride & Silica & $\begin{array}{l}\text { Nitrite plus } \\
\text { nitrate, total } \\
\text { as } \mathbf{N}\end{array}$ & $\begin{array}{c}\text { Ammonia, total, } \\
\text { as N }\end{array}$ & $\begin{array}{l}\text { Phosphorus, } \\
\text { dissolved as P }\end{array}$ & $\begin{array}{c}\text { Orthophosphorus, } \\
\text { as } \mathrm{P}\end{array}$ \\
\hline \multicolumn{10}{|c|}{ Site 5, Logan Creek below Sweetwater Creek (USGS station number 07061840)—Continued } \\
\hline 80 & -- & 22 & -- & -- & 6.4 & -- & -- & -- & -- \\
\hline 124 & -- & 26 & 12 & .9 & 8.6 & -- & -- & -- & -- \\
\hline 120 & -- & 66 & 16 & $<.10$ & 6.4 & -- & -- & -- & -- \\
\hline 34 & -- & 12 & 15 & .1 & 6.0 & -- & -- & -- & -- \\
\hline 44 & -- & $<10$ & 2.9 & .34 & 6.1 & -- & -- & -- & -- \\
\hline 62 & -- & 28 & 1.7 & .1 & 5.6 & -- & -- & -- & -- \\
\hline 26 & -- & 11.2 & -- & .03 & -- & -- & -- & -- & -- \\
\hline 54 & -- & 16 & 16 & .14 & -- & -- & -- & -- & -- \\
\hline 36 & -- & 12 & 9.2 & .18 & -- & -- & -- & -- & -- \\
\hline 100 & -- & 27 & 15 & -- & -- & -- & -- & -- & -- \\
\hline 100 & -- & 44 & 31 & -- & -- & -- & -- & -- & -- \\
\hline 46 & -- & 11 & 10 & -- & -- & -- & -- & -- & -- \\
\hline 50 & -- & 24 & 11 & -- & -- & -- & -- & -- & -- \\
\hline 114 & -- & 82 & 27 & -- & -- & -- & -- & -- & -- \\
\hline \multicolumn{10}{|c|}{ Site 6, Logan Creek at Latter Day Saints (LDS) Church (USGS station number 371754091073901) } \\
\hline 121 & ${ }^{\mathrm{a}} 245$ & 42.4 & 18.5 & 0.2 & 7.0 & E0.03 & $<0.01$ & $<0.04$ & E0.004 \\
\hline \multicolumn{10}{|c|}{ Site 7, Logan Creek at County Road 776 (USGS station number 371703091074201 ) } \\
\hline${ }^{\mathrm{a}} 120$ & ${ }^{\mathrm{a}} 141$ & 73.5 & 31.4 & 0.27 & 3.7 & 0.54 & $<0.04$ & $<0.04$ & $<0.02$ \\
\hline
\end{tabular}


Table 1. Physical properties and concentrations of inorganic chemical constituents in water samples from upper Logan Creek, 1965-2006-Continued.

[All concentrations are dissolved and in milligrams per liter unless noted otherwise; $\mathrm{ft}^{3} / \mathrm{s}$, cubic feet per second; Cond, specific conductance in microsiemens per centimeter at 25 degrees Celsius; Temp, temperature in degrees Celsius; USGS, U.S. Geological Survey; --, no data; <, less than; $\mathrm{CaCO}_{3}$, calcium carbonate; N, nitrogen; $\mathrm{P}$, phosphorus; ND, not detected; E, estimated value; $\mu \mathrm{g} / \mathrm{L}$, micrograms per liter]

\begin{tabular}{|c|c|c|c|c|c|c|c|c|c|c|c|}
\hline $\begin{array}{c}\text { Antimony } \\
(\mu \mathrm{g} / \mathrm{L})\end{array}$ & $\begin{array}{c}\text { Arsenic } \\
(\mu \mathrm{g} / \mathrm{L})\end{array}$ & $\begin{array}{c}\text { Barium } \\
\text { ( } \mu \mathrm{g} / \mathrm{L})\end{array}$ & $\begin{array}{l}\text { Beryllium } \\
\text { ( } \mu \mathrm{g} / \mathrm{L})\end{array}$ & $\begin{array}{l}\text { Boron } \\
(\mu \mathrm{g} / \mathrm{L})\end{array}$ & $\begin{array}{c}\text { Cadmium } \\
(\mu \mathrm{g} / \mathrm{L})\end{array}$ & $\begin{array}{c}\text { Chromium } \\
(\mu \mathrm{g} / \mathrm{L})\end{array}$ & $\begin{array}{c}\text { Cobalt } \\
\text { ( } \mu \mathrm{g} / \mathrm{L})\end{array}$ & $\begin{array}{c}\text { Copper } \\
\text { ( } \mu \mathrm{g} / \mathrm{L})\end{array}$ & $\begin{array}{l}\text { Iron } \\
(\mu g / L)\end{array}$ & $\begin{array}{l}\text { Lead } \\
(\mu \mathrm{g} / \mathrm{L})\end{array}$ & $\begin{array}{c}\text { Lithium } \\
\text { ( } \mu \mathrm{g} / \mathrm{L})\end{array}$ \\
\hline \multicolumn{12}{|c|}{ Site 1, Adair Creek at mouth (USGS station number 372128091072201) } \\
\hline 0.34 & 0.87 & 52 & $<0.2$ & 125 & 0.22 & 0.04 & 2 & 3.2 & 180 & 11.7 & 22 \\
\hline \multicolumn{12}{|c|}{ Site 2, Logan Creek at State Route B (USGS station number 372026091071101) } \\
\hline$<0.2$ & $<1.0$ & 50.3 & $<0.05$ & -- & $<2$ & $<20$ & $<2$ & $<10$ & $<50$ & $<2$ & $<6$ \\
\hline .23 & .54 & 46.3 & E.1 & 95 & .1 & .1 & 1 & 2.5 & 131 & 7.18 & 15 \\
\hline \multicolumn{12}{|c|}{ Site 3, Logan Creek above Sweetwater Creek (USGS station number 07061810) } \\
\hline-- & -- & -- & -- & -- & -- & -- & -- & -- & -- & -- & -- \\
\hline-- & -- & -- & -- & -- & -- & -- & -- & -- & -- & -- & -- \\
\hline-- & -- & -- & -- & -- & -- & -- & -- & -- & -- & -- & -- \\
\hline -- & -- & -- & -- & -- & -- & -- & -- & -- & -- & -- & -- \\
\hline-- & -- & ND & -- & ND & ND & ND & ND & ND & -- & ND & 40 \\
\hline-- & -- & 60 & -- & ${ }^{\mathrm{b}} 1,000$ & -- & -- & -- & -- & -- & -- & ND \\
\hline-- & -- & $\mathrm{ND}$ & -- & ND & -- & 10 & ND & ND & -- & -- & ND \\
\hline-- & -- & ND & -- & 50 & $<2$ & $<20$ & ND & $<10$ & 170 & ND & ND \\
\hline -- & -- & 1,000 & -- & 30 & $<2$ & $<20$ & ND & 10 & 50 & $<2$ & $<6$ \\
\hline-- & -- & 140 & -- & ND & $<2$ & $<20$ & 40 & $<10$ & 40 & $<2$ & ND \\
\hline-- & -- & 800 & -- & 20 & ND & $<20$ & ND & 10 & 50 & ND & ND \\
\hline-- & -- & ND & -- & -- & ND & 20 & $<2$ & 20 & 120 & ND & -- \\
\hline-- & -- & ND & -- & -- & $\mathrm{ND}$ & $<20$ & $<2$ & $<10$ & 100 & ND & -- \\
\hline-- & -- & ND & -- & -- & ND & ND & ND & ND & ND & $<2$ & -- \\
\hline -- & -- & ND & -- & -- & $\mathrm{ND}$ & $<20$ & $<2$ & $<10$ & 60 & $<2$ & -- \\
\hline-- & -- & ND & -- & -- & ND & ND & ND & $<10$ & 110 & ND & -- \\
\hline -- & -- & ND & -- & -- & ND & ND & ND & $<10$ & 20 & $<2$ & -- \\
\hline-- & -- & ND & -- & -- & ND & ND & ND & -- & 40 & $<2$ & -- \\
\hline-- & -- & 200 & -- & -- & $<2$ & ND & $<2$ & $<10$ & 80 & $<2$ & -- \\
\hline-- & -- & $<100$ & -- & -- & ND & ND & $<2$ & $<10$ & 40 & ND & -- \\
\hline-- & -- & $<100$ & -- & -- & ND & $<20$ & $<2$ & $<10$ & $<10$ & ND & -- \\
\hline-- & -- & 200 & -- & -- & ND & $<20$ & $<2$ & $<10$ & $<10$ & ND & -- \\
\hline
\end{tabular}


Table 1. Physical properties and concentrations of inorganic chemical constituents in water samples from upper Logan Creek, 1965-2006-Continued.

[All concentrations are dissolved and in milligrams per liter unless noted otherwise; $\mathrm{ft}^{3} / \mathrm{s}$, cubic feet per second; Cond, specific conductance in microsiemens per centimeter at 25 degrees Celsius; Temp, temperature in degrees Celsius; USGS, U.S. Geological Survey; --, no data; <, less than; $\mathrm{CaCO}_{3}$, calcium carbonate; N, nitrogen; P, phosphorus; ND, not detected; E, estimated value; $\mu \mathrm{g} / \mathrm{L}$, micrograms per liter]

\begin{tabular}{|c|c|c|c|c|c|c|c|c|c|c|c|}
\hline $\begin{array}{c}\text { Antimony } \\
(\mu \mathrm{g} / \mathrm{L})\end{array}$ & $\begin{array}{c}\text { Arsenic } \\
(\mu \mathrm{g} / \mathrm{L})\end{array}$ & $\begin{array}{c}\text { Barium } \\
\text { ( } \mu \mathrm{g} / \mathrm{L})\end{array}$ & $\begin{array}{c}\text { Beryllium } \\
(\mu \mathrm{g} / \mathrm{L})\end{array}$ & $\begin{array}{l}\text { Boron } \\
\text { ( } \mu \mathrm{g} / \mathrm{L})\end{array}$ & $\begin{array}{c}\text { Cadmium } \\
\text { ( } \mu \mathrm{g} / \mathrm{L})\end{array}$ & $\begin{array}{c}\text { Chromium } \\
(\mu \mathrm{g} / \mathrm{L})\end{array}$ & $\begin{array}{c}\text { Cobalt } \\
(\mu \mathrm{g} / \mathrm{L})\end{array}$ & $\begin{array}{c}\text { Copper } \\
(\mu \mathrm{g} / \mathrm{L})\end{array}$ & $\begin{array}{c}\text { Iron } \\
\text { ( } \mu \mathrm{g} / \mathrm{L})\end{array}$ & $\begin{array}{l}\text { Lead } \\
(\mu \mathrm{g} / \mathrm{L})\end{array}$ & $\begin{array}{c}\text { Lithium } \\
\text { ( } \mu \mathrm{g} / \mathrm{L})\end{array}$ \\
\hline \multicolumn{12}{|c|}{ Site 3, Logan Creek above Sweetwater Creek (USGS station number 07061810)—Continued } \\
\hline -- & -- & $<100$ & -- & -- & ND & ND & $<2$ & $<10$ & 70 & $<2$ & -- \\
\hline -- & -- & $<100$ & -- & -- & ND & ND & ND & $<10$ & 30 & $<2$ & -- \\
\hline -- & -- & $<100$ & -- & -- & ND & ND & $<2$ & 10 & $<10$ & $<2$ & -- \\
\hline-- & -- & $<100$ & -- & -- & ND & ND & ND & ND & 40 & $<2$ & -- \\
\hline-- & -- & -- & -- & -- & $<2$ & -- & -- & $<10$ & -- & $<2$ & -- \\
\hline-- & -- & -- & -- & -- & ND & -- & -- & ND & -- & $<2$ & -- \\
\hline-- & -- & -- & -- & -- & $<2$ & -- & -- & $<2$ & -- & $<2$ & -- \\
\hline-- & -- & -- & -- & -- & ND & -- & -- & $<2$ & -- & $<2$ & -- \\
\hline-- & -- & -- & -- & -- & $<2$ & -- & -- & $<2$ & -- & $<2$ & -- \\
\hline
\end{tabular}

Site 5, Logan Creek below Sweetwater Creek (USGS station number 07061840)

\begin{tabular}{|c|c|c|c|c|c|c|c|c|c|c|c|}
\hline -- & -- & 30 & ND & 30 & -- & ND & ND & $\mathrm{ND}$ & -- & ND & -- \\
\hline -- & -- & 35 & -- & 40 & -- & ND & $\mathrm{ND}$ & $<10$ & -- & $<2$ & $<6$ \\
\hline-- & -- & -- & -- & -- & -- & -- & -- & -- & -- & -- & -- \\
\hline-- & -- & 60 & -- & 70 & -- & ND & ND & ND & ND & ND & ND \\
\hline -- & -- & ND & -- & ND & $\mathrm{ND}$ & ND & $\mathrm{ND}$ & $\mathrm{ND}$ & -- & $<2$ & 30 \\
\hline -- & -- & ND & -- & ND & $\mathrm{ND}$ & ND & $\mathrm{ND}$ & ND & -- & ND & ND \\
\hline -- & -- & ND & -- & ND & $\mathrm{ND}$ & 20 & $\mathrm{ND}$ & $\mathrm{ND}$ & -- & ND & $\mathrm{ND}$ \\
\hline-- & -- & ND & -- & 170 & $\mathrm{ND}$ & ND & ND & ND & -- & ND & ND \\
\hline -- & -- & ND & -- & 70 & $<2$ & $<20$ & $\mathrm{ND}$ & 10 & 20 & ND & ND \\
\hline-- & -- & ND & -- & 40 & $<2$ & $<20$ & ND & $<10$ & 20 & $<2$ & $<6$ \\
\hline -- & -- & ND & -- & 10 & $<2$ & ND & 20 & $<10$ & 40 & ND & ND \\
\hline -- & -- & ND & -- & 90 & ND & $<20$ & ND & $<10$ & 20 & ND & $\mathrm{ND}$ \\
\hline-- & -- & ND & -- & -- & $<2$ & 10 & $<2$ & $<10$ & 380 & $<2$ & -- \\
\hline-- & -- & 300 & -- & -- & $\mathrm{ND}$ & $<20$ & $\mathrm{ND}$ & $<10$ & 120 & ND & -- \\
\hline -- & -- & ND & -- & -- & $\mathrm{ND}$ & ND & $\mathrm{ND}$ & ND & ND & ND & -- \\
\hline -- & -- & 1,700 & -- & -- & $\mathrm{ND}$ & $<20$ & $<2$ & $<10$ & 20 & ND & -- \\
\hline-- & -- & 3,300 & -- & -- & ND & $<20$ & ND & $<10$ & ND & ND & -- \\
\hline -- & -- & ND & -- & -- & $<2$ & $<20$ & $\mathrm{ND}$ & $<10$ & ND & $<2$ & -- \\
\hline
\end{tabular}


Table 1. Physical properties and concentrations of inorganic chemical constituents in water samples from upper Logan Creek, 1965-2006-Continued.

[All concentrations are dissolved and in milligrams per liter unless noted otherwise; $\mathrm{ft}^{3} / \mathrm{s}$, cubic feet per second; Cond, specific conductance in microsiemens per centimeter at 25 degrees Celsius; Temp, temperature in degrees Celsius; USGS, U.S. Geological Survey; --, no data; <, less than; $\mathrm{CaCO}_{3}$, calcium carbonate; N, nitrogen; P, phosphorus; ND, not detected; E, estimated value; $\mu \mathrm{g} / \mathrm{L}$, micrograms per liter]

\begin{tabular}{|c|c|c|c|c|c|c|c|c|c|c|c|}
\hline $\begin{array}{c}\text { Antimony } \\
(\mu \mathrm{g} / \mathrm{L})\end{array}$ & $\begin{array}{c}\text { Arsenic } \\
\text { ( } \mu \mathrm{g} / \mathrm{L})\end{array}$ & $\begin{array}{c}\text { Barium } \\
\text { ( } \mu \mathrm{g} / \mathrm{L})\end{array}$ & $\begin{array}{c}\text { Beryllium } \\
(\mu \mathrm{g} / \mathrm{L})\end{array}$ & $\begin{array}{l}\text { Boron } \\
\text { ( } \mu \mathrm{g} / \mathrm{L})\end{array}$ & $\begin{array}{c}\text { Cadmium } \\
(\mu \mathrm{g} / \mathrm{L})\end{array}$ & $\begin{array}{c}\text { Chromium } \\
(\mu \mathrm{g} / \mathrm{L})\end{array}$ & $\begin{array}{l}\text { Cobalt } \\
(\mu \mathrm{g} / \mathrm{L})\end{array}$ & $\begin{array}{c}\text { Copper } \\
(\mu \mathrm{g} / \mathrm{L})\end{array}$ & $\begin{array}{l}\text { Iron } \\
(\mu \mathrm{g} / \mathrm{L})\end{array}$ & $\begin{array}{l}\text { Lead } \\
\text { ( } \mu \mathrm{g} / \mathrm{L})\end{array}$ & $\begin{array}{c}\text { Lithium } \\
\text { ( } \mu \mathrm{g} / \mathrm{L} \text { ) }\end{array}$ \\
\hline \multicolumn{12}{|c|}{ Site 5, Logan Creek below Sweetwater Creek (USGS station number 07061840)—Continued } \\
\hline-- & -- & ND & -- & -- & ND & ND & ND & -- & ND & $<2$ & -- \\
\hline-- & -- & ND & -- & -- & $<2$ & ND & $<2$ & $<10$ & 20 & $<2$ & -- \\
\hline-- & -- & $<100$ & -- & -- & ND & ND & ND & $<10$ & $<10$ & $<2$ & -- \\
\hline -- & -- & $<100$ & -- & -- & ND & $<20$ & $<2$ & $<20$ & 20 & $<2$ & -- \\
\hline-- & -- & $<100$ & -- & -- & ND & $<20$ & $<2$ & $<10$ & $<10$ & ND & -- \\
\hline -- & -- & $<100$ & -- & -- & ND & ND & ND & $<10$ & 50 & $<2$ & -- \\
\hline-- & -- & $<100$ & -- & -- & ND & ND & ND & 20 & 40 & $<2$ & -- \\
\hline -- & -- & $<100$ & -- & -- & $\mathrm{ND}$ & ND & $<2$ & 10 & 30 & $<2$ & -- \\
\hline-- & -- & $<100$ & -- & -- & $<2$ & ND & ND & $<10$ & 50 & $<2$ & -- \\
\hline -- & -- & -- & -- & -- & ND & -- & -- & $<10$ & -- & $<2$ & -- \\
\hline-- & -- & -- & -- & -- & ND & -- & -- & ND & -- & $<2$ & -- \\
\hline-- & -- & -- & -- & -- & ND & -- & -- & $<10$ & -- & $<2$ & -- \\
\hline-- & -- & -- & -- & -- & ND & -- & -- & $<10$ & -- & $<2$ & -- \\
\hline-- & -- & -- & -- & -- & $<2$ & -- & -- & $<10$ & -- & $<2$ & -- \\
\hline \multicolumn{12}{|c|}{ Site 6, Logan Creek at Latter Day Saints (LDS) Church (USGS station number 371754091073901) } \\
\hline$<0.20$ & 0.32 & 58.5 & $<0.2$ & 62 & E0.03 & 0.05 & E0.03 & E0.37 & $<6$ & 0.09 & 4 \\
\hline \multicolumn{12}{|c|}{ Site 7, Logan Creek at County Road 776 (USGS station number 371703091074201) } \\
\hline E0.13 & 0.18 & 49.2 & E0.2 & 72 & 0.06 & 0.07 & 0.3 & 1.5 & $<6$ & 0.17 & 9 \\
\hline
\end{tabular}


Table 1. Physical properties and concentrations of inorganic chemical constituents in water samples from upper Logan Creek, 1965-2006-Continued.

[ft³/s, cubic feet per second; Cond, specific conductance in microsiemens per centimeter at 25 degrees Celsius; Temp, temperature in degrees Celsius; --, no data; <, less than; $\mathrm{E}$, estimated value; CaCO, calcium carbonate; N, Nitrogen; P, phosphorus; $\mu \mathrm{g} / \mathrm{L}$, micrograms per liter; $\mathrm{Ca}: \mathrm{Mg}$, molar ratio of calcium to magnesium; ND, not detected; all concentrations are dissolved and in milligrams per liter unless noted otherwise]

\begin{tabular}{|c|c|c|c|c|c|c|c|c|}
\hline $\begin{array}{c}\text { Manganese } \\
(\mu \mathrm{g} / \mathrm{L})\end{array}$ & $\begin{array}{l}\text { Molybdenum } \\
(\mu \mathrm{g} / \mathrm{L})\end{array}$ & $\begin{array}{l}\text { Nickel } \\
\text { ( } \mu \mathrm{g} / \mathrm{L})\end{array}$ & $\begin{array}{l}\text { Selenium } \\
(\mu \mathrm{g} / \mathrm{L})\end{array}$ & $\begin{array}{l}\text { Silver } \\
(\mu \mathrm{g} / \mathrm{L})\end{array}$ & $\begin{array}{l}\text { Strontium } \\
(\mu \mathrm{g} / \mathrm{L})\end{array}$ & $\begin{array}{l}\text { Thallium } \\
\text { ( } \mu \mathrm{g} / \mathrm{L})\end{array}$ & $\begin{array}{l}\text { Vanadium } \\
(\mu \mathrm{g} / \mathrm{L})\end{array}$ & $\begin{array}{l}\text { Zinc } \\
\text { ( } \mu g / L)\end{array}$ \\
\hline \multicolumn{9}{|c|}{ Site 1, Adair Creek at mouth (USGS station number 372128091072201) } \\
\hline 330 & 5.2 & 8.5 & E0.05 & $<0.2$ & 168 & E0.03 & $<2$ & 34 \\
\hline \multicolumn{9}{|c|}{ Site 2, Logan Creek at State Route B (USGS station number 372026091071101) } \\
\hline 260 & 2 & $<2$ & $<1$ & $<3$ & 80 & $<0.1$ & 0.4 & 20 \\
\hline 138 & 3.6 & 4.97 & .09 & $<.2$ & 135 & E.02 & $<2$ & 21 \\
\hline \multicolumn{9}{|c|}{ Site 3, Logan Creek above Sweetwater Creek (USGS station number 07061810) } \\
\hline ND & -- & -- & -- & -- & -- & -- & -- & -- \\
\hline ND & -- & -- & -- & -- & -- & -- & -- & -- \\
\hline ND & -- & -- & -- & -- & -- & -- & -- & -- \\
\hline -- & -- & -- & -- & -- & -- & -- & -- & -- \\
\hline 30 & -- & $<2$ & -- & -- & 70 & -- & -- & 10 \\
\hline -- & -- & -- & -- & -- & -- & -- & -- & -- \\
\hline 40 & -- & -- & -- & -- & 100 & -- & -- & ND \\
\hline 100 & -- & $<2$ & -- & 30 & 70 & -- & -- & 10 \\
\hline 20 & -- & $<2$ & -- & ND & 70 & -- & -- & 10 \\
\hline$<10$ & -- & $<2$ & -- & 7 & 70 & -- & -- & 10 \\
\hline 60 & -- & ND & -- & ND & -- & -- & -- & 10 \\
\hline 80 & -- & $<2$ & -- & 2 & -- & -- & -- & 20 \\
\hline 10 & -- & ND & -- & ND & -- & -- & -- & 30 \\
\hline 10 & -- & $<2$ & -- & ND & -- & -- & -- & ND \\
\hline 60 & -- & $<2$ & -- & 1 & -- & -- & -- & 30 \\
\hline 40 & -- & $<2$ & -- & ND & -- & -- & -- & ND \\
\hline 10 & -- & $<2$ & -- & 4 & -- & -- & -- & ND \\
\hline 50 & -- & $<2$ & -- & ND & -- & -- & -- & c90 \\
\hline 50 & -- & $<2$ & -- & $\mathrm{ND}$ & -- & -- & -- & ND \\
\hline 30 & -- & $<2$ & -- & $<2$ & -- & -- & -- & ${ }^{\mathrm{c}} 60$ \\
\hline$<10$ & -- & $\mathrm{ND}$ & -- & $\mathrm{ND}$ & -- & -- & -- & $<20$ \\
\hline$<10$ & -- & $<2$ & -- & ND & -- & -- & -- & ND \\
\hline
\end{tabular}


Table 1. Physical properties and concentrations of inorganic chemical constituents in water samples from upper Logan Creek, 1965-2006-Continued.

[ft $3 / \mathrm{s}$, cubic feet per second; Cond, specific conductance in microsiemens per centimeter at 25 degrees Celsius; Temp, temperature in degrees Celsius; --, no data; <, less than; E, estimated value; $\mathrm{CaCO}$, calcium carbonate; N, Nitrogen; P, phosphorus; $\mu \mathrm{g} / \mathrm{L}$, micrograms per liter; Ca:Mg, molar ratio of calcium to magnesium; ND, not detected; all concentrations are dissolved and in milligrams per liter unless noted otherwise]

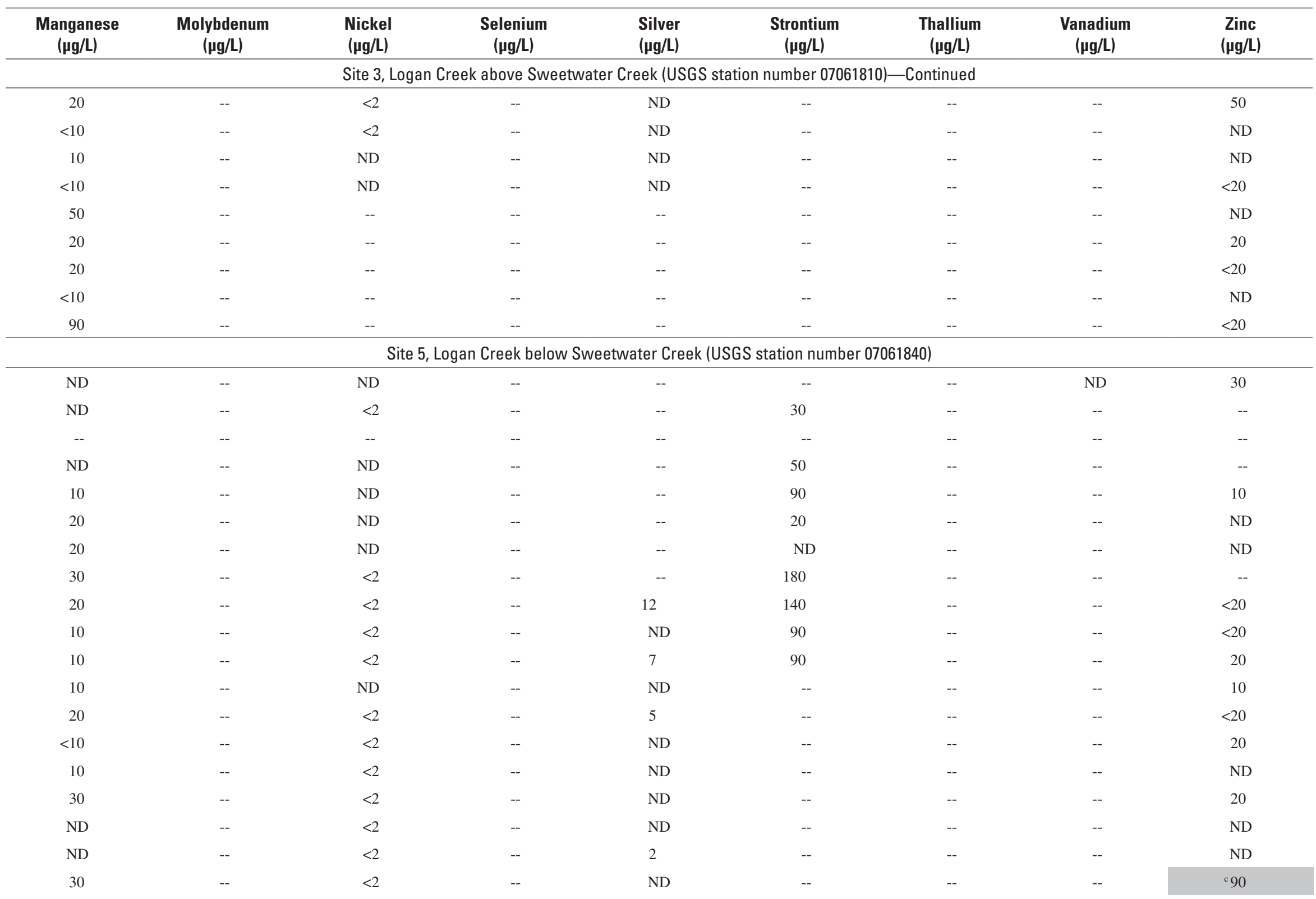


Table 1. Physical properties and concentrations of inorganic chemical constituents in water samples from upper Logan Creek, 1965-2006-Continued.

[ft³/s, cubic feet per second; Cond, specific conductance in microsiemens per centimeter at 25 degrees Celsius; Temp, temperature in degrees Celsius; --, no data; <, less than; $\mathrm{E}$, estimated value; $\mathrm{CaCO}_{3}$, calcium carbonate; N, Nitrogen; P, phosphorus; $\mu \mathrm{g} / \mathrm{L}$, micrograms per liter; $\mathrm{Ca}: \mathrm{Mg}$, molar ratio of calcium to magnesium; ND, not detected; all concentrations are dissolved and in milligrams per liter unless noted otherwise]

\begin{tabular}{|c|c|c|c|c|c|c|c|c|}
\hline $\begin{array}{c}\text { Manganese } \\
(\mu \mathrm{g} / \mathrm{L})\end{array}$ & $\begin{array}{l}\text { Molybdenum } \\
(\mu \mathrm{g} / \mathrm{L})\end{array}$ & $\begin{array}{l}\text { Nickel } \\
(\mu \mathrm{g} / \mathrm{L})\end{array}$ & $\begin{array}{l}\text { Selenium } \\
(\mu \mathrm{g} / \mathrm{L})\end{array}$ & $\begin{array}{l}\text { Silver } \\
(\mu \mathrm{g} / \mathrm{L})\end{array}$ & $\begin{array}{l}\text { Strontium } \\
(\mu \mathrm{g} / \mathrm{L})\end{array}$ & $\begin{array}{l}\text { Thallium } \\
\text { ( } \mu \mathrm{g} / \mathrm{L})\end{array}$ & $\begin{array}{l}\text { Vanadium } \\
(\mu \mathrm{g} / \mathrm{L})\end{array}$ & $\begin{array}{l}\text { Zinc } \\
\text { ( } \mu g / L)\end{array}$ \\
\hline \multicolumn{9}{|c|}{ Site 5, Logan Creek below Sweetwater Creek (USGS station number 07061840)—Continued } \\
\hline 30 & -- & ND & -- & ND & -- & -- & -- & ND \\
\hline 40 & -- & $<2$ & -- & $<2$ & -- & -- & -- & ${ }^{\mathrm{c}} 60$ \\
\hline 120 & -- & $<2$ & -- & ND & -- & -- & -- & 50 \\
\hline 40 & -- & $<2$ & -- & ND & -- & -- & -- & $<20$ \\
\hline 40 & -- & $<2$ & -- & ND & -- & -- & -- & 20 \\
\hline 80 & -- & $<2$ & -- & ND & -- & -- & -- & $<20$ \\
\hline 130 & -- & $<2$ & -- & ND & -- & -- & -- & 30 \\
\hline 50 & -- & $<2$ & -- & ND & -- & -- & -- & 20 \\
\hline 20 & -- & -- & -- & -- & -- & -- & -- & ND \\
\hline$<10$ & -- & -- & -- & -- & -- & -- & -- & 30 \\
\hline 20 & -- & -- & -- & -- & -- & -- & -- & ND \\
\hline 160 & -- & -- & -- & -- & -- & -- & -- & ND \\
\hline 20 & -- & -- & -- & -- & -- & -- & -- & ND \\
\hline \multicolumn{9}{|c|}{ Site 6, Logan Creek at Latter Day Saints (LDS) Church (USGS station number 371754091073901) } \\
\hline 1.6 & 1.8 & 0.14 & E0.07 & $<0.2$ & 102 & $<0.04$ & $<2$ & 1.2 \\
\hline \multicolumn{9}{|c|}{ Site 7, Logan Creek at County Road 776 (USGS station number 371703091074201 ) } \\
\hline 2.7 & 2.2 & 1.44 & 0.08 & $<0.2$ & 107 & $<0.04$ & $<2$ & 3.7 \\
\hline
\end{tabular}

${ }^{a}$ Concentration determined from a filtered sample.

${ }^{\mathrm{b}}$ Concentration is suspect, and cannot be verified.

${ }^{\mathrm{c}}$ Shade and footnote indicates concentration exceeded the 2007 Missouri Department of Natural Resources chronic standard for the protection of aquatic life (Missouri Department of Natural Resources, 2007b). 
Table 2. Water-quality samples from Blue Spring analyzed for major and trace inorganic constituents, 1925-2006.

[All concentrations are dissolved and in milligrams per liter unless noted otherwise; $\mathrm{ft}^{3} / \mathrm{s}$, cubic feet per second; Cond; specific conductance in microsiemens per centimeter at 25 degrees Celsius; Temp, temperature in degrees Celsius; $\mathrm{CaCO}_{3}$, calcium carbonate; --, no data; $\mathrm{f}$, analysis on filtered sample; $\mathrm{N}$, nitrogen; $\mathrm{P}$, phosphorus; $\mu \mathrm{g} / \mathrm{L}$, micrograms per liter; <, less than; $\mathrm{E}$, estimated value; $\mathrm{u}$, analysis on unfiltered sample; $\mathrm{Ca}: \mathrm{Mg}$, molar ratio of calcium to magnesium]

\begin{tabular}{|c|c|c|c|c|c|c|c|c|c|c|c|c|c|}
\hline Date & Time & $\begin{array}{c}\text { Discharge } \\
\left(\mathrm{ft}^{3} / \mathrm{s}\right)\end{array}$ & Cond & Temp & $\mathrm{pH}$ & Hardness & Calcium & $\begin{array}{l}\text { Magne- } \\
\text { sium }\end{array}$ & Sodium & Potassium & $\begin{array}{c}\text { Alkalinity, } \\
\text { total as } \\
\mathrm{CaCO}_{3}\end{array}$ & $\begin{array}{c}\text { Bicarbon- } \\
\text { ate } \\
\text { (total) }\end{array}$ & Sulfate \\
\hline 06/01/1925 & -- & -- & -- & -- & -- & 150 & 29 & 18 & a 3.0 & b3.8 & 142 & 173 & 0.4 \\
\hline 05/23/1952 & -- & -- & -- & -- & 7.6 & 110 & 24 & 13 & 3.1 & ${ }^{\mathrm{b}} 3.9$ & 105 & 128 & 2.5 \\
\hline 08/24/1965 & -- & 73.6 & -- & -- & 8.2 & 160 & 33 & 20 & a2.3 & b3.3 & 157 & 192 & 6.1 \\
\hline 07/24/1969 & -- & 90.9 & 290 & 13 & 7.8 & 150 & 32 & 18 & 5.5 & .8 & 146 & 178 & 6.3 \\
\hline 09/17/1969 & -- & 83 & 312 & 13 & 7.8 & 160 & 33 & 18 & 5.3 & .8 & 153 & 187 & 6.4 \\
\hline 03/30/1970 & -- & 189 & 184 & 10.5 & 7.5 & 81 & 16 & 10 & 4.7 & 1 & 59 & 72 & 22 \\
\hline 04/27/1971 & -- & 122 & 280 & 13 & 7.7 & -- & 29 & 14 & 4.3 & .7 & 128 & 156 & 8.8 \\
\hline 08/17/1971 & -- & 66.7 & 350 & 13.5 & 7.6 & 150 & 34 & 15 & 5.2 & .7 & 154 & 188 & 2.4 \\
\hline 04/06/1973 & 1430 & 290 & 118 & 11.5 & 7.1 & 52 & 11 & 6.1 & 3.0 & 1.1 & 46 & 56 & 7.6 \\
\hline 08/01/1973 & 1730 & 155 & 215 & 14.5 & 7.5 & 110 & 26 & 12 & 2.9 & .9 & 110 & 130 & 6.9 \\
\hline 05/30/2002 & 1130 & -- & 171 & 14 & 7.0 & 76 & 16.4 & 8.5 & 2.7 & 1.2 & 65 & 81 & 7.9 \\
\hline 03/07/2006 & 1540 & 98.6 & 264 & 12.4 & 7.5 & 150 & 29.8 & 18.1 & 6.3 & .91 & 150 & $189 \mathrm{f}$ & 16 \\
\hline 08/15/2006 & 1315 & 88.4 & 310 & 14.1 & 7.8 & 160 & 32.4 & 18.7 & 3.1 & .7 & 152 & $185 \mathrm{f}$ & 4.78 \\
\hline Date & Time & Chloride & Fluoride & Silica & $\begin{array}{l}\text { Total } \\
\text { dissolved } \\
\text { solids }\end{array}$ & $\begin{array}{c}\text { Nitrite plus } \\
\text { Nitrate, } \\
\text { total as N }\end{array}$ & $\begin{array}{c}\text { Ammonia, } \\
\text { total, } \\
\text { as } N\end{array}$ & $\begin{array}{c}\text { Phos- } \\
\text { phorus, } \\
\text { dissolved } \\
\text { as } \mathrm{P} \\
\end{array}$ & $\begin{array}{l}\text { Phospho- } \\
\text { rus, total } \\
\text { as P }\end{array}$ & $\begin{array}{l}\text { Orthophos- } \\
\text { phorus } \\
\text { as P }\end{array}$ & $\begin{array}{c}\text { Antimony } \\
(\mu \mathrm{g} / \mathrm{L})\end{array}$ & $\begin{array}{c}\text { Arsenic } \\
\text { ( } \mu \mathrm{g} / \mathrm{L})\end{array}$ & $\begin{array}{c}\text { Barium } \\
(\mu \mathrm{g} / \mathrm{L})\end{array}$ \\
\hline 06/01/1925 & -- & 3.7 & -- & 5.6 & 146 & 0.11 & -- & -- & -- & -- & -- & -- & -- \\
\hline 05/23/1952 & -- & 1.8 & -- & 2.6 & 116 & .38 & -- & -- & -- & -- & -- & -- & -- \\
\hline 08/24/1965 & -- & 9.0 & -- & 2.6 & 182 & .2 & -- & -- & -- & -- & -- & -- & -- \\
\hline 07/24/1969 & -- & 6.6 & -- & 6.7 & 160 & .41 & -- & -- & -- & -- & -- & -- & -- \\
\hline 09/17/1969 & -- & 6 & -- & 7.3 & 168 & .29 & -- & -- & -- & -- & -- & -- & -- \\
\hline 03/30/1970 & -- & 6.3 & 0.1 & 4.6 & 103 & .63 & -- & -- & -- & -- & -- & -- & -- \\
\hline 04/27/1971 & -- & 8.2 & -- & 6.9 & 161 & .25 & -- & -- & -- & -- & -- & -- & -- \\
\hline 08/17/1971 & -- & 8.2 & .1 & 8.0 & 181 & .23 & -- & -- & -- & -- & -- & -- & -- \\
\hline 04/06/1973 & 1430 & 1.4 & $<.1$ & 6.1 & 70 & .38 & $<0.01$ & $<0.010$ & $<0.010$ & -- & -- & $<10$ & $<100 \mathrm{u}$ \\
\hline 08/01/1973 & 1730 & 3.3 & $<.1$ & 7.1 & 134 & .52 & .07 & $<.010$ & .02 & -- & -- & 5 & $400 \mathrm{u}$ \\
\hline 05/30/2002 & 1130 & 4.1 & $<.08$ & 3.6 & -- & $2.8 \mathrm{f}$ & -- & $<.010$ & -- & -- & $<0.1$ & -- & 33.3 \\
\hline 03/07/2006 & 1540 & 7.7 & .12 & 7.0 & -- & $.46 \mathrm{f}$ & $<.04 \mathrm{f}$ & $<.04$ & -- & $<0.02$ & $<.2$ & -- & 31.6 \\
\hline $08 / 15 / 2006$ & 1315 & 3.4 & $.06 \mathrm{E}$ & 7.49 & -- & $.39 \mathrm{f}$ & $<.01 \mathrm{f}$ & $<.04$ & -- & .008 & $<.2$ & -- & 30.4 \\
\hline
\end{tabular}


Table 2. Water-quality samples from Blue Spring analyzed for major and trace inorganic constituents, 1925-2006.—Continued

[All concentrations are dissolved and in milligrams per liter unless noted otherwise; $\mathrm{ft}^{3} / \mathrm{s}$, cubic feet per second; Cond; specific conductance in microsiemens per centimeter at 25 degrees Celsius; Temp, temperature in degrees Celsius; $\mathrm{CaCO}_{3}$, calcium carbonate; --, no data; f, analysis on filtered sample; N, nitrogen; P, phosphorus; $\mu \mathrm{g} / \mathrm{L}$, micrograms per liter; <, less than; E, estimated value; u, analysis on unfiltered sample; $\mathrm{Ca}: \mathrm{Mg}$, molar ratio of calcium to magnesium]

\begin{tabular}{|c|c|c|c|c|c|c|c|c|c|c|c|c|c|}
\hline Date & Time & $\begin{array}{c}\text { Beryllium } \\
\text { ( } \mu \mathrm{g} / \mathrm{L})\end{array}$ & $\begin{array}{c}\text { Bismuth } \\
(\mu \mathrm{g} / \mathrm{L})\end{array}$ & $\begin{array}{l}\text { Boron } \\
\text { ( } \mu \mathrm{g} / \mathrm{L})\end{array}$ & $\begin{array}{c}\text { Cadmium } \\
(\mu \mathrm{g} / \mathrm{L})\end{array}$ & $\begin{array}{c}\text { Cerium } \\
\text { ( } \mu \mathrm{g} / \mathrm{L})\end{array}$ & $\begin{array}{c}\text { Chromium } \\
(\mu \mathrm{g} / \mathrm{L})\end{array}$ & $\begin{array}{l}\text { Cobalt } \\
\text { ( } \mu \mathrm{g} / \mathrm{L})\end{array}$ & $\begin{array}{c}\text { Copper } \\
(\mu \mathrm{g} / \mathrm{L})\end{array}$ & $\begin{array}{c}\text { Gallium } \\
\text { ( } \mu \mathrm{g} / \mathrm{L})\end{array}$ & $\begin{array}{l}\text { Germani- } \\
\text { um ( } \mu \mathrm{g} / \mathrm{L})\end{array}$ & $\begin{array}{c}\text { Iron } \\
\text { ( } \mu \mathrm{g} / \mathrm{L})\end{array}$ & $\begin{array}{l}\text { Lanthanum } \\
\text { ( } \mu \mathrm{g} / \mathrm{L})\end{array}$ \\
\hline 06/01/1925 & -- & -- & -- & -- & -- & -- & -- & -- & -- & -- & -- & -- & -- \\
\hline $05 / 23 / 1952$ & -- & -- & -- & -- & -- & -- & -- & -- & -- & -- & -- & -- & -- \\
\hline $08 / 24 / 1965$ & -- & -- & -- & -- & -- & -- & -- & -- & -- & -- & -- & 60 & -- \\
\hline $07 / 24 / 1969$ & -- & -- & -- & -- & -- & -- & -- & -- & -- & -- & -- & $<10$ & -- \\
\hline 09/17/1969 & -- & -- & -- & -- & -- & -- & -- & -- & -- & -- & -- & 70 & -- \\
\hline 03/30/1970 & -- & -- & -- & -- & -- & -- & -- & -- & -- & -- & -- & 20 & -- \\
\hline $04 / 27 / 1971$ & -- & -- & -- & -- & -- & -- & -- & -- & -- & -- & -- & 60 & -- \\
\hline 08/17/1971 & -- & -- & -- & -- & -- & -- & -- & -- & -- & -- & -- & 60 & -- \\
\hline $04 / 06 / 1973$ & 1430 & -- & -- & -- & -- & -- & $<20 \mathrm{u}$ & $6 \mathrm{u}$ & -- & -- & -- & 30 & -- \\
\hline 08/01/1973 & 1730 & -- & -- & -- & $<20 \mathrm{u}$ & -- & $20 \mathrm{u}$ & $<20 \mathrm{u}$ & $<20$ & -- & -- & 160 & -- \\
\hline 05/30/2002 & 1130 & $<0.05$ & $<0.01$ & -- & $<.02$ & 0.03 & 1.3 & .04 & $<.5$ & $<0.02$ & $<0.02$ & $<50$ & 0.03 \\
\hline 03/07/2006 & 1540 & $.2 \mathrm{E}$ & -- & 17.3 & $.03 \mathrm{E}$ & -- & .3 & .12 & .9 & -- & -- & $<6$ & -- \\
\hline $08 / 15 / 2006$ & 1315 & $<.2$ & -- & 11.1 & $<.04$ & -- & .3 & $<.04$ & $<.4$ & -- & -- & $<6$ & -- \\
\hline Date & Time & $\begin{array}{l}\text { Lead } \\
(\mu \mathrm{g} / \mathrm{L})\end{array}$ & $\begin{array}{c}\text { Lithium } \\
(\mu \mathrm{g} / \mathrm{L})\end{array}$ & $\begin{array}{c}\text { Manga- } \\
\text { nese ( } \mu \mathrm{g} / \mathrm{L})\end{array}$ & $\begin{array}{c}\text { Mercury } \\
(\mu \mathrm{g} / \mathrm{L})\end{array}$ & $\begin{array}{l}\text { Molybde- } \\
\text { num ( } \mu \mathrm{g} / \mathrm{L})\end{array}$ & $\begin{array}{c}\text { Nickel } \\
(\mu \mathrm{g} / \mathrm{L})\end{array}$ & $\begin{array}{c}\text { Niobium } \\
(\mu \mathrm{g} / \mathrm{L})\end{array}$ & $\begin{array}{c}\text { Rubidium } \\
\text { ( } \mu \mathrm{g} / \mathrm{L})\end{array}$ & $\begin{array}{c}\text { Scandium } \\
(\mu \mathrm{g} / \mathrm{L})\end{array}$ & $\begin{array}{c}\text { Selenium } \\
(\mu \mathrm{g} / \mathrm{L})\end{array}$ & $\begin{array}{l}\text { Silver } \\
(\mu \mathrm{g} / \mathrm{L})\end{array}$ & $\begin{array}{c}\text { Strontium } \\
(\mu \mathrm{g} / \mathrm{L})\end{array}$ \\
\hline
\end{tabular}

\begin{tabular}{lcccc}
\hline $06 / 01 / 1925$ & -- & -- & -- & -- \\
$05 / 23 / 1952$ & -- & -- & -- & -- \\
$08 / 24 / 1965$ & -- & -- & -- & $<10$ \\
$07 / 24 / 1969$ & -- & -- & -- & $<10$ \\
$09 / 17 / 1969$ & -- & -- & -- & $<10$ \\
$03 / 30 / 1970$ & -- & -- & -- & 10 \\
$04 / 27 / 1971$ & -- & -- & -- & $<3$ \\
$08 / 17 / 1971$ & -- & -- & -- & 5 \\
$04 / 06 / 1973$ & 1430 & 2 u & -- & $<10$ \\
$08 / 01 / 1973$ & 1730 & 650 u & -- & $<10$ \\
$05 / 30 / 2002$ & 1130 & $<.05$ & 0.5 & 0.4 \\
$03 / 07 / 2006$ & 1540 & .17 & $1 \mathrm{E}$ & $<.2$ \\
$08 / 15 / 2006$ & 1315 & $<.08$ & $<2$ & .2
\end{tabular}

$\begin{array}{rrr}-- & -- & - \\ -- & -- & - \\ -- & -- & -- \\ -- & -- & -- \\ -- & -- & -- \\ -- & -- & -- \\ -- & -- & -- \\ -- & -- & -- \\ <0.5 & -- & 6 \mathrm{u} \\ <.5 & -- & <20 \mathrm{u} \\ -- & 0.2 & .5 \\ -- & .4 & .9 \\ -- & .4 \mathrm{E} & .11\end{array}$

--
--
--
--
--
--
--
--
--
--
$<0.02$
--
--

--
--
--
--
--
--
--
--
--
--
1
-
--

--
--
--
--
--
--
--
--
--
--
1
-
-

--
--
--
--
--
--
--
--
--
--
$<1$
01
.1

\begin{tabular}{cc}
-- & -- \\
-- & -- \\
-- & -- \\
-- & -- \\
-- & -- \\
-- & -- \\
-- & -- \\
-- & -- \\
$<2 \mathrm{u}$ & -- \\
$200 \mathrm{u}$ & \multicolumn{2}{c}{-} \\
$<3$ & 30 \\
$<.20$ & 41.2 \\
$<.20$ & 33.3
\end{tabular}


Table 2. Water-quality samples from Blue Spring analyzed for major and trace inorganic constituents,

1925-2006.-Continued

[All concentrations are dissolved and in milligrams per liter unless noted otherwise; $\mathrm{ft}^{3} / \mathrm{s}$, cubic feet per second; Cond; specific conductance in microsiemens per centimeter at 25 degrees Celsius; Temp, temperature in degrees Celsius; $\mathrm{CaCO}_{3}$, calcium carbonate; --, no data; $\mathrm{f}$, analysis on

filtered sample; $\mathrm{N}$, nitrogen; $\mathrm{P}$, phosphorus; $\mu \mathrm{g} / \mathrm{L}$, micrograms per liter; <, less than; E, estimated value; $\mathrm{u}$, analysis on unfiltered sample; Ca:Mg, molar ratio of calcium to magnesium]

\begin{tabular}{|c|c|c|c|c|c|c|c|c|c|}
\hline Date & Time & $\begin{array}{c}\text { Thallium } \\
(\mu \mathrm{g} / \mathrm{L})\end{array}$ & $\begin{array}{c}\text { Thorium } \\
\text { ( } \mu \mathrm{g} / \mathrm{L})\end{array}$ & $\begin{array}{c}\text { Titanium } \\
(\mu \mathrm{g} / \mathrm{L})\end{array}$ & $\begin{array}{c}\text { Vanadium } \\
(\mu \mathrm{g} / \mathrm{L})\end{array}$ & $\begin{array}{c}\text { Yttrium } \\
\text { ( } \mu \mathrm{g} / \mathrm{L})\end{array}$ & $\begin{array}{c}\text { Zinc } \\
(\mu \mathrm{g} / \mathrm{L})\end{array}$ & $\begin{array}{c}\text { Zircon } \\
(\mu \mathrm{g} / \mathrm{L})\end{array}$ & Ca:Mg \\
\hline $06 / 01 / 1925$ & -- & -- & -- & -- & -- & -- & -- & -- & 0.98 \\
\hline $05 / 23 / 1952$ & -- & -- & -- & -- & -- & -- & -- & -- & 1.12 \\
\hline $08 / 24 / 1965$ & -- & -- & -- & -- & -- & -- & -- & -- & 1.00 \\
\hline $07 / 24 / 1969$ & -- & -- & -- & -- & -- & -- & -- & -- & 1.08 \\
\hline 09/17/1969 & -- & -- & -- & -- & -- & -- & -- & -- & 1.11 \\
\hline 03/30/1970 & -- & -- & -- & -- & -- & -- & -- & -- & .97 \\
\hline $04 / 27 / 1971$ & -- & -- & -- & -- & -- & -- & -- & -- & 1.26 \\
\hline 08/17/1971 & -- & -- & -- & -- & -- & -- & -- & -- & 1.37 \\
\hline $04 / 06 / 1973$ & 1430 & -- & -- & -- & -- & -- & $30 \mathrm{u}$ & -- & 1.09 \\
\hline 08/01/1973 & 1730 & -- & -- & -- & -- & -- & $1,400 \mathrm{u}$ & -- & 1.31 \\
\hline $05 / 30 / 2002$ & 1130 & $<0.1$ & $<0.005$ & $<0.1$ & 0.5 & 0.1 & 2 & $<0.1$ & 1.17 \\
\hline 03/07/2006 & 1540 & $<.04$ & -- & -- & $<2$ & -- & 1.5 & -- & 1.00 \\
\hline $08 / 15 / 2006$ & 1315 & $<.04$ & -- & -- & $<2$ & -- & .9 & -- & 1.05 \\
\hline
\end{tabular}

a Sodium concentration estimated as 80 percent of reported combined sodium plus potassium.

${ }^{\mathrm{b}}$ Reported value is combined $\mathrm{Na}$ (sodium) plus $\mathrm{K}$ (potassium), in milligrams per liter. 
Table 4. Grain-size, semi-quantitative bulk mineralogy, and optical mineralogy of the non-magnetic heavy mineral (C3) fraction of streambed-sediment samples from Blue Spring, Big Spring, and Greer Spring, 1995-2002.

[All values are in percent, percent should not be used as absolute number, but rather as relative from one sample site to another; relative standard deviation is at best plus or minus 20 percent; --, not detected]

\begin{tabular}{|c|c|c|c|c|c|c|c|c|c|c|c|c|}
\hline & \multicolumn{3}{|c|}{ Blue Spring } & \multicolumn{3}{|c|}{ Current River } & \multicolumn{3}{|c|}{ Big Spring } & \multicolumn{2}{|c|}{ Current River } & \multirow{2}{*}{$\begin{array}{c}\text { Greer Spring } \\
\text { Branch }\end{array}$} \\
\hline & Orifice & Orifice & Branch & $\begin{array}{l}\text { Upstream } \\
\text { Blue Spring }\end{array}$ & $\begin{array}{c}\text { Downstream } \\
\text { Blue Spring } \\
\text { (east side) }\end{array}$ & $\begin{array}{l}\text { Downstream } \\
\text { Blue Spring } \\
\text { (west side) }\end{array}$ & Orifice & Orifice & Branch & $\begin{array}{l}\text { Upstream } \\
\text { Big Spring }\end{array}$ & $\begin{array}{c}\text { Downstream } \\
\text { Big Spring }\end{array}$ & \\
\hline Constituent & $6 / 20 / 95$ & $5 / 30 / 02$ & $6 / 20 / 95$ & $6 / 20 / 95$ & $6 / 20 / 95$ & $6 / 20 / 95$ & $10 / 16 / 97$ & $6 / 11 / 02$ & $10 / 16 / 97$ & $10 / 16 / 97$ & $10 / 16 / 97$ & $6 / 10 / 02$ \\
\hline \multicolumn{13}{|c|}{ Grain size (percent by weight) } \\
\hline Coarse & 89.2 & 92.3 & 93.4 & 97.7 & 98.2 & 99.5 & 98 & 95 & 99.1 & 98.5 & 98.6 & 98 \\
\hline Silt & 4.1 & 5.6 & 3.2 & 1.1 & .6 & .3 & 1 & 4 & .4 & 1 & .8 & 1.6 \\
\hline Clay (by difference) & 6.7 & 2.1 & 3.4 & 1.2 & 1.2 & .2 & 1 & 1 & .4 & .5 & .6 & .4 \\
\hline \multicolumn{13}{|c|}{ Bulk mineralogy (less than 2 millimeter size fraction) } \\
\hline Quartz & 59 & 69 & 65 & 72 & 72 & 72 & 74 & 93.1 & 92 & 98 & 95 & 80.6 \\
\hline Dolomite & 11 & 29.6 & 9 & 2 & 6 & 1 & 26 & 6.4 & 3 & 1 & 2 & 18.7 \\
\hline Kaolinite & 16 & -- & 14 & 8 & 10 & 11 & -- & -- & -- & -- & -- & -- \\
\hline Amorphous silicates and oxides & $5-10$ & -- & $5-10$ & $5-10$ & $5-10$ & $5-10$ & -- & -- & -- & -- & -- & -- \\
\hline Potassium feldspar & 7 & -- & 6 & 8 & 6 & 8 & -- & -- & 5 & 1 & 3 & -- \\
\hline Plagioclase feldspar & -- & .3 & -- & 4 & -- & -- & -- & -- & -- & -- & -- & -- \\
\hline Hornblende & -- & .8 & -- & -- & -- & -- & -- & .5 & -- & -- & -- & .7 \\
\hline Calcite & -- & .3 & -- & -- & -- & 1 & -- & -- & -- & -- & -- & -- \\
\hline $\begin{array}{l}\text { Other carbonates } \\
\quad \text { including kutnahorite and ankerite }\end{array}$ & -- & -- & -- & 1 & -- & -- & -- & -- & -- & -- & -- & -- \\
\hline
\end{tabular}

\begin{tabular}{|c|c|c|c|c|c|c|c|c|c|c|c|c|}
\hline \multicolumn{13}{|c|}{ Optical mineralogy of the non-magnetic heavy-mineral (C3) fraction } \\
\hline Dolomite $^{a}$ & 80 & 90 & 75 & 10 & 40 & 30 & 6 & 99 & 2 & -- & 7 & 90 \\
\hline Limonite/oxidized rock fragments & 10 & 10 & 15 & 10 & 5 & 50 & 4 & -- & 3 & 5 & 4 & 5 \\
\hline Zircon & -- & -- & 5 & 50 & 40 & 10 & 1 & -- & 1 & 1 & 1 & -- \\
\hline Apatite & -- & -- & 1 & 2 & 1 & 1 & 8 & Trace & -- & -- & 11 & 5 \\
\hline Rutile & 1 & -- & 1 & 20 & 4 & 5 & 2 & -- & 7 & 2 & 2 & -- \\
\hline Tourmaline & 1 & -- & 2 & 5 & 10 & -- & 3 & -- & 4 & 4 & 3 & -- \\
\hline Anatase & -- & -- & 1 & -- & -- & -- & -- & -- & -- & -- & 10 & -- \\
\hline Pyrite & Trace & Trace & Trace & Trace & Trace & Trace & 7 & -- & 5 & 6 & 6 & -- \\
\hline Barite & -- & -- & -- & -- & Trace & Trace & 5 & -- & 6 & -- & 5 & -- \\
\hline Cassiterite & Trace & -- & -- & -- & Trace & -- & -- & -- & -- & 8 & -- & -- \\
\hline Sphene & -- & -- & -- & -- & -- & -- & -- & -- & -- & 3 & -- & -- \\
\hline Cerrusite & Trace & -- & Trace & -- & Trace & -- & -- & -- & -- & -- & -- & -- \\
\hline Galena & Trace & -- & Trace & -- & Trace & -- & -- & -- & -- & -- & -- & -- \\
\hline Hematite & -- & -- & -- & -- & -- & -- & -- & Trace & -- & -- & -- & Trace \\
\hline \multicolumn{13}{|c|}{ Anthropogenic contamination } \\
\hline Lead & -- & -- & - & -- & -- & -- & 9 & -- & 8 & -- & 8 & -- \\
\hline Zinc (silvery metal) & -- & -- & -- & -- & -- & -- & 10 & -- & 9 & 7 & 9 & -- \\
\hline Brass & -- & -- & -- & -- & -- & Trace & -- & -- & -- & -- & -- & -- \\
\hline Glass & -- & -- & -- & -- & -- & -- & -- & Trace & -- & -- & -- & -- \\
\hline
\end{tabular}

${ }^{a}$ Dolomite probably is present as a contaminant because its density $\left[2.85\right.$ grams per cubic centimeter $\left.\left(\mathrm{gm} / \mathrm{cm}^{3}\right)\right]$ is similar to that of the bromoform $\left(2.87 \mathrm{gm} / \mathrm{cm}^{3}\right)$ used to concentrate the heavy mineral fraction. 
Table 5. Concentrations of major and trace elements in the fine (less than 0.063-millimeter size) fraction of streambed-sediment samples from Blue Spring, Current River, Big Spring, and Greer Spring, 1995-2002.

$[<$, less than $]$

\begin{tabular}{|c|c|c|c|c|c|c|c|c|c|c|c|c|}
\hline \multirow[b]{3}{*}{ Constituent } & \multicolumn{3}{|c|}{ Blue Spring } & \multicolumn{3}{|c|}{ Current River } & \multicolumn{3}{|c|}{ Big Spring } & \multicolumn{2}{|c|}{ Current River } & \multirow{2}{*}{$\begin{array}{c}\text { Greer Spring } \\
\text { Branch }\end{array}$} \\
\hline & Orifice & Orifice & Branch & $\begin{array}{c}\text { Upstream } \\
\text { Blue } \\
\text { Spring }\end{array}$ & $\begin{array}{l}\text { Downstream } \\
\text { Blue Spring } \\
\text { (east side) }\end{array}$ & $\begin{array}{l}\text { Downstream } \\
\text { Blue Spring } \\
\text { (west side) }\end{array}$ & Orifice & Orifice & Branch & $\begin{array}{l}\text { Upstream } \\
\text { Big } \\
\text { Spring }\end{array}$ & $\begin{array}{l}\text { Down- } \\
\text { stream Big } \\
\text { Spring }\end{array}$ & \\
\hline & 06/20/95 & 05/30/02 & 06/20/95 & $06 / 20 / 95$ & 06/20/95 & 06/20/95 & 10/16/97 & 06/11/02 & 10/16/97 & 10/16/97 & 10/16/97 & 06/10/02 \\
\hline \multicolumn{13}{|c|}{ Major elements, in percent by weight } \\
\hline Aluminum & 6.6 & 5.9 & 5.9 & 5.3 & 4.9 & 5.7 & 6.0 & 4.7 & 4.2 & 4.7 & 4.4 & 4.6 \\
\hline Calcium & 1.82 & 2.8 & 1.47 & 1.28 & 1.44 & .79 & 2.6 & .77 & 1.8 & .89 & 1.1 & 1.4 \\
\hline Iron & 3.3 & 3.0 & 3.0 & 2.3 & 2.6 & 2.7 & 3.3 & 2.3 & 2.2 & 2.5 & 2.4 & 2.6 \\
\hline Potassium & 1.17 & 1.0 & 1.11 & .99 & 1.02 & 1.09 & 1.1 & 1.0 & .86 & 1 & .89 & 1.0 \\
\hline Magnesium & 1.44 & 1.9 & 1.12 & .95 & .82 & .59 & 1.8 & .53 & 1.2 & .47 & .59 & .8 \\
\hline Sodium & .08 & .05 & .14 & .15 & .19 & .19 & .09 & .2 & .13 & .19 & .16 & .14 \\
\hline Phosphorus & .08 & .08 & .09 & .09 & .1 & .08 & .13 & .08 & .1 & .07 & .09 & .09 \\
\hline Titanium & .26 & .24 & .26 & .25 & .24 & .33 & .28 & .31 & .23 & .32 & .29 & .33 \\
\hline \multicolumn{13}{|c|}{ Mississippi Valley Type-related trace elements, in milligrams per kilogram } \\
\hline Arsenic & $<10$ & $<10$ & $<10$ & $<10$ & $<10$ & $<10$ & 17 & 11 & 11 & $<10$ & 10 & 15 \\
\hline Cadmium & 2.40 & $<2$ & 2.10 & 1.60 & 1.70 & 1.20 & $<2$ & $<2$ & $<2$ & $<2$ & $<2$ & $<2$ \\
\hline Cobalt & 36 & 32 & 34 & 20 & 27 & 22 & 28 & 16 & 19 & 26 & 31 & 20 \\
\hline Copper & 52 & 99 & 45 & 35 & 47 & 27 & 40 & 61 & 39 & 29 & 31 & 99 \\
\hline Lead & 41 & 57 & 26 & 27 & 25 & 24 & 53 & 49 & 45 & 33 & 35 & 84 \\
\hline Nickel & 66 & 72 & 57 & 36 & 47 & 36 & 51 & 35 & 35 & 29 & 34 & 42 \\
\hline Zinc & 104 & 121 & 90 & 86 & 86 & 81 & 160 & 114 & 110 & 74 & 87 & 151 \\
\hline \multicolumn{13}{|c|}{ Other trace elements, in milligrams per kilogram } \\
\hline Barium & 272 & 272 & 336 & 278 & 369 & 386 & 230 & 402 & 220 & 370 & 310 & 438 \\
\hline Beryllium & 3.6 & 3.6 & 3.10 & 2.3 & 2.1 & 2.3 & 3 & 2.2 & 2 & 2 & 2 & 2.2 \\
\hline Bismuth & $<10$ & $<10$ & $<10$ & $<10$ & $<10$ & $<10$ & $<10$ & $<10$ & $<10$ & $<10$ & $<10$ & $<10$ \\
\hline Cesium & 93 & 89 & 85 & 64 & 70 & 78 & 95 & 85 & 77 & 84 & 78 & 97 \\
\hline Chromium & 55 & 48 & 52 & 58 & 74 & 62 & 61 & 54 & 54 & 60 & 60 & 66 \\
\hline Europium & $<2$ & 2.5 & $<2$ & $<2$ & $<2$ & $<2$ & 2 & $<2$ & $<2$ & $<2$ & $<2$ & $<2$ \\
\hline Gallium & 17 & 12 & 15 & 13 & 11 & 14 & 24 & 10 & 14 & 12 & 13 & 9.9 \\
\hline Gold & $<8$ & $<8$ & $<8$ & $<8$ & $<8$ & $<8$ & $<8$ & $<8$ & $<8$ & $<8$ & $<8$ & $<8$ \\
\hline
\end{tabular}


Table 5. Concentrations of major and trace elements in the fine (less than 0.063-millimeter size) fraction of streambed-sediment samples from Blue Spring, Current River, Big Spring, and Greer Spring, 1995-2002.-Continued

$[<$, less than $]$

\begin{tabular}{|c|c|c|c|c|c|c|c|c|c|c|c|c|}
\hline \multirow[b]{3}{*}{ Constituent } & \multicolumn{3}{|c|}{ Blue Spring } & \multicolumn{3}{|c|}{ Current River } & \multicolumn{3}{|c|}{ Big Spring } & \multicolumn{2}{|c|}{ Current River } & \multirow{2}{*}{$\begin{array}{c}\text { Greer Spring } \\
\text { Branch }\end{array}$} \\
\hline & Orifice & Orifice & Branch & $\begin{array}{c}\text { Upstream } \\
\text { Blue } \\
\text { Spring }\end{array}$ & $\begin{array}{l}\text { Downstream } \\
\text { Blue Spring } \\
\text { (east side) }\end{array}$ & $\begin{array}{l}\text { Downstream } \\
\text { Blue Spring } \\
\text { (west side) }\end{array}$ & Orifice & Orifice & Branch & $\begin{array}{c}\text { Upstream } \\
\text { Big } \\
\text { Spring }\end{array}$ & $\begin{array}{l}\text { Down- } \\
\text { stream Big } \\
\text { Spring }\end{array}$ & \\
\hline & $06 / 20 / 95$ & 05/30/02 & $06 / 20 / 95$ & $06 / 20 / 95$ & $06 / 20 / 95$ & $06 / 20 / 95$ & 10/16/97 & 06/11/02 & 10/16/97 & 10/16/97 & 10/16/97 & $06 / 10 / 02$ \\
\hline \multicolumn{13}{|c|}{ Other trace elements, in milligrams per kilogram-Continued } \\
\hline Holmium & $<4$ & $<4$ & $<4$ & $<4$ & $<4$ & $<4$ & $<4$ & $<4$ & $<4$ & $<4$ & $<4$ & $<4$ \\
\hline Lanthanum & 54 & 48 & 49 & 40 & 38 & 45 & 57 & 43 & 42 & 42 & 42 & 46 \\
\hline Lithium & 49 & 38 & 44 & 40 & 32 & 37 & 43 & 26 & 28 & 31 & 29 & 27 \\
\hline Manganese & 1,630 & 2,120 & 1,490 & 390 & 1,580 & 840 & 1,300 & 1,190 & 750 & 1,600 & 1,500 & 2,210 \\
\hline Molybdenum & $<2$ & 3.6 & $<2$ & $<2$ & $<2$ & $<2$ & 2 & $<2$ & $<2$ & $<2$ & $<2$ & 2.9 \\
\hline Neodymium & 60 & 55 & 53 & 42 & 34 & 41 & 67 & 40 & 49 & 41 & 41 & 38 \\
\hline Niobium & 15 & 5.5 & 13 & 13 & 12 & 16 & 12 & 11 & 10 & 17 & 16 & 5.7 \\
\hline Scandium & 12 & 11 & 10 & 8.9 & 7.9 & 9.7 & 12 & 8.5 & 8 & 8 & 8 & 7.6 \\
\hline Silver & $<2$ & $<2$ & $<2$ & $<2$ & $<2$ & $<2$ & $<2$ & $<2$ & $<2$ & $<2$ & $<2$ & $<2$ \\
\hline Strontium & 46 & 39 & 50 & 45 & 57 & 48 & 40 & 39 & 38 & 43 & 40 & 37 \\
\hline Tantalum & $<40$ & $<40$ & $<40$ & $<40$ & $<40$ & $<40$ & $<40$ & $<40$ & $<40$ & $<40$ & $<40$ & $<40$ \\
\hline Thorium & 12.4 & 8.3 & 11.9 & 11.3 & 9.1 & 11.9 & 11 & 6.5 & 6 & 10 & 9 & 7.3 \\
\hline Tin & $<5$ & 11 & $<5$ & $<5$ & $<5$ & $<5$ & $<5$ & 7.3 & $<5$ & $<5$ & $<5$ & 18 \\
\hline Uranium & $<100$ & $<100$ & $<100$ & $<100$ & $<100$ & $<100$ & $<100$ & $<100$ & $<100$ & $<100$ & $<100$ & $<100$ \\
\hline Vanadium & 97 & 83 & 89 & 76 & 72 & 81 & 90 & 66 & 62 & 70 & 68 & 74 \\
\hline Ytterbium & 4.6 & 4.4 & 3.8 & 2.1 & 2.5 & 3 & 4 & 2.8 & 3 & 3 & 3 & 2.7 \\
\hline Yttrium & 48 & 46 & 42 & 31 & 26 & 30 & 44 & 30 & 32 & 28 & 28 & 31 \\
\hline
\end{tabular}


Table 6. Semi-quantitative concentrations of major and trace elements in the non-magnetic heavy mineral (C3) fraction of streambed-sediment samples from Blue Spring and the Current River near Blue Spring, 1995.

[All concentrations semi-quantitative; <, less than; >, greater than]

\begin{tabular}{|c|c|c|c|c|c|}
\hline \multirow[b]{2}{*}{ Constituent } & \multicolumn{2}{|c|}{ Blue Spring } & \multicolumn{3}{|c|}{ Current River } \\
\hline & $\begin{array}{l}\text { Orifice } \\
06 / 20 / 95\end{array}$ & $\begin{array}{l}\text { Branch } \\
06 / 20 / 95\end{array}$ & $\begin{array}{c}\text { Downstream } \\
\text { Blue Spring } \\
\text { (east side) } \\
06 / 20 / 95\end{array}$ & $\begin{array}{c}\text { Downstream } \\
\text { Blue Spring } \\
\text { (west side) } \\
\text { 06/20/95 }\end{array}$ & $\begin{array}{c}\text { Upstream } \\
\text { Blue Spring } \\
06 / 20 / 95\end{array}$ \\
\hline \multicolumn{6}{|c|}{ Percent by weight } \\
\hline Calcium & 10 & 7 & 7 & 1 & 1 \\
\hline Iron & 2 & 3 & .5 & 5 & 1.5 \\
\hline Magnesium & 7 & 7 & 3 & .5 & 1.5 \\
\hline Phosphorous & .7 & .7 & .5 & .7 & .5 \\
\hline Sodium & $<.5$ & $<.5$ & $<.5$ & $<.5$ & $<.5$ \\
\hline Titanium & .5 & .7 & 2 & $>2$ & $>2$ \\
\hline \multicolumn{6}{|c|}{ Mississippi Valley Type-related trace elements, in milligrams per kilogram } \\
\hline Arsenic & $<500$ & $<500$ & $<500$ & $<500$ & $<500$ \\
\hline Cadmium & $<50$ & $<50$ & $<50$ & $<50$ & $<50$ \\
\hline Cobalt & $<20$ & $<20$ & $<20$ & $<20$ & $<20$ \\
\hline Copper & 200 & 30 & 50 & 200 & $<10$ \\
\hline Lead & 2,000 & 1,000 & 1,500 & $<20$ & $<20$ \\
\hline Nickel & 20 & 30 & $<10$ & 50 & $<10$ \\
\hline Zinc & $<500$ & $<500$ & $<500$ & $<500$ & $<500$ \\
\hline \multicolumn{6}{|c|}{ Other trace elements, in milligrams per kilogram } \\
\hline Antimony & $<200$ & $<200$ & $<200$ & $<200$ & $<200$ \\
\hline Barium & 200 & 200 & 200 & 700 & 100 \\
\hline Beryllium & $<2$ & $<2$ & $<2$ & $<2$ & $<2$ \\
\hline Bismuth & $<20$ & $<20$ & 50 & $<20$ & $<20$ \\
\hline Boron & 100 & 200 & 1,000 & 300 & 1,000 \\
\hline Chromium & 50 & 50 & 100 & 50 & 30 \\
\hline Gallium & $<10$ & $<10$ & $<10$ & $<10$ & $<10$ \\
\hline Germanium & $<20$ & $<20$ & $<20$ & $<20$ & $<20$ \\
\hline Gold & $<20$ & $<20$ & $<20$ & $<20$ & $<20$ \\
\hline Lanthanum & $<100$ & $<100$ & 100 & $<100$ & $<100$ \\
\hline Manganese & 700 & 300 & 150 & 500 & 150 \\
\hline Molybdenum & $<10$ & $<10$ & $<10$ & $<10$ & $<10$ \\
\hline Niobium & $<50$ & $<50$ & $<50$ & $<50$ & $<50$ \\
\hline Palladium & $<5$ & $<5$ & $<5$ & $<5$ & $<5$ \\
\hline Platinum & $<20$ & $<20$ & $<20$ & $<20$ & $<20$ \\
\hline Scandium & 15 & 10 & 100 & 10 & 70 \\
\hline Silver & $<1$ & $<1$ & 100 & 5 & 20 \\
\hline Strontium & $<200$ & $<200$ & $<200$ & $<200$ & $<200$ \\
\hline Thorium & $<200$ & $<200$ & $<200$ & $<200$ & $<200$ \\
\hline Tin & 200 & $<20$ & 1,000 & $<20$ & $<20$ \\
\hline Tungsten & $<50$ & $<50$ & $<50$ & $<50$ & $<50$ \\
\hline Vanadium & 50 & 70 & 100 & 150 & 70 \\
\hline Yttrium & 50 & 50 & 200 & 100 & 100 \\
\hline Zirconium & 2,000 & $>2,000$ & $>2,000$ & $>2,000$ & $>2,000$ \\
\hline
\end{tabular}



Publishing support provided by:

Rolla Publishing Service Center

For more information concerning this publication, contact:

Director, USGS Missouri Water Science Center

1400 Independence Road

Rolla, M0 65401

(573) 308-3667

Or visit the Missouri Water Science Center Web site at:

http://mo.water.usgs.gov 

